United States Department of Commerce

Technology Administration

National Institute of Standards and Technology

NIST Technical Note 1504

\title{
Precision Spectroscopy, Diode Lasers, and Optical Frequency Measurement Tẹchnology
}

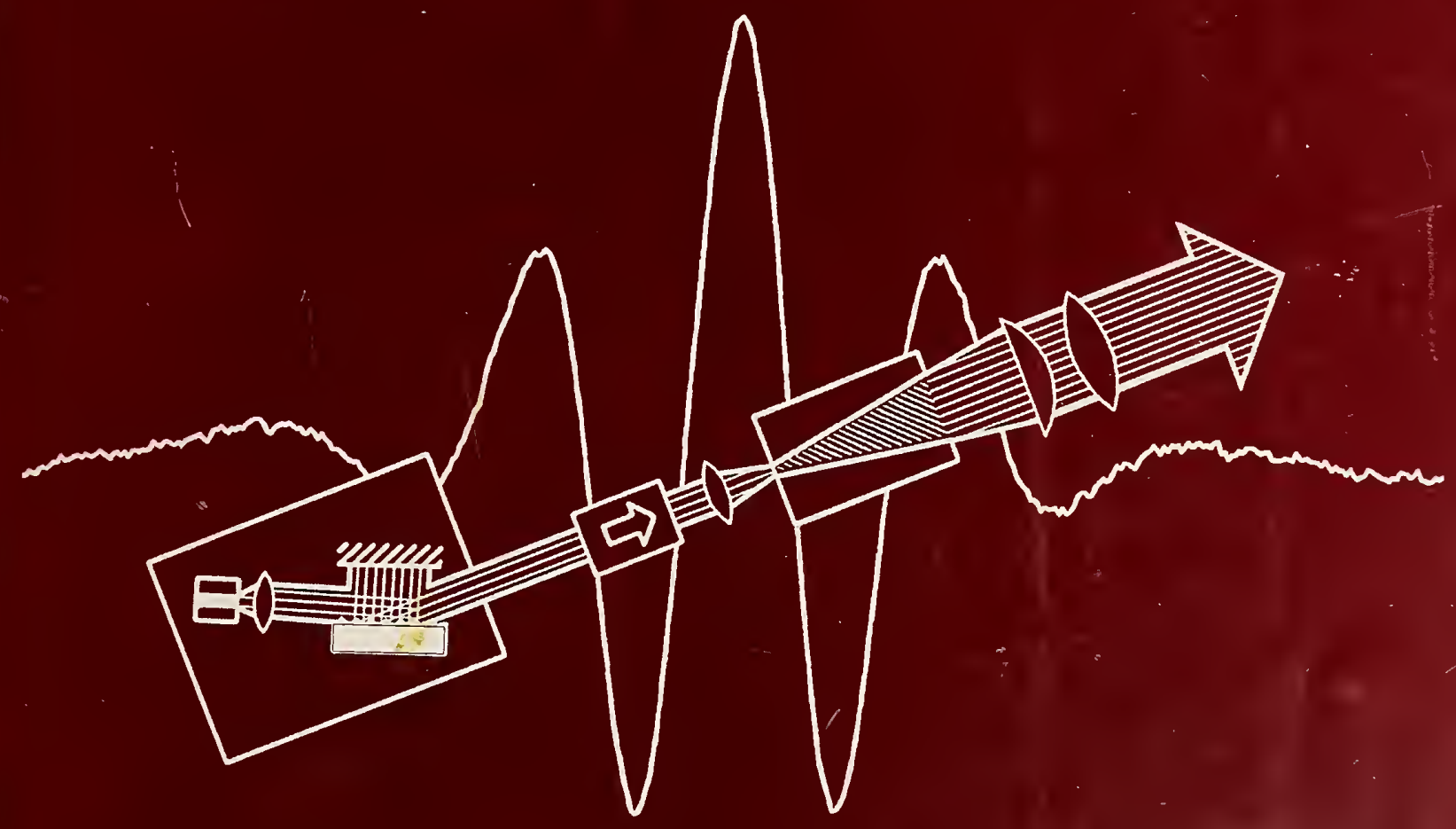

Selected publications of the Optical Frequency Measuremènt Group Time and Frequency Division NIST, Boulder, Colorado
Edited by:

$$
\begin{aligned}
& \text { Leo Hollberg } \\
& \text { Richard Fox } \\
& \text { Steve Waltman } \\
& \text { Hugh Robinson }
\end{aligned}
$$

QC 


\section{DATE DUE}

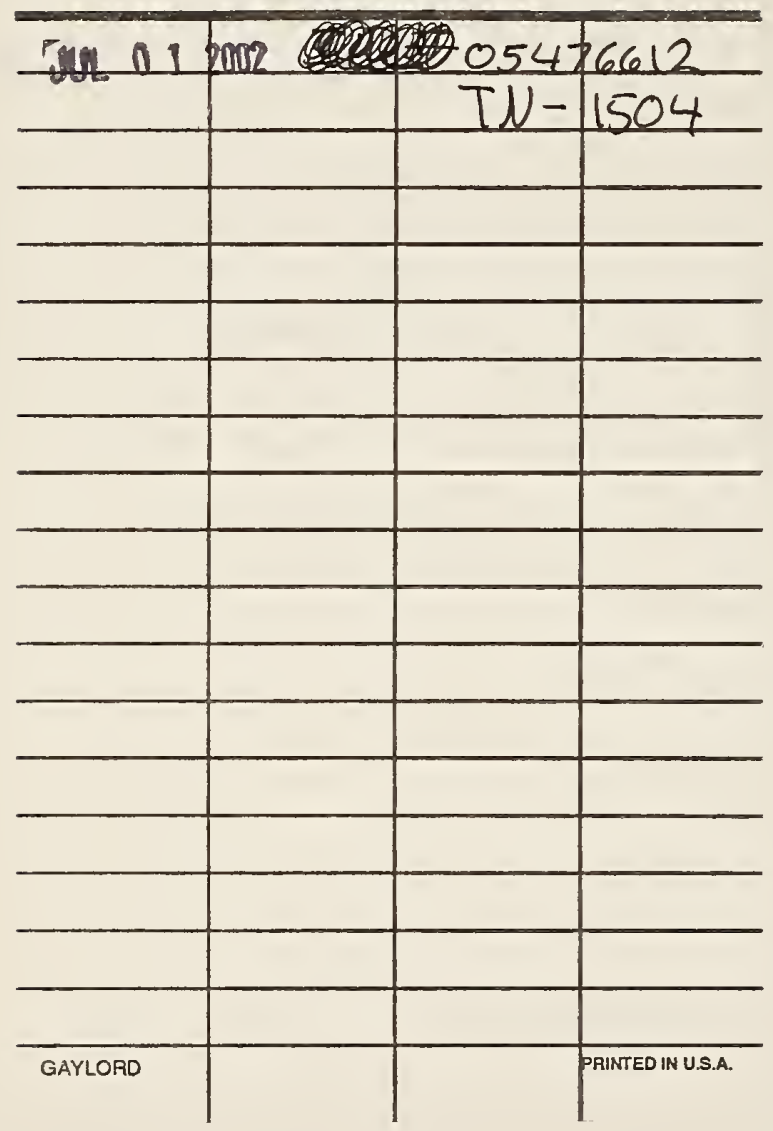




\section{Precision Spectroscopy, Diode Lasers, and Optical Frequency Measurement Technology}

Selected publications of the Optical Frequency Measurement Group of the Time and Frequency Division

Edited by

Leo Hollberg

Richard Fox

Steve Waltman

Hugh Robinson

Time and Frequency Division

Physics Laboratory

National Institute of Standards and Technology

325 Broadway

Boulder, Colorado 80303-3328

Supported in part by

Air Force Office of Scientific Research

and

National Aeronautics and Space Administration

May 1998

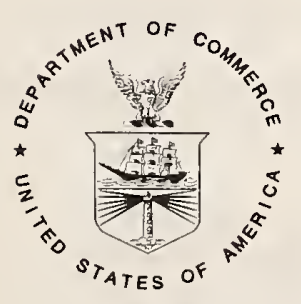

U.S. DEPARTMENT OF COMMERCE, William M. Daley, Secretary 
National Institute of Standards and Technology Technical Note Natl. Inst. Stand. Technol., Tech. Note 1504, 292 pages (May 1998)

CODEN:NTNOEF

\section{U.S. GOVERNMENT PRINTING OFFICE}

WASHINGTON: 1998

For sale by the Superintendent of Documents, U.S. Government Printing Office, Washington, DC 20402-9325 


\section{Precision Spectroscopy, Diode Lasers, and Optical Frequency Measurement Technology}

Preface

This compilation is a selected set of reprints from the Optical Frequency Measurement Group of the Time and Frequency Division of the National Institute of Standards and Technology, and consists of work published between 1987 and 1997 . The two main programs represented here are (1) development of tunable diode-laser technology for scientific applications and precision measurements, and (2) research toward the goal of realizing optical-frequency measurements and synthesis. The papers are organized chronologically in five, somewhat arbitrarily chosen categories: Diode Laser Technology, Tunable Laser Systems, Laser Spectroscopy, Optical Synthesis and Extended Wavelength Coverage, and Multi-Photon Interactions and Optical Coherences. Additional publications mentioned in Section VI are not included here, but are available from the Time and Frequency Division Office. For more information, visit our website at http://www.bldroc.gov/timefreq/ or phone: 303-497-3276, Fax: 303-497-6461.

Other NIST Publications may be purchased from the U.S. Government Printing Office (GPO). The GPO website is http://umw.access.gpo.gov/ or phone: 202-512-1800. Older publications may be purchased from the National Technical Information Service whose website is http://www.ntis.gov/ or phone: 703-487-4650.

Acknowledgments: A number of people have worked with our group during this time period and the results presented here are due in large part to their efforts. Special recognition goes to the many important contributions of $F$. Bondu, J. Kitching, N. Mackie, J. Magyar, J. Marquardt, C. Oates, M. Stephens, C.S. Weimer, J.S. Wells, A.S. Zibrov, and T.P. Zibrova. We have also benefited greatly from collaborations with guest researchers from around the world, including A. Dax, L. D'Evelyn, M.D. Lukin, M. Mfrtz, D.E. Nikonov, K.P. Petrov, O. Pfister, U. Simon, G. Tino, D.A. Van Baak, V.V. Vasiliev, and L. Zink. Particularly important to our research program have been our long-term collaborations with V.L. Velichansky et al. from the Lebedev Institute, Moscow, Russia; F.K. Tittel and R.F. Curl et al. from Rice Univ.; M.O. Scully et al. from Texas A\&M Univ.; M. Inguscio et al. from Firenze, Italy; D. Welch et al. from SDL Company; and A.R. Ravishhankara et al. from NOAA. We gratefully acknowledge the continual guidance and help provided by J.C. Bergquist and J.L. Hall. Our special thanks to Anna Dillon and Wendy Ortega for their diligence and organization in the tedious task of bringing all these papers together into a coherent package. We also acknowledge the partial support of this work from the Air Force Office of Scientific Research, and NASA.

Leo Hollberg

Richard Fox

Steve Waltman

Hugh Robinson

Time and Frequency Division

National Institute of Standards and Technology

325 Broadway, Boulder, CO 80303

December 1997 



\section{CONTENTS}

PART I

DIODE LASER TECHNOLOGY

"Frequency Stabilization of Semiconductor Lasers by Resonant Optical Feedback,"

B. Dahmani, L. Hollberg, and R. Drullinger, Opt. Lett. 12, 876-878 (1987). TN 1

"Modulatable Narrow-Linewidth Semiconductor Lasers," L. Hollberg and M. Ohtsu, Appl. Phys. Lett. $\underline{52}$, 944-946 (1988). TN 4

"Using Diode Lasers for Atomic Physics," C.E. Wieman and L. Hollberg, Rev. Sci. Instrum. $\underline{62}, 1-20$ (1991). TN 7

"High-Sensitivity Spectroscopy with Diode Lasers," R.W. Fox, H.G. Robinson, A.S. Zibrov, N. Mackie, J. Marquardt, J. Magyar, and L.W. Hollberg, Proc. SPIE Conf. on Frequency Stabilized Lasers and Their Applications, 1837 (ed. Y.C. Chung) 360-365 (1992). TN 27

"The Diode Laser as a Spectroscopic Tool," R.W. Fox, C.S. Weimer, L. Hollberg, and G.C. Turk, Spectrochim. Acta Rev. 15, 291-299 (1993). TN 33

"A CPW Phase-Locked Loop for Diode-Laser Stabilization," L. D'Evelyn,

L. Hollberg, and Z.B. Popovic, Proc. 1994 IEEE MTT-S Intl. Microwave Symp., San Diego, CA (1994). TN 42

"Amplitude Modulation on Frequency Locked Extended Cavity Diode Lasers," R.W. Fox, L. D'Evelyn, H.G. Robinson, C.S. Weimer, and L. Hollberg, Proc. SPIE Conference, $\underline{2378}$, 58-62 (1995). TN 46

"High-Accuracy Spectroscopy with Semiconductor Lasers: Application to LaserFrequency Stabilization," L. Hollberg, V.L. Velichansky, C.S. Weimer, and R.W. Fox, in Chapter 3 of Frequency Control of Semiconductor Lasers,

(ed. M. Ohtsu, John Wiley and Sons) 73-93 (1996). TN 51

"Grating-Tuned Semiconductor MOPA Lasers for Precision Spectroscopy," J.H. Marquardt, F.C. Cruz, M. Stephens, C.W. Oates, L.W. Hollberg, J.C. Bergquist, D.F. Welch, D. Mehuys, and S. Sanders, Proc. SPIE Conf. 2834 34-40 (1996).... TN 71

"Semiconductor Diode Lasers," R.W. Fox, L. Hollberg, and A.S. Zibrov, in Atomic, Molecular, and Optical Physics: Electromagnetic Radiation, (ed. F.B. Dunning and R.G. Hulet, Academic Press) 77-102 (1997) of 29C series Experimental Methods in the Physical Sciences. TN 78

\section{PART II TUNABLE LASER SYSTEMS}

"CW Dye Lasers," L. Hollberg, Chapter 5 in Dye Laser Principles: With Applications (ed. F.J. Duarte and Lloyd W. Hillman, Academic Press) 185-238 (1990). 


\section{PART III LASER SPECTROSCOPY}

"Hyperfine Structure of the Metastable ${ }^{5} \mathrm{~S}_{2}$ State of ${ }^{17} \mathrm{O}$ Using an AlGaAs Diode Laser at 777 nm," G.M. Tino, L. Hollberg, A. Sasso, and M. Inguscio, Phys. Rev. Lett. 64, 2999-3002 (1990).

"High-Resolution Diode-Laser Spectroscopy of Calcium," A.S. Zibrov, R.W. Fox, R. Ellingsen, C.S. Weimer, V.L. Velichansky, G.M. Tino, and L. Hollberg, Appl.

Phys. B $\underline{59}, 327-331$ (1994).

TN 163

"Sub-Doppler Frequency Measurements on OCS at $87 \mathrm{THz}(3.4 \mu \mathrm{m})$ with the CO Overtone Laser," A. Dax, J.S. Wells, L. Hollberg, A.G. Maki, and W. Urban, J.

Mol. Spectrosc. 168, 416-428 (1994). TN 168

"Sub-Doppler Frequency Measurements on OCS near 1689 and $1885 \mathrm{CM}^{-1}$," J.S. Wells, A. Dax, L. Hollberg, and A.G. Maki, J. Mol. Spectrosc. $\underline{170}, 75-81$ (1995) TN 181

"Detection of Methane in Air Using Diode-Laser Pumped Difference-Frequency Generation near $3.2 \mu \mathrm{m}$," K.P. Petrov, S. Waltman, U. Simon, R.F. Curl, F.K. Tittel, E.J. Dlugokencky, and L. Hollberg, Appl. Phys. B $\underline{61}$, 553-558 (1995). TN 188

"Precise Measurement of Methane in Air Using Diode-Pumped 3.4- $\mu \mathrm{m}$ DifferenceFrequency Generation in PPLN," K.P. Petrov, S. Waltman, E.J. Dlugokencky, M. Arbore, M.M. Fejer, F.K. Tittel, and L.W. Hollberg, Appl. Phys. B $\underline{64}$, 567-572 (1997). TN 194

"Reactive Uptake of $\mathrm{NO}_{3}$ on Pure Water and lonic Solutions," Y. Rudich, R.K. Talukdar, and A.R. Ravishhankara, J. Geophys. Res. 101, 21,023-21,031 (1996).

"An All-Diode-Laser Optical Frequency Reference Using Laser-Trapped Calcium," C.W. Oates, M. Stephens, and L. Hollberg, Proc. 1997 Intl. Frequency Control Symp., 219-224 (1997).

\section{PART IV OPTICAL SYNTHESIS AND EXTENDED WAVE LENGTH COVERAGE}

"Precise Optical Frequency References and Difference Frequency Measurements with Diode Lasers," S. Waltman, A. Romanovsky, J. Wells, R.W. Fox, L. Hollberg, M.P. Sassi, and H.G. Robinson, Proc. SPIE Conf. on Frequency Stabilized Lasers and Their Applications (ed. Y.C. Chung) 1837, 386-391 (1992).

"Proposed Sum-and-Difference Method for Optical-Frequency Measurements in the Near Infrared," D.A. Van Baak and L. Hollberg, Opt. Lett. $\underline{19}$, 1586-1588 (1994).

"External-Cavity Difference-Frequency Source Near $3.2 \mu \mathrm{m}$ Based on Combining a Tunable Diode Laser with a Diode-Pumped Nd:YAG Laser in AgGaS2," U. Simon, S. Waltman, I. Loa, F.K. Tittel, and L. Hollberg, J. Opt. Soc. Am. B 12, 323-327 (1995). 
"Division by 3 of Optical Frequencies by Use of Difference-Frequency Generation in Noncritically Phase-Matched RbTiOAsO 4 ," O. Pfister, M. Mürtz, J.S. Wells,

L. Hollberg, and J.T. Murray, Opt. Lett. 21, 1387-1389 (1996). TN 229

"Demonstration of a Phase-Lockable Microwave to Submillimeter-Wave Sweeper," S.B. Waltman, L.W. Hollberg, K.A. Mclntosh, and E.R. Brown, Proc. SPIE 2842, 55-58 (1996).

"A 1 GHz Optical-Delay-Line Oscillator Driven by a Diode Laser," J. Kitching,

L. Hollberg, and F.L. Walls, Proc. 1996 Intl. IEEE Freq. Control Symp., 807-814 (1996).

"Continuous-Wave Frequency Tripling and Quadrupling by Simultaneous Three-

Wave Mixings in Periodically Poled Crystals: Application to a Two-Step 1.19-

10.71- $\mu \mathrm{m}$ Frequency Bridge," O. Pfister, J.S. Wells, L. Hollberg, L. Zink,

D.A. Van Baak, M.D. Levenson, and W.R. Bosenberg, Opt. Lett. 22, 1211-1213

(1997).

TN 244

\section{PART V MULTIPHOTON INTERACTONS AND OPTICAL COHERENCES}

"Optical Probing of Cold Trapped Atoms," R.W. Fox, S.L. Gilbert, L. Hollberg,

J.H. Marquardt, and H.G. Robinson, Opt. Lett. 18, 1456-1458 (1993).

"Experimental Demonstration of Laser Oscillation without Population Inversion via Quantum Interference in Rb," A.S. Zibrov, M.D. Lukin, D.E. Nikonov, L. Hollberg, M.O. Scully, V.L. Velichansky, and H.G. Robinson, Phys. Rev. Lett. $\underline{75}, 1499-1502$ (1995). TN 250

"Line Shapes of Cascade Two-Photon Transitions in a Cesium Magneto-Optic Trap," J.H. Marquardt, H.G. Robinson, and L. Hollberg, J. Opt. Soc. Am. B 13, 1384-1393 (1996).

"Population- and Coherence-Induced Gain and Self-Oscillations in Alkali Vapor,"

A.S. Zibrov, H.G. Robinson, V.L. Velichansky, V.V. Vasiliev, L. Hollberg,

E. Arimondo, M.D. Lukin, and M.O. Scully, Proc. Fifth Symp. on Frequency

Standards and Metrology (ed. J.C. Bergquist, World Scientific) 490-492 (1995) TN 264

"Experimental Demonstration of Enhanced Index of Refraction via Quantum Coherence in Rb," A.S. Zibrov, M.D. Lukin, L. Hollberg, D.E. Nikonov, M.O. Scully, H.G. Robinson, and V.L. Velichansky, Phys. Rev. Lett. 76, 3935-3938 (1996).

"Spectroscopy in Dense Coherent Media: Line Narrowing and Interference Effects," M.D. Lukin, M. Fleischhauer, A.S. Zibrov, H.G. Robinson, V.L. Velichansky, L. Hollberg, and M.O. Scully, Phys. Rev. Lett. $\underline{79}$, 2959-2962 (1997). 


\section{PART VI ADDITIONAL PUBLICATIONS (see preface for additional information)}

"Diode Lasers and Spectroscopic Applications," L. Hollberg, R. Fox, N. Mackie, A.S. Zibrov, V.L. Velichansky, R. Ellingsen, and H.G. Robinson, Proc. 10th Intl. Conf. on Laser Spectroscopy (TENICOLS) (eds. M. Ducloy, E. Giacobino, and G. Carry) 347-352 (1991).

"Nonlinear Optics for Optical Frequency Synthesis and an Optical Divide by 3," M. Mürtz, O. Pfister, J.H. Marquardt, M. Stephens, and J.S. Wells, Proc. Fifth Symp. on Frequency Standards, 171-178 (1995).

Diode Lasers and their Application to Spectroscopy," L. Hollberg, in Applied Laser Spectroscopy (ed. W. Demtroder and M. Inguscio, Plenum Press, New York) 117-125 (1990).

"Diode Lasers and Metrology," R. Fox, G. Turk, N. Mackie, T. Zibrova, M.P. Sassi, J. Marquardt, A.S. Zibrov, C. Weimer, and L. Hollberg, Solid State Lasers: New Developments and Applications (ed. M. Ignuscio and R. Wallenstein, Plenum Press, New York), 279-286 (1993).

"Diode Lasers for Frequency Standards and Precision Spectroscopy," L. Hollberg, J. Aman, S. Waltman, J.H. Marquardt, M. Stephens, R.W. Fox, D.A. Van Baak, C.S. Weimer, H.G. Robinson, A.S. Zibrov, N. Mackie, T.P. Zibrova, and L. Pendrill, Proc. 1995 Intl. Frequency Control Symp., 185-190 (1995).

"Experimental Demonstration of Enhanced Index of Refraction via Quantum Coherence in Rb," A.S. Zibrov, M.D. Lukin, L. Hollberg, D.E. Nikonov, M.O. Scully, H.G. Robinson, and V.L. Velichansky, Phys. Rev. Lett. $\underline{76}, 3935$ (1996).

"Lasing without Inversion: The Road to New Short Wavelength Lasers," M.D. Lukin, M.O. Scully, G.R. Welch, E.S. Fry, L. Hollberg, G.G. Padmabandu, H.G. Robinson, and A.S. Zibrov, Laser Phys. $\underline{6}, 436-447$ (1996).

"High-Coherence Diode Laser with Optical Feedback via a Microcavity with 'Whispering Gallery' Modes," V.V. Vasil'ev, V.L. Velichanskii, M.L. Gorodetskii, V.S. Il'chenko, L. Hollberg, and A.V. Yarovitskii, Quant. Electron. 26, 657-658 (1996).

"Absorption Cross Sections and Self-Reaction Kinetics of the 10 Radical," M.H. Harwood, J.B. Burkholder, M. Hunter, R.W. Fox, and A.R. Ravishankara, J. Phys. Chem. A 101, 353-363 (1997). 
Part I

Diode Laser Technology 



\title{
Frequency stabilization of semiconductor lasers by resonant optical feedback
}

\author{
B. Dahmani, * L. Hollberg, and R. Drullinger \\ National Bureau of Standards, Boulder, Colorado 80303
}

Received May 20, 1987; accepted August 12, 1987

\begin{abstract}
With simple optical geometries a separate resonant Fabry-Perot cavity can serve as an optical feedback element that forces a semiconductor laser automatically to lock its frequency optically to the cavity resonance. This method is used to stabilize laser frequencies and reduce linewid ths by a factor of 1000 from $20 \mathrm{MHz}$ to approximately $20 \mathrm{kHz}$.
\end{abstract}

The high sensitivity of diode lasers to optical feedback is a well-known phenomenon ${ }^{1}$ that generally has a disruptive effect on the lasers' output frequency and amplitude stability. Under certain circumstances this sensitivity to feedback can be put to advantageous use. We have discovered an unconventional method that uses a low level of optical feedback to narrow semiconductor-laser linewidths substantially and also automatically to stabilize the laser's oscillation frequency. With the appropriate optical geometry the laser optically self-locks to the resonance of a separate Fabry-Perot reference cavity. The method relies on having optical feedback occur only at the resonance of a high- $Q$ reference cavity. In this case the cavity serves two functions. It provides the optical feedback, which narrows the laser's spectral width, and it provides the center-frequency stabilization to the cavity resonance.

A growing need for lasers with high spectral purity has stimulated the development of a number of semiconductor-laser frequency-stabilization techniques. Methods have been developed based on the techniques of optical feedback, ${ }^{2,3}$ external cavities, ${ }^{4,5}$ injection locking, ${ }^{6}$ and electronic servo control. ${ }^{7,8}$ Another interesting and related system for diode-laser frequency stabilization is a hybrid method that uses electronic as well as optical feedback from a fiber-optic cavity. ${ }^{9}$ Because of the spectral characteristics of semiconductor-laser frequency noise, the only systems that are able to achieve substantial linewidth reduction are those that incorporate some form of optical feedback or use very fast electronic servos ${ }^{10,11}$ (typically requiring servo bandwidths $\geq 20 \mathrm{MHz}$ ).

Our laser locking system contrasts with the previous systems in that the laser sees optical feedback only when its frequency matches the resonant frequency of the reference cavity. We have tested a variety of optical locking geometries; Fig. 1 diagrams a particularly simple and effective version. As shown here, when the confocal reference cavity (mirror separation equal to mirror radius) is operated off axis, it should be viewed as a four-port device. It is important to note that the two ports on the input mirror side (labeled I and II) have different output characteristics. The output beam of type $I$ is a combination of the reflected portion of the input beam with the transmitted portion of the resonant field inside the cavity. This beam has a power minimum when the laser frequency matches a cavity resonance. In contrast, the three outputs of type II contain only the transmitted portion of the cavity resonant field and hence have the desired characteristic of a power maximum on resonance. The geometry of Fig. 1 is one possible method of arranging to have the resonant optical feedback of type II (maximum feedback on resonance) while avoiding the complications inherent in the directly reflected beam contained in output I. For a wide range of feedback conditions this system tends to self-lock stably, thus forcing the laser frequency to match that of the cavity resonance.

Our experiments have been carried out using commercial, single-mode, 850 -nm, GaAlAs lasers ${ }^{12}$ without any special preparation of these devices. The free-running, unperturbed laser linewidths were measured to be approximately $20-50 \mathrm{MHz}$. A variety of reference cavities was used for the optical locking system. These had free spectral ranges varying from 250 $\mathrm{MHz}$ to $7.5 \mathrm{GHz}$ and resonance widths ranging from 4 to $75 \mathrm{MHz}$. As an additional laser diagnostic we mon-

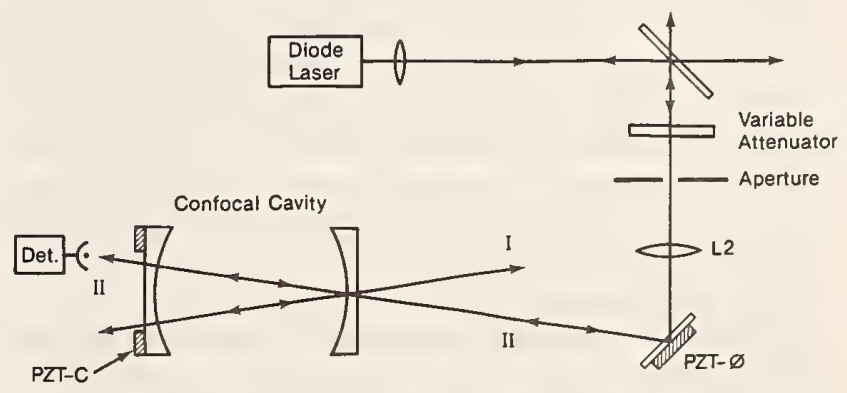

Fig. 1. Schematic of one version of the optical feedback locking system. Lens L2 is used to mode match the laser into the confocal reference cavity. The aperture blocks the unwanted feedback of type I while passing the desired feedback of type II. The variable attenuator is used to study the feedback power dependence of the locking process. The piezoelectric translator PZT- $\phi$ is used to optimize the feedback phase relative to the undisturbed laser. PZT-C is used to scan the reference cavity and in turn the optically locked laser frequency. A photodetector (Det.) monitors the transmitted power. 


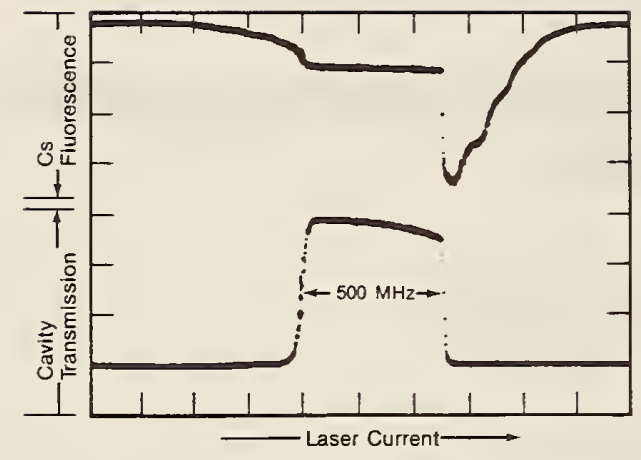

Fig. 2. The lower trace displays the power transmitted through the reference cavity as a function of the laser current. The upper trace, taken simultaneously, shows the fluorescence from a cesium cell as the laser scans from left to right across the 852-nm cesium resonance. The flat portion on the side of the fluorescence signal and on the peak of the cavity resonance occurs when the laser frequency locks to the cavity resonance and no longer scans with the laser current. The indicated locking range $\simeq 500 \mathrm{MHz}$ corresponds to the frequency range that the laser would normally scan without the optical self-locking.

itor the resonance fluorescence from an atomic-cesium vapor cell.

With type II feedback if the laser frequency is far from matching a reference cavity resonance there is no optical feedback and the laser frequency scans as usual with changes in the injection current. However, as the laser frequency approaches a cavity resonance, resonant feedback occurs and the laser frequency locks to the cavity resonance, even if the laser current continues to scan. The actual frequency range over which the diode laser locks is a function of the feedback power level and the phase of the feedback light relative to that of the unperturbed laser. The optical locking of the laser frequency to the reference cavity resonance is observed directly with a photodiode, which monitors the power transmitted through the cavity as a function of the laser current. This signal is displayed as the lower trace in Fig. 2. Here we see an unusual Fabry-Perot transmission function, which has a flat top and a width approximately 10 times larger than the actual cavity resonance width of 50 $\mathrm{MHz}$. This shape is a manifestation of the fact that the laser frequency is not scanning with the laser current but is locking to the frequency of peak transmission of the cavity resonance. The frequency locking is further demonstrated in the upper trace of Fig. 2, which shows a simultaneous record of the fluorescence from a cesium cell as the laser scans over the 852-nm cesium resonance line. This trace shows that when the laser frequency is far from the peak of the cavity resonance (shown in the lower trace) it scans smoothly over the cesium resonance as the laser current is varied. The self-locking, which stabilizes the laser frequency, is evidenced by the abrupt flattening in the scan of the fluorescence signal as well as the cavity transmission function. At the end of the $500-\mathrm{MHz}$ locking range the laser abruptly drops out of lock and continues to scan over the cesium resonance.
For low levels of optical feedback the locking range depends on the feedback power ratio. This is the ratio of the external feedback power, coupled into the laser mode, to the power reflected internally by the output facet. Locking ranges of a few hundred megahertz were observed with feedback power ratios typically in the range of $10^{-4}$ to $10^{-5}$, with a large uncertainty in the coupling efficiency of the feedback power into the laser mode.

In order to determine unambiguously the spectral characteristics of the optically locked semiconductor lasers, we measured the beat note between two lasers independently locked to separate reference cavities. Direct observation of the beat-note spectra avoids many of the uncertainties and errors that plague measurements of laser frequency stability and linewidth using delayed-self-heterodyne and electronic residualerror-signal techniques. Figure 3 shows the beat note detected with a fast photodiode and displayed on a rf spectrum analyzer. The upper trace shows the beat note when one of the lasers is free running without feedback while the other laser is optically locked. The sharply peaked trace is obtained when both of the lasers are optically locked to their independent reference cavities and indicates the dramatic linewidth reduction that is instantly achieved. In the locked case, the width of the displayed beat note is limited not by the laser linewidth but rather by the rf spectrum analyzer's resolution bandwidth of $300 \mathrm{kHz}$. Higher-resolution measurements show that the actual spectral width of the beat note can be less than $20 \mathrm{kHz}$ (Fig. 4). This narrowing of the semiconductor linewdith by a factor of about 1000 (from $20 \mathrm{MHz}$ to $20 \mathrm{kHz}$ ) is obtained by purely optical means without any electronic control.

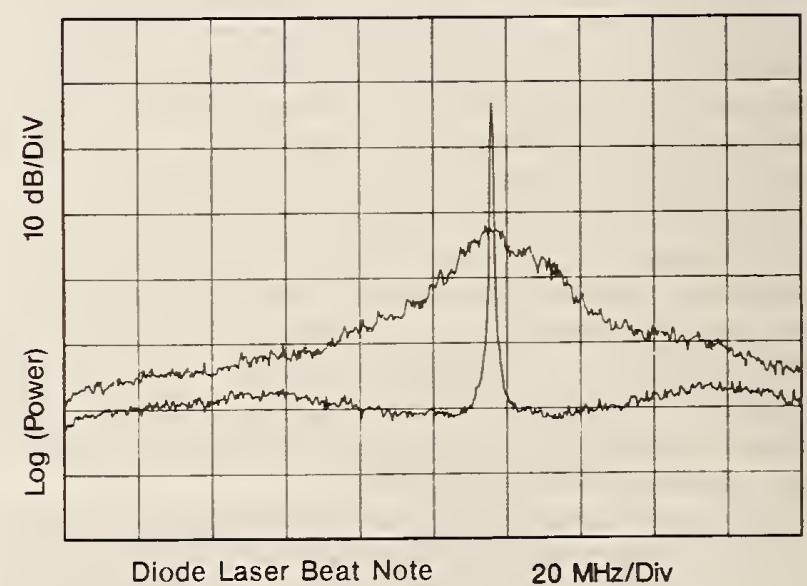

Fig. 3. The beat note between two semiconductor lasers is shown on a rf spectrum analyzer with a sensitivity of $10 \mathrm{~dB} /$ division vertically and $20 \mathrm{MHz} /$ division horizontally and a resolution bandwidth of $300 \mathrm{kHz}$. The upper trace shows the broad beat-note peak that is observed when one of the two lasers is free running and unstabilized. The other, sharply peaked, trace shows the beat note when both of the lasers are optically self-locked to separate Fabry-Perot reference cavities. In the locked case the peak width is limited by the $300-\mathrm{kHz}$ resolution of the spectrum analyzer. 


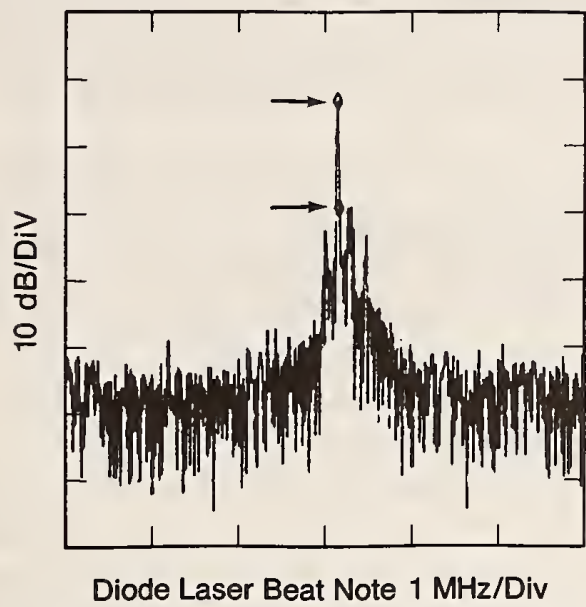

Fig. 4. Diode-laser beat note displayed with higher resolution now shows a very narrow width as measured on a rf spectrum analyzer. The approximately $20-\mathrm{kHz}$ width is inferred from the two markers on the central peak (which are separated by $-16 \mathrm{~dB}$ and $13 \mathrm{kHz}$ ), the spectrum-analyzer resolution of $10 \mathrm{kHz}$, the sweep rate of $30 \mathrm{msec} /$ division, and the repeatability of the measurement.

One advantage of the optically self-locked laser system is that it shows some reduction in sensitivity to other sources of optical feedback. In addition, we find that the excess intensity noise $(\simeq 30 \mathrm{~dB}$ above the expected photodetection shot noise) that we measure on the light from the semiconductor laser is reduced by roughly $10 \mathrm{~dB}$ when the laser is optically locked to the cavity.

For many applications it is important to be able to scan the laser frequency as well as to have a stable long-term frequency lock. Continuous, narrowlinewidth scans of the laser frequency can be made by synchronizing the sweep of the laser current with that of the reference cavity length (PZT-C, Fig. 1) and the feedback phase (PZT- $\phi)$. With this synchronization we have made continuous scans of more than $4 \mathrm{GHz}$; these were limited only by the range of our present PZT- $\phi$. We note that it is necessary to control the laser current only accurately enough that the freerunning laser frequency is within the $\simeq 500-\mathrm{MHz}$ optical-self-locking range. To avoid the inevitable drifts and to ensure long-term locking stability, it is useful to servo control the appropriate feedback phase and laser current relative to the reference cavity. Low-speed electronic feedback circuits are sufficient to accomplish this.

The actual physics that determines the spectral characteristics of semiconductor lasers is a topic of active research and is reviewed in Ref. 13. Initial theoretical analyis ${ }^{14}$ of the self-locking process based on optical feedback theory ${ }^{1,15-17}$ gives reasonable agreement with experimental results, at least in terms of linewidth reduction, locking range, and the phase dependence of the cavity locking.

The optical self-locking system described here has several advantages relative to conventional locking techniques. It is a relatively simple method that provides a vast improvement in the linewidth and direct control of the semiconductor-laser frequency. When the system is locked, the laser's linewidth is reduced dramatically, from $20 \mathrm{MHz}$ to approximately $20 \mathrm{kHz}$, and the laser's oscillation frequency is stabilized to the cavity resonance frequency. Even narrower spectral widths could be expected with better-quality reference cavities. Long-term stability of the reference cavity resonance can be quite high. ${ }^{18}$ We observe that the laser's intensity noise is reduced, as is its sensitivity to other sources of optical feedback. The system is also attractive because it does not require any modification of commercial laser diodes or any extremely fast electronic servo systems. With the increasing interest in semiconductor lasers with high spectral purity and precision optical measurements in general, this optical-self-locking method has the potential for numerous applications.

*Permanent address, Laboratoire Primaire du Temps et des Frequences, 61 avenue de l'Observatoire, 75014 Paris, France.

\section{References}

1. R. Lang and K. Kobayashi, IEEE J. Quantum Electron. QE-16, 347 (1980).

2. K. R. Preston, K. C. Woollard, and K. H. Cameron, Electron. Lett. 17,931 (1981).

3. J. Mark, E. Bodtker, and B. Tromborg, Electron. Lett. 21, 1008 (1985).

4. R. Wyatt and W. J. Devlin, Electron. Lett. 19, 110 (1983).

5. M. Fleming and A. Mooradian, IEEE J. Quantum Electron. QE-17, 44 (1981).

6. S. Kobayashi and T. Kimura, IEEE J. Quantum Electron. QE-17, 681 (1981).

7. Yu. A. Bykovskii, V. L. Velichanskii, I. G. Goncharov, and V. A. Maslov, Sov. Phys. Semicond. 4, 580 (1970).

8. J.-L. Picque and S. Roizen, Appl. Phys. Lett. 27, 340 (1975).

9. F. Favre and D. Le Guen, IEEE J. Quantum Electron. QE-21, 1937 (1985), and references therein.

10. M. Ohtsu and S. Kotajima, IEEE J. Quantum Electron. QE-21, 1905 (1985).

11. Y. Yamamoto, O. Nilsson, and S. Saito, IEEE J. Quantum Electron. QE-21, 1919 (1985).

12. For technical reference, the lasers used were Hitachi HLP 1400's. This mention does not constitute an endorsement, and we expect that other lasers will also be suitable.

13. M. Osinski and J. Buus, IEEE J. Quantum Electron. QE-23, 9 (1987).

14. D. Hjelme, A. Mickelson, L. Hollberg, and B. Dahmani, in Digest of Topical Meeting on Semiconductor Lasers (Optical Society of America, Washington, D.C., 1987), p. 15.

15. C. H. Henry, IEEE J. Lightwave Technol. LT-4, 298 (1986).

16. R. J. Lang and A. Yariv, IEEE J. Quantum Electron. QE-22, 436 (1986).

17. H. Olesen, J. H. Osmundsen, and B. Tromborg, IEEE J. Quantum Electron. QE-22, 762 (1986).

18. Drift rates of $\Delta \nu / \nu \Delta t \simeq 10^{-14} / \mathrm{sec}$ have been reported for gas lasers electronically locked to optical cavities: D. Hils and J. L. Hall, in Digest of XV International Quantum Electronics Conference (Optical Society of America, Washington, D.C., 1987), p. 102. 


\author{
L. Hollberg and M. Ohtsua) \\ National Bureau of Standards, Boulder, Colorado 80303
}

(Received 28 March 1988; accepted for publication 5 July 1988)

\begin{abstract}
We find that using the technique of optical feedback locking, to narrow semiconductor linewidths, does not sacrifice the ability to modulate the laser's frequency via the injection current. The frequency of a laser is stabilized to a separate Fabry-Perot reference cavity using resonant optical feedback and can be modulated efficiently at frequencies related by rational fractions to the free-spectral range of the reference cavity. This system can provide an array of narrow-linewidth, frequency-stable laser lines and shows promise for applications in frequency-division-multiplexed coherent communications, as weil as laser frequency control and precision measurement systems.
\end{abstract}

Optical feedback locking of semiconductor lasers provides a powerful means for stabilizing the frequency and narrowing the linewidth of diode lasers. ${ }^{1-3}$ This optical technique can reduce laser linewidths by more than a factor of 1000 , thus achieving linewidths of a few kilohertz. We consider here the interesting and useful modulation characteristics of these optically stabilized semiconductor lasers (OSSL). Direct modulation of the injection current of these lasers can provide a high FM modulation index while the very narrow linewidth and frequency stabilization produced by the optical feedback lock is maintained.

The high-speed frequency and amplitude modulation capabilities of semiconductor lasers are important attributes of these lasers relative to other optical sources. Unfortunately, the FM capabilities are usually sacrificed when the laser linewidths are reduced by optical means. ${ }^{4}$ With most frequency stabilization schemes there is a trade-off between the diode laser's linewidth and its FM modulation capabilities. Usually a one-to-one correspondence exists between these quantities; that is, if the laser linewidth is reduced by a factor of 10 , its FM sensitivity to injection current (typically $\sim 3$ $\mathrm{GHz} / \mathrm{mA}$ ) is also reduced by roughly this same factor of 10 . In the case of the OSSL the modulation characteristics are definitely altered by the optical locking process, but we find that the modulation sensitivity is not necessarily reduced when the linewidth is narrow. In fact, we find that the modulation characteristics of the OSSL have a predictable resonant frequency dependence.

The concept behind the optical feedback locking technique is to establish weak resonant feedback from a high $Q$ (relative to the $Q$ of the diode laser cavity) optical resonator. Figure 1 is a diagram of a system which gives enhanced optical feedback when the laser frequency matches the cavity resonance and effectively zero-feedback off resonance. Here a beamsplitter picks off a small amount of the laser output $(\sim 4 \%)$ and couples it off axis into a confocal optical resonator. This geometrical arrangement ensures that the laser sees positive optical feedback precisely when the laser frequency matches the cavity resonance frequency. The optical lock usually operates in the low feedback limit and typically requires an optical feedback power of about $10^{-4}$ of the laser

"Permanent adress: Tokyo Institute of Technology, Graduate School at

Nagatsuta. Midori-ku. Yokohama, Kanagawa 227, Japan. output power. ${ }^{2}$ At resonance the spectrally cleaned laser field which has built up in the reference cavity leaks back through the cavity input mirror and returns to the laser where it increases the laser gain at that frequency. This frequency-selective gain enhancement forces the laser to lock to the cavity resonance frequency. The result is that the semiconductor laser's frequency is stabilized to the cavity resonance, and its $20 \mathrm{MHz}$ linewidth is reduced to less than 10 $\mathrm{kHz}$. Related work has also been reported for semiconductor laser frequency control using optical feedback from optical waveguides $^{5}$ and fiber cavities. ${ }^{6}$

We have used a variety of optical geometries and reference cavities for these optical locking systems. The free-spectral ranges (FSR) of the reference cavities were between 7.5 $\mathrm{GHz}$ and $250 \mathrm{MHz}$ with cavity resonance widths varying from $75 \mathrm{MHz}$ to $20 \mathrm{kHz}$. The commercial lasers were singlemode $\mathrm{AlGaAs}$ lasers operating near $850 \mathrm{~nm}$ and were not modified in any way. For applications requiring higher spectral purity and low residual phase noise at high frequencies, it is useful to send all of the laser power into and through the high-efficiency reference cavity. The cavity then acts as a

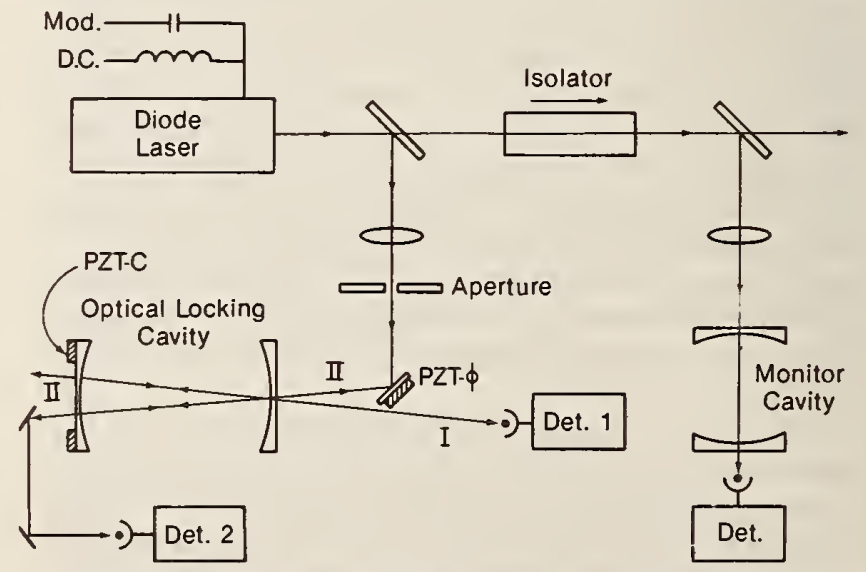

FIG. 1. Experimental diagram of an optically stabilized semiconductor laser (OSSL). Approximately $4 \%$ of the laser output power is coupled offaxis into the confocal optical locking cavity. In this geometry resonant optical feedback returns to the laser and automatically locks the laser frequency to the cavity resonance. Piezoelectric ceramics PZT-C and PZT $\phi$ are used to control the cavity frequency and optimize the feedback phase, respectively. An additional monitor cavity is used for spectral analysis and is separated from the laser by an optical isolator. $\mathrm{rf}$ modulation is added to the dc injection current through the modulation port (mod.). Photodiode detectors (det.) monitor the transmission and reflection signals from the cavities. 
bandpass filter and removes the excess laser phase noise at very high frequencies. This scheme uses the reference cavity as a spectral and spatial filter as well as the frequency discriminator for the optical feedback lock using the weak reflection.

Modulation of the injection current of nonstabilized semiconductor lasers results in both amplitude and frequency modulation of the laser output. At least in the limit of low modulation index the temporal characteristics of the laser electric field can be represented by

$E(t)=A\left[1+m \cos \left(\omega_{m} t\right)\right] \sin \left[\omega t+\beta \cos \left(\omega_{m} t+\theta\right)\right]$.

Here $A$ is the field amplitude, $\omega /(2 \pi)$ is the laser carrier frequency, $\omega_{m} /(2 \pi)$ is the modulation frequency, and $m$ and $\beta$ represent the amplitude and frequency modulation indices, respectively. ${ }^{7,8}$ The resulting frequency-domain spectra can be described by the usual sum of AM plus FM sidebands (with Bessel function amplitudes) except that the relative phase $\theta$ between AM and FM is frequency dependent. When a diode laser is operating well above threshold, a small modulation of the injection current results in a modulation of the laser field that is predominantly FM with the ratio of $\beta$ to $m$ typically in the range of 10 to 20 . However, when the modulation index is large $(\beta>2)$, the situation can be considerably more complicated.

When the OSSL is modulated at low frequencies, typically $\omega_{m}<200 \mathrm{MHz}$, the frequency modulation is suppressed by the optical lock relative to that of an unstabilized laser. ${ }^{9}$ On the other hand, there are special modulation frequencies that strongly affect the frequency modulation characteristics of the OSSL. With certain modulation conditions some or all of the modulation sidebands can resonate with the reference cavity (with or without the carrier), in which case they return to the laser and reinforce the optical lock. In particular optical sidebands at frequencies that correspond to the FSR (or its harmonics) of the reference cavity are simultaneously resonant with the cavity and are fed back to the laser with the carrier. In this way it is possible to modulate the laser current with a high modulation index $\beta$ and generate many sidebands without disrupting the frequency stabilization and linewidth narrowing provided by the optical feedback lock.

Figure 2 shows the resonances in the optical locking process that one observes as a function of the applied rf modulation frequency. The tallest, evenly spaced, sharp resonances in this figure occur when the modulation is at frequencies integrally related to the cavity FSR of $245 \mathrm{MHz}$. Good optical locking is observed at the modulation frequencies corresponding to these resonances. Also, the power transmitted through the cavity is maximized because the laser carrier and the sidebands match cavity resonances.

All of the cavity transmission resonances shown in Fig. 2 correspond to modulation frequencies that are related to the reference cavity FSR by rational numbers, that is, when the modulation frequency is

$$
\omega_{m} /(2 \pi)=(a / b)(\mathrm{FSR}), \quad a, b=1,2,3, \ldots .
$$

The smaller resonances shown in Fig. 2 occur when the ratio $(a / b)$ is not an integer. This rational relationship between

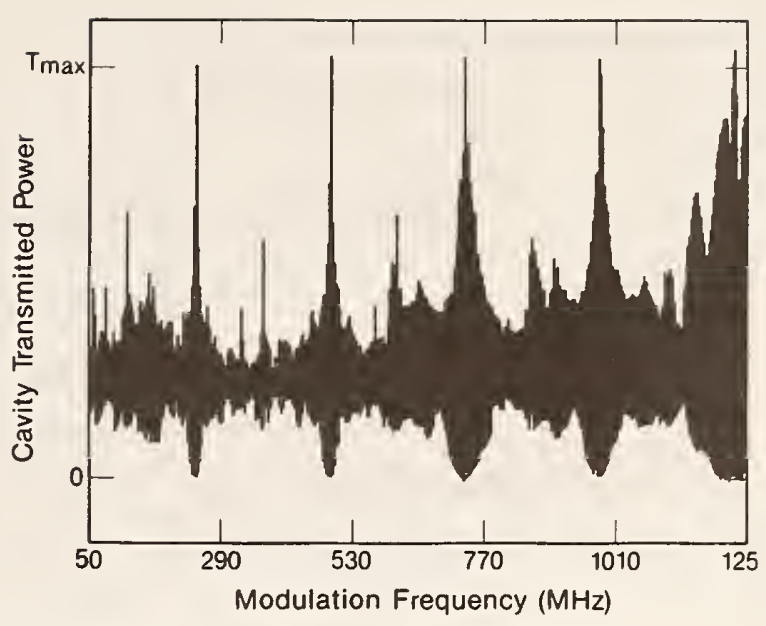

FIG. 2. Power transmitted through the optical locking cavity (FSR $=245$ $\mathrm{MHz}$, finesse $=10$ ) as seen by detector 2 (Fig. 1) as a function of the frequency of the modulation applied to the injection current. The modulation frequency is swept slowly from 50 to $1250 \mathrm{MHz}$. Points of zero and maximum transmission are indicated on the vertical axis. The sharp peaks indicate the resonant nature of the stability of the optical lock as a function of modulation frequency. The large evenly spaced peaks correspond to modulation at the frequency of the cavity free-spectral range (245 $\mathrm{MHz}$ ) and its harmonics. The smaller resonant peaks occur when the modulation frequency is related to the cavity FSR by a noninteger rational number. $A$ modulation current of $\sim 5 \mathrm{~mA}$ was added to the dc current of $95 \mathrm{~mA}(\sim 1.7$ times threshold).

the modulation frequency and the cavity FSR for stable optical locking is easily understood: that is, under the conditions of Eq. (2) every $b$ th sideband can resonate with every $a$ th cavity mode. For example, modulation at $367 \mathrm{MHz}$ corresponds to $a / b=3 / 2$ and means that when the carrier is resonant with a cavity mode, every other sideband will be simultaneously resonant with every third cavity mode. This situation gives stable optical locking with roughly half of the available power resonant with the cavity. We have observed these resonant enhancements in the optical lock for many modulation frequency to FSR ratios, including those with $a=1-9$ and $b=1-5$. In principle, some optical locking occurs for any modulation frequency that is related to the cavity FSR by a rational number $(a / b)$, but in general as the denominator $b$ gets larger, less and less of the available optical power is resonant with the cavity modes and hence the optical lock is less effective. The strength of a particular resonance depends on the number and size of the sidebands that are resonant with cavity modes and thus depends strongly on the modulation index $\beta$ and the value of $b$.

The frequency domain power spectra of an optically locked laser that is modulated at one of the resonances [consistent with Eq. (2)] consist of an array of frequency stabilized, narrow-linewidth laser lines separated by the modulation frequency. Figure 3 shows sideband spectra of a modulated OSSL taken with an optical spectrum analyzer (see Fig. 1). The optical spectrum analyzer that is used to tisplay the modulation sidebands is not capable of measuring the very narrow laser linewidth because of the limited resolving power when a large FSR $(7.5 \mathrm{GHz})$ is required. In order to measure the laser linewidth we use heterodyne methods to detect the beat note between two OSSL's. ${ }^{2}$ We find that the locked laser linewidths are less than $10 \mathrm{kHz}$ and 

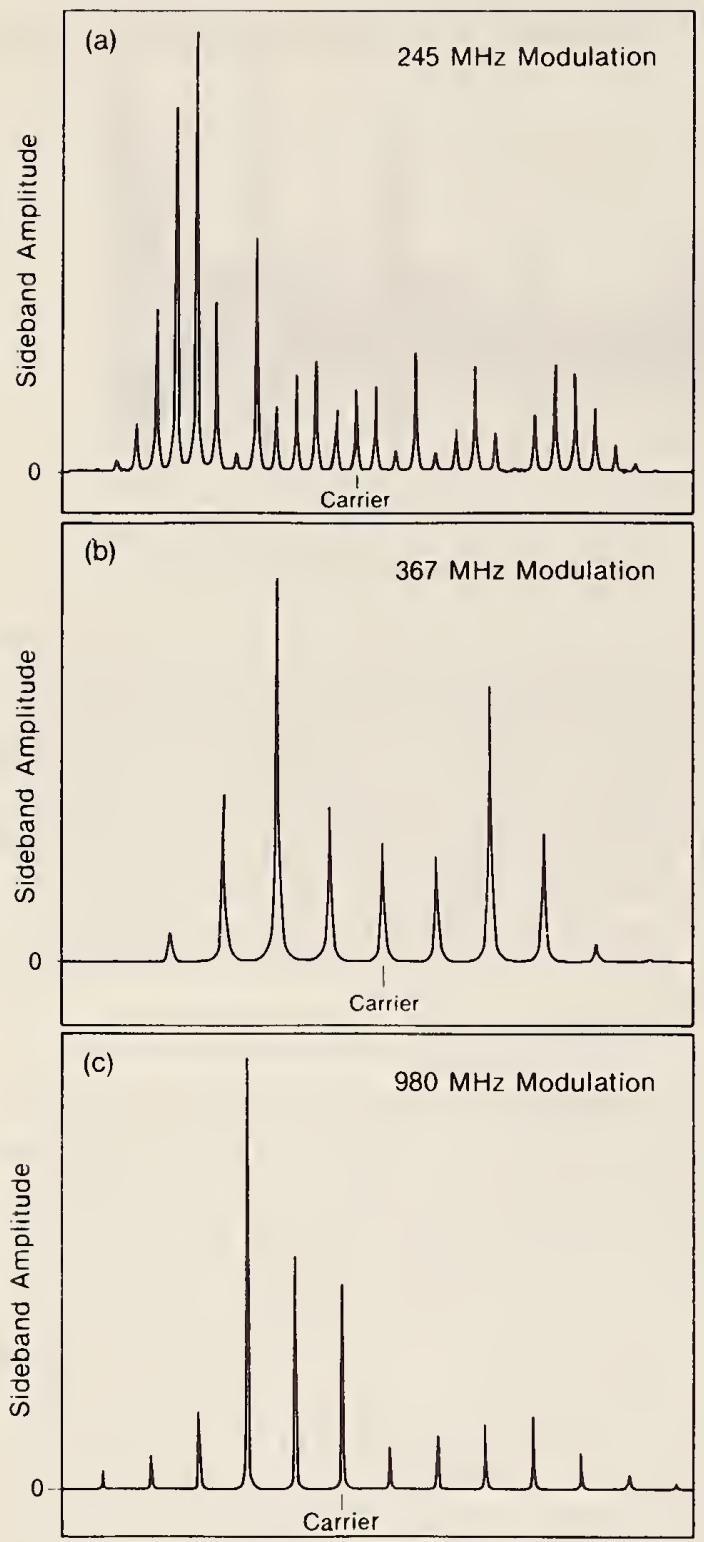

Optical Spectrum

FIG. 3. Sideband st ructure of a modulated, optically locked, semiconductor laser taken with an optical spectrum analyzer (FSR $=7.5 \mathrm{GHz}$, finesse $=150$ ) for three of the resonant modulation frequencies. In all of these cases a high-modulation index $\beta$ is obtained while maintaining the narrow laser linewidth and center frequency stabilization. In (a) the modulation frequency is equal to the locking cavity FSR of $245 \mathrm{MHz}$. Here a modulation current of $\sim 7 \mathrm{~mA}$ is added to the dc bias of $97 \mathrm{~mA}$. We see that more than 20 narrow-linewidth, frequency stable, sidebands are produced. In (b) the $4 \mathrm{~mA}$ of modulation current is at a frequency of $367 \mathrm{MHz}$ which is $3 / 2$ the cavity FSR. In (c) the laser is modulated with $\sim 10 \mathrm{~mA}$ at $980 \mathrm{MHz}$ (four times the FSR), and the laser generates more than ten useful sidebands covering a total frequency range of more than $10 \mathrm{GHz}$ (in this case the overlapping sidebands from the adjacent $7.5 \mathrm{GHz}$ FSR have been removed for clarity).

that the linewidth is unchanged when the laser is modulated at frequencies that correspond to the locking resonances that are shown in Fig. 2. This is to be compared with linewidths that are measured to be $20 \mathrm{MHz}$ when the laser is not locked or is strongly modulated at a frequency that is not an optical locking resonance frequency. When modulated at such nonlocking frequencies the OSSL's linewidth degrades progres- sively from $10 \mathrm{kHz}$ to $20 \mathrm{MHz}$ as the modulation index is increased.

A good optical lock occurs when the laser is modulated at a resonance frequency consistent with Eq. (2). However, these resonances are fairly narrow in modulation frequency and depend directly on the $Q$ of the cavity, the modulation index, and the optical feedback level. For example, with a locking cavity $Q \sim 10^{7}$ (corresponding to a cavity finesse of 10 and a FSR of $245 \mathrm{MHz}$ ) and an optical feedback power ratio of about $10^{-4}$, the modulation frequency range for good optical locking is approximately $6 \mathrm{MHz}$ wide (centered at the modulation resonance frequency at $245 \mathrm{MHz}$; see Fig. 2). This range is for a modulation index $\beta \simeq 8$ and increases, as we might expect with smaller modulation index. This resonant character of the laser's FM modulation response limits the modulation formats that can be employed with the OSSL.

Using the technique of optical feedback locking, we find that it is possible to simultaneously have a narrow-linewidth semiconductor laser with a high-sensitivity frequency modulation capability. Frequency modulation induced via the injection current is compatible with the optical stabilization (and narrow linewidth) when the ratio of the modulation frequency to cavity FSR is a rational number. One of the promising applications is to use the modulated OSSL to provide an array of frequency-stabilized local oscillators for frequency-division-multiplexed coherent communication systems. There is also the potential for measuring optical absorption and dispersion with high sensitivity using the techniques of optical heterodyne spectroscopy ${ }^{10}$ as well as applications in precision laser measurements and frequency control. ${ }^{11.12}$

We would like to thank J. L. Hall, R. Drullinger, B. Dahmani, and A. Clairon for constructive discussions, and J. L. Hall and the Joint Institute for Laboratory Astrophysics for making this collaboration possible.

'D. Hjelme, A. Mickelson, L. Hollberg, and B. Dahmani, in Digest of Topical meeting on Semiconductor Lasers (Optical Society of America, Washington, DC, 1987), p 15.

'B. Dahmani, L. Hollberg, and R. Drullinger, Opt. Lett. 12, 876 (1987). ${ }^{3} \mathrm{H}$. R. Telle and H. Li, IEEE J. Quant um Electron. (to be published). ${ }^{4}$ M. Ohtsu and S. Kotajima, IEEE J. Quantum Electron. 21, 1905 (1985). ${ }^{5}$ N. A. Olsson, C. H. Henry, R. F. Kazarinov, H. J. Lee, B. H. Johnson, and K. J. Orlowsky, Appl. Phys. Lett. 51, 1141 (1987); R. F. Kazarinov and C. H. Henry, IEEE J. Quantum Electron. 231401 (1987); R. F. Kazarinov, C. H. Henry, and N. A. Olsson, IEEE J. Quantum Electron. 23, 1419 (1987).

${ }^{6}$ F. Favre and D. Le Guen, IEEE J. Quantum Electron. 21, 1937 ( 1985 ).

${ }^{7}$ S. Kobayashi, Y. Yamamoto, M. Ito, and T. Kimura, IEEE J. Quantum Electron. 18, 582 (1982).

${ }^{x}$ W. Lenth, Opt. Lett. 8, 575 (1983).

${ }^{4}$ A. Clairon, B. Dahmani, Ph. Laurent, and Ch. Breant, in Proceedings of the 2 nd European Frequency and Time Forum, Neuchatel, Switzerland, 1988; and $\mathrm{Ph}$. Laurent, A. Clairon, and $\mathrm{Ch}$. Breant (unpublished).

${ }^{10}$ J. L. Hall, L. Hollberg, T. Baer, and H. G. Robinson, Appl. Phys. Lett. 39, 680 ( 1981 ).

''R. W. P. Drever, J. L. Hall, F. V. Kowalski, J. Hough, G. M. Ford, A. J. Munley, and H. Ward, Appl. Phys. B 31, 97 (1983).

${ }^{12}$ R.G. Devoe, C. Fabre, K. Jungmann, J. Hoffnagle, and R. G. Brewer, Phys. Rev. A 37, 1802 (1988). 


\title{
Using diode lasers for atomic physics
}

\author{
Carl E. Wieman \\ Joint Institute for Laboratory Astrophysics, University of Colorado and National Institute of Standards \\ and Technology, and Physics Department, University of Colorado, Boulder, Colorado 80309-0440 \\ Leo Hollberg \\ National Institute of Standards and Technology, Boulder. Colorado 80303
}

(Received 20 April 1990; accepted for publication 2 August 1990)

\begin{abstract}
We present a review of the use of diode lasers in atomic physics with an extensive list of references. We discuss the relevant characteristics of diode lasers and explain how to purchase and use them. We also review the various techniques that have been used to control and narrow the spectral outputs of diode lasers. Finally we present a number of examples illustrating the use of diode lasers in atomic physics experiments.
\end{abstract}

\section{INTRODUCTION}

Much of current atomic physics research involves the interaction of atoms and light in some way. Laser sources that can be tuned to particular atomic transitions are now a standard tool in most atomic physics laboratories. Traditionally this source has been a tunable dye laser. However, semiconductor diode lasers have been steadily improving in reliability, power, and wavelength coverage, while steadily decreasing in cost. It is now possible to have a diode laser system that will produce more than $10 \mathrm{~mW}$ of tunable light with a bandwidth of $100 \mathrm{kHz}$ for a cost of less than $\$ 1000$. Furthermore, the diode laser and power supply will fit in a $1-\ell$ box, require very little power or cooling water, and, under the right conditions, will be within a few $\mathrm{MHz}$ of the desired wavelength as soon as it is turned on. A further virtue is that the amplitude is very stable compared to most other laser sources so that it is relatively simple to make sensitive absorption or fluorescence measurements. These features make diode lasers increasingly attractive alternatives for a variety of uses.

In this review we present something of a users' guide aimed at atomic physicists who might want to start using diode lasers in their work. In this spirit we present the advantages of diode lasers as well as their negative features (there are several serious ones) and the best ways to deal with them. We begin in Sec. II by discussing relevant basic laser characteristics. Section III addresses some of the most important issues involved in actually setting up and using diode lasers in atomic physics experiments, such as purchasing the correct laser, tuning it to the desired frequency, and how to avoid destroying it. Section IV discusses optical and electronic feedback to control the laser output frequency. In the past, one of the main drawbacks to using diode lasers in atomic physics was the difficulty in obtaining narrowband, easily tunable output. However, the developments covered in Sec. IV have improved this situation considerably. Section $\mathrm{V}$ then discusses a selected set of applications of diode lasers in atomic physics, focusing primarily on work that has been made possible by these new wavelength control techniques.

To keep this article to a reasonable length there are necessarily many things we do not discuss. For example, we do not cover progress made on producing special diode lasers that work in marvelous ways, because these developmental lasers are not available to most atomic physicists. We restrict ourselves to lasers that are standard commercial devices. Another notable omission is the extensive work in molecular spectroscopy that has been done using lead salt diode lasers in the middle and far infrared regions of the spectrum.

Camparo' completed an extensive review on the use of diode lasers in atomic physics in 1985. That review covered much of the basic physics and characteristics of diode lasers, and the various uses that had been made of them. At that time most devices were simple free-running lasers without feedback, and they had been used principally for optical pumping and low resolution spectroscopy. In the present review, although there will necessarily be some overlap of that discussion, we will emphasize the recent developments including "stabilized lasers" (ones with feedback for frequency control), and new types of applications. There is also an excellent review entitled "Coherence in Semiconductor Lasers" by Ohtsu and Tako ${ }^{2}$ that discusses applications in a variety of areas.

\section{BASIC LASER CHARACTERISTICS}

The basic physics of diode lasers is presented in many references $^{3}$ and will not be repeated here. However, we do wish to single out a few features that are relevant to much of the later discussion. The construction of a typical semiconductor diode laser is shown in Fig. 1. The devices are extremely small and yet are capable of reasonable $\mathrm{cw}$ output powers with high electrical to optical efficiency. The laser light is generated by sending a current (the "injection current") through the active region of the diode between the $n$ and $p$-type cladding layers. This produces electrons and holes, which in turn recombine and emit photons. The laser's emission wavelength is determined by the band gap of the semiconductor material and is very broadband relative to atomic transitions. The spatial mode of the laser is defined by a narrow channel in the active region that confines the light. This confinement of the transverse laser mode is achieved either through the spatial variation of injection current density (gain guided) or by spatial variations in the index of 


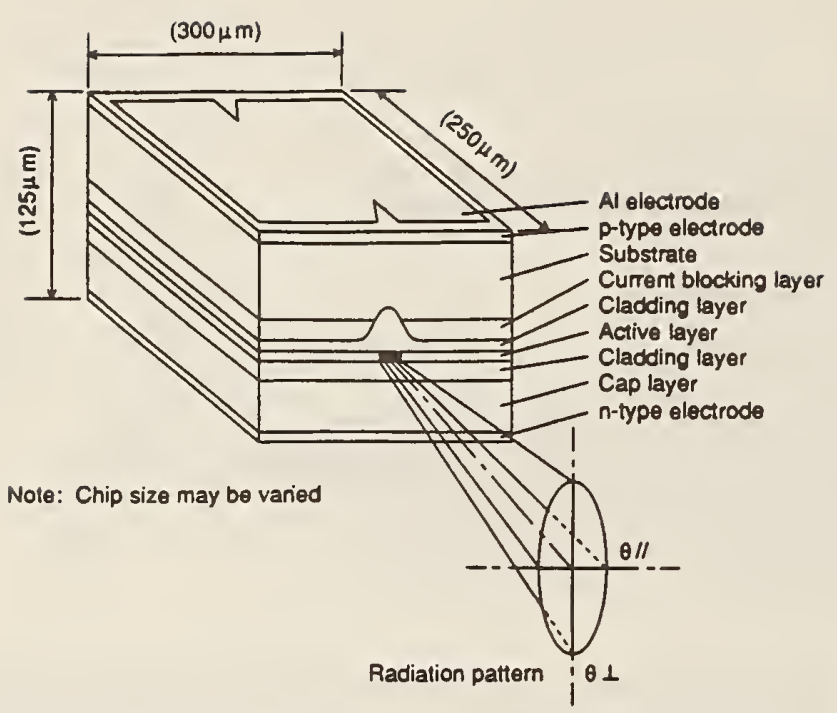

FIG. 1. Illustration of the different layers of semiconductor and the typical dimensions of a diode laser. The rectangular shape of the gain region leads to the oval radiation pattern. (Figure adapted from Ref. 4, with permission.)

refraction due to changes in the materials used in the laser's construction (index guided).

A wide variety of laser designs have been used. Most of the atomic physics done with diode lasers has been carried out using index-guided GaAlAs lasers in the near infrared region of the spectrum. Unless otherwise noted, this type will be assumed in the subsequent discussion. These lasers typically produce single-mode (spatial and longitudinal) output powers of 5-15 $\mathrm{mW}$ with just the cleaved facets of the semiconductor serving as the laser's mirrors. Output powers up to $\approx 50 \mathrm{~mW}$ are commercially available from what are essentially the same devices with the addition of a high re-

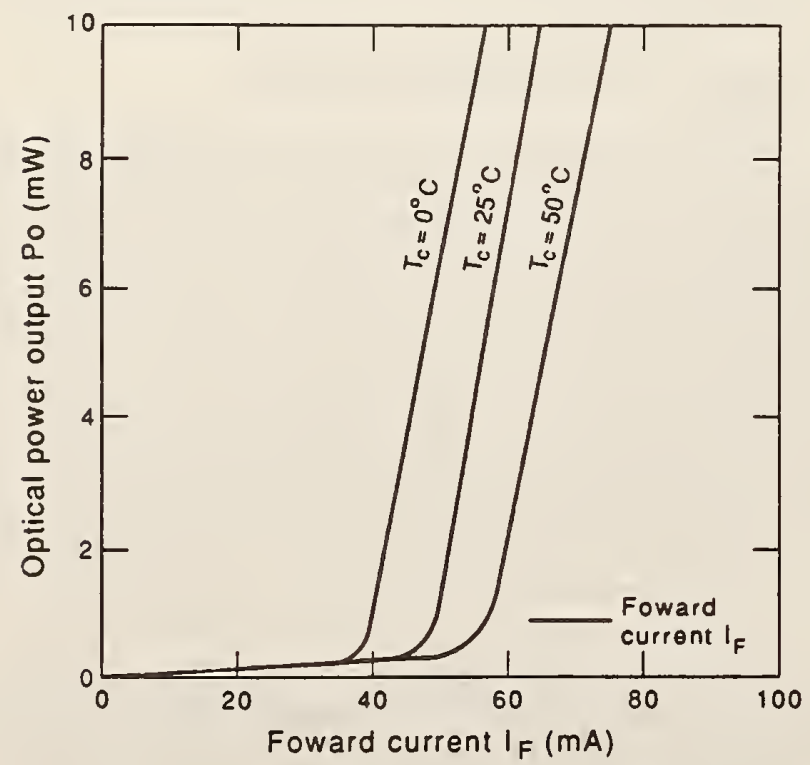

FIG. 2. Output power vs injection current for a typical laser. The sudden change in the slope of each curve marks the onset of laser action, and the current at that point is the threshold current for the laser. The different curves show how the temperature affects the laser output. (Figure adapted from Ref. 4, with permission.)

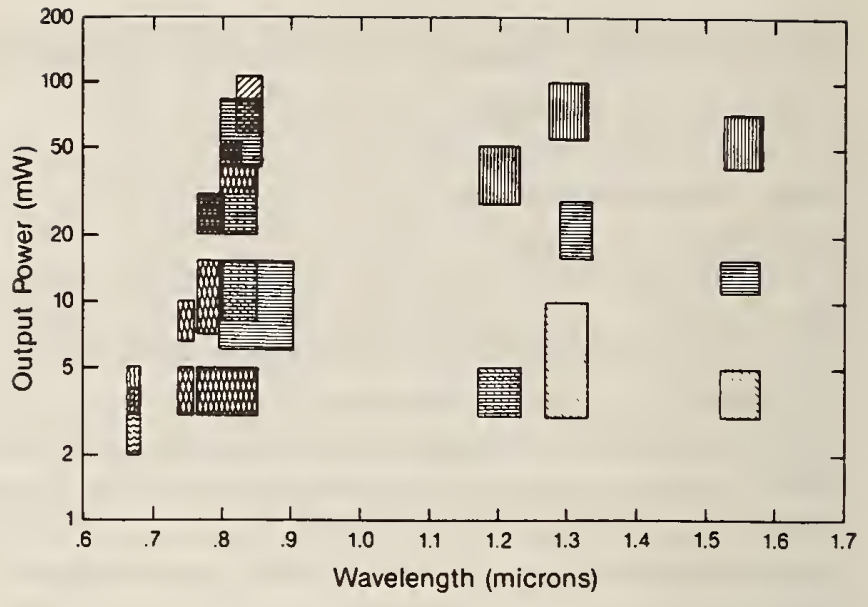

FIG. 3. cw diode laser output powers displayed as a function of wavelength. The various cross hatched boxes are taken from manufacturers catalogs and represent the distributions in wavelength and power that are advertised. In some cases it is possible to obtain lasers outside of these normal distributions. The range shown here is the distribution of lasers from the manufacturers and should not be confused with the tuning range of any specific laser which is much smaller. (The manufacturers shown in this table include; Hitachi, Mitsubishi, Nec, Oki, Sharp, and Spectra Diode and are listed only as being representative, not as an endorsement; other sources should be equally suitable. We have experience with some of these devices but we have not verified all devices nor that the manufactures will deliver all of the devices as advertised.)

flectance coating on the back surface and a reduced reflectance coating on the output facet. Higher power lasers that have special designs such as multistripes or very wide single stripes are also available. These will likely be useful in the future, but at the present time the difficulty and expense in obtaining these special designs at a desired wavelength is a formidable problem.

The output power of a typical semiconductor diode laser as a function of the injection current and temperature is shown in Fig. 2. This shows the abrupt onset of laser action at the threshold current and the increase in the threshold with temperature. Alternatively, for fixed injection current, the laser's output increases rapidly as the temperature is lowered.

Figure 3 shows the power and wavelength characteristic of $\mathrm{cw}$ diode lasers that are readily available from commercial sources. These are divided into five main bands as driven by commercial applications. The two bands in the infrared, at 1300 and $1500 \mathrm{~nm}$, have been developed primarily for fiber optic systems and are based on quartenary InGaAsP semiconductors. The lasers in the $750-890 \mathrm{~nm}$ region are based on $\mathrm{AlGaAs}$ and have applications in consumer and commercial electronics as well as some fiber-optic applications. The relatively new visible diode lasers near $670 \mathrm{~nm}$ are based on InGaAlP semiconductors and have many projected applications in commercial optoelectronic systems. In applying these various lasers to spectroscopy we find that their spectral characteristics vary considerably. Those in the $750-870$ $\mathrm{nm}$ region have the best overall characteristics.

\section{A. Beam spatial characteristics}

Because the light is emitted from a small rectangular region (on the order of $0.1 \mu \mathrm{m}$ by $0.3 \mu \mathrm{m}$ ) the output of a 
diode laser has a large divergence (see Fig. 1). A typical output beam will have a divergence angle (full width at $50 \%$ intensity) of $30^{\circ}$ in the direction perpendicular to the junction, and $10^{\circ}$ in the parallel direction. Normally the beam is collimated using a lens with a small $f$ number. If the laser is operating in a single transverse mode, the collimated beam will be elliptical, but it can be made nearly radially symmetric using anamorphic prisms. In addition to the different divergence angles, the output beam of most diode lasers is astigmatic. This astigmatism can also be compensated when necessary. ${ }^{5}$ A typical diode laser wavefront collimated in this fashion will often have a significant amount of structure. This is primarily a function of the quality of the first collimating lens and the amount it apertures the beam. However, with relatively inexpensive lenses and spatial filtering, one can obtain wavefronts with good Gaussian profiles. This can be done with relatively little loss in power (10\%), but sometimes the optical feedback from the filtering elements can be a problem. If one is forced to work with a laser that is emitting light in a number of transverse modes, a highly structured beam is unavoidable unless one sacrifices a substantial amount of power.

\section{B. Amplitude spectrum}

For most applications, the amplitude and spectral characteristics of the light are more important than the spatial characteristics. When compared to other tunable laser sources, the amplitude noise on diode lasers is relatively small, but it can vary considerably depending on the actual laser and its operating conditions. Figure 4 shows the amplitude noise spectrum of a typical single-mode diode laser taken with a fast photodiode and an rf spectrum analyzer. The natural scale for the measurement of laser amplitude noise is the quantum-limited shot-noise level that is indicated on the figure for this detected power. The general structure of the amplitude noise shows peaking at the lowest Fourier frequencies $(f \leqslant 500 \mathrm{kHz})$. At intermediate frequencies ( 500

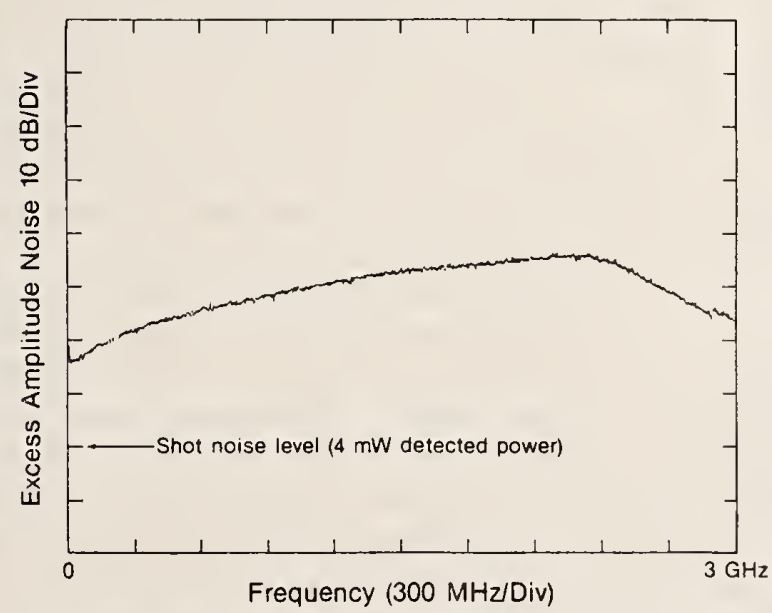

FIG. 4. Spectral distribution of amplitude noise on a typical commercial diode laser operating near $780 \mathrm{~nm}$. The logarithmic vertical scale shows the excess noise on the laser relative to quantum limited shot-noise level for the same detected power level of $4 \mathrm{~mW}$. The laser is operating well above threshold and the peak near $2.4 \mathrm{GHz}$ is due to the lasers relaxation oscillation.
$\mathrm{kHz}<f<3 \mathrm{GHz}$ ) there is a broad plateau that rises to a peak at the laser's relaxation frequency $(2-3 \mathrm{GHz})$, and then drops to the shot-noise level for frequencies above the relaxation frequency. The fundamental noise level is a function of both the injection current and the laser's temperature. ${ }^{6}$ The amplitude noise decreases as the injection current is increased or the temperature decreased. Both of these changes move the relaxation oscillation to higher frequency. Special laser systems have been built to bring the amplitude noise of diode lasers down to, and even below, ${ }^{7}$ the shot-noise level but this performance cannot be expected from standard commercial diode laser systems.

Most atomic physics experiments are done with detection bandwidths centered in the low or intermediate frequency range. In this range, the amplitude noise for a good quality diode laser can be quite low relative to that of a dye laser, typically 10 to $20 \mathrm{~dB}$ above the quantum-limited shot-noise level. This translates into power fluctuations for a typical diode laser of one part in $10^{5}$ for a $10-\mathrm{kHz}$ detection bandwidth. The situation can be much worse with some lasers, particularly when the laser runs multimode in only a few modes; the resulting noise is called mode competition noise. External factors such as fluctuations in the injection current, the temperature, and the optical feedback can increase the amplitude noise substantially. Each of these influence the gain of the diode laser and hence its output power.

The suppliers of diode lasers occasionally provide some information about the lasers' intensity noise, but this information is usually specified in a way that is most useful for applications to consumer electronics. For example, the specifications may give a signal-to-noise ratio $(S / N)$ of 80 $\mathrm{dB}$. This is a measure of the laser's amplitude noise taken in a $10 \mathrm{kHz}$ bandwidth centered at $750 \mathrm{kHz}$. This information is useful but insufficient for many scientific applications.

The peak of the noise at the relaxation oscillation frequency is characteristic of many solid state laser systems ${ }^{8}$ and can be a problem for some applications. The fraction of laser power that is contained in the relaxation oscillation peaks varies considerably from one type of laser to another and even varies for a given laser depending on its operating conditions. For example, the power in the relaxation sidebands can vary by as much as a factor of 100 with variations in optical feedback. We find that good single mode AlGaAs lasers operating near $850 \mathrm{~nm}$ will have relaxation sidebands that are down from the carrier by a factor of $\approx 1000$. In contrast, some of the visible lasers $(670 \mathrm{~nm})$ we have tested show relaxation sidebands that are roughly $10 \%$ of the carrier. The relaxation oscillation sidebands are not always detrimental. As discussed in Sec. V A, they can provide useful optical pumping.

\section{FM spectrum}

There are two natural scales to the oscillation spectrum of diode lasers. The first scale is set by the spacing of the cavity modes of the laser which is on the order of $160 \mathrm{GHz}$ $(\approx 0.35 \mathrm{~nm}$ ). The second is the linewidth of a single cavity mode. At the present time, it is quite routine to obtain "single-mode" lasers in the near infrared region ( 750 to $850 \mathrm{~nm}$, AlGaAs lasers) although the visible $(670 \mathrm{~nm}$, InGaAlP la- 


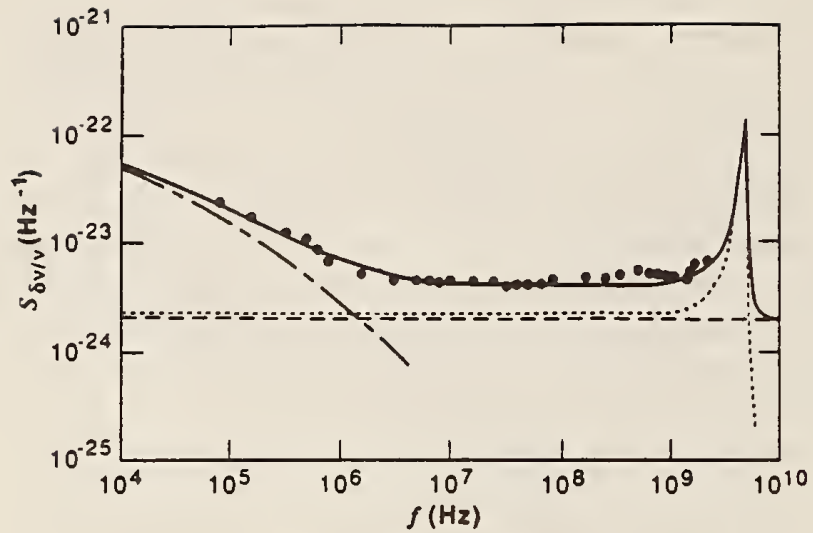

FIG. 5. Spectral density of laser frequency noise for an AIGaAs diode laser. The dots are experimental data which are compared to theory as represented by the lines. The frequency noise peaks at low Fourier frequencies and again at the relaxation oscillation frequency. The theory shows that current induced temperature fluctuations are responsible for the low frequency peak, carrier induced index changes cause the high frequency resonance. and spontaneous emission contributes a flat background noise. (Figure adapted from Ref. 11, with permission.)

sers) and infrared lasers (1300 and $1500 \mathrm{~nm}$, InGaAsP lasers) ordinarily will have their power distributed in many modes. The distributed feedback (DFB) lasers in the infrared will run in a single mode but have a limited tuning range. We might mention a few caveats about nominally single-mode lasers. First, they are not absolutely single mode in that there are small but readily observable amounts of power in numerous other modes that can occasionally be a problem. Second, a laser that has been sold as a single-mode laser will often not run in a single mode for all injection currents. The lasers will always be multimode for very low injection currents, but even at much higher currents there are often ranges of current and temperature where the laser will continue to operate in several modes.

For the remainder of this section we will consider only the linewidth of a single mode. For some applications, in-

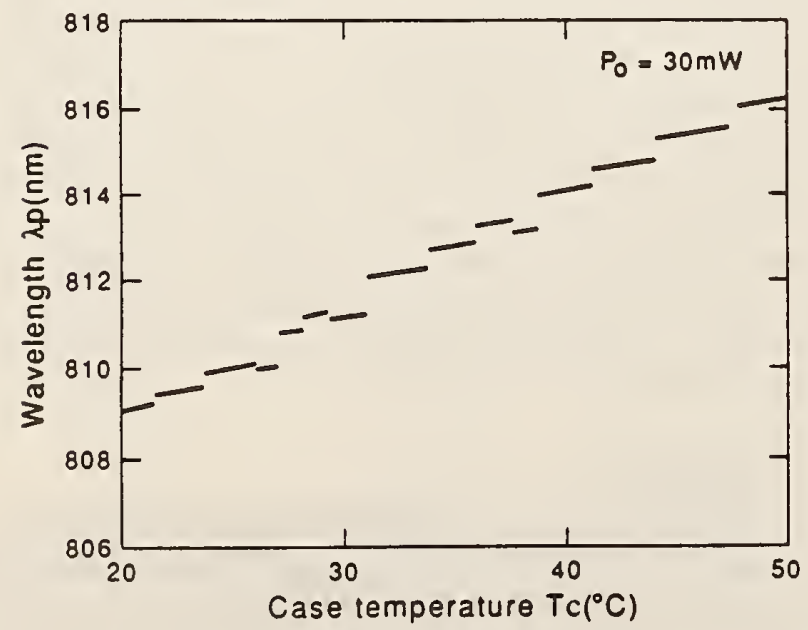

FIG. 6. Laser output wavelength vs the temperature of the laser case. The short continuous segments indicate the tuning of the optical length of the cavity for a given longitudinal mode. When the peak of the gain medium has shifted too far, the laser jumps to another mode. This is indicated by the breaks in the curve. (Figure reproduced from Ref. 4, with permission.) cluding laser spectroscopy, coherent communications, and precision measurements, the diode laser's linewidth can be a serious problem. Many factors contribute to the linewidth of diode lasers; the most fundamental of tnese is that the laser cavities are so short that the Schawlow-Townes linewidth is significant (typically a few $\mathrm{MHz}$ ). 9 The linewidths of common single mode diode lasers are larger than the SchawlowTownes value because of fluctuations in the carriers, the temperature, and in the complex susceptibility of the laser ${ }^{10}$ (typically linewidths vary from about 10 to $500 \mathrm{MHz}$ ). Linewidths of 20 to $50 \mathrm{MHz}$ are typical for low power indexguided lasers. The spectrum of the frequency noise is similar (and coupled) to the spectrum of the amplitude noise. An example of a frequency noise spectrum of a diode laser ${ }^{\prime \prime}$ is shown in Fig. 5. Unfortunately, we see that the frequency noise is large and extends to very high frequencies. The high frequency FM noise is a problem for two reasons: (1) it is difficult to make electronics that are fast enough to correct the frequency fluctuations and (2) even when the linewidth is narrowed by some optical or electronic means there is usually residual high frequency phase noise that contaminates some types of measurements.

\section{Tuning characteristics}

A diode laser's wavelength is determined primarily by the band gap of the semiconductor material and then by the junction's temperature and current density. ${ }^{12}$ The band gap that determines the general range of the laser's wavelength is unfortunately not under the control of the laser user. The range of wavelengths that are readily available is shown in Fig. 3, but a given commercial laser will only (and incompletely) tune over a wavelength interval of about $20 \mathrm{~nm}$. Thus the user must buy a laser that is doped to operate within the tuning range of the wavelength of interest.

The laser frequency tunes with temperature because both the optical path length of the cavity and the wavelength dependence of the gain curve depend on temperature. Unfortunately these temperature dependencies are quite different. For example, in the AlGaAs devices the optical length of the cavity changes about $+0.06 \mathrm{~nm} / \mathrm{K}$, while the gain curve shifts about $+0.25 \mathrm{~nm} / \mathbf{K}$. This results in a temperature tuning curve which, in an ideal device, is a staircase with sloping steps (see Fig. 6). ${ }^{12}$ The slope of each step is just the tuning of that cavity mode, while the jump between steps corresponds to hopping from one longitudinal mode to the next ( $\approx 0.35 \mathrm{~nm}$ ) due to the shifting of the gain curve. The spectral "gaps" encountered in the laser tuning as it jumps from one step to the next are the biggest drawback to using diode lasers in atomic physics. We will discuss below some empirical techniques that have been developed for dealing with this behavior in an isolated laser, as well as techniques for using external optical feedback to reduce or eliminate the problem. In practice, although the overall tuning follows the staircase pattern described, often a laser may jump several cavity modes at once, and then perhaps jump back at a slightly higher temperature as shown in Fig. 6. The choice of which mode the laser will hop to next is often extremely sensitive to optical feedback.

"Room temperature" commercial diode lasers are typi- 
cally rated to operate in a range of $\pm 30 \mathrm{~K}$ about room temperature. This provides a tuning range of about $21 \mathrm{~nm}$ for AlGaAs lasers. The elevated temperatures generally cause noticeable degradation in the laser's lifetime. For example, the data sheets indicate that a typical lifetime of about $10^{5} \mathrm{~h}$ is reduced by a factor of 5 when the temperature is increased by $10 \mathrm{~K}$. Other performance characteristics may also be degraded at higher temperatures. The temperature dependence of the laser characteristics varies substantially for different models and types of lasers; however, detailed information is often provided by the manufacturer. Lasers can actually be cooled far more than $30 \mathrm{~K}$ (experience shows that at least some of the commercial lasers can be operated down to liquid He temperatures); the lower temperatures bring some additional complications such as the need to protect the laser from water condensation and mechanical stress due to large temperature changes. For very large temperature changes one must use the Varshni equation ${ }^{13}$ to predict the wavelength, rather than the linear approximation given above.

In addition to depending on the temperature, the laser wavelength also depends on the injection current. Changes in the injection current affect both the diode temperature and change the carrier density which also changes the index of refraction, and these in turn affect the wavelength. For time scales longer than about $1 \mu$ s the current tuning can simply be thought of as a way to change the temperature rapidly, because the carrier density contribution to the index-of-refraction tuning is relatively small. The current affects the junction temperature because of Joule heating, and the resulting tuning curve for wavelength versus current looks much the same as that versus temperature. Only for times shorter than $-1 \mu \mathrm{s}$ is the temperature rise small enough that the carrier density effect is not overwhelmed. ${ }^{1+}$ For typical AlGaAs diode lasers the variation in the lasers' frequency is $\approx-3 \mathrm{GHz} / \mathrm{mA}$ for frequencies below about 1 $\mathrm{MHz}$, then it drops to $\approx-300 \mathrm{MHz} / \mathrm{mA}$ for frequencies from 1 to $3000 \mathrm{MHz}$. It rises again to $\approx-1 \mathrm{GHz} / \mathrm{mA}$ at the frequency of the relaxation oscillation (typically $\sim 3 \mathrm{GHz}$ ) and then drops off rapidly above this frequency. The crossover behavior between the different regions of FM response can cause considerable complication in high-speed modulation and frequency control systems for diode lasers.

One of the important advantages of diode lasers over other optical sources is that their amplitude and frequency can be modulated very easily and rapidly by changing the injection current. Unfortunately, when the injection current is modulated, one obtains both AM and FM, and these are not independent. The simplest useful picture of the modulation response of diode lasers is that the AM and FM are both present but with different sensitivities. ${ }^{15}$ Also modulation can be complicated by the fact that the relative phase between the AM and the FM changes as a function of modulation frequency. To a good approximation, the AM and the FM are linear with the injection current but the FM modulation index can be more than ten times the AM index. This means that the amplitude change can be ignored in many atomic physics applications. Thus the laser can be scanned over spectroscopic features and/or jumped back and forth to specific frequencies just by applying the appropriate modulation to the injection current. Applications of various modulation capabilities are discussed in Sec. V B.

\section{E. Visible lasers}

The new visible diode lasers ${ }^{16}$ which operate near 670 $\mathrm{nm}$ are exciting for spectroscopic and other applications. Because of the newness of these devices we have had only limited experience with them. The primary problem with these lasers is their broad spectral width. Most are gain-guided rather than index-guided, which typically means that they will have very poor spectral characteristics. We find that the visible lasers usually run multimode, with limited regions of single-mode operation. In addition, even in the regions of single-mode operation (and with very limited data) the linewidths of unstabilized devices are $300 \mathrm{MHz}$ or larger. They also have a transverse mode structure that has a very large and asymmetric divergence $\left(7^{\circ}\right.$ and $\left.40^{\circ}\right)$. Without compensation, this produces a laser beam with a linear shape rather than a round spot. This structure could be corrected, but it is still difficult to obtain optics designed specifically for these lasers. Finally, the output power of visible diode lasers has a very steep dependence on temperature and injection current which can lead one easily to a light intensity induced laser failure!

\section{PRACTICAL GUIDE TO USING DIODE LASERS}

Having discussed the basic behavior of diode lasers, we will now present some of the practical issues involved in setting up and using diode lasers for atomic physics. Although we do not discuss lasers with feedback in this section most of the discussion applies to that case as well.

\section{A. Purchasing diode lasers}

Probably the single biggest frustration in using diode lasers in atomic physics experiments has nothing to do with scientific or technical issues. Instead it is the difficulty in purchasing diodes that have been doped to emit at the desired wavelength (or close enough to reach it with temperature tuning). Because of this unfortunate fact, it seems worth while to provide some discussion to prepare the potential user for some of the problems that must be handled. These arise because the atomic physics market is an utterly insignificant fraction of the total diode laser market, and diode laser production is overwhelmingly Japanese. Therefore, unless you are fortunate enough to have some direct contact, you will probably be dealing with a distributor who is unfamiliar with your special requirements and rather distant from the supplier.

With this background we will now explain what is involved in getting the laser you desire. In the manufacturing process there will normally be a target wavelength which the company is trying to produce, but there will be some scatter in what is actually produced, and they will advertise this as the range over which lasers are available. Thus the first issue is to get the company to screen the lasers and select the desired wavelength. Some manufacturers of lasers will do this and some will not. However, it is quite common for the local distributor for a company that does select to say that it is 
impossible to get wavelength selected lasers, or simply refuse to handle them. Sometimes extended phone discussions with the national headquarters and/or the distributor will solve this problem. Once you have found a distributor willing to order selected lasers for you, there are still some additional problems. First, if you want a wavelength that is well out on the tail of the distribution of wavelengths produced, you could have a substantial delay. Several years ago there were many anecdotes about 1.5-2 year waits for delivery, but our impression is that in recent years this has happened less frequently, and the quoted delivery times (typically 2-3 months) have been fairly reliable. However, there is still the problem that after waiting several months for lasers, one may receive lasers that have been selected for a wavelength that is not the requested wavelength. Based on our considerable direct and anecdotal experience concerning this phenomenon, the problem usually seems to be with the distributors and their lack of familiarity with handling wavelength selected lasers.

There are two alternatives to going through the time consuming and frustrating purchasing process just described. The first is to buy a wafer doped to your specifications that will then produce a very large number of lasers. The second, and more common, approach is to find a secondary supplier of diode lasers who will provide wavelength selected lasers. There are a number of suppliers who buy large numbers of lasers from various manufacturers and then resell them, often with optional packages including power supplies and/or optics. Frequently such companies will wavelength select lasers from their stock. Of course this is usually more expensive than obtaining them directly from the manufacturer. Electronics distributors are also often willing to select lasers from their stock.

In concluding this discussion we strongly recommend that if you are starting an experiment with diode lasers, buy several lasers at once. Because of the difficulties and delays in purchasing lasers, combined with the possibility that some lasers may never reach the desired transition and others may die abruptly, it is highly advisable to keep a number of spares on hand. Since the typical cost of AlGaAs lasers is $\$ 100$ $\$ 300$, this is a worthwhile investment. However, with the special high power and long wavelength lasers that are more expensive, it may not be feasible to afford this luxury.

\section{B. Mounting of diodes and related optical elements}

Once the laser has arrived, it must be set up for use. Two important initial considerations about mounting the laser, regardless of application, are optical feedback and temperature control. One needs to consider the best arrangement to minimize unwanted optical feedback and, if desired, provide controlled optical feedback for frequency control. This usually determines the overall physical layout. The details of the mounting are then determined by considerations of how to keep the temperature of the laser as stable as possible. This is discussed below. Normally, the optimum design in terms of temperature and mechanical stability, as with any laser system, is one that is as compact as possible and reasonably rigid. However, because diode lasers and associated optics can be much smaller and lighter than other types of lasers,

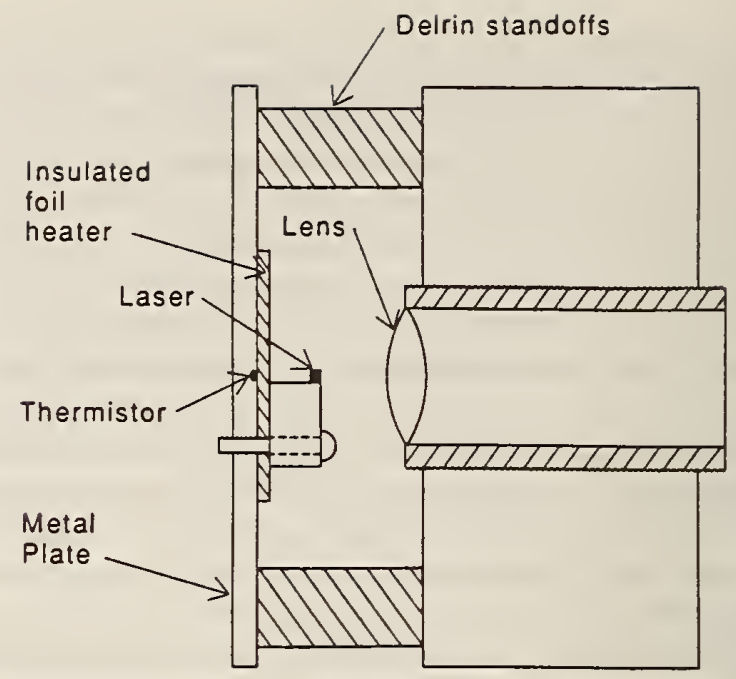

FIG. 7. A simple mount for a diode laser. The heater and thermistor are for stabilizing the temperature. The threaded mount for the collimating lens makes focusing simple.

the scale of the assembly is often quite different.

In Fig. 7 we show a simple laser mount. The laser itself comes attached to a small heat sink. This is mounted with good thermal contact to a small metal plate to which a heater (or thermoelectric cooler) and sensing thermistor are bonded. This plate is then attached to a solid block through a connection that is mechanically rigid, but a poor thermal conductor. The collimating lens is then mounted directly onto this block. A vast variety of types (and prices) of lens are available. Making a rather sweeping generalization, larger lenses that will be mounted farther away from the laser tend to cause less feedback, but it is more difficult to keep the feedback constant than with a small lens close to the laser. The transverse lens position is relatively unimportant; one can simply translate it to the correct position by hand and then tighten down the mounting screw. However, the focusing (longitudinal) adjustment is much more critical, so it is desirable to have some sort of fine screw adjustment on it.

A specific warning to the beginning diode laser user about microscope objectives for collimating lenses: Standard objectives may seem to be a desireable choice because they are so readily available, but they are usually quite lossy for infrared wavelengths, and the optical feedback problems are often quite severe. A superior, low cost alternative is one of the many plastic aspheric lenses made specifically for diode lasers. Another choice when beam quality is not important is the use of inexpensive gradient index (GRIN) lenses.

\section{Temperature and current control}

As a general rule, the day-to-day repeatability of the laser system performance is determined primarily by the temperature stability. The most direct factor is the temperature dependence of the laser output frequency. However, we have found that the benefits of good temperature stability extend beyond the diode itself. If there is any external feedback, the laser frequency is always somewhat sensitive to the positions of the optical elements and these change with temperature. One almost unavoidable example is back scattering 
from the collimating lens that feeds back to the laser. If this is not too large and is fairly constant in phase, often it is not even noticeable. However, if variations in the temperature of the apparatus start causing the distance and hence the phase of the feedback light to vary, it can cause quite unwelcome and, at the time, highly mysterious variations in the laser wavelength or linewidth. Thus, poor temperature control can affect the laser performance in a variety of ways. Although it is adequate for some purposes to use only a single stage of temperature control on the laser, we have found that in the long run it is usually worth the small extra effort to add a second stage of temperature control on the base of the mount or on the box enclosing the laser assembly.

A detailed discussion of temperature control servoloops has been given by Williams ${ }^{17}$ and we will not dwell on them here. The basic idea is to use a thermistor as one leg in a balanced bridge circuit, and any voltage across the bridge is amplified and used to drive a heater or cooler. As discussed in Ref. 17, one must carefully consider the thermal time delays and the time constants in the electronics to achieve optimum performance. However, we have found that if effort is made to reduce the thermal mass and the thermal delays by mounting the sensing thermistor and the laser in close proximity to the heater, a relatively simple circuit will achieve stabilities on the order of $1 \mathrm{mK}$. A circuit diagram for such a simple controller is shown in Fig. 8. To achieve this kind of performance, the reference voltage and the bridge resistors must have low temperature coefficients. In addition because of thermal radiation and air currents, it is advisable to enclose the laser mount in some sort of metal container. This has the added benefits of keeping dust out of the system and isolating the laser system from acoustic vibrations.

The last important element in setting up a diode laser is the current control circuit. It is difficult to recommend a generally useful circuit because the requirements of various experiments can be so different. In every case one needs to start with a low-noise current source and some protection against unwanted transients that can destroy the laser. Such a setup can range from a battery and a potentiometer plus a few diodes and capacitors, to quite elaborate circuits. Often the primary consideration in selecting a circuit is its current modulation capabilities. In Fig. 9 we show two sample circuits. See also Bradley and co-workers. ${ }^{17}$ As discussed in Sec. III G, it is important to mount the protective diode right on the laser mount.

\section{Tuning to an atomic transition}

The main difficulty in using diode lasers in most atomic physics experiments is the problem of tuning them to the desired wavelength, usually that of some atomic transition. We have developed some empirical procedures for addressing this problem. To find a transition we set the laser current at the value we would like to operate, and then tune the temperature of the laser to near the correct value by observing the laser's wavelength on a spectrometer. (A note of warning-if one follows the usual procedure of sending a collimated beam into the spectrometer, which no doubt has metal slits, the backscattered light from the slits will often cause the laser wavelength to shift and make the measure-

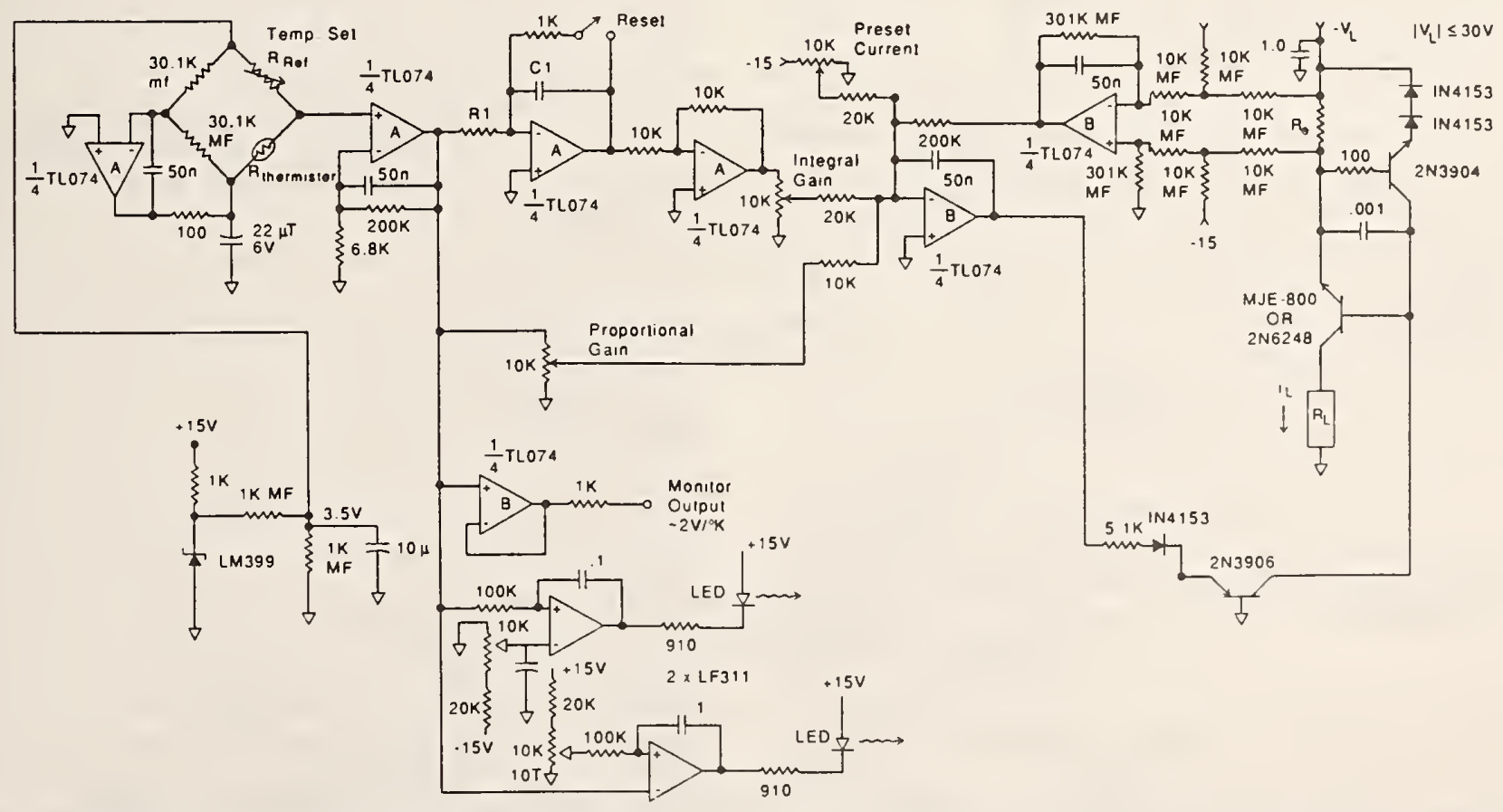

FIG. 8. This simple temperature controller uses a variable combination of proportional and integral feedback and can work as a diode laser controller. The circuit acts to match the resistance of the thermistor with the value of the reference resistor in the bridge. The controller only requires 1 wo IC chips ( 2 - Quad op amps) and a few readily available components. The load $R$, can be either resistive or a Peltier element for heating or cooling. $R I$ and $C l$ determine the integration time constant and are chosen for a given application based on the appropriate system response time. The current is limited to a maximum of 2.4 $V / R_{\text {.. }}$ The LEDs and associated circuitry are optional but useful to indicate whether the temperature is above or below the set point. (This circuit adapted from that of J. Magyar, NIST, Boulder.) 
ment meaningless.) Once the laser is near the correct temperature, the current is then rapidly ramped back and forth over a large range, while the mount temperature is adjusted by small increments. The fluorescence or absorption from an atomic absorption cell is used to determine when the desired transition wavelength is reached. Because of the tuning gaps, there may be no combination of current and temperature that produces the desired wavelength. However, there may also be several and one must choose which is most desirable. If maximum laser power is needed, a temperature should be chosen where the laser is on the transition at a high current. On the other hand, if only a small amount of power is needed it is better to pick a low current point so that the diode lifetime will be maximized (although the linewidth and amplitude noise will be somewhat larger). We repeat our previous warning: Never buy a single laser and expect to do an experi-

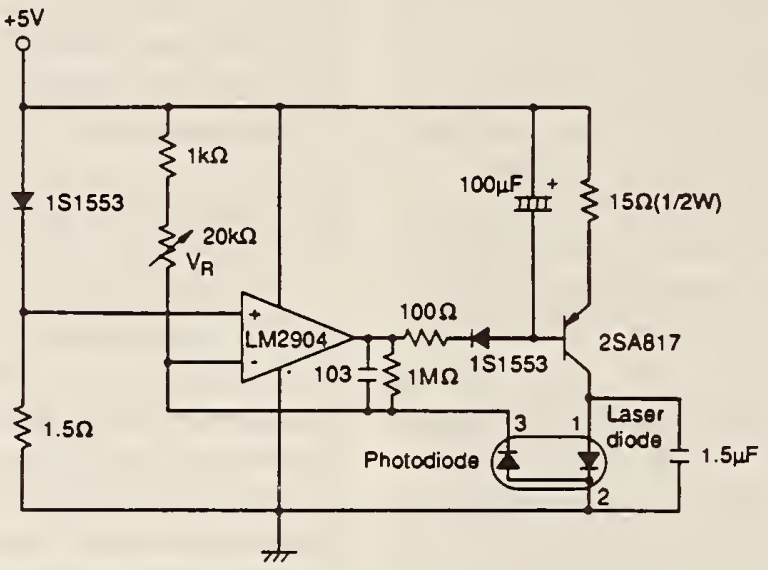

\section{LASER DIODE CURRENT CONTROLER}

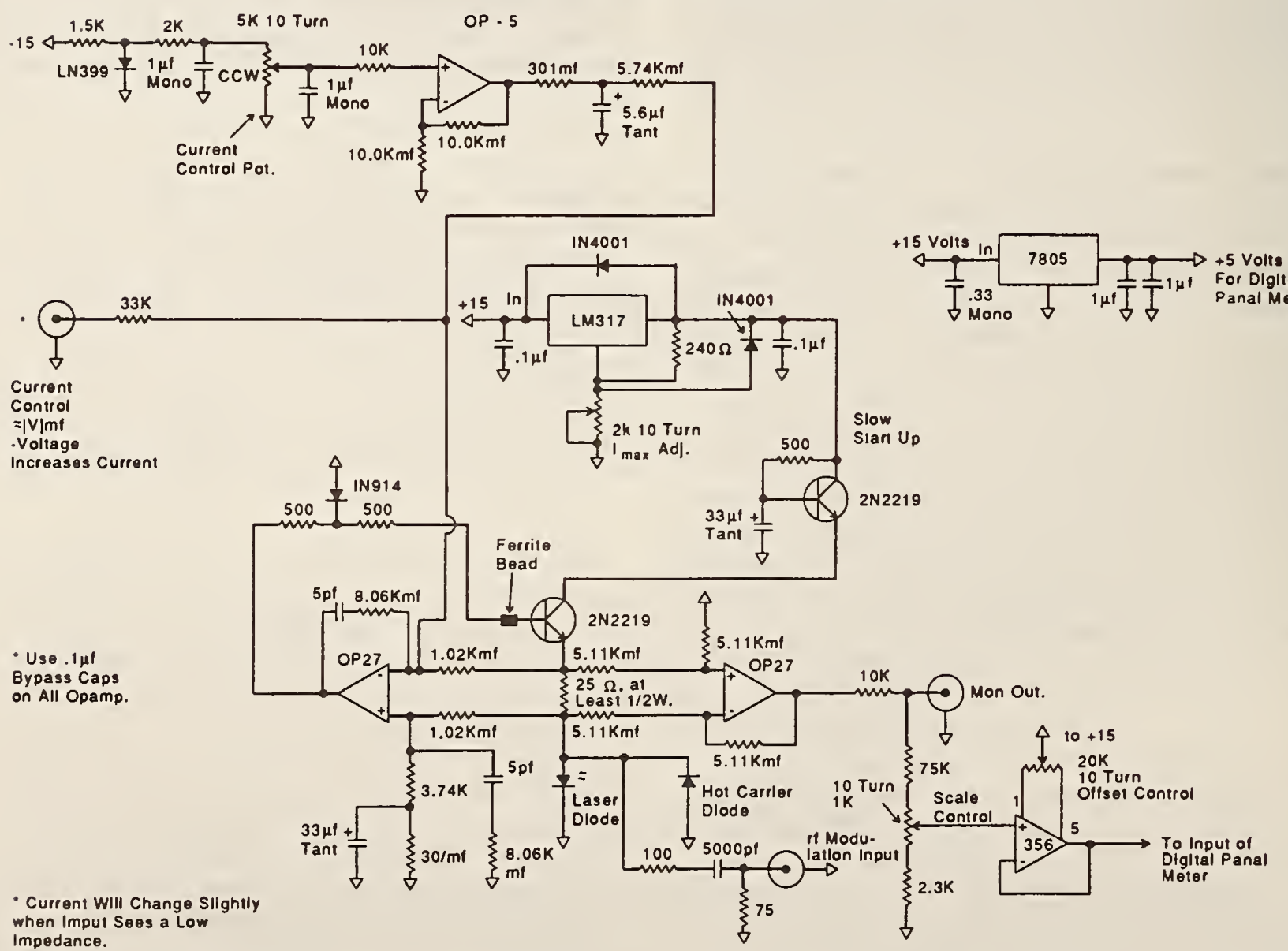

FIG. 9. Two examples of diode laser current sources. (a) A simple diode laser current source that is adapted with permission from the Sharp Corp. (Ref. 4). This circuit has automatic feedback control from a photodiode to maintain constant laser output power. (b) A low-noise diode laser current source that has high speed modulation capabilities. (This circuit with permission from S. Swartz and J. L. Hall.) 
ment with it. There is always a significant probability that a given laser will have a tuning gap just at the output wavelength of interest. We have found that, if no external feedback is used, this probability varies between about $30 \%$ and $60 \%$ for different batches of lasers operating near $852 \mathrm{~nm}$.

As discussed below, additional optical feedback to tune the laser can almost always force it to oscillate at the desired wavelengths, but of course this results in a more complicated device and procedure.

\section{E. Avoiding unwanted optical feedback}

We have mentioned several times that the laser performance is affected by optical feedback. One of the unique features of diode lasers relative to most other lasers is their astonishing sensitivity to such feedback. This can be both a blessing and a curse. In Sec. IV we will discuss the blessing aspect; here we will consider the curse.

As discussed in several references, ${ }^{18}$ the sensitivity of diode lasers to optical feedback arises from a combination of factors. First, the gain curve is very flat as a function of wavelength; second, the cavity finesse is quite low; and third, the cavity is very short. As a result, the overall gain of the system has an extremely weak dependence on wavelength and there are relatively few photons in the cavity, so that the lasing frequency is very easily perturbed. In addition, when light is returned to the laser it acts as a photodetector, generating more carriers in the junction and affecting the net laser gain. Detailed quantitative discussions are given in the references, but it is probably more useful here to give some general rules for laboratory work using a free-running laser with uncoated output facets. We have already mentioned the problem with scattering from spectrometer slits. In general, we can say that if the laser beam is collimated and hits a surface which scatters strongly, such as burnished metal or a white lab wall, there will be a significant effect on the laser wavelength (frequency shifts on the order of $10 \mathrm{MHz}$ or greater, and easily observable variations in the laser amplitude as the distance changes), but if the surface has a dull black appearance there will probably be little effect. If the beam is going through a focus a larger fraction of the scattered light will be focused back into the laser thus increasing feedback sensitivity. We have found that, as a general rule, one cannot put anything, even good optical glass, at a focus without causing a large perturbation on the laser. These rules must be considered only as very crude guides since there are significant differences in sensitivity among the various types of lasers. How much feedback can be tolerated generally depends on how the laser is being used and the stability of the feedback. If the amplitude and phase of the feedback are constant, it can often be ignored unless one needs to scan the laser's wavelength. Lasers with an output facet coated for reduced reflectance are more sensitive to optical feedback than uncoated lasers. On the other hand, lasers set up to have strong optical feedback to control the wavelength are correspondingly less sensitive to stray feedback.

When using a laser in an experiment where simple positioning of the optical elements does not avoid feedback, one must use some form of optical isolator. The simplest and least expensive isolator is an attenuator in the beam, but this of course is useful only when one can afford to waste most of the laser power. An alternative is a circular polarizer that does not waste light, but only works if the incident and feedback light have the same polarization. This is still fairly inexpensive. When the polarization of the return light is different from the incident light, which happens if there are any birefringent elements in the beam, it is necessary to use a Faraday isolator. ${ }^{4}$ Unfortunately these usually cost several times as much as the laser itself.

\section{F. Aging behavior}

A key consideration for any experimenter is the reliability of the laser. With diode lasers this has two different aspects. The first is euphemistically referred to as catastrophic failure, which usually means the experimenter did something to destroy the laser. The second is a gradual change in the behavior of the laser, referred to as "aging." Normally one must gain considerable experience with diode lasers before aging becomes relevant compared to catastrophic failure. We will discuss aging first. Although we have not done careful statistical studies of aging behavior we can provide some observations based on our experiences with on the order of 100 lasers. First, while operation at high temperatures and/or high currents can contribute to aging, the original manufacturing process seems to be the dominant factor. We have had some lasers that we used extensively for two or three years before they showed any aging while others changed markedly in just a few weeks. Usually all the lasers in a given manufacturing run will age in a similar fashion.

There are a variety of ways that the characteristics will change with age. One that is hardly noticeable because it is usually slow is that the tuning steps will gradually move. This means that one will occasionally have to adjust the current and/or temperature to keep the laser on the atomic resonance. This becomes serious if eventually the shift causes one to lose the transition entirely, but the opposite can also happen: namely, a laser which formerly could not be tuned to the resonance may age until it can. Aging drift rates can be as large as $+30 \mathrm{MHz} / \mathrm{h}$ and may be due in part to increases in the thermal conductivity of the laser to the heat $\operatorname{sink}^{2.16 .19}$ and changes in the laser due to nonradiative recombination. There are two other symptoms of aging that are more immediately detrimental. The first of these is an increasing tendency for the laser to emit in more than one longitudinal mode, which often means that higher and higher currents must be used to obtain single mode output. The second characteristic is that the spectral width of the single mode output can gradually increase from its initial 20-30 MHz up to several hundred megahertz.

\section{G. Catastrophic failure modes, or 1001 ways to kill a laser}

In a lab where diode lasers are applied to a variety of problems and there is a constant flux of students and guest workers, it is a highly unusual laser that can age gracefully and die of the "natural causes" listed above. It is far more common for the lasers to be abruptly destroyed. We have 
discovered many ways this can be accomplished, and we list these here in the hope it may save others from repeating our experiences. A major weakness of diode lasers is that a very brief transient, which causes too much current to flow or produces too large a back voltage across the junction, can be fatal. A common way this can happen is switching transients when turning the laser on or off. Although one obviously must put in protective circuitry to deal with transients when the laser power supply is switched on and off intentionally, it is also necessary to be concerned with the potential effects of accidental disconnection of the power supply, or intermittent connections in power cables or the ac power line. Other sources of transients are discharges of static electricity and high voltage arcs in other parts of the lab. These last two are minimized by good grounding and shielding procedures. Also the protection diode normally used to prevent excessive back voltage should be as close to the laser as possible. If there is even a fraction of a meter of moderately shielded cable between the laser and the protective diode, a high voltage spark nearby will often destroy the laser. We should point out that a laser which has been electrocuted will often continue to emit laser light, but the wavelength, threshold, and output spectrum will be quite different.

Although electrocution is by far the most common form of death for diode lasers, the lasers that are not mounted in a protective can are also quite delicate and one must be careful not to touch the laser itself, or the tiny current leads, while mounting the laser or associated optics.

Considering all of the possible failure modes for the diode lasers, we find that, on the average, the lasers need to be replaced approximately every six months. This average includes lasers dying for whatever reason or becoming impossible to tune to the appropriate wavelength. Presumably the replacement rate would be much less if the lasers were left alone and not always being tampered with in evolving experiments. This average rate is for moderately experienced users and failure is much more frequent with inexperienced users.

\section{TECHNIQUES FOR CONTROLLING AND NARROWING LASER OUTPUT SPECTRA}

Several techniques, both optical and electronic, have been devised to narrow the linewidth and control the center frequency of diode lasers. As mentioned earlier, the effects of optical feedback on semiconductor diode lasers are both profound and complicated, but they have been studied in detail. ${ }^{18}$ In particular, the effects of optical feedback on the spectral properties of these lasers can be found in Ref. 20 . However, the essence of the optical feedback methods is the simple idea that by increasing the quality factor $(Q)$ of the laser's resonator, the linewidth will be reduced. The simplest implementation of the optical method for spectral narrowing is just to reflect back to the laser a small fraction of its output power. The basic electronic method uses feedback to the laser's injection current to control the laser's frequency. Both the simple optical and simple electronic methods have severe limitations in terms of general applicability.

More elaborate frequency control systems are usually necessary to deal with the variety of diode lasers that are commercially available. No one method has been found to narrow the linewidth and control the frequency of all types of diode lasers. Here we will briefly review some of the more successful methods.

We should mention that there are many errors that one will find in the literature relative to measurements of diode laser linewidths and frequency stability. The most common mistake is that the experimenters will measure the residual noise on an error signal inside a servo loop and use this information to infer a frequency stability. Such measurements do not guarantee that the error signal actually represents laser frequency fluctuations, and they are fraught with systematic errors. Picque and co-workers ${ }^{21}$ and Pevtschin and Eze$\mathrm{kiel}^{22}$ have made more realistic measurements and pointed out some of the possible pitfalls.

\section{A. Simple feedback}

Some limited success in narrowing the linewidth of a diode laser has been achieved by using simple optical elements to feed back to the laser some of its output power. Among the optical elements that have been used for feedback are simple mirrors, ${ }^{23}$ etalons, ${ }^{24}$ gratings, ${ }^{25}$ fiber cavities, ${ }^{26}$ and phase conjugate mirrors. ${ }^{27}$ The resulting systems must be described as lasers with complex resonators where the diodes' facets and the external optical elements all play a role in creating the net resonator structure as seen by the semiconductor gain medium. By providing optical feedback from a small mirror or glass plate placed close to one of the laser facets, sometimes one can force a multimode laser to oscillate on a single mode, or induce a single-mode laser to tune across the forbidden "gaps." ${ }^{28}$ The limitations to this method are that it does not work with all lasers, and still requires some sort of frequency reference to stabilize the laser's frequency. Also, as with any complex resonator, it can be difficult to achieve long-term stable performance with such systems.

\section{B. External cavity lasers}

The method of the "external cavity laser" uses antireflection (AR) coatings on the diode laser chip, and some external optics to provide the laser resonator. The external optics may contain frequency selective elements such as a grating ${ }^{29}$ and/or etalons, ${ }^{30}$ with the grating being the least expensive and most common. A variety of geometries are possible and one example of an external cavity laser is shown in Fig. 10. For the nonspecialist these systems can be a challenge because they require having the laser diode facets $A R$ coated, which is usually an expensive and nontrivial procedure. In addition one may need access to both output facets, which is often difficult given the packaging of commercial devices. In principle, this method should work with all types of diode lasers, although only a limited number of systems have been built, and they are not commercially available.

A number of variations on the basic external cavity idea have been demonstrated. These include the use of prisms, birefringent filters, and various other clever optical schemes (of particular interest is the paper by Belenov et al. ${ }^{31}$ ). An example is putting AR coatings on one facet of the laser chip and building pseudo-external cavity lasers as discussed in 


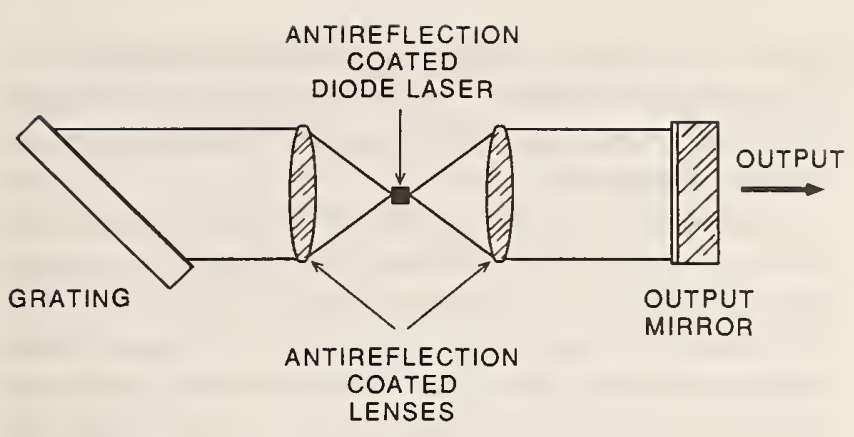

FIG. 10. External-cavity diode laser. This schematic shows an AR coated diode laser chip used in an external cavity with a grating for wavelength selectivity. (Figure from Fleming and Mooradian, Ref. 29, reproduced with permission.)

the next section. This technique has been useful in controlling the spectral properties of $670-\mathrm{nm}$ diode lasers. ${ }^{32}$

\section{Pseudo-external cavity lasers}

Various laboratories have shown that if only one of the facets of a diode laser is AR coated, the laser's linewidth can be narrowed and its oscillation frequency controlled by providing external frequency selective optical feedback. Fortunately, many of the high power ( $>15 \mathrm{~mW}$ ) commercial lasers already have reduced reflectance coatings on the output facet and high reflectors on the back facet. Gibble and Swann and collaborators have demonstrated that without modification these commercial lasers with coatings can be spectrally narrowed and tuned effectively with grating feedback. ${ }^{33}$ An example of such a system is shown in Fig. 11. This method is a combination of the two listed above in that it is not really an external cavity laser, since the laser oscillates without the extra feedback, but the system can operate so that external feedback dominates that from the low reflectance facet. These devices have fundamental linewidths on the order of $100 \mathrm{kHz}$ or less, but the true linewidth is usually dominated by the mechanical and thermal instabilities in the length of the grating-laser cavity. This linewidth can be several hundred kilohertz or larger if the cavity length is not electronically stabilized. We and others have found such lasers to be quite well suited for a variety of applications. Their virtues are that they use standard commercial lasers and inexpensive components, and the grating allows tuning over a range larger than could be easily covered by temperature tuning. Also the tuning can be continuous over much larger spectral ranges, and it is quite easy to set and keep the laser frequency on particular atomic transitions. Minor difficulties in the design are that the grating alignment is moderately sensitive, and to achieve the necessary degree of collimation of the laser light, the distance between the laser and the collimating lens must be adjusted to within a few micrometers or less. In practice it is adequate to have the lens position adjusted with a fine-pitch screw, with the position set so that the beam appears the same diameter to the eye over a path of several meters.

There are tradeoffs one can make in the detailed design between greater feedback, and hence more frequency con-

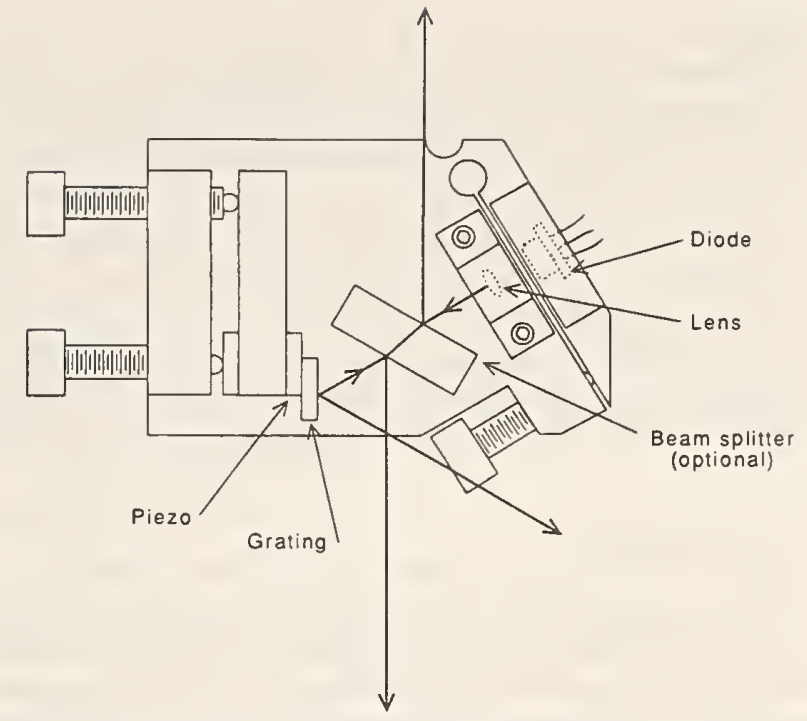

FIG. 11. A pseudo-external cavity laser using a standard commercial laser and a diffraction grating for feedback. The flex pivot is used to obtain the precise focusing of the collimating lens which is necessary. The peizoelectric transducer under the grating is used for fine frequency tuning by changing the length of the cavity. Other tuning options are to have the piezo-electric transducer on the other side of the mount so that it rotates as well as translates the grating, or to do the tuning using an uncoated piece of glass which is mounted on a galvanometer near Brewster's angle and is precisely rotated. Not shown is the temperature control elements (sensors and heaters or coolers) that must be used on the diode and may be used on the baseplate.

trol, versus coupling out more power. The exact performance will depend strongly on the reflectance of the output facet of the laser. For illustrative purposes, we will describe the behavior of such a laser system using a Sharp LT015 laser (mention of this product does not represent a recommendation and we expect other lasers to behave similarly), with $\approx 70 \%$ feedback to the laser. As the grating is rotated, the laser will tune continuously over a region of about 80 $\mathrm{GHz}$ spanning the peak of the semiconductor laser cavity resonance. To obtain continuous tuning, the grating is mounted so that, as it rotates, the change in the cavity resonant frequency due to the change in the cavity length matches the angle tuning of the grating. ${ }^{34}$ A small $(2 \mathrm{~cm})$ piezoelectric speaker disk placed between the grating tilt screw and the block on which the grating is mounted is a convenient and inexpensive way to rotate the grating smoothly. There will then be a gap in the tuning of 70-80 $\mathrm{GHz}$ until one reaches the next mode of the small semiconductor cavity. This pattern repeats itself as the laser is tuned farther from its gain peak, with the range of continuous tuning gradually decreasing. The maximum range the laser wavelength can be pulled from its gain peak is about \pm 10 $\mathrm{nm}$, at which point the tuning range per diode mode is about $10 \mathrm{GHz}$. With the feedback reduced to $25 \%$ to increase the output coupling, the maximum range of continuous tuning is about $\pm 6 \mathrm{GHz}$, and the farthest the frequency can be pulled is about $6 \mathrm{~nm}$. Gaps in the tuning are easily filled by adjusting the laser current or temperature to shift the frequency of the laser diode modes. The output power is about $10 \mathrm{~mW}$ with this output coupling. 


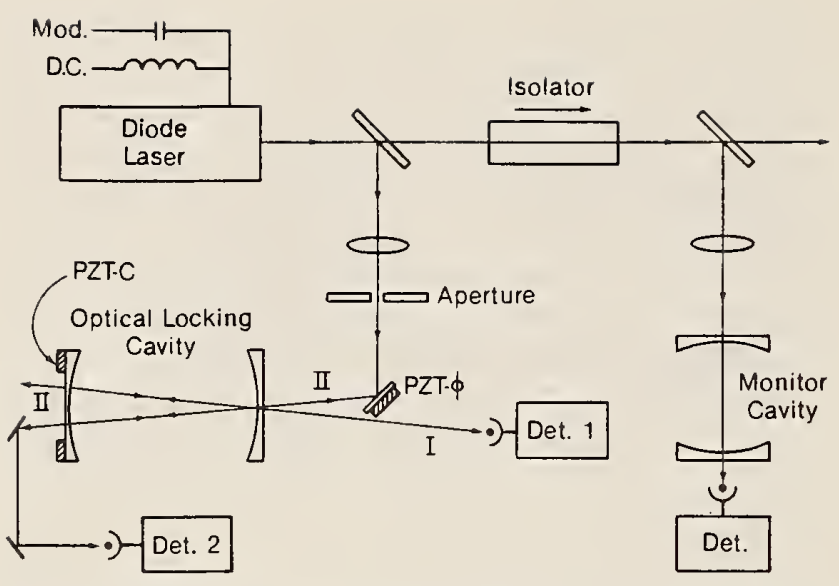

FIG. 12. Schematic diagram of an optical feedback locking system for laser diodes. In the geometry shown optical feedback (type II) occurs when the lasers frequency matches the resonance frequency of the optical locking cavity. This type of feedback locks the laser's frequency to the cavity resonance frequency and narrows the laser's linewidth. The monitor cavity is used as a diagnostic and does not affect the laser's frequency because of the optical isolator. (Figure from Ref. 59, reproduced with permission.)

\section{Feedback from high- $Q$ optical cavities}

Another optical feedback locking system that we have used quite successfully is diagrammed in Fig. 12. In this system we use weak optical coupling of the laser's output to a high- $Q$ optical resonator. ${ }^{35}$ The geometry is such that the laser sees optical feedback from the Fabry-Perot cavity only when the laser's frequency matches a resonance of this cavity. The result is that the coupled (laser plus cavity) system has lower losses at a cavity resonance and the laser's frequency automatically locks up to the cavity resonance. In this way the laser's linewidth can be reduced to a few kilohertz and the laser's center frequency is stabilized to the cavity. One of the limitations of this system is that it requires some additional slow electronics to keep the laser locked to the same cavity mode for long times and to keep the laser synchronized with the cavity for long scans (greater than about $1 \mathrm{GHz}$ ). Without the additional electronics, the laser will stay locked to the same cavity mode for times that vary from less than 1 to as much as $30 \mathrm{~min}$, depending on the stability of the cavity and the temperature and current controls for the laser. By reducing the distance between laser and cavity to a few centimeters and mounting the cavity and laser in a pressure-tight temperature-controlled enclosure, we have been able to extend this to several hours without electronic control. We and others use this system with good results with unmodified commercial lasers operating in the diode laser wavelength bands at 780,850 , and $1300 \mathrm{~nm}$. With rather limited experience, we have been disappointed in the results of this method for unaltered gain-guided lasers operating at $670 \mathrm{~nm}$. However, the method appears to work with the new index-guided versions that have some AR coating. ${ }^{36}$ The major advantages of this system of frequency control are that the linewidth is very narrow and the stability is determined by the external cavity, which can be made very rigid and insensitive to changes in temperature and atmospheric pressure. Also, the laser retains some high speed modulation ca- pabilities. The disadvantages are the sensitivity to cavity laser separation mentioned above, and the fact that laser tuning range (and gaps) is still essentially the same as that of the basic unstabilized laser.

There are a number of optical configurations that can produce resonant optical feedback from a high $Q$ resonator, but in practice the $V$ configuration shown in Fig. 12 is a very convenient one. The alignment procedure is straightforward and after a little practice can be done fairly quickly when one recognizes the proper signal shapes. During the alignment and afterwards it is best to sweep the laser's frequency over several orders of the Fabry-Perot and to monitor the power transmitted through the cavity. The effects of feedback from the cavity to the laser will be obvious in the shape of the transmitted fringe. By sweeping many cavity modes the effects of the feedback can be observed. The system requires only a small amount of the laser's output power $(\sim 1 \%)$ for locking but for alignment it is easier to start with higher powers $(\sim 10 \%)$ so that it is easy to see the beams. After one becomes familiar with aligning the optical feedback lock, the feedback power can be reduced. This $10 \%$ of the output is split off and sent through an attenuator and a lens that approximately mode-matches the beam into the high- $Q$ FabryPerot cavity. An easy way to determine that the mode matching is correct is to ensure that the return beam reflected from the Fabry-Perot cavity is about the same size as the beam that is incident on the cavity. Next, one looks to see that the input beam is centered on the Fabry-Perot's input mirror and that the reflected beam is at a small angle relative to the input beam. The divergence angle can be quite small but it is necessary that the return beam is a few spot diameters away from the input beam by the time it returns to the laser. One should also observe that there are two output beams transmitted through the cavity on resonance (see Fig. 12 ). By setting the attenuator for very low powers one will see the usual Fabry-Perot peak structure in the transmitted light; then as the feedback is increased the laser will begin to lock to the cavity resonance and the Fabry-Perot shape will broaden and become squarish. The fringe will typically broaden to a few hundred megahertz, corresponding to the range over which the cavity controls the laser's frequency. The feedback power can be increased to enhance the locking range and to reduce further the laser's linewidth. When the feedback becomes too strong the system will become unstable. ${ }^{37}$

The phase of optical feedback (distance from the laser to the cavity) is optimum when the power transmitted through the cavity is maximum. One can force the lock to the optimum condition by modulating the path length from the laser to the cavity (with a PZT) and detecting the transmitted power. This signal is then demodulated with a lock-in amplifier and fed back electronically to control the position of the PZT. Variations in the optical feedback phase can be minimized by good mechanical stability and by making the distance from the laser to the cavity small. In fact we often operate these optically locked systems without active electronic control.

Although commercial cavities are often adequate it is useful to be able to make simple inexpensive tunable Fabry- 
Perot cavities. The simplest method is to glue laser quality mirrors onto a cylindrical Invar or quartz tube, with a PZT disk under one of the mirrors so that the cavity can be scanned. If one of the mirrors can slide inside a tubular mount, the cavity can be aligned and set to the confocal condition by aligning it with a laser before the last mirror is glued in place. For the best stability it is useful to have a hermetically sealed cavity. In these simple cavities this can be accomplished with a glue that does not unnecessarily stress the mirrors (such as Tracon 2143D epoxy, mention of this product does not represent a recommendation). An alternative design uses a fine thread on the Invar spacer to set the cavity to the proper length. For most of our diode laser spectroscopy we use reference cavities with finesses of about 100 and free-spectral ranges of $0.25-8 \mathrm{GHz}$.

\section{E. Other optical feedback techniques}

Other monolithic or semimonolithic extended cavity diode lasers with good spectral qualities have been reported in the literature, ${ }^{38}$ but they are usually laboratory test devices that are not commercially available and usually not tunable. There are indications that some of these systems will become commercially available in the near future, but it is not likely there will be broad spectral coverage anytime soon.

\section{F. Electronic feedback}

The other competing method for laser frequency stabilization is electronic feedback. It is straightforward to use electronic feedback to lock the center frequency of diode lasers to cavities or atomic absorption lines using standard laser stabilization methods..$^{39}$ On the other hand, narrowing the laser's linewidth with electronic servos is a much more difficult task. The problem is that the frequency noise spectrum extends to such high frequencies that most servosystems are not able to act fast enough to correct the laser's frequency fluctuations. A few groups ${ }^{4()-42}$ have been successful in developing very fast (few ns delay time) electronic servosystems that can be used to narrow the linewidth of diode lasers if the laser's intrinsic linewidth is not too broad to start with. Linewidths on the order of $100 \mathrm{kHz}$ have been achieved with these techniques. Electronic systems have the advantage over most of the optical methods in that they do not degrade the modulation characteristics of the lasers. However, a disadvantage is that they do not extend the laser's tuning range. Unfortunately, considerable expertise in electronics is required to design and build the necessary circuits.

\section{G. Hybrid systems}

Hybrid designs are of two basic types. In the first case, electronic feedback is simply used to tune a cavity that controls the laser frequency by optical feedback. In the designs described in Secs. IV B, IV C, and IV D, for example, this would entail moving the end mirror or grating using a piezoelectric transducer. Typically this is done to achieve longterm frequency stability by locking the cavity frequency (and thereby the laser) to an atomic or molecular transition.

Promising results have also been obtained with a second type of hybrid system which uses electronic feedback in addition to optical feedback to reduce the laser's linewidth. ${ }^{43}$ In this hytrid optical-electronic system the optical feedback is used to initially narrow the laser to the point that less sophisticated electronics (than that mentioned in Sec. IV F) can further narrow the linewidth and stabilize the laser's frequency. With such systems it should be possible to achieve frequency stabilities that are limited only by one's ability to provide an adequate frequency discriminator. Using this type of servosystem, phase locks between diode lasers have been demonstrated. ${ }^{43}$

\section{H. Injection locking}

It is also possible to stabilize the frequency of diode lasers by optical injection locking. ${ }^{44}$ The frequency of an unstabilized diode laser can be locked to a spectrally narrow, master laser oscillator by coupling a small amount of power from the master laser into the slave diode laser. This technique may be useful for some applications (e.g., generating higher power) but obviously does not meet many spectroscopic needs, because if a good master laser were available the diode laser would not be needed. The notable exception is that injection locking allows one to use the high powers available from arrays or wide channel lasers and still maintain the precise frequency control which can be obtained with a feedback-stabilized diode ${ }^{45}$

\section{Summary}

For some spectroscopic applications the spectral characteristics of commercial diode lasers are good enough as they stand, but for many others that is not the case. Also, unstabilized lasers can be quite difficult to tune in a controlled manner. Unfortunately, none of the existing frequency stabilization methods (perhaps with the exception of the external cavity system) are able to narrow the linewidth and control the frequency of all of the types of semiconductor lasers that are potentially useful for atomic physics. We do not mean to discourage the potential user of high resolution lasers, because at many wavelengths the existing solutions work very well and provide tunable narrow linewidth lasers from commercially available diodes. Such devices have the advantages of very narrow linewidths and excellent amplitude stability, and they are much simpler and more reliable to tune to a particular frequency than are unstabilized lasers.

\section{APPLICATIONS}

Having discussed how to build and use diode lasers, we will now discuss some of their applications in atomic physics. Of the numerous possibilities, we have chosen to describe a few novel applications that demonstrate some of the new capabilities of diode lasers that have not previously been appreciated. Many of the examples are related to our own work. We do not wish to imply that this is the only work in this field; rather these are simply a number of representative samples that are most familiar to us.

\section{A. Optical pumping}

One of the earliest applications of a diode laser to atomic physics was as the light source for optical pumping and prob- 
ing of a polarized medium. Many applications of this type are reviewed by Camparo.' Here we want to mention briefly some interesting new optical-pumping applications using traditional laser approaches.

Diode lasers have been used extensively in frequency standards and atomic clocks. In these applications they optically pump the atoms of interest, and then detect the atoms that have undergone the microwave frequency clock transitions. Fortuitously, the atoms used in some of our best frequency standards (rubidium and cesium) have resonanceline wavelengths that are easily produced by semiconductor lasers. The work in this area started in earnest in the mid70 ' $\mathrm{s}^{46}$ and continues with promising results today. ${ }^{47.48} \mathrm{Sig}$ nificant research and development efforts to incorporate diode lasers into rubidium, cesium, and other atomic frequency standards are under way around the world. These efforts are driven by vast improvements in signal-to-noise ratios, ultimate accuracy, and the relative simplicity obtained by using diode lasers. Figure 13 is a schematic diagram of a diode-laser-pumped cesium atomic clock. The lasers are first used to prepare the atomic beam by putting all the atoms into the appropriate hyperfine level using optical pumping. Then, after the atoms have passed through the microwave region, a laser excites the initially depleted level. Thus the microwave transitions are detected by the increase in the downstream fluorescence.

In an optical-pumping experiment of a different sort, Streater, Mooibroek, and Woerdman ${ }^{49}$ used the light from a diode laser to drive very successfully a rubidium "optical piston." The optical piston is an atomically selective drift that is driven by laser light. We list this under optical pumping because a key element in the experiment was the novel scheme of using the relaxation oscillation sidebands of the diode laser to assist in optical pumping of Rb atoms. In this particular case the 3-GHz ground state hyperfine splitting of ${ }^{85} \mathrm{Rb}$ matches the relaxation oscillation frequency of the 780 $\mathrm{nm}$ lasers. Thus a single laser allowed them to excite atoms in both hyperfine ground states, which greatly enhanced the operation of the "piston."

\section{B. Applications using fast frequency modulation}

One of the unique aspects of semiconductor diode lasers is the ease with which their amplitude and frequency can be modulated. The amount of modulation can be both large and very fast. For common diode lasers the modulation response extends out to a few gigahertz, with a few special lasers capable of modulation rates of more than $10 \mathrm{GHz}$. This modulation capability has applications in spectroscopy as well as other fields. As mentioned earlier, modulation of the diode laser's injection current produces both AM and FM, but in many cases of atomic transitions the frequency modulation is the dominant effect and we will focus attention on it here. The frequency modulation is dominant simply because the atomic linewidth corresponds to such a small fractional change in frequency.

We can picture the frequency modulation of the laser either in the time domain or in the frequency domain, depending on the particular application. If we first think in the frequency domain, modulation of the injection current at high frequency produces a laser spectrum with modulation sidebands. The application of modulation sidebands to all types of lasers has proven very useful for spectroscopy and other applications. ${ }^{50}$ The principal advantage of these optical heterodyne methods is that they increase the signal-tonoise ratio in detecting absorption and dispersion signals. These techniques have been extended to diode lasers by Bjorklund, ${ }^{51}$ Lenth, ${ }^{52}$ and others. ${ }^{53}$ The diode laser case is complicated somewhat by the additional AM that accompanies the desired FM. Lenth in particular has sorted out the effects of having both AM and FM modulation simultaneously. ${ }^{52}$

We have used optical heterodyne methods with frequency modulated diode lasers to detect alkali atoms and optical cavity resonances with high sensitivity. External frequency modulators have been also used with InGaAsP lasers to detect $\mathrm{NH}_{3}$ using FM spectroscopy. ${ }^{54} \mathrm{Ohtsu}^{55}$ and collaborators have achieved excellent results with optically pumped $\mathrm{Rb}$ clocks by using frequency-modulated laser diodes for

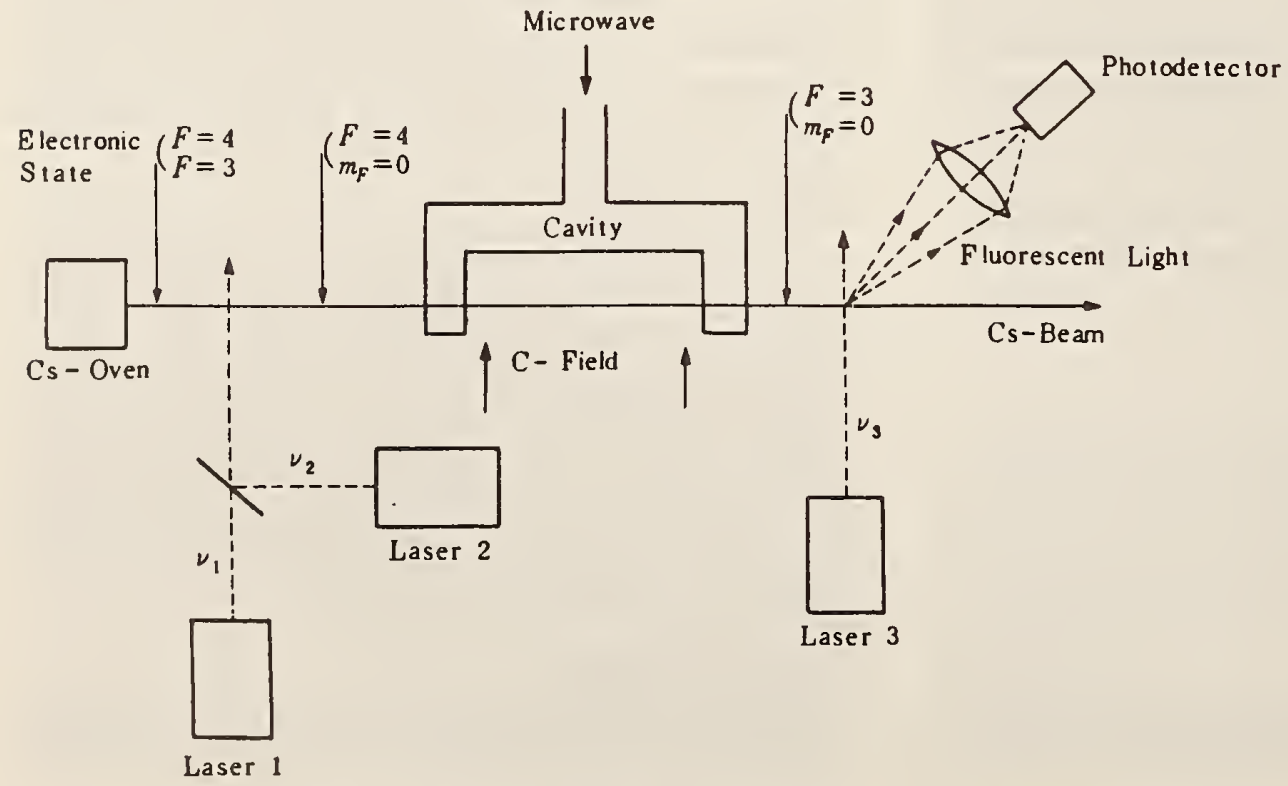

FIG. 13. Diagram of an optically pumped cesium atomic frequency standard. The cesium atomic beam travels in a vacuum through the first laser interaction region where the atoms are optically pumped, it then traverses the microwave cavity region and then on to the second laser interaction region where the atoms are detected. The population in the ground state hyperfine levels is indicated along the path by the $F$ and $m$, values. The microwave clock transition is between the $F=4, m_{l}=0$, and the $F=3, m_{1}=0$ states. As shown here the system uses two lasers with different frequencies for optical pumping and a third laser for detection. (Figure from Ref. 2. reproduced with permission.) 


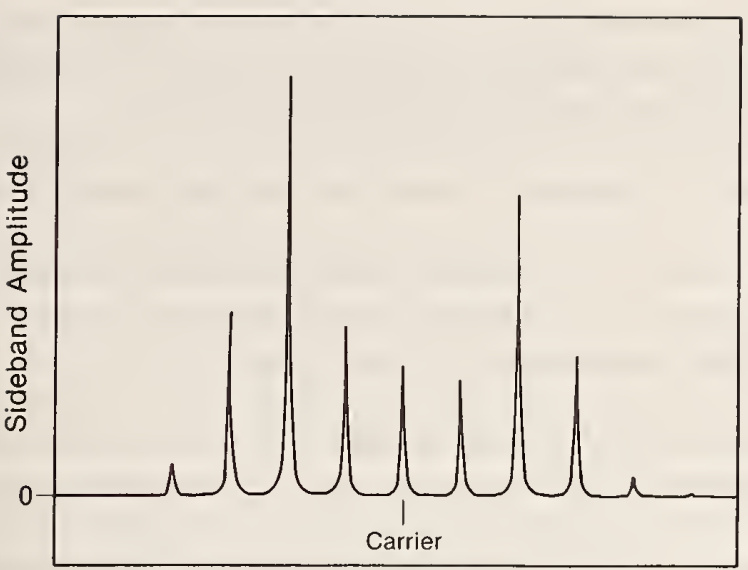

Optical Spectrum, $367 \mathrm{MHz}$ Modulation

FIG. 14. Modulation response of an optically locked diode laser. The spectrum shown here is taken with the detector (Det.) and monitor cavity system shown in Fig. 12. The laser's injection current is modulated at $367 \mathrm{MHz}$ through the modulation port (Mod.). The modulation frequency is rationally related ( case the optical lock is stable and provides an array of narrow linewidth laser side bands each spaced by the modulation frequency. Lasers without optical locking have similar modulation response but with broad linewidths. (Figure from Ref. 57, reproduced with permission.)

optical pumping and high sensitivity detection. Other applications of frequency-modulated diode lasers include communication systems, length measurements, range finding, and laser radar.

A great deal of spectroscopic work with diode lasers and frequency modulation has been done with the lead salt diode lasers operating in the infrared. Methods have been developed using modulation at two frequencies ("two-tone" modulation) to detect molecular species with high sensitivity. ${ }^{56}$ The techniques employed with these lasers are also generally applicable to the GaAs lasers.

If we increase the strength of the modulation of the injection current, it is possible to spread out the diode laser's spectrum into many discrete sidebands. These sidebands can cover spectral windows of many gigahertz. Figure 14 shows a spectrum of a diode laser whose injection current $(97 \mathrm{~mA}$ dc) is modulated at $367 \mathrm{MHz}$ with an amplitude of $4 \mathrm{~mA}$. This modulation produces eight discrete sidebands spaced by the modulation frequency, in addition to the original carrier, and does not otherwise broaden the spectrally narrow laser $(\approx 10-\mathrm{kHz}$ linewidth). Potential applications of this high modulation index case include laser frequency control, fixed local oscillators for coherent communication systems, and atomic and molecular physics experiments where numerous frequencies are required.

Some applications of diode laser frequency modulation are best viewed in the time dcmain. For example, rapid chirping or jumping of the output frequency has been used successfully in several experiments. Watts and Wieman ${ }^{58}$ demonstrated that the output frequency of a laser can be changed by $10 \mathrm{GHz}$ and become stable to within a few megahertz in a time of $2 \mu \mathrm{s}$. This was accomplished by simply changing the injection current through the laser using an appropriately tailored pulse. With this high speed switching it was possible to optically pump all the atoms in a cesium beam into a specific magnetic sublevel $\left(F, M_{F}\right)$ using a single laser. Normally this would be impossible because cesium has two ground state hyperfine levels that are separated by 9.2 $\mathrm{GHz}$. However, in a fraction of the time it took the atomic beam to pass through the laser beam, the laser frequency was switched to excite both levels. Robinson ${ }^{59}$ has also used this frequency jump technique to polarize a rubidium cell with a single laser. This capability to rapidly sweep the laser frequency over a large range in a controlled manner is unique. In these experiments it was used to reduce the number of lasers needed for optical pumping, but it may find greater utility in studies of various types of transient phenomena. ${ }^{1}$

A recent application of the frequency chirping capability of diode lasers is the stopping of atomic beams. Ertmer et $a l .{ }^{60}$ showed that by frequency chirping the light from a dye laser it was possible to slow and stop a beam of sodium atoms. To achieve the necessary frequency scan a sophisticated broadband electro-optic modulator was developed. A corresponding frequency chirp is very easy to produce with a diode laser by merely adding a small sawtooth ramp to the injection current. Watts and Wieman used this technique to stop a beam of cesium atoms ${ }^{\text {6t }}$ with a surprisingly simple and inexpensive apparatus. Salomon et $a l^{62}$ have carried out a similar experiment to study the optimization of the cooling. They showed that by adding $110-\mathrm{MHz}$ sidebands to the laser, they could enhance the number of stopped atoms by $50 \%$. Recently Sheehy et al. ${ }^{63}$ have used frequency chirped diode lasers to stop rubidium atoms. These various experiments demonstrated that, in addition to slowing atoms, the frequency modulation capability of diode lasers is also well suited to probing atomic velocity distributions rapidly enough to avoid distortion.

The resulting $15-\mathrm{m} / \mathrm{s}$ velocity spread of the nearly stopped atoms reported in Ref. 61 was limited by the $30-$ $\mathrm{MHz}$ linewidth of the diode laser. To improve on this, Sesko, Fan, and Wieman ${ }^{64}$ stopped atoms in a similar manner using a cavity-stabilized laser of the type discussed in Sec. IV D. In this case, the frequency was ramped by changing both the laser current and the length of the stabilization cavity (using a piezoelectric transducer). The speed of the cavity response was less than that of the laser, but was still adequate for atom stopping. With a cavity-stabilized stopping laser, the number of stopped atoms was found to improve dramatically and the final velocity spread was substantially smaller. We have obtained similar results by stopping atoms with the light from grating cavity lasers of the type discussed in Sec. IV C.

\section{High resolution spectroscopy}

We mentioned briefly the improvements that cavity locked lasers have made for atom stopping. This is only one of a large number of experiments made possible by optical feedback stabilization. This technique provides a very narrow bandwidth source which is valuable for high resolution spectroscopy and other experiments where narrow linewidth is important. Although often neglected in the English literature, a great deal of forefront work on diode laser stabilization and applications to high resolution spectroscopy has been done by Velichanski and co-workers. ${ }^{65}$ Other examples of high resolution and high sensitivity spectrosco- 


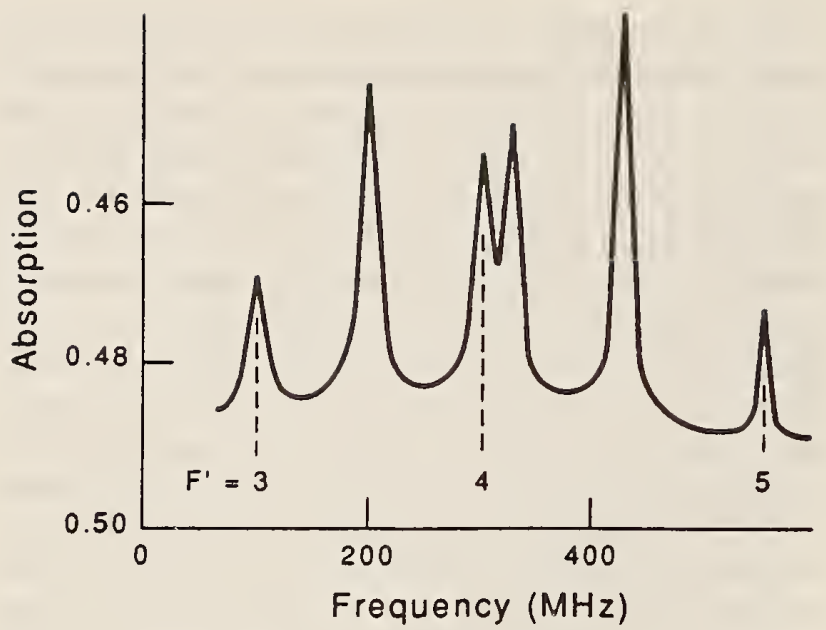

FIG. 15. A saturated absorption spectrum of the $6 S$ to $6 P_{3} / 2$ transition in cesium obtained using a stabilized diode laser. This spectrum was a single oscilloscope trace with no signal processing except the subtraction of the Doppler broadened absorption line. This demonstrates the signal-to-noise ratios that can be obtained with diode lasers. The diode linewidth gave a negligible contribution to the resolution ( $10 \mathrm{MHz}$ for the narrowest. line).

py are described in Refs. 66-69. Figure 15 illustrates the capabilities of cavity stabilized lasers for high resolution spectroscopy. This figure shows a fluorescence spectrum of the $6 S-6 P_{3 / 2}$ transition in cesium observed in a single $100-\mathrm{ms}$ oscilloscope trace. The spectral linewidths were limited entirely by the $6-\mathrm{MHz}$ natural linewidth of the transition, and had no contribution from the laser linewidth. Figure 15 also illustrates how the amplitude stability of the laser leads to very good signal-to-noise ratios. This laser was used to make precise measurements of the Stark shifts and the hyperfine structure of the $6 P_{3 / 2}$ state of cesium. These measurements had an uncertainty of only $20 \mathrm{kHz}$.

Good examples of applications that use the unique modulation capabilities of diode lasers for precision spectroscopy

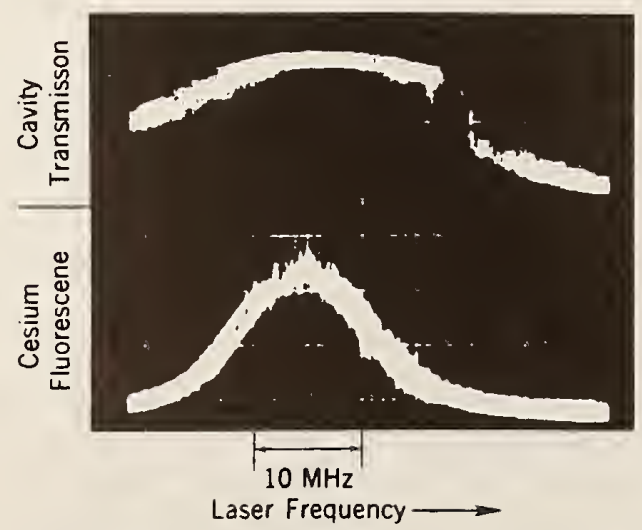

FIG. 16. Fluorescence measurements of the $6 S F=4$ to $6 P_{1,2} F=5$ resonance line in a cesium atomic beam. The laser's frequency noise causes excess amplitude noise on the cesium fluorescence signal. This noise is surprisingly irregular as can be seen by comparing it to the noise observed on the Fabry-Perot cavity transmission signal that was taken simultaneously. We see the frequency noise shows up on the sides of the cavity transmission fringe whereas it is enhanced on the peak and one side of the fluorescence signal. This noise disappears when the lasers linewidth is narrowed. are the work of Martin and co-workers. ${ }^{68}$ They have demonstrated time-resolved spectroscopy of $\mathrm{BaCl}$ and velocitymodulation spectroscopy of $\mathrm{N}_{2}^{+}$.

A vila et al.$^{69}$ have used diode lasers to make a high precision measurement of the absolute energy of the $6 P_{3 / 2}$ state in cesium. The laser frequency was locked to the cesium resonance line by observing fluorescence from a collimated cesium beam. A wavemeter was then used to measure the absolute frequency to $12 \mathrm{MHz}$ ( 3 parts in $10^{8}$ ).

In the work of Gibble and Gallagher, ${ }^{67}$ a pseudo-external cavity laser with grating feedback was used to study velocity changing collisions in rubidium. Using a saturated absorption technique and this continuously tunable narrowband laser, they were able to measure collisional distortions of the saturated absorption line shapes which were a small fraction of the $10-\mathrm{MHz}$ natural linewidth.

Feedback-stabilized diode lasers are uniquely simple and inexpensive sources of very narrowband tunable light. For this reason, they will undoubtedly be widely used for high resolution spectroscopy in the future.

\section{High sensitivity spectroscopy}

Another developing area of application of diode lasers is to high sensitivity spectroscopy. This includes the application to optical pumping, optical heterodyne or FM spectroscopy,${ }^{51-53}$ ultrasensitive detection, ${ }^{70}$ optogalvanic spectroscopy, ${ }^{71}$ and other areas. ${ }^{68}$ Also, significant work in sensitive spectroscopy has been done using the lead salt diode lasers ${ }^{72}$ which we have not addressed in detail in this article. Often these applications are just extensions (to diode lasers) of well-established techniques of spectroscopy. On the other hand, there are a few applications that utilize the unique capabilities of the diode lasers.

Diode lasers stabilized by optical feedback have been found to be much better than unstabilized lasers for high precision measurements of fluorescence or absorption where the signal-to-noise ratio is important. In some cases the feedback not only reduces amplitude noise on the laser, but also reduces some previously unexpected noise on atomic spectra associated with the laser FM spectrum. In using diode lasers for precision spectroscopy some workers have reported that the measured signal-to-noise ratios are much lower than expected. ${ }^{73}$ For example, we observe large amounts of excess noise in diode laser-induced fluorescence and direct absorption measurements of the $F=4$ to $F=5$ resonance line in cesium (see Fig. 16). This noise results from the frequency fluctuations in the diode laser and is eliminated when the diode laser's linewidth is reduced by using the high- $Q$ cavity feedback lock. Two surprising features are observed in this excess noise relative to what one would expect from simple rate equation analysis of the laser induced transition: first, it is much too large; and second, the noise can also be asymmetric with respect to laser detuning from the resonance. By properly treating coherence and atomic saturation with a noisy laser source, Zoller and co-workers ${ }^{74}$ have provided an explanation of these observations.

Two significant instances in which the elimination of this noise has been a major concern are the new optically pumped cesium clocks being const ructed at NIST and other 
national standards labs ${ }^{48}$ and the precise measurement of parity nonconservation in cesium using an optically pumped beam. ${ }^{75}$ In these experiments the use of feedback-stabilized lasers has been essential because of the reduced noise in the atomic fluorescence.

\section{E. Self-locking power buildup cavities}

Another application of feedback from high $Q$ cavities is the self-locking power-buildup cavity, which can provide large enhancements in laser power. One obvious shortcoming of diode lasers is the small output power, but buildup cavities allow one to overcome this limitation in some experiments where there is little optical absorption. The basic idea of such a cavity is the same as discussed in Sec. IV D, but the optics are arranged so that a large fraction of the laser power is coupled into the locking cavity. This results in the intracavity power becoming much higher than the output power of the laser. Buildup cavities have been used with lasers for many years; however these always required some electronic feedback system to keep the cavity and laser frequencies the same. With a diode laser, the need for electronic feedback can be eliminated because the optical feedback locks the laser to the cavity resonance. Enhancements as high as 1500 in the one-way power buildup ${ }^{76}$ have been obtained with such "self-locking buildup cavities." These have provided a circulating power of $45 \mathrm{~W}$.

Tanner, Masterson, and Wieman ${ }^{76}$ used a self-locking buildup cavity to study how a strong blue-detuned standing wave can collimate a cesium atomic beam. Using this technique they were able to reduce the divergence of the beam from 15 to $4 \mathrm{mrad}$ using only a $10-\mathrm{mW}$ laser source.

In another application, over $1 \mathrm{~mW}$ of tunable light at $432 \mathrm{~nm}$ was produced by placing a potassium niobate crystal inside such a self-locking buildup cavity to produce the second harmonic of the diode laser light. ${ }^{77}$ With the proper lasers and crystals, it may be possible to use this approach to achieve several milliwatts of narrowband tunable light over much of the blue region of the spectrum. When this technology is combined with injection-locked diode arrays or arrays that are themselves optically locked to buildup cavities, ${ }^{78}$ many tens of milliwatts will be available. On the other hand, for applications not requiring a large amount of second-harmonic light, Ohtsu ${ }^{79}$ has shown that it is possible to do spectroscopy with the small amount of second-harmonic light that is emitted from ordinary diode lasers.

\section{F. Trapping and cooling atoms using diode lasers}

Another area in which stabilized diode lasers have been used very successfully is in cooling and trapping neutral atoms. Such experiments often require several different laser frequencies with linewidths of less than $1 \mathrm{MHz}$, and thus they are quite expensive to set up using dye lasers. This expense precludes the use of dye laser-cooled and/or trapped atoms in a wide range of atomic physics experiments for which they would otherwise be well suited. The use of cavityand grating-stabilized diode lasers overcomes this problem. Sesko and co-workers ${ }^{64}$ have recently cooled cesium atoms using a three-dimensional standing wave tuned to the red side of the cesium resonance line ( so called "optical molas- ses"). This has produced atomic samples containing $5 \times 10^{7}$ atoms with a temperature of $100 \mu \mathrm{K}$. In a related experiment he and co-workers then observed the cesium "clock" transition between the two ground hyperfine states in this cold sample. ${ }^{80}$ The low temperature allowed an interaction time of $20 \mathrm{~ms}$ and hence a linewidth of only $44 \mathrm{~Hz}$, in an interaction volume of less than $1 \mathrm{~cm}^{3}$.

In other experiments Sesko et al. ${ }^{81}$ have succeeded in trapping atoms using a Zeeman shift spontaneous force trap created with diode lasers. Using four stabilized lasers, samples of up to $4 \times 10^{8}$ atoms were trapped, and trapping times of greater than $100 \mathrm{~s}$ were obtained. ${ }^{81}$ A variety of interactions between the trapped atoms were studied. In both the optical molasses experiments and these trapped atom experiments, features of the diode lasers other than their cost have been important. There were a number of probe studies where very low amplitude noise and ease of rapid frequency changes were invaluable.

Inexpensive diode lasers are proving to be valuable tools for atomic spectroscopy. They offer continuously tunable sources of radiation with linewidths under $100 \mathrm{kHz}$ and powers of $30 \mathrm{~mW}$ or more. They can cover much of the near infrared region of the spectrum and are moving into the visible. These devices can (though they may not) be simple and highly reliable. Their low cost and ease of use make multilaser experiments quite feasible, and more importantly, diode lasers allow laboratories with limited resources to still be involved in "state-of-the-art" laser-atomic physics experiments. These include the areas of laser cooling and trapping, nonlinear optics, and high precision spectroscopy.

\section{ACKNOWLEDGMENTS}

The authors acknowledge valuable discussions with, and contributions made by J. Camparo, J. L. Hall, G. Tino, V. L. Velichanski, M. Ohtsu, H. Robinson, R. Drullinger, R. Hulet, K. Gibble, W. Swann, C. Tanner, B. Masterson, D. Sesko, and R. Watts. Readers can obtain a copy of the references including the titles of the articles by contacting the JILA Publications Office. C. Wieman is pleased to acknowledge support in this work from the Office of Naval Research and the National Science Foundation through the University of Colorado.

' J. C. Camparo, Contemp. Phys. 26, 443 ( 1985).

${ }^{2} \mathrm{M}$. Ohtsu and T. Tako, in Progress in Optics XXV, edited by E. Wolf (Elsevier, Amsterdam, 1988).

M. B. Panish, Proc. IEEE 64, 1512 (1976); H. Kressel and J. K. But'er, in Semiconductor Lasers and Heterojunction LEDs (Academic, New York, 1977); H. C. Casey, Jr. and M. B. Panish, in Heterostructure Lasers Part A and B (Academic, New York, 1978); G. H. B. Thompson, in Physics of Semiconductor Laser Devices (Wiley, New York, 1980); Y. Suematsu, Phys. Today, 32 (1985); W. T. Tsang, Ed., Semiconductors and Semimetals, (Academic, New York, 1985), Vol. 22, Part E; K. Petermann, in Laser Diode Modulation and Noise (Kluwer, Dordrecht, 1988); A Yariv, Quantum Electronics, 3rd ed. (Wiley, New York, 1989).

+Laser Diode User's Manual, Sharp Corporation (1988), p. 17.

${ }^{5}$ D. Kuntz, Laser Focus/Electro-Optics, March 1984, p. 44.

"D. E. McCumber, Phys. Rev. 141, 306 (1966); S. Raab, K. Hoffmann, W. Brunner, and H. Paul, Opt. Commun. 32, 133 (1980); R. Lang and K. Kobayashi, IEEE J. Quantum Electron. QE-16, 347 (1980); C. Henry, IEEE J. Quantum Electron. 18, 259 (1982); Y. Yamamoto, IEEE J. Quantum Electron. 19, 34 (1983); Y. Yamamoto. S. Saito, and T. Mukai, IEEE J. Quantum Electron. 19, 47 (1983); T. Mukai and Y. Yamamoto, 
Electron. Lett. 20, 29 (1983); K. Sato, IEEE J. Quantum Electron. 19. 1380 (1983); K. Vahala and A. Yariv, IEEE J. Quantum Electron. 19, 1096, 1102 (1983); P. Hill, R. Olshansky, J. Schafer, W. Powazinik, P-L. Liu, G. Eisenstein, R. S. Tucker, and I. P. Kaminow, Appl. Phys. Lett. 44, 481 (1984); R. J. Land, K. J. Vahala, and A. Yariv, IEEE J. Quantum Electron. QE-21, 443 (1985); R. J. Lang, K. J. Vahala, and A. Yariv, IEEE J. Quantum Electron. QE-21, 443 (1985). R. J. Lang and A. Yariv, IEEE J. Quantum Electron. 22, 436 (1986); P. Hill, R. Olshansky, J. Schafer, and W. Powazinik, Appl. Phys. Lett. 50, 1400 (1987).

Y. Yamamoto, S. Machida, and O. Nilsson, Phys. Rev. A 34, 4025 (1986): S. Machida, Y. Yamamoto, and Y. Itaya, Phys. Rev. Lett. 58, 1000 (1987); S. Machida and Y. Yamamoto, Phys. Rev. Lett. 60, 792 (1988).

${ }^{\star}$ A. Siegman, in Lasers (University Science Books, Mill Valley, CA, 1986), Chap. 25.

Ref. 8, Chap. 11.

M. W. Fleming and A. Mooradian, Appl. Phys. Lett. 38, 511 (1981); D. Welford and A. Mooradian, Appl. Phys. Lett. 40, 865 (1982); C. H. Henry, IEEE J. Quantum Electron. QE-19, 1391 (1983); K. Kikuchi and T. Okoshi, IEEE J. Quantum Electron. QE-21, 1814 (1985); M. Osinski and J. Buus, IEEE J. Quantum Electron. 23, 9 (1987). This last paper contains an extensive list of references.

Y. Yamamoto, T. Mukai, and S. Saito, Electron. Lett. 17, 327 (1981).

M. Nakamura, K. Aiki, N. Chinone, R. Ito, and J. Umeda, J. Appl. Phys. 49, 4644 (1978); A. Dandridge and L. Goldberg, Electron. Lett. 18, 302 (1982); T. Sato, S. Yashima, and M. Shimba, Electron. Lett. 22, 979 (1986); C. M. Klimcak and J. C. Camparo, J. Opt. Soc. Am. B 5, 211 (1988)

Y. P. Varshni, Physica 34, 149 (1967); H. C. Casey and M. B. Panish, in Heterostructure Lasers Part A and B (Academic. New York, 1978).

H. Kressel and J. K. Butler, in Semiconductor Lasers and Heterojunction LEDs (Academic, New York, 1977); P. Melman and W. J. Carlsen, Appl. Opt. 20, 2694 (1981)

S. Kobayashi, Y. Yamamoto, M. Ito, and T. Kimura, IEEE J. Quantum Electron. QE-18, 582 (1982); W. Lenth, IEEE J. Quantum Electron. QE-20, 1045 (1984); D. Welford, IEEE J. Quantum Electron. QE-21, 1749 ( 1985 ); O. Doyle, P. B. Gallion, and G. Debarge, IEEE J. Quantum Electron. 24, 516 (1988).

K. Kobayashi, S. Kawata, A. Gomto, I. Hino, and T. Suzuki, Electron. Lett. 21. 931 (1985); M. Ishikawa, Y. Ohba, H. Sugawara, M. Yamamoto, and T. Nakanisi, Appl. Phys. Lett. 48, 207 (1986); A. Gomyo, K. Kobayashi. S. Kawata, I. Hino, and T. Suzuki, Electron. Lett. 23, 85 (1987); M. Ikeda, H. Sato, T. Ohata, K. Nakano, A. Toda, O. Kumagai, and C. Kojima, Appl. Phys. Lett. 51, 1572 (1987); T. Tawaka, S. Minagawa, and T. Kajimura, Appl. Phys. Lett. 54, 1391 (1989).

J. Williams, Electronic Design, June, 1977; C. C. Bradley, J. Chen, and R. G. Hulet, Rev. Sci. Inst. 61, 2097 (1990).

P. J. Brannon. Appl. Opt. 15, 1119 (1976); R. Lang and K. Kobayashi, IEEE J. Quantum Electron. QE-16, 347 (1980); K. Peterman and G. Arnold, J. Quantum Electron. QE-18, 543 (1982): K. E. Stubkjaer and M. B. Small, IEEE J. Quantum Electron. QE-20, 472 (1984): G. P. Agrawal, IEEE J. Quantum Electron. QE-20, 468 ( 1984 ); G. Acket, D. Lenstra, A. J. Den Boef, and B. H. Verbeek, IEEE J. Quantum Electron. QE-20. 1163 (1984); P. Spano, S. Piazzolla, and M. Tamburrinı, IEEE J. Quantum Electron. QE-20, 350 (1984); M. Yamada, IEEE J. Quantum Electron. QE-22, 1052 (1986); J. Mork, B. Tromborg, and P. L Christiansen, IEEE J. Quantum Electron. 24, 123 (1988); J. Cohen, R. R. Drenten, and B. H. Verbeek, IEEE J. Quantum Electron. 24, 1989 (1988)

F. Favre and D. LeGuen, Electron. Lett. 19, 663 (1983).

W. J. Burke, M. Ettenberg, and H. Kressel, Appl. Opt. 17, 2233 (1978); T. Kanada and K. Nawata, IEEE J. Quantum Electron. QE-15, 559 (1979); H. A. Haus, J. Appl. Phys. 51, 4042 (1980); R. Lang and K Kobayashi, IEEE J. Quantum Electron. QE-16, 347 (1980); F. Favre, D. Le Guen, and J. C. Simon, IEEE J. Quantum Electron. QE-18, 1712 (1982); K. Kikuchi and T. Okoshi. Electron. Lett. 18, 10 (1982); P-L. Liu, G. Eisenstein, R. S. Tucker, and I. P. Kaminow. Appl. Phys. Lett. 44. 481 (1984); C. H. Henry and R. F. Kazarinow, IEEE J. Quantum Elec. tron. QE-22, 294 (1986); G. Duan, P. Gallion, and G. Debarge, Opt. Lett. 12, 800 (1987); R. F. Kazarinov and C. H. Henry, IEEE J. Quantum Electron. QE-23, 1401 (1987); B. Tromberg, H. Olesen, X. Pan, and S. Saito, IEEE J. Quantum Electron. QE-23, 1875 (1987); R. Lang and A. Yariv, IEEE J. Quantum Electron. QE-24, 66 (1988); J. O. Binder and G. D. Cormack, IEEE J. Quantum Electron. 25, 2255 ( 1989 ); D-S. Seo, J-D. Park, J. G. McInerney, and M. Osinski, IEEE J. Quantum Electron.
25. 2229 (1989)

'J-L. Picque and S. Roizen, Appl. Phys. Lett. 27, 340 (1975); M. Arditi and J.-L. Picque, Optics Comm. 15. 317 (1975).

V. Pevtschin and S. Ezekiel, Opt. Lett. 12, 172 (1987).

N. Chinone, K. Aiki, and R. Ito, Appl. Phys. Lett. 33, 990 (1978); T Kanada and K. Nawata, Opt. Commun. 31. 81 (1979); R. P. Salathe, Appl. Phys. 20, I (1979); L. Goldberg, H. F. Taylor, A. Dandridge, J. F. Weller, and R. O. Miles, IEEE J. Quantum Electron. QE-18, 555 (1982); L. A. Coldren and T. L. Koch, J. Lightwave Tech. LT-2, 1045 ( 1984); D. R. Hjelme and A. R. Mickelson, IEEE J. Quantum Electron. QE-23, 1000 (1987); C. J. Chang-Hasnain, J. Berger, D. R. Scifres, W. Streifer, J. R. Shinnery, and A. Dienes, Appl. Phys. Lett. 50, 1465 (1987). This last work used a diode array, rather than a single diode.

${ }^{4}$ H. Hemmati, A ppl. Phys. Lett. 51, 224 (1987); P. B. Coates, J. Phys. E 21,812 (1988).

R. Ludeke and E. P. Harris, Appl. Phys. Lett. 20, 499 ( 1972): R. P. Salathe, Appl. Phys. 20, I (1979) (this reference is a review of theory and experiment for all types of external cavities); M. Ito and T. Kimura, IEEE J. Quantum Electron. QE-16, 69 (1980); H. Sato, H. Asakura. M. Fukai, and N. Suzuki, IEEE J. Quantum Electron. QE-18, 155 (1982); S. Saito, O. Nilsson, and Y. Yamamoto, IEEE J. Quantum Electron. QE-18, 961 (1982) (this paper presents the theory as well as experimental results); H. Olesen, S. Saito, T. Mukai, T. Saitoh, and O. Mikami, Jpn. J. Appl. Phys. 22, L664 (1983).

L. Figueroa, K. Y. Lau, H. W. Yen, and A. Yariv, J. Appl. Phys. 51, 3062 (1980): A. Dandridge and R. O. Miles, Electron. Lett. 17. 273 (1981); F. Favre and D. LeGuen, IEEE J. Quantum Electron. 21, 1937 (1985).

K. Vahala, K. Kyuma, and A. Yariv, Appl. Phys. Lett. 49, 1563 (1986). J. R. Andrews. Appl. Phys. Lett. 47, 71 (1985); Y. Shutong and X. Linzhen, in Laser Diode Optics (SPIE, Bellingham, WA, 1987), p. 8.

L. A. Glasser, IEEE J. Quantum Electron. QE-16, 525 (1980); M. W. Fleming and A. Mooradian, IEEE J. Quantum Electron. QE-17, 44 (1981); R. Wyatt and W. J. Devlin. Electron. Lett. 19, 110 (1983); M. de Labachellerie and P. Cerez, Opt. Commun. 55, 174 (1985); F. Favre, D. Le Guen, J. C. Simon, B. Landousies, Electron. Lett. 22, 795 (1986); A. T. Schremer and C. L. Tang, 1EEE Photo. Tech. Lett. 2.3 (1990).

C. Voumard, Opt. Lett. 1, 61 (1977); W. Fuhrmann and W. Demtroder, Appl. Phys. 49, 29 ( 1989 ); J. Harrison and A. Mooradian, IEEE J. Quantum Electron. 25, 1152 (1989)

E. M. Belenov, V. L. Velichanskii, A. S. Zibrov, V. V. Nikitin, V. A. Sautenkov, and A. V. Uskov, Sov. J. Quantum Electron. 13, 792 (1983) (good summary of frequency stabilization techniques); A. Hardy, W. Streifer, and M. Osinski, Appl. Phys. Lett. 49, 185 (1986); S. Tai, K. Kyuma, K. Hamanaka, and T. Nakayama, Opt. Acta 33, 1539 (1986); M. Ohtsu, K.-Y. Liou, E. C. Burrows, C. A. Burrus, and G. Eisenstein, Electron. Lett. 23, 1111 (1987); M. S. Whalen, K. L. Hall, D. M. Tennant, U. Koren, and G. Raybon, Electron. Lett. 23, 313 (1987); G. A. Coquin and K. W. Cheung, Electron. Lett. 24, 599 (1988).

M. Boshier and E. Hinds (Yale Univ.) (private communication): G. Tino and L. Hollberg (NIST, Boulder) (unpublished).

K. Gibble and W. Swann (Joint Institute for Laboratory Astrophysics, Univ. of Colorado, Boulder) (private communication).

P. McNichol and H. J. Metcalf, Appl. Opt. 24, 2757 ( 1985); F. Favre, D. Le Guen, J. C. Simon, B. Landousies, Electron. Lett. 22, 795 (1986).

"B. Dahmani, L. Hollberg, and R. Drullinger, Opt. Lett. 12, 876 (1987); $\mathrm{Ph}$. Laurent, A. Clairon, and Ch. Breant, IEEE J. Quantum Electron. 25, 1131 (1989); H. Li and H. R. Telle, IEEE J. Quantum Electron. 25, 257 (1989).

R. Hulet (Rice Univ.) (private communication).

B. Tromborg, J. H. Osmundsen, and H. Olesen, IEEE J. Quantum Elec. tron. QE-20, 1023 (1984); H. Olesen, J. H. Osmundsen, and B. Tromborg, IEEE J. Quantum Electron. QE-22, 762 (1986); P. Zorabedian, W. R. Trutna, Jr., and L. S. Cutler, IEEE J. Quantum Electron. QE-23, 1855 ( 1987); J. Mork, B. Tromborg, and P. L. Christiansen, IEEE J. Quantum Electron. 24, 123 (1988); J. Mork, P. L. Christiansen, and B. Tromborg, Electron. Lett. 24, 1065 (1988); H. Li and N. B. Abraham, IEEE J. Quantum Electron. 25, 1782 (1989); Ph. Laurent, A. Clairon, and Ch. Breant, IEEE J. Quantum Electron. 25, 1131 (1989); J. Mork and B. Tromborg. IEEE Photo. Tech. Lett. 2, 21 (1990).

F. Heismann, R. C. Alferness, L. L. Buhl, G. Eisenstein, S. K. Korotky, J. J. Veselka, L. W. Stulz, and C. A. Burrus, Appl. Phys. Lett. 51, 164 (1987); G. Eisenstein, U. Koren, R. S. Tucher, G. Raybon, A. G. Dentai, L. W. Stulz, and B. I. Miller, Appl. Phys. Lett. 50, 1567 (1987); G. A. Coquin and K. W. Cheung. Electron. Lett. 24, 599 ( 1988); N. A. Olsson, C. H. Henry, R. F. Kazarinov, H. J. Lee, K. J. Orlowsky, B. H. Johnson, 
R. E. Scotti, D. A. Ackerman, and P. J. Anthony, IEEE J. Quantum Electron. 24, 143 (1988).

3"' T. Yabuzaki, A. Ibaragi, H. Hori, M. Kitano, and T. Ogawa, Jpn. J. Appl. Phys. 20, L451 (1981); S. Yamaguchi and M. Suzuki, Appl. Phys. Lett. 41, 1034 (1982); H. Hori, Y. Kitayama, M. Kitano, T. Yabuzaki, and T. Ogawa, IEEE J. Quantum Electron. QE-19, 169 (1983); T. Yanagawa, S. Saito, S. Machida, Y. Yamamoto, and Y. Noguchi, Appl. Phys. Lett. 47, 1036 (1985); M. de Labachellerie and P. Cerez, 1986 European Conference on Optics, Optical Systems and Applications, edited by S. Sottini and S. Trigari (SPIE, Bellingham, WA, 1986), Vol. 701, p. 182; Y. C. Chung and R. W. Tkach, Electron. Lett. 24, 804 (1988); Y. C. Chung and C. B. Roxlo, Electron. Lett. 24, 1048 (1988); S. Koizumi, T. Sato, and M. Shimba, Electron. Lett. 24, 13 (1988); S. Sudo, T. Sakai, H. Yasaka, and T. Ikegami, IEEE Photo. Tech. Lett. 1, 392 (1989); S. Sudo, Y. Sakai, H. Yasaka, and T. Ikegami, IEEE Photo. Tech. Lett. 1, 281 (1989).

${ }^{41}$ S. Saito, O. Nilsson, and Y. Yamamoto, Appl. Phys. Lett. 46, 3 (1985); Y. Yamamoto, O. Nilsson, and S. Saito, IEEE J. Quantum Electron. QE-21, 1919 (1985).

${ }^{41}$ M. Ohtsu and S. Kotajima, IEEE J. Quantum Electron. QE-21, 1905 (1985); M. Ohtsu and N. Tabuchi, J. Lightwave Tech. 6, 357 (1988); M. Ohtsu, Opt. Quantum Electron. 20, 283 (1988); M. Ohtsu, J. Lightwave Tech. 6, 245 (1988).

42S. Kobayashi and T. Kimura, IEEE J. Quantum Electron. 17, 681 (1981); H. R. Telle and B. Lipphardt, in Frequency Standards and Metrology, edited by A. DeMarchi (Springer, Berlin, 1989), p. 436.

${ }^{+3}$ S. Swartz and J. Hall (JILA, Univ. of Colorado) (to be published)

${ }^{+4}$ G. R. Hadley, IEEE J. Quantum Electron. QE-22, 419 ( 1986); P. Spano, S. Piazzolla, and M. Tamburrini, IEEE J. Quantum Electron. QE-22, 427 (1986); V. L. Velichanskii, A. S. Zibrov, V. I. Malakhova, V. A. Sautenkov, and N. V. Senkov, Sov. Phys.-Lebedev Inst. Rep. 5, 44 (1986); H. R. Telle, Electron. Lett. 22, 150 (1986).

${ }^{15}$ G. R. Hadley, A. Ow young, and J. P. Hohimer, Opt. Lett. 11, 144 ( 1986); L. Goldberg and M. K. Chun, Appl. Phys. Lett. 53, 1900 (1988); M. K. Chun, L. Goldberg, and J. F. Weller, Opt. Lett. 14, 272 (1989); S-Q. Shang and H. J. Metcalf, Appl. Opt. 28, 1618 (1989).

${ }^{\text {th }}$ M. Arditi and J.-L. Picque, Opt. Commun. 15, 317 (1975); M. Arditi, I. Hiramo, and P. Tougne, J. Phys. D 11, 2465 (1978)

${ }^{47}$ G. Avila, E. de Clercq, M. de Labachelerie, and P. Cerez, IEEE Trans. Instrum. Meas. IM-34, 139 ( 1985); J. C. Camparo and R. P. Frueholz, J. Appl. Phys. 59, 301 (1986); J. C. Camparo and R. P. Frueholz, J. Appl. Phys. 59, 3313 (1986); M. de Labachelerie and P. Cerez, in 1986 European Conference on Optics, Optical Systems and Applications, edited by S. Sottini and S. Trigari (SPIE, Bellingham, WA, 1986), p. 182; G. Avila, V. Giodrano, V. Candelier, E. de Clercq, G. Theobald, and P. Cerez, Phys. Rev. A 36, 3719 (1987); J. C. Camparo and R. P. Frueholz, IEEE Trans. Ultrason. Ferroelectr. Freq. Control UFFC-34, 607 ( 1987); H. K. Haugen, W. P. Hess, and S. R. Leone, in Advances in Laser Science - 1l, Optical Science and Engineering Series 8, edited by M. Lapp, W. C. Stwalley, and G. A. Kenney-Wallace (AIP Conf. Proc. No. 160, 1987); V. Giordano, A. Hamel, G. Theobald, P. Cerez, and C. Audoin, Metrologia 25, 17 (1988); S-I. Ohshima, Y. Nakadan, T. Ikegami, Y. Koga, R. Drullinger, and L. Hollberg, IEEE Trans. Instrum. Meas. 38, 533 (1989); S. Ohshima, Y. Nakadan, and K. Koga, IEEE Trans. Instrum. Meas. IM37, 409 (1988)

${ }^{\text {tx }}$ L. L. Lewis, F. L. Walls, and D. A. Howe, in Precision Measurements and Fundamental Constants II, edited by B. N. Taylor and W. D. Phillips, Natl. Bur. Stand. Spec. Publ. 617 (1984); L. L. Lewis, D. A. Howe, S. R. Stein, C. Manney, M. Mohler, J. C. Bergquist, D. J. Wineland, and D. F. Walls; R. E. Drullinger, Proc. 1EEE 74, 140 (1986); R. E. Drullinger, J. Shirley, D. J. Glaze, L. W. Hollberg, and A. DeMarchi, in Proc., 40th Annual Symposium on Frequency Control, Philadelphia, 428 (1986); J. C. Camparo and R. P. Frueholz, J. Appl. Phys. 59, 3313 (1986); J. C. Camparo and R. P. Frueholz, IEEE Trans. Ultrason. Ferroelectr. Freq. Control UFFC-34, 607 (1987).

4" A. D. Streater, J. Mooibroek, and J. P. Woerdman, Appl. Phys. Lett. 52, $602(1988)$

${ }^{50}$ G. C. Bjorklund, Opt. Lett. 5, 15 ( 1980); J. L. Hall, L. Hollberg, T. Baer, and H. G. Robinson, Appl. Phys. Lett. 39, 680, 1981; L. Hollberg, M. Long-sheng, M. Hohenstatt, and J. L. Hall, in Proceedings of the International Society for Optical Engineering, Laser-based Ultrasensitive Spectroscopy and Detection V, Aug. 23 and 24, edited by R. A. Keller (San Diego, CA, 1983), Vol. 426.

${ }^{5}$ D. T. Cassidy and J. Reid, Appl. Phys. B 29, 279 (1982); P. Pokrowsky, W. Zapka, F. Chu, and G. C. Bjorklund, Opt. Commun. 44, 175 (1983). ${ }^{2}$ W. Lenth, Opt. Lett. 8, 575 (1983); W. Lenth, IEEE J. Quantum Elec- tron. QE-20, 1045 (1984)

${ }^{53}$ M. Hashimoto and M. Ohtsu, J. Opt. Soc. Am. B 6, 1777 (1989); L.-G Wang, D. A. Tate, H. Riris, and T. F. Gallagher, J. Opt. Soc. Am. B 6, 871 (1989).

${ }^{54}$ T. Yanagawa, S. Saito, S. Machida, and Y. Yamamoto, Appl. Phys. Lett. 47, 1036 (1985).

${ }^{5}$ M. Hashimoto and M. Ohtsu, IEEE J. Quantum Electron. 23, 446 (1987); M. Hashimoto and M. Ohtsu (unpublished); H. Furuta and M. Ohtsu, Appl. Opt. 28, 3737 (1989)

J. Jeid, M. El-Sherbiny, B. K. Garside, and E. A. Ballik, Appl. Opt. 19, 3349 ( 1980); D. T. Cassidy and J. Reid, Appl. Phys. B 29, 279 (1982); D. E. Cooper and J. P. Watjen, Opt. Lett. 11, 606 (1986); D. E. Cooper and R. E. Warren, J. Opt. Soc. Am. B 4, 470 ( 1987); C. Clinton and D. Cooper, Opt. Lett. 14, 1306 (1989).

${ }^{57}$ L. Hollberg and M. Ohtsu, Appl. Phys. Lett. 53, 944 (1988).

${ }^{5} \mathrm{R}$. Watts and C. Wieman, Opt. Commun. 57, 45 (1986)

${ }^{64} \mathrm{H}$. Robinson (Duke Univ.) (private communication).

${ }^{60}$ W. Ertmer, R. Blatt, J. L. Hall, and M. Zhu, Phys. Rev. Lett. 54, 996 (1985).

'R. N. Watts and C. E. Wieman, Opt. Lett. 11, 291 (1986)

${ }^{\prime 2}$ C. Salomon, J. Dalibard, A. Aspect, H. Metcalf, and C. Cohen-Tannoudji, Phys. Rev. Lett. 59, 1659 (1987); C. Salomon, H. Metcalf, A. Aspect, and J. Dalibard, in Laser Spectroscopy VII, edited by W. Persson and S. Svanberg (Springer, Berlin, 1987), p. 404; C. Salomon and J. Dalibard, C. R. Acad. Sci. Paris 306, 1319 (1988); C. Salomon and J. Dalibard, in Frequency Standards and Metrology, edited by A. De Marchi (Springer, Berlin, 1989); J. Dalibard, C. Salomon, A. Aspect, E. Arimondo, R. Kaiser, N. Vansteenkiste, and C. Cohen-Tannoudji, in Proceedings of the 11th International Conference on Atomic Physics, edited by S. Haroche, G. Grynberg, and J. C. Gay (World Scientific, Singapore, 1989)

B. Sheehy, S-Q. Shang, R. Watts, S. Hatamian, and H. Metcalf, J. Opt. Soc. Am. B 6, 2165 (1989).

${ }^{+4}$ D. Sesko, C. G. Fan, and C. E. Wieman, J. Opt. Soc. Am. B 5, 1225 (1988).

"Yu. A. Bykovskii, V. L. Velichanskii, I. G. Goncharov, and V. A. Maslov, Sov. Phys. Semicond. 4, 580 (1970); V. L. Velichanskii, A. S. Zibrov, V. S. Kargopol'tsev, O. R. Kachurin, V. V. Nikitin, V. A. Sautenkov, G. G. Kharisov, and D. A. Tyurikov, Sov. J. Quantum Electron. 10, 1244 (1980); V. A. Sautenkov, V. L. Velichanskii, A. S. Zibrov, V. N. Luk'yanov, V. V. Nikitin, and D. A. Tyurikov, Sov. J. Quantum Electron. 11, 1131 (1981); Y. A. Bykovskii, V. L. Velichanskii, I. G. Goncharov, A. P. Grachev, A. S. Zibrov, S. I. Koval, and G. T. Pak, Sov. J. Quantum Electron. 19, 730 (1989).

${ }^{6}$ C. E. Tanner and C. E. Wieman, Phys. Rev. A 38, 162 (1988); C. E. Tanner and C. E. Wieman, Phys. Rev. A 38, 1616 ( 1988); G. M. Tino, L. Hollberg, A. Sasso, M. Inguscio, and M. Barsanti (to be published).

${ }^{17}{ }^{7} \mathrm{~K}$. E. Gibble and A. C. Gallagher, in XVI ICPEAC (in press).

${ }^{\mathrm{w} x}$ T. Gustavsson and H. Martin, Phys. Scripta 34, 207 (1986); B. Lindgren, H. Martin, and U. Sassenberg, in Laser Spectroscopy VIII, edited by W Persson and S. Svanberg (Springer, Berlin, 1987), p. 402; G. Gustafsson, H. Martin, and P. Weijnitz, Opt. Commun. 67, 112 (1988)

${ }^{\prime \prime \prime}$ G. A vila, P. Gain, E. de Clercq, and P. Cerez, Metrologia 22, 111 (1986). ${ }^{71)}$ J. Reid, M. El-Sherbiny, B. K. Garside, and E. A. Ballik, Appl. Opt. 19, 3349 (1980); W. Lenth and M. Gehrtz, Appl. Phys. Lett. 47, 1263 (1985); L. G. Wang, H. Riris, C. B. Carlisle, and T. F. Gallagher, Appl. Opt. 27, 207d (1988); C. Clinton and D. Cooper, Opt. Lett. 14, 1306 (1989); L.-G. Wang, D. A. Tate, H. Riris, and T. F. Gallagher, J. Opt. Soc. Am. B 6, 871 (1989)

S. Yamaguchi and M. Suzuki, Appl. Phys. Lett. 41, 597 (1982); S. Yamaguchi and M. Suzuki, IEEE J. Quantum Electron. QE-19, 1514 (1983). D. T. Cassidy and J. Reid, Appl. Phys. B 29, 279 (1982); M. Gehrtz, G. C. Bjorklund, and E. A. Whittaker, J. Opt. Soc. Am. B 2, 1510 (1985); J. S. Wells, A. Hinz, and A. G. Maki, J. Mol. Spectrosc. 114, 351 (1985); D E. Cooper and J. P. Watjen, Opt. Lett. 11, 606 (1986); D. E. Cooper and R. E. Warren, J. Opt. Soc. Am. B 4, 470 (1987); D. E. Cooper and R. E. Warren, Appl. Opt. 26, 3726 (1987); A. Hinz, J. S. Wells, and A. G. Maki, Z. Phys. D 5, 351 (1987); D. E. Cooper and C. B. Carlisle, Opt. Lett. 13, 719 (1988); C. B. Carlisle and D. E. Cooper, Appl. Opt. 28, 2567 (1989); C. Clinton and D. Cooper, Opt. Lett. 14, 1306 (1989).

V. Giordano, A. Hamel, G. Theobald, P. Cerez, and C. Audoin, Metrologia 25, 17 (1988): L. Hollberg, Applied Laser Spectroscopy, NATO ASI series, edited by M. Inguscio and W. Demtroder (Plenum, New York) (in press).

${ }^{74}$ Th. Haslwanter, H. Tirsch, J. Cooper, and P. Zoller, Phys. Rev. A 38 , 
5652 (1988); H. Ritsch. P. Zoller, and J. Cooper, Phys. Rev. A 41, 2653 (1990).

"S. L. Gilbert and C. E. Wieman, Phys. Rev. A 34, 792 (1986); M. C. Noecker, B. P. Masterson, and C. E. Wieman, Phys. Rev. Lett. 61, 310 (1988).

${ }^{76}$ C. E. Tanner, B. P. Masterson, and C. E. Wieman, Opt. Lett. 13, 357 (1988). In subsequent work we have achieved higher buildup (1500) and higher circulating power ( $45 \mathrm{~W})$.
"G. J. Dixon, C. E. Tanner, and C. E. Wieman, Opt. Lett. 14. 731 (1989). ${ }^{7 *}$ M. K. Chun, L. Goldberg, and J. F. Weller, Appl. Phys. Lett. 53, 1170 (1988).

${ }^{74} \mathrm{M}$. Ohtsu, Electron. Lett. 25, 22 (1989).

N1 D. W. Sesko and C. E. Wieman, Opt. Lett. 14, 269 (1989).

" D. Sesko, T. Walker, C. Monroe, A. Gallagher, and C. Wieman, Phys. Rev. Lett. 63, 961 (1989); T. Walker, D. Sesko, and C. Wieman, Phys. Rev. Lett. 64, 408 (1990). 


\title{
High-Sensitivity Spectroscopy with Diode Lasers
}

\author{
R. W. Fox, H. G. Robinson ${ }^{\dagger}$ A. S. Zibrov ${ }^{\ddagger}$, N. Mackie, J. Marquardt, \\ J. Magyar, L. W. Hollberg \\ National Institute of Standards and Technology \\ 325 Broadway, Boulder, CO 80303
}

\begin{abstract}
Linewidth reduction of an extended cavity diode laser at $657 \mathrm{~nm}$ was accomplished by negative feedback to an intra-cavity ADP crystal. High resolution ( $170 \mathrm{kHz}$ wide) saturated absorption signals were recorded of the calcium intercombination line which is of interest for a frequency standard. The spectrum of the red $6^{2} p_{3 / 2}-9^{2} s_{1 / 2}$ cesium line in a magneto-optical cell trap was also investigated.
\end{abstract}

\section{INTRODUCTION}

The advance of diode lasers into the visible offers opportunities in the area of frequency standards and frequency metrology. Diode lasers stabilized to known spectral features have the potential to be low-cost, high precision frequency markers and length standards. The calcium intercombination line at $657 \mathrm{~nm}$ is one such feature that has generated much interest $^{1,2}$.

An optical frequency reference may in general be realized by first reducing the short term laser frequency fluctuations by locking to a Fabry-Perot cavity, then locking the cavity to an atomic or molecular line. This approach allows enough signal-to-noise in the short term $(<1 \mathrm{~s})$ for the performance to be limited by systematics associated with measuring the spectral feature, such as field shifts or time of flight broadening. A caveat to this is that the lock to the optical cavity must be relatively immune from offset drifts. Offsets may occur, for instance, when the injection current of a diode system is modulated to produce sidebands for a if heterodyne lock. Some fraction of the inherent amplitude modulation (am) is detected along with the desired signal, leading to an intensity dependent $\mathrm{dc}$ term at the output of the phase sensitive detector. Strategies to deal with this include demodulating at $3 \mathrm{f}$ and/or sampling the laser intensity prior to the reference cavity in order to subtract any fluctuations out of the cavity transmission or reflection. However there are both fundamental limits (with regard to the power that must be expended in the sampling channel), and practical limits such as the inhomogeneity of the am across the beam that will induce drift at some level. Thus it is of interest to start with as low residual am from the modulation as possible.

Employing a phase modulator external to the laser cavity to apply sidebands and to reduce the frequency/phase fluctuations is a method that has met with much success. ${ }^{3}$ We have positioned the phase modulator inside the extended cavity, where it serves as a frequency modulator. In comparison an external modulator has much lower gain as a frequency transducer. Although external multiple pass configurations have been used, the poor quality of the diode laser beam makes this approach increasingly difficult. A disadvantage of placing the crystal in the laser cavity is the coupling of the output power to the frequency modulation (fm), albeit at a much lower level than direct injection current modulation. However the injection current channel is now available to be part of a closed loop for intensity control, with a servo bandwidth independent of the frequency control loop. If the intensity control loop bandwidth is extended to cover the fm sideband frequency, the residual am and subsequent dc lock drift will be further decreased. Similar approaches using negative feedback to externally placed modulating crystals to eliminate residual am have worked well. ${ }^{4}$

\section{LASER FREQUENCY NOISE AND LINEWIDTH REDUCTION}

The extended cavity laser consisted of an anti-reflection coated ${ }^{5}$ red diode laser operating near $660 \mathrm{~nm}$, a f/1 collimating lens, a $29 \mathrm{~mm}$ long Brewster-cut ADP modulator crystal, and a high diffraction efficiency holographic grating. ${ }^{6}$ The laser was built on an invar baseplate and enclosed in an aluminum box to reduce acoustics. The grating was mounted on an invar

\section{Contribution of NIST, not subject to copyright}

Proc. SPIE Conference on Frequency Lasers \& their Applications, 1837 (edited by Y.C. Chung) pp. 360-365 (1992). 
support with a simple hinge and a piezo-electric crystal, allowing about $12 \mathrm{Ghz}$ of tuning without mode hops. The zero-order beam from the grating was reflected from a mirror mounted perpendicular to the grating, and served as the output. This configuration reduces the output beam's angular sweep with tuning. The output power was $1 \mathrm{~mW}$ to $1.5 \mathrm{~mW}$, depending on the lasing wavelength.

The spectral linewidth of the unlocked (and locked) laser may be examined by a heterodyne measurement using a similar or more stable laser as the local oscillator. In lieu of building two complete systems, the linewidth may also be studied by observing the laser's frequency noise spectrum. This provides information on the spectral distribution and magnitude of the frequency fluctuations that generate the linewidth. The noise spectrum can be measured by using an independent optical cavity as a discriminator. Locking the laser and discriminator cavity together with a low bandwidth $(-1 \mathrm{~Hz})$ side-lock conveniently cancels drift and permits faster differential frequency fluctuations between the laser and cavity to be accurately converted to intensity fluctuations.

The $12 \mathrm{~cm}$ extended cavity length resulted in a measured frequency noise level of approximately $8000 \mathrm{~Hz} / \mathrm{Hz}$ (see fig. 1). Below about $50 \mathrm{kHz}$ the fluctuations increase in a typical $1 / \mathrm{f}$ fashion, obliging the noise density to rise as $1 / \mathrm{f}^{2}$. A number of distinct acoustic peaks are evident in figure 1; these result from structural resonances of the grating mount. The frequency noise data was taken with a $\Delta v=8 \mathrm{MHz}$ linewidth discriminator cavity in transmission. The data shows the cavity roll-off $(-40 \mathrm{~dB} /$ decade) at $8 \mathrm{MHz}$, indicating that the laser's frequency noise spectrum is flat out to at least $30 \mathrm{MHz}$. At about 30 $\mathrm{MHz}$ the measurement noise floor causes a deviation from $-40 \mathrm{~dB} /$ decade. The fast frequency noise level apparent between $50 \mathrm{kHz}$ and $30 \mathrm{MHz}$ indicates an inherent Lorentzian laser linewidth of about $25 \mathrm{kHz}(\Delta \mathrm{v}=\pi \cdot 8000 \mathrm{~Hz} / \mathrm{Hz})$. This is additionally broadened by the low frequency noise.
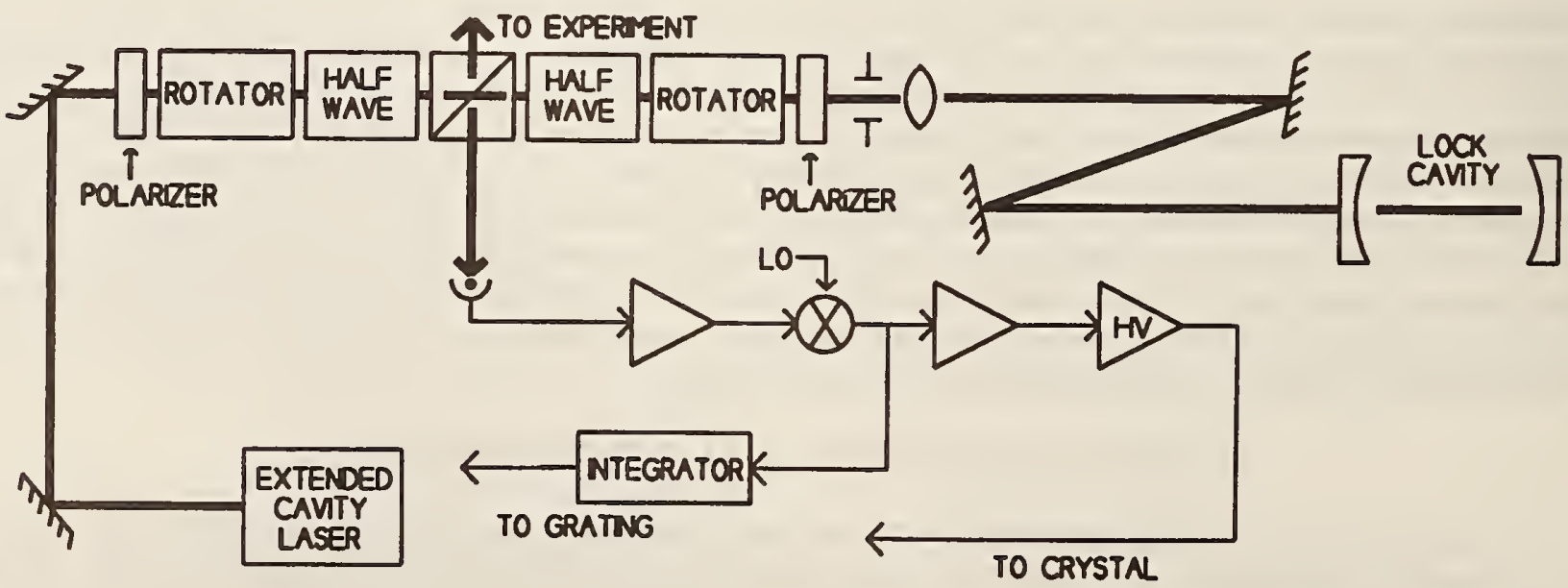

Figure 2. The extended cavity laser was locked to a $2.5 \mathrm{MHz}$ linewidth optical cavity by the rf heterodyne technique. The rotatorpolarizer pairs provide $35 \mathrm{~dB}$ of isolation each.

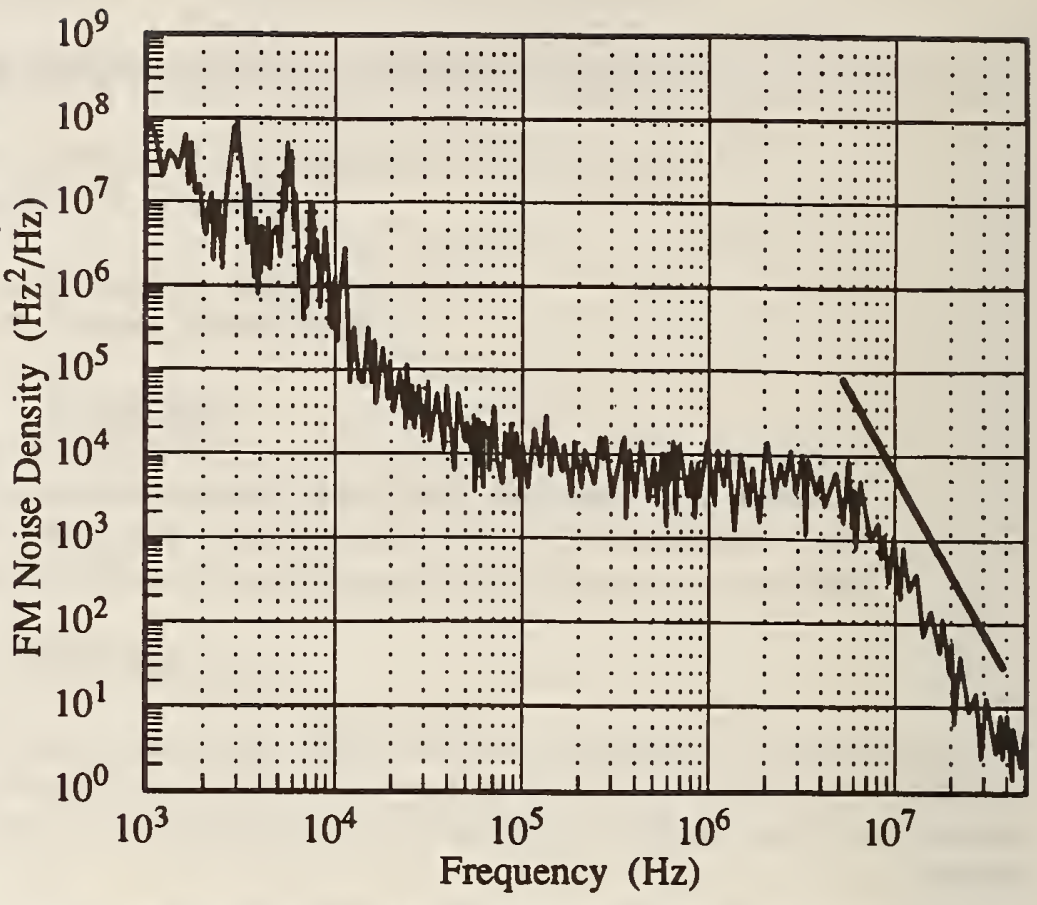

Fig. 1 The unlocked extended cavity laser frequency noise spectrum. The line indicates the discriminator cavity roll-off slope.
TO CRYSTNL 
The frequency fluctuations at rates above 100 $\mathrm{kHz}$ comprise relatively little of the laser linewidth. This is a consequence of the fast frequency noise level, $\mathrm{H}_{0}$, and the corresponding integrated phase noise. For a white fm noise spectrum, the phase noise integrated ${ }^{7}$ beyond a given arbitrary frequency $f$, i.e. integrated from $f$ to infinity, is $H_{d} / f$. Note that significant contributions to the laser linewidth appear only when the integrated phase noise density approaches $1 \mathrm{rad}^{2}{ }^{(8)}$ The measured noise level indicates the integrated phase fluctuations above $100 \mathrm{kHz}$ are on the order of $H_{d} / f \sim 0.1$. Therefore we expect a sharp reduction of the linewidth when the noise below $100 \mathrm{kHz}$ is reduced, in contrast to the situation of a solitary diode laser where the frequency noise in the $\mathrm{MHz}$ range is important.

The non-confocal cavity used for the lock had a linewidth of $2.5 \mathrm{MHz}$, a free spectral range of 1 $\mathrm{GHz}$ and a piezo-electric transducer (pzt) for tuning. A conventional if heterodyne set-up ${ }^{9}$ provided the error signal as shown in figure 2. Two negative feedback loops were implemented to reduce the frequency noise. An integrating loop $\left(f_{0 A B} \sim 300 \mathrm{~Hz}\right)$ to the grating pzt primarily served to limit the necessary dynamic range of the faster loop. This rather low bandwidth was dictated by mechanical resonances of the grating structure, the fundamental resonance being at $3 \mathrm{kHz}$. The faster (ADP) loop had approximately $80 \mathrm{~dB}$ of gain below $100 \mathrm{~Hz}$, which was then rolled-off with double-pole and single-pole sections to a unity gain frequency of $\sim 1 \mathrm{MHz}$. Compensation was made for the lock-cavity phase shift and much of the crystal high-voltage driver's phase shift. The bandwidth and consequently the attainable low-frequency gain were limited by the residual uncompensated phase shift of the high-voltage driver. Higher frequency fluctuations could be corrected by using a low-voltage higher-bandwidth feedback path to the other side of the crystal.

Most of the laser power was reserved for the saturation spectroscopy, leaving $30 \mu \mathrm{W}$ incident on the detector used for the lock channel. This incident power was just sufficient to allow intensity noise limited operation. The $1 \mathrm{MHz}$ bandwidth allowed enough low frequency gain to impart this limiting noise floor onto the laser at frequencies below $10 \mathrm{kHz}$.

The ADP crystal transfer function was $\sim 0.78$ $\mathrm{MHz} /$ volt. The crystal was bonded on one side to a ceramic mount and acoustically damped to reduce piezo-electric resonances in the $90 \mathrm{kHz}$ range. The modulation frequency was chosen to be well above the cavity bandwidth to produce a broad lock acquisition range. Modulation frequencies of $12 \mathrm{MHz}$ and $26 \mathrm{MHz}$ were used. However the modulation index was limited by the available synthesizer power, so the crystal was driven as a series resonant LC circuit at the modulation frequency. Q's of about 10 yielded

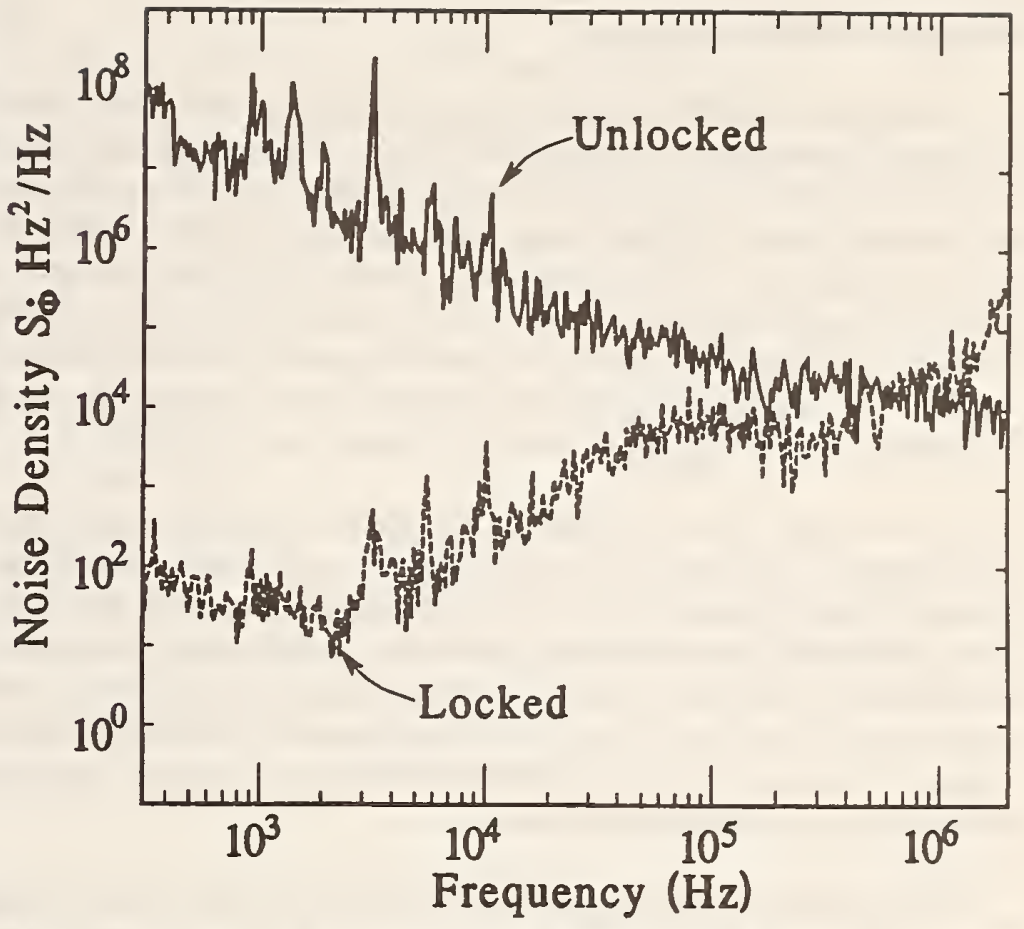

Fig. 3 The unlocked and locked error signal out of the mixer. The noise density values agree well with an independent measurement, fig. 4

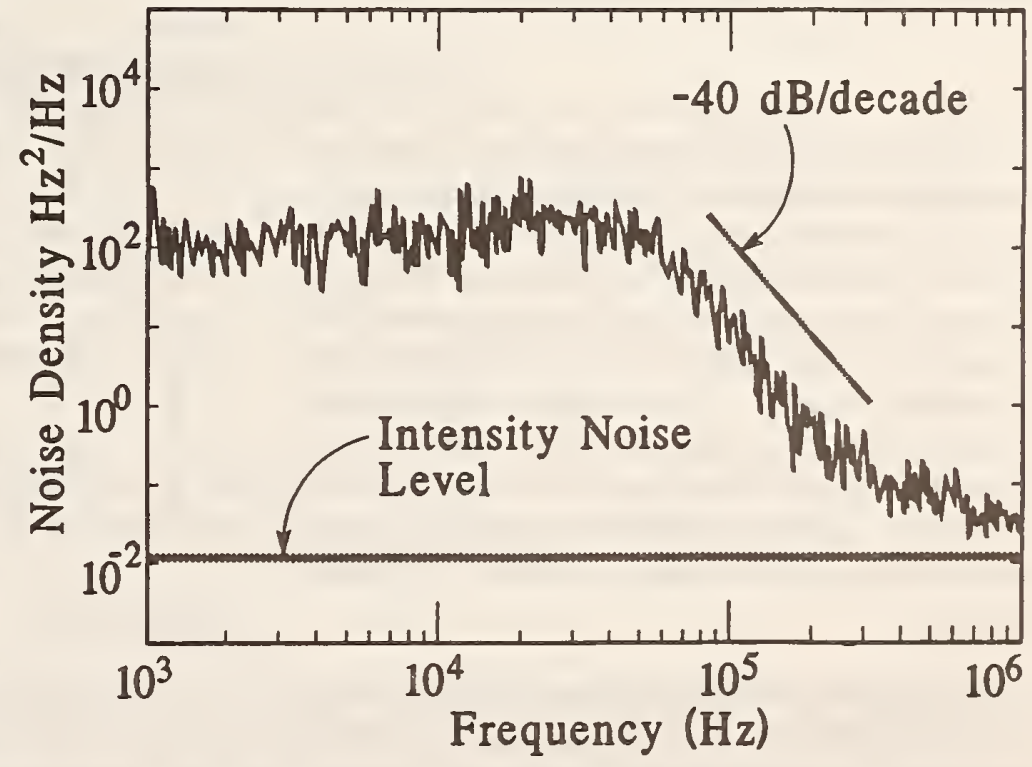

Fig. 4 The locked extended cavity laser frequency noise spectrum. The data was obtained with a $50 \mathrm{KHz}$ linewidth discriminator cavity. 
adequate sideband amplitudes.

Reduction of the frequency noise and attendant linewidth were observed with an independent high quality reference cavity used as a discriminator. The reference cavity is constructed from a ULE glass cylinder with optically contacted mirrors. It has a $50 \mathrm{kHz}$ linewidth, a $3 \mathrm{GHz}$ free spectral range, and is mounted inside a vacuum housing $\left(-10^{-6} \mathrm{~Pa}\right)$ to reduce index drifts and acoustic noise. Frequency noise measurements were obtained by side-locking to this high finesse reference cavity with slow feedback $\left(\mathrm{f}_{0 \mathrm{~dB}} \sim 1 \mathrm{~Hz}\right)$ to the $\Delta v=2.5 \mathrm{MHz}$ "lock" cavity pzt. The resultant fm to am conversion is indicative of the laser's frequency fluctuations only to the extent that the two cavities do not exhibit common mode vibrations. The hermetically sealed lock cavity was hard-mounted to the optical table and the reference cavity was supported in a "cradle" fashion by 2 fine wires that allowed pendulum modes but strongly damped high frequency oscillations. We believe that the frequency region of "common mode" vibrations was $\ll 100 \mathrm{~Hz}$.

The locked laser exhibits frequency fluctuations (see fig. 4) at the $200 \mathrm{~Hz}^{2} / \mathrm{Hz}$ level out to several hundred $\mathrm{kHz}$. At approximately $400 \mathrm{kHz}$ the increasing laser noise (due to the falling servo gain) and measurement noise floor (due to detector/intensity noise) combine to deviate the slope from $-40 \mathrm{~dB} / \mathrm{decade}$. The laser noise increases to the meet the open-loop level of $8000 \mathrm{~Hz}^{2} / \mathrm{Hz}$ as the servo gain drops to unity at about $1 \mathrm{MHz}$. Inadequate servo-loop phase margin causes an increase at this point over the open-loop noise, but in terms of fluctuations that contribute to the linewidth this is not significant since the integral of the phase noise contribution from this frequency region is negligible compared that of the lower frequency region. This noise spectrum indicates ${ }^{10}$ a linewidth reduced to less than $1 \mathrm{kHz}$, sufficient for high resolution saturated spectroscopy of Calcium in an oven.

Modulating the laser frequency with the ADP crystal was accompanied by measurable residual amplitude modulation. The level of am depended slightly upon the particular mode and wavelength. This can be understood by noting that the residual am will be a function of the slope of the composite gain curve $G(v)$ of the extended cavity laser at the lasing wavelength. The grating reflection and the residual etalon of the AR coated chip are the two primary contributors to $\mathrm{dG}(v) / \mathrm{dv}$. A modulation index of $\beta=1.0$ at $12 \mathrm{MHz}$ was accompanied by $0.015 \%$ amplitude modulation. Modulation via the injection current at the same modulation index (as determined by the average of the induced sideband amplitudes) resulted in $1.7 \%$ amplitude modulation.

The above mentioned residual am could be reduced by a simple feedback loop to the injection current. However, this may in general cause a change in $\beta$ and the phase of the modulation, which is a cause for concern if it effects the lock discriminator. Although to first order the lock discriminator is insensitive to small changes in the signal phase, ${ }^{11}$ for highquality locks this may be a noise term.

\section{CALCIUM SATURATION SPECTROSCOPY}

The calcium intercombination line at $657 \mathrm{~nm}$ exhibits a number of desirable properties of a spectral reference line. Among other attributes it has a $400 \mathrm{~Hz}$ natural linewidth and a simple ground state configuration $\left({ }^{1} \mathrm{~S}_{0}\right)$ that should allow nearly all of the atoms present to take part in the absorption process. A laser cooled calcium beam or neutral atom trap approach may well be required to take advantage of it's full potential. However calcium saturated absorption in an oven provides excellent frequency stability in a simple system.

A high density calcium beam about $6 \mathrm{~cm}$ long was created in an oven with uncoated bk-7 glass windows. The background gas pressure was kept at less than $1.3 \cdot 10^{-3} \mathrm{~Pa}$ (10 ${ }^{-5}$ Torr) to avoid pressure broadening. The windows were partially

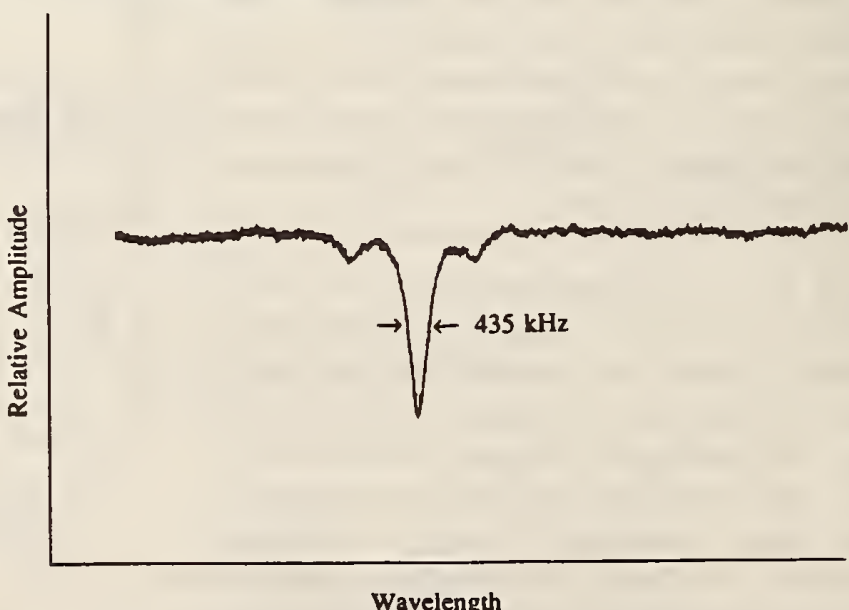

Fig. $5435 \mathrm{kHz}$ wide absorption of the calcium intercombination line at 657 $\mathrm{nm}$. The linewidth is limited by time-of-flight broadening. The sidebands are spaced by $2.3 \mathrm{MHz}$. 
protected from calcium deposition by the oven design.

A pair of coils provided a transverse magnetic field that was aligned with the laser polarization. This allows only $\Delta \mathrm{M}_{\mathrm{l}}=0$ transitions, which have only a very small quadratic Zeeman sensitivity $\left(-10^{8} \mathrm{~Hz} / \mathrm{T}^{2}\right)$. The saturating beam power was approximately $400 \mu \mathrm{W}$, and was reflected by a flat mirror to serve as the probe beam. The Doppler absorption at the operating temperature was $10 \%$ and $1.4 \mathrm{GHz}$ wide. The Doppler curve was narrowed somewhat due to a directional calcium flux in the oven. The saturation feature (see fig. 5) was about $5 \%$ of the Doppler absorption. The hot calcium and a $2 \mathrm{~mm}$ diameter laser beam size resulted in a short interaction time $(\sim 1 \mu \mathrm{s})$, limiting the observed linewidth to $435 \mathrm{kHz}$. The laser's spatial profile was shaped by a pair of anamorphic prisms and a telescope to provide a $7 \mathrm{~mm}$ beam, with which $170 \mathrm{kHz}$ linewidths were obtained. Further reductions will require a better spatial mode. Lock-in detection at $40 \mathrm{kHz}$ resulted in signal-to-noise ratios of $-10^{3}(1 \mathrm{~Hz}$ bandwidth) at modulation levels that produced no modulation broadening.

\section{CESIUM TRAP SPECTROSCOPY}

Cesium magneto-optic cell traps may well serve as the basis for the next generation of atomic frequency and time standards. There is a cesium transition $\left(6 \mathrm{p}_{3 / 2}\right.$ to $\left.9 \mathrm{~s}_{1 / 2}\right)$ at $658.8 \mathrm{~nm}$ which is close to calcium in wavelength and within the tuning range of the laser. We note that the essentially Doppler-free absorption from the $6 p_{3 / 2}$ levels is a potentially useful indication of trap characteristics. The $852 \mathrm{~nm}$ cooling laser in this trap is typically locked several linewidths to the red of the ground state $\mathrm{F}=4$ to $\mathrm{F}=5$ transition (see fig. 6), and a second laser tuned $9 \mathrm{Ghz}$ away re-pumps atoms in the lower ground state level back into the cycling transition.

The red absorption from the upper trapping level was measured with a weak probe beam $\left(-10 \mu \mathrm{W}, 0.05 \mathrm{~mW} / \mathrm{cm}^{2}\right)$ approximately the same diameter as the cluster of trapped atoms. An optical fiber was used as a spatial filter and conveniently transmitted the red probe light from the locked laser to the trap. Etalon fringes with a period of $4 \mathrm{MHz}$ and spurious low frequency amplitude noise resulting from polarization changes also caused by the fiber were reduced by a double beam subtraction configuration. FM modulation at $50 \mathrm{kHz}$ and lock-in detection with a $1 \mathrm{~ms}$ time constant were employed, yielding signal-tonoise ratios of $\sim 200$. The absorption detection limit extrapolated to $1 \mathrm{~s}$ was $3 \cdot 10^{-6}$, limited by mismatching of the double beam configuration that allowed laser and fiber noise to be detected. The $1 \%$ absorption (see fig. 7) of the $6 \mathrm{p}_{3 / 2} \mathrm{~F}=5 \rightarrow 9 \mathrm{~s}_{1 / 2}$ $\mathrm{F}=4$ transition indicates a trap population of $\sim 2 \cdot 10^{8}$ atoms, which agrees well with $852 \mathrm{~nm}$ fluorescence measurements.

We observe a double peak absorption spectrum which is preliminarily attributed to Autler-Townes splitting (AC Stark effect) caused by the $852 \mathrm{~nm}$ cooling laser fields. A frequency separation that varies as the square root of the intensity and an asymmetry that depends on detuning from line center is expected ${ }^{13}$ and is consistent with our observations.

\section{SUMMARY}

A $12 \mathrm{~cm}$ long extended cavity laser at $657 \mathrm{~nm}$ stabilized to a precision reference cavity by electronic feedback to an intracavity ADP crystal has been described. The residual am that accompanies fm is approximately 100 times less when the laser is modulated with the crystal rather than the injection current. This laser was used to measure linear doppler-free absorption from the cesium $6^{2} \mathrm{p}_{3 / 2}$ state in a magneto-optical trap. In addition we used the laser for non-linear absorption experiments of the calcium intercombination line. Linewidths of $170 \mathrm{kHz}$ were observed, limited by laser spatial mode size and quality. 


\section{Acknowledgements}

We acknowledge useful discussions with C. Weimer, J. Berquist, J. L. Hall, J. Helmcke, F. Riehle, and V. Velichansky. This work was supported in part by NASA and the AFOSR.

${ }^{\dagger}$ Present address: Dept. Physics, Duke University, Durham, N. C.

${ }^{\ddagger}$ Permanent address: P. N. Lebedev Physics Institute, Leninsky Prospect, 53, 117924, Moscow, Russia

\section{REFERENCES}

1. R. L. Barger, J. C. Berquist, and D. J. Glaze, "High-Resolution Saturated Absorption of the ${ }^{1} S_{0}{ }^{3} P_{1}$ Calcium Transition in an Atomic Beam," J. Opt. Soc. Am., Vol. 68, No. 11, p. 1634, 1978.

2. J. Helmcke, A. Morinaga, J. Ishikawa, and F. Riehle, "Optical Frequency Standards," IEEE Trans. Inst. Meas., Vol. 38, No. 2, p. 524, 1989.

3. Ch. Salomon, D. Hils, and J. L. Hall, "Laser stabilization at the millihertz level," J. Opt. Soc. Am. B, Vol. 5, No. 8, p. 1576, 1988

4. N. C. Wong and J. L. Hall, "Servo control of amplitude modulation in frequency-modulation spectroscopy: demonstration of shot-noise-limited detection," J. Opt. Soc. Am. B, Vol. 2, No. 9, p. 1527, 1985

5. The coating was $\mathrm{Al}_{2} \mathrm{O}_{3}$ and hafnium oxide, which nearly doubled the solitary laser's threshold current.

6. M. S. Soskin and V. B. Taranenko, "Holographic total-internal-reflection selector for tunable lasers," Sov. J. Quan. Elect., Vol 7, No. 3, 1977.

7. This follows immediately from the fact that the phase noise density in $\mathrm{rd}^{2} / \mathrm{Hz}$ is equal to the frequency noise density in $\mathrm{Hz}^{2} / \mathrm{Hz}$ divided by $\mathrm{f}^{2}$.

8. E. Bava, A. DeMarchi, and A. Godone, "Spectral analysis of synthesized signals in the mm wavelength region," IEEE Trans. Inst. and Meas., Vol IM-26, No. 2, 1977

9. R. W. P. Drever, et. al, "Laser phase and frequency stabilization using an optical resonator," Appl. Phys. B 31, p. $97,1983$. 10. D. S. Elliot, Rajarshi Roy, and S. J. Smith, "Extracavity laser band-shape and bandwidth modification," Phys. Rev. A, Vol. 26, No. 1, p. 12, 1982

11. J. L. Hall, H. G. Robinson, T. Baer, and L. Hollberg, "The lineshapes of subdoppler resonances observable with fm sideband (optical heterodyne) laser techniques," Proc. Nato Advanced Study Institute on General Relativity and Quantum Electronics, 1981

12. E. Arimondo, M. Inguscio, and P. Violino, "Experimental determinations of the hyperfine structure in the alkali atoms," Rev. Mod. Phys., Vol. 49, No. 1, p. 31, 1977.

13. R. M. Whitley and C. R. Stroud, Jr.,"Double optical resonance," Phys. Rev. A, Vol. 14, No. 4, p. 1498, 1976. 


\title{
The diode laser as a spectroscopic tool
}

\author{
R. W. Fox ${ }^{*}$ C. S. Weimer, ${ }^{*}$ L. Hollberg ${ }^{*}$ and G. C. TUrk $\dagger$ \\ -National Institute of Standards and Technology, Boulder, CO, 80303, U.S.A. \\ +National Institute of Standards and Technology, Gaithersburg, MD 20899, U.S.A.
}

\begin{abstract}
The properties of diode lasers that make them attractive as spectroscopic sources are discussed, along with the use of extended cavities to enhance their tuning range and reduce their linewidths. Semiconductors can now provide efficient, tunable, narrow linewidth laser light over much of the red and near-i.r. region of the spectrum. The progress in using nonlinear optics to extend the useful wavelength range of diodes lasers is also considered. Our recent analytical work with diode laser spectroscopy provides examples of their application.
\end{abstract}

\section{Introduction and Basic Properties}

INHERENT low noise, low cost, tunability, and simplicity make diode lasers unique spectroscopic sources, and their use in this respect has been reviewed several times [1-5]. Here we will focus briefly on general characteristics and then explore some of the new technologies and applications, with particular interest in spectroscopy and ultrasensitive detection.

There are two main types of laser diodes available. The type we will deal with here are the visible and near-infrared lasers fabricated from the group III-V semiconductor compounds (for example: AlGaInP, AlGaAs, InGaAs and InGaAsP). The approximate distribution of wavelength and power for commercial lasers of this type are shown in Fig. 1. The other basic type of diode lasers are commonly known as lead-salt lasers which operate at wavelengths between 3 and $30 \mu \mathrm{m}$. These lasers are distinct in that they are only operated at cryogenic temperatures, and are not considered here [6].

Although red cw diodes with nominal wavelengths as short as $635 \mathrm{~nm}$ are commercially available, there have been diode lasers developed at shorter wavelengths $[7,8]$. However, coincidences between strong atomic or molecular lines and wavelength ranges of available diodes remain the exception rather than the rule. The visible and near-i.r. diodes can be tuned roughly $3-4 \mathrm{~nm}$ from the nominal wavelength with a $20^{\circ} \mathrm{C}$ temperature change. The tuning is in a step-wise fashion; for instance, smooth

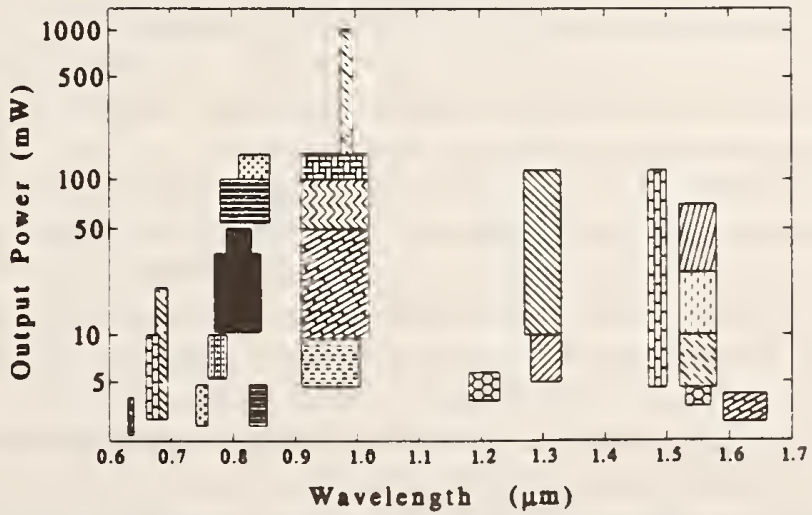

Fig. 1. Commercially available $\mathrm{cw}$ laser diodes in the $600-1600 \mathrm{~nm}$ wavelength range. The data bars represent families of laser products as advertised by a number of different manufacturers. 
tuning for typically one fourth of a nanometer. followed by a transition (mode-hop) in wavelength to another mode. Tuning with injection current $(\sim 0.01 \mathrm{~nm} / \mathrm{mA})$ exhibits similar behavior, and hysteresis is often observed at the mode hops in either case. Thus it is difficult to ensure that a given diode will reliably reach all wavelengths near the nominal. This problem is usually overcome by either having a sample of several diodes to choose from or by using optical feedback to control the lasing frequency. A common example of the latter method is the use of a diffraction grating in an extended cavity laser, as discussed in the next section. A second example is the use of a small mirror placed close to one of the laser facets, effectively forming an etalon which forces the laser to operate in a specific longitudinal mode [9]. The mode may then be wavelength tuned with current or temperature; success in achieving complete wavelength coverage near the nominal has been reported [10.11] with this technique.

These comments on tuning apply mainly to single-mode Fabry-Perot type diodes as opposed to distributed-feedback (DFB) lasers. DFB lasers have superior tuning characteristics (but restricted wavelength range), typically tuning $\sim 10-12 \mathrm{~nm}$ with temperature before encountering a mode-hop. Unfortunately, they are as yet widely available only in the 1.3 and $1.55 \mu \mathrm{m}$ telecommunications band wavelengths and are relatively expensive.

Many of the diode lasers available now are designed to operate in a single longitudinal mode. This is of course preferable for absorption measurements. since power in side modes (off the absorption feature) does not contribute to the signal but does add shot noise and mode competition noise to the detected photocurrent. A laser running with significant power in more than one mode may exhibit greatly increased amplitude noise (AM) due to mode competition [12]. Lasers with a few modes running usually have the highest level of mode-partition-noise whereas those with either a large number of modes or one mode have low AM noise. In fact the detected photo-current of a typical near-i.r., single-mode laser driven with a good low noise current source will show amplitude fluctuations that are within a factor of 10 of the shot noise limit in the frequency range above $100 \mathrm{kHz}$. These low intrinsic noise levels are just one of the reasons that diode lasers are attractive as spectroscopic sources.

Many groups have reported impressive sensitivities in detecting atoms and molecules by exploiting modulation techniques to take advantage of the low amplitude noise exhibited in the higher frequency range [13-15]. Several studies have compared detection sensitivities obtained at different modulation frequencies [16-18]. A separate approach is to work closer to the base-band frequency range but use negative electronic feedback to suppress the amplitude fluctuations inherent at the lower frequencies [ 19 , 20]. With these methods it is possible to approach the shot noise limit and greatly enhance the signal-to-noise ratio in absorption detection. It is important to realize that the low intrinsic noise does not, however, mean that diode lasers are free from systematic amplitude changes as their frequency is swept or modulated. The linear power vs. current curve exhibited by diodes leads to a sloping baseline if direct detection is used. A more serious problem that limits detection sensitivities is the residual AM that accompanies frequency modulation.

The spectral linewidth of even a typical solitary diode laser is quite narrow with respect to Doppler or atmospheric pressure-broadened lines. AlGaAs, near-i.r. lasers operating well above threshold have linewidths in the $10-40 \mathrm{MHz}$ range. Some highpower long-cavity solitary lasers have reported linewidths of a few megahertz. The visible AlGaInP lasers exhibit broader linewidths, typically $150 \mathrm{MHz}$. For high resolution work a number of methods have been developed to achieve greatly reduced linewidths. The most common method is to employ an extended cavity (see Section 2 ), but resonant optical feedback from a confocal cavity has also been successfully used in a number of experiments [21,22]. Techniques to achieve substantially narrower linewidths than the hundreds of kilohertz afforded by standard extended cavities are discussed in a number of articles [23-25]. 


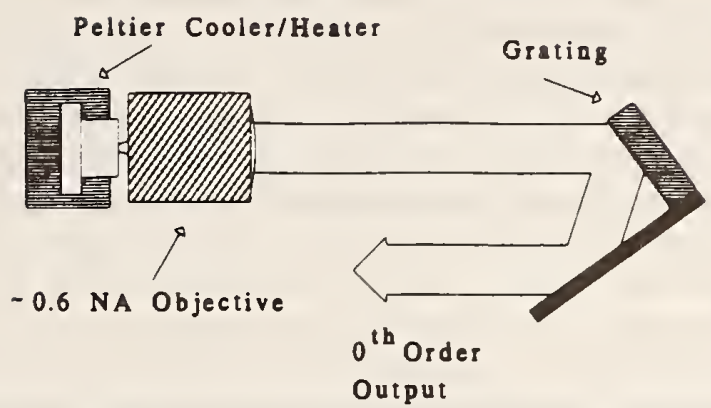

Fig. 2. Basic form of an extended cavity diode laser system. In this example an optical grating in a Littrow configuration is used as a wavelength selective tuning element. A piezoelectric element (not shown) on the grating assembly provides for fine wavelength sweeping. Angular sweep of the output beam with tuning is eliminated with a mirror attached at $90^{\circ}$ to the grating as shown.

\section{Methods for Broad Tuning}

At NIST most of our work with diode lasers has concentrated on high resolution spectroscopy of narrow atomic resonances, but there is a much wider spectrum of applications that will require broadly tunable sources without stringent linewidth requirements. For most of these applications it is also necessary to reach a specific wavelength of interest. This may require some form of wavelength-selective optical feedback in order to extend and even fill in a laser's tuning range [26]. The most common method is to use an optical grating for selective feedback in an extendedcavity-diode-laser (ECDL) configuration. An example ECDL system is shown in Fig. 2. Extended cavity lasers are relatively simple, and up to now many groups have been building these systems for their own use. The number of users and applications can be expected to increase as more advanced commercial diode laser sources become available.

Many variations on the basic ECDL scheme are possible, and a variety of different tuning elements have been used, although gratings seem to be the most common. Grating tuning of $\pm 20 \mathrm{~nm}$ from the nominal diode wavelength is possible with neari.r. AlGaAs lasers without changing the diode temperature. However, this represents rather optimum results, dependent upon the device and laser anti-reflection (AR) coating. A tuning range of $\pm 10 \mathrm{~nm}$ is more common.

Other important characteristics of a diode used in an extended cavity include a moderate loss of power and a much narrower linewidth as compared to the solitary diode. The usable single-frequency, tunable output power can be as low as $30 \%$ of the rated single-mode diode power. This is due in part to a change in the output coupling, but is also often due to lower injection currents required to stay in a stable operating regime. The observed linewidth will depend upon the cavity length as well as the lasers' environment. Although the fundamental linewidth decreases as the square of the cavity length, low frequency $(\leq 100 \mathrm{kHz}$ ) environmental and $1 / f$ noise soon become the dominant frequency noise contributions. This leads to observed linewidths that range from ters to hundreds of kilohertz depending mostly on construction and external perturbations. We also note, that with this sort of frequency fluctuations, the "linewidth" that one measures depends on the observation time of the measurement, and is thus somewhat ill-defined [25].

With simple grating feedback and average anti-refiection coatings an ECDL will not usually tune single-frequency with no mode hops over the laser's entire gain bandwidth. This is due to a number of factors, paramount of which is the residual output facet reflectance of the laser chip (as discussed below). For single-frequency scanning without mode-hops it is necessary to synchronously scan the extended cavity length with the pass-band of the wavelength selective element(s) (such as a grating and/or etalon). Several techniques that accurately change the cavity length together with the 
grating angle have accomplished single-mode tuning over $1000 \mathrm{GHz}[27,28]$. Again these are exceptional results rather than typical. which might show scanning from 10 to $100 \mathrm{GHz}$ depending on a number of factors.

Special regimes of ECDL operation called "autostabilization" also exist [29] where single-mode tuning ranges of more than $20 \mathrm{GHz}$ are possible with just cavity length changes and no change of the grating angle. This broad tuning is the result of semiconductor device non-linearities that help to maintain single-mode operation. We note that this is typical for $\sim 5-20 \mathrm{~cm}$ long ECDLs, and is in fact single mode scanning of about ten free spectral ranges of the extended cavity with no mode hops.

ECDLs generally operate in a straight-forward, single-mode manner but it is also easy to reach unacceptable operating conditions (multimode or chaotic) that depend on parameters both internal and external to the laser. Spectroscopic applications are particularly sensitive to outside factors that affect the laser's frequency, such as stray optical feedback, temperature and current fluctuations, and even vibrations for ECDLs. A more fundamental cause of instabilities in ECDLs is the fact that the laser is a coupled-cavity system with potentially incommensurate boundary conditions [30-32]. In applying these lasers we have to find ways to avoid regions of instability. Three techniques are useful to increase the stable operating regions of grating tuned lasers: (1) operate at slightly lower powers, (2) use short extended cavities [33.34] and (3) improve the AR coatings on the output facet so that the system is dominated by the grating feedback. All of these techniques are helpful in achieving broad tuning and stable operation even though they have the inherent disadvantages of low output power, broader linewidths, and complications of additional processing respectively. A compelling reason to strive for very low output facet reflectance $\left(R<10^{-3}\right)$, is that with such AR coatings it is possible to obtain complete spectral coverage over the laser's gain profile without having to change the laser's temperature [35].

Fortunately, many of the higher power lasers that are commercially available already have some AR coating on their output facet. These lasers will work as supplied in ECDL systems, but they can also be improved. For our extended-cavity systems we generally add additional coatings to the output facet in order to reduce the reflectance. After some experience we routinely achieve facet reflectances of $10^{-3}$ on ordinary commercial lasers. Lower reflectance is possible but is difficult to achieve because of a number of factors; not the least of which is the fact that we lack detailed knowledge of the structures and materials of specific commercial lasers. In addition, most of the lasers come from the manufacturers with coatings already on the facets. We typically find single layer $\lambda / 4$ coatings with reflectance of $\sim 5 \%$ for high power lasers and $\lambda / 2$ with $30 \%$ reflectance for low power devices. These coatings are for passivation as well as tailored reflectance and generally make it more difficult to reach very low reflectance with additional coatings. Quite a lot of information on coating lasers is available in the literature [36-39], particular'y with regard to making optical amplifiers. Our coatings at NIST achieve good reliable results with common optical coating materials $\left(\mathrm{HfO}_{2}, \mathrm{Al}_{2} \mathrm{O}_{3}\right.$ and $\left.\mathrm{SiO}\right)$ and techniques (thermal, e-beam and $\mathrm{RF}$-sputtering) [40].

\section{Higher Power and Access to Other Spectral Regions}

The main problem in using diode lasers for spectroscopic applications is the limited number of wavelengths that are accessible. Using only semiconductors based on various derivatives of $\mathrm{GaAs}$ and $\operatorname{In} \mathrm{P}$, the wavelength coverage could be nearly complete over the spectral region from $630 \mathrm{~nm}$ to $1.7 \mu \mathrm{m}$. In practice this is not the case (see Fig. 1) because the manufacturers concentrate production at certain wavelengths. Other wavelength regions may be accessible with different semiconductor materials; for example blue-green diode lasers based on $\mathrm{ZnSe}$ have been reported recently [7]. Also some research laboratories have produced i.r. lasers in the 2.0-2.3 $\mu \mathrm{m}$ region based on GaInAsSb [41, 42]. These spectral regions will see important spectroscopic applications if development is successful and if there is sufficient commercial demand to support production. 
An alternative to direct semiconductor sources for other spectral regions is to use diode lasers in conjunction with nonlinear optical techniques to generate new wavelengths. This requires high intensity beams and materials with large nonlinear coefficients. Until recently we have been limited to a few tens of milliwatts of singlemode power from diode lasers in the best cases. Consequently, resonant cavities are useful to increase the intensity and thus generate nonlinear products more efficiently. The future promises to make this much easier with new higher power lasers and better nonlinear materials.

Two basic techniques have demonstrated high-power single-mode single-frequency operation of semiconductor diode lasers. These are (1) injection-locking of high-power broad-area lasers with a single-mode laser [43-45] and (2) single-mode masteroscillator-power-amplifier systems (MOPAs) [46]. The distinction between these two methods is just whether the power device operates above or below threshold without the master laser's input. Injection locking can be done with low power single-mode master lasers to lock the frequency of the higher power (hundreds of milliwatts) semiconductor laser sources. MOPA systems have been reported with pulsed outputs of $27 \mathrm{~W}$ and $\mathrm{cw}$ outputs of up to $2 \mathrm{~W}$. At present many of these high power systems are relatively expensive which we expect to change with time. The question remains of how broad a wavelength coverage will be available with these very high power systems.

There are already a few nonlinear optical techniques that work particularly well with diode lasers. One of these is the fortuitous match of the wavelength of the highpower $\mathrm{AlGaAs}$ lasers and $90^{\circ}$ phase-matching in potassium niobate $\left(\mathrm{KNbO}_{3}\right)$. High efficiency second-harmonic generation (SHG) at fundamental wavelengths from about 840 to $930 \mathrm{~nm}$ can be achieved in this material with temperature tuning of the crystal from about -40 to $180^{\circ} \mathrm{C}$ [47]. As much as $40 \mathrm{~mW}$ of blue light has been generated with a diode laser and $\mathrm{KNbO}_{3}$ [48]. Using less infrared power, others [49,50] have reported conversion efficiencies from diode power to blue light in the range of $1-10 \%$. These results are consistent with our experience in using a ring buildup cavity that generates $30 \mathrm{~mW}$ of blue light at $423 \mathrm{~nm}$ for $150 \mathrm{~mW}$ of diode laser power. The main limitation in our system is the spatial mode of the diode laser that limits the coupling efficiency of the laser into the ring. Improvements in the mode matching and laser power should increase the overall conversion efficiency since $\mathrm{KNbO}_{3}$ is capable of producing much higher blue light powers [51].

A promising technique that has demonstrated excellent results for SHG and sumfrequency mixing is the fabrication of periodic domains in the crystal structure [52-54]. This involves reversing properties of the crystal on a periodic basis, which allows the fundamental and harmonic waves to retain a constructive phase relationship over much longer distances than would otherwise be the case. Yet another approach to tunable blue light from semiconductor sources is to phase-match for sum-frequency mixing of a diode laser output with the second harmonic of a diode pumped Nd-Yag laser. Output powers of $4 \mathrm{~mW}$ at $459 \mathrm{~nm}$ have been demonstrated [55] in this manner, using a monolithic KTP resonator and approximately $30 \mathrm{~mW}$ of input power from each laser.

Nonlinear mixing to longer wavelengths has also been recently demonstrated using diode lasers. Using difference frequency mixing SimON [56] and collaborators have generated tunable mid-i.r. light from diode lasers. The i.r. output powers are low at present $(\sim 3 \mathrm{nW})$, but the principle of difference frequency generation from diode laser sources has been proven. In this case the non-linear materials that seem the most appropriate are $\mathrm{AgGaS}_{2}$ and $\mathrm{AgGaSe}_{2}$, which can potentially cover the wavelength range from 4 to $18 \mu \mathrm{m}$. 


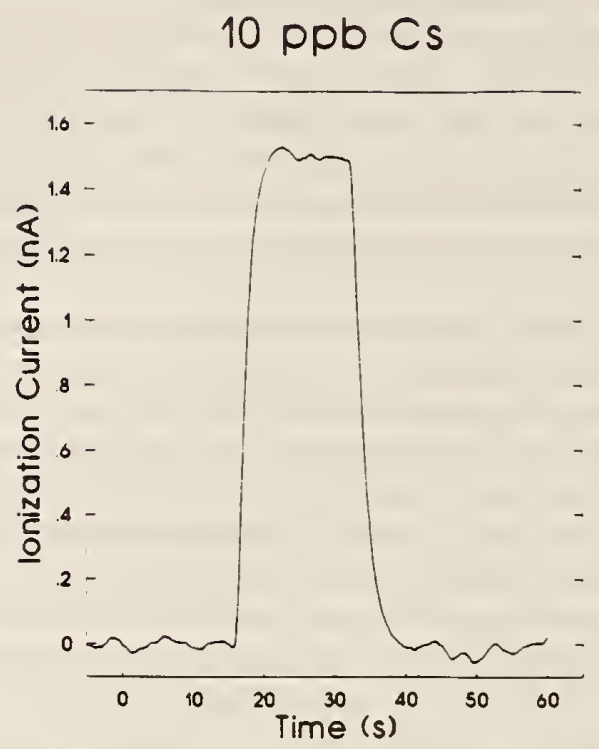

Fig. 3. Diode laser-enhanced ionization (LEI) signal obtained with $10 \mathrm{ppb}$ of $\mathrm{Cs}_{\mathrm{s}}$ in water. The laser was tuned to the strong peak of the $C_{s} D_{2}$ line. The limiting background noise was contamination by easily ionized elements in the burner equipment, which led to an increased flame background current.

\section{Applications}

\subsection{Atomic spectroscopy}

The use of diode lasers as spectroscopic sources in the setting of analytical chemistry for trace detection of atoms has been reported by several groups. Absorption, fluorescence and ionization-based methods have all been demonstrated, using a variety of atom sources including flames [57], a graphite furnace [58, 59], a thermionic diode [60], a glow discharge [61], and a thermal filament [62].

Along these lines we have used diode lasers for the detection of trace levels of Rb and $\mathrm{Cs}$ in solutions using laser-enhanced ionization spectroscopy (LEI) in a flame. Our very elementary set-up consisted of a water-cooled cathode in a premixed air-hydrogen flame with a pneumatic nebulizer for liquid sample introduction $[63,64]$. The cathode was held at a potential of about $-100 \mathrm{~V}$ with respect to the burner, and the laser beam chopped and directed through the flame. Resonant absorption on the $S_{1 / 2}$ to $P_{3 / 2}$ transition in the alkalis changes the charge density in the flame since there is a differential ionization potential between the $\mathrm{S}$ and $\mathrm{P}$ states. This causes a change in the current flowing between the burner and the cathode, which is phase sensitively detected at the laser chopping frequency.

Figure 3 shows the signal obtained with a water solution of $\mathrm{Cs}$ at a concentration of $10 \mathrm{ppb}$ (an atomic density of approximately $10^{8} \mathrm{~cm}^{-3}$ in the flame). The baseline is obtained by aspirating deionized water into the flame. An extended-cavity grating tuned laser was used, which produce $1.9 \mathrm{~mW}$ of output power with a $2 \mathrm{~mm}$ diameter beam that was passed through the flame twice. In this example the use of a relatively narrow linewidth $(\sim 100 \mathrm{kHz})$ extended-cavity laser as opposed to a solitary diode laser is not significant (other than for ease of tuning) since the absorption is homogeneously broadened by atmospheric pressure. The laser beam was positioned for maximum signal, approximately one beam diameter below the flame electrode.

Limits of detection for $\mathrm{Cs}$ and $\mathrm{Rb}$ were calculated by extrapolating the signals obtained for $10 \mathrm{ppb}$ solutions down to the concentration that would give a signal equal to three times the r.m.s. noise level of the deionized water baseline. A $1 \mathrm{~s}$ time constant on the lock-in amplifier was used for these measurements. For Cs at $852 \mathrm{~nm}$ a limit of detection of $0.25 \mathrm{ppb}$ was achieved. For $\mathrm{Rb}$ at $780 \mathrm{~nm}$ the limit of detection 
was $0.3 \mathrm{ppb}$. The limiting source of noise at the detection limit was contamination by easily ionized elements in the burner equipment, which led to an increased flame background current. For both $\mathrm{Cs}$ and $\mathrm{Rb}$, the sensitivity was diminished by the high fraction of normal flame ionization of these low ionization potential elements, which reduces the atomic fraction available for LEI. In the case of Cs, approximately $90 \%$ of the population is thermally ionized before laser excitation.

Our limit of detection for Rb compares well with that reported by Havrilla [65], using LEI in the air-hydrogen flame with a dye laser pumped by a cw krypton ion laser. They reported a limit of detection of $0.09 \mathrm{ppb}$, with a laser power approximately two orders of magnitude higher. The laser system used in that work was considerably more complex and expensive, requiring $30 \mathrm{~kW}$ of power and cooling water at $501 \mathrm{~min}^{-1}$. No comparable LEI measurement of $\mathrm{Cs}$ has been reported. Future development of LEI using diode lasers depends on expansion of wavelength availability. Several attempts were made to make LEI measurements of other elements using transitions beginning from excited states with near-i.r. wavelengths where diode lasers were available. The very low Boltzmann population of the lower levels of these transitions in the flame prevented our observation of LEI.

\subsection{Molecular spectroscopy}

The detection of molecular species instead of constituent atom concentrations is often of interest. Although in general the molecular absorption bands in the near-i.r. are weak compared to the vibrational bands at longer wavelengths, the room temperature operation of the near-i.r. lasers makes them very attractive nevertheless. Many groups have applied the 1.3 and $1.5 \mu \mathrm{m}$ lasers to probing various molecular resonances, including $\mathrm{CH}_{4}[66,67], \mathrm{C}_{2} \mathrm{H}_{2}$ [68], $\mathrm{CO}_{2}$ and $\mathrm{CO}$ [69], $\mathrm{NH}_{3}$ and $\mathrm{H}_{2} \mathrm{O}$ [70], $\mathrm{HCN}$ [71], $\mathrm{HDO}$ [72] and $\mathrm{HCl}$ [73]. Particularly important due to its role in atmospheric pollution is $\mathrm{NO}_{2}$, which has been investigated by several groups $[14,15,74,75]$.

In some on going work at NIST in collaboration with NOAA [76], red diode lasers are being used to detect $\mathrm{NO}_{3}$ radicals. This molecule plays an important role in atmospheric photochemistry. In the stratosphere its photochemistry affects ozone depletion, and it acts as a strong oxidizer in the troposphere. $\mathrm{NO}_{3}$ has a strong electronic absorption band at $662 \mathrm{~nm}$ that can be reached with commercial red diode lasers. In these experiments a solitary diode laser is used to detect the time-dependent concentration of $\mathrm{NO}_{3}$ in an excimer-laser photodissociation experiment. Good detection sensitivities are achieved with simple direct absorption measurements (noise-limited absorption sensitivity $\sim 10^{-4}$ at $\mathrm{DC}$ with a $200 \mathrm{kHz}$ detection bandwidth). This corresponds to about $5 \times 10^{10} \mathrm{NO}_{3}$ molecules $/ \mathrm{cm}^{3}$ and a $1 \mathrm{~m}$ single-pass absorption path length. The detection sensitivity could be improved with: subtraction of residual low-frequency AM noise, or high frequency modulation, or multi-passing the absorption cell. This experiment illustrates the potential for using very simple diode laser systems to detect reactive molecular species that play an important role both in the laboratory and in the earth's atmosphere.

\section{Summary and Projections}

The rapid growth in the field of diode laser spectroscopy is the direct result of the simplicity and good performance that can be achieved with inexpensive commercial semiconductor lasers. These lasers are becoming more readily available, and in addition we now have a better understanding of how to control them and apply them to a variety of scientific and technical problems. With some acknowledged limitations we can now have low cost, portable, tunable, low noise, narrow linewidth laser sources at a number of wavelengths. Very high power devices are becoming available which will extend the opportunities with diode lasers in part by accessing other wavelength regions. Important spectroscopic and analytic roles are recognized for both simple solitary laser systems as well as broadly tunable extended cavity laser systems. Areas of precision optical physics such as atomic clocks, frequency and length standards. 
and laser cooling of atoms are also being strongly affected by diode lasers. The capabilities of these systems continues to increase as does the number of applications. The time is not far in the future that we will consider diode laser systems as standard research tools.

Acknowledgements-We acknowledge the important contributions to this work by N. Mackie. T. Zibrova, A. S. Zibrov, J. H. Marquardt, J. S. Wells and the NOAA group [76]. Our thanks to U. Simon for information about difference frequency generation. This work has been supported in part by the NASA upper atmospheric research program, and the Air Force office of scientific research.

\section{REFERENCES}

[1] J. C. Comparo, Contemp. Phys. 26, 443 (1985).

[2] J. Lawrenz and K. Niemax. Spectrochim. Acta. 44B, 155 (1989).

[3] C. Wieman and L. Hollberg, Rev. Sci. Instrum. 62, 1 (1991).

[4] D. E. Cooper and R. U. Martinelli. Laser Focus World Nov., 133 (1992).

[5] M. Ohtsu and K. Nakagawa, Spectroscopy by Semiconductor Lasers, In Coherence. Amplification. and Quantum Effects in Semiconductor Lasers, Ed. Y. Yamamoto, Chap. 5. John Wiley, New York (1991).

[6] Tunable Diode Laser Applications, Eds. A. I. Nadezhdinskii, A. M. Prokhorov, Proc. SPIE 172A (1992); and C. B. Carlisle, D. E. Cooper and H. Preier, Appl. Opt. 28. 2567 (1989).

[7] M. A. Haase, J. Qiu, J. M. DePuydt and H. Cheng, Appl. Phys. Lent. 59, 1272 (1991).

[8] H. Jeon, J. Ding, W. Patterson, A. V. Nurmikko, W. Xie, D. C. Grillo, M. Kobayashi and R. L. Gunshor, Appl. Phys. Lett. 59, 3619 (1991)

[9] A. P. Bogatov, P. G. Eliseev, L. P. Ivanov, A. S. Logginov, M. A. Manko and K. Y. Senatorov, IEEE J. Quant. Elect. QE-9, 392 (1973).

[10] L. J. Bonnell and D. T. Cassidy, Appl. Opt. 28, 4622 (1989).

[11] D. T. Cassidy, D. M. Bruce and B. F. Ventrudo, Rev. Sci. Instrum. 62. 2385 (1991).

[12] K. Ogawa, IEEE J. Quant. Elect. QE-18, 849 (1982).

[13] W. Lenth, Opt. Lett. 8, 575 (1983).

[14] W. Lenth and M. Gehrtz, Appl. Phys. Letr. 47, 1263 (1985).

[15] G. R. Janik, C. B. Carlisle and T. F. Gallagher, J. Opt. Soc. Am. B3, 1070 (1986).

[16] L. Wang, H. Riris, C. B. Carlisle and T. F. Gallagher, Appl. Opt. 27, 2071 (1988).

[17] D. B. Bomse, A. C. Stanton and J. A. Silver, Appl. Opt. 31, 718 (1992).

[18] F. S. Pavone and M. Inguscio, Appl. Phys. B 56, 118 (1993).

[19] G. Björk, Phys. Rev. A 45, 8259 (1992).

[20] L. Hollberg, R. Fox. N. Mackie, A. S. Zibrov, V. L. Velichansky, R. Ellingsen and H. G. Robinson, In Tenth International Conference on Laser Spectroscopy. Eds. M. Ducloy, E. Giacobino and G. Camy, World Scientific, Singapore, New Jersey, London, Hong Kong (1992).

(21) B. Dahmani, L. Hollberg and R. Drullinger, Opt. Lett. 12, 876 (1987).

[22] A. Hemmerich, D. H. McIntyre, D. Schropp, Jr, D. Meschede and T. W. Hänsch, Opt. Commun. 75, $118(1990)$.

[23] H. R. Telle, Appl. Phys. B49, 217 (1989).

[24] M. Ohtsu, Highly Coherent Semiconductor Lasers, Artech House, Boston (1992).

[25] R. W. Fox, H. G. Robinson, A. S. Zibrov, N. Mackie, J. Marquardt, J. Magyar and L. Hollberg, Proc. SPIE, Vol. 1837, 360-365 (1993).

[26] E. M. Belenov, V. L. Velichansky, A. S. Zibrov, V. V. Nikitin, V. A. Sautenkov and A. V. Uskov, Sov. J. Quant. Elect. 13, 792 (1983).

[27] F. Favre, D. Le Guen, J. C. Simon and B. Landousies. Elect. Letr. 22, 795 (1986).

[28] A. T. Schremer and C. L. Tang, IEEE Phot. Tech. Letr. 2, 3 (1990).

[29] A. M. Akul'shin, V. Y. Bazhenov, V. L. Velichanski, M. V. Zverkov, A. S. Zibrov, V. V. Nikitin, O. G. Okhotnikov, V. A. Sautenkov, N. V. Senkov and E. K. Yurkin, Sov. J. Quunt. Elect. 16, 912 (1986).

[30] H. Olesen, J. H. Osmundsen and B. Tromborg, IEEE J. Quant. Elect. QE-22, 762 (1986).

[31] R. W. Tkach and A. R. Chraplyvy, J. Lightwave Tech. LT 4, 1655 (1986).

[32] D. R. Hjelme, A. R. Mickelson and R. G. Beausoleil, IEEE J. Quant. Elect. 27, 352 (1991).

[33] N. Schunk and K. Petermann, IEEE Photon. Tech. Letr. 1, 49 (1989).

[34] Y. A. Bykovski, V. L. Velichanski, I. G. Goncharov, A. P. Grachev, A. S. Zibrov, S. I. Koval and G. T. Pak, Sov. J. Quant. Elect. 19, 730 (1989).

[35] P. Zorabedian, W. R. Trutna, Jr and L. S. Cutler, IEEE J. Quant. Elect. QE-23, 1855 (1987).

[36] D. R. Kaplan and P. P. Deimel, AT\&T Bell Lab. Tech. J. 63, 857 (1984).

[37] T. Saitoh, T. Mukai and O. Mikami, J. Lightwave Tech. 3, 288 (1985).

[38] G. Eisenstein, G. Raybon and L. W. Stulz, J. Lightwave Tech. 6, 12 (1988).

[39] V. M. Baev, J. Eschner, E. Paeth, R. Schüler and P. E. Toschek, Appl. Phys. B55, 463 (1992).

[40] R. Fox, G. Turk, N. Mackie, T. Zibrova, S. Waltman, M. P. Sassi, J. Marquardt, A. S. Zibrov, C. Weimer and L. Hollberg, Solid State Lasers: New Developments and Applications, Proc. Nato Advanced Study Institute, Elba Italy, Plenum (1993). 
[41] A. N. Baranov, A. N. Imenkov, M. P. Mikhailova, A. A. Rogachev and Y. P. Yakovlev, Proc. SPIE 1408. 188 (1989)

[42] S. J. Eglash and H. K. Choi, Appl. Phys. Lett. 57, 1292 (1990).

[43] K. Soichi and K. Tatsuya, IEEE J. Quant. Elect. QE-17, 681 (1981).

[44] P. Isabelle, G. Philippe, D. Guy and C. Claude, IEEE J. Quant. Elect. QE-24, 148 (1988)

[45] L. Goldberg and M. K. Chun, Appl. Phys. Letr. 53, 1900 (1988).

[46] R. Parke, D. F. Welch, A. Hardy, R. Lang, D. Mehuys, S. O'Brien, K. Dzurko and D. Scifres, IEEE Phot. Tech. Letl. 5, 297 (1993).

[47] J. C. Baumert, P. Günter and H. Melchior, Opt. Commun. 48, 215 (1983)

[48] W. J. Kozlovsky, W. Lenth, E. E. Latta. A. Moser and G. L. Bona, Appl. Phys. Letl. 56. 2291 (1990).

[49] G. J. Dixon, C. E. Tanner and C. E. Wieman, Opt. Lett. 14, 731 (1989).

[50] A. Hemmerich, D. H. Mclntyre, C. Zimmermann and T. W. Hänsch, Opt. Lett. 15, 372 (1990)

[51] E. S. Polzik and H. J. Kimble, Opt. Lett. 16, 1400 (1991).

[52] J. Webjörn, F. Laurell and G. Arvidsson, IEEE Phot. Tech. Letr. 1, 316 (1989)

[53] F. Laurell, J. B. Brown and J. D. Bierlein, Appl. Phys. Lett. 60, 1064 (1992).

[54] E. J. Lim, M. M. Fejer, R. L. Byer and W. J. Kozlovsky, Elect. Lett. 25, 731 (1989)

[55] W. P. Risk and W. J. Kozlovsky, Opt. Lett. 17, 707 (1992).

[56] U. Simon, C. E. Miller, C. C. Bradley, R. G. Hulet, R. F. Curl and F. K. Tittel, Opt. Letr. 18, 1062 (1993).

[57] P. E. Walters, T. E. Barber, M. W. Wensing and J. D. Winefordner, Spectrochim. Acta 46B, 1015 (1991).

[58] A. Zybin. C. Schnürer-Patshan and K. Niemax, Spectrochim. Acta 47B, 1519 (1992).

[59] P. A. Johnson, J. A. Vera, B. W. Smith and J. D. Winefordner, Spectrosc. Lett. 21, 607 (1988).

[60] J. Lawrenz, A. Obrebski and K. Niemax, Anal. Chem. 59, 1232 (1987).

[61] J. Lawrenz and K. Niemax. Spectrochim. Acta 44B, 155 (1988).

[62] R. W. Shaw, J. P. Young and D. H. Smith, Anal. Chem. 61, 695 (1989).

[63] G. C. Turk, Anal. Chem. 53, 1187 (1981).

[64] J. C. Travis, G. C. Turk, J. R. DeVoe, P. K. Schenck and C. A. Van Dijk, Prog. Anal. At. Spectrosc. 7. 199 (1984).

[65] G. J. Havrilla, S. J. Weeks and J. C. Travis, Anal. Chem. 54, 2566 (1982).

[66] Y. Shimose, T. Okamoto, A. Maruyama, M. Aizawa and H. Nagai, IEEE Phot. Tech. Lett. 3, 86 (1991).

[67] K. Uehara and H. Tai, Appl. Opt. 31, 809 (1992).

[68] S. Sudo, Y. Sakai, H. Yasaka and T. Ikegami, IEEE Phot. Tech. Lett. 1, 281 (1989).

[69] D. T. Cassidy and L. L. Bonnell, Appl. Opt. 27, 2688 (1988).

[70] M. Ohtsu, H. Kotani and H. Tagawa, Jap. J. Appl. Phys. 22, 1553 (1983).

[71] H. Sasada and K. Yamada, Appl. Opt. 29, 3535 (1990).

[72] T. Ohshima and H. Sasada, J. Mole. Spec. 136, 250 (1989).

[73] A. C. Stanton and J. A. Silver, Appl. Opt. 27, 5009 (1988).

[74] M. Gehrtz. G. C. Bjorklund and E. A. Whittaker, J. Opt. Soc. Am. B2, 1510 (1985).

[75] J. Reid, M. El-Sherbiny, B. K. Garside and E. A. Ballik, Appl. Opt. 19, 3349 (1980).

[76] R. J. Yokelson. J. B. Burkholder. A. R. Ravishankara anc C. J. Howard, National Oceanic and Atmospheric Administration Aeronomy Laboratory, Boulder, Colorado. 


\title{
A CPW PHASE-LOCKED LOOP FOR DIODE-LASER STABILIZATION*
}

\author{
L. D'Evelyn ${ }^{\mathrm{a}}$, L. Hollberg ${ }^{\mathrm{a}}$, Z.B. Popovic ${ }^{\mathrm{b}}$ \\ ${ }^{a}$ National Institute of Standards and Technology \\ Boulder, Colorado 80303 \\ ${ }^{b}$ Department of Electrical and Computer Engineering \\ University of Colorado \\ Boulder, Colorado 80309-0425
}

\begin{abstract}
A low-cost, phase-lock circuit for slaving an extended-cavity diode laser to a stabilized reference laser has been developed. Grounded coplanar waveguide and surface mount technology have been used. An internal mixing stage allows continuous tuning of the laser difference frequency between $5 \mathrm{MHz}$ and $1.5 \mathrm{GHz}$. Absolute phase locks exceeding 8 hours have been demonstrated.
\end{abstract}

\section{INTRODUCTION}

Precise control of the frequency and/or phase of diode lasers opens up a wide variety of scientific and commercial applications. Consequently, several research laboratories have recently demonstrated phase-stabilized diode-laser systems [1-4] similar to the one presented in this paper. The diodelaser phase-lock circuit described below has been developed to address a number of scientific applications at the National Institute for Standards and Technology. These applications require both extreme stability and frequency control of extended cavity lasers and include: high resolution spectroscopy, optical frequency synthesis, and laser cooling and trapping. In combination with new, commercially available extended-cavity lasers [5], our phase-lock servo could also be integrated into numerous commercial applications, such as laser ranging and distance measurements and coherent communications systems. In the future, this circuit may serve as a fundamental building block for a tunable optical synthesizer. A precision optical synthesizer would allow optical heterodyne and RF conversion techniques to be applied to the optical regime

\section{OPTICAL PHASE-LOCKED LOOP}

The phase-lock circuit serves to slave an extended-cavity diode-laser to a highly stable reference laser. Grounded coplanar waveguide (CPW) on a FR4/G10 circuit board and

* Contribution of NIST, not subject to copyright surface mount technology have been employed for all microwave and RF sections to maximize performance while minimizing cost. Only low-cost, commercial-grade parts have been used in the design. Figure 1 shows a photograph of the $11.7 \times 14.2 \mathrm{~cm}(4.6 \times 5.6 \mathrm{in})$ circuit.

The basic operation can be understood as follows (Figure 2). The beat note between the two lasers is detected with a fast photodiode, then input to the microwave mixer. An external synthesized LO source allows continuous tuning of the difference frequency between the two lasers between $5 \mathrm{MHz}$ and $1.5 \mathrm{GHz}$. The filtered IF signal is first converted to an ECL signal and then detected by a low phase-error ECL phase/frequency detector [6-7]. High speed op-amps perform active loop filtering on the low pass filtered error signal and load compensation for the combined FM response of the current source and extended-cavity laser. The final error signal is fed back to the slave laser via two ports: a wide bandwidth channel ( $\mathrm{DC}$ to $\approx 1 \mathrm{MHz}$ ) to the laser's injection

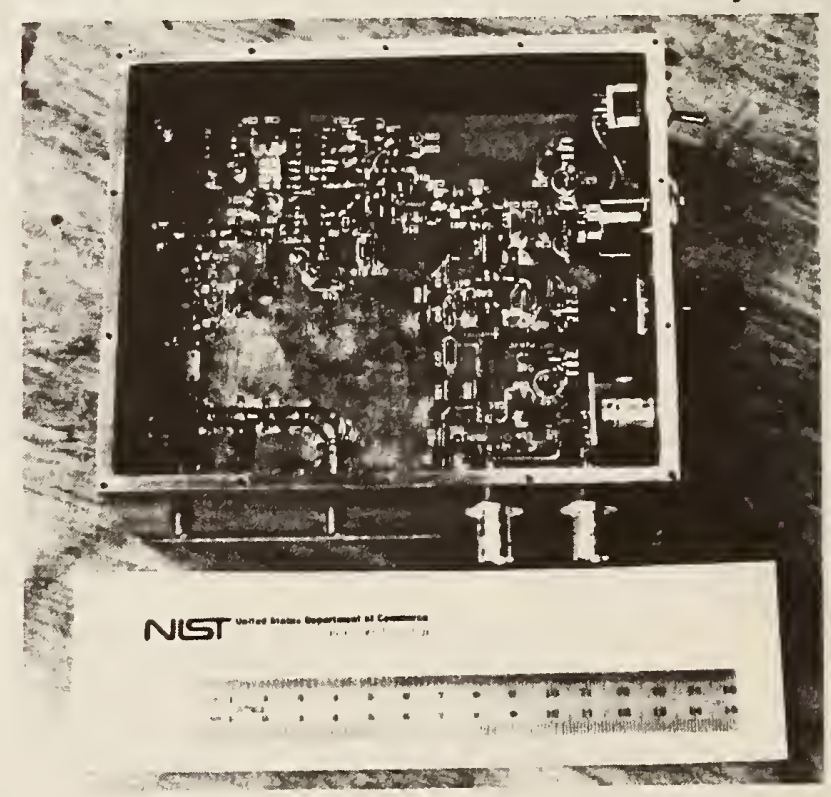

Figure 1: $\quad$ Phase-lock loop for diode-laser stabilization. CPW lines and surface mount technology allow input frequencies to $1.5 \mathrm{GHz}$.

Proc. of 1994 IEEE MTT-S International Microwave Symp., San Diego, CA (1994). 
current controls fast frequency jitter, while a high dynamic range channel ( $\mathrm{DC}$ to $\approx 2 \mathrm{kHz}$ ) to a piezo-electric transducer (PZT) adjusts the laser's cavity length to compensate for drift.

The design requirements were determined, in part, by our desire to have a simple system that allows offset frequency sweeps from near DC to at least $1.5 \mathrm{GHz}$ with a resolution of $\leq 1 \mathrm{~Hz}$. The $1.5 \mathrm{GHz}$ specification was dictated by our requirement of complete spectral coverage and the $3 \mathrm{GHz}$ free spectral range of the Fabry-Perot reference cavity that we use to lock the master laser. The maximum tuning range is not fundamentally limited, but is rather naturally set by the availability and cost of microwave synthesizers capable of $\leq 1 \mathrm{~Hz}$ resolution. The grounded CPW lines on the low-cost FR4/G10 printed circuit board perform adequately to the required $1.5 \mathrm{GHz}$. Extension of the sweep range beyond 1.5 $\mathrm{GHz}$, however, would require more accurate transmission-line design equations and/or improved circuit board fabrication. Alternatively, external microwave mixing stages can be used to extend the maximum offset frequency. Special Schottky diodes can be used as harmonic mixers, for example, to detect beat notes between near-IR diode lasers to frequencies higher than $600 \mathrm{GHz}[8]$.

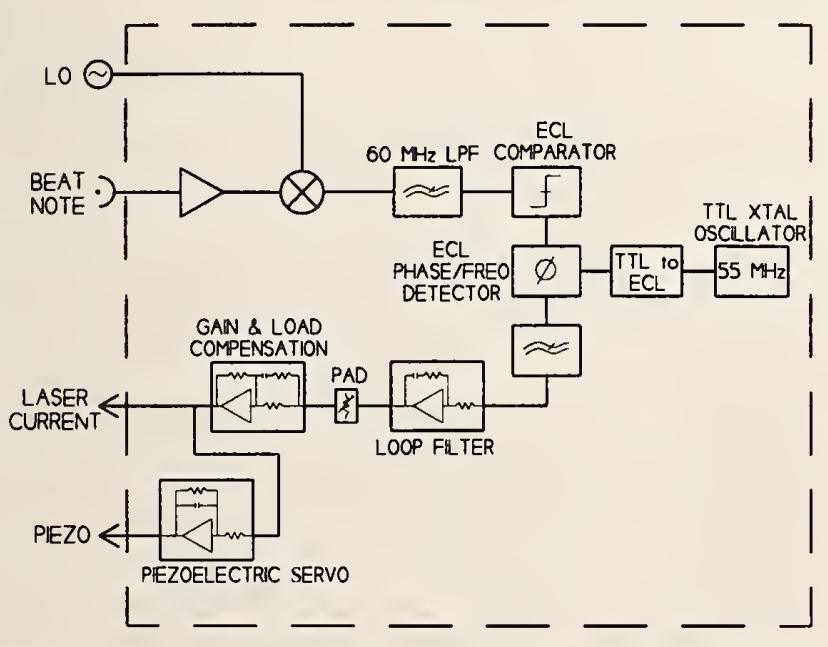

Figure 2: Block diagram for phase-locking extendedcavity diode lasers. The $55 \mathrm{MHz}$ IF signal is detected by an ECL phase/frequency detector. High-speed op-amps provide loop filtering and compensation.

Figure 3 shows the extended-cavity laser [9-10] controlled by the phase-servo circuit. A commercially available diode-laser (with the output facet AR coated) is used together with a high-efficiency holographic diffraction grating [11] in a Littrow geometry. The rear facet of the diode and the grating form an "extended cavity." Cavity lengths ranging from 6 to $50 \mathrm{~cm}$ have been used in our experiments. The PZTadjustable mirror provides a precise adjustment of the optical length of the cavity. Optical feedback of the first-order beam from the grating narrows the linewidth from greater than
$30 \mathrm{MHz}$ to about $50 \mathrm{kHz}$ and allows tuning of the laser's wavelength/frequency. The narrowed spectral characteristics of the extended-cavity greatly facilitate locking and control of the slave laser.

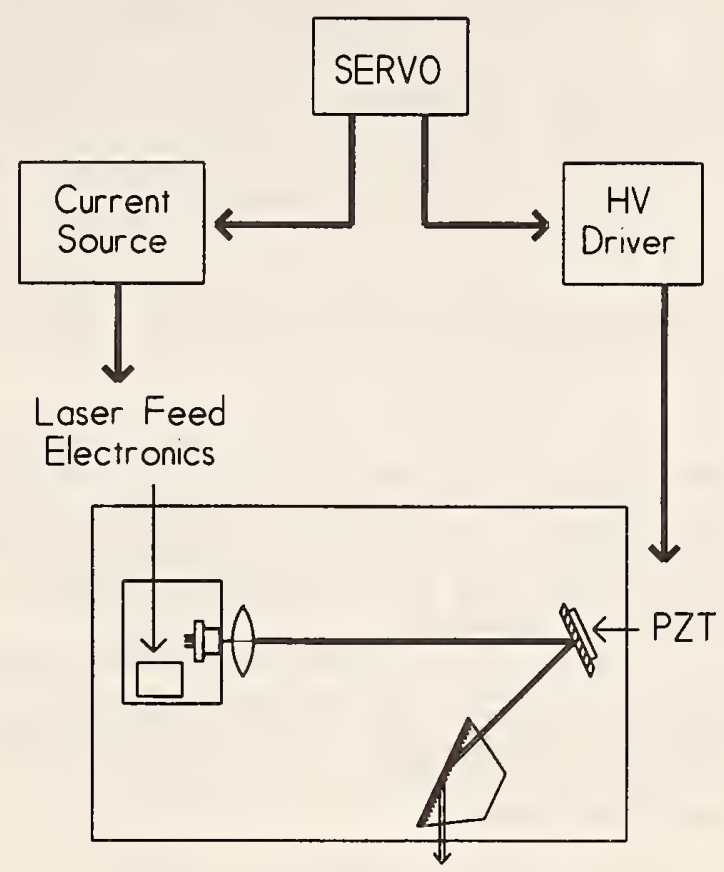

Figure 3: Extended-cavity laser. The rear facet of the laser-diode and the holographic diffraction grating form an "extended cavity." The narrowed spectral characteristics greatly facilitate locking the slave laser.

No additional auto-locking features have been added to the electronics. The PZT provides a $20 \mathrm{GHz}$ continuous tuning range and can easily be used to tune a laser within the -20 to $+100 \mathrm{MHz}$ capture range of the electronic circuit. Broader stepwise tuning can be accomplished by mechanical rotation of the grating. Laser mode-hopping remains the greatest impediment to initially locking the long $(50 \mathrm{~cm})$ cavity lasers (free spectral range $\approx 300 \mathrm{MHz}$ ). An etalon in the extended laser cavity or a clamp on the maximum correction current [3] should solve this complication.

\section{APPLICATIONS}

The laser phase-lock circuit is now used in two experiments with different laser setups. In both cases, the servo-loop bandwidth of about $1 \mathrm{MHz}$ results in robust phase-locks. Locking times exceeding 8 hours have been demonstrated. Unfortunately, the sensitive nature of the experimental laser systems preclude longer tests. Both laser systems will be described to illustrate the flexibility of the phase-lock circuit. Plans to include this circuit in a laser cooling and trapping experiment will also be outlined. 
The first experiment uses two phase-locked AlGalnP lasers at $657 \mathrm{~nm}$ for high resolution spectroscopy of calcium

(Figure 4). Phase-locking allows the stability of a nontunable reference laser to be transferred to the offset-tunable slave laser. Extremely narrow laser linewidths are required to observe and lock the laser to narrow saturated-absorption signals. The "master" extended-cavity laser, electronically locked to a stable Fabry-Perot cavity, has a fast linewidth of only $500 \mathrm{~Hz}$ and a residual frequency jitter of $5 \mathrm{kHz}$ [12]. Figure 5 shows a spectral plot of the locked beat note between the two lasers. In future experiments the master laser will be referenced to a very high $Q\left(\approx 10^{10}\right)$, stable FabryPerot cavity. Such a laser system locked to the calcium resonance could serve as a high accuracy transfer standard of optical wavelength/frequency.

In the second example, the phase-locked loop (PLL) locks an AlGaAs extended-cavity laser $(792 \mathrm{~nm})$ to an $\mathrm{Ar}^{+}$laser pumped Ti: $\mathrm{Al}_{2} \mathrm{O}_{3}$ laser. Here, the offset frequency of $47 \mathrm{GHz}$ is established using an external harmonic mixing stage. This system is being used by J.C. Bergquist of NIST for experiments on laser-cooled

$\mathrm{Hg}^{+}$[13]. Because of the ground-state hyperfine structure splitting, laser cooling of $\mathrm{Hg}^{199}$ requires two lasers (at $194 \mathrm{~nm}$ ) separated by $47 \mathrm{GHz}$. The $194 \mathrm{~nm}$ is generated by summing the $792 \mathrm{~nm}$ light with the second harmonic of an $\mathrm{Ar}^{+}$laser line $(514 \mathrm{~nm})$.

Future plans include incorporating the phase-lock circuit into a laser system for high resolution spectroscopy of lasercooled, trapped cesium atoms. As demonstrated by Kasevich et al [14], this can lower the effective temperature of the cooled cesium atoms from about $500 \mu \mathrm{K}$ to less than $1 \mu \mathrm{K}$.

\section{CONCLUSION}

The CPW phase-lock circuit described in this paper has consistently demonstrated robust locks of several hours duration between diode-lasers. Measured results in two different laser systems illustrate the circuit's potential for advancing many scientific experiements, especially those involving high-resolution spectroscopy and laser cooling and trapping. This low-cost, highly reproducible circuit could also be used in many additional applications. For example. the PLL could be utilized to reduce the number of oscillators required in an optical frequency synthesis system [15-16]. In short, the laser phase-lock circuit promises future viability of coherent optical applications of diode lasers.

\section{ACKNOWLEDGEMENTS}

We thank J.L. Hall, J.C. Bergquist, and R.W. Fox for important contributions to the development of this technology. This work was supported in part by the Air Force Office of Scientific Research.

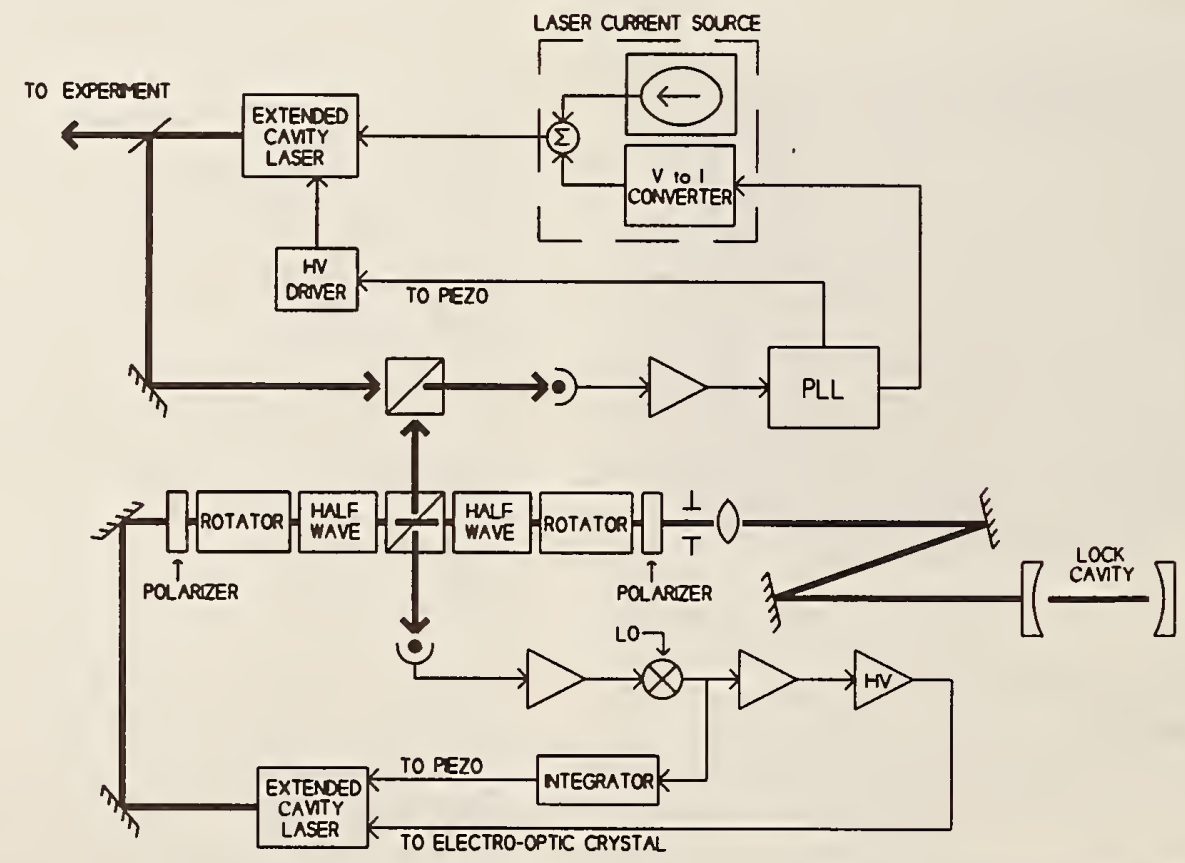

Figure 4: Laser setup for high resolution calcium spectroscopy. Multi-hour phaselocks between two AlGaInP diode-lasers have routinely been demonstrated. 


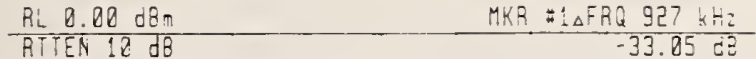

$10.08 \mathrm{~dB} / 01 \mathrm{U}+\ldots \mathrm{COS}$ P.

MARKEA

$927 \mathrm{kHz}$
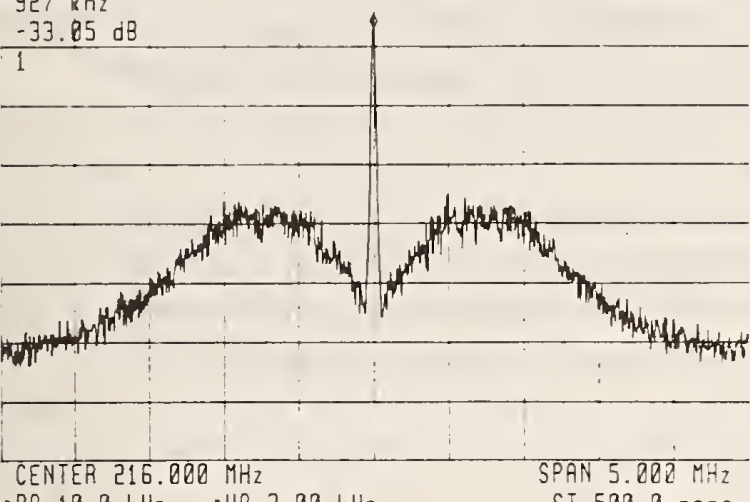

- RB $10.0 \mathrm{kHz}$.UB $3.80 \mathrm{kHz}$ ST $500.8 \mathrm{msec}$

Figure 5: Beat-note between locked diode-lasers. The circuit's loop bandwidth of about $1 \mathrm{MHz}$ results in robust phase-lock

\section{REFERENCES}

[1] H.R. Telle, H. Li, "Phase-Locking of Laser Diodes", Electronic Letters, vol. 26, pp. 858-859, 1990.

[2] M. Ohtsu, K. Nakagawa, M. Kourogi, and W. Wang, "Frequency Control of Semiconductor Lasers", J. Appl. Phys., vol. 73, no. 12, pp. R1-R17, June 1993.

[3] G. Santarelli, A. Clairon, S.N. Lea, and G.M. Tino, "Heterodyne Optical Phase-Locking of ExtendedCavity Semiconductor Lasers at $9 \mathrm{GHz}$ ", Optics Communications, vol. 104, pp. 339-344, January 1994.

[4] S. Schwartz, J.L. Hall, K. Gibble, D. Weiss, "Robust Phase-Locking of Diode Lasers", submitted to JOSA B 1993.

[5] "NIST, SDL Develop High-Power Laser", Optics \& Photonics News, pp. 50, January 1994.

[6] Analog Devices AD9901 Product Data Sheet, Norwood, MA.

[7] W. Egan and E. Clark, "Test Your Charge-Pump Phase Detectors", Electronic Design, vol. 12, pp. 134-137, June 1978.

[8] S. Waltman, A. Romanovsky, J. Wells, R.W. Fox, and L. Hollberg, "Precise Optical Frequency References and Difference Frequency Measurements with Diode Lasers, Proc. Soc. Photo.-Opt. Instrum. Engrs., vol. 1837, pp. 386, 1992. (Note: range now extended to at least $620 \mathrm{GHz}$ )
[9] C. Wieman, L. Hollberg, "Using Laser Diodes for Atomic Physics". Rev. Sci. Instrum., vol. 62. pp. 1-20, January 1991.

[10] M. Ohtsu, Highly Coherent Semiconductor Lasers, Artech House, Norwood MA, 1992.

[11] M.S. Soskin and V.B. Taranenko, "Holographic TotalInternal Reflection Selector for Tunable Lasers". Soviet Journal of Quant. Elect., vol. 7, no. 2. pp. 298-304, 1977.

[12] A.S. Zibrov, R.W. Fox, R. Ellingsen. C.S. Weimer. V.L. Velichansky, G.M. Tino, and L. Hollberg, "High Resolution Diode Laser Spectroscopy of Calcium", submitted to Appl. Phys. B 1993.

[13] J.C. Bergquist, W.M. Itano, D.J. Wineland, "Laser Stabilization to a Single Ion", Proceedings of the International School of Physics $<<$ Enrico Fermi $>$, edited by T. Hansch and M. Inguscio, North Holland, Amsterdam, July 1992.

[14] M. Kasevich and S. Chu, "Laser Cooling Below a Photon Recoil with Three-Level Atoms", Physics Review Letters, vol. 69, pp. 1741-1744, 1992.

[15] J. Helmcke, A. Morinaga, J. Ishikawa, and F. Riehle. "Optical Frequency Standards", IEEE Trans. on Instrumentation and Measurement, vol. 83, no. 2, pp. 524-532, April 1989.

[16] K. Nakagawa, M. Kourogi, and M. Ohtsu. "Proposal of a Frequency-Synthesis Chain Between the Microwave and Optical Frequencies of the $\mathrm{Ca}$ Intercombination Line at $657 \mathrm{~nm}$ Using Diode Lasers", Appl. Phys. B, vol. 57, pp. 435-430, 1993. 


\title{
Amplitude Modulation on Frequency Locked Extended Cavity Diode Lasers
}

\author{
R. W. Fox, L. D'Evelyn, H. G. Robinson, C. S. Weimer, and L. Hollberg \\ National Institute for Standards and Technology, 325 Broadway, Boulder, CO, 80303
}

\begin{abstract}
The increase in low frequency amplitude noise on several extended cavity diode lasers was measured when frequency or phase lock servos were applied using the injection current as the feedback channel. The AM noise increase inside the FM servo bandwidth is approximately that expected from the suppression of frequency noise uncorrelated with the inherent amplitude noise of the laser.
\end{abstract}

\section{Introduction}

The noise characteristics ${ }^{1}$ of semiconductor diode lasers are relevant to a number of applications, including communications and spectroscopy. The noise can be quite complex due to optical feedback and the well known AM to FM coupling, and is an area of active research. ${ }^{2}$ Diode lasers are often used with extended cavities to provide wavelength tunability and to narrow the linewidth. It is now common practice to use active (electronic) control systems to suppress portions of either the AM or FM noise. The coupling between $A M$ and FM in the semiconductor laser complicates the noise characteristics in these active feedback control systems. For example, in many systems the feedback path is via the laser's injection current, which simultaneously alters both the amplitude and frequency of the laser.

In previous experiments we studied some of the limitations of using active electronic control of the injection current to suppress AM noise on the output of an extended cavity diode laser (ECDL). ${ }^{3}$ The fundamental shot-noise-limit may be approached (fig. 1), but only by using most of the laser output for the feedback control channel. The photo-current signal-to-noise $(\mathrm{s} / \mathrm{n})$ of the useful experimental beam reaches a maximum in between the extremes of using most of the light for the experiment (in which case the

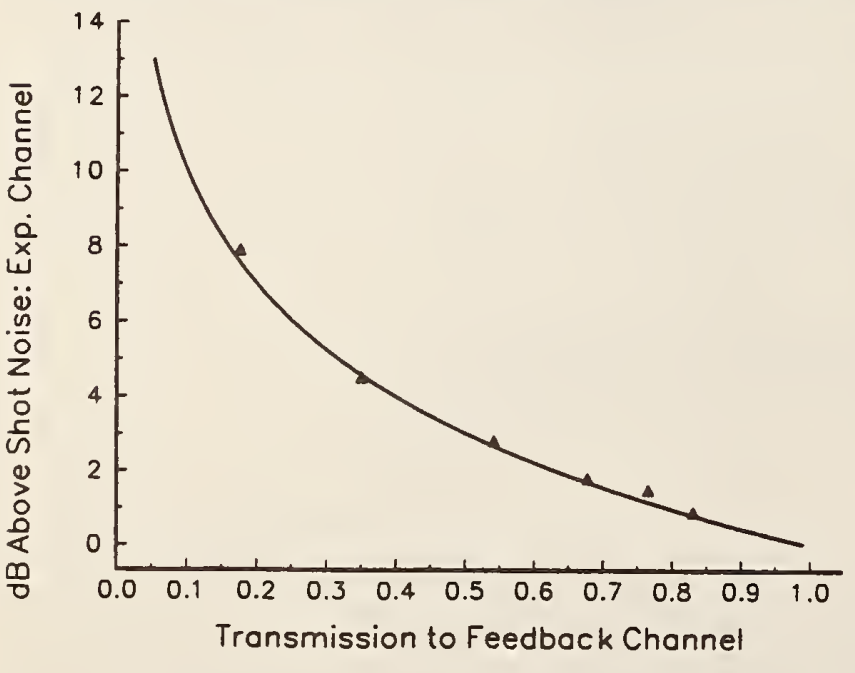

Fig. 1 The performance of an AM feedback loop to the injection current of an ECDL. The residual AM noise of the experimental beam photo-current is plotted versus the beamsplitter transmission to the servo detector. technical AM is not suppressed) or for the servo control (in which case the shot $s / n$ level is relatively high).

In some applications both the AM and FM laser characteristics are important. For instance in very high-resolution spectroscopy, it is desirable to have a narrow linewidth laser with low AM noise, zero frequency drift, and precise tunability. Towards this goal we have phase-locked (with a tunable offset frequency) an ECDL to a stable low-drift reference ECDL, by using feedback to the slave injection current. However the injection current feedback path cannot also be used for AM control, and furthermore the FM control adversely affects the laser's AM. In this paper we report measurements of the power fluctuations when frequency control is employed.

In the case of a frequency servo acting through the injection current, the output power is affected primarily by the injection current 
fluctuations. More generally, for any method of feedback the changes induced in the FM noise by the servo will also be mapped to amplitude noise by any element in the extended cavity that has a frequency dependent gain or loss. The amount of FM to AM conversion is dependent on the dispersion of the longitudinal mode selector (usually a diffraction grating), and the lasing wavelength with respect to the mode selector, as well as the chip facet reflectance and alignment effects.

When diode lasers are built into extended cavities of moderate lengths $(\approx 10 \mathrm{~cm})$, the inherent frequency noise is reduced so that a relatively low bandwidth $(\approx 1 \mathrm{MHz})$ electronic feedback loop is sufficient to frequency lock the laser. ${ }^{4}$ At low Fourier frequencies piezo-electric elements may be used to adjust the cavity length, while higher bandwidths require feedback either to the laser injection current, or to intra-cavity tuning elements. ${ }^{5}$ Most often the slow piezo feedback loop is necessary to keep the higher bandwidth loop in the center of it's dynamic range.

We can make an estimate of the $\mathrm{AM}$ induced by a frequency control servo that uses the injection current by assuming no correlation between the inherent FM and AM fluctuations of the ECDL. In this case the induced $A M$ will add in a root mean square fashion to the inherent amplitude noise. We can estimate the induced power fluctuation $\triangle P(f)$ (in $\mathrm{mW} / \sqrt{\mathrm{Hz}}$ ) from the frequency noise density $S_{\dot{\varphi}}(f)$ that is suppressed when the loop is closed. The dependence of the output power with current $\Delta \mathrm{p} / \Delta \mathrm{i}$, and the dependence of the laser frequency with injection current $\Delta v / \Delta i$ are used to estimate the induced $A M$. The ratio $\Delta \mathrm{p} / \Delta \mathrm{i}$ is assumed flat with frequency, which is justified by noting that we are only interested in the effects within the servo bandwidth (relatively low frequencies). We write

$$
\Delta P(f)=\frac{\sqrt{S_{\dot{\phi}}(f)} \cdot \frac{\Delta p}{\Delta i}}{\left|\frac{\Delta v}{\Delta i}(f)\right|}
$$

as an estimate for the maximum increase in the output power noise spectrum within the servo bandwidth. The increase of the relative intensity noise $(R I N)$ is then $\left[\Delta P(f) / P_{0}\right]^{2}$.

\section{Experiment}

The increase in the AM noise due to frequency control loops using current feedback was measured in two separate experiments. A Littrow type extended cavity laser at $657 \mathrm{~nm}$ was frequency locked to an optical cavity by electronic feedback to the injection current and the changes induced in the AM spectrum were measured. Secondly, two grazing incidence ECDL's were slaved to reference lasers with a phase-lock by feeding back to the slave injection current ports. ${ }^{6}$ The changes induced in the AM spectrum of the slaves were measured.

The Littrow laser was built using an anti-reflection coated $3 \mathrm{~mW}$ laser, and frequency locked to the optical cavity with a Pound-Drever-Hall RF lock scheme. ${ }^{7}$ The RF modulation was applied with an external modulator resonant at $20 \mathrm{MHz}$. In the case of injection-current locking the feedback bandwidth was approximately $500 \mathrm{kHz}$. The power versus injection current had a slope of $0.09 \mathrm{~mW} / \mathrm{mA}$, as shown in figure 2. Also shown in figure 2 is the dc change in frequency with injection current $\Delta v / \Delta \mathrm{i}$, measured at different bias current levels by sweeping the reference cavity and monitoring the correction signal to the injection current. The laser would lock for a frequency interval which could easily be determined with the help of the modulation sidebands, and during this interval the change of the injection current correction signal was measured. The laser cavity length was $12 \mathrm{~cm}$, and the power output at the normal operating point of $I_{1} \approx$ 1.16 was about $1.0 \mathrm{~mW}$. 
At this bias-current level the complex frequency dependence of $\Delta v / \Delta i$ was measured out to $4 \mathrm{MHz}$ with a network analyzer. The response was flat out to several hundred $\mathrm{kHz}$, decreasing $10 \mathrm{db}$ in magnitude by $1.5 \mathrm{MHz}$. The phase of the response was nearly constant to $100 \mathrm{kHz}$, but lagged by approximately $90^{\circ}$ at $1.5 \mathrm{MHz}$.

An alternative method for frequency stabilization of this laser was also used for comparison. An electro-optic modulator (a $1 \mathrm{~cm}$ aperture ADP crystal) was used in the extended laser cavity $^{5}$ to provide a feedback path to the cavity length that is independent of the injection current (and hence to first order the power). For this path the feedback bandwidth was approximately $1 \mathrm{MHz}$.

The AM noise with the laser unlocked and locked with the two feedback paths is shown in

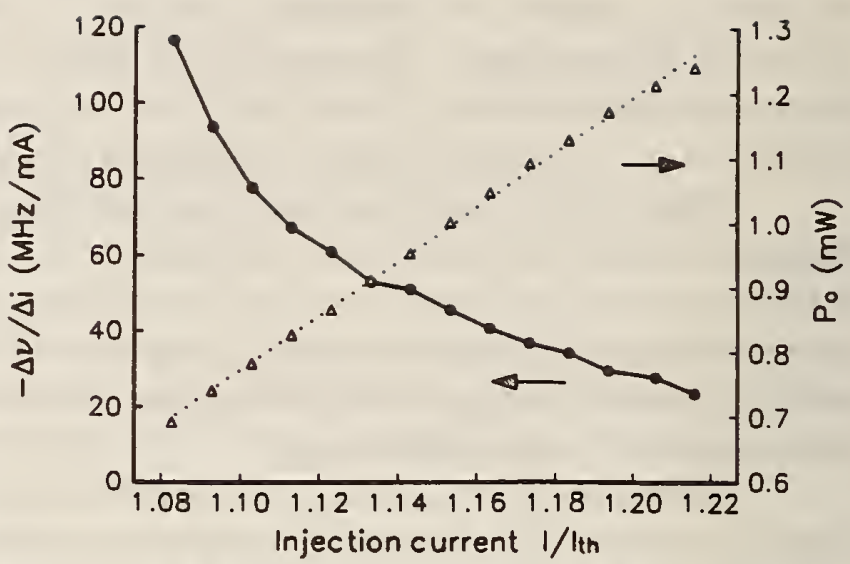

Fig.2 The output power of the Littrow AlGalnP extended cavity laser above threshold and the dc change in frequency with injection current, $\Delta v / \Delta i$. The frequency response was also measured (see text).

figure 3. The laser was operating with a dc injection current of $\mathrm{L}_{\mathrm{l}}=1.16$, and $\Delta \mathrm{v} / \Delta \mathrm{i} \approx 40 \mathrm{MHz} / \mathrm{mA}$ when the locked data was recorded. No change in AM is observed with feedback to the intra-cavity EO crystal.

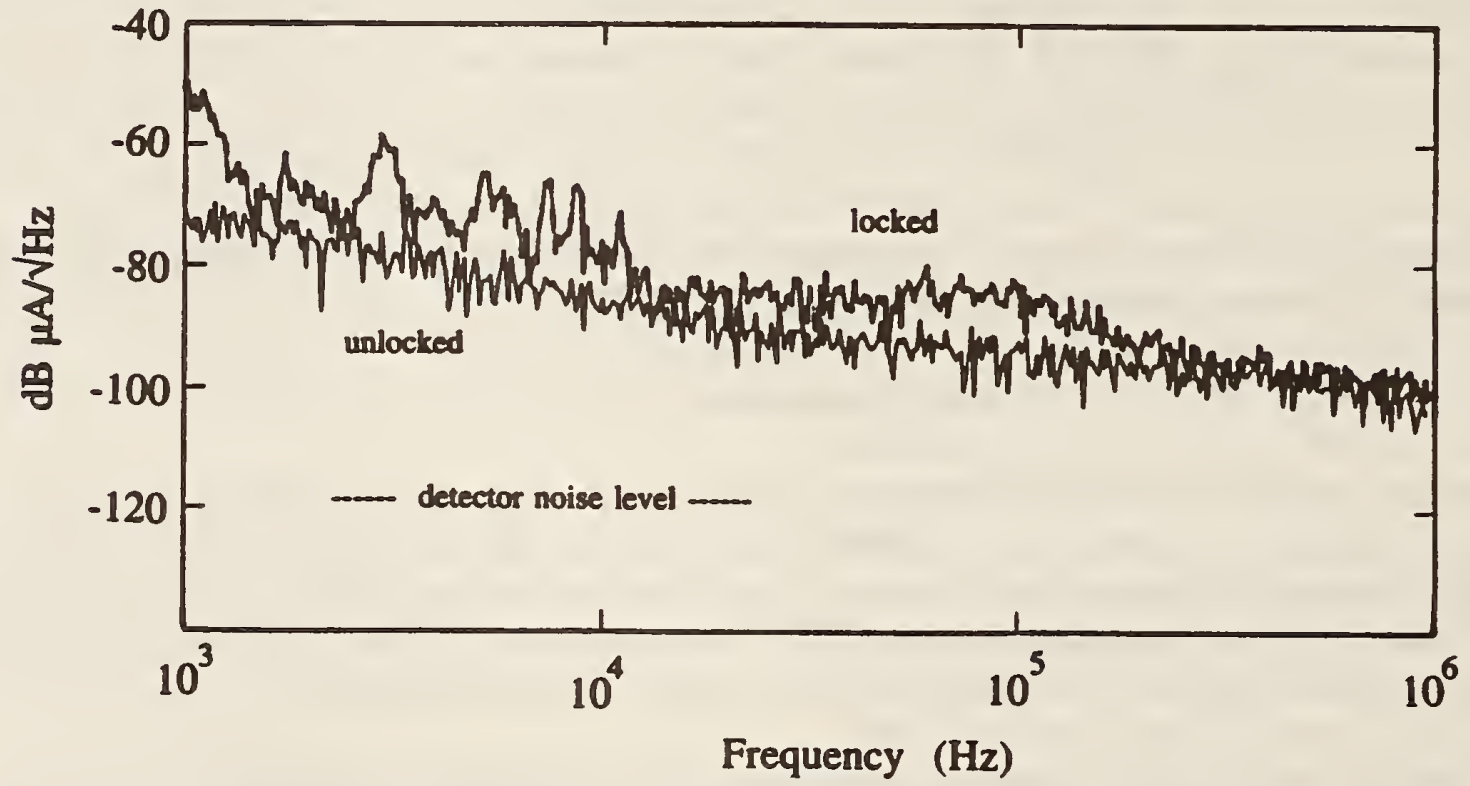

Fig. 3 The low frequency amplitude noise spectrum of the Littrow AlGaInP ECDL. The upper-most trace is the laser locked to an optical cavity by feedback to the injection current. The lower trace is the amplitude noise of the unlocked laser, which on this scale does not change when the laser is locked to the cavity with the intra-cavity electro-optic crystal. The detector noise level is indicated, and the dc photo-current is $\approx 210 \mu \mathrm{A}$.

In the second experiment, a slave ECDL was constructed with an anti-reflection coated $5 \mathrm{~mW}$ red diode laser. A diffraction grating with $1800 \mathrm{~V} / \mathrm{mm}$ was used at a grazing incidence angle of about 85 degrees, and the cavity length was approximately $8.8 \mathrm{~cm}$, resulting in a free spectral range of $1.7 \mathrm{GHz}$. The output power at the normal operating point was about $2 \mathrm{~mW}$. This laser was phase-locked to the Littrow laser discussed above, and the beat note is shown in figure 4. A significant AM noise increase in 
the frequency region near the phase-lock servo's unity gain point was observed, i.e. where the noise shoulders appear in the beat note. An example of this induced AM is shown in figure 5 .

The other AM measurement of a phase-locked slave was also with a grazing incidence type ECDL, except the laser was a $100 \mathrm{~mW} \mathrm{AlGaAs}$ laser operating at $792 \mathrm{~nm}$. This ECDL was slaved to a Ti:Sapphire laser with a feedback bandwidth of approximately $800 \mathrm{kHz}$. The phase-lock on the AlGaAs laser was implemented with a similar degree of phase margin, and a similar increase in the slave's AM noise spectrum was observed.

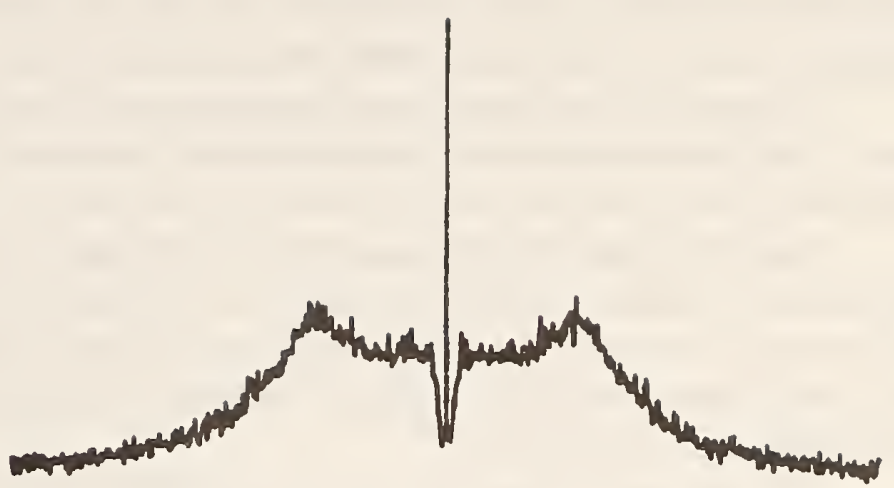

Fig. 4 A beat note between the $657 \mathrm{~nm}$ Littrow laser and the phase-locked slave. The offset frequency is approximately 200 $\mathrm{MHz}$. The trace is over a $10 \mathrm{MHz}$ band, and the noise peaks are at $1.5 \mathrm{MHz}$. The noise close to the carrier peak is $-60 \mathrm{dBc}$.

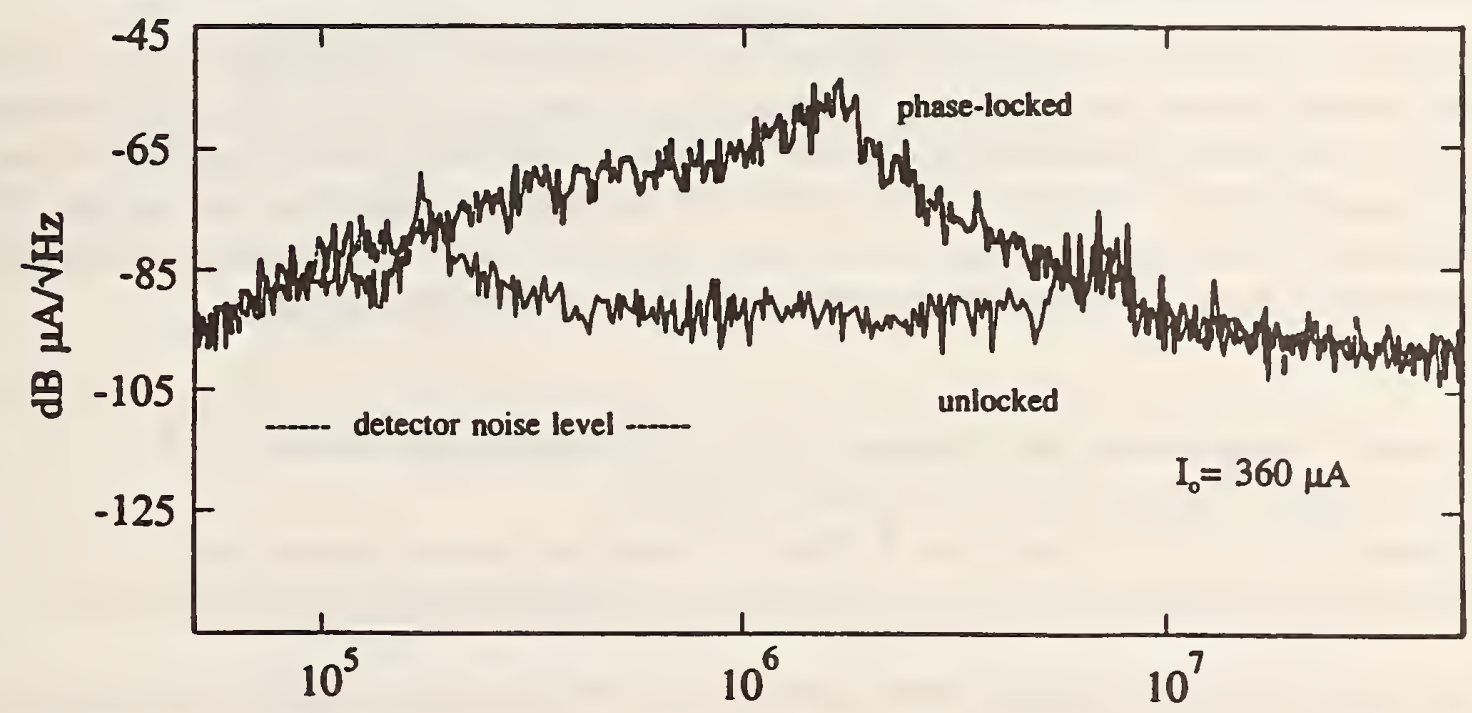

Frequency $(\mathrm{Hz})$

Fig. 5 The AM noise of the phase-locked slave laser (upper trace). The broad noise peak at $1.5 \mathrm{MHz}$ corresponds to the increase in phase noise at the FM servo's unity gain frequency. The peak in the phase noise at this point, combined with the decreasing sensitivity of the laser frequency to injection current, acts to increase the AM noise.

\section{Discussion}

The frequency locked ECDL noise data (fig. 3) clearly shows a broad-band increase in the AM noise level, and further increases at distinct frequencies. The FM noise spectrum typically exhibited by an ECDL operating in the single-mode regime has a 1/f frequency dependence, reaching a spectrally-white noise-floor at approximately $100 \mathrm{kHz}$. Structural resonances will cause narrow-band frequency noise, and the FM servo response to this narrow-band noise shows up as AM peaks in the locked spectrum. A noise floor of approximately $10^{4} \mathrm{~Hz}^{2} / \mathrm{Hz}$ in the frequency noise density was measured in the $12 \mathrm{~cm}$ long ECDL. ${ }^{5}$ The network analyzer measurement indicates that the current to frequency transfer function value at $d c(\Delta v / \Delta i \approx$ $40 \mathrm{MHz} / \mathrm{mA}$ ) is valid at $100 \mathrm{kHz}$. The induced $\mathrm{AM}$ noise can then be estimated at $100 \mathrm{kHz}$. 
Approximately $50 \%$ of the laser output was incident on the AM noise detector, and the detector responsivity at $657 \mathrm{~nm}$ was measured to be $0.43 \mathrm{~mA} / \mathrm{mW}$. Using equation (1), at $100 \mathrm{kHz}$ the estimated maximum photo-current noise level for the induced $\mathrm{AM}$ is $-86.3 \mathrm{~dB} \mu \mathrm{A} / \sqrt{\mathrm{Hz}}$.

The data of fig. 3 shows good agreement with this level, even though we have used a very simplistic model. The current servo-loop bandwidth used was about $500 \mathrm{kHz}$, and the data shows the locked AM noise approaching the unlocked case at about this frequency.

The AM noise of the phase-locked slave laser (figure 5) displays a large increase in the frequency region near $1.5 \mathrm{MHz}$. The phase margin of the FM loop at the unity gain frequency was such that the beat note between the two lasers had broad noise sidebands centered at approximately $1.5 \mathrm{MHz}$ from the carrier peak. Only the dc value of the transfer function $\Delta v / \Delta \mathrm{i}(f)$ of the slave was measured, which was $40 \mathrm{MHz} / \mathrm{mA}$ at the operating current level.

However as the laser chip is of the same type ${ }^{8}$ and the extended cavity is a similar length as the Littrow laser, we assume that the transfer function $\Delta v / \Delta i(f)$ of the slave is similar to that of the Littrow laser. If so, the $10 \mathrm{~dB}$ decrease of $\Delta \mathrm{v} / \Delta \mathrm{i}(f)$ together with a $20 \mathrm{~dB}$ increase in the phase noise near the FM servo's unity gain point would account for the increase in the AM noise observed in figure 5.

\section{Summary}

An increase in the AM noise when an extended cavity diode laser is frequency or phase locked by feedback to the injection current was measured. The increased AM was consistent with a simple model that relates FM noise to AM through the sensitivity of the laser frequency, and power to injection current. The most prominent increases in the AM noise were from low frequency mechanical vibrations that the FM servo suppressed, and from the servo gain peaking near the bandwidth limit, which is accentuated in this frequency region by a decrease in $\Delta v / \Delta \mathrm{i}$, the dependence of frequency on the injection current.

\section{References}

1. Coherence, Amplification, and Quantum Effects in Semiconductor Lasers, Ed. Y. Yamamoto, Wiley \& Sons (1991), and references therein.

2. J. Kitching, R. Boyd, A. Yariv, and Y. Shevy, "Amplitude noise reduction in semiconductor lasers using weak optical feedback with dispersive loss," Opt. Lett. 19, 1331 (1994).

3. L. Hollberg, R. Fox, N. Mackie, A. S. Zibrov, V. L. Velichansky, R. Ellingsen, and H. G. Robinson, "Diode lasers and spectroscopic applications," in Tenth International Conference on Laser Spectroscopy, Eds. M. Ducloy, E. Giacobino, and G. Gamy, World Scientific, 347 (1992).

4. H. R. Telle and H. Li, "Phase-locking of laser diodes," Elect. Lett. 26, 858 (1990).

5. R. W. Fox, H. G. Robinson, A. S. Zibrov, N. Mackie, J. Marquardt, J. Magyar, L. W. Hollberg, "High sensitivity spectroscopy with diode lasers," SPIE Vol. 1837, 360 (1992).

6. L. D'Evelyn, L. Hollberg, and Z. Popovic, "A CPW phase-locked loop for diode laser stabilization," IEEE MTT-S Int. Microwave Symp. Digest, San Diego, 65 (1994).

7. R. W. P. Drever, J. L. Hall, F. V. Kowalski, J. Hough, G. M. Ford, A. J. Munley, and H. Ward, "Laser phase and frequency stabilization using an optical resonator," Appl. Phys. B 31, 97 (1983).

8. The lasers used were Toshiba TOLD9421 and TOLD9321. This is mentioned only to allow repeatability of experimental results, and does not imply endorsement of these products.

\section{Contribution of NIST, not subject to copyright.}




\section{High-Accuracy Spectroscopy with Semiconductor Lasers: Application to Laser- frequency Stabilization}

\section{LEO HOLLBERG, V. L. VELICHANSKY," C. S. WEIMER, ${ }^{\dagger}$} and R. W. FOX

United States Department of Commerce, National Institute of Standards and Technology, Boulder, Colorado

\subsection{INTRODUCTION}

In this chapter, we focus on the performance of diode lasers used for high-precision spectroscopy, with an eye toward optical frequency and wavelength references in the visible and near-infrared spectral region. A brief summary is given of the existing optical references and the major physical processes that limit the performance of optical

-Currently, Lebedev Institute of Physics, Moscow, Russia.

tCurrently, Ophir Corp., Littleton, Colorado.

Frequency Control of Semiconductor Lasers, Edited by Motoichi Ohtsu. ISBN 0-471-013412 (C) 1996 John Wiley \& Sons, Inc. 
frequency-wavelength standards. Present state-of-the-art high-stability diode laser systems and laser technology are outlined. Particular attention is given to our present results in high-resolution diode-laser spectroscopy of calcium and the prospects for the future.

For many years there has been the promise of future optical frequency standards that could augment or even replace the now traditional microwave frequency standards. In terms of reproducibility, the best proven standard is still the microwave frequency stan. dard based on the cesium ground state hyperfine structure. The present performance of the best cesium standards is a frequency uncertainty of $(\delta v) / v \approx 1 \times 10^{-14}$. None of the proposed optical standards in the visible region bave been tested at a level that is comparable to this. Nevertheless, projected performance is quite impressive, and significant progress has been made in the past few years by using cold trapped ions and neutral atoms.

Prior to the present era of laser cooling and trapping, the highest resolution that had been achieved in the visible was the work of Barger and Bergquist, who in the late 1970 s demonstrated optical Ramsey-fringe widths of approximately $2 \mathrm{kHz}$ on the $657-\mathrm{nm}$ calcium transition $[1,2]$. Now with laser cooling. Bergquist and collaborators have demonstrated an approximately $50-\mathrm{Hz}$-wide optical transition at $563 \mathrm{~nm}$ in a single, trapped mercury ion [3]; Hansch's group has demonstrated approximately $7 \cdot \mathrm{kHz}$-wide lines at $486 \mathrm{~nm}$ on the very important hydrogen 15 to $2 S$ transition [4]; Ertmer's group has detected approximately $2-\mathrm{kHz}$-wide lines on the $457 \mathrm{~nm}$ transition in magnesium in a magnetooptical trap [5]; and Riehle and collaborators have also shown approximately $2-\mathrm{kHz}$-wide resonances on the $657 \mathrm{~nm}$ line in calcium in a magnetooptical trap [6].

In the visible, the most common wavelength-frequency reference is the $\mathrm{I}_{2}$-stabilized helium-neon laser at $633 \mathrm{~nm}$. This system provides very good performance, has been carefully studied, and is well understood at the approximately $3-\mathrm{kHz}$ level of precision. Achieving much higher performance with the $633 \mathrm{~nm}$ helium-neon seems improbable, given the width of the transition $(\sim 5 \mathrm{MHz})$, the celldependent systematic shifts, and the amount of time that has gone into optimizing the system. However, it is an excellent stabilized laser and its widespread use is not only because of its high accuracy, but 
also because of practical considerations: it is compact, relatively low cost, easy to use, and it lases in the visible. The excellent performance is due in a large part to the many years of developmental work that have been invested throughout the world.

Relative newcomers to the field of high-performance optical references are diode lasers stabilized to the rubidium 2-photon transition at $778 \mathrm{~nm}$ [7], and the $\mathrm{I}_{2}$-stabilized frequency-doubled Nd:YAG-laser at $532 \mathrm{~nm}$ [8]. Both of these systems look very promising and are already competitive with existing standards, but neither has been developed, nor tested to the degree that prior systems have, so the ultimate performance is yet to be determined.

In the infrared spectral region there are two other well-established standards for quality frequency references, the $\mathrm{CH}_{4}$-stabilized helium-neon laser at $3.39 \mu \mathrm{m}$, and the $\mathrm{OsO}_{4}$-stabilized $\mathrm{CO}_{2}$ lasers at $10 \mu \mathrm{m}$. In the best cases, frequency stabilities have been reported at the level of a few hertz. In terms of fractional frequency uncertainty, the $\mathrm{CH}_{4}$ system is now known by optical frequency measurements to $(\delta v) / v \approx 3 \times 10^{-12}$, and the $\mathrm{OsO}_{4}$ system is known to $50 \mathrm{~Hz},(\delta v) /$ $v \approx 2 \times 10^{-12}$ [9].

For a new optical reference to be useful, it needs to provide something that cannot be achieved with existing standards. For example, it could feature better stability, higher accuracy, access to different spectral regions, lower cost, portability, or better reliability, or it might be more easily realized in the lab or transferred to other locations. For a combination of these reasons, diode lasers are beginning to play an important role in high-precision spectroscopy. They are obviously lower cost and more compact than other tunable laser sources, but importantly they give us access to different spectral regions. Until recently, the best stabilized lasers have either been dye lasers or gas lasers. Because of their transportability the gas lasers have served the role of wavelength and frequency-transfer standards. But stabilized gas lasers have only limited tunability. so we have had to rely on a few fortuitous overlaps of narrow molecular lines with common gas-laser wavelengths. With some limitations. tunable diode lasers now give us the opportunity to choose the best atomic or molecular transitions and, at the same time, have the option of a transportable instrument. Some of the interesting possibilities that 
are now being pursued with diode lasers are the alkaline earth atoms (calcium, strontium, and barium) and ions $\left(\mathrm{Ca}^{+}\right.$and $\left.\mathrm{Sr}^{+}\right)$.

\subsection{LIMITS TO SPECTROSCOPIC ACCURACY}

In evaluating the merits of various optical references, it is easy to focus on the linewidth and signal-to-noise ratio that can be obtained with a given transition. These are clearly important parameters, but they do not necessarily guarantee high accuracy. More often than not, the accuracy, and even the stability, are limited by systematic effects, rather than the signal size, the fundamental noise processes, or the linewidth. The effects that limit the precision and accuracy in optical frequency references are discussed in many places [9]. An early article by Hall [10] provides a clear insight into the physical processes that limit the performance of high-resolution saturatedabsorption spectroscopy. Precise theoretical treatments of many of the important effects were done by Bordé and collaborators [11-14].

In the highest performance neutral-atom systems, we are almost always limited by velocity-dependent shifts and broadening due to limited observation times. Even with the thermal velocity reduced by laser cooling and trapping, gravitational acceleration limits the practical observation times. Trapped ion systems in contrast have essentially unlimited observation times but are limited in signal size by a relatively small number of ions. With neutrals we can use large numbers of atoms and hence have large signals, but we do not have unperturbed atoms that can be observed for times much longer than approximately $1 \mathrm{~s}$.

Some of the important physical effects that limit the resolution and accuracy in optical spectroscopy are listed in Table 3.1. For any specific system, one effect or another may be the dominant one. But because of optical phase uncertainties and second-order Doppler effects, it is clearly difficult to push beyond $10^{-12}$ fractional frequency uncertainly using fast atoms. Surprisingly, even at low pressures. collision with other atoms actually limit the accuracy that can be achieved in many frequency standards. For example, at the highest levels of accuracy, collision effects are significant even in cesium 
atomic clocks, some microwave trapped-ion systems, and $I_{2}$-stabilized lasers.

\subsection{DIODE LASER TECHNOLOGY}

\subsubsection{Extended Cavity Diode Lasers}

The sensitivity of diode lasers to optical feedback, can be an advantage for stabilizing the laser frequency. When used judiciously, optical feedback can easily narrow the linewidth of a solitary laser from tens of megahertz to tens of kilohertz (extended cavity diode lasers, ECDLs, are a good example of this).

In the case of ECDLs (as with optically locked lasers), the natural wavelength of the solitary laser chip is independent of the extended cavity; it is a function of temperature, injection current, and semiconductor properties. For stable ECDL operation at a specific wavelength we have to suppress the modes of the solitary chip with good antireflectance coatings, or we must rely on stable current and temperature control to keep the natural frequency of the laser chip within the control range of the grating. This control range depends critically on the dispersion, the feedback level, and the reflectance of the output facet. For a typical ECDL with a facet reflectance of a few percent, the control range of the grating might be approximately $10 \mathrm{GHz}$, whereas the solitary mode spacing might be approximately $120 \mathrm{GHz}$. Thus we are faced with the fact that the ECDL laser can still jump modes if the environment is not stable or as the laser ages. If, on the other hand, the facet reflectance is reduced with a broadband coating to a power reflectance of $10^{-4}$, the grating can control the wavelength over the entire gain curve $(\sim 40 \mathrm{~nm}$ for $\mathrm{AlGaAs}$ lasers), and broad single-mode scans are possible.

\subsubsection{Amplitude Noise}

Even though the amplitude-modulated (AM) noise level on diode lasers is very low, it does increase at low Fourier frequencies. Because we are greedy for a low signal-to-noise ratio in our experiments, we 


\begin{tabular}{|c|c|c|c|c|}
\hline Effect & $\begin{array}{c}\text { Dependence, } \\
\text { or Frequency } \\
\text { Uncertainty } \delta \omega / \omega_{0}\end{array}$ & Hot Atoms ${ }^{6}$ & Cold Atoms ${ }^{6}$ & Comments \\
\hline $\begin{array}{l}\text { Uncertainty in } \\
\text { size of signal }\end{array}$ & $(\mathrm{N} \gamma)^{-1 / 2}$ & & & $\begin{array}{l}\text { Amplitude uncertainty improves } \\
\text { with number of atoms } N \text {, and } \\
\text { linewidth } \gamma\end{array}$ \\
\hline Natural linewidth & $\gamma / \omega_{0}$ & $\Delta 10^{-8} 1010^{-14}$ & $\diamond 10^{-8}$ to $10^{-14}$ & $\begin{array}{l}\text { Frequency uncertainty improves } \\
\text { with } \omega_{0} \text { and } 1 / \gamma\end{array}$ \\
\hline $\begin{array}{l}\text { First-order } \\
\text { Doppler }\end{array}$ & $k \cdot v / \omega_{0}$ & $\begin{array}{l}\diamond 10^{-6} \\
* 10^{-12}\end{array}$ & $\begin{array}{l}\Delta 10^{-10} \\
* 10^{-15}\end{array}$ & $\begin{array}{l}\text { Need Doppler-free method! } \\
\text { Uncertainty is method dependent }\end{array}$ \\
\hline $\begin{array}{l}\text { Second-order } \\
\text { Doppler }\end{array}$ & $v^{2} / c^{2}$ & $\begin{array}{l}\diamond 10^{-12} \\
* 10^{-13}\end{array}$ & $\begin{array}{l}\diamond 10^{-17} \\
* 10^{-18}\end{array}$ & $\begin{array}{l}\text { Requires knowledge of velocity } \\
\text { distributions or cold atoms }\end{array}$ \\
\hline $\begin{array}{l}\text { Ramsey method } \\
\text { interaction }\end{array}$ & $\Delta \phi k \cdot v / \omega_{0}$ & $\begin{array}{l}\diamond 10^{-11} \\
* 10^{-12}\end{array}$ & $\begin{array}{l}\diamond 10^{-13} \\
* 10^{-13}\end{array}$ & $\begin{array}{l}\text { Optical geometry dependent and } \\
\text { relative Ramsey-phase } \Delta \phi \\
\text { dependent }\end{array}$ \\
\hline $\begin{array}{l}\text { Wavefront } \\
\text { curvature }\end{array}$ & $v /\left(w_{0} \omega_{0}\right)$ & $\diamond 10^{-11}$ & $\diamond 10^{-15}$ & $\begin{array}{l}\text { Geometry dependent. Need flat } \\
\text { wavefronts and low velocities }\end{array}$ \\
\hline $\begin{array}{l}\text { Power } \\
\text { broadening }\end{array}$ & $\left(\gamma / \omega_{0}\right) \sqrt{ }\left(1+I / I_{0}\right)$ & $\diamond 10^{-8}$ to $10^{-13}$ & $\diamond 10^{-8}$ to $10^{-13}$ & $\begin{array}{l}\text { Need low intensity } I \text { relative to } \\
\text { saturation intensity } I \text {, }\end{array}$ \\
\hline $\begin{array}{l}\text { Light shift, } \\
\text { (a.c.-Stark shift) }\end{array}$ & $\left(\mu_{n} \cdot \mathbb{E}\right)^{2} \sum_{n} \omega_{0} h^{2}\left(\omega-\omega_{n}\right)$ & $\diamond 10^{-9}$ to $10^{-16}$ & $\diamond 10^{-9}$ to $10^{-16}$ & $\begin{array}{l}\text { Significant for atoms with } \\
\text { hyperfine structure and for } \\
\text { two-photon transitions. Need } \\
\text { low power and isolated lines }\end{array}$ \\
\hline Gas lens & $L /\left(k w_{0}^{2}\right)$ & $\Delta 10^{-12}$ to $10^{-14}$ & $\diamond 10^{-12}$ to $10^{-14}$ & $\begin{array}{l}\text { Significant in cells. Depends on } \\
\text { geometry, absorption path length } \\
L \text {, wavelength } 1 / k \text {, and mode } \\
\text { size } w_{0}\end{array}$ \\
\hline $\begin{array}{l}\text { d.c. E\&B field shifts } \\
\text { Stark }\end{array}$ & $\left(\mu_{\infty} \cdot E\right)^{2} / h \omega_{0}$ & $\diamond 10^{-10} 1010^{-17}$ & $\diamond 10^{-10}$ to $10^{-17}$ & $\begin{array}{l}\text { State dependent } \\
\text { Lower lying states and } S \text { states better }\end{array}$ \\
\hline Zeeman & $\mu_{0} \cdot B / h \omega_{0}$ & $\begin{array}{l}\diamond 10^{-4} / \mathrm{T} \\
\diamond 10^{-7} / \mathrm{T}^{2}\end{array}$ & $\begin{array}{l}\diamond 10^{-4} / \mathrm{T} \\
\diamond 10^{-7} / \mathrm{T}^{2}\end{array}$ & $\begin{array}{l}\text { Use } \Delta m=0 \text {, or first-order } \\
\text { field-independent Iransition }\end{array}$ \\
\hline \multicolumn{5}{|l|}{ Collisional } \\
\hline Broadening & $\left(N \sigma_{b} v\right) /\left(V \omega_{0}\right)$ & $\diamond 10^{-10} / \mathrm{Pa}$ & $?$ & \multirow{2}{*}{$\begin{array}{l}\text { State and collision partner } \\
\text { dependent; cross section } \sigma \text {, } \\
\text { magnitude often unknown }\end{array}$} \\
\hline Shift & $\left(N \sigma_{0} v\right) /\left(V \omega_{0}\right)$ & $\diamond 10^{-11} / \mathrm{Pa}$ & $?$ & \\
\hline $\begin{array}{l}\text { Transit } \\
\text { broadening }\end{array}$ & $\left(v /\left(w_{0} \omega_{0}\right)\right.$ & $\diamond 10^{-10}$ & $\diamond 10^{-13}$ & $\begin{array}{l}\text { Requires large beam waits } w_{0} \\
\text { or low velocities } v\end{array}$ \\
\hline $\begin{array}{l}\text { Adjacent } \\
\text { transition } \\
\text { line pulling }\end{array}$ & $\begin{array}{l}\gamma_{n} /\left(\omega_{0}-\omega_{a}\right) \text { or } \\
\gamma_{a}^{2} /\left(\omega_{0}-\omega_{0}\right)^{2}\end{array}$ & $\begin{array}{c}\Delta 10^{-10} 10 \\
<10^{-13}\end{array}$ & $\begin{array}{c}\Delta 10^{-10} 10 \\
<10^{-15}\end{array}$ & $\begin{array}{l}\text { Perturbation by nearby lines } \\
\text { with transition rates } \gamma_{n} \text {. } \\
\text { Need well-isolated lines }\end{array}$ \\
\hline Recoil shift & $h \omega_{0} / m c^{2}$ & $\diamond 10^{-11}$ & $\diamond 10^{-11}$ & \multirow{3}{*}{$\begin{array}{l}\text { Depends on atomic mass } m \\
\text { Horizontal laser beam. Need } \\
\text { large mode } w_{0} \text { or fountain }\end{array}$} \\
\hline Gravity acceleration, & & & & \\
\hline $\begin{array}{l}\text { Broadening } \\
\text { Frequency shift }\end{array}$ & $\begin{array}{l}g^{1 / 2} w_{0}^{-1 / 2} \omega_{0}^{-1} \\
\left(w_{0} / g\right)^{1 / 2} k \cdot g / \omega_{0}\end{array}$ & $\begin{array}{l}\diamond 10^{-13} \\
\diamond 10^{-13}\end{array}$ & $\begin{array}{l}\diamond 10^{-13} \\
\diamond 10^{-13}\end{array}$ & \\
\hline
\end{tabular}

SOURCE: Parts of this table have been adapted from a similar table presented by J. L. Hall [10], and some of the data comes from Sergstock et al. [5] and Kisters et al. [6].

- Listed here are the Beneral dependencies of the various physical effecte, and some typical values found in neutral-atom spectroscopy. Trapped-ion systems have similar effocts, but typical values can be quite different.

The magnitudes of the fractional frequency shifts and broadening coefficients are indicated by $\diamond$, and the uncertainties designated with a . These values are not for a spocific system, but are given for order-of-magnitude comparisons. 
frequently try to reduce the noise even further. One approach is to sample the laser's amplitude noise with a beam splitter and photodetector, then to feedback to the injection current with an electronic servo to reduce the amplitude noise. This has the appearance of simplicity and does work, but the results depend on the degree to which the sampled beam represents the experiment beam. In the limit where the sampled beam is simply some fraction of the experiment beam, the fluctuations of the experiment beam, expressed in decibels above the shot-noise limit, may be reduced to $-10 \log S$. $S$ is the fraction of the power sampled (e.g., with a $50 \%$ beam splitter, a servo can put the experiment beam noise within $3 \mathrm{~dB}$ of the shot-noise limit) [15]. To reliably attain this level of noise reduction, care must be taken to ensure that the sampled beam accurately represents the experiment beam. Many lasers are packaged with an internal photodiode, which is used to detect the beam out of the laser's rear facet. Caution should be exercised in using this signal for the sampling channel, because it is not an accurate representation of the intensity fluctuations on the front facet beam.

\subsubsection{Frequency Stabilization}

Because diode laser cavities are small and facet reflectances are low, the residence time for a photon in the cavity is short ( $\$ 1 \mathrm{~ns}$ ), which results in a substantial amount of frequency noise and hence broad spectral linewidths. Linewidths of solitary diode lasers can range from approximately $10 \mathrm{MHz}$ for the near-infrared $\mathrm{AlGaAs}$ lasers, up to $200 \mathrm{MHz}$ for the visible AlGaInP lasers. Good discussions of the various contributions to diode laser noise are given in Yamamoto et al. [16-19] .Of particular interest is the paper by Telle [19], which gives a clear and concise overview of diode laser frequency noise and stabilization. Also $\mathrm{Zhu}$ and $\mathrm{Hall}$ [20] give a good detailed description of how laser frequency noise contributes to a laser's spectral line shape.

Quite a lot of useful information about the spectral quality of diode lasers can be inferred from the measurements of the frequency-noise spectral density, an example of which is shown in Figure 3.1. The frequency noise on solitary lasers is largest at low frequencies and decreases monotonically toward higher frequencies, 


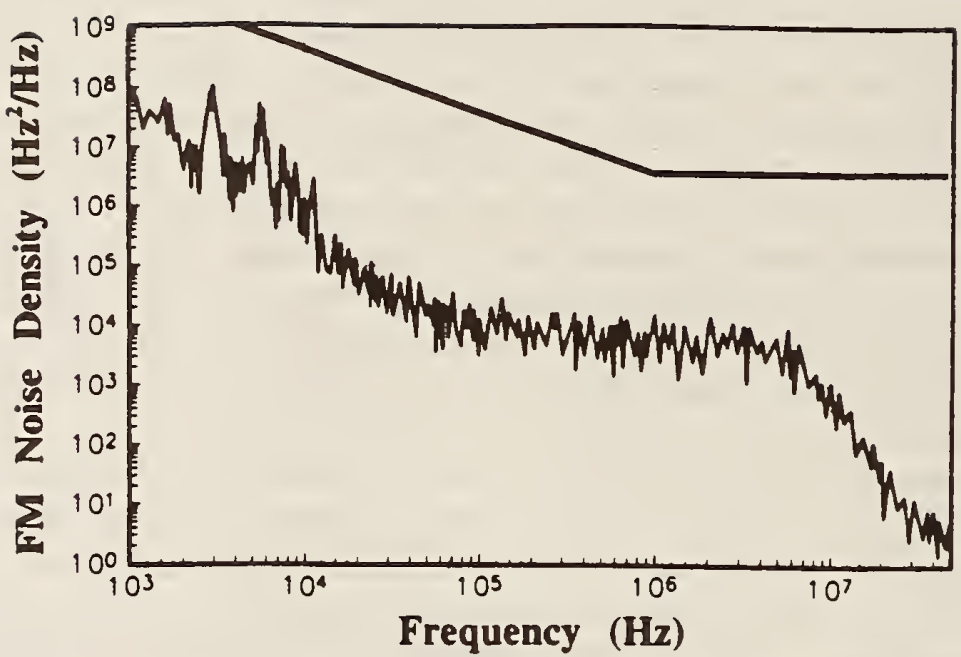

FICURE 3.1 Frequency-noise spectral density of diode lasers. The frequency noise of an ECDL was measured by using the transmission fringe of a Fabry-Perot cavity as a frequency discriminator. The bandwidth of the cavity fringe is responsible for the roll-off in the measured noise near $8 \mathrm{MHz}$ The data was taken with an InGaAlP laser $(657 \mathrm{~nm})$ and was adapted from Fox et al. [21]. For comparison, the approximate noise level of a solitary diode laser is indicated as a solid line.

reaching a plateau that extends from approximately $1 \mathrm{MHz}$ out to many gigahertz. For typical $\mathrm{AlGaAs}$ lasers the level of this plateau in the frequency noise is on the order of $10^{7} \mathrm{~Hz}^{2} / \mathrm{Hz}$. In the case of extended cavity lasers the spectral characteristics depend on the cavity length, optical feedback ratio, and mechanical stability of the cavity $[16-19,21]$. The frequency-noise density is reduced by a large factor (typically in the range of 100 to 1,000 ) from the noise level of a solitary laser. However, we still find that the laser's frequency fluctuations are largest at low frequencies, and then they level off to a plateau at intermediate frequencies (a typical spectral density is approximately $10^{4} \mathrm{~Hz}^{2} / \mathrm{Hz}$ between $50 \mathrm{kHz}$ and $100 \mathrm{MHz}$ ). For extended cavities approximately $10 \mathrm{~cm}$ long, the fast linewidth will be near $50 \mathrm{kHz}$. Low-frequency fluctuations of the cavity length that will modulate the inherent fast linewidth, effectively broadening it. The low-frequency vibrations show up as resonant peaks in the frequency 
noise as can be easily seen in Figure 3.1. As with any oscillator that exhibits increasing noise at lower frequencies, the actual linewidth will depend on the averaging time that is used to measure it, making linewidth statements very ambiguous.

It is very clear from the Figure 3.1 curves that the frequency noise on solitary diode lasers is large in magnitude and broadband. The ECDLs have a much lower level of frequency noise, but it still extends to fairly high Fourier frequencies. As is usually the case, the magnitude of the frequency noise diverges toward low frequencies. Fluctuations in carrier density, localized temperature fluctuations, and mode competition noise are believed to be the main contributions to the frequency noise at low frequencies; whereas spontaneous emission [enhanced by a factor of $\left(1+\alpha^{2}\right)$, with $2<\alpha<12$ ] contributes throughout.

In the literature we often find simple theoretical models (such as the phase diffusion model) of diode laser frequency noise, which are then used to declare that diode lasers have Lorentzian line-shapes. This is not our experience in the laboratory. Actual diode laser line shapes are complicated by fundamental interactions that introduce excess frequency noise (some mentioned above) as well as other less interesting, but important, technical details (like even small amounts of optical feedback) that dramatically alter the spectral character of diode laser light. The lasing frequency of ECDLs is also perturbed by thermal and vibrational changes in the cavity length, which lead to peaks in the frequency noise as can be seen in Figure 3.1. Only in hypothetical cases where the frequency-noise spectral density depends in a simple way on Fourier frequency can the line shape be described by simple analytical functions. In most practical diode laser systems, the frequency noise does not satisfy these conditions and the resulting line-shape is complicated.

As mentioned above, we are usually faced with the situation that even the laser's line width depends on how long an observation time we use to measure it. Nonetheless, it is still useful to have some simple, qualitative, one- or two-parameter measure of the spectral width of a laser. Because the actual linewidth depends on the observation time, one approach is to define a fast linewidth and then lump fluctuations that occur at lower frequencies into frequency jitter 
and drift (which are often conveniently neglected). Although imprecise, one prescription for determining the fast linewidth comes from using the spectrally flat portion of the frequency-noise spectral density (e.g., the region between approximately $50 \mathrm{kHz}$ and $5 \mathrm{MHz}$ in Figure 3.1). It turns out that, if the frequency noise is spectrally flat, then the line shape is really Lorentzian and the linewidth is given by $\Delta v=\pi S_{\mathrm{f}}(f)$. So applying this definition to the ECDL data in Figure 3.1 gives a fast linewidth of approximately $30 \mathrm{kHz}$, and a fast linewidth of approximately $15 \mathrm{MHz}$ for the solitary laser. Alternative methods to measure the line shape are to make a beat note with a narrow linewidth laser, or, in the case of spectrally broad lasers, an optical delay line can be used for a self-heterodyne measurement. These techniques can also be used in the time domain by measuring the amount of time it takes for the optical phase to become ambiguous by $\pi$ radians; this serves as another indicator of laser linewidth.

The usual models of semiconductor laser gain predict that the plateau of frequency noise at high frequencies extends out to the laser's relaxation frequency (typically a few $\mathrm{GHz}$ ) and then drops rapidly toward higher frequencies [16]. Although we have not carefully measured the frequency (phase) noise out to very bigh Fourier frequencies, we have inferred from noise measurements in atomic absorption experiments that the frequency noise density can be almost flat to frequencies as large $9 \mathrm{GHz}$ away from the laser carrier. This admittedly indirect measurement implies that these lasers AlGaAs Fabry-Perot lasers operating at $I / I_{\mathrm{tb}} \approx 3.0$ ) have frequency noise that is almost spectrally flat (for frequencies between 1 and $9 \mathrm{GHz}$ ) with a spectral density that is approximately $-40 \mathrm{~dB}_{\mathrm{c}}$ in a $1 \mathrm{MHz}$ bandwidth. Although it has not been proven, we could speculate that this level is dominated by the background spontaneous emission in the spatial mode of the coherent field of the laser.

To have a spectrally narrow laser that is also precisely tunable relative to a stable reference frequency, we follow the general approach developed some time ago by J. L. Hall and others. With this method, a nontunable Fabry-Perot cavity is used to stabilize the frequency of a reference laser; a second laser is then phase-locked to the reference laser but with a frequency offset. The frequency offset is 


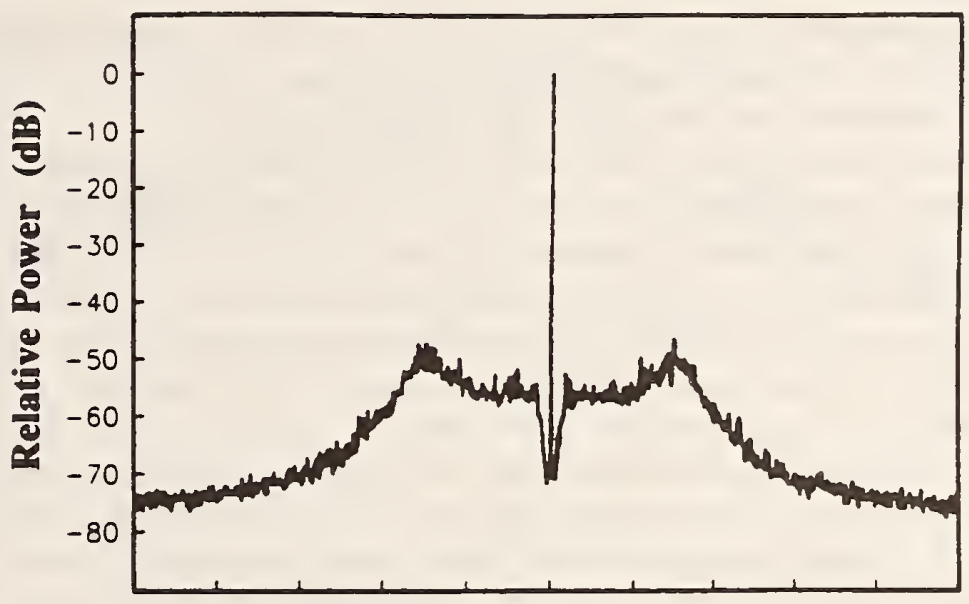

Beatnote Frequency (1 MHz/Div)

FICURE 3.2 Beat-ncte between phase-locked diode lasers operating at $657 \mathrm{~nm}$. Curve shows the $\log$ of the power spectral density of the beat note between the reference laser and the frequency ofiset laser when the system is phase locked. Noise close in to the carrier is down $-60 \mathrm{~dB}_{\mathrm{c}}$ from the peak. The resolution bandwidth is $1 \mathrm{kHz}$ and the horizontal axis has a full span of $10 \mathrm{MHz}$ The frequency offset of the phase-locked laser, relative to the reference laser, is continuously tunable between $10 \mathrm{MHz}$ and $2 \mathrm{GHz}$ with sub-Hertz precision.

provided by a RF-microwave synthesizer, which thus allows precise tunability of the laser frequency. Figure 3.2 shows the beat note between the reference laser and the tunable phase-locked ECDL under locked conditions.

To achieve the highest accuracy in the lock of the reference laser to the Fabry-Perot savity we use the Pound-Drever-Hall optical-heterodyne technique. Frequency-modulated sidebands at approximately $20 \mathrm{MHz}$ are generated with an external, resonant, phase modulator. Two feedback loops are used to frequency stabilize the reference ECDL, a low-frequency path to a piezo-crystal (PZT) that controls the ECDL length, and a high-frequency path to an $A D * P$ phase modulator within the laser cavity. Servo-loop bandwidths of 
approximately $1 \mathrm{MHz}$ result in a fast linewidth of approximately $500 \mathrm{~Hz}$ for the stabilized reference laser. More details on the stabilized reference laser can be found in Fox et al. [21].

In the laser phase locked to the reference laser, there are also two feedback channels, a slow one to a PZT, and a faster feedback channel to the laser's injection current $[22,23]$. In this ECDL, the injection current tunes the frequency of the laser with a coefficient of approximately $40 \mathrm{MHz} / \mathrm{mA}$. Unfortunately, changes in the laser's injection current also change the laser's output power, with a coefficient of approximately $0.09 \mathrm{~mW} / \mathrm{mA}$. It was not clear a priori whether the injection-current loop for frequency stabilization would reduce the amplitude noise or increase it. In studying a few of these ECDL systems, we have found that using the injection current to stabilize the laser's frequency always degrades the laser's amplitude noise. This can be understood from the tuning coefficients and the relative magnitudes of the frequency-and amplitude-modulated noise on ECDLs. The diode laser's amplitude noise is very small, but the frequency noise is significant. Thus, the injection current that removes the frequency noise causes an increase in the amplitude noise. The dependence of the relative phase between the amplitude and the frequency modulation for ECDLs (also a function of Fourier frequency) is an interesting question, but it is almost irrelevant.(because of the difference in magnitude) in the usual regime of frequency stabilization by using the injection current. An example of this effect is the degradation of the amplitude noise of the red diode laser when it is phase locked to the reference laser. Figure 3.3 shows the amplitude noise of the laser with, and without the phase lock. This amplitude-modulation data corresponds to the same lasers and conditions as the beat note curve in Figure 3.2.

In an analogous manner to locking to a Fabry-Perot cavity, an ECDL can also be stabilized to an atomic or molecular resonance such as absorption, fluorescence, saturated absorption. or an optogalvanic signal. The easiest way to lock to the peak of a resonance is to use a modulation lock [24]. In this case, the laser's frequency is modulated by dithering either the PZT that controls the cavity length or the injection current. The optical power transmitted through the resonance is detected, demodulated with a locking amplifier, and fed 


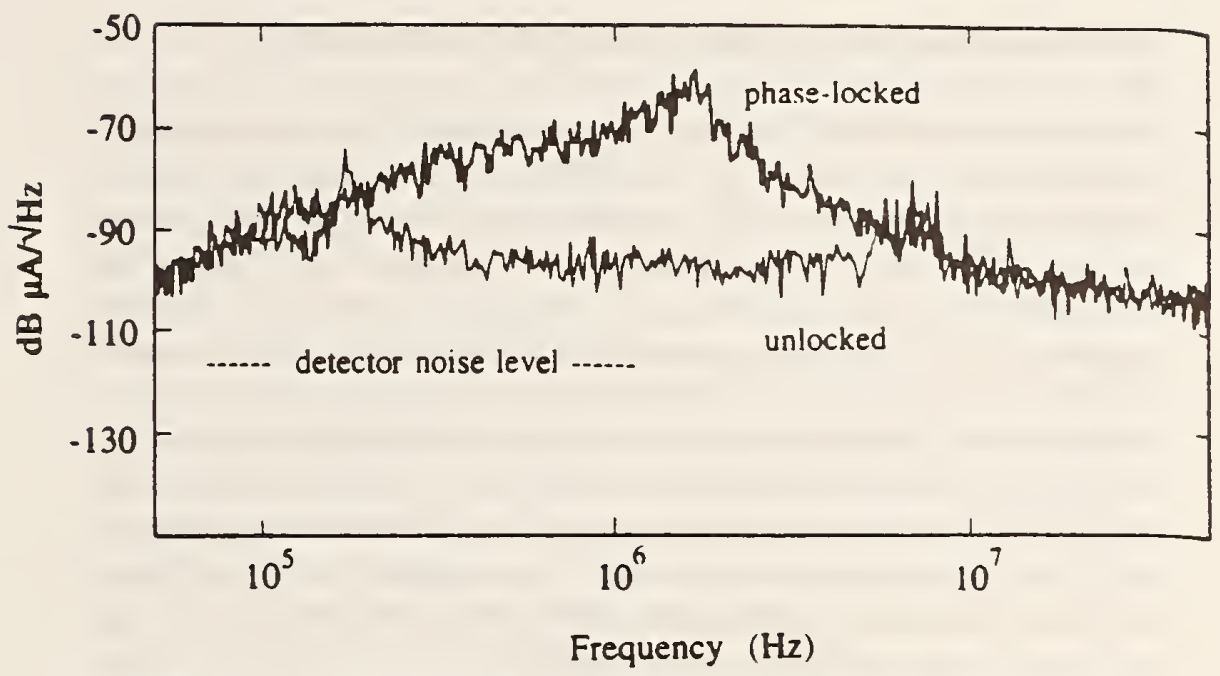

FICURE 3.3 Amplitude-noise of the phase-locked laser for the lock conditions shown in Figure 3.2. The phase-lock servo to the injection current of the ECDL removes the frequency noise, but it simultaneously degrades the laser's amplitude noise. The upper curve shows the amplitude noise on the phase-locked laser when the system is phase locked, and the lower trace shows the intrinsic amplitude noise on the laser when the system is not locked. The equivalent shot-noise for the detected photo current of $360 \mu \mathrm{A}$ is approximately $-100 \mathrm{~dB}_{\mu} \mathrm{A} / \sqrt{\mathrm{Hz}}$ Adapted from Fox et al. [23].

back to the PZT to control the laser frequency. These lockin systems that require modulation of the laser's frequency are often contaminated by unwanted amplitude modulation that comes along with the frequency modulation. In many cases, this is not significant, but the problem has to be addressed for high-accuracy frequency control. For some applications that require a spectrally clean output beam the frequency modulation can itself be a problem. In these cases, it is possible to lock to the side of a resonance without using modulation, or it is sometimes possible to derive an error signal by modulating the atomic resonance rather than the laser's frequency. A good example of this is the use of an a.c. magnetic field to Zeeman modulate atomic resonances. 


\subsection{CALCIUM SPECTROSCOPY WITH DIODE LASERS}

The calcium intercombination line at $657 \mathrm{~nm}$ has been thoroughly studied using dye lasers with atomic beams for use as a wavelengthfrequency reference $[2,6,25]$. The availability of red diode lasers at this wavelength, and also high-power diode lasers at $846 \mathrm{~nm}$ has stimulated renewed interest in calcium as a wavelength-frequency reference [26]. The whole system can be greatly simplified by use of diode lasers; with reduced size and power requirements a compact, transportable, transfer standard seems feasible. The $846 \mathrm{~nm}$ lasers can be frequency-doubled efficiently to produce the $423 \mathrm{~nm}$ light that is required for laser cooling and trapping. Similar research efforts and results in high-resolution spectroscopy of calcium with diode lasers have also been reported from the PTB group in Germany [27], and by Kurosu et al. at NRLM in Japan [28].

We have been working to improve diode laser technology to the performance level required for very-high-precision spectroscopy of calcium. This includes precise tuning, amplitude noise reduction, and frequency stabilization. The laser that probes the $657 \mathrm{~nm}$ line needs to be narrower than the width of the transition, and so requires precision in the frequency control that is approximately $\$ 1 / 10$ tb of the $400 \mathrm{~Hz}$ natural width, i.e., approximately $40 \mathrm{~Hz}$ Our present system does not achieve this required level of performance, but rather has a fast linewidth of $500 \mathrm{~Hz}$, and residual frequency jitter that causes laser frequency excursions of approximately $5 \mathrm{kHz}$ at acoustical frequencies. In addition, the system is presently plagued by frequency drifts in the reference cavity that amount to approximately $1 \mathrm{kHz} / \mathrm{s}$ at the optical frequency. The jitter and the drift are solely due to instabilities in the reference cavity. The laser frequency is locked tightly to the cavity resonance and so follows the fluctuations inherent in the cavity resonance frequency. With a better cavity and using the vibration isolation and stabilization techniques that have already been demonstrated with dye lasers [3], we can expect to reach the required diode laser frequency stability.

At $657 \mathrm{~nm}$, the diode lasers are a little challenging (large initial linewidths and relatively low powers) but most of the difficult problems have now been solved. In terms of ultrahigh-resolution 
spectroscopy, we have not done quite as well with the diode lasers as the previous results with dye lasers, but the diodes are fairly new and improving rapidly. The semiconductor lasers themselves do not seem to present a fundamental limit, and we expect equal performance will be achieved with diodes or dye lasers. The one remaining challenge is that we would like to have more optical power than is readily available from single-frequency tunable ECDLs. This is particularly relevant for time-domain Ramsey spectroscopy of cold-trapped atoms. In this case, the optimum power at $657 \mathrm{~nm}$ will be approximately $50 \mathrm{~mW}$ delivered to the atoms in a repetitive pulse sequence. The power limitation of diode lasers should not be a serious long-term problem, because there are at least two possible solutions: (a) higher power single-mode lasers in the red appear to be on the near term horizon, and (b) we can use injection locking of broadband lasers to increase the usable single-mode power.

We have constructed two very different vacuum systems to do the saturated absorption in calcium. In earlier work [26], we developed a high-flux atomic beam-cell that allowed detection of the saturated absorption signal by optical heterodyne saturation spectroscopy. That system is relatively compact and achieves good spectral resolution with an exceptionally good signal-to-noise ratio. The detected saturated-absorption signal was not large (approximately $1 \%$ of the laser power), but the linewidth was narrow (approximately $60 \mathrm{kHz}$ FWHM) and, using heterodyne detection, gave a background noise that was near shot-noise limited. The linewidth and noise-limited signal-to-noise ratio gave a projected frequency stability of $3 \times 10^{-14} \tau^{-1 / 2}$. With this level of short-term stability, it may even be possible to lock directly to the calcium resonance without the use of any reference cavity.

The second calcium system that we constructed is a more traditional atomic-beam optical Ramsey-fringe spectrometer. Although the present system is not designed to be portable, it is reasonably compact (total length $\sim 1.0 \mathrm{~m}$ ), has good power efficiency (requiring only $20 \mathrm{~W}$ for the beam source), and the vacuum is maintained with a single $201 / \mathrm{s}$ ion pump. With this system, we have observed four-zone saturated-absorption, optical Ramsey-fringes with high sensitivity on the $657 \mathrm{~nm}$ line (Figure 3.4). To capture the narrow Ramsey-fringe structure and at the same time display the whole 


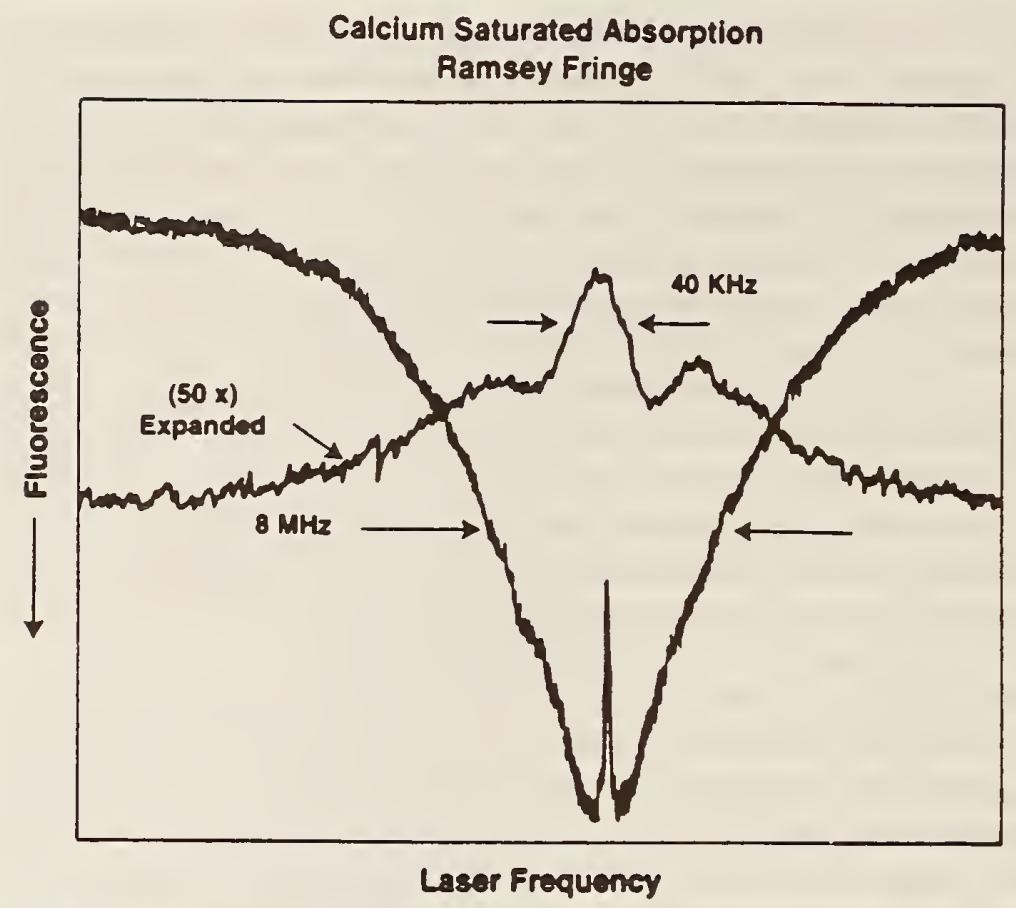

FICURE 3.4 Calcium Ramsey-fringes taken with a diode laser at $657 \mathrm{~nm}$. The lower of the two traces is a broad scan over the entire calcium resonance in the atomic beam. The laser interaction region in the atomic beam was configured in the usual way for four-zone, saturated-absorption Ramsey-fringes. Expanding this same data set by a factor of 50 along the horizontal axis produces the upper trace, where the Ramsey interference fringes now becomes apparent. In this example, the width of the central Ramsey-fringe is $40 \mathrm{kHz}$.

Doppler-broadened lineshape this spectra was taken with a scan time of $10 \mathrm{~s}$ but used a detection bandwidth of $10 \mathrm{kHz}$ Lower detection bandwidths give very clean Ramsey-fringe lineshapes.

Higher spectral resolution is achieved in the traditional atomicbeam system than is obtained in the beam-cell system because of the longer interaction time provided by the Ramsey interrogation method. However, there is some penalty in the signal size. The narrowest lines that we have observed up to now are $10 \mathrm{kHz}$ FWHM, 
which is sufficient to resolve the photon-induced atomic recoil splitting of $23 \mathrm{kHz}$. Unfortunately, as is well known, the Ramsey line shape becomes quite complicated in the higher resolution limit where the effects of atomic recoil, second-order Doppler, and Ramsey-fringe resolution are all interconnected [29]. A very important simplification of the spectrum (usually at the expense of a more complex apparatus) can be achieved by eliminating one of the atomic recoil components as has been described in previous work $[5,11,25,30]$. However, the limitation in the accuracy of this system will be the same as that previously realized in other fast atomic-beam systems. The ultimate accuracy will likely be limited by imprecise knowledge of the atomic velocity distribution and hence the second-order Doppler shift and also by the atomic velocity-dependent opticalphase sensitivity of the Ramsey interferometer. Projected accuracy of these systems is approximately $500 \mathrm{~Hz},(\delta v) / v \sim 1 \times 10^{-12}$.

Real improvements in the frequency accuracy require low-velocity atoms. Fortunately laser cooling and trapping of calcium has already been demonstrated with dye lasers, and, recently, high-resolution spectra of cold neutral atoms have been achieved $[5,6]$. It is also now feasible to do the trapping and cooling of calcium with frequencydoubled diode lasers. We have built a doubling system that uses $\mathrm{KNbO}_{3}$ in a resonant ring-cavity. Starting with an 846-nm grating-tuned extended-cavity laser, we injection lock a $150 \mathrm{~mW}$, single-, spatial-mode, diode laser. The resulting high-power beam is collimated and sent through an anamorphic prism pair and optical isolator before it is mode-matched into the ring build-up cavity. The resonance of the ring is electronically locked to the laser frequency. With $110 \mathrm{~mW}$ of $846-\mathrm{nm}$ light incident on the ring we have been able to generate as much as $45 \mathrm{~mW}$ of useful output at $423 \mathrm{~nm}$. This should be sufficient for laser cooling and trapping of calcium. Thus far, we have demonstrated one-dimensional transverse-cooling of the calcium beam, which enhances the Ramsey-fringe signal by a factor of two to three.

It is also interesting that some other transitions in calcium are accessible with diode laser sources. Some of these may play an important role in improving the performance of this standard in the future. For instance, we have used a $672-\mathrm{nm}$ diode in conjunction with the $423-\mathrm{nm}$ light to probe the calcium ${ }^{1} \mathrm{D}(4 \mathrm{~s} 3 \mathrm{~d})$ to ${ }^{1} \mathrm{P}(4 \mathrm{~s} 5 \mathrm{p})$ 
transition. This gives us additional information about (and may enhance) the 423-nm cooling process and may also be useful to suppress the large background $(657 \mathrm{~nm})$ emission that is present in the cold trapped-atom systems. Other transitions in calcium are also compatible with diode lasers, including the $612-\mathrm{nm}$ transition that can be used to suppress a recoil component. Similarly, 423- or 444 nm light, from double diodes, could be used to improve the trapping or to suppress a recoil component.

\section{ACKNOWLEDGMENTS}

Our thanks to A. Zibrov, L. D'Evelyn, H. G. Robinson, J. Aman and J. Bergquist for important contributions to the results presented here.

\section{REFERENCES}

1. R. L. Barger, J. C. Bergquist, and D. J. Glaze, High-resolution Saturated Absorption of the ${ }^{1} \mathrm{~S}_{0}-{ }^{3} \mathrm{P}_{1}$ Calcium Transition in an Atomic Beam. $J$. Opt. Soc. Am. 68(11), 1634 (1978).

2. J. C. Bergquist, R. L. Barger, and D. J. Glaze, High resolution spectroscopy of calcium atoms. In Laser Spectroscopy IV $(\mathrm{H}$. Walther and $\mathrm{K}$. W. Rothe, eds. p. 120. Springer-Verlag, Berlin, 1979.

3. J. C. Bergquist, W. M. Itano, and D. J. Wineland, Laser stabilization to a siagle ion, in Frontiers in Laser Spectroscopy (T. W. Hänscb and M. Inguscio, eds.), p. 359 North-Holland Publ., Amsterdam, 1994.

4. T. W. Hänsch, Precision spectroscopy of atomic bydrogen. At. Phys. 14, 63 (1993).

5. K. Sengstock, U. Sterr, J. H. Muller, V. Rieger, D. Bettermann, and W. Ertmer, Optical Ramsey spectroscopy on laser-trapped and thermal $\mathrm{Mg}$ atoms. Appl. Phys. B59, 99-115 (1994).

6. T. Kisters, K. Zeiske, F. Riehle, and J. Helmcke, High-resolution spectroscopy with laser-cooled and trapped calcium atoms. Appl. Phys. B59, 89-98 (1994).

7. F. Nez, F. Biraben, R. Felder, and Y. Millerioux, Optical frequency determination of the hyperfine components of the $5 S_{1 / 2}-5 D_{3 / 2}$ two photon transitions in rubidium. Opt. Commun. 102, 432-438 (1993). 
8. A. Arie and R. L. Byer, Laser heterodyne spectroscopy of ${ }^{127} I_{2}$ hyperfine structure near $532 \mathrm{~nm}$. J. Opt. Soc. Am., B. 10, 1990-1997 (1993).

9. A. Clairon, O. Acef, C. Chardonnet, and C. J. Bordé in Frequency Standards and Metrology (A. De Marchi, ed.), Springer-Verlag, Berlin, 212-221, 1989; G. Kramer, C. O. Weiss, and B. Lipphardt, ibid, 181-186; M. A. Gubin, A. V. Nikulchin, and D. A. Tyunkov, ibid, 206-211; B. G. Whitford, ibid, 187-190.

10. J. L. Hall, Sub Doppler spectroscopy, methane hyperfine spectroscopy. and the ultimate resolution limits. Colloq. Int. C.N.R.S. 217, 105-125 (1974).

11. C. J. Bordé, New sub-Doppler interaction techniques, in Laser Spectroscopy III (J. L. Hall and J. L. Carlsten, eds.), p. 121-134. Springer-Verlag, Berlin, 1977.

12. C. J. Bordé, J. L. Hall, C. V. Kunasz and D. G. Hummer, Saturated absorption line shape; Calculation of the transit time broadening by a perturbation approach. Phys. Rev. A 14, 236-263 (1976).

13. C. J. Bordé, C. Salomon, S. Avrillier, A. Van Lerberghe, C. Bréant, D. Bassi, and G. Scoles, Optical Ramsey fringes with traveling waves. Phys. Rev. $A$ 30, 1836-1848 (1984).

14. C. J. Bordé, The physics of optical frequency standards using saturation methods. In Frequency Standards and Metrology (A. De Marchi ed.). pp. 196-205, Springer-Verlag, Berlin, 1989.

15. L. Hollberg, R. W. Fox, N. Mackie, A. S. Zibrov, V. L. Velichansky, R. Ellingsen, and H. G. Robinson, Diode lasers and spectroscopic applications. Int. Conf. Laser Spectrosc., 10th, p. 347 (1992).

16. Y. Yamamoto, ed., Coherence, Amplification and Quantum Effects in Semiconductor Lasers. Wiley, New York, 1991.

17. K. Peterman, Laser Diode Modulation and Noise. Kluwer, Dordrecht, The Netherlands, 1988.

18. M. Ohtsu, Highly Coherent Semiconductor Lasers. Artech House, Boston, 1992.

19. H. R. Telle, Stabilization and modulation schemes of laser diodes for applied spectroscopy. Spectrochim. Acta Rev. 15, 301-327 (1993).

20. M. Zhu and J. L. Hall, Stabilization of optical phase/frequency of a laser system: Application to a commercial dye laser with an external stabilizer. J. Opt. Soc. Am., B 10, 802 (1993).

21. R. W. Fox, H. G. Robinson, A. S. Zibrov, N. Mackie, J. Marquardt, J. Magyar, and L. Hollberg, High-sensitivity spectroscopy with diode lasers. Proc. - Int. Soc. Opt. Eng. SPIE 1837, 360 (1992). 
22. L. D'Evelyn, L. Hollberg, and Z. Popovic, A cpw phase-lock loop for diode-laser stabilization. IEEE MTT-S Int. Microwave Symp. Dig. San Diego, p. 65 (1994).

23. R. W. Fox, L. D'Evelyn, H. G. Robinson, C. S. Weimer, and L. Hollberg, Amplitude modulation on frequency-locked extended cavity diode lasers. Proc. SPIE - Int. Soc. Opt. Eng. 2378, 58-62 (1995).

24. L. Hollberg, in Dye Laser Principles: with Applications (F. J. Duarte and L. W. Hillman, eds. pp. 185-238. Academic Press, Boston, 1990.

25. T. Kurosu and A. Morinaga, Suppression of the high-frequency recoil component in optical Ramsey-fringe spectroscopy. Phys. Rev. A 45, 4799 (1992).

26. A. S. Zibrov, R. W. Fox, R. Ellingsen, C. S. Weimer, V. L. Velichansky, G. M. Tino, and L. Hollberg, High-resolution diode-laser spectroscopy of calcium. Appl. Phys. B59, 327-331 (1994).

27. A. Celikov, F. Riehle, V. L. Velichansky, and J. Helmcke, Diode laser spectroscopy in a Ca atomic beam. Opt. Commun. 107, 54-60 (1994).

28. T. Kurosu, R. W. Fox, J. Ishikawa, and N. Ito, High-resolution diode laser spectrometer for $\mathrm{Ca}^{1} \mathrm{~S}_{0}-{ }^{3} \mathrm{P}_{1}$ transition. Proc. SPIE - Int. Soc. Opt. Eng. 2378, 236-244 (1995).

29. R. Barger, Influence of second-order Doppler effect on optical Ramsey fringe profiles. Opt. Lett. 6, 145 (1981).

30. F. Riehle, J. Ishikawa, and J. Helmcke, Suppression of a recoil component in nonlinear Doppler-free spectroscopy. Phys. Rev. Lett. 61, 2092 (1988). 
Grating-tuned semiconductor MOPA lasers for precision spectroscopy*

\author{
J. H. Marquardt, F. C. Cruz, M. Stephens, C. W. Oates, L. W. Hollberg, J. C. Bergquist \\ National Institute of Standards and Technology \\ Boulder, CO 80303
}

\author{
D. F. Welch, D. Mehuys, and S. Sanders \\ SDL, Inc. \\ 80 Rose Orchard Way, San Jose, CA 95134
}

\begin{abstract}
A standard grating-tuned extended-cavity diode laser is used for injection seeding of a tapered semiconductor laser/amplifier. With sufficient injection power the output of the amplifier takes on the spectral characteristics of the master laser. We have constructed master-oscillator power-amplifier (MOPA) systems that operate near $657 \mathrm{~nm}, 675 \mathrm{~nm}, 795 \mathrm{~nm}$, and $850 \mathrm{~nm}$. Although the characteristics vary from system to system, we have demonstrated output powers of greater than $700 \mathrm{~mW}$ in a single spatial mode, linewidths less than $1 \mathrm{kHz}$, coarse tuning greater than $20 \mathrm{~nm}$, and continuous single-frequency scanning greater than $150 \mathrm{GHz}$. We discuss the spectroscopic applications of these high power, highly coherent, tunable diode lasers as applied to $\mathrm{Ca}, \mathrm{Hg}^{+}, \mathrm{I}_{2}$, and two-photon transitions in Cs.
\end{abstract}

Keywords: diode lasers, tapered amplifiers, injection locking, high resolution spectroscopy, MOPA

${ }^{*}$ Contribution of NIST and not subject to copyright

\title{
1. INTRODUCTION
}

Many scientific and technical applications of tunable diode lasers require higher powers than have been available from semiconductor laser sources. Recent advances in tapered gain structures have opened a new frontier in single-mode, singlefrequency, tunable, serniconductor laser capabilities. ${ }^{1}$ Tapered amplifiers (TA) can have broad gain-bandwidths, providing tremendous potential for tunable lasers with high power. They provide roughly one order of magnitude more power than previous single-spatial-mode devices. However, these tapered amplifiers/oscillators usually operate with many simultaneous longitudinal modes, which limits their usefulness for coherent and high-resolution applications. Using tapered amplifiers in extended cavities for wavelength control (such as optical feedback from diffraction gratings) allows good coarse tuning at high power, ${ }^{2}$ but it is difficult to maintain true single-frequency operation. Extended-cavity TAs often exhibit instabilities which can cause jumps among a few nearby longitudinal modes or result in lasing on a few modes simultaneously.

One powerful solution to these problems is to separate the function of the high-power generation from that of the highprecision single-frequency tuning. A few modern examples of this type of system can be found in the literature and include: distributed Bragg reflector (DBR) laser diode plus tapered amplifier, ${ }^{3}$ solitary diode laser plus tapered amplifier, ${ }^{4}$ and grating-tuned extended-cavity diode laser (ECDL) plus tapered amplifier. In this paper we describe the applications of extended-cavity diode-laser plus master-oscillator power-amplifier (ECDL-MOPA) systems that are currently under way at NIST. The characteristics of these systems are discussed from a user's perspective, where our applications are primarily in high resolution spectroscopy. Other related systems and applications have been described by Zimmermann et al. ${ }^{5}$ and Goldberg and Burns. ${ }^{6}$

\section{EXPERIMENT}

By using several different experimental setups we gain some insight into the general characteristics of injection-locked MOPAs. A typical experimental setup is shown in Figure 1 ; it consists of a standard grating-tuned ECDL in the Littman- 
Metcalf (grazing incidence) configuration, which acts as the master laser to inject the TA slave. The light from the master laser is sent through an optical isolator and spatially mode-matched to the beam emitted from the back facet of the TA. Since the front output aperture of the TA is very asymmetric $(\sim 1 \mu \mathrm{m}$ by $100 \mu \mathrm{m})$, a cylindrical lens, in addition to the standard spherical lens is needed to collimate the output beam. (In addition the output beam can have large astigmatism which may be compensated.)

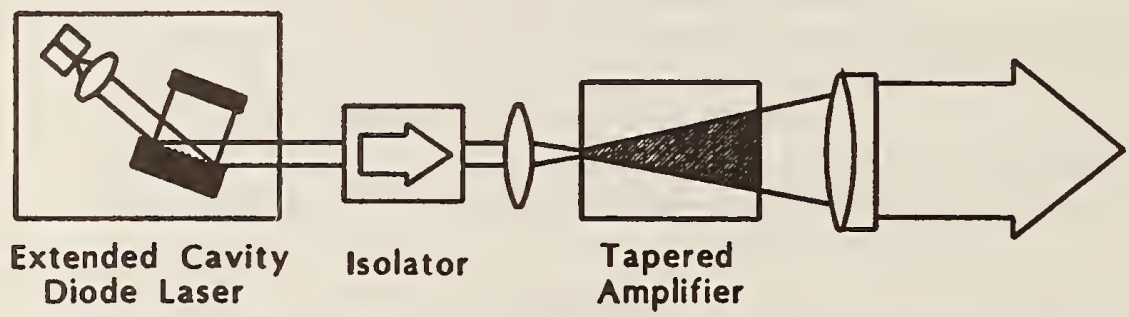

Figure 1. General ECDL-MOPA set-up. The ECDL output is sent through an isolator and then mode-matched to the beam emitted from the rear facet of the tapered amplifier. The collimating optics consist of one spherical and one cylindrical lens.
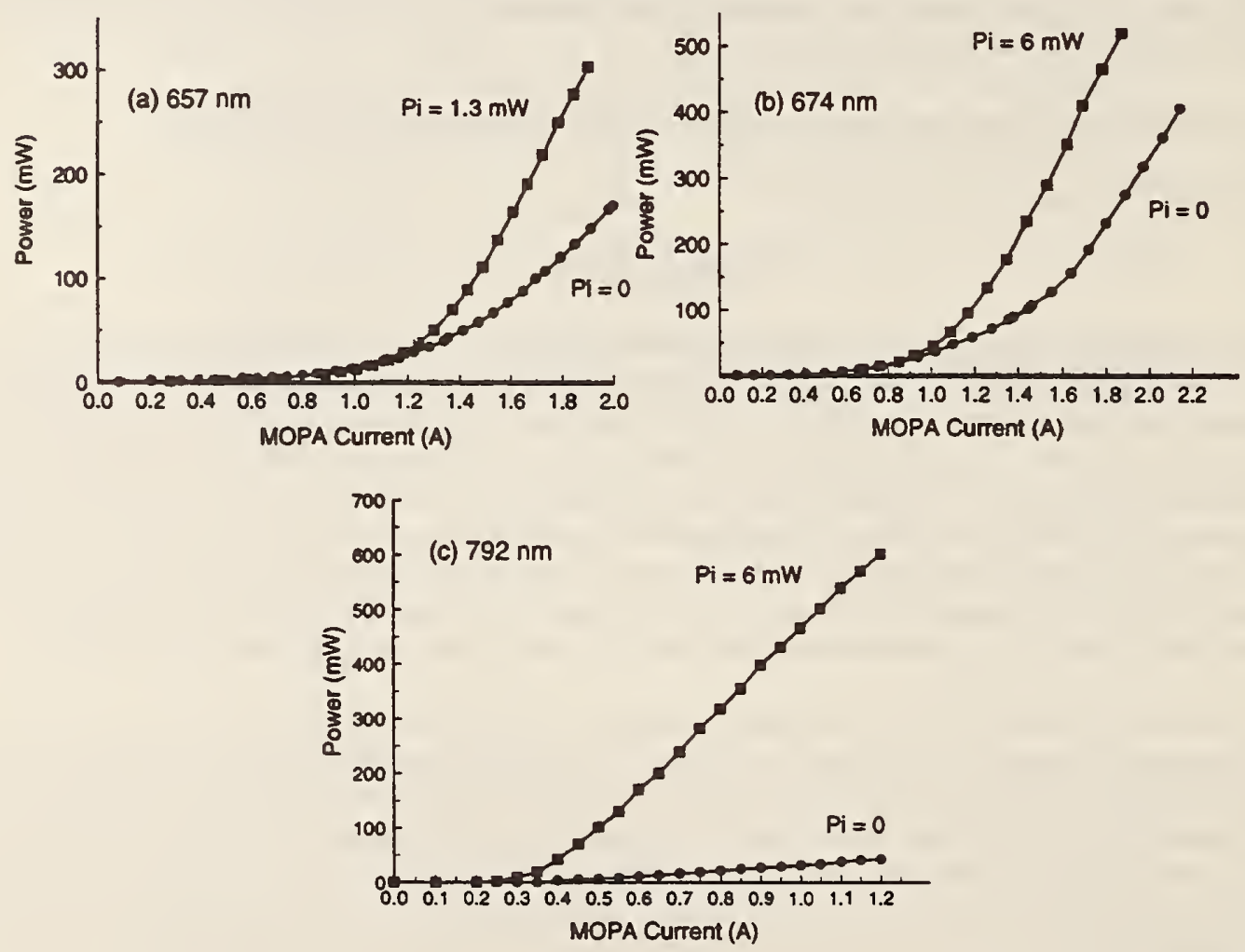

Figure 2. This figure shows the power versus injection current for three of our ECDL-MOPA systems (a) $657 \mathrm{~nm}$, (b) $674 \mathrm{~nm}$, and (c) $792 \mathrm{~nm}$. Each graph is shown with and without optical injection power. Graph (a) and (b) show clearly a lasing threshold of the TA at high currents even without optical injection. The thresholds of all of the systems are lowered, and the slope efficiencies increase with increasing injection power. The optical injection power $P_{i}$ used in these systems is (a) $1.3 \mathrm{~mW}$, (b) $6 \mathrm{~mW}$, and (c) $6 \mathrm{~mW}$. 
With optical injection from the master laser into the TA, the threshold of the TA decreases, resulting in more emitted power and the slope efficiency increases to as much as 0.7 W/A for the $674 \mathrm{~nm}$ and $792 \mathrm{~nm}$ systems (Figure 2). The MOPA output power quickly saturates with injection power from the master laser. Figure 3 shows the output power of the MOPA as a function of injected optical power. With good mode matching of the master into the TA, the output power saturates with about 2-3 mW of injected power. The different ECDL-MOPA systems vary slightly in this characteristic but they all saturate with less than $-5 \mathrm{~mW}$ of injection power due to the high gain and strongly asymmetric nature of the TA.

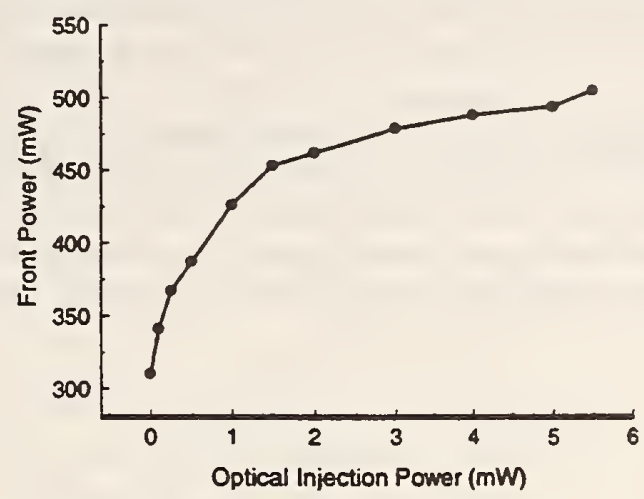

Figure 3. Dependence of output power with optical injection power for the $674 \mathrm{~nm}$ system. The output power saturates quickly with as little as $2-3 \mathrm{~mW}$ (solid circles).

The isolation required between the master and the slave depends somewhat on system parameters such as master laser power, mode matching, and the power emitted out of the back of the amplifier. We often use another isolator on the output of the amplifier to eliminate feedback into the high power amplifier. Although good quality isolators are needed between the master and the slave for relatively low injection powers $(<5 \mathrm{~mW})$, this requirement may be relaxed with higher injection powers. With optical injection from the master laser we have observed a decrease in temperature of the TA due to the large increase in optical power and high efficiency of the TA.

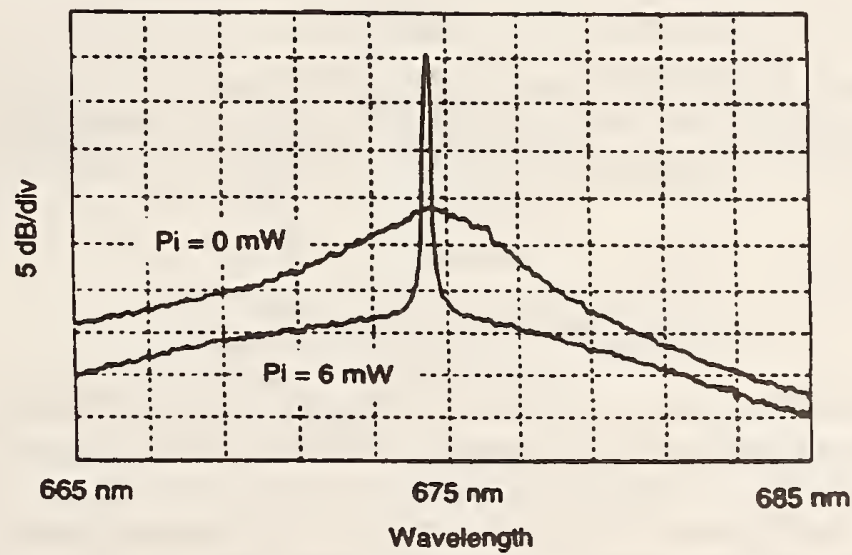

Figure 4. Optical spectrum analyzer trace showing the output of the ECDL-MOPA with and without $6 \mathrm{~mW}$ of injection power. The vertical scale is $5 \mathrm{~dB} /$ div and the horizontal scale is 2 $\mathrm{nm} /$ div. The instrument resolution is $0.1 \mathrm{~nm}$.

The MOPA output power can be quite high (up to $700 \mathrm{~mW}$ ), in a single spatial mode, while retaining the spectral properties of the master laser. Figure 4 shows an optical spectrum of the TA at $674 \mathrm{~nm}$ with and without injection. With injection the broad spectrum collapses into the single strong mode of the master laser. The single-frequency output takes power away from nearby regions and rises $25-30 \mathrm{~dB}$ above the background pedestal as measured with a $0.1 \mathrm{~nm}$ resolution bandwidth. As the master laser is tuned, the single-mode, coherent output of the MOPA follows exactly. 
The residual facet reflectance of the TA can significantly affect the tuning characteristics of the MOPA and varies somewhat with each system. When injection current in the TA is brought above its own lasing threshold, the residual chip modes of the amplifier become important. Since these chip modes tune with slave current and temperature, it is straightforward to reach the desired wavelength and be centered on a "good" MOPA chip mode as well. When the TA shows no threshold behavior (as for the $792 \mathrm{~nm}$ system), these chip modes can be suppressed by the optical injection. For those TAs that can operate well above their threshold, the modulation is always present. These characteristics represent two different operating regimes for the MOPA: below the threshold of the TA the system acts like an optical amplifier, while above the threshold of the TA the system behaves like an injection locked laser. Using the $674 \mathrm{~nm}$ system near its own lasing threshold $(-1.2 \mathrm{~A})$ and with $\sim 6$ $\mathrm{mW}$ of injection power, we were able to scan continuously over $150 \mathrm{GHz}$ with an output power of $-100 \mathrm{~mW} .^{7}$ As a demonstration of this capability, Figure 5 shows absorption of $\mathrm{I}_{2}$ near $674 \mathrm{~nm}$. Although there is some modulation in the power, the sweep remains single mode for greater than $150 \mathrm{GHz}$. Even when the chip modes are strong, the system can be scanned $-20-30 \mathrm{GHz}$ without mode jumps. This performance is comparable to that of many single-frequency tunable dye lasers. For most high resolution spectroscopy, this scan range is more than adequate, but some applications, such as molecular spectroscopy, may need longer single mode scanning ranges. In addition, active control of the injection current of the slave can be used to suppress the power modulation due to the residual facet reflectance.

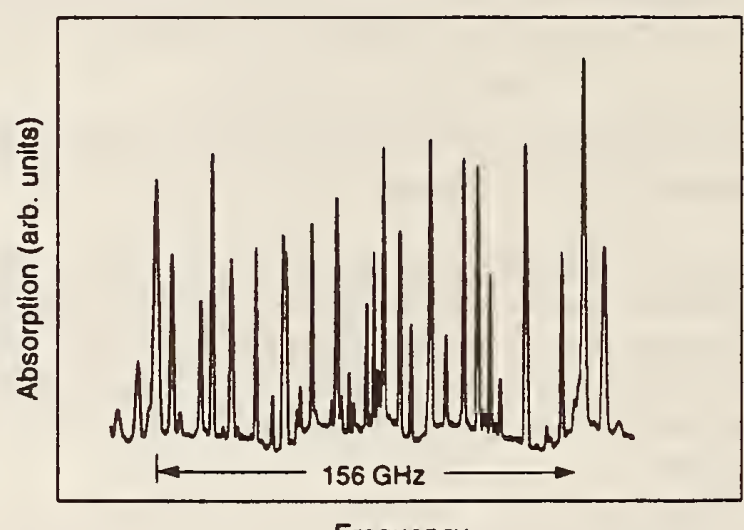

Frequency

Figure 5. Absorption spectrum of $\mathrm{I}_{2}$ near $674 \mathrm{~nm}$ taken with an ECDL-MOPA system (adapted from ref (7)). A specially designed long-scanning ECDL was used as the master oscillator to obtain this spectrum. The peak absorption was about $30 \%$ for a $5 \mathrm{~cm}$ long cell with a temperature of about $80^{\circ} \mathrm{C}$.

\section{APPLICATIONS}

Applications of these ECDL-MOPAs provide "real-world" tests of the practicality and usefulness of these systems. The following examples from our laboratory are used to illustrate the performance characteristics of these systems.

The first system at $792 \mathrm{~nm}$ is used to generate $194 \mathrm{~nm}$ light for use in a $\mathrm{Hg}^{+}$frequency standard. In this experiment $\mathrm{Hg}^{+}$ions are held in electromagnetic traps and laser-cooled to temperatures on the order of $1 \mathrm{mK}$. Single-frequency radiation at 194 $\mathrm{nm}$, required for laser-cooling the ions, is generated by sum-frequency mixing of radiation at 257 and $792 \mathrm{~nm}$ in an external build-up cavity. Radiation at $257 \mathrm{~nm}$ is obtained by frequency-doubling $515 \mathrm{~nm}$ radiation from an $\mathrm{Ar}^{+}$laser. Singlefrequency radiation at $792 \mathrm{~nm}$ is generated from the ECDL-MOPA system. More than $700 \mathrm{~mW}$ of light at $792 \mathrm{~nm}$ is generated with optical injection between $3 \mathrm{~mW}$ and $20 \mathrm{~mW}$ from the master laser. Greater than $90 \%$ of the slave laser power can be mode-matched into the sum-frequency cavity, and negligible variation of the mode quality is observed as the slave laser's output power or frequency is varied. The ability to couple such a high percentage of light into the external ring cavity illustrates the excellent spatial mode these systems can provide. Two isolators (net isolation of about $60 \mathrm{~dB}$ ) were used between the master and the slave laser, but no isolator was used on the output of the ECDL-MOPA. This high level of isolation was required to prevent instabilities of the ECDL-MOPA caused by a small amount of optical feedback from the 
build-up cavity.

Using the $792 \mathrm{~nm}$ system an experiment to check for the relative coherence between the injection beam and the TA output was performed. We split off part of the master laser beam, frequency-shifted it in an acousto-optic modulator (AOM), and then heterodyned this beam with the output of the TA (Figure 6). The beat signal at the modulation frequency was then heterodyned with the signal driving the AOM, and the resulting DC signal (proportional to the relative optical phase between the master and the slave) was observed on an oscilloscope. In this way phase variations between the master and slave lasers were observed directly. Phase locking between the master and slave laser was observed. The phase difference never exceeded $\pi$ rad for times of the order of minutes. Similar results have also been reported by Kikuchi et al. ${ }^{8}$

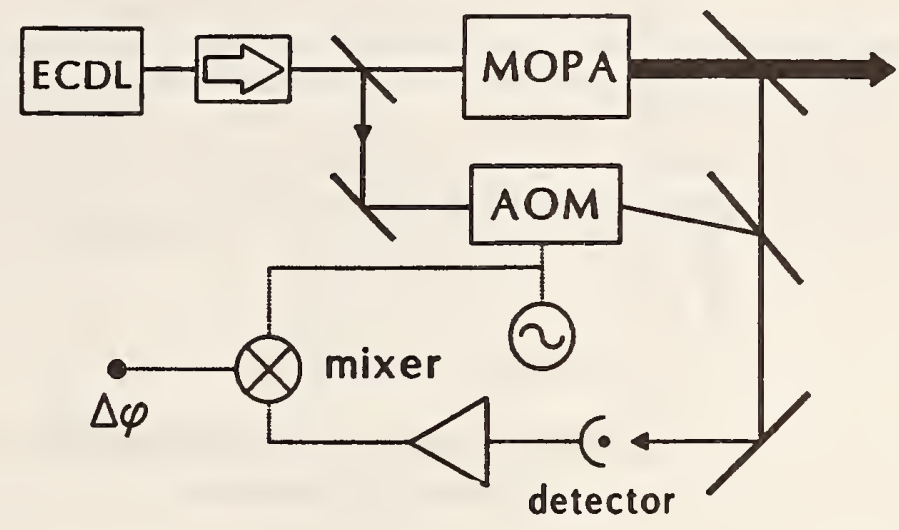

Figure 6. Schematic diagram of the method used to measure the relative phase coherence between the master laser and the slave.

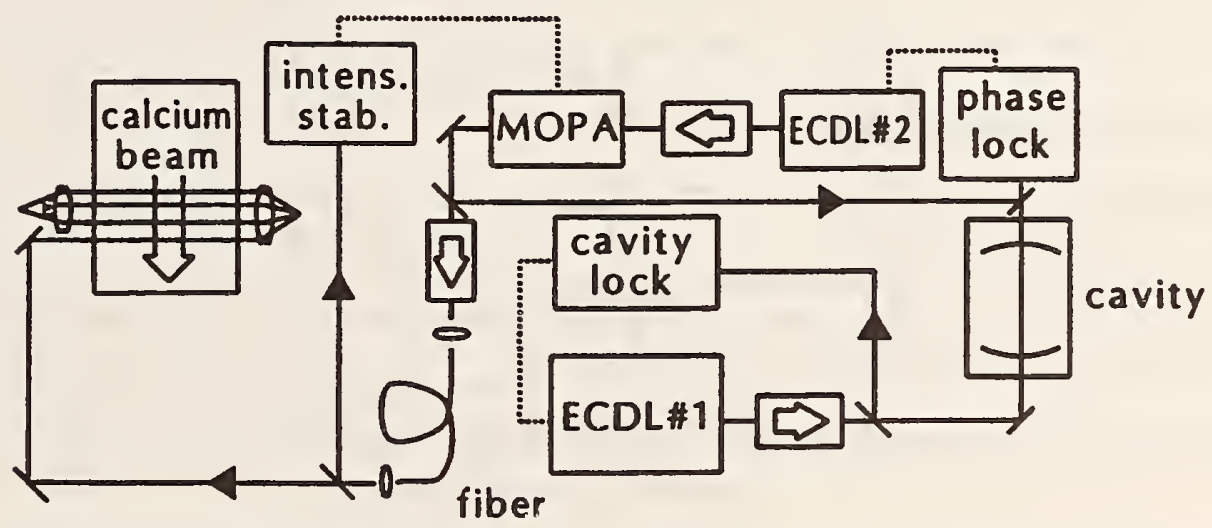

Figure 7. Calcium spectroscopy set-up. The solid lines represent the laser light and the dotted lines represent the servo loops.

A good demonstration of the narrow linewidths and high optical resolution that can be achieved with these ECDL-MOPAs is provided by the $657 \mathrm{~nm}$ system we use for Ca spectroscopy. The system is shown in Figure 7. ECDL1 is locked to an ultra-stable high finesse Fabry-Perot cavity (fringe width $\sim 50 \mathrm{kHz}$ FWHM) using the Pound-Drever-Hall technique. The beam transmitted through the cavity is used to phase-lock the $657 \mathrm{~nm}$ ECDL2-MOPA. This ECDL-MOPA output is then spatially filtered using an optical fiber which delivers the light to the $\mathrm{Ca}$ atomic beam system. In addition, the output of the fiber is intensity-stabilized by active feedback control to the MOPA current. This is done to compensate for the intensity fluctuations due to polarization changes and thermal drifts of the fiber system. The very high resolution achievable with this system is evident in Figure 8. This figure shows the optical Ramsey spectra of the $657 \mathrm{~nm}$ calcium line. The FWHM of 
approximately $11.5 \mathrm{kHz}$, with a signal-to-noise ratio of about $30 \mathrm{in}$ a $1 \mathrm{~Hz}$ bandwidth, corresponds to what would be a noiselimited frequency stability $\delta v / v$ of about $8 \times 10^{-13} \tau^{-1 / 2}$ (where $\tau$ is the averaging time). The resolution is limited here by the physical size of the windows on the atomic beam apparatus which limits the distance between interaction regions. Using the $\mathrm{Ca}$ lineshape and the Fabry-Perot cavity as frequency discriminators we measure the residual FM noise of the ECDL-MOPA to be $s 1 \mathrm{kHz}$ dominated by the acoustical mode coupling between the laser table and the reference Fabry-Perot cavity (at about 1-2 Hz).

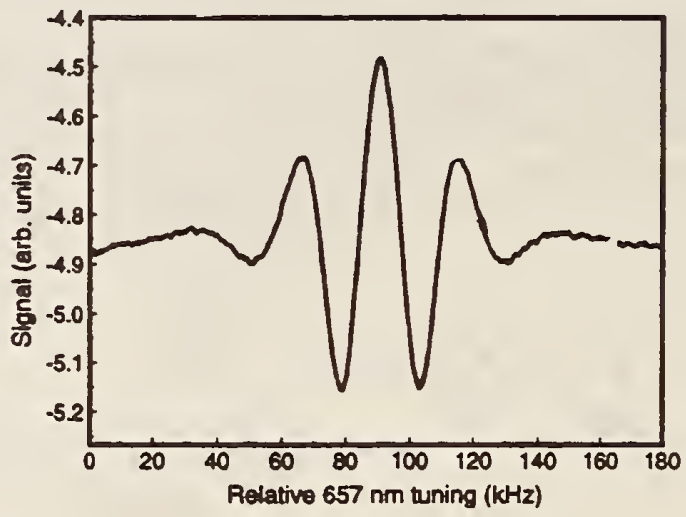

Figure 8. Ramsey fringe of calcium using the ECDL-MOPA setup shown in Figure 7. The FWHM of the fringe is $\sim 11.5 \mathrm{kHz}$, at an optical frequency of $456 \mathrm{THz}$.

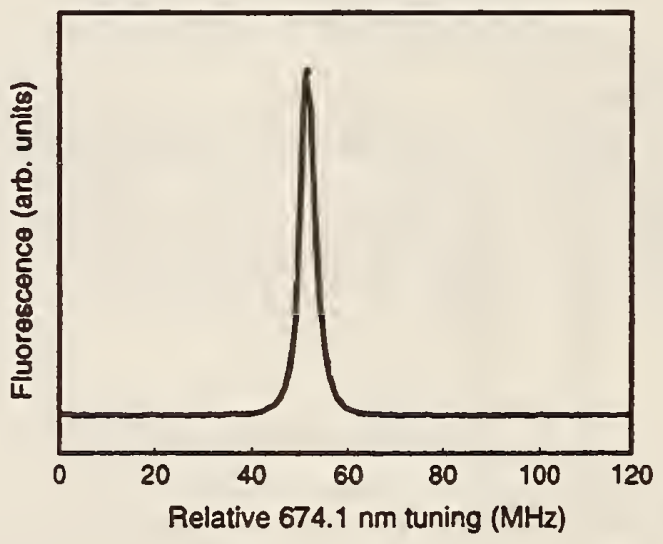

Figure 9. Fluorescence spectroscopy of the $6 S_{12}, F=4->12 S_{12}, F=5$ two-photon transition in cesium (1 $\mathrm{Hz}$ bandwidth).

A third ECDL-MOPA system is used for high resolution and high sensitivity nonlinear spectroscopy in cesium atoms. The $674 \mathrm{~nm}$ system provides $500 \mathrm{~mW}$ of usable output power which is sufficient for two-photon spectroscopy on weak optical transitions without the need of a build-up cavity. The $674 \mathrm{~nm}$ light is scanned over the $6 S_{1 / 2} \rightarrow 12 S_{1 / 2}$ two-photon transition in a cesium vapor cell heated to $-80^{\circ} \mathrm{C}$; the transition is detected by the decay fluorescence from the $12 \mathrm{~S}_{1 / 2}$ state to the $6 \mathrm{P}_{1 / 2}$ state at $541 \mathrm{~nm}$ (Figure 9). The nonlinear signal is quadratic in laser power, yet the signal-to-noise ratio is excellent (>1000 in a $1 \mathrm{~Hz}$ bandwidth). This demonstrates that the MOPA output has low AM noise. 


\section{LIMITATIONS}

This new technology is exciting and appears to have a bright future. However, there are some limitations. One limitation is that the ECDL-MOPA systems are roughly one order of magnitude more costly to build than the standard low power counterparts. Although more expensive they are still an order of magnitude less expensive than many other high-power tunable laser systems such as dye and $\mathrm{Ti}: \mathrm{Al}_{2} \mathrm{O}_{3}$ lasers. As with low power diode lasers, these ECDL-MOPAs are very susceptible to optical feedback and often require good quality isolators.

\section{CONCLUSIONS}

With these ECDL-MOPA lasers a new era in diode laser spectroscopy is opened. Power is increased by an order of magnitude or more over conventional diode lasers while the systems retain the good spectral characteristics and tunability of the lower power ECDL lasers. We have demonstrated the ability to use these lasers in several different experiments including the high resolution spectroscopy of $\mathrm{Ca}, \mathrm{Hg}^{+}, \mathrm{I}_{2}$, and $\mathrm{Cs}$. Although the systems have slightly different characteristics, our experiments demonstrate that tunable, highly coherent, spatially single-mode, high power laser systems are achievable. These lasers will undoubtedly play an increasingly important role in the high resolution spectroscopy and nonlinear optics of the future.

\section{ACKNOWLEDGMENTS}

We acknowledge the support of the U.S. Air Force Office of Scientific Research.

\section{REFERENCES}

1. J. N. Walpole, "Semiconductor amplifiers and lasers with tapered gain regions," Opt. Quantum Electron., 28, pp. 623-645 (1996).

2. D. Mehuys, D. Welch, and D. Scifres, "1 W CW, Diffraction-Limited, Tunable External-Cavity Semiconductor Laser," Electron. Lett., 29, pp. 1254-1255 (1992).

3. S. OBrien et al., "Operating characteristics of a high-power monolithically integrated flared amplifier master oscillator power amplifier," IEEE J. Quantum Electron., 29, 2052 (1993).

4. D. Mehuys, D.F. Welch, and L. Goldberg, "2.0 W CW, diffraction-limited tapered amplifier with diode injection," Elec. Lett., 28, 1944 (1992).

5. C. Zimmermann, V. Vuletic, A. Hemmerich, and T.W. Hänsch, "All solid state laser source for tunable blue and ultraviolet radiation," Appl. Phys. Lett., 66, pp. 2318-2320 (1995).

6. Lew Goldberg and W. K. Burns, "Deep UV to mid-IR generation with laser diodes and non-linear frequency conversion, in Proc. LEOS '95, edited by T. Fan, pp. 459-460 (IEEE, Piscatoway, NJ, 1995).

7. L. Hollberg, J. Marquardh, M. Stephens, and R. W. Fox, "Diode lasers and spectroscopy," in Proc. LEOS '95, edited by T.

Y. Fan, pp. 327-328 (IEEE Piscatoway, NJ, 1995).

8. K. Kikuchi, C.-E. Zah, and T.-P. Lee, "Measurement and analysis of phase noise generated from semiconductor optical amplifiers," IEEE J. Quantum Electron., 27, 416 (1991). 


\title{
4. SEMICONDUCTOR DIODE LASERS*
}

\author{
R. W. Fox and L. Hollberg \\ National Institute of Standards and Technology \\ Boulder, Colorado
}

A. S. Zibrov

Lebedev Institute of Physics

Moscow, Russia

\subsection{Introduction}

This chapter deals with the technology of applying semiconductor lasers to scientific and technical fields. Diode lasers are prevalent in many applications because of their well-known attributes: high reliability, miniature size, relative simplicity of use, and relatively low cost. These factors are important, but the use of diode lasers in technical fields is also increasing because of their unique capabilities, such as: tunability, high efficiency, useful power levels, reasonable coherence, and excellent modulation capabilities. Volumes of information are already available on the technology of semiconductor lasers [1-8]. Their application to scientific fields has been discussed in a few review articles [9-13], and at least one special issue of a journal focused on the spectroscopic detection of atoms and molecules using diode lasers [14]. As in any rapidly advancing field, this information becomes outdated quickly and will need to be supplemented with current journal publications. We will concentrate here on the basic laser characteristics and general principles of operating single-frequency, tunable, diode laser systems.

\subsection{General Characteristics of Diode Lasers}

A wide variety of diode lasers are now commercially available. These range from low-power/high-speed communications lasers to high-power/wide-stripe devices that run multimode (both spatial and temporal) and are used mainly for pumping solid-state lasers. For most scientific applications it is much easier to use

*Contribution of NIST, not subject to copyright. 
single spatial-mode lasers, and we will concentrate on these devices here. In addition to the usual Fabry-Perot type lasers, distributed feedback (DFB) and distributed Bragg reflector (DBR) lasers are now commercially available at some wavelengths. These lasers have a more complex resonator structure that incorporates an optical grating fabricated within the semiconductor chip. Bragg reflection from the internal grating provides wavelength-selective optical feedback that forces single-longitudinal-mode operation at a wavelength within the reflection bandpass of the grating.

Figure 1 gives a rough sketch of the current (1995) distribution of commercially available continuous wave ( $\mathrm{CW}$ ) single spatial-mode semiconductor lasers. There are gaps in the wavelength coverage (and actual availability) and significant differences in performance across this distribution. Some typical characteristics of semiconductor lasers are outlined in Table I. More details can be found in manufacturer's catalogs and an excellent recent handbook [15].

Another family of semiconductor lasers (outside the scope of the present chapter) is based on group IV-VI elements (most common are Pb-salt lasers). These lasers span the wavelength range from 3.3 to $30 \mu \mathrm{m}$ and operate at cryogenic temperatures. The lead-salt lasers have not experienced the same degree of commercialization as the room-temperature devices, primarily because they are much more expensive and they have poor mode quality and relatively low output powers $(\leq 5 \mathrm{~mW})$. However, high-quality devices using Sb-based semiconductors that operate at wavelengths that range from $\sim 2$ to $4.5 \mu \mathrm{m}$ have been developed [16]. These promising new devices, unfortunately, are not yet commercially available.

\subsubsection{Tuning Characteristics}

Often of paramount importance for scientific applications are the tuning characteristics of a laser. A diode laser's wavelength is determined by the semiconductor material and structure, and is a function of both temperature and carrier density. For a typical single-mode laser the wavelength increases monotonically with increasing temperature and then suddenly jumps to another mode at a longer wavelength. Although this jump is most often to the next cavity mode $(\sim 0.3 \mathrm{~nm}$ cavity mode spacing), it is not at all unusual for one or several modes to be skipped. The tuning range of each mode is typically on the order of $25 \%$ of the mode spacing, but might vary by a factor of 5 or more. Subsequently decreasing the temperature causes the operating wavelength to shift downward with similar behavior, but hysteresis will be observed at the mode transitions. Details of the tuning characteristics are available in the general references [1-8] and [17].

Operating a simple diode laser at the wavelength of an atomic or molecular transition usually requires iterative selection of injection current and temperature settings. However, for any given laser the optimum tuning to a specific wavelength cannot always be achieved. Possible alternatives include trying several lasers or 


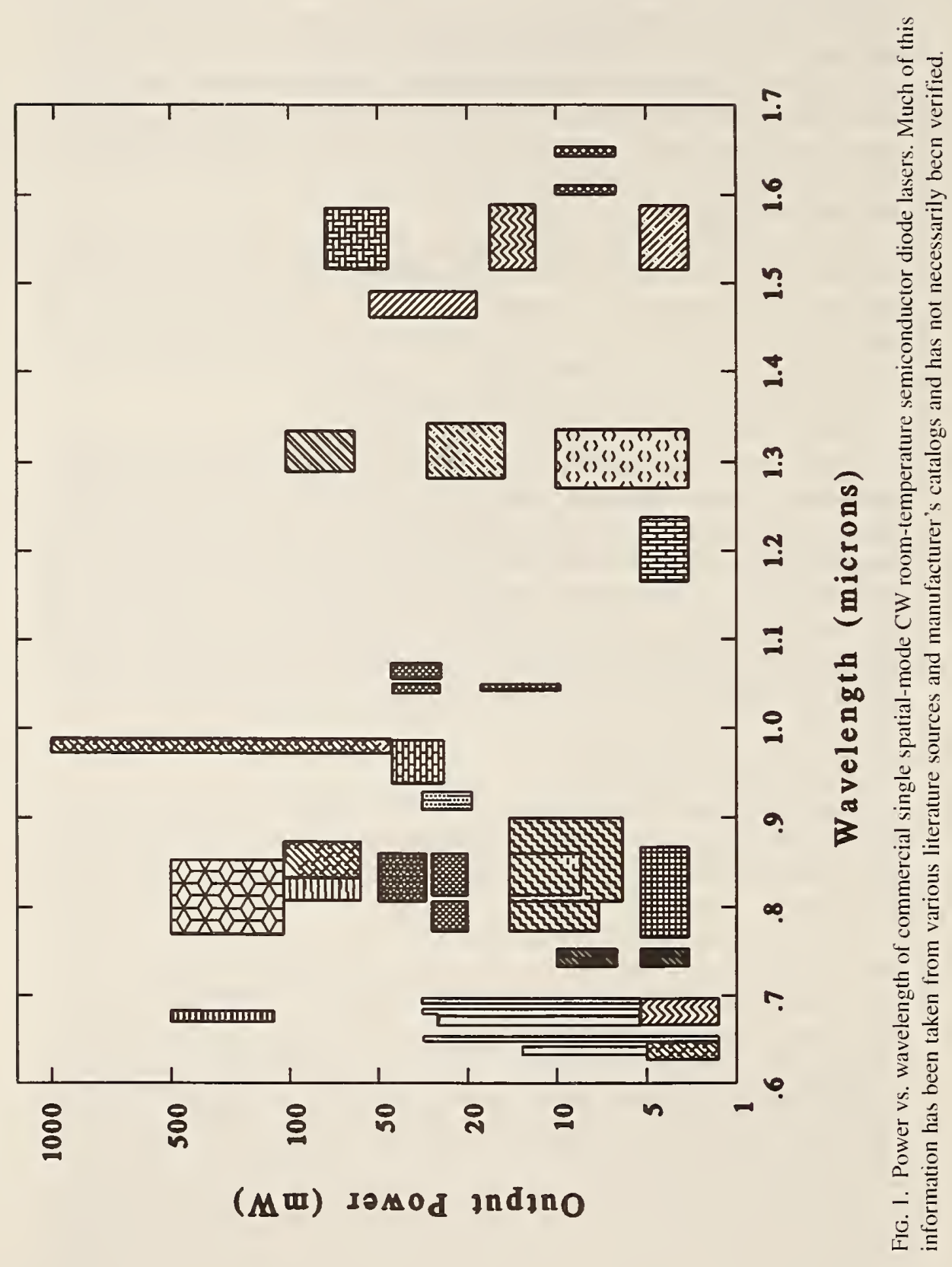


TABlE 1. Typical Semiconductor Laser Parameters ${ }^{a}$

\begin{tabular}{|c|c|c|c|c|}
\hline Semiconductor & $\left(\mathrm{Al}_{x} \mathrm{Ga}_{1-x}\right)_{y} \mathrm{In}_{\mathrm{I}_{-\rightarrow}} \mathrm{P}$ & $\mathrm{Al}_{x} \mathrm{Ga}_{1-x} \mathrm{As}$ & $\mathrm{Ga}_{x} \mathrm{In}_{1-x} \mathrm{P}_{3} \mathrm{As}_{1-y}$ & MOPA \\
\hline Wavelength & $635-670 \mathrm{~nm}$ & $750-850 \mathrm{~nm}$ & $1.3-1.5 \mu \mathrm{m}$ & $\begin{array}{l}670,780-850, \\
980 \mathrm{~nm}\end{array}$ \\
\hline Output power (mW) & $3-30$ & $5-200$ & $3-100$ & $500-1000$ \\
\hline $\begin{array}{l}\text { Far-field divergence } \\
\text { FWM1 (degrees) }\end{array}$ & $8 \times 40$ & $11 \times 33$ & $30 \times 35$ & $0.3 \times 35$ \\
\hline $\begin{array}{l}\text { Waveguide mode } \\
\text { dimensions }(\mu \mathrm{m})\end{array}$ & $4 \times 1$ & $3 \times 1$ & $1.25 \times 1$ & $1 \times 100$ \\
\hline Astigmatism $(\mu \mathrm{m})$ & $\sim 10$ & $1-5$ & $1-5$ & $\sim 500$ \\
\hline Threshold current & $30-90 \mathrm{~mA}$ & $20-60 \mathrm{~mA}$ & $20-50 \mathrm{~mA}$ & $\sim 0.5 \mathrm{~A}$ \\
\hline Operating current & $50-120 \mathrm{~mA}$ & $50-200 \mathrm{~mA}$ & $40-120 \mathrm{~mA}$ & $-2.5 \mathrm{~A}$ \\
\hline$I_{2}=I_{1} e^{(T 2-T 1) / T 0}$ & $T_{0} \approx 100 \mathrm{~K}$ & $T_{0} \approx 150 \mathrm{~K}$ & $T_{0} \approx 60 \mathrm{~K}$ & \\
\hline Slope efficiency $(\mathrm{mW} / \mathrm{mA})$ & $0.5-0.7$ & 0.7 & 0.2 & 1 \\
\hline Refractive index & $3.1-3.5$ & $3.3-3.6$ & $3.2-3.5$ & $3.3-3.6$ \\
\hline $\begin{array}{l}\text { Frequency vs. injection } \\
\text { current }(\mathrm{GHz} / \mathrm{mA})\end{array}$ & -5 & -3 & 1 & \\
\hline \multicolumn{5}{|l|}{ Frequency vs. temperature } \\
\hline Large-scale $(\mathrm{nm} / \mathrm{K})$ & $\sim 0.2$ & $\sim 0.25$ & $\sim 0.3$ & \\
\hline Small-scale $(\mathrm{GHz} / \mathrm{K})$ & -30 & -30 & $\sim 10$ & \\
\hline Gain bandwidth (nm) & 20 & 30 & 50 & 20 \\
\hline Typical linewidth $(\mathrm{MHz})$ & 200 & $5-20$ & 100 & \\
\hline Alpha factor $(\alpha)$ & & $3-6$ & $4-8$ & \\
\hline
\end{tabular}

"These values are representative examples and can vary significantly with structure and composition. The data applies to index-guided single spatial-and spectral-mode Fabry-Perot lasers. Much of this information has been taken from various literature sources and manufacturer's catalogs and has not necessarily been verified.

using optical feedback techniques to control the wavelength. Extended-cavity lasers (Section 4.3) allow tuning to any wavelength within the gain curve of the laser. Another option is to place a small mirror near $(\sim 100 \mu \mathrm{m}$ away) the laser's facet, forming an etalon that acts as a mode selector [18]. An attractive alternative (but improbable for other than a few standard wavelengths) is to find a monolithic DBR laser that operates at the desired wavelength.

\subsubsection{Output Power}

A typical output power vs. injection current $(P-I)$ curve for a $\mathrm{CW}$ diode laser is shown in Fig. 2. The slope of the $P-I$ curve above threshold gives the laser's slope-efficiency (in $\mathrm{mW} / \mathrm{mA}$ ). Semiconductor lasers operate as forward-biased diodes with the voltage drop fixed by the bandgap and an additional series resistance of about 2 to $50 \Omega$. As expected for a semiconductor diode, the laser's 
LTO 26 AC Laser

$\mathrm{Al} \mathrm{OO} 3+\mathrm{HFO} 2$ Coated

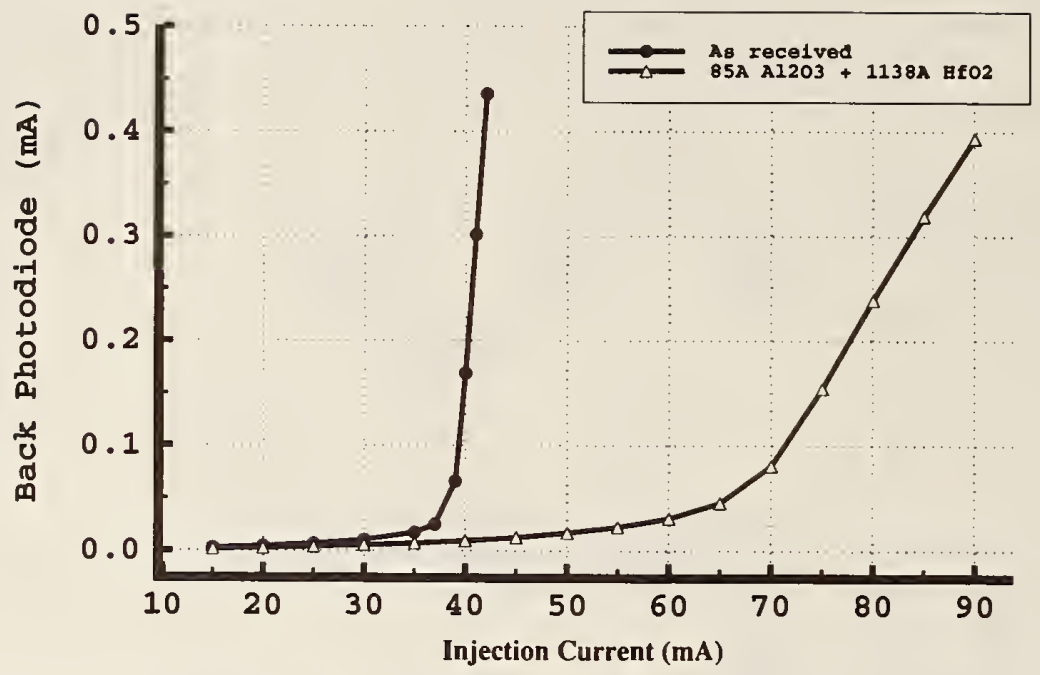

FIG. 2. Output power vs. injection current for a typical AIGaAs diode laser operating near $780 \mathrm{~nm}$. The vertical axis is photocurrent from the photodiode that monitors power out of the laser's back facet. The solid dots show the $P-I$ curve of the standard commercial laser as received $\left(I_{t h} \approx 38 \mathrm{~mA}\right)$, while the open triangles show the $P-I$ curve of the laser after we coated the front facet with an antireflectance $(\mathrm{AR})$ coating $\left(I_{t h} \approx 68 \mathrm{~mA}\right)$. As fractionally more power is coupled out the front facet, the slope efficiency of the back facet power is decreased.

operating characteristics are strongly temperature-dependent. Using a simple model for a diode laser $[3,5]$ gives a threshold gain of

$$
g_{t h}=a_{L}+\frac{1}{L} \ln \left[\frac{1}{R_{1} R_{2}}\right],
$$

and a total output power above threshold of

$$
P_{\text {total }}=\frac{\eta_{i} h v\left(I-I_{t h}\right)}{e g_{t h} L} \ln \left[\frac{1}{R_{1} R_{2}}\right],
$$

where $R_{1}$ and $R_{2}$ are the facet reflectances, $a_{L}$ is the loss internal to the laser, $h v$ is the photon energy, $I$ is the injection current, $I_{t h}$ is the threshold injection current, $\eta_{i}$ is the internal quantum efficiency, $e$ is the electron charge, and $L$ is the laser length. The threshold will vary with temperature as $\left.I_{2}=I_{1} \exp \left[T_{2}-T_{1}\right) / T_{0}\right]$, where 
$T_{0}$ is a characteristic temperature for the laser. A more precise theoretical treatment allows parameters in these equations to depend on wavelength, carrier density, semiconductor material, and laser structure. If the injection current is increased to excessively high values, the $P-I$ curve becomes sublinear, with the output power eventually decreasing due to gain saturation, heating, or damage resulting from high optical powers on the facets. Operation at overly high currents shortens the lifetime and can seriously damage or destroy the laser. An important measure of a laser's operating characteristics is the pumping rate, $R=\left(I-I_{t h}\right) / I_{t h}$, which is the injection current above threshold normalized to the threshold value. Generally in the safe operating region of temperature and current, the laser's characteristics improve with pumping rate: the power increases, the linewidth decreases, and the relative amplitude noise decreases.

Recent advances in diode laser technology have produced impressive results in high-power devices based on tapered amplifier designs. By starting with a narrowstripe single-mode waveguide and then expanding the width of the waveguide out to larger dimensions, the output power can be increased while retaining single spatial-mode operation. Thus, monolithic master-oscillator/power-amplifier (MOPA) systems can be integrated into a single semiconductor chip [19]. The increase in power obtained with tapered amplifiers scales roughly as the ratio of the widths of the output to input waveguide dimensions. Single spatial-mode devices with watt-level output powers are now appearing on the commercial market. Obviously, high-power devices will open up many new possibilities. Of particular interest will be their use with nonlinear optical materials to access other spectral regions.

\subsubsection{Beam Quality}

The spatial mode produced by diode lasers depends on the laser structure and the collimating optics. Since the output beam diverges rapidly from the small semiconductor waveguide, a high-numerical-aperture lens is needed to collect the light into a collimated beam. Beam quality is not always what we might hope for, but at least in the case of single-mode lasers it can be corrected to be nearly cylindrically symmetric Gaussian. Output beams are typically asymmetric, with a larger divergence in the direction perpendicular to the junction (see Table I). Well above threshold, most are strongly polarized with the electric field parallel to the junction. The modes can also have astigmatism, which manifests itself as the apparent axial separation of the source of rays in the planes parallel and perpendicular to the junction. Astigmatic distances for Fabry-Perot lasers are generally in the range of 1 to $10 \mu \mathrm{m}$, while for tapered amplifiers the astigmatism can be $-1 \mathrm{~mm}$, about half the length of the chip. Left uncompensated, this aberration may degrade the beam quality and result in an aberrated far-field intensity distribution. A cylindrical lens can be used to correct the astigmatism, while the asymmetry in 
divergence is often corrected with a pair of anamorphic prisms that are oriented to angularly magnify the smaller divergence and hence produce a nearly circular beam [20]. Alternatively, a cylindrical telescope can be used to correct the angular asymmetry. In addition, there are sometimes small changes in the spatial mode parameters with large changes in the pumping rate $R$.

\subsubsection{Laser Amplitude Noise}

An important attribute of semiconductor lasers is that they typically have much lower amplitude noise then other tunable laser systems. For example, a singlemode laser operating well above threshold might typically exhibit amplitude fluctuations that approach the shot-noise level for frequencies above $\sim 1 \mathrm{MHz}$. The noise does increases toward lower frequencies with an approximately $1 / f$ dependence. A common measure of the amplitude noise is the relative intensity noise: RIN $=\left\langle\Delta P^{2}\right\rangle\left\langle\langle P\rangle^{2} \approx\left[2 \cdot \Delta f S_{p}(f)\right]^{2} /\langle P\rangle^{2}\right.$, where $\Delta f$ is the detection bandwidth, $S_{p}(f)$ is the spectral density of the power fluctuations, and $\langle P\rangle$ is the mean power. In general, RIN will decrease with increasing pumping rate. If the laser is operated in a multimode regime, the amplitude noise may be much more pronounced, especially when there are only a small number of modes oscillating. Optical feedback can also cause increased amplitude noise.

\subsubsection{Optical Feedback}

The effects of optical feedback on diode lasers can be profound and depend on a number of factors: the amount of feedback, the facet reflectance, the pumping rate $R$, and the distance from the laser to the source of feedback (feedback time delay). In some regions of this multidimensional space, the feedback can narrow the linewidth and stabilize the laser's wavelength, while in other regions optical feedback causes such instabilities as erratic mode jumping and coherence collapse [5, 21-24]. In general, optical feedback (even small amounts $P_{\text {feedback }} / P_{\text {out }} \leq 10^{-5}$ ) from uncontrolled sources should be avoided. For many applications this simply means tilting optical elements to avoid direct reflections. In other cases we are forced to use Faraday isolators, which unfortunately tend to be bulky and expensive for wavelengths less than $1 \mu \mathrm{m}$. In some of the next sections we discuss ways to use optical feedback to advantage.

Even though the terminology used to describe diode lasers is inconsistent and imprecise, it may be useful to review some of the nomenclature. For some reason the description "solitary laser" has come to mean a semiconductor diode laser that has not been modified by external effects such as optical or electronic feedback. "External cavity laser," on the other, hand describes a system that lases only because of optical feedback from external optical elements [25]. The name "extended-cavity diode laser" (ECDL) has come to mean something between these 
two cases; that is, when the laser is operated in a regime where strong feedback from one side of the chip (from an external element such as a grating) is used to control the laser's mode. Sometimes the nomenclature and jargon are more confusing than useful.

\subsubsection{Laser Degradation}

When properly protected from electrical damage, semiconductor diode lasers are reliable and long-lived. However, there are variations from laser to laser and some variations of laser characteristics with time. Quite a lot of research has been done on the degradation of laser diodes (there is even a book on the subject [26]). However, we still lack detailed understanding of some aspects of laser aging, such as the change in laser wavelength with time. Some characteristic modes of laser degradation are now recognized, including "dark line defect" and facet degradation (which can be caused by gradual photochemical effects or catastrophic optical damage, COD). There seems little that users can do about these changes other than providing good electrical protection and a clean environment for lasers whose facets are open to the air. High currents and high temperatures will increase the rate of degradation. When treated properly, modern lasers have very long natural lifetimes $(-10,000 \mathrm{~h})$, but unnatural lifetimes can be quite short!

\subsection{Extended-Cavity Lasers}

Tuning the wavelength of diode lasers and narrowing their linewidth can be accomplished using some form of dispersive optical feedback [27]. Designs that employ relatively high optical feedback power, $P_{\text {feedback }} / P_{\text {out }} \gtrsim 0.05$ (which is the power fed back into the laser's waveguide mode relative to the output power without feedback), can have a large tuning range and be very stable. In addition to broad tuning, extended-cavity lasers typically have much narrower linewidths than solitary lasers (fast linewidths $\sim 50 \mathrm{kHz}$ as opposed to $\sim 20 \mathrm{MHz}$ ). Diffraction gratings are the most commonly used dispersive feedback element, although intracavity etalons, prisms, and birefringent filters have also been used successfully.

Good-quality commercial extended-cavity diode laser systems are now available from a few manufacturers, but distribution in terms of power and wavelength are still somewhat limited. Because of special requirements or cost constraints, users may find it desirable to build their own extended-cavity systems. This can be done relatively simply, as described in a number of papers $[7,12,28,29]$ and as we outline below.

\subsubsection{ECDL Construction}

There are many good designs for extended-cavity lasers, but no particular design that is optimum for all applications. In Fig. 3 we see five different configu- 
a)

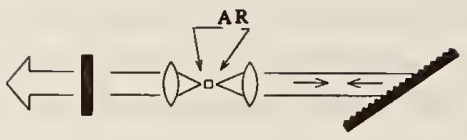

b)

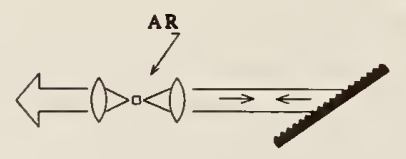

c)

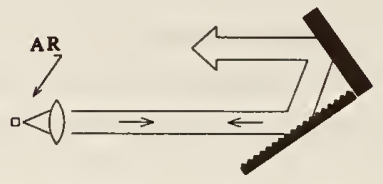

d)

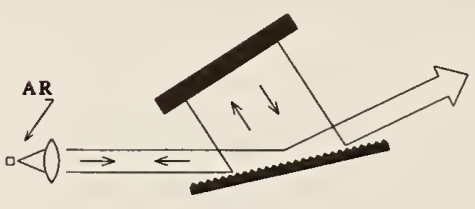

e)

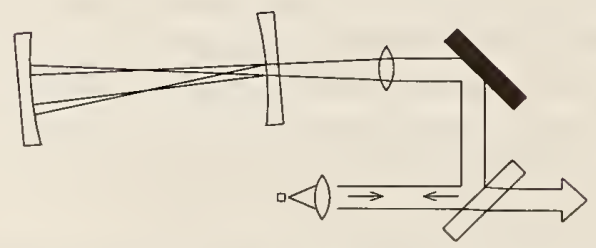

FIG. 3. Various optical feedback configurations. The first design (a) is a two-sided external-cavity design, $(\mathrm{b}, \mathrm{c})$ are single-sided extended-cavity designs in Littrow configuration, (d) is an extended-cavity laser that uses a grating in a grazing-incidence configuration, and (e) is a resonant optical-locking configuration using a confocal Fabry-Perot cavity. Design (b) is appealing because there is no movement of the output beam when the grating is rotated to tune the wavelength, and it can also be optimized to deliver high output power. On the other hand, it has the disadvantage that it requires a special laser package (or a rather severe modification of standard laser packages) to gain unobstructed access to both facets. In present usage, the two most common tunable laser designs are (c) and (d) because they are relatively easy to implement using commercial lasers. 
rations of optical feedback that can be used to control diode lasers. For optimum performance many of these designs require good antireflection coatings on the output facet of the chip (see Section 4.5).

When designing a laser cavity, it is important to remember that the diffraction efficiency of typical gratings has a strong polarization dependence. For beams polarized perpendicular to the rulings we expect high diffraction efficiency, while for beams polarized parallel the efficiency can be significantly reduced. In the Littrow configuration (Fig. 3b,c), the number of grating lines covered by the laser mode is constrained by the focal length and numerical aperture of the collimating lens, the orientation of the laser spatial mode, and the line spacing and corresponding cutoff wavelength of the grating. Since the beam from a laser waveguide is normally polarized in the direction parallel to the junction, in the far field the laser beam is polarized along the narrow dimension of the spatial mode. When the laser's asymmetric mode is incident on a diffraction grating, there is a compromise between high resolution and high diffraction efficiency. The user has very little freedom of design, which means that the resolution is sometimes not as high as we would like. In some cases it can be advantageous to use a half-wave plate between the laser and the grating to decouple the polarization (hence diffraction efficiency) from the spatial mode orientation. An alternative approach is to use prisms to expand the small direction of the spatial mode $[20,24,30]$. With the one-sided Littrow configuration (Fig. 3c) there is also the disadvantage that the beam moves as the laser's wavelength is tuned. A remedy to the problem of beam movement with tuning (present in the Fig. $3 c$ configuration) is to use a mirror mounted with its surface perpendicular to the grating surface so as to form a retroreflector. Rotating both the grating and mirror together leaves the output beam direction unchanged, although there will be some beam displacement if the axis of rotation is not defined by the intersection of the grating and the mirror. This arrangement is not easily compatible with long continuous scans.

Even with these limitations we often use the standard Littrow configuration (Fig. 3c) because of its simplicity, and most of the time it works reasonably well. For many applications (atomic physics in particular) large tuning ranges are not very important. In practice one can achieve continuous scans of $\sim 2$ to $10 \mathrm{GHz}$ by simply arranging the grating pivot point so that the grating angle tunes synchronously with the laser's frequency as controlled by the extended-cavity length. Coarse tuning can be accomplished manually with a fine pitched screw. A typical 800-nm system might consist of an 8-mm focal length collimating lens (N.A. = 0.5 ), an 1800 -line/mm grating in Littrow configuration with a cavity length of about $5 \mathrm{~cm}$. In a typical Littrow system we normally orient the laser mode so that the larger dimension is orthogonal to the direction of the grating rulings. This orientation gives better resolution and better output power than the opposite orientation. 


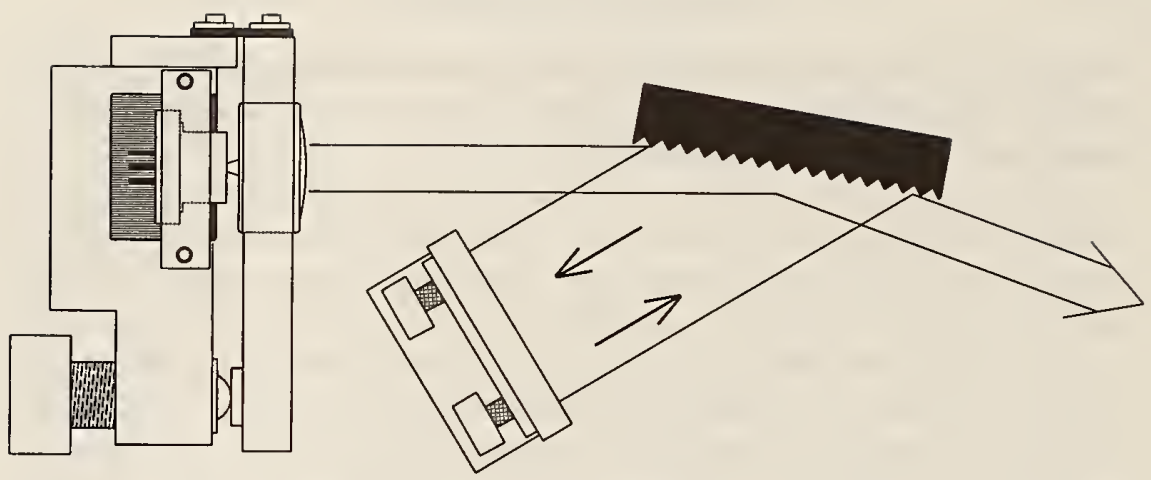

FIG. 4. Diagram of a simple grazing-incidence grating-tuned extended-cavity diode laser. This extended-cavity system is constructed of three basic units: the laser mount, the grating mount, and the feedback mirror. The diode laser, Peltier cooler, and collimating objective are all integrated together in a stable laser mount that uses a flexure and fine-pitched screw for precise focus adjustment. The feedback mirror and the angle of the grating are adjusted relative to laser beam direction to provide just enough optical feedback power for stable single-mode operation.

The grazing incidence arrangement (Fig. 3d) as proposed by Harvey and Myatt [31] was shown to have the important advantages of significantly higher spectral resolving power and no movement of the output beam when the laser is tuned. This design, implemented with diode lasers by Harvey, is often called the Littman (or Littman-Metcalf) configuration because they introduced the use of grazing-incidence gratings to control dye lasers [32]. Good discussions of the grazing incidence design have also been given by Day [33]. A simple mechanical layout for a grazing-incidence grating-tuned ECDL is shown in Fig. 4.

In designing all of these systems we need to address a few basic requirements. We need to precisely align the laser cavity, to tune the wavelength, and to precisely control the laser chip temperature and extract excess heat. Care must be taken to ensure a stable thermal and mechanical structure by using good materials and kinematic design principles. Ironically, ECDLs provide narrow spectral linewidths, but they are also much more susceptible to external perturbations than were the solitary lasers that we started with! For stable single-frequency operation, ECDLs need to be isolated from vibrations and pressure fluctuations. A useful principle to keep in mind in this design is: if something can move, it will. Allowing a large dynamic range of motion competes directly with stability.

The simple optomechanical design sketched in Fig. 4 is an example of a versatile system that satisfies most (but never all) of our basic requirements. It consists of a copper baseplate (not shown) that acts as a rigid backbone for the laser resonator and at the same time serves as the heat sink for the temperature 
control system. A commercial laser is mounted in a small copper fixture that bridges the Peltier cooler and attaches to the laser base mount (also copper). The base mount is then rigidly attached to one end of the baseplate. The collimating lens is connected to the laser base mount by a stiff spring-steel flexure that is clamped in place using locating pins and multiple screws. The lens is mounted in an eccentric ring that is clamped in place after initial coarse alignment. The transverse alignment can be set accurately enough with careful hand alignment and visual inspection of the beam direction and shape. In the example of Fig. 4, the eccentric on the lens mount is used to adjust the vertical height of the lens, while the horizontal position is set by the transverse displacement of the laser on the base mount. Critical adjustment of the focus is done with a high-quality fine-pitched screw that translates the lens against the restoring force provided by the flexure. We typically use $6.3-\mathrm{mm}$ diameter screws with 3.1 threads/mm (1/4-80 screw). The lever arm from the flexure pivot point (Fig. 4) gives further drive reduction. This simple design tilts the lens slightly as it is translated for focusing; however, this is not a serious limitation because the lens can be set very close to the optimum position before it is clamped in place. In constructing this system we tolerate the thermal expansion of copper in order to have the good thermal conductivity and low mechanical $Q$. For very-high-frequency stability the cavity length can be controlled with a PZT. For some high-power lasers we use water cooling in the laser base mount. The water can be circulated without pump vibrations by using a simple thermal syphon.

One of the requirements for stable operation of ECDLs is to return sufficient optical feedback power to the diode laser waveguide mode. The optimal feedback power depends on the characteristics of the laser and, in particular, the reflectance of the output facet. Typically feedback power ratios $\left(p_{\text {feedback }} / p_{\text {out }}\right)$ of from 5 to $50 \%$ are appropriate. In grazing-incidence systems the feedback mirror and the angle of the grating (relative to the laser beam direction) are adjusted to provide just enough feedback power for stable single-mode operation (typical incidence angles for the grating might be $\sim 75-85^{\circ}$ ). Good-quality high-numerical-aperture objectives (N.A. 0.5) are recommended for the collimating lens. In most cases it is not necessary to correct for the laser's astigmatism for the laser to function properly in extended-cavity mode. However, the output beam will have some aberration if only spherical optics are used. Objectives lenses specifically designed for diode laser collimation often assume that there is a thin window between the laser chip and the lens. For best mode quality, the lens design needs to take into account any window on the laser mount.

\subsubsection{Alignment}

The optical alignment of extended-cavity lasers requires precision, but with practice alignment can be accomplished in a simple common-sense manner. Infra- 
red-sensitive cards that fluoresce in the visible are extremely useful when working with near-IR lasers. Electronic IR viewers or CCD cameras can also be used. For the initial alignment, the objective lens is carefully centered on the laser's output so that the beam propagates in the same direction as the unperturbed laser emission. The beam should appear roughly collimated over a distance of several meters. The grating is then positioned so that the first-order diffracted beam returns to the laser (taking care that the grating blaze is toward the lens). A card with a small aperture held in front of the lens is useful to roughly position the returned beam on the outgoing beam. Fine tuning of the focus and alignment will then be necessary to obtain stable single-mode operation.

The method that we use most often to align ECDLs was derived from work describing the output power-vs.-injection current characteristics of ECDLs [2], 34, 35]. The method is simple and requires only monitoring the output power as a function of the swept injection current. Following the coarse alignment procedure described above and with the laser operated near threshold, a triangular ramp is applied to the injection current. Monitoring the output power of the ECDL with a large-area photodiode and an oscilloscope (synchronized to the triangular wave) yields a classic diode laser $P-I$ curve with no optical feedback (see Fig. 2). When there is feedback from the extended cavity, there will be abrupt discontinuous changes in threshold behavior. Iterative adjustment of the focus and extended-cavity alignment will result in oscilloscope traces similar to those in Fig. 5.

\subsubsection{Tuning}

The maximum tuning range of an ECDL depends on the design of the cavity and on the detailed characteristics of the diode laser chip. Factors that affect the tuning include: the facet reflectances, the degree to which the spectral broadening is homogeneous, the side-mode suppression ratio, the pumping rate $R$, the spatial mode control (how well the mode is index guided), and the propensity for nonlinear coupling between modes (for example, four-wave mixing). As users, the facet reflectance is almost the only parameter that we have any control over. If the reflectance of the laser facet that faces the grating is very low $\left(<10^{-4}\right)$, the maximum tuning range can be as large as the laser's gain curve (approximately $\pm 20 \mathrm{~nm}$ for AlGaAs lasers and even larger for InGaAsP lasers). Continuous single-mode scans over many nanometers require very low facet reflectance and synchronization of the tuning of the grating passband with change in cavity length.

\subsection{Electronics}

The electronics required to operate semiconductor lasers are relatively straightforward and can be very simple if we do not require precise tuning. Since the 


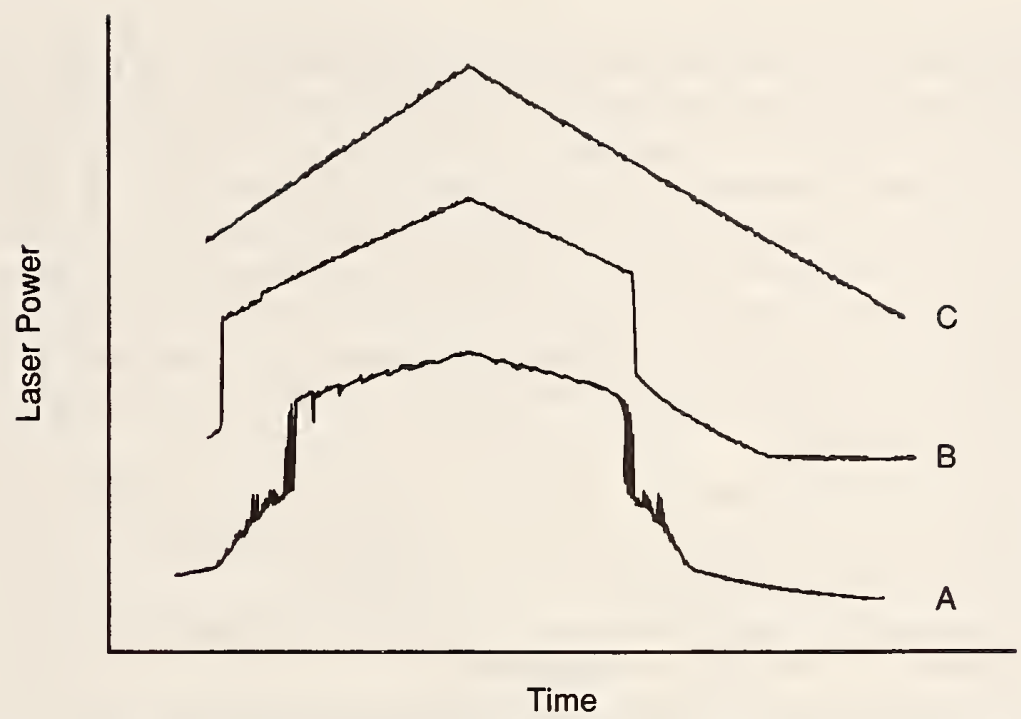

FIG. 5. Output power of ECDL vs. injection current, which is swept near threshold with a triangle wave. Three different $P-I$ curves are shown for different cavity alignments; the curves have been offset vertically for clarity. The horizontal axis (labeled here as time) is synched with a triangle wave ramp, with maximum injection current corresponding to maximum output power. The characteristic features of the $P-I$ curve are a good indicator of the quality of the extended-cavity alignment. As the focus of the collimating lens and feedback from the grating are aligned, the output power increases in abrupt steps. The rounded corners and noise on curve A corresponds to poor cavity alignment and are indicative of multilongitudinal mode operation. Curve B shows the type of abrupt jumps that are indicative of single-mode operation. Curve $\mathrm{C}$ is near-optimum alignment, where the injection current has very little effect on the laser's frequency and no mode jumps are apparent.

laser's output power and frequency depend sensitively on temperature and injection current, we need to control both with some precision. The typical laser parameters in Table I can be used to estimate the stability and noise performance that will be required of the current source and temperature controller for a given application.

\subsubsection{Current Sources}

The most important requirement of current sources used for diode lasers is that they MUST BE FREE of electrical transients that can seriously damage the laser. CW narrow-stripe lasers require an injection current of about 20 to $200 \mathrm{~mA}$ and have a forward voltage drop of about $2 \mathrm{~V}$. In contrast, some of the high-power devices-such as wide-stripe lasers, MOPAs, and diode laser arrays-might re- 
quire a few amperes of injection current. The simplest diode laser current supply is just a battery and a current-limiting resistor (or potentiometer). In practice we usually want to do more than just turn the laser on, and so the current source becomes somewhat more complicated. A number of good diode laser current sources are now commercially available. These range from simple integrated circuit chips that are designed for low-cost consumer electronics to very elaborate microprocessor-controlled systems. If a laboratory with a limited budget is planning on operating a significant number of precision diode laser systems, it can be cost-effective to produce some simple circuit boards for current sources and temperature controllers. Following this approach, we have found a few general principles to be useful.

- When possible do not put the AC-to-DC power converter in the same box with the current source electronics; very good filtering and regulation of the DC supplies are also important.

- It is important to keep the diode laser current path, both to and from the laser, independent of other signal and return paths.

- Pay careful attention to avoiding spikes on startup, shutdown, and even power failures. Before endangering a valuable laser, time is well invested in testing for transients (under all conceivable operating conditions) with an inexpensive imitation laser load (such as 10 LEDs in parallel and these in series with a $1 \Omega$ current sensing resistor to ground; you can also use one or more rectifier diodes in place of the LEDs).

- To protect against damage caused by transients and operator errors, it is important to attach some passive protection near the laser chip (a typical protection circuit is shown in Fig. 6). Protection circuits can also have adverse effects, such as slowing down modulation response and increasing thermal instability due to diode leakage current. This last effect can be eliminated when necessary by controlling the temperature of the protection diodes by placing them on the laser heat sink.

- Watch for potential ground loop problems, particularly when connecting multiple cables between the laser, current source, sweep source, temperature controller, optical table, detectors, oscilloscope, etc. Our approach has been to keep the laser heat sink at ground potential but to isolate it from all other grounds (including that of the box that we use to enclose the laser), except at the laser current source where there is a common ground.

- Look for other useful hints and examples in catalogs supplied by the laser manufacturers.

An example of a good-quality diode laser current source is in Fig. 6. Other examples can be found in the literature $[36,37]$. This circuit is designed to have good stability, low noise, and modulation capability. 

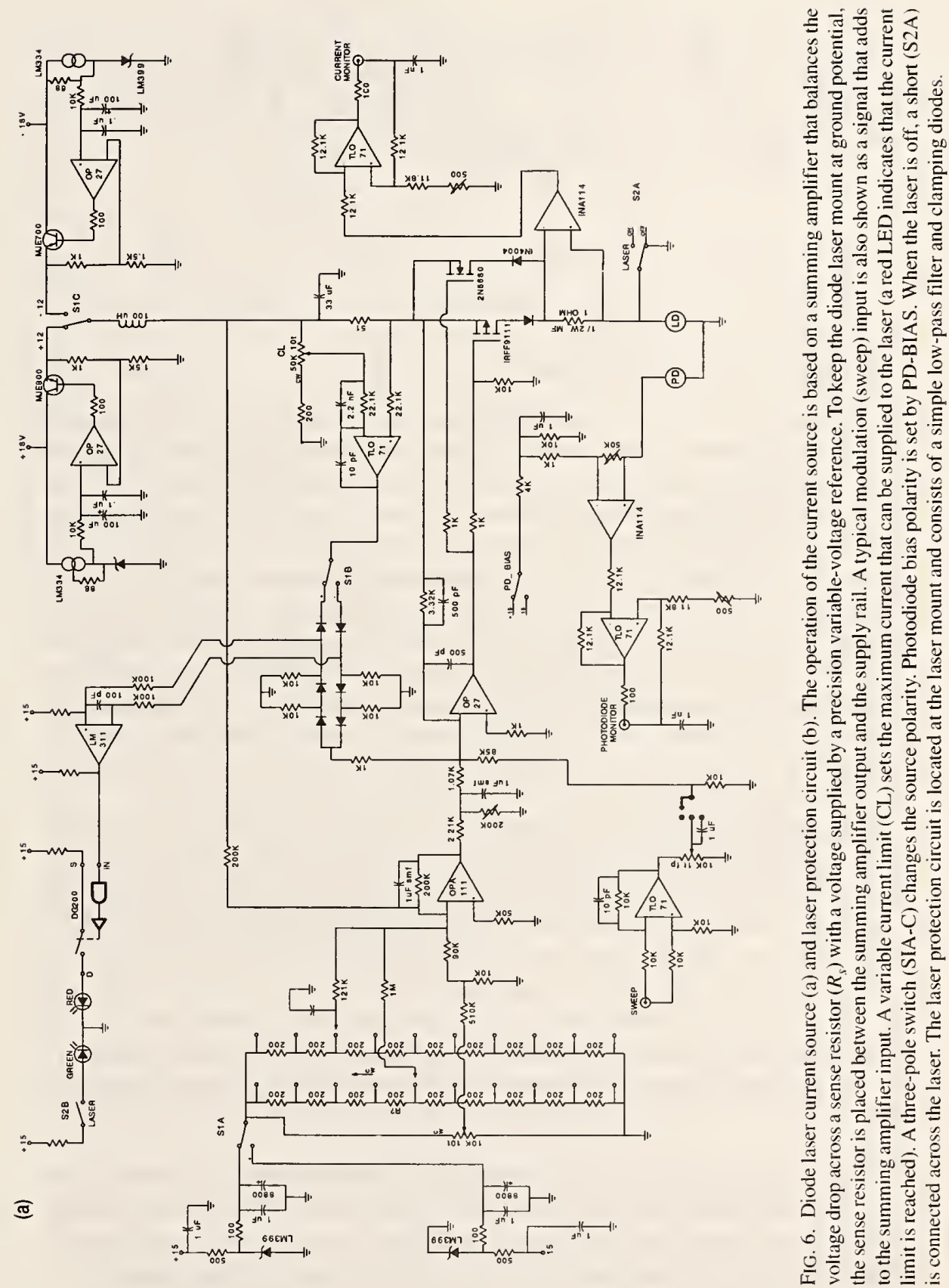
(b)

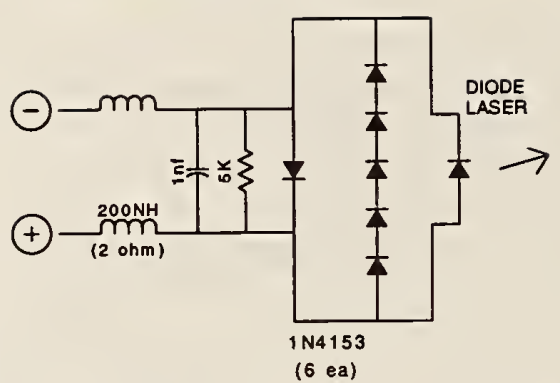

Fig. 6b

\subsubsection{Temperature Controllers}

The high sensitivity of diode laser wavelengths to temperature $(\sim 30 \mathrm{MHz} / \mathrm{mK})$ places stringent requirements on their temperature control. For fixed-wavelength operation, solitary lasers require better temperature stability than ECDLs. With ECDLs the temperature stability is more of a concern because of thermal expansion of materials than it is because of the temperature sensitivity of the laser's gain. In practice the most difficult task in maintaining temperature stability is not the electronics, but rather it is the design of the thermal environment. A stable thermomechanical environment will reduce problems associated with such important, but often less obvious, perturbations as: beam-pointing stability (remember the angular magnifying properties of the short-focal-length collimating objective) and fluctuations of even the small optical feedback that is invariably present. Putting ECDLs in hermetically sealed containers greatly improves both thermal and optomechanical stability.

Important tradeoffs have to be balanced in designing temperature control systems for diode lasers. For instance, we would like to have the thermistor very close to the laser to accurately measure the laser's temperature, but we also want the thermistor close to the thermoelectric transducer so that the time delay in the servo is small and the loop can have fast response and high gain. These considerations indicate that a very small thermal mass should be used to support the laser. But the mount should also be mechanically stable and have a large thermal mass so that rapid temperature fluctuations (outside the loop bandwidth, e.g., from moving air) do not perturb the laser's temperature. For practical reasons we would also like the entire system to be relatively light and compact. Obviously, compromises are in order.

Figure 7 depicts an example of a temperature controller that uses a Peltier cooler to control the temperature of a diode laser; others can be found in the literature $[12,29]$. An excellent analysis of the use of Peltiers in temperature control systems has been provided by Van Baak [38]. As with current sources, there are some basic design principles that are useful in constructing thermal control systems: 


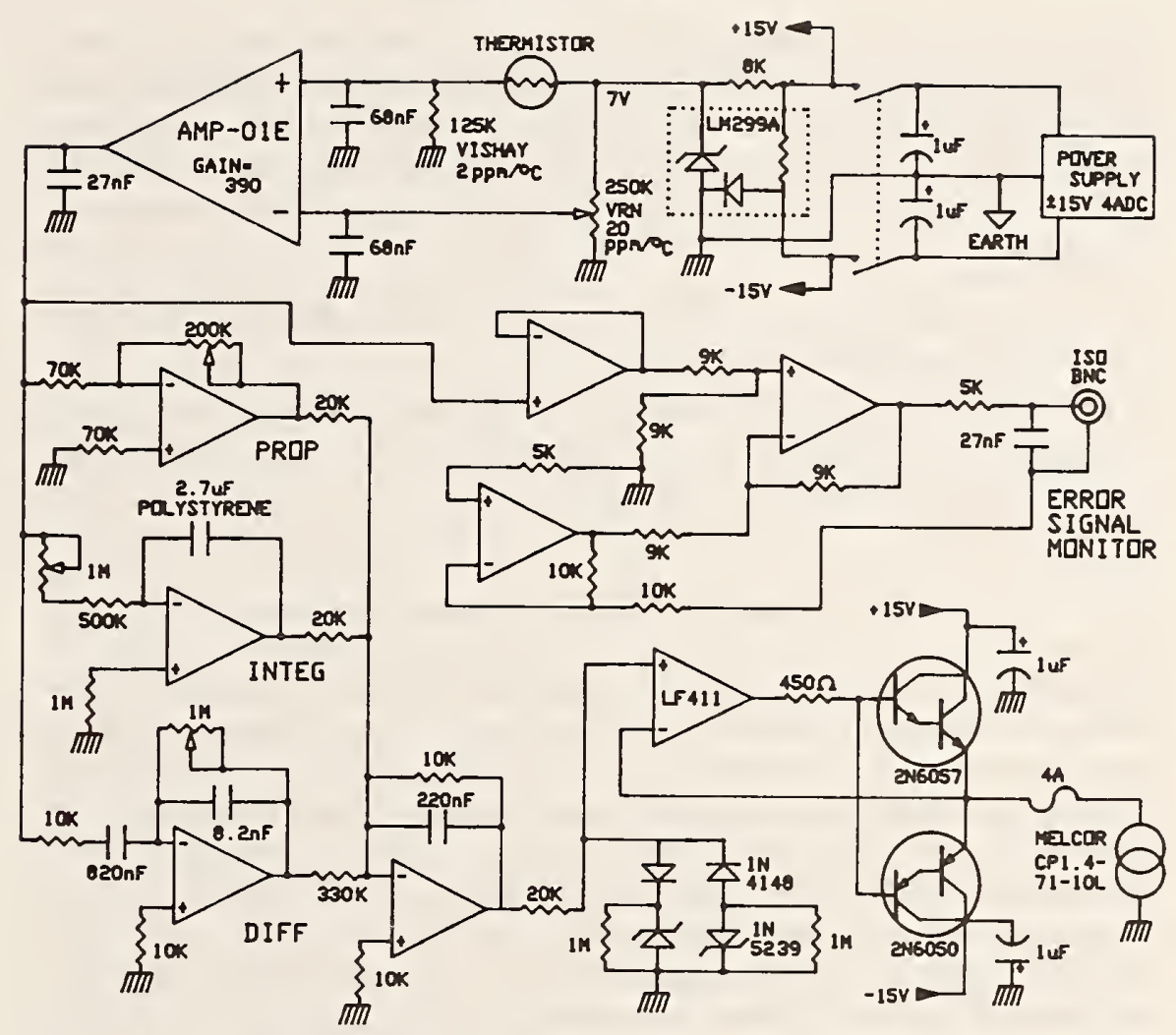

FIG. 7. Diode laser temperature controller. A precision thermistor (nominal value $125 \mathrm{k} \Omega$ ) is used in a resistance bridge to sense the laser's temperature, and a Peltier (thermoelectric) cooler is used for cooling or heating. The servo-loop is a PID (proportional integral differential) control that processes the error signal and then sends it to a driver amplifier which provides sufficient power to run the Peltier cooler (typically a few volts and a few amperes). This circuit is from reference [36].

- Two-stage thermal control can be very effective in improving the stability of ECDLs. One controller is used for the extended cavity and the other for the laser chip.

- Thermistors should be buried in holes below the surface $(\& 5 \mathrm{~mm})$ to avoid perturbations from temperature differences with the surrounding air.

- High-thermal-expansion materials (e.g., plastics, aluminum, copper) require better temperature control to maintain mechanical stability and alignment. Invar and fused silica can be useful for some components. 
- The combined thermomechanical properties of the materials and construction must be considered. This includes the dynamic response of the material used within the thermal control loop (aluminum, copper, and silver have large coefficients of thermal diffusivity, which is good for control systems, but they also have large coefficients of thermal expansion, which is bad for mechanical stability).

- Peltier coolers are not very efficient, so a relatively large thermal reservoir may be required to dissipate excess heat.

- Watch out for thermal drifts and emfs in the electronics. Even good-quality resistors and capacitors can have significant temperature coefficients. Potentiometers can be particularly troublesome.

\subsection{Optical Coatings on Laser Facets}

Operating a diode laser in an extended-cavity configuration works best if the reflectance of the laser's output facet is very low. ECDLs are inherently coupled cavity systems and are known to have operating regions of stability and instability [21-23]. By reducing the reflectance of the output facet the performance of the ECDL is improved: the size of the stable operating region (in temperature, current, and feedback power) is increased, the usable output power is increased, and, most importantly, the laser's tuning range is increased.

In Eq. (1) we see the strong dependence of the laser threshold current on the reflectance of the facets. This is illustrated in Fig. 2, which shows the $P-I$ curves of a laser before and after the output facet is antireflection (AR) coated. Almost all commercial lasers come from manufacturers with coatings already on the laser's facets. These coatings serve two purposes: first, they protect the facets from degradation, and, second, they adjust the facet reflectance to optimize the output power. Since the early days of diode lasers, $\mathrm{Al}_{2} \mathrm{O}_{3}$ coatings have been used to reduce facet degradation and increase the lifetime of diode lasers. We typically find that commercial low-power $(P .10 \mathrm{~mW})$ AlGaAs lasers have single-layer coatings on both facets that are approximately $\lambda / 2$ in optical thickness. In most cases we do not know with certainty what coating material has been used. Empirically we often (but not always) find that the laser coatings have an index of refraction of about 1.60 , which is consistent with $e$-beam vapor-deposited $\mathrm{Al}_{2} \mathrm{O}_{3}$. Higherpower lasers $(\& 15 \mathrm{~mW}$ ) typically have a similar coating on the output facet, except that the thickness is approximately $\lambda / 4$ (which results in a reflectance of a few percent). The back facet of the high-power lasers typically has a multilayer coating with a reflectance of $90 \%$ or more.

Most commercial lasers can be improved for use in extended-cavity configurations by reducing the reflectance of the output facet. The majority of laser manu- 
facturers are not interested in putting special coatings on diode lasers for those users who need a few inexpensive lasers. In order to transform general-purpose lasers into ECDLs, we have found it advantageous to develop some simple coating capabilities in our own lab. Fortunately, the equipment needed to coat diode lasers is readily available from the optical coating/semiconductor industry. For example, inexpensive (can opener-like) tools are available for removing the caps from the standard hermetically sealed semiconductor packages. Otherwise, all that is really needed is a simple vacuum coating system with electrical feedthroughs.

Our coating techniques are based on traditional dielectric coating methods but are directed toward the specific problem of modifying the facet reflectance of semiconductor lasers. We generally use common coating materials (e.g., $\mathrm{Al}_{2} \mathrm{O}_{3}$, $\mathrm{HfO}_{2}, \mathrm{SiO}$ ) and well-established techniques (thermal evaporation, electron beam deposition, and RF sputtering). If one does not want to spend time characterizing the unknown coatings on the lasers and carefully calibrating thickness monitors, it is easiest to do the coatings by monitoring the change in threshold while the laser is being coated. During the coating process the laser injection current is ramped through threshold with a repetitive triangular sweep and the power out of the back facet is monitored. Many lasers already have a monitor photodiode behind them that can be used for this purpose.

Diode laser coatings have been studied in detail, and good-quality AR and mirror coatings have been developed. Some very useful information can even be found in the published literature, but often details are lacking because they are proprietary in nature. The lowest-reflectance coatings that have been reported on diode lasers range from $10^{-4}$ to $10^{-5}$. These results have often been achieved by laboratories working on optical amplifiers, in which case it is necessary to suppress lasing altogether while maintaining very high optical gain.

Two relatively simple AR coatings that work reasonably well on commercial lasers are: (i) thermally evaporated silicon monoxide and (ii) $e$-beam-deposited $\mathrm{HfO}_{2}$ and/or $\mathrm{Al}_{2} \mathrm{O}_{3}$. Either of these coatings can achieve reflectances below $10^{-3}$, even on commercial lasers that already have some coating on their facets. Silicon monoxide is convenient because the equipment required for thermal evaporation is relatively simple. It also has the useful (but challenging) property that changing the oxygen pressure in the coating chamber changes the oxygen composition $(x)$ in the film $\left(\mathrm{SiO}_{x}\right)$, and hence the index of refraction from about 1.6 to 2.0. With all coatings, but with $\mathrm{SiO}_{x}$ in particular, the apparent index of refraction of the coating can change over time as the laser is exposed to and operated in air.

An AR coating that we use regularly for semiconductor lasers is a dual coating of $\mathrm{Al}_{2} \mathrm{O}_{3}$ and $\mathrm{HfO}_{2}$. We apply this coating using a standard electron beam evaporation source. Both of these materials produce good-quality optical coatings that are compatible with most of the commercial lasers that we have tested. To reduce the facet reflectance of commercial lasers we first add $\mathrm{Al}_{2} \mathrm{O}_{3}$ (when needed) to bring the laser's base coating thickness to approximately $\lambda / 2$, and then we put 
down a $\lambda / 4$ layer of $\mathrm{HfO}_{2}$ (deposited index approximately 1.89). The optical thickness of coatings is monitored by watching the change in laser threshold while the laser is being coated. This particular two-layer coating is relatively easy to monitor because the threshold reaches an extremum at or near the proper thickness for both layers.

\subsection{Diode Laser Frequency Noise and Stabilization}

We draw a clear distinction between two aspects of laser frequency stabilization. The first concerns the reduction in the drift and low-frequency fluctuations (jitter) of the laser's center frequency, and the second concerns the faster fluctuations that are responsible for the "fast linewidth" of the laser. The general aspects of frequency stabilization of lasers are addressed in other chapters (Zhu and Hall, Chapter 5 in this volume) and elsewhere. We will concentrate here on those aspects particularly relevant to diode lasers [5-7]. An excellent discussion of the subject is given by Telle [39].

First, the optical cavity of a solitary diode lasers is very small and the facet reflectance is not very high, which means that the $Q$ of the resonator is low and the resulting linewidth of the laser is relatively large (typically $10-200 \mathrm{MHz}$ ). In addition, a number of physical processes affect the spectral width of diode lasers. These include carrier density and temperature fluctuations, pump fluctuations, and spontaneous emission. These effects can be amplified by the strong coupling between the phase and amplitude of the lasing field. This coupling causes the linewidth of diode lasers to be increased above the fundamental SchawlowTownes limit by a factor of $\left(1+\alpha^{2}\right)$, where $\alpha$ (typically $\left.3-10\right)$ is the ratio of the change in the real to the imaginary part of the susceptibility of the gain mediun.

\subsubsection{Electronic Control}

An inexact but useful rule of thumb for frequency control servo systems is that the bandwidth of the servo needs to be somewhat greater than the linewidth of the laser before the servo can actually reduce the laser's linewidth. Solitary lasers have linewidths that are typically tens of $\mathrm{MHz}$ wide, which means that it is difficult, but not impossible, to achieve servo-loop bandwidths that are high enough to narrow the linewidth. Even though the frequency of the laser light responds on nanosecond time scales to changes in the injection current, other factors limit the loop response time. Very fast electronics must be used, and the physical size of the system must be small to minimize propagation delays. It is necessary to compensate phase shifts in the transfer function of injection current to laser frequency and also the time delays inherent in the frequency discriminator (for example, a Fabry-Perot cavity). 


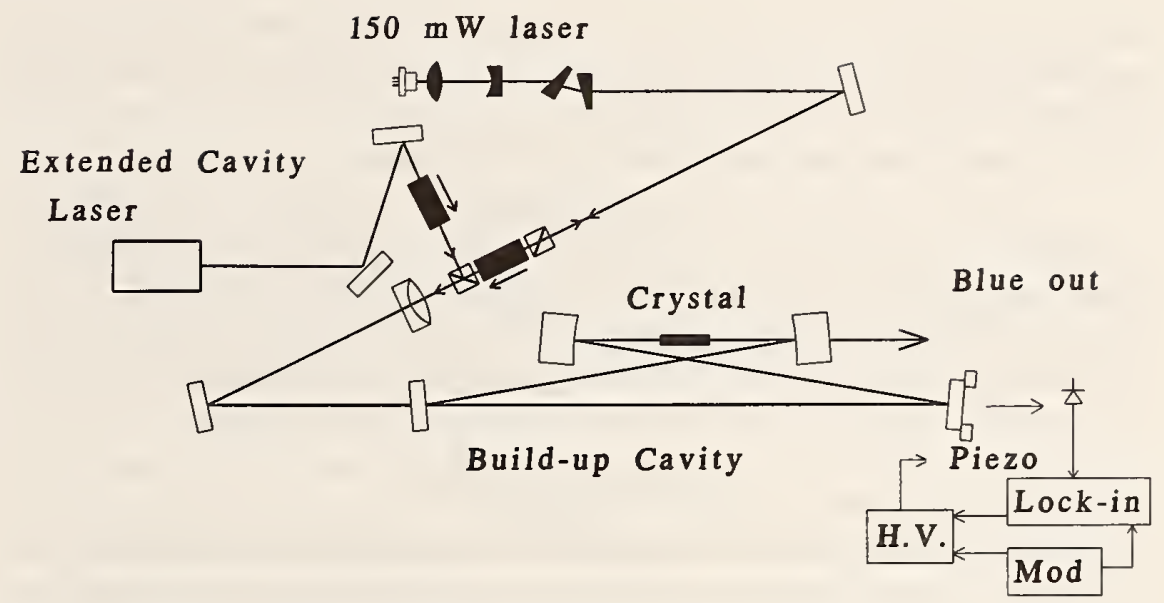

FIG. 8. Frequency-doubled diode laser. In this system a grating-tuned ECDL provides tuning and high spectral purity, which is then used to control the frequency of a higherpower diode laser by injection locking. Injection about $5 \mathrm{~mW}$ from the ECDL into the slave laser provides about $150 \mathrm{~mW}$ of tunable output power. The spatial mode of the slave laser is asymmetric $\left(\theta_{\perp} / \theta_{\|} \sim 3\right)$ and has a small amount of astigmatism (about $3 \mu \mathrm{m}$ ); these are corrected by using an anamorphic prism pair and a cylindrical lens. After spatial mode correction and passing through an optical isolator, there are about $105 \mathrm{~mW}$ remaining in the beam that is incident upon the ring buildup cavity that contains the $\mathrm{KNbO}_{3}$ crystal. The combined mode matching and coupling efficiency of this beam into the ring is about $75 \%$. Usable blue output powers as high as $45 \mathrm{~mW}$ are obtained in a good stable spatial and temporal mode near $425 \mathrm{~nm}$.

\subsubsection{Optical Control}

Resonant optical feedback from a confocal cavity (Fig. 3e) can be used to narrow the linewidth and simultaneously stabilize the center frequency of diode lasers [2]. With this method optical feedback occurs only in the narrow spectral windows defined by the confocal Fabry-Perot resonances. When the solitary laser's frequency is tuned within the optical capture range of a Fabry-Perot resonance, the resulting resonant feedback causes the laser to lock its frequency to the nearby cavity resonance. The laser's fast linewidth is thus reduced by a factor of about 1000 (to $<10 \mathrm{kHz}$ ), and the center frequency is then automatically stabilized to the cavity resonance. This system does not require modification of the solitary diode laser and typically operates in the weak feedback regime, $P_{\text {feedback }} / P_{\text {out }} \cdot 10^{-3}$. A limitation of this optical locking technique is that the tuning range of the laser is still restricted to those wavelengths accessible by the solitary laser alone. Although no active control is needed to optically lock a laser to a 
cavity, electronic servos will be necessary for stable long-term operation at a specific frequency.

\subsubsection{Combined Optical and Electronic Control}

As with most tunable lasers, the lasing frequency of ECDLs is controlled by a mechanical system (the grating angle and cavity length) that is susceptible to perturbation and drift. Active frequency stabilization can be done using some sort of frequency reference (such as a Fabry-Perot cavity or an atomic resonance) and an electronic control system. The error signal from the frequency reference is processed by a loop filter and fed back to control the laser frequency. The main feedback path is usually to a PZT that controls the laser cavity length. The frequency stability that can be achieved using only a PZT loop is usually adequate for most applications, but when linewidths less than about $50 \mathrm{kHz}$ are needed it is relatively easy to use an additional feedback loop to the injection current (or to an intracavity modulator) to further narrow the laser linewidth. These additional feedback paths have higher servo bandwidths because they are not limited in speed by having to move the mass of a mirror or grating. With servo bandwidths of $\geq 1$ $\mathrm{MHz}$ it is even possible to phase-lock a diode laser to a reference laser source.

\subsection{Extending Wavelength Coverage}

Present technology is now reasonably good at controlling the spectral characteristics of diode lasers, but these lasers may not be available at the wavelengths that we require (Fig. 1). Fortunately, new wavelengths are on the horizon. There are promising research results with blue lasers using $\mathrm{ZnSe}$ and $\mathrm{GaN}$, and the Sb-based IR lasers also have great potential. In the meantime, we can use the existing diodes and nonlinear optical techniques to extend wavelength coverage.

Good results have been reported by using diode lasers and difference-frequency generation (DFG) to produce single-frequency tunable infrared light [40]. The chalcopyrite crystals $\mathrm{AgGaS}_{2}$ and $\mathrm{AgGaSe}_{2}$ and periodically poled lithium niobate are well suited to this application because they have large nonlinear coefficients and they phase match for diode laser wavelengths. In fact, it appears that almost the entire spectral region between 2 and $18 \mu \mathrm{m}$ could be covered by using these crystals and diode lasers as the input.

In the opposite wavelength direction there is a need for good tunable lasers in the blue and UV based on solid-state sources. Fortunately, nonlinear crystals such as $\mathrm{KNbO}_{3}, \mathrm{KTP}, \mathrm{LiNbO}_{3}, \mathrm{BBO}$, and $\mathrm{LiIO}_{3}$ can be used for second-harmonic generation (SHG) and sum frequency mixing of diode laser light [41]. With these techniques and materials the region between 200 and $500 \mathrm{~nm}$ is accessible. For example, the SHG system developed in our lab by C. Weimer is diagrammed in Fig. 8. This system uses $\mathrm{KNbO}_{3}$, which is a particularly good example because it 
has a large nonlinear coefficient, and it noncritically phase matches for AlGaAs diode laser wavelengths. Starting with an injection-locked $150 \mathrm{~mW}$ diode laser, this system produces more than $40 \mathrm{~mW}$ of tunable light near $425 \mathrm{~nm}$. An analogous system that uses angle phase matching in $\mathrm{LiIO}_{3}$ produces about $200 \mu \mathrm{W}$ of 405 $\mathrm{nm}$ light for an input power of $40 \mathrm{~mW}$ at $810 \mathrm{~nm}$.

From recent publications and conference proceedings we can anticipate that higher-power lasers will soon be available in many wavelength regions. As the technology improves we will be able to incorporate these lasers and MOPAs with various nonlinear optical materials to greatly extend useful wavelength coverage.

\section{Acknowledgments}

Our thanks to V. L. Velichansky, C. W. Weimer, N. Mackie, T. Zibrova, H. G. Robinson, J. Marquardt, S. Waltman, M. Stephens, and L. Mor for contributions to this work.

\section{References}

Given the length and general nature of this chapter, we provide only a very basic list of references that might serve as a starting point for further study.

1. Kressel, H., and Butler, J. K. (1977). Semiconductor Lasers and Heterojunction LEDs, Academic Press, New York.

2. Casey Jr., H. C., and Panish, M. B. (1978). Heterostructure Lasers, Parts A and B, Academic Press, New York.

3. Thompson. G. H. B. (1980). Physics of Semiconductor Laser Devices, Wiley, New York.

4. Yariv, A. (1989). Quantum Electronics, 3rd edition, Wiley, New York.

5. Petermann, K. (1988). Laser Diode Modulation and Noise, Kluwer Academic, Dordrecht, The Netherlands.

6. Yamamoto, Y., ed. (1991). Coherence, Amplification, and Quantunı Effects in Seniconductor Lasers, Wiley, New York.

7. Ohtsu, M. (1992). Highly Coherent Semiconductor Lasers, Artech House, Boston.

8. Zory. P. S.. ed. (1993). Quantun Well Lasers, Academic Press, Boston.

9. Camparo, J. (1985). Contemp. Plyss. 26, 443.

10. Ohtsu, M., and Tako, T.'(1988). In Progress in Optics, Volume 25, E. Wolf (ed.), Elsevier, Amsterdam.

11. Lawrenz, J., and Niemax, K. (1989). Spectrochim. Acta 44B, 155.

12. Wieman, C., and Hollberg, L. (1991). Rev. Sci. Instrum. 62, 1, and the references therein.

13. Tino, G. (1994). Phys. Scripta. T51, 58-66. 
14. Niemax, K., ed. (1993). Spectrochim. Acta Rev. 15(5).

15. Suematsu, Y., and Adams, A. R., eds. (1994). Handbook of Semiconductor Lasers and Photonic Integrated Circuits, Chapman and Hall, London.

16. Choi, H. K., Walpole, J. N., Turner, G. W., Eglash, S. J., Missaggia, L. J., and Connors, M. K. (1994). IEEE Photon. Tech. Lett. 6, 7, and the references therein.

17. Franzke, J., Schnell, A., and Niemax, K. (1993). Spectrochim. Acta Rev. 15(5), 379-395; see also general diode laser references [1-7, 14].

18. Bonnell, L. J., and Cassidy, D. T. (1989). Appl. Opt. 28, 4622-4628; Ruprecht, P. A., and Brandenberger, J. R. (1992). Opt. Comm. 93. 82-86.

19. Welch, D. F., Parke, R., Mehuys, D., Hardy, A., Lang, R., O’Brien, S., and Scifres, D. S. (1992). Electron. Lett. 28, 2011-2013.

20. Kasuya, T., Suzuki. T., and Shimoda. K. (1978). Appl. Phys. 17, 131-136.

21. Lang, R., and Kobayashi, K. (1980). IEEE J. Quantum Electronics QE-16, 347-355.

22. Tkach, R., and Chraplyvy, A. (1986). J. Lightwave Tech. LT-4, 1655-1661.

23. Tromborg, B., and Mørk, J. (1990). IEEE J. Quantum Electronics 26, 642-654; Mørk, J., Tromborg, B., Mark, J., and Velichansky, V. L. (1992). Proc. SPIE 1837, and the references therein.

24. Zorabedian, P. (1994). IEEE J. Quantum Electronics 30, 1542, and the references therein.

25. Fleming, M. W., and Mooradian. A. (1981). IEEE J. Quantum Electronics QE-17, 44-59.

26. Fukuda, M. (1991). Reliability and Degradation of Semiconductor Laser and LEDs, Artech House, Boston.

27. Belenov, E. M., Velichansky, V. L., Zibrov, A. S., Nikitin, V. V., Sautenkov. V. A., and Uskov, A. V. (1983). Sov. J. Quantum Electronics 13, 792: Akul'shin. A.. Bazhenov. V., Velichansky, V., Zverkov, M., Zibrov. A., Nikitin, V., Okhotnikov, O., Sautenkov. V., Senkov, N., and Yurkin, E. (1986). Sov: J. Quantum Electronics 16, 912.

28. Boshier, M. G., Berkeland, D. J., Hinds, E. A., and Sandoghadar, V. (1991). Opt. Comm. 85, 355-359.

29. MacAdam, K. B., Steinbach, A., and Wieman, C. (1992). Am. J. Phys. 60, 1098-1111.

30. Duarte, F. J. (1993). Laser Focus World. pp. 103-109. February.

31. Harvey. K., and Myatt. C. (1991). Opt. Lett. 16. 910.

32. Liu. K.. and Littman. M. G. (1981). Opt. Lett. 6. 117: McNicholl. P.. and Metcalf. H. J. (1985). Appl. Opt. 24. 2757.

33. Day, T., Luecke, F., and Brownell, M. (1993). Lasers Optronics. pp. 15-17, June: Day, T., Brownell, M., and Wu. 1.-Fan (1995). Proc. SPIE 2378, 37-41.

34. Velichansky, V. L., Zibrov, A. S., Molochev, V. I., Nikitin, V. V., Sautenkov, V. A., Tyurikov, D. A., and Kharisov, G. G.. (1981). Sov. J. Quantum Electronics 11. 1165-1171.

35. Bazhenov, V. Yu., Bogatov, A. P.. Eliseev, P. G., Okhotnikov, O. G., Pak. G. T., Rakhvalsky, M. P., Soskin. M. S., Taranenko. V. B.. and Khairetdinov, K. A. (1982). IEEE Proc. 129(1), 77-82. 
36. Bradley, C. C., Chen, J., and Hulet, R. G. (1990). Rev. Sci. Instrum. 61, 2097.

37. Libbrecht, K. G., and Hall, J. L. (1993). Rev. Sci. Instrum. 64, 2133-2135.

38. Van Baak, D. A. (1992). Am. J. Phys. 60, 803-815.

39. Telle, H. (1993). Spectrochim. Acta Rev. 15, 301-327.

40. Simon, U., Waltman, S., Loa, I., Tittel, F. K., and Hollberg, L. (1994). J. Opt. Soc. Am. B 12, 323-327; Petrov, K. P., Waltman, S.. Dlugokencky, E. J., Arbore. M., Fejer, M. M., Tittel, F. K., and Hollberg, L. (1997). Appl. Phys. B, and the references therein, to be published.

41. Risk, W. P., and Kozlovsky, W. J. (1992). Opt. Lett. 17, 707-709, and the references therein. 


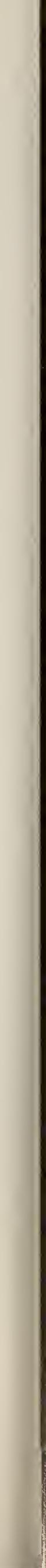


Part II

Tunable Laser Systems 



\title{
Chapter 5
}

\section{CW DYE LASERS}

\author{
Leo Hollberg \\ National Institute of Standards and Technology \\ (formerly National Bureau of Standards) \\ Boulder, Colorado
}

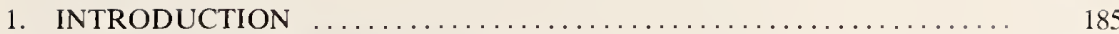

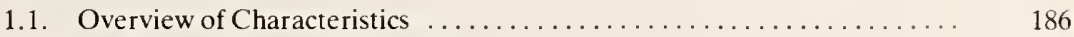

1.2. Brief History of the CW Dye Laser . . . . . . . . . . . . . . . . . . . . 186

1.3. Other Related Laser Systems . . . . . . . . . . . . . . . . . . . . . . . . . . 188

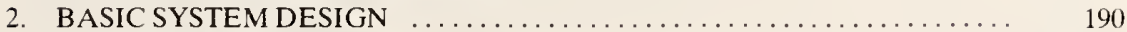

2.1. Example Systems ... . . . . . . . . . . . . . . . . . . . . . . . . . . . . 190

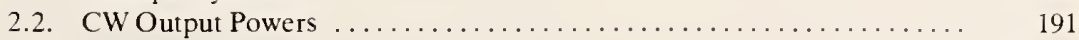

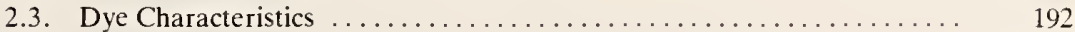

2.4. Pump Sources ... . . . . . . . . . . . . . . . . . . . . . . . . . . . . . . . . 197

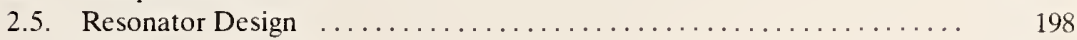

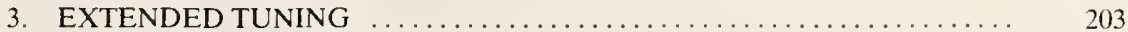

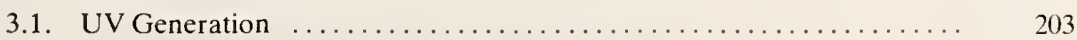

3.2. IR Generation by Difference-Frequency Mixing ............... 205

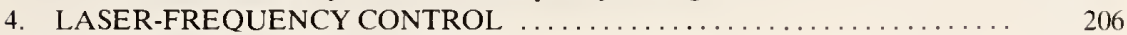

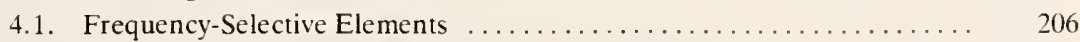

4.2. Frequency-Stabilization Methods ....................... 214

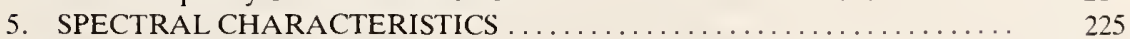

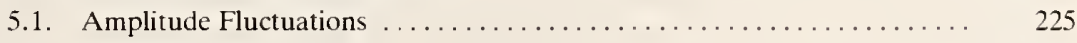

5.2. Frequency-Fluctuations ............................... 228

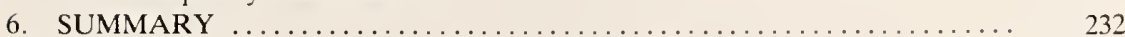

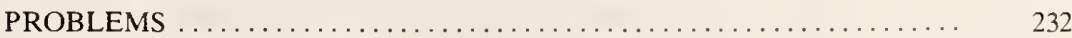

REFERENCES ........................................ 233

\section{INTRODUCTION}

The cw dye laser is a well-established tool of optical science. It plays the premier role as a source of tunable radiation in the visible and near-visible regions of the spectrum. This laser has a unique set of capabilities that include broad tunability, high power, and the potential for extremely high

${ }^{*}$ I gratefully acknowledge the helpful discussions and contributions made to this chapter by Dr. J.C. Bergquist, Dr. T.F. Johnston. Jr., Dr. J.L. Hall. Dr. S.L. Gilbert, and Dr. M. Young. 
resolution. Because of these capabilities we overlook its high cost, sometimes complicated design, and the requirement of a high-power optical pump. No other optical source can provide a comparable combination of tunability, resolution, and power.

\subsection{Overview of Characteristics}

The most important attribute of the dye laser is its tunability, which gives the user access to essentially any wavelength in the visible and near-visible spectrum. The spectral range of ion-laser-pumped $\mathrm{cw}$ dye lasers is essentially complete coverage from 365 to $1000 \mathrm{~nm}$. It is even possible to extend their $\mathrm{cw}$ tuning range by using nonlinear optical methods to generate wavelengths further into the ultraviolet and infrared. With the addition of these methods of nonlinear optical synthesis, the spectral range for complete coverage with $\mathrm{cw}$ radiation has been extended to $250 \mathrm{~nm}$. Radiation at even shorter wavelengths has been generated for special applications. In addition nonlinear methods have been used to generate radiation in the $2-5 \mu \mathrm{m}$ infrared region. This range of spectral coverage will certainly be extended in the future.

The power levels available from cw dye lasers are generally more than adequate for spectroscopic applications. The output power of cw dye lasers varies with the type of dye, but typical cw systems produce between .1 and 1 watt of output power. As with most things, there are some applications with almost unlimited demand for power. Special high-power cw-dye-laser systems have been developed that can produce tens of watts of tunable visible radiation.

With broad tuning ranges and narrow linewidths, single-mode cw dye lasers can provide an impressively large number of resolution elements. For example, a standard single-frequency dye laser (linewidth $\approx 1 \mathrm{MHz}$ ) operating with rhodamine $6 \mathrm{G}$ dye (tuning range $\approx 100 \mathrm{~nm}$ or $\approx 10^{14} \mathrm{~Hz}$ ) is capable of resolving $\approx 10^{8}$ spectral elements across its tuning curve. If we considered one of the special high-resolution lasers that have been demonstrated (linewidths less than $1 \mathrm{kHz}$ ) the number of resolution elements is $\approx 10^{11}$, probably more than any application can actually use and certainly one of the highest of any electromagnetic source.

\subsection{Brief History of the CW Dye Laser}

Historically, the development of the $\mathrm{cw}$ dye laser was an outgrowth of research into the photophysics of dye-laser action in pulsed dye lasers (Schäfer et al., 1966; Snavely and Schäfer, 1969). The first flashlamppumped dye lasers were developed in 1967 (Schmidt and Schäfer 1967; Sorokin and Lankard, 1967), just one year after the first successful pulsed 
dye lasers, which were achieved using ruby lasers as the pump source (Sorokin and Lankard, 1966: Spaeth and Bortfeld, 1966; Stepanov et al. 1967). With flashlamp pumping, sufficient population inversion could be attained with surprisingly long output-pulse lengths. The pulse lengths were longer than expected because the trapping of molecular excitation in the unwanted triplet states was less probable than expected. The other important contribution that allowed $\mathrm{cw}$ operation was the development of a system to flow the dye rapidly through the pumping region in order to reduce the optical inhomogeneities induced in the dye by heating from the cw pump laser. In 1970 Peterson et al. (1970) demonstrated the first cw lasing of an organic dye. That laser used the dye, rhodamine $6 \mathrm{G}$, with an argon-ion laser as the pump. Two decades later, the combination of the argon-ion laser and the rhodamine $6 \mathrm{G}$ dye is the most common, and in some ways, the best cw dye-laser system that exists.

That first $\mathrm{cw}$ dye laser appears extremely simple, but the design is very clever in solving some of the serious technical problems. Figure 5.1 is a simplified diagram of the type of laser design that was used in that first successful demonstration of a $\mathrm{cw}$ dye laser. This laser has the dye flowing between two dichroic mirrors that form the laser resonator as well as part of the containment cell for the dye. The argon-ion laser pumping beam was injected collinearly through one of the dichroic mirrors. The Peterson et al. (1970) laser of this type produced an output power of $30 \mathrm{~mW}$ in a spectral width of $3 \mathrm{~nm}$ when pumped with $1 \mathrm{~W}$ of 514-nm (argon ion) laser light. We can get some idea of the technological evolution of dye lasers by

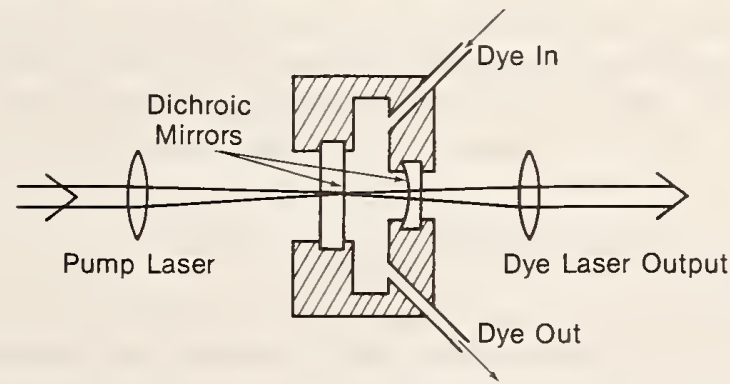

Fig. 5.1 Cross section of the type of dye laser used for the first successful cw operation. The two dichroic mirrors serve the dual role of containing the dye fluid and acting as the dye-laser resonator. The dichroic mirrors transmit the blue-green pump laser beam but are high reflectors for the reddish fluorescence from the dye. The pump-laser beam is derived from an $\mathrm{Ar}^{+}$laser. A lens is used to focus the pump beam into the dye cell at the dye-laser resonator waist which is on the flat dichroic mirror. The dye flows rapidly through the cell to remove the excess heat and triplet state population. With this type of laser Peterson et al. (1970) achieved $\approx 30 \mathrm{~mW}$ of $\mathrm{cw}$ dye-laser output power at $597 \mathrm{~nm}$ when the laser was pumped with $\simeq 1 \mathrm{~W}$ of $514-\mathrm{nm}$ light. 
comparing these results with the performance characteristics of modern lasers, which can produce $1 \mathrm{~W}$ of output in a spectral width of about $10^{-6} \mathrm{~nm}(\approx 1 \mathrm{MHz})$ with a pump power of $6 \mathrm{~W}$. More detailed discussion of the early cw dye lasers can be found in Tuccio and Strome (1972).

Development of the $\mathrm{cw}$ dye laser since 1970 has been extensive and continues in many labs around the world today. As in most technologies, progress in the development of the $\mathrm{cw}$ dye laser has involved a gradual improvement in the design of each of its components, combined with a few important discoveries that have significantly altered the basic system. The important achievements will be discussed in later sections, but it is useful to have some historical perspective of the development. One of the important developments that improved the performance of the dye laser was the introduction in 1972 (Runge and Rosenberg, 1972) of the jet-stream dyecirculation system, which gave more reliable high-power operation. About that same time techniques were developed that allowed frequency stabilization of these lasers and thus greatly enhanced their spectral resolving power (Barger et al., 1973, 1975; Liberman and Pinard, 1973; Schröder et al., 1973, 1975; Wu et al. 1974, Hall and Lee, 1976b). The widespread use of traveling-wave ring lasers started in the late $70 \mathrm{~s}$ and improved both the spectral characteristics and the power output from the $\mathrm{cw}$ dye lasers (Schröder et al., 1977; Jarret and Young, 1979; Johnston et al., 1982). The incorporation of computer control has provided automatic long-range tuning and enhanced data-collection capabilities (Marshall et al., 1980; Williams et al., 1983; Clark et al., 1986). In addition to the continual improvement of laser dyes the ' 80 s have seen the application nonlinear optical techniques that extended the spectral coverage that was possible with $\mathrm{cw}$ lasers into the UV and IR regions (Blit et al., 1977, 1978; Couillaud, 1981; Couillaud et al., 1982; Bergquist et al., 1982; Hemmati et al., 1983; T. F. Johnston, Jr., 1987; T. Johnston, 1988; Pine, 1974, 1976; Oka, 1988).

\subsection{Other Related Laser Systems}

Dye lasers are often the only choice or the best choice of laser for a given application, particularly if it is necessary to tune to a specific wavelength. In the dye laser's region of operation, only a few laser sources are competitive and those are only in limited parts of the dye laser's spectral region. A few new tunable lasers are beginning to threaten some of the traditional domains of the dye laser. These include semiconductor, color-center, Ti-sapphire, alexandrite, and a few optically pumped solid-state lasers. Other nontunable lasers exist that oscillate in the visible and near-visible region of the spectrum; for example, the $\mathrm{Kr}$-ion and Ar-ion lasers have many useful lasing lines, and the optically pumped dimer lasers (sodium, 
lithium, iodine, etc.) have hundreds of lines, but the lines are relatively narrow and, even when combining all of these lasers, the actual fractional coverage of the available spectrum is small.

Semiconductor lasers are now available in some parts of the near-IR and red regions of the spectrum. As of the late 1980s, in comparison with dye lasers the semiconductor lasers generally have large linewidths and low powers. But the characteristics of semiconductor lasers are improving and their future looks quite bright.

The broad class of optically pumped lasers shares many of the characteristics of the cw dye laser-for example, color-center lasers (Mollenauer, 1985; Mollenauer and White, 1987). Two important, and relatively new optically pumped solid-state lasers are the Ti-sapphire laser (Schulz, 1988) and the LNA (Lanthamide hexa-aluminate) laser (Schearer et al., 1986). The designs of these lasers, as well as that of color center lasers, are very similar to the designs of $\mathrm{cw}$ dye lasers. The Ti-sapphire laser is an interesting example of a laser competing for some of the domain dominated by the dye laser (Ti-sapphire tuning range $\simeq 700-1000 \mathrm{~nm}$ ). These lasers use the same pumping source and essentially the same cavity design, but they have the dye medium replaced by a solid-state material. Most of the design features discussed here for dye lasers apply equally well to other optically pumped lasers. True, some types of lasers produce higher power, and some yield higher spectral purity, but the dye laser combines both of these traits with broad tunability and the ability to change dyes for even broader spectral coverage.

This chapter is not intended to be a comprehensive review of cw dye lasers but rather a description of the basic principles of some common $\mathrm{cw}$ dye lasers. Keep in mind that the descriptions are simply examples and the possible variations and extensions are large and often unexplored. This material is but a glimpse at the field from one perspective, where I have additionally chosen to cover in more detail those aspects that have been neglected in other sources, and to give less emphasis to material that is covered well elsewhere. Effort is made to give reference to other sources of material on cw dye lasers but the reference list is not complete. It is rather a place to start for further study. In this light it is important to note some of the review articles and books that are particularly relevant to the subject of cw dye lasers, including Shank (1975), Snavely (1977), Schäfer (1977), Peterson (1979), and Mollenauer and White (1987). Of paramount interest is the excellent article by T. F. Johnston (1987) which is a review of dye lasers. Applications of dye lasers are discussed by Hänsch (1976) and Hall (1978). In addition, a great deal of useful information about the design and characteristics of cw dye lasers can be obtained from the instruction manuals of commercial laser systems. 


\section{BASIC SYSTEM DESIGN}

\subsection{Example Systems}

The years since 1970 have seen the development of a large number of $\mathrm{cw}$ dye-laser designs. Two examples of dye-laser systems that have seen widespread use are the three-mirror folded-linear cavity and the four-mirror unidirectional-ring cavity. These systems are diagrammed in Fig. 5.2. We see some features that are common to both designs; for example, both use

a)

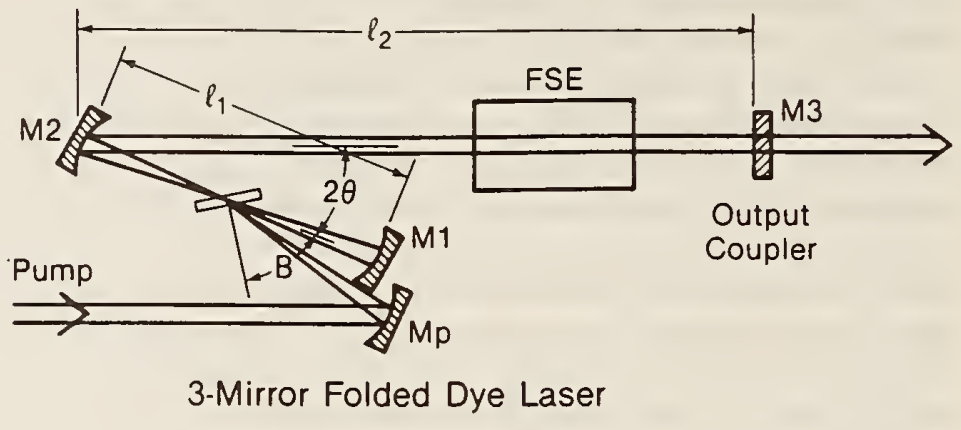

b)

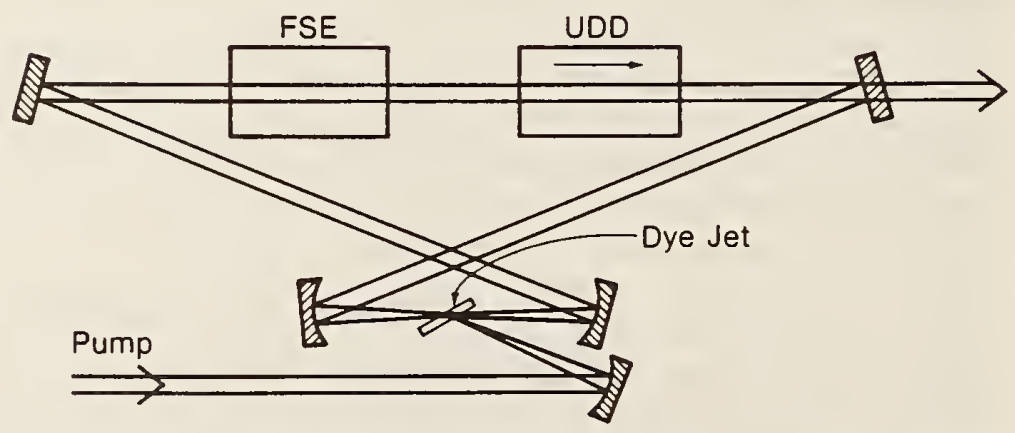

4-Mirror Unidirectional Ring Laser

Fig. 5.2 Two popular $\mathrm{cw}$ dye-laser resonator configurations. The standing wave threemirror resonator shown in (a) has a folded geometry with two short-radius curved mirrors (M1 and M2) and a flat output coupler, M3. The curved pump mirror, Mp. focuses the pump beam on the dye jet, which is placed between M1 and M2 at the waist of the dye-laser resonator. FSE represents any frequency-selective elements that might be used inside the laser's resonator. In (b), another flat mirror has been added and the resonator is transformed into a figure-8-shaped ring. This resonator also has a unidirectional device, which ensures that the laser oscillates as a traveling wave, propagating in only one direction around the ring. 
a free-flowing jet stream for the dye gain medium and a tightly focused ion-laser beam for the pump source. The two mirrors with short radii of curvature, which surround the dye jet, match the dye-laser cavity mode to the small pump spot. This strongly pumped region of the dye provides a high gain over a very short interaction length. The high gain allows flexibility in designing the other parts of the dye-laser resonator. The remaining components of these two resonators are chosen for specific attributes or applications and many variations of these basic systems are found. The frequency-selective elements, FSE, and the unidirectional device, UDD, in the ring laser, indicated schematically in Fig. 5.2, are components that control the frequency and lasing direction, respectively, and will be discussed in detail in a later section.

Some of the early cw dye-laser designs (which have not survived the test of time) used glass cells to contain the gain medium. In almost all modern cw systems the dye cells have been replaced by free-flowing dye jets. The introduction of the dye jet (Runge and Rosenberg, 1972) and its subsequent development (for example, Harri et al., 1982) has been a major contribution to the success of the $\mathrm{cw}$ dye laser. The jets do not have the problem of the pump laser burning the dye on the cell windows. Also, much higher output powers can be obtained with dye jets, rather than cells, because more rapid flow rates are possible. Dye-cell lasers could have some advantage in low-power applications because they might have less frequency noise than the high-power dye-jet lasers. But in general the problems outweigh the advantages of the dye cells.

\subsection{CW Output Powers}

In the beginning it is useful to have a rough idea of the performance characteristics that can be expected from a modern cw dye laser. Curves for laser output power as a function of laser wavelength are shown in Fig. 5.3. These outstanding results are obtained for single-frequency cwlaser oscillation using a number of dyes, pump-laser lines, and changes of laser optics. One of the important things to notice here is that it is possible to have reasonably high output powers and single-frequency operation anywhere in the visible and near-visible spectrum (in fact from about 365 to $1000 \mathrm{~nm}$ ). The broad spectral coverage demonstrates the versatility of the dye laser. With different ion-laser pump sources and 11 different dyes it is possible to generate high-intensity, coherent radiation with powers of up to several watts. In exceptional cases output powers as high as $33 \mathrm{~W} c w$ have been obtained from dye lasers (Anliker et al., 1977).

The portion of the spectrum covered by the $\mathrm{cw}$ dye laser extends for more than an octave. The requirement of a large number of dyes is usually 


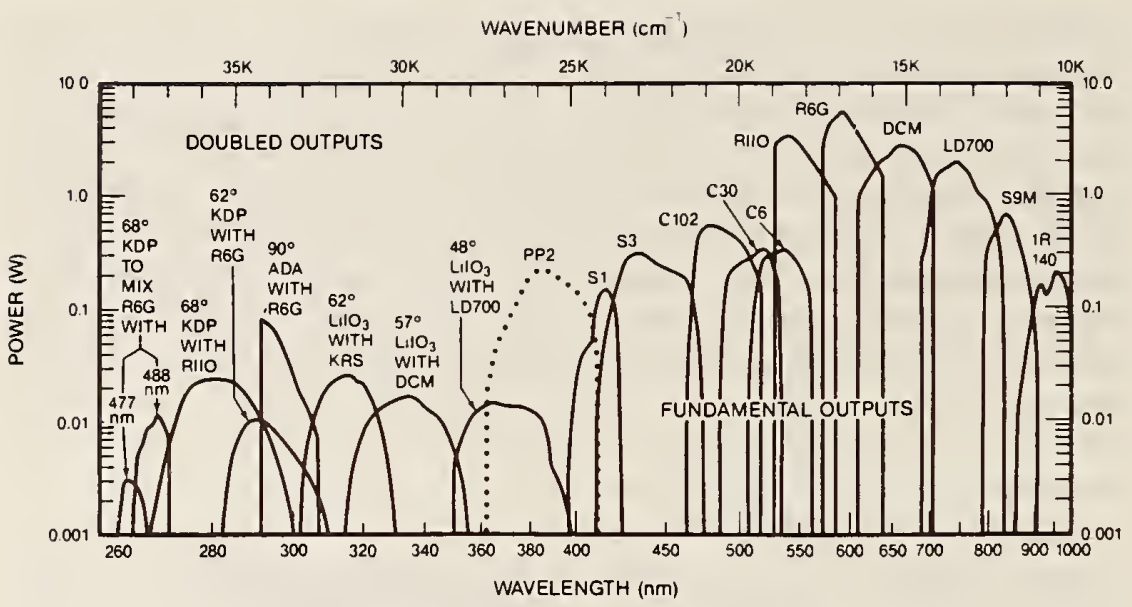

SINGLE FREQUENCY DYE TUNING SPECTRUM

Fig. 5.3 Tuning curves of single-frequency dye-laser radiation, including frequencydoubled and sum frequency mixed outputs. The vertical axis is single-frequency power output displayed as a function of wavelength. The direct dye-laser output spans the spectral region from the blue end of the dye PP2 at $=365 \mathrm{~nm}$ to the red end of the dye IR $1+0$ at $=1 \mu \mathrm{m}$. The outputs from the intracavity frequency doubled radiation extends from $\approx 395$ to $270 \mathrm{~nm}$. The two curves at the extreme blue and end show the single-frequency power obtained by intracavity sum-frequency-mixing the R6G dye laser radiation with argon-ion laser lines at 488 and $477 \mathrm{~nm}$. These curves are taken with permission from Johnston, T. F. (1987) and have been modified slightly to include the results from Johnston, T. (1988) for the dye PP2 (polyphenl 2).

not a problem for most applications because spectroscopic studies usually have spectral windows that are easily covered by one dye. In fact, with the high resolution (approximately one $\mathrm{MHz}$ ) of a single-frequency dye laser, there is often more spectroscopic resolution than is needed. Most of the UV outputs indicated in this figure $(260-400 \mathrm{~nm})$ are generated by using the strong visible dye-laser outputs and nonlinear optical-mixing methods.

\subsection{Dye Characteristics}

\subsubsection{Absorption and Emission Spectra}

The basic design of the $\mathrm{cw}$ dye laser is naturally constrained in fundamental ways by the photophysics and chemistry of the dye molecules. In Fig. 5.4 we see the representative absorption and emission spectra of a common laser dye. One of the important things to notice here is that the emission band is lower in frequency (red shifted) and is nearly the mirror image of the absorption band. In addition both the absorption and emission spectra are broadband features without sharp lines, indicative of a multitude of broadened mechanisms and overlapping energy levels. This is 


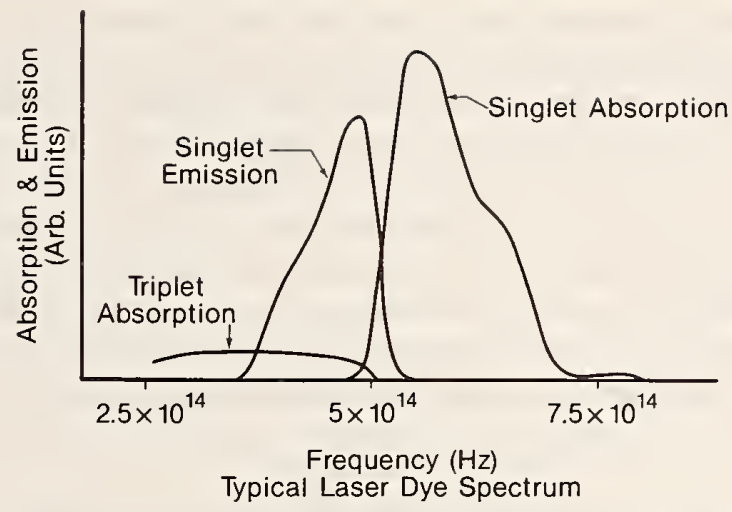

Fig. 5.4 Representation of typical absorption and emission spectra of a laser dye. The singlet emission spectrum is to the red and nearly the mirror image of the singlet absorption spectrum. Triplet absorption is smaller and typically extends even farther to the red than the singlet emission spectrum.

characteristic of large organic molecules in the liquid state and is depicted by a plausible energy-level diagram shown in Fig. 5.5. Here we see two manifolds of states, the singlets and the triplets, as designated by the net

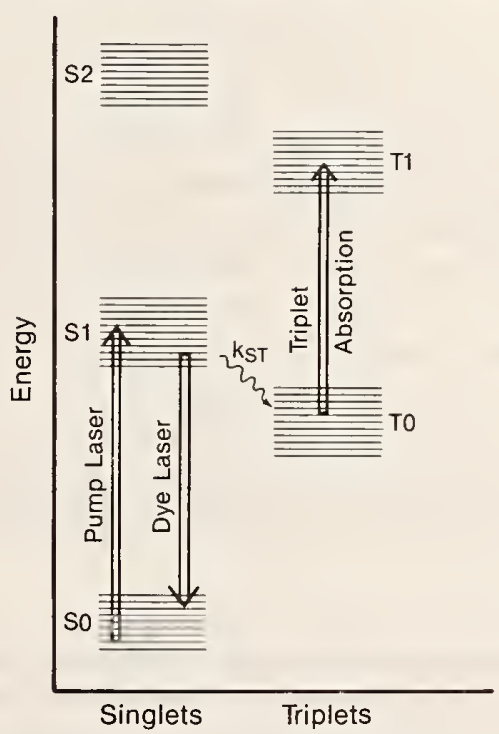

Fig. 5.5 Schematic picture of the energy levels of a generic laser dye, showing the singlet and triplet manifolds of states. The double lined arrows represent the optical redistribution of excitation by the pump laser, by the dye-laser emission (and dye fluorescence). and by triplet-state absorption. The arrow labeled $\mathrm{k}_{\mathrm{st}}$ represents the transfer of excitation from the singlet to the triplet manifolds. 
electronic spin. The transitions from the singlet manifold to the triplet manifold have low probability because they require an electron spin flip. The large organic-dye molecules have many internal degrees of freedom (vibration and rotation) that give rise to broad overlapping energy levels and spectra. The transfer of excitation within these energy bands can be very rapid, with typical time scales on the order of a picosecond. This energy transfer within a band is much faster than the spontaneous decay rates for interband transitions which have nanosecond time scales.

For laser oscillation the dye molecule's absorption band is excited by the intense pump laser from the lower part of the ground state, S0, to higher energy regions of the first excited singlet state S1 (Fig. 5.5). This excitation energy is then rapidly redistributed within the S1 state and reappears predominantly as fluorescence photons as the molecule decays by an electric-dipole transition from the S1 state to the S0 state. This transition in the singlet manifold from the first excited-electronic state S1 to the upper regions of the ground state $\mathrm{SO}$ is the lasing transition. It is obviously advantageous to have efficient conversion of the pump photons into fluorescence photons and to avoid the loss of the pump energy to other channels. The primary loss mechanism (indicated on Fig. 5.5) is the transition from the S1 state in the singlet manifold to the T0 state in the triplet manifold. This excitation, which reaches the triplet states, is detrimental for two reasons. First, it is a loss of available energy from the lasing singlet system. Second, the population in the $\mathrm{T} 1$ state can absorb photons by making transitions to higher levels in the triplet manifold, thereby creating additional loss for the laser system. The characteristic time scale for the transfer of population from the S1 state to the T0 state is typically $100 \mathrm{~ns}$. This needs to be compared with the time scales for other processes that affect the excited-state population. These are the fluorescence lifetime for the S1-S0 transition (typically a few nanoseconds) and the time scale for transfer of population within the Sl state (typically one ps). With the good laser dyes available today, the conversion efficiency, in terms of fluorescence photons ( $\mathrm{S} 1$ decays to $\mathrm{S} 0$ ) for pump photons, is in the range of 0.8 to 1.0. Note that this efficiency is not the energy-conversion efficiency because of the difference in color between the pump and fluorescence photons.

\subsubsection{Dye-Laser Gain and Threshold}

Following the treatments of Shank (1975) and Johnston (1987) and making some simplifying assumptions that ignore the actual spatial distribution of the pump and the dye laser intensities we can write the small-signal gain of the laser as

$$
\alpha_{0} \ell=\frac{N \ell \beta_{\mathrm{p}} I_{\mathrm{p}}\left(\sigma_{\mathrm{e}}-k_{\mathrm{st}} \tau \sigma_{\mathrm{t}}-\sigma_{\mathrm{a}}\right)}{h \nu_{\mathrm{p}}+\beta_{\mathrm{p}} I_{\mathrm{p}}\left(1+k_{\mathrm{st}} \tau\right)} .
$$


Here, $\beta_{\mathrm{p}}=\sigma_{\mathrm{p}} \tau \phi$, h is Planck's constant, $\nu_{\mathrm{p}}$ is the pump frequency, $\tau$ is the spontaneous emission lifetime and $\phi$ is fluorescence efficiency. This efficiency is the ratio of the fluorescence decay rate to the total decay rate for the S1 state and is usually near 1 for most good laser dyes. The quantities $\sigma_{\mathrm{p}}, \sigma_{\mathrm{a}}$, and $\sigma_{\mathrm{e}}$ are the singlet pumping, absorption, and emission cross sections, respectively. $N$ is the density of dye molecules, $\ell$ is the dye-jet interaction length, and $I_{\mathrm{p}}$ is the intensity of the pump laser. The factor $k_{\mathrm{st}}$ is the rate of transfer of population from the singlet to the triplet manifold and multiplies the triplet absorption cross section $\sigma_{\mathrm{t}}$. Above threshold the dye laser will show laser-intensity-dependent saturation as is usual for a homogeneously broadened system; that is,

$$
\alpha \ell=\frac{\alpha_{0} \ell}{1+I_{\mathrm{e}} / I_{\mathrm{s}}} .
$$

The saturation intensity $I_{\mathrm{s}}$ is a characteristic of the dye and is a function of the pump intensity and limits the gain as the dye laser intensity $I_{\mathrm{e}}$ increases.

For example, we can make the reasonable assumptions that the loss terms $\sigma_{\mathrm{a}}$ (which represents absorption of the dye-laser radiation by the dye itself) and $k_{\mathrm{st}}$ (the singlet-to-triplet transfer rate) are small enough to be ignored. Then Eq. (5.1) becomes

$$
\alpha_{0} \ell=\frac{N \ell \sigma_{\mathrm{p}} I_{\mathrm{p}} \tau \sigma_{\mathrm{e}}}{h \nu_{\mathrm{p}}+\sigma_{\mathrm{p}} I_{\mathrm{p}} \tau \phi} .
$$

The pump intensity at threshold can be estimated from Eq. (5.3) by equating the gain to the resonator losses. We shall represent the total resonator loss as $T$, which is the sum of the output coupling transmission and the actual scattering and absorption losses. The threshold pump intensity is then

$$
I_{\mathrm{p}, \mathrm{th}}=\left(\frac{h \nu_{\mathrm{p}}}{\sigma_{\mathrm{p}} \tau \phi}\right)\left(\frac{T}{N \ell \sigma_{\mathrm{e}}-T}\right) .
$$

The optimum transmittance of the output coupler for most cw dye lasers ranges from 1 to $15 \%$ and is almost always the dominant loss term for the laser resonator.

By using typical values for the dye rhodamine $6 \mathrm{G}$ and the parameters in Eq. (5.4), we can estimate the minimum required pump intensity for laser oscillation. For rhodamine $6 \mathrm{G}$ we expect $T \simeq 15 \%, \ell=.01 \mathrm{~cm}$, $N \simeq 10^{18} \mathrm{~cm}^{-3}, \sigma_{\mathrm{e}}=\sigma_{\mathrm{p}} \simeq 1 \times 10^{-16} \mathrm{~cm}^{2}, \nu_{\mathrm{p}}=5.8 \times 10^{14} \mathrm{~Hz}, \tau \simeq 3 \mathrm{~ns}$, and $\phi \simeq 1$. This gives a rough value for the pump intensity at threshold of $I_{\mathrm{p}, \mathrm{th}} \simeq 2 \times 10^{5} \mathrm{~W} / \mathrm{cm}^{2}$. Such high intensities can be obtained continuously only at the focus of a strong pump laser. Actual operation of a cw dye laser will often be at pump intensities that are several times the threshold value. 
In these simple considerations we have neglected many factors that strongly influence the gain and tuning characteristics of dye lasers. Two of the important omissions here are the spatial dependence of the pump and dye laser modes (Tuccio, 1971), and the wavelength dependence of the gain and loss cross sections. A more complete discussion of dye-laser gain can be found in Chapters 1, 3, and 7 as well as in articles by Peterson et al. (1971), Peterson (1979), Danielmeyer (1971), Snavely (1977), Shank (1975), and Kuhlke and Dietel (1977).

Two conclusions that we can glean from this calculation are that with practical pump powers the useful gain in laser dyes will be achieved in a very small active region and that the gain will be large. High gain is an asset in that it allows design flexibility because the laser is relatively insensitive to small amounts of extra loss. This, in part, is the reason that there are so many different dye-laser designs. Almost anything works if you have enough gain. Thus dye lasers can be designed with characteristics optimized for specific applications. Even though high pump powers are often needed to reach threshold with dye lasers, the high slope efficiencies that are possible result in laser-conversion efficiencies from pump power to dye-laser power that can be quite acceptable (even as high as $50 \%$ in some cases). However, at the highest pump powers the effects of gain saturation and dye heating limit the ultimate powers that are achieved.

\subsubsection{Broadening - Homogeneous versus Inhomogeneous}

The most important aspect of the gain of organic dyes is that it spectrally broad. The processes that determine the spectral width are categorized according to whether they are homogeneous or inhomogeneous. Operationally there are important distinctions between the two classes of broadening. Homogeneous broadening means that all of the gain medium can contribute power to the oscillating laser mode. In contrast, for inhomogeneously broadened systems, only a fraction of the total gain is available for a specific oscillating mode. The physics of a specific broadening mechanism will determine whether the broadening is homogeneous or inhomogeneous. In the case of homogeneous broadening. all of the excited molecules (or atoms, or excited carriers, etc.) are effectively equivalent and can emit radiation at any frequency within the fluorescence bandwidth. In inhomogeneously broadened systems, on the other hand, the emission spectrum of a specific group of excited molecules is different from the emission spectrum of the system as a whole. When the broadening is inhomogeneous the molecules can be divided into groups that have distinct absorption and emission spectra. The Doppler shift is the best example of inhomogeneous broadening; atoms with different velocities have different emission spectra and hence contribute gain to a laser 
oscillator at different frequencies. On the other hand, the broadening due to collisions (for example, in a dense liquid) is homogeneous broadening.

The homogeneous broadening of the gain of organic dyes is both a blessing and a curse. It is a blessing in the sense that most of the available gain can be used for a single oscillation frequency and because the broad emission spectrum provides the laser's tunability. It is a curse because the broad spectral width means that the excited-state lifetime is short and hence intense pump powers are required in order to achieve sufficient population inversion for laser oscillation.

\subsubsection{Dye Lifetimes}

An annoying characteristic of organic dyes is that the dyes have limited productive lifetimes. (Unfortunately this is also the case for laser scientists!) The factors that limit the lifetime of laser dyes are thought to be the chemical and photochemical degradation of the dye in solution. The lifetime of the gain of a dye is often specified in terms of watt-hours, based on empirical data. This power-lifetime product is a measure of the pump-laser energy that has been used to excite the dye. Thus the degradation must be at least in part due to thermally activated chemistry (resulting from the pump-laser heating of the dye) and/or actual laser-induced photochemistry in the dye solution. The chemistry of the dyes can be quite complicated as is evidenced by the fact that the lifetime of the dyes' gain can even be affected by the type of metal plumbing components used in the dye-circulating system. The lifetimes of the typical $\mathrm{cw}$ dyes range from $\simeq 75 \mathrm{~W} \cdot \mathrm{hr}$ (coumarin 480$)$ to several $1000 \mathrm{~W} \cdot \mathrm{hr}(\mathrm{Rh} 6 \mathrm{G})$. Obviously the predicted lifetimes are estimates of typical performance and cannot be depended upon without further specification of the conditions. For example, the lifetimes usually assume a typical dye-pumping system with approximately one liter of dye solution.

\subsection{Pump Sources}

We require strong, broad fluorescence from the dye for broadly tunable $\mathrm{cw}$ laser operation. This in turn requires an intense optical-pump source tuned to some part of the absorption band of the dye. Incoherent optical pumping of a cw dye laser to threshold has been demonstrated by Thiel et al. (1987) using high-pressure arc lamps; but in order to have sufficient intensity for a practical system, the pump sources are almost always cw ion-lasers. In practice, we are presently limited to the argon and krypton-ion lasers. The obvious reason is that they are the only cw lasers that can produce high enough power ( $>4 \mathrm{~W}$, say) in a good single-spatial mode and in the visible or UV region of the spectrum. Recall that the dye's emission band is to 
Table 5-1

Powers Available from High-Power Ion Lasers

\begin{tabular}{ccc}
\hline & Wavelength $(\mathrm{nm})$ & Power $(\mathrm{W})$ \\
\hline Argon ion & 528.7 & 1.5 \\
$51+.5$ & 10.0 \\
501.7 & 1.5 \\
496.5 & 2.5 \\
488.0 & 7.0 \\
476.5 & 2.8 \\
472.7 & 1.2 \\
465.8 & 0.75 \\
457.9 & 1.4 \\
454.5 & 1.0 \\
& Multiline visible & $18-25$ \\
& Multiline UV & 5.0 \\
$351.1-385.8$ & 3.0 \\
$333.6-363.8$ & 5.0 \\
$275.4-305.5$ & 0.6 \\
& Multiline & - \\
$752.5-799.3$ & 1.6 \\
$647.1-676.4$ & 4.6 \\
$520.8-568.2$ & 3.6 \\
$468.0-530.9$ & 2.5 \\
$406.7-422.6$ & 1.3 \\
$337.5-365.4$ & 2.0 \\
\hline
\end{tabular}

the red of the absorption band; this means that for visible dye lasers we need strong blue and UV pump lasers. As of 1989, we are stuck with the very inefficient high-power ion-lasers for dye-laser pumping. Some of the strongest lines that are available from high-power ion-lasers are listed in Table 5-1. To be useful as a laser dye, a compounds-absorption band must overlap one of these strong ion laser lines. Other pump sources are certainly possible. For example, dye lasers can be pumped with a $\mathrm{HeNe}$ laser at $633 \mathrm{~nm}$ (Runge and Rosenberg 1972; Thiel et al. 1986) and also the future holds promise for pumping with high-power green light $(532 \mathrm{~nm})$ obtained from frequency doubling the output of $\mathrm{cw}$ YAG lasers. There has also been some progress in designing dye lasers that could be pumped with concentrated solar radiation (Lee et al., 1988).

\subsection{Resonator Design}

A good way to understand the performance characteristics of modern $\mathrm{cw}$ dye lasers is to study an example laser that is typical of modern designs. 


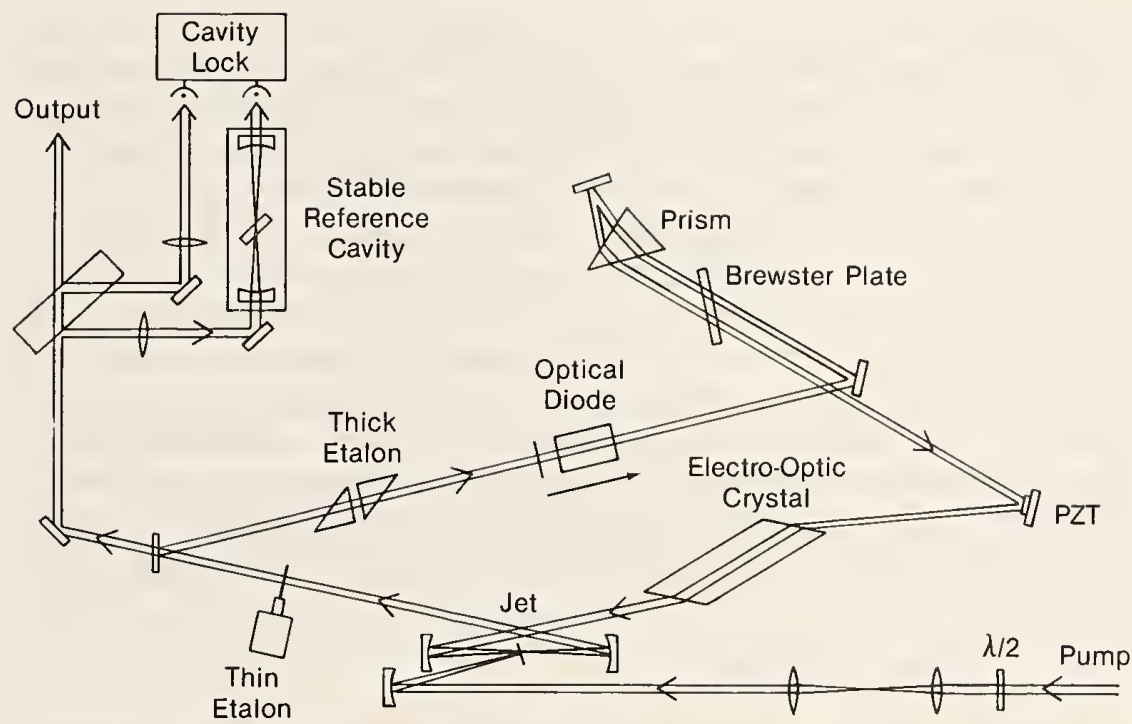

Fig. 5.6 Diagram of a frequency-stabilized ring dye laser. The basic laser resonator is formed with four flat mirrors and two short radius curved mirrors $(\simeq 5 \mathrm{~cm})$. The two curved mirrors form a tight waist that overlaps the focused pump spot in the dye jet. The optical diode is made from a Faraday rotator and a thin quartz plate. It forces the ring laser to oscillate in one direction as a traveling wave. The hierarchy of frequency-selective elements are the prism (double passed for added dispersion) the thin etalon (driven with a galvo motor) and the air-spaced thick etalon (constructed from two Brewster prisms and scanned with a PZT, piezo-electric ceramic). The frequency of the laser is lock to the resonance of a stable reference cavity using the cavity side lock method. The resonance frequency of the stable reference cavity is scanned using an intracavity Brewster plate that is rotated by a galvo motor. To stabilize the laser's frequency the cavity lock feeds back to the galvo-driven Brewster plate, the PZT translated mirror, and the intracavity electro-optic crystal. This laser system can scan continuously for about $80 \mathrm{Ghz}$ and has a linewidth of about one $\mathrm{kHz}$. Figure reprinted with permission from Hollberg (1984).

The goal of this reverse engineering is to understand how and why dye lasers have evolved to their present state. We choose here a laser design that has very good performance and is in regular use today.

Figure 5.6 shows a schematic diagram of a ring dye laser based on a six-mirror ring-cavity resonator. The two mirrors close to the dye jet have short radii of curvature $(\simeq 5 \mathrm{~cm})$ in order to produce the small laser-cavity mode that is necessary in the dye jet. The remaining mirrors are flat. They close the optical cavity in a manner that also provides space within the resonator for other optical elements. The organic dye is dissolved in a liquid solvent (for example, ethylene glycol) and squirted as a small ribbon of liquid dye into the space between the two short-radius mirrors. The 
pump radiation from ion laser is focused by the pump mirror to a small spot size $\left(w_{\mathrm{o}} \simeq 15 \mu \mathrm{m}\right)$ on the flowing jet of dye. Because of the heat produced in the dye by the focused pump laser and because of triplet-state trapping, it is necessary to have the dye molecules traverse the pump spot very rapidly, where "rapidly" in this case is determined by the heating rate and by the rate of transfer of excitation to the triplet state (typically about $1 \times 10^{-7} \mathrm{~s}$ ). To avoid significant loss into the triplet manifold the dye fluid needs to traverse the pumping region at a velocity of about $10 \mathrm{~m} / \mathrm{s}$. Usually the dye jet is a nearly rectangular ribbon with a cross section of 0.1 by $3 \mathrm{~mm}$ and is oriented at Brewster's angle (to minimize reflection loss) relative to the laser-cavity mode and near Brewster's angle to the pump beam. The laser mode passes through the thin dimension of the dye jet. The concentration of the organic dye in the transporting fluid is chosen to be high enough that the dye absorbs about $85 \%$ of incident pump radiation.

\subsubsection{Spatial Hole Burning}

The first question we need to address about the design of the laser cavity is why have we abandoned the linear three-mirror cavity of Fig. 5.2 in favor of the ring resonator of Fig. 5.6. The problem with the linear cavity is spatial hole burning. This is easily understood by thinking about the longitudinal mode structure of a linear dye-laser cavity. The dye laser's oscillating mode traverses the dye jet twice, in opposite directions: this sets up a standing wave field in the gain medium. The standing wave is a strong periodic spatial modulation of the laser intensity across the gain medium. Because of the intensity-dependence of stimulated emission, a singlefrequency laser mode is only able to use the gain that is available where the field strength is high. But we recall that the pumping field is a traveling wave that provides gain throughout the jet. Thus gain at the nodes of the standing wave cannot be used by the single-mode oscillatory field. The laser can, and will, use this available gain by oscillating simultaneously at a different frequency so that the nodes of the new oscillating mode overlap the antinodes of the original mode. The effect of spatial hole burning is to cause the linear standing-wave cavity to oscillate with more than one frequency (longitudinal mode).

\subsubsection{Unidirectional Ring Laser}

The problem of spatial hole burning can be alleviated by designing a laser resonator that oscillates in a traveling wave rather than a standing wave. Both the effect of spatial hole burning and its solution have been known for quite some time (Tang et al., 1963; Danielmeyer, 1971; Pike, 1974; Green et al., 1973; Kuhlke and Dietel, 1977), but it was not until about 1977 (Schröder et al., 1977) that the travelling-wave ring dye lasers 


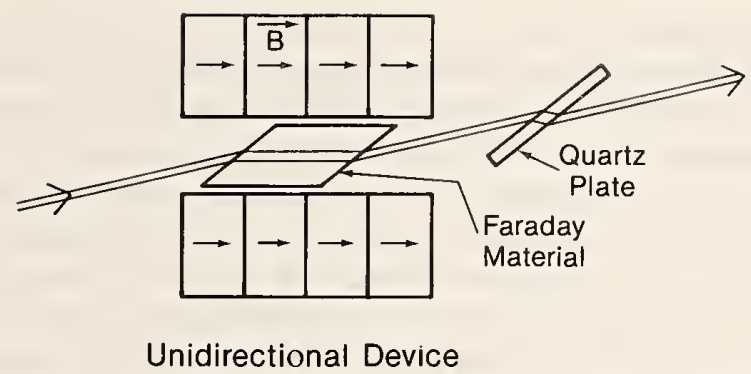

Fig. 5.7 Cross-section diagram of a unidirectional device (optical diode). A stack of permanent magnets creates a strong magnetic field (a few $\mathrm{kG}$ ) within its central bore which contains Faraday material. The quartz "rotate-back" plate uses optical activity to rotate the polarization of the laser field. Thus, the laser-propagation direction inside the quartz plate must be along the $z$-axis of the crystal. The configuration shown here is designed for minimum loss with both the Faraday material and the quartz plate oriented at Brewster's angle relative to the laser-propagation direction. For the beam propagating in the direction shown, the net rotation of the polarization is $0^{\circ}$, whereas the other direction has a net rotation of about $4^{\circ}$.

came into prominence (Jarrett and Young, 1979; Johnston et al., 1982). An interesting discussion of using prisms to create ring resonators for dye lasers is given by Marowsky and Zaraga (1974).

To avoid lasing in both directions, the ring laser's cavity must have more loss for one of the two propagation directions. This increased loss for one direction is provided by a nonreciprocal loss element, depicted in Fig. 5.6 as an optical diode. This optical diode (or unidirectional device) provides the directionally specific loss by using the axial vector nature of magnetic fields. A typical unidirectional device is diagrammed in Fig. 5.7. The device works by rotating the polarization of the propagating laser mode for only one of the two propagation directions. The rotated polarization encounters extra loss in the remaining Brewster-angle elements of the ring cavity. The rotation is made nonreciprocal by the Faraday effect of a crystal or glass in a magnetic field (applied parallel to the direction of propagation of the laser mode). The direction of polarization rotation due to the Faraday effect is independent of the direction of propagation of the light through the material. This Faraday rotation can then be combined with a reciprocal polarization rotation to produce an optical diode that rotates the direction of polarization for a beam from one direction but has no net rotation for a beam from the other direction. The reciprocal rotation is easily obtained from the optical activity in a thin quartz plate. A small differential loss of about $\simeq 0.5 \%$ is sufficient for unidirectional operation of most ring dye lasers. This loss is achieved from a one-way polarization rotation of about $4^{\circ}$ in a laser cavity that has six Brewster surfaces or more. The design criteria for unidirectional devices are discussed by Biraben (1979) and by Johnston and Proffitt (1980). 
Unidirectional ring lasers with traveling wave fields have advantages over standing-wave lasers because of more efficient use of the available gain and better spectral characteristics. On the other hand, with ring lasers there is reduced gain per pass because the radiation field only traverses the gain medium once for each incidence on the output coupler. The net result is that with sufficient pump power the traveling-wave ring dye lasers can provide almost twice the single-mode output power of the standing-wave lasers. For this extra power and mode stability ring lasers have the disadvantage of slightly higher cavity loss because of the extra optical components. This means that the thresholds are typically higher for ring cavities. Some of the very low gain dyes actually work better in linear cavities because of their lower threshold.

From the gain arguments just given and knowledge of the pump powers that are available, we know that the gain medium will occupy a small volume (about $10^{-7} \mathrm{~cm}^{3}$ ). To optimally use the available gain the dye laser's spatial mode is approximately matched to the pump-laser spatial mode in the dye medium. "Approximately matched" here indicates that the optimal size of the pump and dye laser modes is actually a function of the saturation of the gain medium and is thus a weak function of the pump power (Snavely, 1977; Johnston, 1987). In practical cases, where the laser operates well above threshold, this dependence is weak and is usually not worth the trouble of readjusting the laser mode when the pump power is changed. We usually have a pump-laser spot size of about $w_{0}=15 \mu \mathrm{m}$, which is consistent with the available pump powers and the threshold intensity of the dye. We can then use Gaussian mode analysis to calculate the transverse mode structure for a laser resonator (Yariv. 1975; Siegman, 1986). This analysis can give us the information that is necessary to select the mirror spacings, orientations, and radii of curvature that will approximately match the dye-laser mode to the gain region.

\subsubsection{Resonator Aberrations}

Up to now we have naively assumed that the natural fundamental transverse mode structure of the dye laser is the round TEM $\mathrm{O}_{00}$ mode. But in fact the orientation of the curved mirrors in the laser cavity relative to the incidence angle of the laser beam alters the transverse structure of the $\mathrm{TEM}_{00}$ mode. Having a laser beam incident on spherical mirrors at an angle other than normal incidence causes the beam to be aberrated by astigmatism and coma. The dye jet is also a source of astigmatism and coma because it intercepts the strongly focused Gaussian laser mode at an angle. These aberrations distort the laser mode from the desired $\mathrm{TEM}_{\mathrm{0}}$ mode, and this degrades the resonator's stability and reduces the laser's conversion efficiency. In most modern designs the astigmatism is compen- 
sated by the appropriate choice of angles of the resonator's mode at the curved mirrors or by the addition of other optical elements that introduce an astigmatism of the opposite sign. It is even possible to simultaneously compensate for both astigmatism and coma. Details of the compensation methods can be found in Kogelnik et al. (1972), Johnston and Runge (1972), and Dunn and Ferguson (1977).

Using the geometry shown in Fig. 5.2a, we can follow the analysis of Kogelnik et al. (1972), and Mollenauer and White (1987) to calculate the astigmatism introduced by the two curved mirrors and the dye jet. The dye jet with thickness $t$ is located between the two short-radius curved mirrors (M1, M2) and is tilted at Brewster's angle $B$ relative to the beampropagation direction. In this geometry there are two sources of astigmatism; the first is that due to the dye jet crossing the laser mode at an angle and the second due to the curved mirror M2 that is tilted at the angle $\theta$ relative to the laser-mode-propagation direction. Fortunately, the sign of the astigmatism is opposite for these two sources, and the angles can be chosen so that the mirror astigmatism cancels that of the jet. The condition for zero astigmatism is

$$
f(\sin \theta)(\tan \theta)=t\left(n^{2}-1\right) \frac{\sqrt{n^{2}+1}}{n^{4}},
$$

where $f$ is the focal length of mirror M2 and $n$ is the index of refraction of the gain medium (Kogelnik et al. 1972). Since the dye jets are quite thin $(\approx 0.1 \mathrm{~mm})$ and the mirrors have radii of about $5 \mathrm{~cm}$, the angle $\theta$ must be small. These small angles are not always practical, so other methods have been used to compensate the astigmatism. These includes lenses or additional curved mirrors that are titled off normal incidence, a Brewster plate between the dye jet and the short-radius mirrors, and rhombi with Brewster-cut surfaces that are placed in an auxiliary beam waist in the laser cavity.

\section{EXTENDED TUNING}

\subsection{UV Generation}

Nonlinear optical methods can be used with dye lasers to extend the already broad tuning range of $\mathrm{cw}$ dye lasers. If we look back to Fig. 5.3 we see the UV-tuning curves that were obtained by T. F. Johnston (1987) and T. Johnston (1988) using a high-power single-mode cw dye laser. This cw single-frequency UV output is generated either by frequency doubling the output of the dye laser or by sum-frequency mixing the output of the dye 
laser with the output of a single-frequency argon-ion laser. Using these methods, they have generated single-frequency cw UV radiation across the frequency range between 260 and $400 \mathrm{~nm}$. The excellent UV powers obtained in these experiments were typically about $10 \mathrm{~mW}$. Both the doubling and the frequency mixing were done inside the resonator of a ring dye laser. For the sum-frequency mixing experiments the two laser resonators were actually arranged to overlap at the nonlinear crystal. Thus the mixing occurred with buildup on both the input frequencies. Other systems have been developed to generate $\mathrm{cw}$ ultraviolet light in this frequency range and beyond (Frölich et al., 1976; Blit et al., 1977, 1978; Couillaud, 1981; Couillaud et al., 1982; Marshall et al., 1980; Bergquist et al., 1982; Hemmati et al., 1983). The transparency of the nonlinear crystals limits $\mathrm{cw}$ UV generation to wavelengths longer than approximately $160 \mathrm{~nm}$. That is not the case for UV generation in atomic vapors. Four-wave mixing has been used in strontium vapor to generate $\simeq 10 \mathrm{pW}$ of broadband $(\simeq 6 \mathrm{GHz})$ cw UV radiation at $170 \mathrm{~nm}$ (Freeman et al., 1978).

Noteworthy among these efforts to generate single-frequency cw UV radiation is the work of Bergquist and collaborators(Bergquist et al., 1982; Hemmati et al., 1983), who have combined the second harmonic of argonion laser radiation (fundamental $=514 \mathrm{~nm}$ ) with tunable dye-laser radiation $(\simeq 792 \mathrm{~nm})$ to generate UV radiation at $194 \mathrm{~nm}$. Other colors could certainly be generated by these methods but the 194-nm radiation is of particular interest for high-resolution spectroscopy of $\mathrm{Hg}$ ions. Their system is diagrammed in Fig. 5.8. It serves as good example of the techniques and the 1980s state of the art for synthesizing single-frequency cw, UV radiation. Their system uses the nonlinear crystal KB5 (potassium pentaborate) to generate the sum frequency of $792-$ and $257-\mathrm{nm}$ radiation. The $792 \mathrm{~nm}$ radiation comes from a cw ring dye laser (LD700 dye), and the $257 \mathrm{~nm}$ radiation is from the frequency-doubled $514 \mathrm{~nm}$ argon laser. One of the techniques they use to advantage is putting the nonlinear crystals in external ring buildup cavities. This enhances the optical power in the nonlinear crystals and avoids the problem of the crystal's loss in the laser resonator. A ring cavity containing a Brewster-cut ADP (ammonium dihydrogen phosphate) crystal is locked to and enhances the power of the $514 \mathrm{~nm}$ radiation by a factor of $\simeq 33$. Thus $700 \mathrm{~mW}$ of $514 \mathrm{~nm}$ radiation generates about $30-40 \mathrm{~mW}$ of $257 \mathrm{~nm}$ radiation. Then, as shown in Fig. 5.8, they use two buildup cavities around the KB5 summing crystal; one ring cavity builds up the $792-\mathrm{nm}$ radiation and the second ring resonator builds up the $257-\mathrm{nm}$ radiation. Both these cavities have buildup factors of $\simeq 15$. With input powers of $\simeq 120 \mathrm{~mW}$ at $792 \mathrm{~nm}$ and $\simeq 30 \mathrm{~mW}$ at $257 \mathrm{~nm}$ they obtain $\simeq 20 \mu \mathrm{W}$ of $\mathrm{cW}$ single-frequency radiation at $194 \mathrm{~nm}$. The output power at $194 \mathrm{~nm}$ is not limited by the availability of input powers 


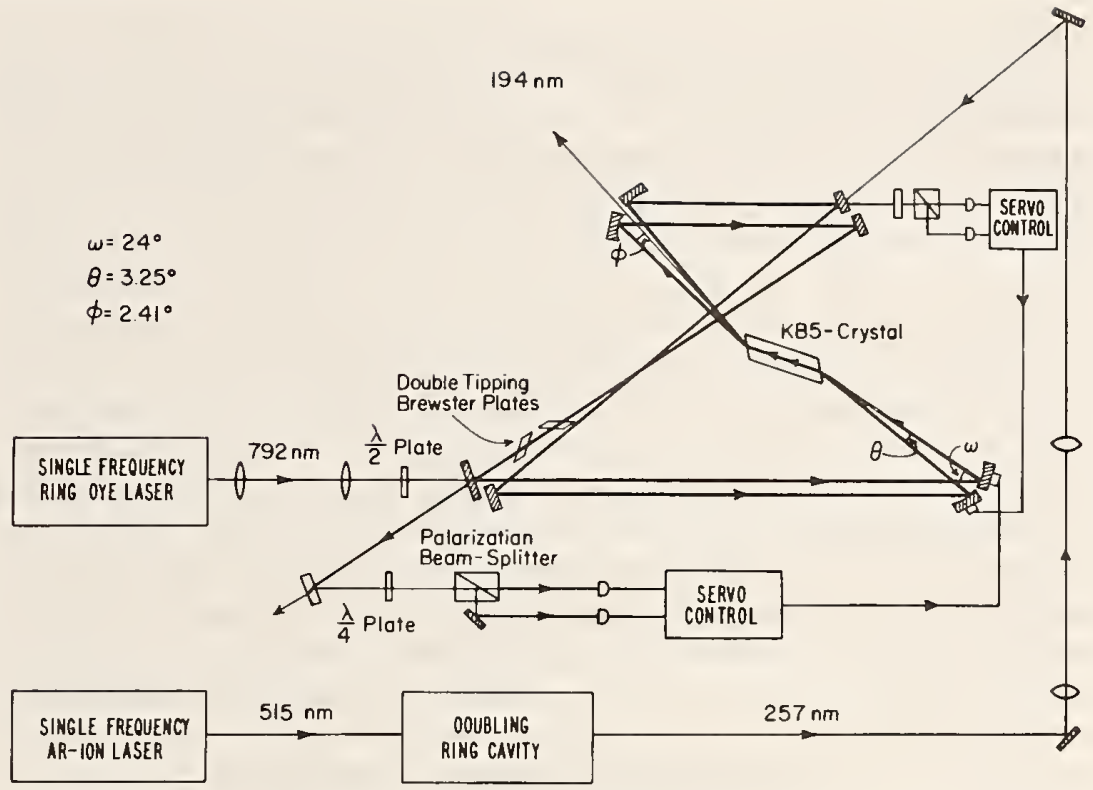

Fig. 5.8 Sum frequency generation of 194-nm radiation. This system, developed by Hemmati et al. (1983), and Bergquist (1989) uses the nonlinearity of the KB5 crystal to generate the sum frequency of 257 - and $792-\mathrm{nm}$ radiation. The power in both of these input beams is enhanced in ring buildup cavities whose waists intersect in the KB5 crystal. Servo-control loops (using the polarization lock method) keep the two buildup cavities locked to their respective input beams. The 194-nm radiation leaves the KB5 crystal at a slightly different angle and thus escapes from the two buildup cavities. The 792-nm input radiation comes from a frequency-stabilized ring dye laser, while the $257-\mathrm{nm}$ is generated by frequency doubling the 514-nm radiation from a single-frequency argon-ion laser. The frequency doubling of the argon-ion laser radiation is done in a ring buildup cavity that is similar to the two used around the KB5 crystal shown here. With input powers of $=30 \mathrm{~mW}$ at $257 \mathrm{~nm}$ and $120 \mathrm{~mW}$ at $792 \mathrm{~nm}$, this system generates up to $20 \mu \mathrm{W}$ of single-frequency radiation at $194 \mathrm{~nm}$. Unpublished figure reprinted with permission from Bergquist (1989).

but rather by the effects of thermal blooming due to nonuniform optical heating of the KB5 crystal. Another important technique of nonlinear optics that is used in this system is electronic servo-control systems to maintain the resonance condition for the buildup cavities (see section 4 on laser frequency control).

\subsection{IR Generation by Difference-Frequency Mixing}

The useful tuning range of dye lasers can be similarly extended into the infrared by difference-frequency mixing (Pine, 1974. 1976; Oka, 1988). The technique is analogous to the sum-frequency generation, but now the 
crystal's nonlinearity is used to generate the difference frequency between an ion-laser beam and a tunable $\mathrm{cw}$ dye laser. Using $\mathrm{LiNbO}_{3}$ as the nonlinear crystal Pine $(1974,1976)$ has demonstrated that one can produce narrowband $(\simeq 15-\mathrm{MHz}$ linewidth) radiation with wavelengths in the range of 2.2 to 4.2 microns and with power levels of a few microwatts. The tuning range can be extended by using other nonlinear crystals such as lithium iodate (Oka, 1988). Somewhat higher power levels can also be achieved.

One problem with describing the late 1980 s state of the art is that there is a tendency to report the best performances rather than what is typically achieved. The output-power curves shown in Fig. 5.3 are an example of what is possible with an optimized dye laser with very powerful pump lasers. The performance that you should expect from a typical commercial dye laser system is probably a factor of two less power than shown there. Nevertheless the record performances are always interesting, because research continues to expand our possibilities and the best results today are indicative of the tools of the future. In addition, the extreme limits are often dominated by new and interesting science waiting to be explored.

\section{LASER-FREQUENCY CONTROL}

High-resolution dye lasers are common laboratory tools today because of early research efforts in laser frequency control. The broad bandwidth of the gain medium, combined with the mechanical instabilities of the rapidly flowing dye jet, conspire to make frequency selection and stabilization a challenge. Nont theless, the technology required to control the frequency of dye lasers has been developed. Many years ago, Soffer and McFarland (1967) demonstrated that the wide bandwidth of pulsed dye lasers could be compressed to a relatively narrow bandwidth by inserting an optical grating inside the cavity. In addition to reducing the laser's linewidth, the frequency selectivity of the grating allowed the laser's wavelength to be tuned. This demonstration of spectral compression, as well as more recent techniques, takes advantage of the homogeneous broadening of the dye laser gain to produce a narrower spectral linewidth without sacrificing output power.

\subsection{Frequency-Selective Elements}

The laser's oscillation frequency can also be controlled by providing frequency-selective loss inside the resonator. To achieve this frequencyselective loss, we have freedom to control both the spatial and polarization boundary conditions of the resonator. Dispersive optical elements that create boundary conditions for the oscillating mode provide the appropri- 
ate frequency-selective loss. The dispersion can come from interferometry, which is solely wavelength-selective, or it can be provided by polarizationsensitive elements which act on the vector nature of the optical field. Some of the dispersive elements that have been used to control the frequency of cw dye lasers include gratings, prisms, birefringent filters, and Fabry-Perot etalons, as well as Michelson, Fox-Smith, and Mach-Zehnder interferometers (Smith, 1972).

Usually the addition of one frequency-selective element into a dye laser's cavity is not sufficient to uniquely determine the laser's oscillation frequency. The conventional technique to achieve mode-stable singlefrequency operation is to use a hierarchy of frequency-selective elements inside the laser's cavity. Most single-mode lasers use three separate levels of frequency selectivity to force the laser to oscillate on a specific longitudinal mode of the laser's cavity. Lacking this hierarchy of frequency selectivity, the laser may oscillate with many modes, or it may oscillate with a single mode that is unstable to small perturbations and jump from mode to mode as a function of time.

Due to the homogeneous broadening of the gain, once the laser starts to oscillate on a specific mode, this mode quickly depletes the gain that is otherwise available for the other longitudinal modes. In a traveling-wave ring dye laser the homogeneous broadening causes strong mode competition, which is usually enough to ensure that the laser will oscillate on a single longitudinal mode. Unfortunately, without frequency selectivity in the cavity, the oscillating mode is unstable to small perturbations and will change in time. Thus even though the laser oscillates on a single mode, additional frequency-selective elements inside the cavity are necessary to select a particular frequency.

A typical hierarchy of frequency-selective elements might start on the least selective end with a birefringent filter, which typically has a halfpower bandwidth in the laser resonator of about $1800 \mathrm{GHz}\left(60 \mathrm{~cm}^{-1}\right)$. This is usually followed by two etalons, with progressively higher resolution; for example, a thin etalon with a free spectral range (FSR) of $200 \mathrm{GHz}$ and a finesse of $\simeq 3$, then a thick etalon with a FSR of $10 \mathrm{GHz}$ and again a finesse of $\simeq 3$. The overlap of the transmittance peaks of the three frequencyselective elements provides a net transmittance bandwidth that is narrow enough to uniquely determine a single mode for laser oscillation. The role of the various frequency-selective elements can be seen in Fig. 5.9, which shows the transmission functions of these elements overlaid with the laser longitudinal mode structure. The resolving powers of the three levels of frequency-selectivity are chosen so that the resolution of one level is sufficient to select a unique resolution element of the next higher level of selectivity. Thus, the birefringent filter has enough resolution to select a 
|a|

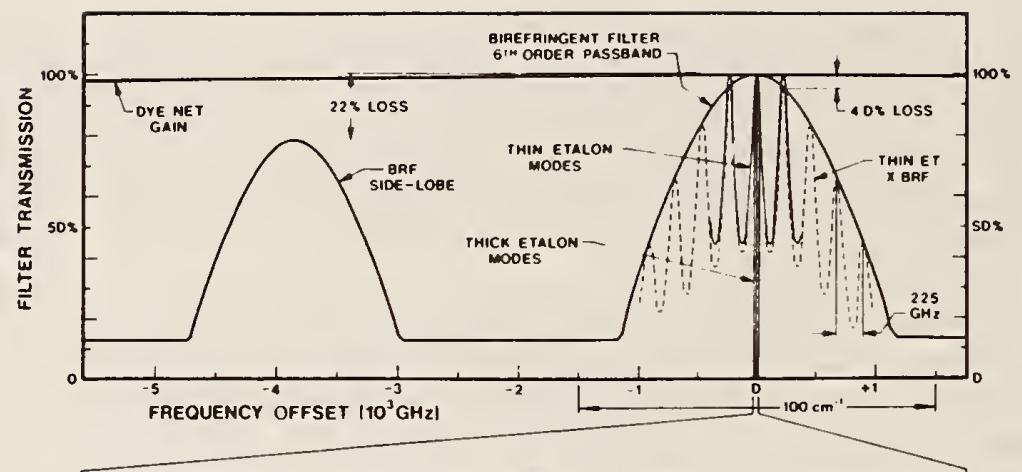

$|b|$

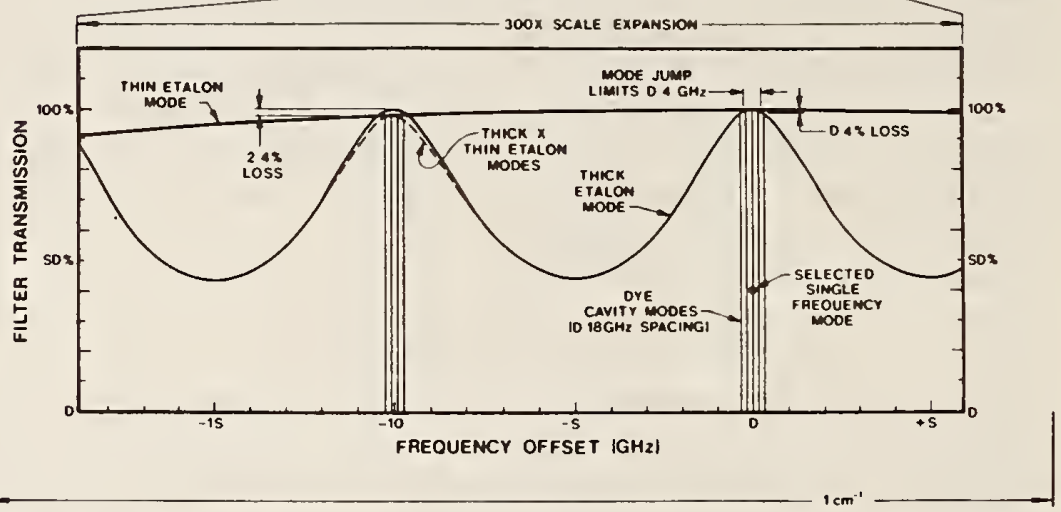

Fig. 5.9 Filter transmission functions of the various frequency-selective elements of a single-frequency dye laser. The traces show the bandpasses of the frequency-selective elements relative to the dye gain curve and the laser's cavity-mode structure. The upper half of the figure has a broad spectral range and shows the birefringent filter and thin-etalon transmission functions. The lower half of the figure shows the thick etalon's transmission function relative to the longitudinal mode spacing ( $c / L$ with $L=$ cavity round-trip length) of the laser cavity. Also indicated are the losses created for radiation away from the central transmission peaks. This figure is reprinted with permission from Coherent Inc. and Johnston, T. F. (1987).

specific thin-etalon mode, the thin-etalon selects a specific thick-etalon mode, and the thick etalon has enough resolution to select a specific longitudinal mode of the laser cavity. For the system to work in an optimum way, the bandpasses of these filters need to be at least roughly centered on a specific cavity mode. Figure 5.6 is an example of a singlefrequency dye laser with a slightly different hierarchy of frequencyselective elements, in this case a prism and two etalons.

The frequency stability of such single-frequency lasers is now determined by the mechanical stability of the optical elements, the gain 
medium, and the resonator's superstructure. We shall see in the spectral characteristics section (5.2) that "single frequency" is a bit optimistic, in that the spectral linewidth of single-mode lasers can still be substantial.

The birefringent filter is the most common coarse tuning element and is composed of quartz plates placed in the laser cavity inclined at Brewster's angle relative to the direction of propagation (Bloom, 1974; Bonarev and Kobtsev, 1986). The thickness of each plate in the filter is integrally related to the others (for example, $1: 4: 16$ for a common three-plate birefringent filter). The quartz plates are cut with the optic axis parallel to the surface of the plates. The design of the birefringent filter for a laser follows the traditional Lyot filter design (Jenkins and White, 1957), with the important difference that the polarizers between the birefringent elements have been removed. The role of the polarizers is played by the Brewster-angle surfaces of the plates themselves and other Brewster surfaces in the cavity. The optical frequency selectivity of this filter results from the birefringence of the quartz waveplates combined with the polarization-dependent reflection loss from the Brewster surfaces of the plates. The plates are mounted together in the laser cavity so that they can be rotated about the normal to the plates, thereby changing the angle of the crystal axis with respect to the laser polarization (parallel to the plane of incidence) while maintaining the Brewster-angle orientation of the quartz surface relative to the laser mode propagation direction. For a given plate thickness and orientation of the optic axis, the plate will act as a wavelength-dependent waveplate, which in general changes the polarization character of the input laser mode. At some wavelength the polarization of the light that exits each plate will be the same linear polarization ( $p$-polarization) that entered and, thus, will pass with no loss through the subsequent Brewster surfaces. In general the polarization of the beam will elliptical upon exiting each of the plates and will therefore experience reflection loss at the Brewster surfaces that it encounters in traversing the rest of the laser cavity. Birefringent plates with integrally related thicknesses can provide enough selectivity that lasing will be restricted to one color (a few cavity modes) within the dye gain curve. It is interesting to note the frequency selectivity of the birefringent filter, in the laser cavity, is higher than that of a traditional Lyot filter with polarizers. The net spectral width of a cw dye laser with only a three-plate birefringent filter can vary significantly depending on alignment; widths can vary from 1 to more than $100 \mathrm{GHz}$ (Nieuwesteeg et al., 1986). The loss provided by a single Brewster surface for a beam incident with a polarization that is orthogonal to Brewster's polarization is

$$
\text { loss }=1-\left(\frac{2 n}{n^{2}+1}\right)^{2}
$$


Here $n$ represents the index of refraction of the Brewster plate, and the loss is the fractional reduction in power of the transmitted beam. A net gain reduction of less than $1 \%$ is usually sufficient to suppress other modes from lasing. A typical birefringent filter will have a free spectral range of $\approx 100 \mathrm{~nm}$. A dye laser with a three-plate birefringent filter has at least eight Brewster surfaces (including the jet surfaces) that can provide a net loss of $\simeq 4 \%$ for a $200-\mathrm{GHz}$ detuning (this corresponds to the FSR of the thin etalon, see Fig. 5.9).

Usually etalons provide the next two higher levels of frequency selectivity. These parallel plate interferometers have the standard Fabry-Perot transmittance (Born and Wolf, 1964),

$$
\mathrm{I}(\delta)=\frac{I_{0}}{1+F \sin ^{2}\left(\frac{\delta}{2}\right)}
$$

where $F=\frac{4 R}{(1-R)^{2}}$ and $\delta=\frac{4 \pi n L}{\lambda} \cos \theta$.

Here $R$ represents the power reflectance of the etalon's surfaces (assumed equal), $n$ its index of refraction. $L$ its thickness, and $\theta$ the angle between the etalon normal and the optical propagation direction inside the etalon. $\lambda$ is the vacuum wavelength. A useful measure of the etalon's resolving power is its finesse, $\mathscr{F}$, which is given by,

$$
\mathscr{F}=\pi \frac{\sqrt{F}}{2} \text {. }
$$

A cw ring dye laser might have one thin etalon $(L=200 \mu \mathrm{m}$ with $R=$ $30 \%$ ) and one thick etalon ( $L=1 \mathrm{~cm}$ and $R=30 \%$ ). which, when combined with the birefringent filter. provide sufficient frequency-selectivity to force the laser to oscillate on a single longitudinal mode.

The frequency-selective components that we consider here are typical of single-frequency dye lasers used in 1989, but other frequency-control systems have also been developed. As noted earlier, the high gain of the dye laser allows some experimental flexibility in testing ideas about laser design. The system with a birefringent filter and two etalons is the most popular because of its dependability and relative ease of construction. That is not to imply that this system is optimum. The tuning elements that are alternatives to the birefringent filter are prisms and the tuning wedge that is now rarely used. A variety of interferometric methods have also been used to control the frequency of dye lasers. These include the Michelson, Fox-Smith, and Mach-Zehnder interferometers and multiple implementations of these. Interferometers generally compete for the higher selectivity roles normally filled by the etalons. 


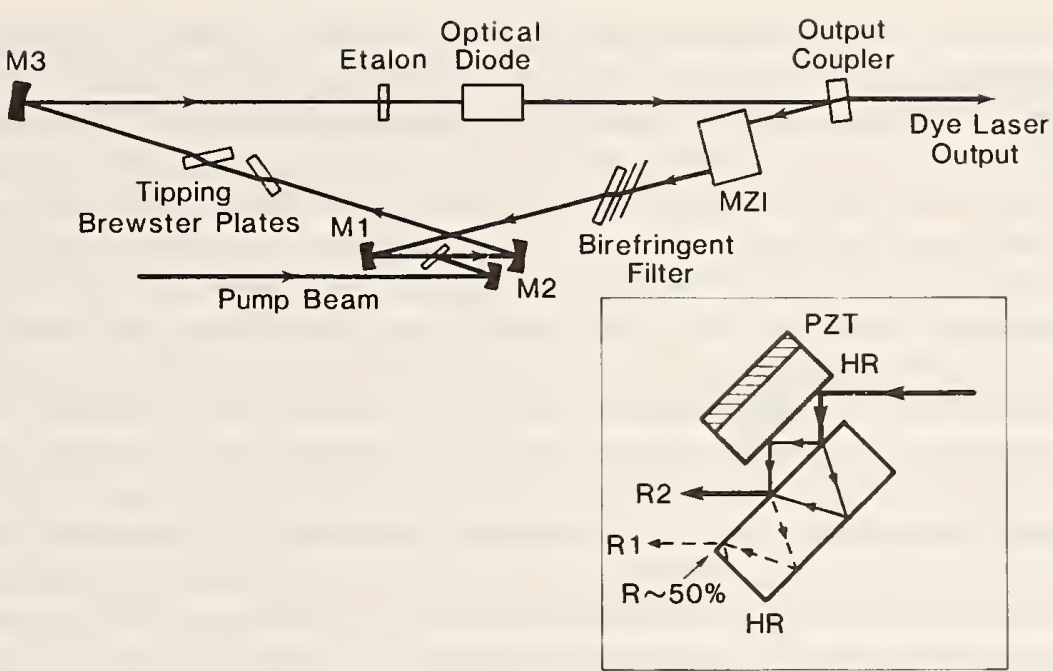

Fig. 5.10 Diagram of a single-frequency ring dye laser that uses a Mach-Zehnder interferometer for longitudinal mode control. This laser also uses a birefringent filter and a thin etalon for frequency selectivity. An optical diode is used for unidirectional operation and tipping Brewster plates are used for frequency scanning. The inset shows the structure of one type of intracavity Mach-Zennder. This interferometer is constructed from two high-quality optical flats. One piece has a $50 \%$ reflectivity coating on its front surface and a high reflectivity coating (HR) on the back surface. The partial reflecting surface splits the input beam into two paths through the interferometer. The other optical flat is a high-reflectivity mirror and is mounted on a PZT translator so that the passband of the interferometer can be changed. The Mach-Zehnder has two output ports (R1 and R2). If the reflectivity of the partial reflector is $50 \%$, and the losses in the two paths through the interferometer are matched, the contrast in the output ports can be $100 \%$. With good low-loss coatings, the net insertion loss of this device in the laser cavity can be very small. Figure reprinted with permission from Bergquist and Burkins (1984).

One of the most intriguing of the interferometric frequency selectors for use with dye lasers is the Mach-Zehnder system that was proposed and developed by Bergquist and Burkins (1984). An example of one of the several possible implementations is diagrammed in Fig. 5.10. The advantage of the Mach-Zehnder interferometer is that, in principle, it can provide the necessary frequency-selectivity with substantially less excess loss than that of a comparable etalon. The reason for the unavoidable loss with intracavity etalons is that it is necessary to tilt the etalons away from normal incidence to avoid the disruptive effects of optical feedback on the oscillating mode of the laser. Tilting the etalon eliminates the feedback but we pay the penalty that power is lost from the laser mode. In laser jargon, this "etalon walkoff loss" results from the multiple reflections within the tilted etalon that bounce laser power out of the spatial mode of the laser 
cavity. A properly balanced Mach-Zehnder system does not have this limitation because the only additional optical output port (beam R1 in Fig. 5.10) has zero output under proper operating conditions. This occurs when the Mach-Zehnder interferometer is set for a interference maximum on beam R2 (which corresponds to a minimum for beam R1) and when the interference maximum is tuned to a longitudinal mode of the laser cavity. In this case the only excess loss introduced by the Mach-Zehnder to the laser is that due to the absorption and scattering from the optical coatings, which can be made negligibly small. In practice the Mach-Zehnder system has seen only limited application but appears to have great potential.

The most important attribute of dye lasers is their tunability. In addition to reaching a particular wavelength, we frequently want to scan the frequency of the laser over some region near this wavelength. Depending on the spectral resolution that is required, it may not be necessary for the laser to oscillate on a single longitudinal mode. For example, the spectral features observed in solid-state spectroscopy are relatively broad, which often means that the resolution provided by multimode dye lasers is adequate. In this case a dye laser with only a birefringent filter provides enough resolution, and the laser can be scanned simply by tuning the bandpass of the birefringent filter. On the other hand, the technology required to tune and continuously scan the frequency of single-mode (single-frequency) dye lasers is complicated by the many frequencyselective elements. It is necessary to individually center and then scan all of these elements synchronously. Such scanning systems have been developed and some even actively optimize the centering of the bandpasses of the frequency-selective elements. The actively controlled systems provide long-term mode stability and repeatable scans. More sophisticated dyelaser control systems incorporate computer-controlled wavelength calibration, frequency scanning, and data acquisition (Marshall et al., 1980; Williams et al., 1983; Clark et al., 1986).

For a deeper understanding of the methods of laser-frequency control we focus attention on a typical tuning system for a single-mode ring dye laser (such as in Fig. 5.6). We assume that the frequency-selective elements are able to force the dye laser to operate on a single longitudinal mode of the laser cavity. The optical length of the cavity then determines the fine tuning of the laser frequency. The laser frequency can be adjusted over a small range by using the piezoelectrically translated mirror, PZT (see Fig. 5.6), and larger-frequency changes are induced by rotation of the Brewster plate. Changing the optical length of the cavity will scan the laser but only within the bandpass of the thick etalon. Since the thick etalon has enough resolution to select a single mode it also limits the scan to less than the laser cavity FSR. In order to achieve longer scans, the thick etalon 
must be scanned synchronously and likewise eventually the thin etalon must also be scanned. Usually these etalons are scanned with a piezoelectric translator to change the spacing between the plates of the thick etalon and by using a galvo-motor to rotate the thin etalon.

The alignment of the thick-etalon transmission peak to the cavity mode is critical and usually requires an automatic electronic servo to keep its bandpass properly aligned with the laser-cavity mode. This can be accomplished by using a conventional "modulation lock" which will be discussed shortly. For long scans it is necessary to tilt the thin etalon to track the thick etalon, but because of its low resolving power it is usually not necessary to have automatic feedback control-although such systems have been developed (Biraben and Labastie 1982). Tracking of the thinetalon bandpass to a scanning laser frequency is also complicated by the fact that the bandpass of a tilted etalon scans approximately quadratically as a function of the tilt angle (see Eq. 5.2). This nonlinearity can be electronically compensated by using an electronic square-root-function module. Centering the passband of the birefringent filter is usually done manually by finding the maximum laser output power on a given mode. Automatic control systems for the birefringent filter can also be made by monitoring the polarization of the laser's output, which becomes slightly elliptical when the birefringent filter's bandpass is not properly centered on the cavity mode (Biraben, 1989).

A typical dye-laser scanning system thus consists of a Brewster plate rotated by a galvo-motor, a piezoelectrically driven thick etalon to track the specific cavity mode, and a thin etalon that is tilted by a galvo-motor to track the thick-etalon scan. All of these scanned elements are synchronized by a master control circuit that thus controls the laser's frequency. Commercial single-mode dye-laser systems can be scanned continuously with direct electromechanical control over ranges of about $30 \mathrm{GHz}$. Longer scans are certainly possible but they would put more stringent requirements on the electromechanical stability of the tuning elements. For example, with $1-\mathrm{MHz}$ resolution, a $30-\mathrm{GHz}$ scan already provides $3 \times 10^{4}$ resolution elements, which is usually adequate for most applications. There are some limits to the mechanical and electrical stability, linearity, and reproducibility of the electromechanical transducers used to position the tuning elements. Instability in these systems translates directly to laser-frequency fluctuations. In practice it is difficult to achieve 10 parts per million resolution in the electromechanical positioning. One can reconstruct a broader spectrum by computer-driven piecewise scans of about $30 \mathrm{GHz}$ each. The total scan is then limited only by the bandwidth of the dye, but care must be taken to maintain some frequency reference in putting all of the separate scans together. 
Pressure scanning of dye lasers is an alternative approach to mechanical scanning. As of the late 1980 s, pressure scanning is not very popular, but it has some advantages. For example, it does not require moving parts in the laser. The idea is to enclose the entire laser inside a pressure-tight vessel so that when the pressure is changed the optical path length changes (due to changes in the index of refraction of the gas) and this causes the laser's frequency to change. If the etalons are designed with an open air space between the etalon surfaces, then when the pressure is changed, the etalons scan synchronously with the laser frequency.

\subsection{Frequency-Stabilization Methods}

The nature of the frequency fluctuations of lasers (as with other oscillators) is such that the measured frequency-stability depends on the time scale over which the measurement is made. The spectrum of dye laser frequency. fluctuations is strongly peaked at low Fourier frequencies and then tapers down to a white noise level at high frequencies. In addition strong resonant peaks in the spectrum of the frequency noise result from a variety of technical problems (for example, noise at the AC line frequency). It is common practice to specify a laser's frequency-stability by a short-term "linewidth" and a longer-term center-frequency stability or drift rate. The implicit assumption here is that short-term fluctuations of the laser's phase determine the "linewidth," while slower fluctuations and drift dominate the stability the laser's center frequency. This model of the lasers frequency fluctuations has enough validity to be useful but it is far from the complete picture.

The frequency of the single-mode dye laser is determined by the boundary conditions imposed by the optical length of its resonator, which are usually arbitrary and generally lack long-term stability. The solution to the problem of frequency instability and inevitable drift is to lock the laser to a frequency reference such as a stable reference cavity or a molecular resonance. In principle the ultimate stability of the laser frequency can match that of the reference cavity if the control system is properly designed. Usually systematic errors degrade this performance considerably. Standard stabilized dye-laser systems have linewidths of $\simeq 1 \mathrm{MHz}$ with a center frequency of $\simeq 5 \times 10^{14} \mathrm{~Hz}$. Typical drift rates are about $10 \mathrm{kHz} / \mathrm{sec}$, which is adequate for most experiments. The best cavitystabilized laser systems have laser-frequency drift rates of about $1 \mathrm{~Hz} / \mathrm{s}$ (Salomon et al., 1988; Hils and Hall, 1989; Helmcke et al., 1987). This outstanding performance exceeds the precision that is required for all but the most demanding metrology and physics experiments.

The other compelling reason to have a stable reference cavity is that it 
can act as a fast frequency discriminator with a very large signal-to-noise ratio. The error signal derived from the cavity is then used in an electronic servo system to narrow the laser's linewidth and hence improve its resolution. The resulting linewidth of a laser stabilized to a reference cavity will depend on the laser's intrinsic frequency-fluctuation spectrum, the reference cavity's resolution, the servo system's bandwidth, and even the observation time. Some knowledge of the methodology of frequencycontrol systems is useful in developing an understanding of the operational characteristics of high-resolution dye lasers (see also Balykin et al. (1987) and Helmcke et al. $(1982,1987)$. Some of the common frequencystabilization techniques are outlined in the following section.

\subsubsection{Cavity-Side-Lock}

One of the easiest and most generally applicable frequency-control methods is the cavity-side-lock system. It has been with us at least since the early days of the dye laser. This system uses a spectrally sharp Fabry-Perot transmittance peak to derive an electronic error signal that can be used to lock the laser to the side of the cavity resonance (see Fig. 5.11). The error signal is the difference between the photocurrents of the cavity transmittance peak (signal channel detected by D1) and the laser power (reference channel detected by D2). The light levels are adjusted so that the output from the difference amplifier is 0 when the laser frequency is tuned to the side of the cavity resonance. This provides a smoothly varying monotonic frequency discriminator for laser frequency excursions less than $1 / 2$ of the

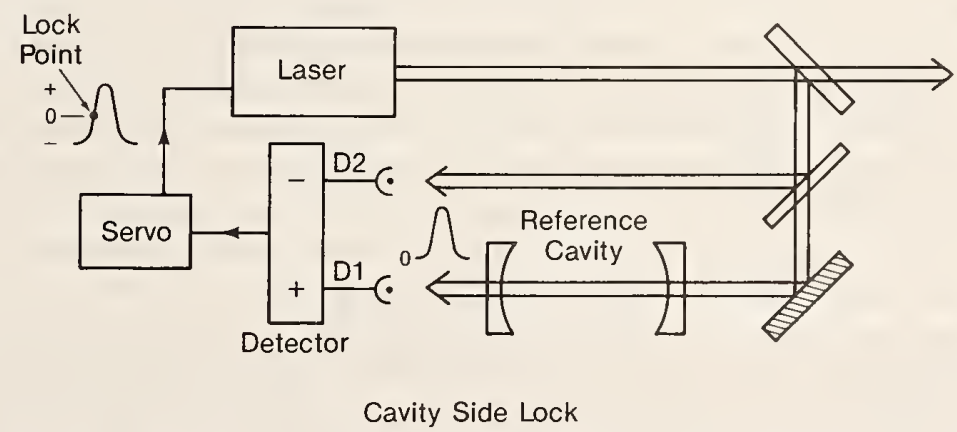

Fig. 5.11 Laser frequency control by the cavity side lock method. Part of the laser's output beam is directed into a Fabry-Perot cavity and onto a photodetector D1. A reference beam is directed onto detector D2 which measures the laser's power. The transmission function of the Fabry-Perot cavity is shown schematically near D1. The cavity acts as a frequency discriminator for the laser. The error signal is generated from the difference between the signals from D1 and D2, and is shown with the lock point indicated at zero voltage. A servo amplifier uses the error signal to control the laser's frequency. 
cavity-resonance width. Depending on the overall sign of the correction signal, the laser frequency can be locked to one side of the fringe or the other. The zero crossing of the error signal is set at the point of fringe half maximum by adjusting a variable attenuator in the reference channel. The DC offset of the reference channel is derived from the laser power rather than from a voltage reference for a good reason. This reference signal generated by the laser power cancels laser-amplitude fluctuations that would otherwise cause an error in the lock signal. This is an important correction because, as we shall see, the amplitude fluctuations on dye lasers are often relatively large. Details of an advanced side-lock frequency-control systems can be found in Helmcke et al. (1982).

\subsubsection{Modulation Lock}

A second and very useful method of laser-frequency stabilization is the modulation lock. This system incorporates frequency modulation and synchronous detection to generate an error signal that is then used to lock the laser to the peak of a resonance. The resonance can be a Fabry-Perot cavity transmission signal but it can equally well be an atomic or molecular resonance. This method is very general and it allows us to stabilize any variable parameter to the maximum or a minimum of a response function. Because of the simplicity of the method and because we often want to maximize (or minimize) some response, the technique is widely used in servo-control systems. We described this method in terms of locking the oscillation frequency of a laser to the peak of a Fabry-Perot transmission fringe. The important features of the modulation lock are illustrated in Fig. 5.12a, 5.12b. The various electronic signals are shown in Fig. 5.12b where the Fabry-Perot transmission fringe is displayed as a function of the laser frequency. If the laser frequency is sinusoidally modulated about the peak of the cavity resonance, the transmitted light intensity varies as the second harmonic of the modulation frequency. However if it is modulated about the side of the cavity resonance, the transmitted power will vary at the modulation frequency with a phase that depends on which side of the fringe the laser is tuned. There is a $180^{\circ}$ relative phase shift of the modulation response on the two sides of the fringe. The power of the laser light transmitted through the cavity is then detected and demodulated using a lock-in amplifier. The lock-in output is then lowpass filtered to produce the discriminator-shaped output (see Fig. 5.12b). This discriminator signal is used as the error signal for the servo-control loop. The phase reversal of the error signal across the fringe changes the sign of the output from the lock-in and produces an error signal that crosses 0 at the peak of the resonance. For small-modulation amplitudes, the shape of the discriminator signal is approximately the first derivative (with respect to frequency) 


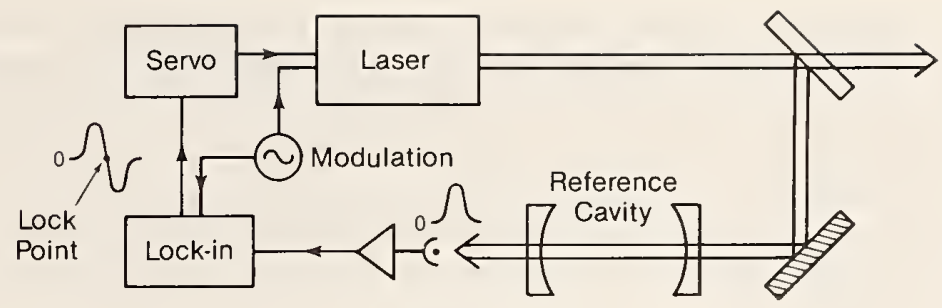

\section{Modulation Lock}

Fig. 5.12a Laser frequency control by the modulation lock method. A Fabry-Perot reference cavity is used as a frequency discriminator for the laser. The cavity's transmission function is indicated schematically near the photodetector and amplifier. The signal from the detector goes to the lock-in amplifier where it is compared with the modulation signal that is used to modulate the frequency of the laser. The lock point is indicated on the error signal that is shown schematically as the output of the lock-in. A servo amplifier uses the error signal to control the laser's frequency.

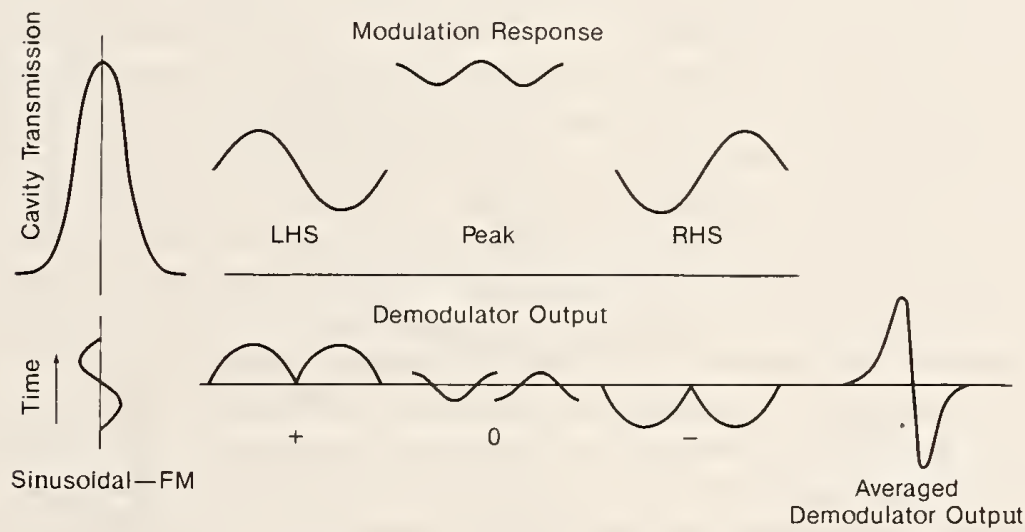

\section{Modulation Lock Signals}

Fig. 5.12b Signals used in the modulation locking method. The cavity transmission function and the modulation of the laser's center frequency are displayed on the left-hand edge of the figure. The center of the figure shows the variation of the cavity's transmission due to the modulation and is displayed as a function of the laser's center frequency relative to the cavity resonance. The demodulator (lock-in) inverts the modulation response synchronously every half-cycle of the modulation. Its output is shown at the bottom of the figure. When the demodulator's output is averaged for times that are long compared with the modulation frequency, the result is an output signal that is approximately the derivative of the cavity transmission function. This output is shown at the bottom right of the figure and is displayed as a function of the laser's center frequency. 
of the transmission function. Similarly, high-order approximate derivative signals are obtained by demodulating the transmitted power at harmonics of the modulation frequency. For example, a third derivative locking signal is obtained by demodulating at the third harmonic of the modulation frequency and it has some advantages with respect to systematic errors. These frequency modulation-demodulation ideas are also useful for reducing noise and background problems in spectroscopy (e.g., laser derivative or FM spectroscopy).

\subsection{3. rf-Optical Heterodyne Lock}

Another laser stabilization system has been developed using rf heterodyne techniques (Drever et al., 1983; Hough et al., 1984; Helmcke et al., 1987). Of all of the methods, this "rf-optical heterodyne" locking system has demonstrated the best performance, in terms of both center frequency control and the narrowest linewidths. The method is similar to the modulation lock described previously, with the distinction that it uses radio- or microwave phase modulation on the laser s output to generate laser sidebands that lie outside the resolution width of the resonance of interest. The high modulation frequencies are advantageous in suppressing noise, in achieving high-servo-control bandwidths, and in achieving good transient response characteristics. An example of an optical heterodyne system used for laser-frequency stabilization is diagrammed in Fig. 5.13. Here the system consists of a laser, an electro-optic phase modulator ( $\phi$ mod, made from a crystal of ADP, ammonium dihydrogen phosphate), an optical directional coupler (made from two polarizers and a Faraday rotator), a precision reference cavity, a photodetector (det), some filters. a balanced mixer, and a servo-control amplifier. The cavity resonance is detected in reflection by this heterodyne technique and can provide two types of signals. These signals are derived from the two detection quadratures of the balanced mixer (determined by the phase of the reference signal) and correspond to the cavity transmission and dispersion functions. The cavity resonance acts to alter the pure phase modulation that was imposed on the laser light by the electro-optic modulator. This alteration of the phase modulation comes from the transmittance and phase shift of the laser light by the cavity. The cavity thus converts some of the phase modulation into amplitude modulation, which is then detected by the photodetector and subsequently demodulated by the mixer to generate a baseband (DC) response. The phase of the reference signal (derived from the original modulation signal) that is applied to the mixer determines the detection quadrature and, thus, whether we detect the effect of cavity transmittance or dispersion. Typical response signals for the two detection quadratures are shown in Fig. 5.14. The discriminator shape of the dispersion signal provides an excellent error 


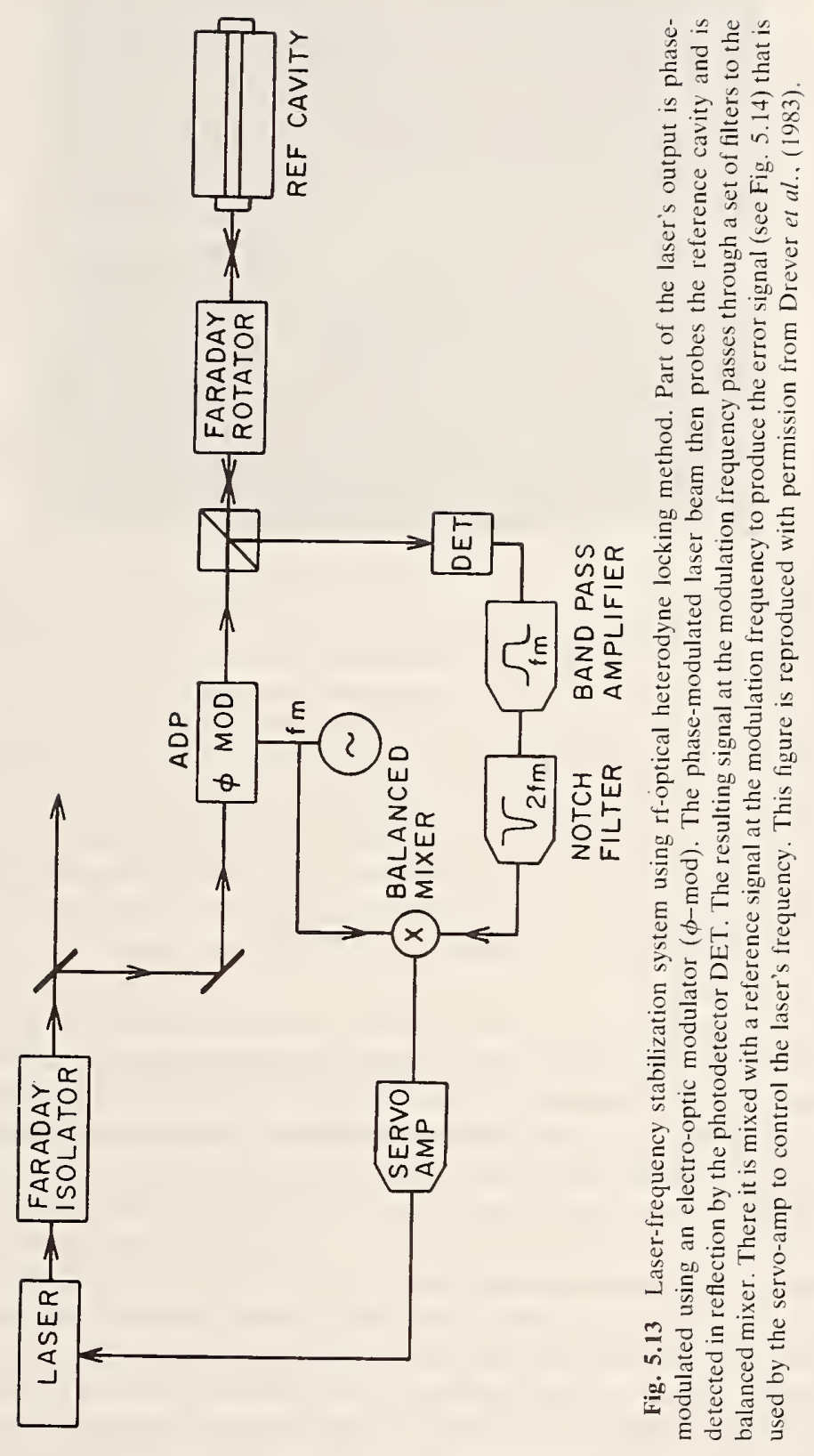




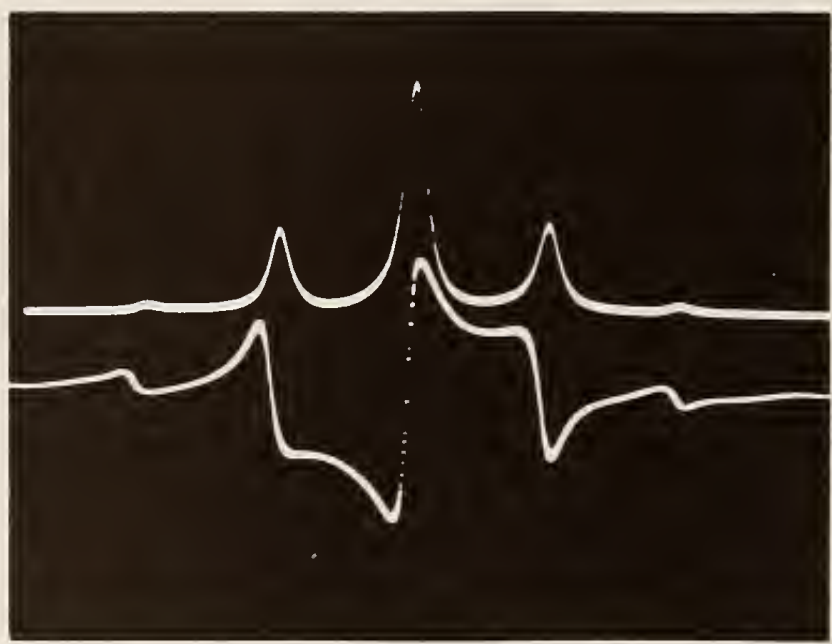

Fig. 5.14 Cavity signals generated by the rf-optical heterodyne locking method. The output of the mixer (from Fig. 5.13) is shown as a function of the laser's frequency which is scanned. The upper trace shows the output when the phase of the reference signal (applied to the mixer) is set to detect the cavity's transmission. The multiple-peaked structure results from the carrier and sidebands being transmitted into the cavity when they come into resonance. The carrier (large peak in the center) and the two adjacent sidebands are separated by the modulation frequency. Small peaks due to the second-order sidebands are also visible at a spacing that is twice the modulation frequency. The lower trace shows the output when the phase of the reference signal is shifted by $\pi / 2$. It represents the effect of cavity dispersion as seen by the phase-modulated laser beam. The dispersion signal has a steep discriminator shape that crosses 0 when the carrier is tuned to the peak of the cavity resonance. This discriminator is used as the error signal in the frequency-control loop. This figure is reprinted with permission from Drever et al. (1983).

signal for laser-frequency control. Some of the components in this system can be compared directly to those of the modulation lock; the phase modulator serves the role of the laser FM, and the mixer replaces the lock-in amplifier. One important advantage of the heterodyne system is that the high modulation frequencies and the detection of the cavity in reflection allow the time scales of the detection process to be very short. This allows the response time of a servo system to be shorter than the characteristic response time of the cavity (Drever et al., 1983) while maintaining a good signal-to-noise ratio. This is an important consideration for the high-speed servo systems that are required for very narrow laser linewidths. This method has been used by Bergquist and collaborators to observe $\simeq 50-\mathrm{Hz}$-wide mercury-ion resonances; this indicates that the dye laser's linewidth and center frequency stability are at least that good (Wineland et al., 1989). 


\subsubsection{Polarization Lock}

Noteworthy because of its good performance and simplicity is a related laser-frequency-stabilization method that was developed by Hänsch and Couillaud (1980). Analysis of the polarization state of the light reflected from the cavity is used to detect the dispersive nature of the cavity resonance. This method has the advantages that it produces a discriminatorshaped signal without modulation and it has a very large capture range. This results in a robust laser-locking method that is useful for laserfrequency stabilization and optical buildup cavities.

\subsubsection{Postlaser Stabilization}

An exciting and relatively new development in laser-frequency control is the implementation of a postlaser-frequency-correction system (Hall and Han̈sch, 1984; Hall et al. 1988). The concept is that with the appropriate acousto-optic and electro-optic transducers, we can correct the frequency errors on the laser beam after the beam exits the laser. A system of this type is diagrammed in Fig. 5.15. The external frequency correction is implemented with an acousto-optic frequency shifter (driven by a voltagecontrolled oscillator) and an electro-optic modulator (EOM). The EOM acts as frequency transducer through its ability to induce a time-dependent optical phase shift via its electric-field-dependent index of refraction. In this case the change in the laser frequency is proportional to the derivative with respect to time of the applied electric field. Thus the circuitry for the electronic feedback to the EOM is complicated by the fact that adding a fixed frequency shift to the laser requires that a voltage ramp be applied to the EOM. The EOM used in this way operates as an optical phase shifter and hence only works well for removing high-frequency fluctuations. The acousto-optic transducer is required for the low frequency fluctuations and fixed frequency offsets. Another challenge is the fact that the time scales for the response of the acousto-optic and electro-optic transducers are very different and appropriate crossover compensation must be designed. Clever solutions to these challenges have been developed by Hall and Hänsch (1984). The error signal for this system can be derived from any of the methods described previously, but perhaps the most appropriate is the rf-optical heterodyne method. Impressive performance has been achieved with these postlaser stabilization systems but, unfortunately, they are not available commercially as of 1989 . Starting with a single-mode dye laser that had an intrinsic linewidth of $\simeq 1 \mathrm{MHz}$, the postlaser stabilization methods have produced linewidths on the order of $1 \mathrm{kHz}$. Even better performance can be anticipated. Another application of this method has been to make an optical phase lock between a cw dye laser and a HeNe laser 


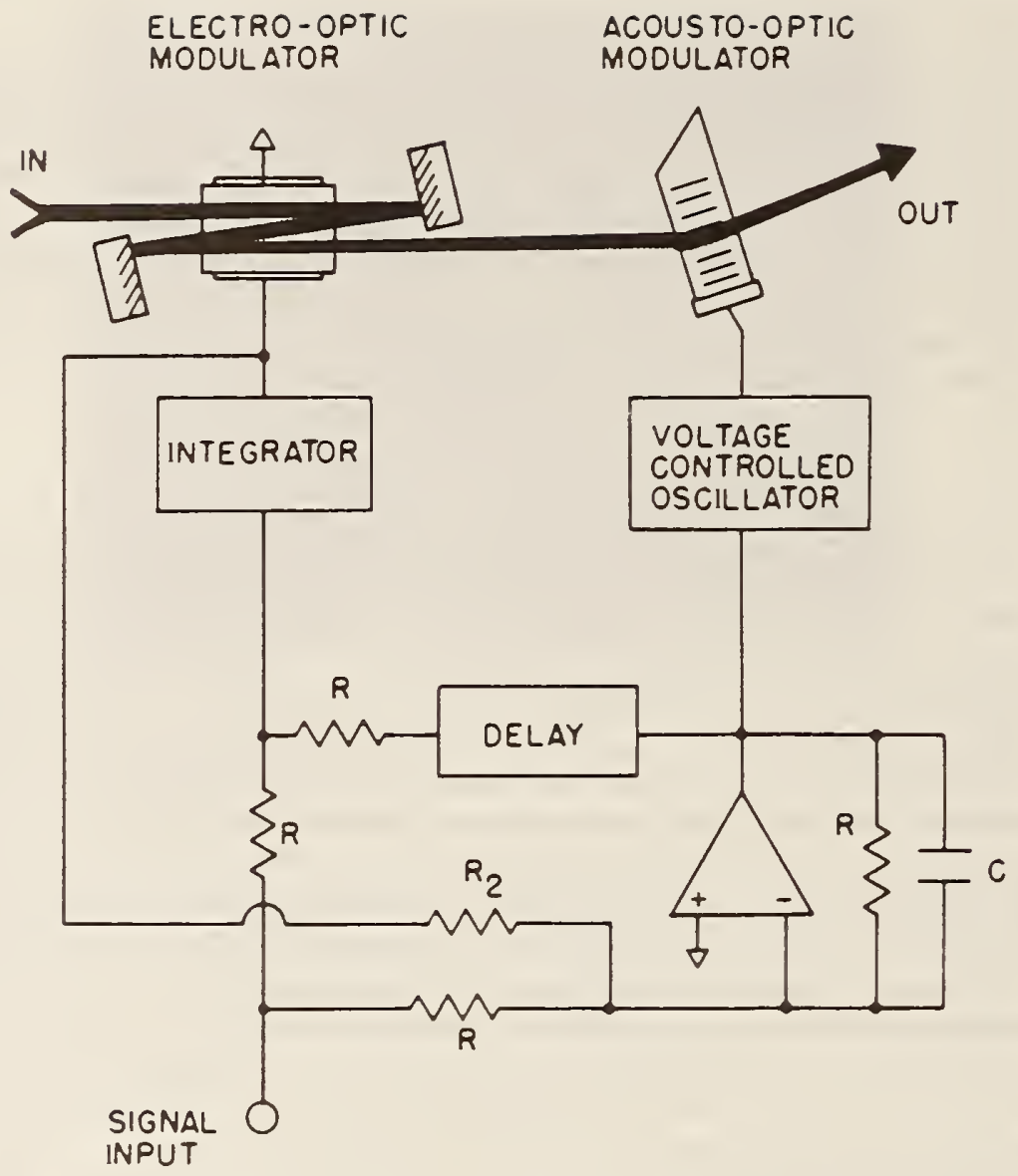

Fig. 5.15 Laser-frequency control by the post-laser-stabilization method. In this system the frequency of the laser's output is changed outside the laser by an electro-optic modulator and an acousto-optic modulator. A time rate of change of voltage applied to the electro-optic crystal will change the frequency of the laser beam by a small amount. Larger frequency shifts are generated by the acousto-optic modulator which is driven by a voltage-controlled oscillator. To optimally use the two transducers the input error signal is first processed electronically as indicated. Figure reprinted with permission from Hall and Hänsch (1984).

that was used as the reference oscillator (Hils and Hall, 1989; Salomon et al., 1988).

It is humbling for laser scientists to learn that many of the basic principles in these methods had been developed in the prelaser days by scientists working with rf and microwave systems. The techniques were subsequently "reinvented" with laser technology. 
Up to this point I have outlined various general techniques for deriving an error signal from a resonance, but have not discussed the requirements for a feedback loop that can use this error signal to control the laser frequency. The loop needs to process the error signal and drive the appropriate transducers to correct the laser's frequency fluctuations. The loop will thereby null (or attempt to null) the error signal. Common dye laser frequency transducers are galvo-motor-driven Brewster plates, piezoelectrically mounted mirrors, and electro-optic crystals (see Fig. 5.6). Each of these transducers can change the optical path length of the laser cavity and hence the laser frequency. Typical commercial frequencystabilized dye-laser systems use a galvo-driven Brewster plate and a piezomounted mirror for their frequency-control transducers. These systems routinely achieve laser linewidths on the order of one $\mathrm{MHz}$. With faster feedback control and better reference cavities it is possible to achieve laser linewidths of about $20-100 \mathrm{kHz}$ with a piezo-mounted mirror and a galvodriven Brewster plate. For very high resolution applications an electrooptic modulator (EOM) can be included in the laser cavity. With the EOM. dye-laser linewidths on the order of 50 hertz have been demonstrated and linewidths of one hertz or less are anticipated. These are truly impressive high-resolution oscillators when we recall that the oscillation frequency is $\simeq 5 \times 10^{14} \mathrm{~Hz}$. The narrow linewidths achieved with the intracavity EOMs are due to the high-speed response of the crystal and the high servo bandwidths that are possible. In general the higher the servo bandwidth. the narrower the resulting laser linewidth. The actual performance of the frequency-stabilization system depends on the spectral density of the frequency noise on the laser and the characteristics of the servo loop. In principle, and in some cases in practice, laser linewidths as narrow as you desire can be achieved with late 1980s technology (Hils and Hall, 1989). Often the limitation to the resolution is not the laser at all but rather the spectroscopic sample of interest.

Optimization of the filter characteristics of the feedback loop can be a difficult task given the complexity of the system with the multiplicity of transducers. In addition we need to take into account the spectrum of the dye laser's frequency noise and the laser's dynamics. The simplest control loops (termed proportional locks) use purely proportional control, where the feedback is linearly related (by the gain) to the error signal. These control systems are very stable but are lacking in both accuracy and dynamic response. A significant improvement in performance is obtained by integrating the error signal with respect to time before feeding back to the transducers. An integrating loop filter has gain that rolls off toward higher frequencies at a rate of $6 \mathrm{~dB}$ per octave for each stage of integration. Even a single-stage integrating-loop filter has the advantage that is 
guarantees zero error at DC and only a fixed, finite error for a ramping error signal. In contrast, the proportional control loop will in general always have a finite error. Adding some derivative feedback in the loop can often improve the dynamic response. It is common practice to use some combination of proportional, integral, and derivative feedback. These systems are best optimized with careful system analysis, but empirical adjustment of the gains and time constants is often productive.

As we have discussed, most of these laser-locking methods can be applied equally well to stabilizing the frequency of lasers to a cavity or to atomic or molecular resonances. In the latter case the most applicable techniques are the modulation lock and the rf heterodyne lock. The ability to stabilize the laser to an atomic resonance is important for some spectroscopic applications and is crucial for high-accuracy metrology and laser-frequency standards. The atomic and molecular resonances cannot provide the same signal-to-noise ratio that is available from a cavity, but their long-term (say, for times longer than a few seconds) stability is generally much better. For dye lasers to have high resolution and high accuracy, it is customary to lock the laser's frequency to a high-Q reference cavity (to achieve the narrow linewidths) and then to lock the cavity to an atomic or molecular resonance (which then provides the long-term stability).

The transformation of a broadband dye laser into a frequency-stabilized narrow-linewidth laser adds considerably to the cost and complexity of the laser system. There is also a penalty to be paid in the available output power when we add intracavity optical elements (this typically amounts to about a factor of 2). If we use an electro-optic crystal, the upper power limit may also be reduced due to the limited optical-power density that the crystals can endure.

In summary, commercial stabilized dye-laser systems can provide high resolution $(\simeq 1 \mathrm{MHz})$ with tunability and good power levels. Their tuning range extends throughout the visible and near-visible part of the spectrum. These high-resolution systems are more than adequate for most spectroscopic applications. For applications that demand higher resolution, very narrow-linewidth dye-laser systems have been developed in a number of research laboratories. The systems with the best reported resolution generally use optical heterodyne techniques and EOMs to achieve laser linewidths below $100 \mathrm{~Hz}$. I see no reason that the linewidths could not be reduced further to below one $\mathrm{Hz}$ in the future. So while the performance levels achieved in 1989 are truly impressive, they are merely a glimpse of the present state of the art and they will certainly be surpassed. 


\section{SPECTRAL CHARACTERISTICS}

The complexity of dye lasers encourages the user to have some diagnostic instruments to monitor the laser's output characteristics. Useful diagnostic tools include power meters as well as wavelength- and linewidthmeasuring devices.

With dye tuning ranges of about $100 \mathrm{~nm}$ and linewidths of about $10^{-6} \mathrm{~nm}$ it is not a trivial task to determine, with precision, the lasing wavelength of $\mathrm{cw}$ dye lasers. Many interferometric instruments have been developed to measure the laser's wavelength relative to known standards; these include the lambda or wave meter, the sigma meter, the Fizeau wavemeter, calibrated Fabry-Perot interferometers, and dispersive birefringent filters (Juncar and Pinard, 1975; Kowalski et al., 1976, 1978; Hall and Lee, 1976a; Woods et al., 1978; Snyder, 1980; Williams et al., 1983; Licthten 1985, 1986; DeVoe et al., 1988). The accuracy of these interferometers varies from 5 to 8 digits. Special standards-laboratory instruments are capable of 10 digits of accuracy in wavelength measurement. Also extremely useful for general laboratory spectroscopy are the wavelength tabulations that exist for molecular spectra of some simple molecules, such as those for $\mathrm{I}_{2}$ (in the red) (Gerstenkorn and Luc, 1978) and $\mathrm{Te}_{2}$ (in the blue) (Cariou and Luc, 1980). These tabulations can be used to determine a dye laser's wavelength to about $3 \times 10^{-7}$.

We need certain mathematical and experimental tools to characterize the spectral properties of lasers. These tools certainly include the frequency and amplitude-noise spectral densities and maybe the Allan variance. The Allan variance was developed to quantify the frequency stability of oscillators and can be applied directly to lasers. It is discussed here because of its usefulness and its popularity in the literature and also because it is not found in the usual textbooks.

\subsection{Amplitude Fluctuations}

The spectral distribution of amplitude noise in the output of a singlefrequency dye laser contains a great deal of useful information. It can be measured directly by monitoring the laser light with a fast photodetector and a rf-spectrum analyzer. The quantum theory of the laser predicts that the fluctuations in the power from an ideal laser source will be determined by the statistics of the quantum mechanical generation of the photons (Sargent et al., 1974; London, 1983). In this model the ideal laser would produce light with a time distribution of photons that is Poissonian. Upon 


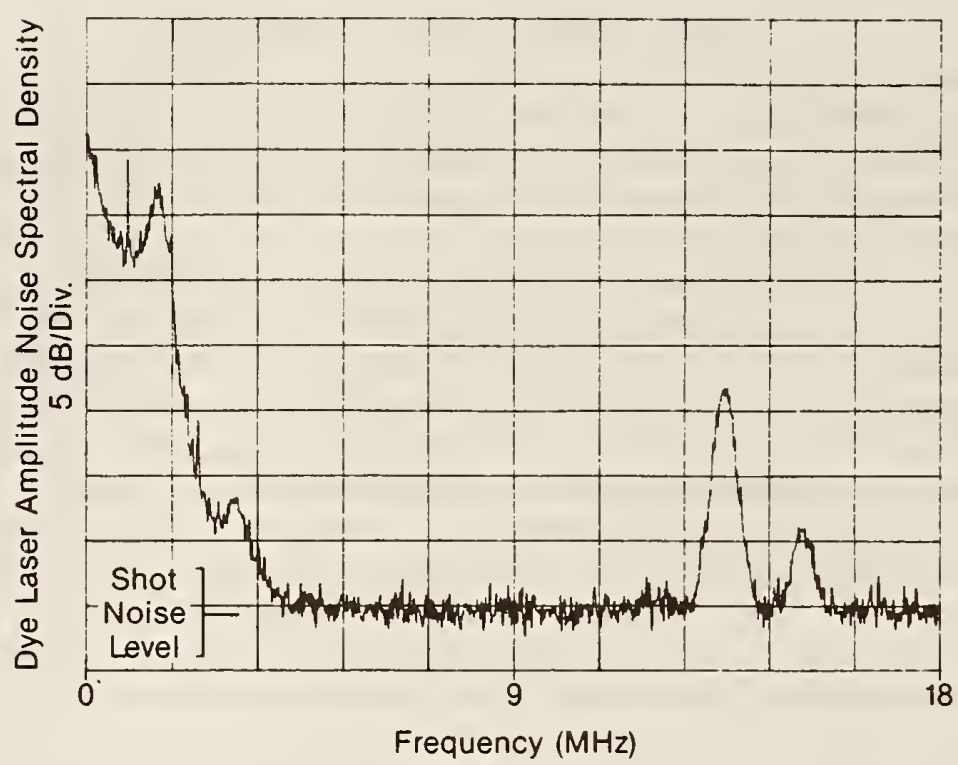

Fig. 5.16a Spectral density of the amplitude noise of a single-mode ring dye laser. The noise on the laser's output power is strongly peaked at the lower frequencies and finally reaches the shot-noise level at a frequency of about $4.5 \mathrm{MHz}$. The broad peaks in the noise come from small amounts of power oscillating in transverse modes in the argon pump laser. These pump-laser-related noise peaks extend out to hundreds of megahertzes but spectral regions can be found where the dye laser s amplitude noise is shot-noise-limited.

detection this photon distribution generates a Poissonian photoelectron distribution and hence produces the usual "shot-noise" fluctuations in the photocurrent,

$$
i_{\mathrm{SN}}=(2 e i B W)^{1 / 2} .
$$

Here $i$ is the detected photocurrent, $e$ is the electron charge. and $B W$ is the detection bandwidth. The shot-noise distribution is spectrally flat. Figure 5.16a shows the measured spectral density of the amplitude noise of a good single-mode dye laser. The figure also indicates the expected shot-noise level for the same average optical power on the detector. Clearly, there is more going on in real lasers than is predicted by the quantum mechanical model of the ideal laser. Characteristically, the amplitude noise is very large at low Fourier frequencies and decreases progressively to higher frequencies until it reaches the shot-noise level at a frequency of approximately $4.5 \mathrm{MHz}$. We also see that there are many large resonant noise peaks throughout the spectrum. These are due to various technical noise 


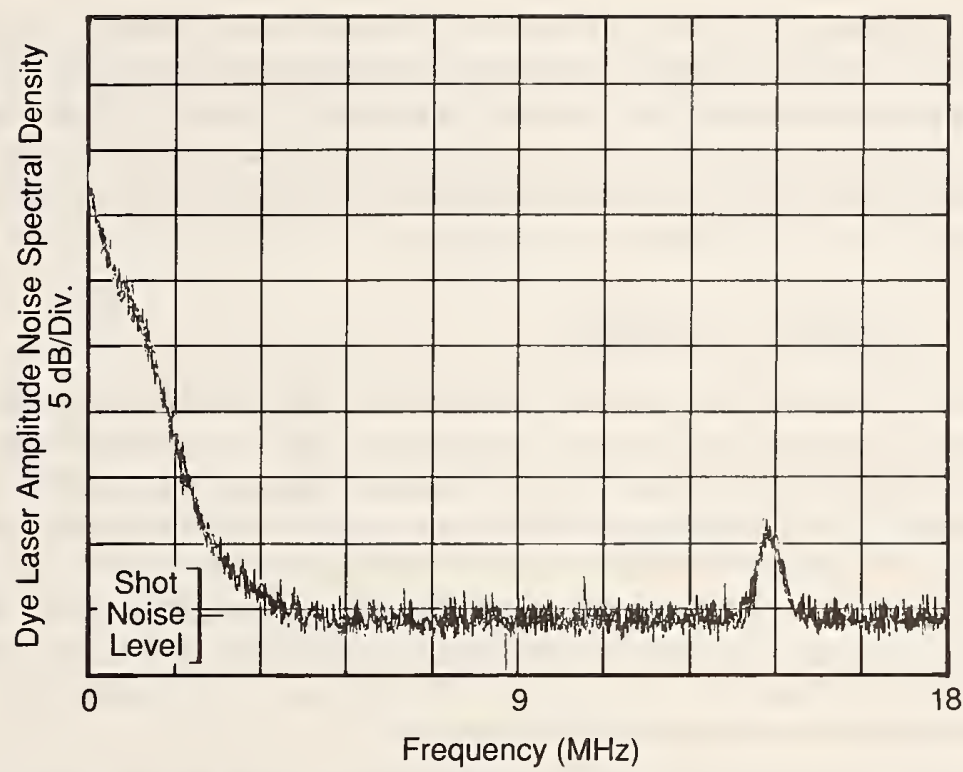

Fig. 5.16b Spectral density of the amplitude noise of the same dye laser as shown in Fig. 5.16a, but now with the aperture in the argon laser carefully adjusted to elliminate transverse modes. The dye laser's amplitude noise is improved, but the noise is still strongly peaked at low frequencies.

sources, the most important of which are the argon laser's intermode beats that are transferred through the gain of the dye jet to the dye laser's power (these intermode beats are observed out to several hundreds of megahertz). In Fig. 5.16b the spectral density of the amplitude noise of the same dye laser is shown but now with the argon laser resonator apertured to eliminate transverse modes. This removes most of the resonant peaks in the noise (in this frequency range) and significantly improves the dye laser's amplitude noise spectrum.

One of the important things we learn from the spectrum of amplitude noise is that any measurements that are made with a detection bandwidth centered at low frequencies (or near one of the resonant noise peaks) will be contaminated by excess noise on the laser. As seen in Fig. 5.16 this excess noise is not negligible. In this example there is $\simeq 35 \mathrm{~dB}$ of excess noise at a detection frequency near $100 \mathrm{kHz}$ (resolution bandwidth was $3 \mathrm{kHz}$ ). The noise is even greater at lower frequencies. Not surprisingly the signal-to-noise ratio can be improved by moving the center of the detection bandwidth to higher frequencies by using high-frequency modulation methods. If we look to high enough frequencies it is possible to reach the 
shot-noise level in some limited-detection bandwidth. In fact, it is even, possible to achieve noise levels below this shot-noise level by using nonlinear optical methods to generate nonclassical forms of light such as squeezed states. This regime of sub-shot-noise limited detection is a hot topic in modern optics with two compilations of papers on this and related subjects ("Squeezed States," 1987).

\subsection{Frequency Fluctuations}

One method for determining the frequency stability and linewidth of a dye laser is to monitor the laser's output with a high-resolution Fabry-Perot cavity (optical spectrum analyzer). These instruments are available commercially. Using a scanning Fabry-Perot cavity to measure the spectral distribution of light from a multimode standing-wave dye laser (with only a birefringent filter as the tuning element) shows the optical power distributed in a large number of discrete longitudinal modes. The spectral width of this array of modes can vary from 1 to $100 \mathrm{GHz}$. In this multimode case the distribution of modes is often unstable.

If the dye laser is made to run on a single mode, a high-finesse cavity can be used to determine the laser's linewidth and the spectral distribution of the residual frequency fluctuations. Another method for measuring the frequency stability of a laser is to use a fast photodiode to observe the beat note between the unknown laser and a narrow-linewidth reference laser. There is also the "self-heterodyne" method, which uses a fiber delay-line to measure the laser's linewidth.

The frequency noise spectrum of a single-mode laser can be measured best by detecting the rf spectrum of the heterodyne beat note between two lasers: the unknown laser and a reference laser that has a narrower spectral width (Hough et al., 1984). This heterodyne method is generally preferred for the very high resolution systems but it requires an additional very stable reference laser to act as the local oscillator. A limitation here is that the linewidth of the very best lasers must be determined by deconvolution of two equally good lasers or by other methods (cavities or special narrow spectral lines).

The Fabry-Perot cavity method of measuring a laser's frequency-noise spectrum relies on using the cavity's transmittance or reflectance function as a frequency discriminator. By tuning the laser's center frequency to the side of the cavity resonance and measuring transmitted power, the cavity then acts to convert frequency fluctuations directly into amplitude fluctuations at the detector. A cavity (or a molecular resonance) can be an excellent frequency discriminator but care must be taken to avoid systematic errors (for example, detection bandwidth limits, cavity response time, 
and laser amplitude noise). The literature is full of "outstanding" results based on improper measurements. A good example of a very popular mistake is to lock a laser's frequency to some cavity or atomic resonance (by the methods described previously) and then monitor the error signal within the servo-loop to provide a measure of the residual frequency noise. This appears reasonable at first glance, but usually the error signal is contaminated at some level by systematic errors (such as a ground loop). These systematic error signals are then corrected by the servo system as if they were laser-frequency fluctuations. This puts these artificial errors directly on the laser frequency. Any measurement of the error signal would indicate that the laser's frequency fluctuations are small, because the servo loop is acting to cancel the error signal. This gives a misleading result when the measured error signal is then used to calculate the laser's frequency fluctuations. However, it is true that monitoring the residual error signal is useful in providing information about how well the servo electronics are working. It does not answer the question of how well the error signal represents the laser's frequency fluctuations. To make a definitive statement about the frequency fluctuations of a stabilized laser it is imperative to have a measure of the laser's frequency that is independent of the stabilization system.

The self-heterodyne method of measuring a laser's linewidth uses a time delay and a heterodyne detector to produce the autocorrelation of the laser's frequency noise at a given time delay (Okoshi et al., 1980). This method compares the oscillation frequency of a laser at an earlier time with its oscillation frequency now. Self-heterodyne systems are very easily implemented by sending some of the laser's output through a long fiber (for the delay) and then combining this fiber-delayed output with the direct output on a fast photodetector to observe the beat note. In these systems one of the two beams is usually frequency shifted by an acousto-optic modulator (for example, by $80 \mathrm{MHz}$ ) to avoid technical noise at zero frequency difference upon detection. It is difficult to make accurate measurements with this method if the laser has a very narrow linewidth, and in general care must be used to avoid misleading results (Kikuchi and Okoshi, 1985).

A spectrum of frequency fluctuations can be used to provide an effective laser linewidth (Elliot et al., 1982). In two limiting cases, which depend on the analytical character of the frequency noise, the linewidth can be calculated trivially. The important factor here is whether the Fourier frequency of the dominant frequency fluctuations is high or low, compared with the rms frequency fluctuations of the laser. That is, are the main frequency excursions caused by low frequencies with a high modulation index, or high frequencies with a low modulation index, where high and 
low are measured relative to the laser's rms frequency fluctuations. For example, if we make the assumption that the frequency-noise spectrum is rectangular with a bandwidth $B$, we can calculate the linewidth in these two limiting cases. If the laser's rms frequency fluctuations are large compared to the bandwidth of the frequency fluctuations, the lasers lineshape is Gaussian and the linewidth is given by

$$
\Delta_{\mathrm{FHM}}=2(2 \ln 2)^{1 / 2} \delta_{\mathrm{rms}} .
$$

Here $\delta_{\mathrm{rms}}^{2}$ is the rms frequency deviation. On the other hand, when the bandwidth of frequency fluctuations is larger than the laser's rms frequency fluctuations, the laser's lineshape will be Lorentzian and the linewidth will be given by

$$
\Delta_{\mathrm{FWHM}}=\pi \frac{\left(\delta_{\mathrm{rms}}\right)^{2}}{B} .
$$

Here we have again simplified the problem by assuming that the spectrum of frequency fluctuations is rectangular in shape with a bandwidth $B$. In the more general case when the frequency fluctuations are not spectrally flat, with important contributions coming from both high and low Fourier frequencies, the lineshape and linewidth could be determined from the spectrum of frequency fluctuations by numerical integration of the equations given by Elliot et al. (1982).

As with the amplitude noise, the spectrum of frequency fluctuations for dye lasers is large at low frequencies and then falls off at higher frequencies. There are often numerous large resonances throughout the spectrum. Figure 5.17 shows the spectrum of frequency fluctuations of a single-mode ring dye laser that was measured with a high finesse cavity and a rf-optical heterodyne detection system. Also shown is the reduction in the frequency noise that is achieved when the frequency-control loop is turned on. In this case, the servo is able to reduce the frequency noise (as measured by the servo-loop error signal) by as much as $50 \mathrm{~dB}$. An independent frequency discriminator would be required for an accurate determination of the laser's linewidth.

A mathematical tool that is genuinely useful in describing the frequency stability of lasers (and other oscillators) is the Allan variance (Allan, 1966; Allan et al., 1974). It is the variance of the second difference of a series of consecutive frequency measurements. The Allan variance is defined

$$
\sigma(\tau)=\frac{1}{\nu_{0}}\left(\frac{1}{2(M-1)} \sum_{i=1}^{M-1}\left(\nu_{i+1}-\nu_{i}\right)^{2}\right)^{1 / 2}
$$




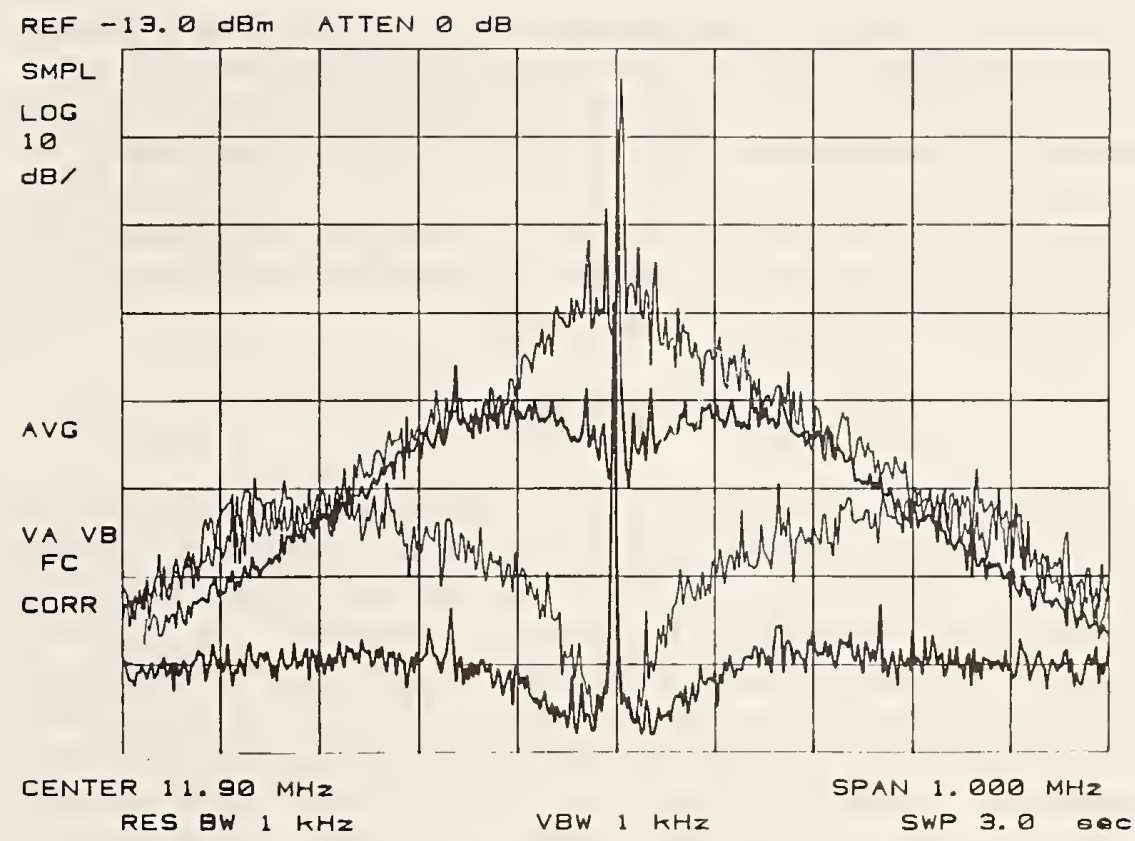

Hall et al Summary for IQEC - 88 Tokyo, Japan July 18-22, 1988

Fig. 5.17 Spectral density of the frequency noise of a single-mode ring dye laser with and without fast frequency control. The frequency fluctuations are measured using the rf-optical heterodyne locking method (modulation frequency $\simeq 12 \mathrm{MHz}$ ) diagrammed in Fig. 5.13. The vertical scale displays the dye laser's frequency noise with a sensitivity of $10 \mathrm{~dB} /$ div. The horizontal scale is $100 \mathrm{kHz} /$ div and the data were taken with a resolution bandwidth of one $\mathrm{kHz}$ and a video bandwidth of one $\mathrm{kHz}$. The largest peak shows the frequency noise on the laser without fast frequency control. When a fast postlaser frequency control loop (of the type diagrammed in Fig. 5.15) is implemented, the frequency fluctuations are reduced. The three lower curves show the effect of incrementing the control loop gain by about $10 \mathrm{~dB}$ per step. The frequency noise remaining in the lowest trace is near the noise level of the measurement system. It would correspond to a dye-laser linewidth of less than one $\mathrm{Hz}$ if the measurement of the error signal was an accurate measure of the laser's frequency fluctuations. Figure is reproduced with permission from Hall et al. (1988).

Here $M$ is the number of successive frequency measurements (assuming no dead time between measurements), $\nu_{0}$ is the mean laser oscillation frequency, and $\nu_{i}$ is the $i$ th frequency measurement made during the sample interval time $\tau$. The Allan variance can also be calculated from the power spectral density of the frequency noise $\mathrm{S}(f)$ as follows:

$$
\sigma^{2}(\tau)=2 \int_{0}^{\infty} d f \frac{S(f)}{\nu_{0}^{2}}\left(\frac{\sin ^{2} \pi f \tau}{\pi f \tau}\right)^{2}
$$


One of the wonderful properties of this function is that it is well behaved for common types of frequency noise, such as $1 / f$ noise. This is not true for the usual standard deviation which is often divergent. The Allan variance serves as a one-parameter figure of merit for the frequency stability of oscillators. Without measuring the spectrum, it can provide a diagnostic of the type of frequency fluctuations that are present. For example, an Allan variance that decreases as $1 / \tau$, where $\tau$ is the averaging time, indicates that the frequency noise spectral density is white.

\section{SUMMARY}

$\mathrm{Cw}$ dye lasers are a unique source of laser radiation with broad tunability, high spectral purity and good output-power levels. We have seen that these lasers can provide complete spectral coverage from 365 to $1000 \mathrm{~nm}$ with typical power levels that range from 0.1 to more than $1 \mathrm{~W}$. Nonlinear optical techniques have been used to extend this spectral coverage into the UV (260-400 $\mathrm{nm})$ by harmonic generation and sum frequency mixing and into the near-IR (2.2-5 microns) by difference frequency mixing. Other specific wavelengths not included here have been generated by nonlinear methods for special applications. Continued improvements in the pump lasers, the dyes, and the nonlinear optical materials will undoubtedly provide higher output powers and extended spectral coverage.

The spectral characteristics of cw dye lasers are both good and bad: good with respect to their linewidths and. hence, spectral-resolving capabilities, but generally poor with respect to their amplitude noise properties. The spectral density of amplitude noise on cw dye lasers is large for Fourier frequencies below a few megahertz. Because of necessity, techniques have been developed to alleviate the problems associated with this amplitude noise. Typical linewidths of $\mathrm{cw}$ frequency-stabilized dye lasers are $\simeq 1 \mathrm{MHz}$, which is more than adequate for most applications. The technology is available to reduce the dye laser's linewidth to $\approx 1 \mathrm{~Hz}$ for those special applications that require it.

\section{PROBLEMS}

1. By making simple assumptions about the dye gain and available pump power, design a three-mirror folded linear dye laser cavity of the type shown in Fig. 5.2. For this cavity calculate the expected difference in frequency between the main longitudinal mode and the spatial holeburning mode that could oscillate simultaneously.

2. Calculate the expected temperature rise of a laser dye (such as R6G in ethylene glycol) when it is pumped with $10 \mathrm{~W}$ of $\mathrm{cw}$ ion-laser radiation 
at $514 \mathrm{~nm}$. Make reasonable assumptions about the optical and physical properties of the dye and the dye-circulation system.

3. Estimate the feasibility of pumping a $\mathrm{cw}$ dye laser with solar radiation. For this estimate propose some simple and realistic design for the laser.

4. Assuming the spectrum of frequency noise shown in Fig. 5.17 is an accurate representation of the dye laser's frequency fluctuations, make an estimate of the contributions to the laser's linewidth due to the various spectral regions of the noise. To calibrate the vertical scale, use the fact that a perfectly coherent laser would produce a signal of $-3 \mathrm{dBm}$ (at $12 \mathrm{MHz}$ ) when the laser's center frequency was tuned to the half-power transmission point of the reference cavity. Note that the rf-heterodyne detection method transfers the frequencyfluctuation information from zero frequency to the modulation frequency $(12 \mathrm{MHz}$ in this case).

5. Assume that the Fourier spectrum of a laser's frequency fluctuations is described by a simple power law dependence (that is, $\mathrm{S}(f)=H_{a} \cdot f^{-a}$ : $a=2,3 / 2,1,1 / 2,0,-1 / 2$, and -1 , and $H_{a}$ is a constant).

(a) Find the functional dependence of the Allan variance on the averaging time $\tau$.

(b) Determine which simple power laws also give a standard deviation (using the usual definition) that is well behaved.

6. What sort of length stability is required for a Fabry-Perot reference cavity that is used to stabilize the frequency of a visible laser to one Hz? Can length stabilities with this performance be achieved given the physical properties of realistic materials?

\section{REFERENCES}

Allan, D.W. (1966). Statistics of atomic frequency standards, Proc. IEEE 54, 221-230.

Allan, D.W., Shoaf, J.H., and Halford, D. (1974). Time and Frequency: Theory and Fundamentals (Blair, B.E.. ed.) NBS (National Bureau of Standards). Monograph 140. U.S Government Printing Office, Washington. D.C., pp. 151-166.

Anliker, P., Luthi, H.R., Seelig, W., Steinger, J., Weber. H.P., Leutwyler, S., Schumacher, E., and Woste, L. (1977). 33-W cw dye laser. IEEE J. Quant. Elect. QE-13, 547-548.

Balykin, V.I., Ovchinnikov, Yu. B., and Sidorov. A.I. (1987). Single- and double-frequency cw dye laser with active frequency stabilization and electronic spectral scanning. Sov. $J$. Quant. Elect. 17, 1535-1539.

Barger, R.L., Sorem. M.S., and Hall, J.L. (1973). Frequency stabilization of a cw dye laser. Appl. Phys. Lett. 22, 573-575.

Barger, R.L., West. J.B., and English, T.C. (1975). Fast frequency stabilization of a cw dye laser. Appl. Phys. Lett. 27, 31-33.

Bergquist, J.C., (1989). Private communication. 
Bergquist, J.C.. and Burkins, L. (1984). Efficient single mode operation of a $\mathrm{cw}$ ring dye laser with a Mach-Zehnder interferometer. Opt. Comm. 50, 379-385.

Bergquist, J.C., Hemmati, H., and Itano. W.M. (1982). High power second harmonic generation of $257 \mathrm{~nm}$ radiation in an external ring cavity. Opt. Comm. 43, 437-442.

Biraben, F. (1979). Efficacité des systemes unidirectionnels utilisables dans les lasers en anneau. Opt. Comm. 29, 353-356.

Biraben, F. (1989). Private communication.

Biraben, F.. and Labastie, P. (1982). Balayage d'un laser a colorant continu monomode sur $150 \mathrm{GHZ}$. Opt. Comm. 41, 49-51.

Blit, S., Weaver, E.G., Dunning, F.B., and Tittel, F.K. (1977). Generation of tunable continuous-wave ultraviolet radiation from 257 to $320 \mathrm{~nm}$. Opt. Lett. 1, 58-60.

Blit, S., Weaver, E.G., Rabson,. T.A., and Tittel, F.K. (1978). Continuous wave UV radiation tunable from $285 \mathrm{~nm}$ to $400 \mathrm{~nm}$ by harmonic and sum frequency generation. Appl. Optics, 5, 721-723.

Bloom, A.L. (1974). Modes of a laser resonator containing tilted birefringent plates. J. Opt. Soc. Am. 64, 447-452.

Bonarev, B.V., and Kobtsev. S.M. (1986). Calculation and optimization of a birefringent filter for a cw dye laser. Opt. Spectrosc. 60, 501-504.

Born, M. and Wolf, E. (1964). Principles of Optics, 2nd ed. Macmillan. New York, pp. 323341 .

Cariou, J., and Luc, P. (1980). Atlas du Spectre d'Absorption de la Molecule de Tellure. Laboratoire Aime-Cotton C.N.R.S. II, Orsay, France.

Clark, D.L., Martin, A.G., Dutta, S.B., and Rogers, W.F. (1986). A high resolution computer controlled dye laser system. Advances in Laser Science-1, AIP Conf. series 146, Optical Sci. and Eng. subseries 6, (Stwalley, W.C., and Lapp, M.. eds.) American Institute of Physics, New York.

Couillaud, B. (1981). Generation of cw coherent radiation in the near U.V.J. de Physique, colloque $\mathrm{C} 8$, supplement 12 . tome $42,115-125$.

Couillaud, B., Dabkiewicz, Ph., Bloomfield, L.A., and Hänsch, T.W. (1982). Generation of continuous-wave ultraviolet radiation by sum-frequency mixing in an external ring cavity. Opt. Lett. 7, 265-267.

Danielmeyer, H.G. (1971). Effects of drift and diffusion of excited states on spatial hole burning and laser oscillations. J. Appl. Phys. 42, 3125-3132.

DeVoe, R.G., Fabre, C., Jungmann. K.. Hoffnagle, J., and Brewer, R.G. (1988). Precision optical-frequency difference measurements. Phys. Rev. A 37, 1802-1805.

Drever, R.W.P., Hall, J.L., Kowalski. F.V., Hough, J., Ford. G.M., Munley. A.J.. and Ward, H. (1983). Laser phase and frequency stabilization using an optical resonator. Appl. Phys. B 31, 97-105.

Dunn. M.H., and Ferguson, A.I. (1977). Coma compensation in off-axis laser resonators. Opt. Comm. 20, 214-219.

Elliot, D.S., Roy, R., and Smith, S.J. (1982). Extracavity laser band-shape and bandwidth modification. Phys. Rev. A 26, 12-18.

Freeman. R.R., Bjorklund. G.C., Economou. N.P., Liao. P.F., and Bjorkholm. J.E. (1978). Generation of cw VUV coherent radiation by four-wave sum frequency mixing in $\mathrm{Sr}$ vapor. Appl. Phys. Lett. 33, 739-742.

Frölich, D., Stein, L., Schröder, H.W., and Welling, H. (1976). Efficient frequency doubling of $\mathrm{cw}$ dye laser radiation. Appl. Phys. 11, 97-101.

Gerstenkorn, S., and Luc, P. (1978). Atlas du Spectre d'Absorption de la Molecule d'lode. Laboratoire Aime Cotton, C.N.R.S. II, Orsay, France.

Green, J.M., Hohimer, J.P., and Tittel F.K. (1973). Traveling-wave operation of a tunable cw dye laser. Opt. Comm. 7, 349-350. 
Hall, J.L. (1978). Stabilized lasers and precision measurements. Science 202, 147-156.

Hall, J.L., and Hänsch, T.W. (1984). External dye-laser frequency stabilizer. Opt. Lett. 9, 502-504.

Hall, J.L., and Lee, S.A. (1976a). Interferometric real-time display of cw dye laser wavelength with sub-doppler accuracy. Appl. Phys. Lett. 29, 367-369.

Hall, J.L., and Lee, S.A. (1976b). Control techniques for cw dye lasers. In Tunable Lasers and Applications (Mooradian, A., Jaeger, T., and Stokseth, P., eds.). Springer-Verlag, Berlin, pp. 361-366.

Hall, J.L., Zhu, M., Shimizu, F.J., and Shimizu, K. (1988). External frequency stabilization of a commercial dye laser at the Hertz level. XVI International Quantum Electronics Digest (Japan Society of Applied Physics. Tokyo, 1988), pp. 4-5.

Hänsch, T.W. (1976). Spectroscopic applications of tunable lasers. In Tunable Lasers and Applications. (Mooradian, A., Jaeger, T., and Stokseth, P., eds.), Springer-Verlag, Berlin, pp. 326-339.

Hänsch, T.W., and Couillaud, B. (1980). Laser frequency stabilization by polarization spectroscopy of a reflecting reference cavity. Opt. Comm. 35, 441-444.

Harri, H.-P., Leutwyler, S., and Schumacher, E. (1982). Nozzle design yielding interferometrically flat fluid jets for use in single-mode dye lasers. Rev. Sci. Instrum. 53, $1855-1858$.

Helmcke, J., Lee, S.A., and Hall, J.L. (1982). Dye laser spectrometer for ultrahigh spectral resolution: Design and performance. Appl. Opt. 21, 1686-1694.

Helmcke, J., Snyder, J.J., Morinaga, A., Mensing, F., and Glaser, M. (1987). New ultra-high resolution dye laser spectrometer utilizing a non-tunable reference resonator. Appl. Phys. $B$ 43, 85-91.

Hemmati, H., Bergquist, J.C., and Itano, W.M. (1983). Generation of continuous-wave 194-nm radiation by sum-frequency mixing in an external ring cavity. Opts. Lett. 8, 73-75, and Hemmati, H., and Bergquist, J.C. (1983). Generation of continuous-wave 243-nm radiation by sum-fiequency mixing. Opt. Comm. 47, 157-160, and Wineland, D.J., Itano, W.M., Bergquist, J.C., Bollinger, J.J., and Prestage, J.D. (1985). In Spectroscopy of stored atomic ions. Atomic Physics 9, (Vandyck Jr., J.S., and Fortson, E.N., eds.). World Scientific, Singapore.

Hills, D., and Hall, J.L. (1989). Ultra stable cavity-stabilized lasers with subhertz linewidth. In 4th Symposium on Frequency Standards and Metrology, Ancona, Italy, Sept. 1988) De Marchi, A. ed.). Springer-Verlag, Heidelberg.

Holberg, L.W. (1984). Measurement of the Atomic Energy Level Shift Induced by Blackbody Radiation. Thesis. U. of Colorado, Boulder.

Hough, J., Hils, D., Rayman, M.D., Ma, L.s., Hollberg, L., and Hall, J.L. (1984). Dye-laser frequency stabiiization using optical resonators. Appl. Phys. B 33, 179-185.

Jarrett, S.M., and Young, J.F. (1979). High-efficiency single-frequency $\mathrm{cw}$ ring dye laser. Opt. Lett. 4, 176-178

Jenkins, F.A., and White, H.E. (1957). Fundamentals of Optics, 3rd ed. McGraw-Hill, New York, p. 565.

Johnston. T. (1988). High power single frequency operation of dyes over the spectrum from $364 \mathrm{~nm}$ to $524 \mathrm{~nm}$ pumped by an ultraviolet argon ion laser. Opt. Comm. 69, 147-152.

Johnston, T.F., Jr. (1987). Tunable dye lasers. In Encyclopedia of Physical Science and Technology 14, Academic Press, Orlando, Fla., pp. 96-141.

Johnston, T.F., JR., and Proffitt, W. (1980). Design and performance of a broad-band optical diode to enforce one-direction traveling-wave operation of a ring laser. IEEEJ. Quant. Elect. QE-16, 483-488.

Johnston, T.F. Jr., Brady, R.H., and Proffitt, W. (1982). Powerful single-frequency ring dye laser spanning the visible spectrum. Appl. Phys. 21, 2307-2316. 
Johnston, W.D., Jr., and Runge, P.K. (1972). An improved astigmatically compensated resonator for $\mathrm{cw}$ dye laser. IEEE J. Quant. Elect. QE-8 (Corresp.), 724-725.

Juncar, P., and Pinard, J. (1975). A new method for frequency calibration and control of a laser. Opt. Comm. 14, 438-441.

Kikuchi, K., and Okoshi, T. (1985). Dependence of semiconductor laser linewidth on measurement time: Evidence of predominance of $1 / \mathrm{f}$ noise. Electron. Lett. 21, 10111012.

Kogelnik, H.W., Ippen, E.P., Dienes, A., and Shank, C.V. (1972). Astigmatically compensated cavities for $\mathrm{cw}$ dye lasers. IEEE J. Quant. Elect. QE-8, 373-379.

Kowalski, F.V., Hawkins, R.T., and Schawlow, A.L. (1976). Digital wavemeter for cw lasers. J. Opt. Soc. Am. 66, 965-966.

Kowalski, F.V., Teets, R.E., Demtroder, W., and Schawlow, A.L. (1978). An improved wavemeter for cw lasers. J. Opt. Soc. Am. 68, 1611-1613.

Kuhlke, D., Dietel, W. (1977). Mode selection in cw laser with homogeneously broadened gain. Opt. Quant. Elect. 9, 305-313.

Lee, J.H., Kim, K.C., and Kim K.H. (1988). Threshold pump power of a solar-pumped dye laser. Appl. Phys. Lett. 53, 2021-2022.

Liberman, S., and Pinard, J. (1973). Single-mode $\mathrm{cw}$ dye laser with large frequency range tunability. Appl. Phys. Lett. 24, 142-144.

Lichten, W. (1985). Precise wavelength measurements and optical phase shifts. I. general theory. J. Opt. Soc. Am. A 2, 1869-1876.

Lichten. W. (1986). Precise wavelength measurements and optical phase shifts II. Applications. J. Opt. Soc. Am. A 3, 909-915.

Loudon, R. (1983). The Quantum Theory of Light, 2nd ed. Clarendon Press, Oxford. England.

Marowsky, G., and Zaraga, F. (1974). A comparative study of dye prism ring lasers. IEEE J. Quant. Elect. QE-10, 832-837.

Marshall, C.M., Stickel, R.E., Dunniway, F.B., and Tittel, F.K. (1980). Computer controlled intracavity SHG in cw ring dye laser. Appl. Optics 19, 1980-1983.

Mollenauer, L.F. (1985). Color center lasers. In Laser Handbook, vol. 4 (Stitch, M.L., and Bass, M., eds.). North-Holland, Amsterdam, pp. 143-228.

Mollenauer, L.F., and White, J.C. (1987). General principles and some common features. In Tunable Lasers (Mollenauer, L.F., and White, J.C., eds.). Springer-Verlag, Berlin, Heidelberg, pp. 1-18.

Nieuwesteeg, K.J.B.M., Hollander, Tj., and Alkemade. C.Th.J. (1986). Spectral properties of an Ar-ion laser-pumped tunable cw dye laser. Opt. Comm. 59, 285-289

Oka, T. (1988). Report of IR difference frequency generation in the 2 to $5 \mu \mathrm{m}$ range using the crystal lithium iodate and the method developed by Pine (1974). Presentation at the conference, Coherent Sources for Frontier Spectroscopy, International Center for Theoretical Physics, Aug. 1988, Triesta, Italy.

Okoshi, T., Kikuchi, K., and Nakayama, A. (1980). Novel method for high resolution measurement of laser output spectrum. Electron. Lett. 16, 630-631.

Peterson, O.G. (1979). Dye lasers. In Quantum Electronics. part A. Methods of Experimental Physics, 15, (Tang, C.L., ed.). Academic Press, New York, pp. 251-359.

Peterson, O.B., Tuccio, S.A., and Snavely, B.B. (1970). Cw operation of an organic dye solution laser. Appl. Phys. Lett. 17, 245-247.

Peterson, O.G., Webb, J.P., McColgin, W.C., and Eberly, J.H. (1971). Organic dye laser threshold. J. Appl. Phys. 42, 1917-1928.

Pike, C.T. (1974). Spatial hole burning in cw dye lasers. Opt. Comm. 10, 14-17.

Pine, A.S. (1974). Doppler-limited molecular spectroscopy by difference-frequency mixing. J. Opt. Soc. Am. 64, 1683-1690. 
Pine, A.S. (1976). High-resolution methane $\nu_{3}$-band spectra using a stabilized tunable difference-frequency laser system. J. Opt. Soc. Am. 66, 97-108.

Runge, P.K., and Rosenberg, R. (1972). Unconfined flowing-dye films for cw dye lasers. IEEE J. Quant. Elect. QE-8, 910-911.

Salomon, Ch., Hils, D., and Hall, J.L. (1988). Laser stabilization at the millihertz level. J. Opt. Soc. Am. B 5, 1576-1587.

Sargent, M., Scully, M.O., and Lamb, W.E. (1974). Laser Physics. Addison-Wesley, London.

Schäfer, F.P. (1977). Dye Lasers, 2nd ed. Vol. 1 of Topics in Applied Physics. SpringerVerlag, Berlin, Heidelberg. (Good summary of dye lasers as of 1977.)

Schäfer, F.P., Schmidt, W., and Volze, J. (1966). Organic dye solution laser. Appl. Phys. Letl. 9, 306-309.

Schearer, L.D., Leduc, M., Vivien, D., Lejus, A.M., and Thery, J. (1986). LNA: A new cw Nd laser tunable around 1.05 and $1.08 \mu \mathrm{m}$. IEEE J. Quant. Elect. QE-22, 713-717.

Schmidt, W., and Schäfer, F.P. (1967). Blitzlampengepumpte farbstofflaser. Z. Naturforschg. 22a, $1563-1566$.

Schröder, H.W., Welling, H., and Wellegehausen, B. (1973). A narrowband single-mode dye laser. Appl. Phys. 1, 343-348.

Schröder, H.W., Dux, H., and Welling, H. (1975). Single mode operation of cw dye lasers. Appl. Phys. 7, 21-28.

Schröder, H.W., Stein, L., Frölich, D., Fugger, B., and Welling, H. (1977). A high-power single-mode cw dye ring laser. Appl. Phys., 14, 377-380.

Schulz, P.A. (1988). Single-frequency Ti: $\mathrm{Al}_{2} \mathrm{O}_{3}$ ring laser. IEEE J. Quant. Elect. 24, 10391044.

Shank, C.V. (1975). Physics of dye lasers. Rev. Mod. Phys. 47, 649-657.

Siegman, A.E. (1986). Lasers. University Science Books, Mill Valley, calif.

Smith, P.W. (1972). Mode Selection in Lasers. Proc. IEEE 60, 422-440.

Snavely, B.B. (1977). Continuous-wave dye lasers. In Dye Lasers, vol. 1, 2nd ed. Topics in Applied Physics (Schäfer, F.P., ed.). Springer-Verlag, Berlin, Heidelberg, pp. 86-120.

Snavely, B.B., and Schäfer, F.P. (1969). Feasibility of CW Operation of dye-lasers. Phvs. Lett. 28A, 728-729.

Snyder, J.J. (1980). Algorithm for fast digital analysis of interference fringes. Appl. Opts. 19, 1223-1225.

Soffer, B.H., and McFarland, B.B. (1967). Continuously tunable, narrow-band organic dye lasers. Appl. Phys Lett. 10, 266-267.

Sorokin, P.P., and Lankard, J.R. (1966). Stimulated emission observed from an organic dye, chloro-aluminum phthalocyanine. IBM J. Res. Dev. 10, 162-163.

Sorokin, P.P., and Lankard, J.R. (1967). Flashlamp excitation of organic dye lasers: A short communication. IBM J. Res. Dev. 11, 148.

Spaeth, M., and Bortfeld, D.P. (1966). Stimulated emission from polymethine dyes. Appl. Phys. Lett. 9, 179-181.

"Squeezed States" (1987). J. Opt. Am. B 4 (10), whole issue, and J. Mod. Optics $34(6,7)$, whole issue.

Stepanov, B.I., Rubinov, A.N., and Mostovnikov, V.A. (1967). Optic generation in solutions of complex molecules. JETP Lett. 5, 117-119.

Tang, C.L., Statz, H., and deMars, G. (1963). Regular spiking and single-mode operation of ruby laser. Appl. Phys. Lett. 2, 222-224. And Tang. C.L.. Statz, H., and de Mars, G. (1963). Spectral output and spiking behavior of solid state lasers. J. Appl. Phys. 34. 2289-2295

Thiel, E., Zander. C., and Drexhage, K.H. (1986). Continuous wave dye laser pumped by a HeNc laser. Opt. Cornm. 60, 396-398. 
Theil, E., Zander, C., and Drexhage. K.H. (1987). Incoherently pumped continuous wave dye laser. Opt. Comm. 62, 171-173.

Tuccio, S.A., and Strome, F.C., Jr. (1972). Design and operation of a tunable continuous dye laser. Appl. Optics 11, 64-73.

Williams, G.H., Hobart, J.L., and Johnston, T.F., Jr. (1983). A 10-THz scan range dye laser, with $0.5-\mathrm{MHz}$ resolution and integral wavelength readout. In Laser Spectroscopy VI (Weber, H.P., and Luthy, W., eds.). Springer-Verlag, Heidelberg, pp. 422-423.

Wineland, D.J., Berquist, J.C., Bolinger, J.J., Itano. W.M., Gilbert, S.L., and Hulet, R.G. (1989). Quantum jumps, ion crystals and solid plasmas. CLEO/QELS conference plenary session JA2, Baltimore, Md., unpublished.

Woods, P.T., Shotton, K.C., and Rowley, W.R.C. (1978). Frequency determination of visible laser light by interferometric comparison with upconverted $\mathrm{CO}_{2}$ laser radiation. Appl. Opt. 17, 1048-1054.

Wu, F.Y., Grove, R.E., and Ezekial, S. (1974). Cw dye laser for ultrahigh-resolution spectroscopy. Appl. Phys. Lett. 25, 73-75.

Yariv, A. (1975). Quantum Electronics, 2nd ed. Wiley, New York. 
Part III

Laser Spectroscopy 



\title{
Hyperfine Structure of the Metastable ${ }^{5} S_{2}$ State of ${ }^{17} \mathrm{O}$ Using an AlGaAs Diode Laser at $777 \mathrm{~nm}$
}

\author{
G. M. Tino, ${ }^{(a)}$ L. Hollberg, ${ }^{(b)}$ A. Sasso, ${ }^{(a)}$ and M. Inguscio ${ }^{(a)}$ \\ European Laboratory of Nonlinear Spectroscopy (LENS), Largo E. Fermi 2, 1-50125 Firenze, Italy \\ M. Barsanti \\ Scuola Normale Superiore, Piazza dei Cavalieri 7, 1-56100 Pisa, Italy
}

(Received 12 February 1990)

\begin{abstract}
By exploiting a narrow-linewidth diode-laser source we measure the hyperfine structure of the ${ }^{5} S_{2}$ state of ${ }^{17} \mathrm{O}$. Nuclear parameters can be calculated from the measured hyperfine structure. Recorded collision-free linewidths allow an estimate of the lifetime of the levels involved in a new scheme proposed for the cooling of atomic oxygen.
\end{abstract}

PACS numbers: $35.10 . F k, 32.30 . J c$

Nuclear effects in the spectrum of atomic oxygen are of great interest. Recently, a possible nuclear volume effect on the isotope shift of optical transitions was observed.' This effect is usually negligible in light elements but it is enhanced in this case by the "doubly magic" structure of the ${ }^{16} \mathrm{O}$ nucleus which has both proton and neutron closed shells. A deeper understanding of these effects requires more detailed information about the atomic structure. For example, information is required about the electron density at the nucleus which can be obtained from the analysis of the hyperfine structure. ${ }^{17} \mathrm{O}$ is the only stable oxygen isotope which has hyperfine structure since it has one neutron in the $d_{5 / 2}$ orbital outside the doubly closed ${ }^{16} \mathrm{O}$ nucleus; this produces a nuclear magnetic moment of $-1.894 \mu_{N}{ }^{2}$ In this paper we report the first measurement of the hyperfine structure of atomic oxygen performed by high-resolution laser spectroscopy. In fact, the only existing data on the hyperfine structure for this atom were obtained in a paramagnetic-resonance experiment on the ground state. ${ }^{3}$

The level we have investigated is the excited $3^{5} S_{2}$ level (Fig. 1). The interest of this particular level is twofold. First, it is the lowest in energy $(9.14 \mathrm{eV})$ for the excited configuration $1 s^{2} 2 s^{2} 2 p^{3} 3 s$, so stronger nuclear effects can be expected. Since optical transitions start from this level, combined measurements of the isotope shift and the hyperfine structure represent a stringent test for $a$ priori Hartree-Fock calculations, which are not easy for this multielectron atom. Second, the $3{ }^{5} S_{2}$ state is metastable (lifetime $\tau=180 \mu \mathrm{s}$ ) and is connected to the $3^{5} P_{1.2 .3}$ states by three strong transitions at $\lambda=777 \mathrm{~nm}$; as we discuss below, radiative cooling of atomic oxygen could be achieved using these transitions and the subsequent decay of the cooled atoms into the ground state would produce very cold ground-state oxygen. This metastabie cooling is the only realistic cooling scheme for oxygen since no allowed transition at visible wavelengths is available from the ground state. A critical parameter for cooling is the lifetime of the upper $3^{5} P$ state; it determines the time required for the deceleration of the atoms which must be shorter than the lifetime of the $3^{5} S_{2}$ state. From the present results an estimate for the $3^{5} P$-state lifetime is obtained, which supports this proposed cooling scheme. In addition, $\mathrm{GaAlAs} / \mathrm{GaAs}$ heterostructure diode lasers emitting at $\lambda \sim 780 \mathrm{~nm}$ are now available with enough power to perform nonlinear highresolution spectroscopy. Interest in diode-laser spectroscopy has increased since the development of several techniques which improve these lasers' spectral purity and tunability ${ }^{4-8}$ making them suitable for laser-cooling experiments. In this work a diode laser mounted in an extended cavity configuration is used to perform saturation spectroscopy on the $3 s^{5} S_{2}-3 p^{5} P_{3}$ transition at 777.1 $\mathrm{nm}$.

The experimental arrangement is shown in Fig. 2. The laser we used was a commercial $\mathrm{AlGaAs} / \mathrm{GaAs}$ diode laser emitting at $780 \mathrm{~nm}$ at room temperature. Coarse tuning over a range of more than $10 \mathrm{~nm}$ was achieved using optical feedback from a 1200-lines/mm

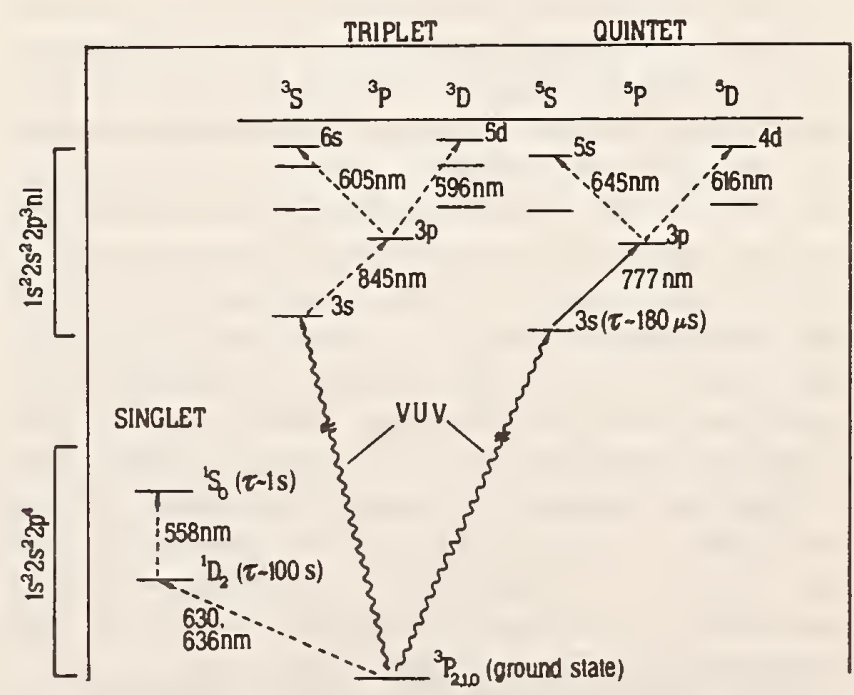

FIG. 1. A simplified energy-level scheme of OI showing the optical transition investigated in this work (solid line) and other transitions of spectroscopic interest (dashed lines). 


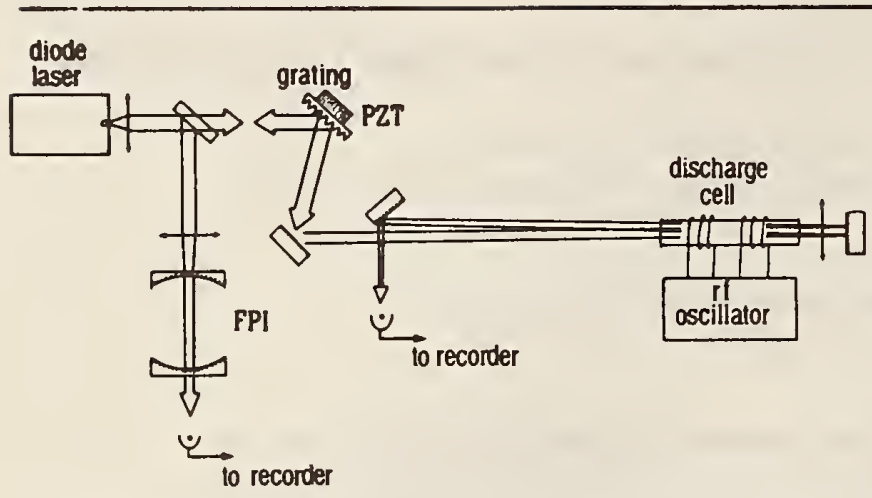

FIG. 2. Scheme of the experimental apparatus. Laser frequency stabilization is achieved by optical feedback from a grating which also acts as the output coupler. The beam is retroreflected through the sample cell to observe sub-Doppler saturation spectra. Double-headed arrows represent lenses. A Fabry-Perot interferometer (FPI) is used to monitor the laser's frequency.

ruled grating. The first-order diffracted beam was coupled back into the diode laser which had a reducedreflection coating on the output facet. The optical feedback was strong enough to select a single mode of the cavity. Tuning was achieved by changing the temperature and the injection current of the diode along with the grating angle. The zero-order reflection from the grating was used for the spectroscopy. The power available in the zero order was $6 \mathrm{~mW}$ in a single mode (jitter -1 $\mathrm{MHz}$ ). Light from an intracavity beam splitter ( $R$ $=20 \%$ ) was used for diagnostics. Frequency scans of - $5 \mathrm{GHz}$ were accomplished by synchronously changing the length of the cavity [with a piezoelectric transducer (PZT)] and the injection current of the laser. The calibration of the frequency scan was provided by a FabryPerot cavity with a free spectral range of $310 \mathrm{MHz}$. The low finesse of the cavity and feedback effects on the laser frequency are the main cause of uncertainty in the experiment. Direct absorption could be detected using a photodiode to measure the intensity of the light transmitted through the discharge cell as the laser frequency was scanned over the Doppler profile. The saturation dip could be observed with good signal-to-noise ratio by retroreflecting the laser beam (beam size $\sim 2 \mathrm{~mm} \times 4$ $\mathrm{mm}$ ) back through the cell at a small angle. A longfocal-length lens provided a better interaction of the probe beam with the saturating beam in the cell and helped in separating the two after it. This simple configuration has the advantage of exploiting all the laser power to saturate the transition. Derivative saturation spectra were obtained by introducing a $20-\mathrm{kHz}$ modulation on the cavity length by means of the piezoelectric transducer on the grating.

The fast sweep rate and low noise of the diode laser allowed us to detect saturated absorption signals in real time on an oscilloscope (detection bandwidth $\sim 50 \mathrm{kHz}$ ).

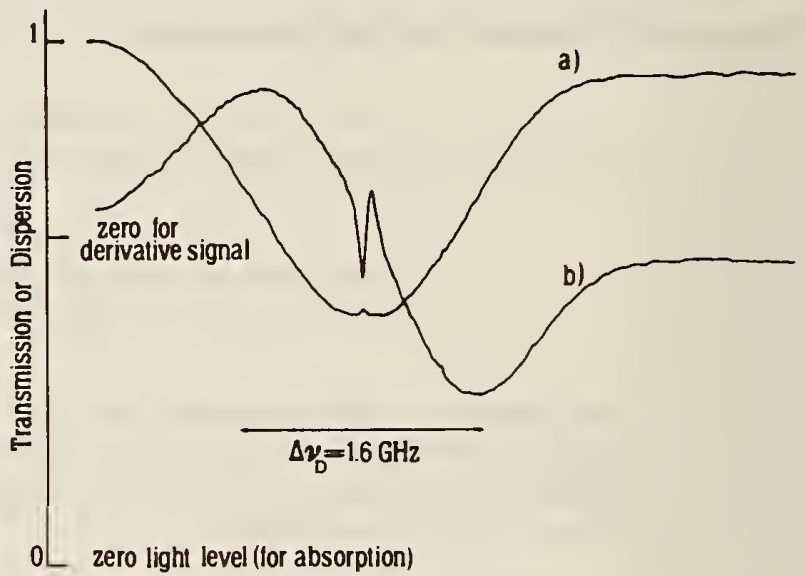

FIG. 3. Absorption profile of the $3 s^{5} S_{2}-3 p^{5} P_{3}$ oxygen transition at $777.1 \mathrm{~nm}$ observed in a natural-abundance sample using the experimental configuration shown in Fig. 2. (a) The saturation dip can be observed on the peak of the Doppler profile. (b) The saturation signal is enhanced relative to the Doppler background with derivative spectroscopy using FM modulation of the laser. The derivative signal is displayed with arbitrary units on the vertical axis.

Also, the derivative spectra could be obtained rapidly, limited only by the 1-ms time constant of the lock-in amplifier used for signal demodulation.

The radio-frequency discharge in which atomic oxygen is produced has been described elsewhere. ${ }^{9}$ As discussed below, thanks to the higher sensitivity achieved in the present experiment, lower pressure could be used both for oxygen and for the buffer gas (argon in this case). A typical $\mathrm{O}_{2} / \mathrm{Ar}$ pressure ratio was $1 / 20$ and sub-Doppler signals could be detected for total pressure ranging from about 150 down to $0.6 \mathrm{~Pa}$. For the measurement of the hyperfine structure, a $50 \%{ }^{17} \mathrm{O} /{ }^{18} \mathrm{O}$ enriched sample was used. Natural abundances are ${ }^{17} \mathrm{O}=0.04 \%,{ }^{18} \mathrm{O}=0.2 \%$, and the enriched sample enhances the relative signal sizes.

In Fig. 3(a) we show the absorption profile for the $3 s^{5} S_{2}-3 p{ }^{5} P_{3} 777.1-\mathrm{nm}$ transition recorded in a natural-abundance sample with the experimental configuration shown in Fig. 2. The small saturation dip can be observed on the top of the broad Doppler profile. This corresponds to the most abundant ${ }^{16} \mathrm{O}$ isotope and is easily observed because of the very low noise on the diode laser. In fact, this feature allowed us to detect oxygen in direct absorption at very low concentrations and with a short $(\sim 2-\mathrm{cm})$ interaction length. However, observation of sub-Doppler spectra at very low pressures using enriched samples required an increase of sensitivity and resolution. These were obtained by recording the derivative of the absorption profile, as shown in Fig. 3(b). As described before, this was done adding a small dither on the voltage tuning the grating position and using the same dither as the reference signal for the lock-in 


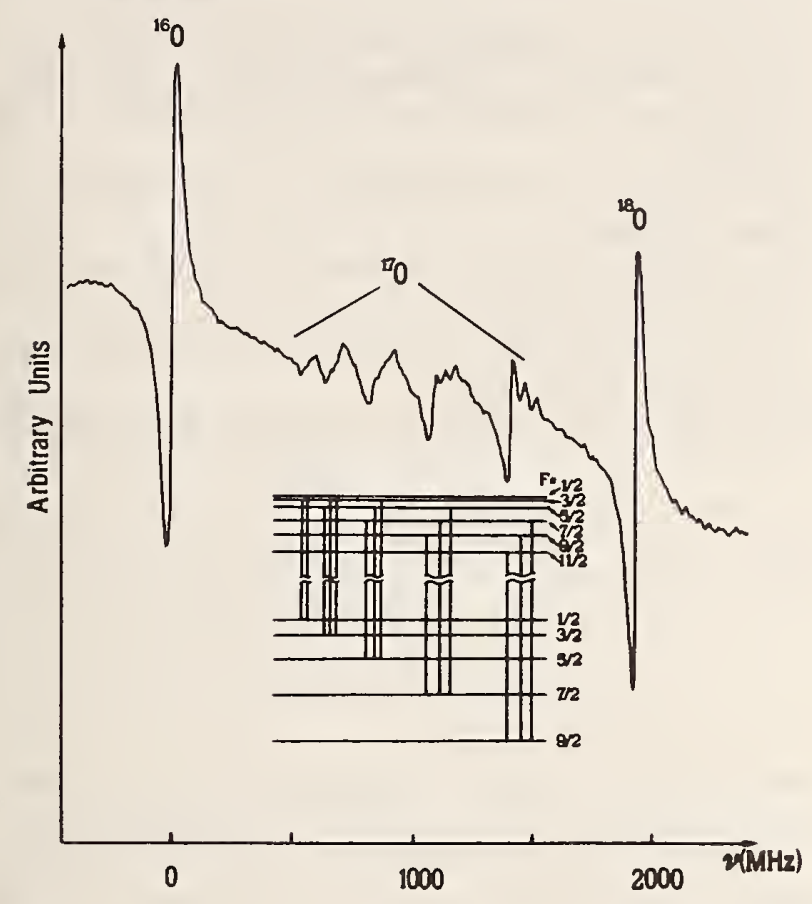

FIG. 4. Resonances of the three stable isotopes of oxygen as observed in an isotopically enriched sample. Hyperfine structure of the ${ }^{5} S_{2}$ level is fully resolved for ${ }^{17} \mathrm{O}$. Each of the five expected peaks also shows finer structure due to the upperstate hyperfine splittings, which are partially resolved. These data represent a single scan taken with a l-ms time constant. Inset: The ${ }^{17} \mathrm{O}$ hyperfine levels (not to scale).

amplifier. The amplitude of the dither was optimized to reduce the amplitude of the broad Doppler signal relative to the saturation dip. In Fig. 4 we show such a spectrum observed in an enriched sample. Besides the individual resonances of ${ }^{16} \mathrm{O}$ and ${ }^{18} \mathrm{O}$, the spectrum shows that the five hyperfine components of the lower ${ }^{5} S_{2}$ level are fully resolved for ${ }^{17} \mathrm{O}$. The hyperfine structure of the upper ${ }^{5} P_{3}$ level is also almost resolved in this case, while in previous measurements on transitions starting from this level the hyperfine structure was unresolved for the ${ }^{17} \mathrm{O}$ resonance.

The measured splittings for the ${ }^{5} S_{2}$ level are reported in Table I. Comparison of these data with the theoretical expression $E_{F}=E_{J}+\frac{1}{2} h A K$, with $K=F(F+1)-I(I$ $+1)-J(J+1)$, gives a value for the magnetic coupling constant of $A=74.3(4) \mathrm{MHz}$. The quadrupole interaction is neglected because of the insufficient accuracy of the data.

The results we obtained are of interest for a deeper understanding of nuclear effects on the atomic spectra. From the measured $A$ constant we can estimate the electron density at the nucleus $|\Psi(0)|^{2}$. We obtain $a_{0}^{3}$ $x|\Psi(0)|^{2}=0.12$, where $a_{0}$ is the Bohr radius. A more refined approach would require Hartree-Fock calculations, which are not presently accurate enough. Our experimental results provide a useful test for such theoreti-
TABLE I. Measured hyperfine splittings of the ${ }^{5} S_{2}$ level of ${ }^{17} \mathrm{O}$ obtained by averaging over ten different recordings. Uncertainties correspond to 1 standard deviation.

\begin{tabular}{cc}
\hline \hline & $\begin{array}{c}\text { Measured hf splittings } \\
(\mathrm{MHz})\end{array}$ \\
\hline$\Delta_{F, F}$ & $118.3 \pm 18$ \\
$\Delta_{1 / 2.3 / 2}$ & $172.0 \pm 22$ \\
$\Delta_{3 / 2.5 / 2}$ & $253.6 \pm 20$ \\
$\Delta_{S / 2.7 / 2}$ & $342.0 \pm 18$ \\
$\Delta_{7 / 2.9 / 2}$ & \\
\hline \hline
\end{tabular}

cal calculations.

The resonances recorded in this experiment are nearly free from collisional broadening and correspond to a linewidth of $60 \mathrm{MHz}$ FWHM, a factor of 2 or 3 narrower than those previously obtained for different transitions by intermodulated optogalvanic ${ }^{10}$ or polarization ${ }^{1}$ spectroscopy with a dye laser. From measurements of the linewidth as a function of pressure down to $0.1 \mathrm{~Pa}$ we can give a lower limit to the radiative lifetime $\tau$ of the $3^{5} P_{3}$ level of $3 \mathrm{~ns}$. Taking into account the effect of broadening due to residual pressure and saturation effects, we estimate a radiative lifetime of 6 ns. In anticipation of the radiative cooling of oxygen this value is of fundamental importance. In the scheme we propose, in fact, it is necessary that the deceleration time $t_{\text {decel }}$ is shorter than the lifetime of the lower $3{ }^{5} S_{2}$ state. Assuming a starting velocity for $O$ atoms of $4 \times 10^{2} \mathrm{~m} / \mathrm{s}$ and a final velocity near zero, we get the necessary number of cycles of laser absorption and spontaneous emission, $N_{\text {ph }}$ $=12000$. The deceleration time is then $t_{\text {decel }}=2 N_{\mathrm{ph}} \tau$ $-150 \mu \mathrm{s}$, which is within the time available of $180 \mu \mathrm{s}$. This demonstrates the possibility of cooling a thermal oxygen beam by resonant scattering of $777-\mathrm{nm}$ radiation. Very cold oxygen atoms could be produced in the ground state with a nonequilibrium distribution among the finestructure levels populations. This opens the possibility of performing experiments of metrological interest since the ground state is connected by visible transitions to the ${ }^{1} D_{2}$ state, whose lifetime is $-100 \mathrm{~s}$. Also of interest are the magnetic-dipole fine-structure transitions in the ground state at 63 and $145 \mu \mathrm{m}$.

L.H. thanks the Scuola Normale Superiore di Pisa for financial support for his visit. The authors also thank $F$. T. Arecchi for a critical reading of the manuscript and E. Andreoni and P. Barnini of the Laboratorio of Scuola Normale Superiore di Pisa for technical support. This work was performed as part of a cooperation agreement between LENS and the Scuola Normale Superiore di Pisa.

(a)Permanent address: Dipartimento di Scienze Fisiche dell'Universitá di Napoli, I-80125 Napoli, Italy.

(b) Permanent address: Time and Frequency Division, National Institute of Standards and Technology, 80303 Boulder. 
$\mathrm{CO} 80303$.

'L. Gianfrani, A. Sasso, G. M. Tino, and M. Inguscio, Opt. Commun. (to be published).

${ }^{2}$ F. Ajzenberg-Selove, Nucl. Phys. A375, 1 (1982).

3J. S. M. Harvey, Proc. Roy. Soc. London A 285, 581 (1965).

${ }^{4} \mathrm{M}$. W. Fleming and A. Mooradian, IEEE J. Quantum Electron. 7, 44 (1981).

${ }^{5}$ V. L. Velichanskii, A. S. Zibrov, V. V. Nikitin, V. A. Sautenkov, and A. V. Uskov, Kvantovaya Elektron (Moscow) 10,
1232 (1983) [Sov. J. Quantum Electron. 13, 792 (1982)].

${ }^{6}$ R. N. Watts and C. E. Wieman, Opt. Lett. 11, 291 (1986).

'B. Dahmani, L. Hollberg, and R. Drullinger, Opt. Lett. 12, 876 (1987).

${ }^{8} \mathrm{M}$. Ohtsu, Opt. Quantum Electron. 20, 283 (1988), and references therein.

${ }^{9}$ M. Inguscio, P. Minutolo, A. Sasso, and G. M. Tino, Phys. Rev. A 37, 4056 (1988).

${ }^{10} \mathrm{~K}$. Ernst, P. Minutolo, A. Sasso, G. M. Tino, and M. Inguscio, Opt. Lett. 14, 554 (1989). 
Appl. Phys. B 59. 327-331 (1994)

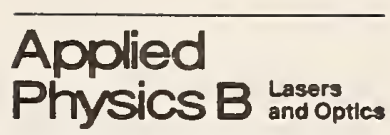

(C) Springer-Verlag 1994

\title{
High-resolution diode-laser spectroscopy of calcium
}

\author{
A. S. Zibrov*, R. W. Fox, R. Ellingsen**, C. S. Weimer, V. L. Velichansky*, G. M. Tino***, L. Hollberg \\ National Institute of Standards and Technology, Boulder, CO 80303, USA \\ (Fax: $+1-303$ 497-7845)
}

Received 16 November 1993/Accepted 15 May 1994

\begin{abstract}
Saturated-absorption signals on the calcium $657 \mathrm{~nm}$ transition are observed by direct absorption using diode lasers and a high flux atomic-beam cell. Linewidths as narrow as $65 \mathrm{kHz}$ are observed with a high signal-to-noise ratio. Prospects for using this system as a compact wavelength/frequency reference are considered.
\end{abstract}

PACS : 42.55.Px, 32.30.Jc

The intercombination transitions of the alkaline-earth atoms have been recognized as important optical frequency/wavelength references. Prominent among these, in terms of development effort, is the calcium transition at $657 \mathrm{~nm}$ which has a $400 \mathrm{~Hz}$ natural linewidth. Important advances in research on this system have come from a number of laboratories including saturated-absorption signals [1], atomic-beam optical Ramsey fringes [2-6], resolution of photon recoil [7], observation of second-order Doppler perturbation of Ramsey fringes [8], and suppression of one recoil component [9]. Laser cooling of calcium has also been demonstrated and promises significant improvement in accuracy and precision in the future [10-12].

Recent experiments on very high resolution spectroscopy of laser-cooled magnesium atoms have provided beautiful results that now demonstrate some of this promise [13]. In addition, diode-laser spectroscopy of barium [14] and strontium [15] show optical resonances with the potential to be used as wavelength references. All of these alternative systems have inherent advantages and disadvantages that could favor one for specific applications.

* Permanent address: Lebedev Institute of Physics, Moscow, Russia

** Permanent address: SINTEF DELAB, N-7034 Trondheim, Norway

*** Permanent address: Dipartimento di Scienze Fisiche, Univ. di Napoli, 1-Napoli. Italy
The reasons to choose the calcium $(657 \mathrm{~nm})$ transition are mostly well known; some are fundamental and others are of a practical nature. These include relatively small field shifts, no ground-state hyperfine structure, potential for laser cooling, visible wavelength (hence, easy to use for interferometry etc.), recognition by standards community [16], projected accuracy much higher than $I_{2}$ stabilized lasers, and the expectation of an actual frequency measurement that will provide absolute calibration of frequency/wavelength [17]. A renewed interest in calcium is the result, at least in part, of its compatibility with existing semiconductor diode-laser technology. This technology will allow a significant reduction in size and cost that may lead to construction of a compact portable transfer standard. Not only are high performance diodelasers possible at $657 \mathrm{~nm}$ [18], but high-power diode lasers can also be frequency-doubled efficiently to produce the $423 \mathrm{~nm}$ light required for laser cooling [19].

In striving for high accuracy and stability in optical references we need unperturbed transitions with very narrow linewidths and high signal-to-noise ratios. The quantum nature of atomic transitions results in a fundamental tradeoff between the linewidth and signal-tonoise ratio for spectroscopic measurements. This is readily apparent in trapped-ion experiments that use single ions for the highest accuracy [20]. With neutral atoms we can hope to achieve higher signal-to-noise ratios by using more atoms. but we must pay the very serious penalty of limited observation time, and hence linewidth. Even using laser cooling/trapping/fountain methods with neutrals has limitations that result from the perturbing effects of the trapping fields. These cannot be ignored and generally require that we turn off the trapping light. Present best-case neutral-atom fountains have unperturbed observation times of $\approx 1 \mathrm{~s}$; whereas trapped-ion experiments have nearly unlimited observation times. We anticipate that to achieve the highest accuracy laser cooling will be required, but we report here about a system in which ultimate accuracy is traded for short term stability, compactness and the future potential for portability. Such a system could serve as a relatively 
simple transfer standard of optical wavelength/frequency.

\section{Experimental system}

Our simple diode-laser saturated-absorption system is diagrammed in Fig. 1. To conserve the optical power we use a minimum number of components in the optical beam path. In contrast to previous high resolution studies of calcium we have done the saturated-absorption in a high-flux hybrid beam-cell rather than in a traditional low-density atomic beam. The idea here is to take advantage of the low amplitude noise on the diode laser by detecting the absorption directly. The high-flux beam provides narrow resonances and high signal-to-noise ratios and avoids the problems associated with buffer gases. As usual, absorption has advantages and disadvantages compared to the common fluorescence detection systems. For fast calcium atoms the traditional fluorescence detection methods have detection efficiencies (signal-to-noise ratios) that are limited by the $20 \mathrm{~cm}$ long decay length of the $657 \mathrm{~nm}$ fluorescence. The signalto-noise ratio of our absorption signal was limited by the diode's amplitude noise power which was within a factor of 5 of the shot-noise limit at the $1.5 \mathrm{MHz}$ detection frequency we chose.

Our high-flux beam is generated from calcium metal that is heated in a stainless steel boat (internal dimensions $1 \times 1.1 \times 10 \mathrm{~cm}^{3}$ ). The boat is capped with a collimating nozzle which is formed by a narrow channel ( $480 \mu \mathrm{m}$ wide) that is cut through a $4.5 \mathrm{~mm}$ thick stainless steel plate. A wire-EDM (Electric Discharge $\mathrm{Ma}$ chine) was used to cut the continuous channel in a pattern that forms a rectangular zig-zag across the surface of the plate. The rectangular zig-zag pattern is contained in an area of $3 \mathrm{~mm}$ wide by $8 \mathrm{~cm}$ long and consists of 106 interconnected parallel slots. The result is a net nozzle area of $\approx 115 \mathrm{~mm}^{2}$. When the calcium vapor pressure is low $(\mathrm{T} \leq 800 \mathrm{~K})$ the nozzle should produce an atomic

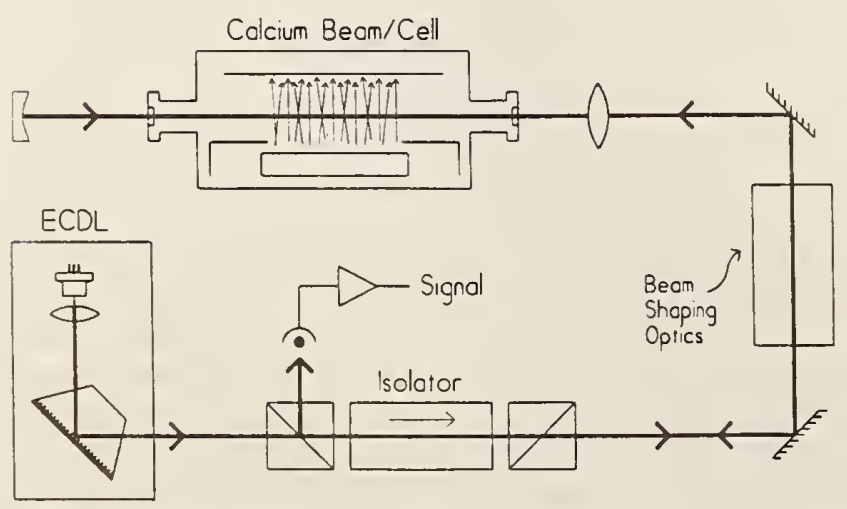

Fig. 1. Saturated-absorption spectrometer for calcium. The output from an extended-cavity diode laser passes through a two-stage optical isolator (isolation $\approx 60 \mathrm{~dB}$ ), beam-shaping optics and then on to the calcium beam cell. The return beam is deflected by the isolator and detected with a photodiode beam divergence (in the direction of the laser propagation) of about 12 degrees. With a single fill of calcium the beam-cell can be operated for about $15 \mathrm{~h}$. Counter propagating laser beams intersect the atomic beam $\approx 3 \mathrm{~cm}$ downstream from the nozzle. The calcium beam then condenses on a water cooled surface $\approx 4 \mathrm{~cm}$ beyond the interaction region. Under normal operating conditions the source temperature is $\approx 900 \mathrm{~K}$ and produces a Doppler-broadened absorption of about $3 \%$. In this case the calcium pressure in the nozzle is higher than necessary for effusive flow; hence we observe a Doppler width of about $700 \mathrm{MHz}$. The present beam-cell has a volume of about $4 \mathrm{l}$ and is now pumped with a turbo pump, although ion pumping seems feasible. With some minor design modifications of the nozzle and thermal/ vacuum enclosure the beam-cell should be portable and have a much longer calcium source lifetime.

In this experiment the extended-cavity diode lasers [21. 22] use commercial index-guided lasers in conjunction with a special holographic optical selectors [23] for wavelength control. Although other good optical designs are possible, the holographic selectors are attractive because they provide both high diffraction efficiency $(60-90 \%)$ and high resolving power $(\approx 3600$ lines $/ \mathrm{mm})$. In addition, and in contrast to traditional ruled gratings, the high diffraction efficiency is obtained when the laser polarization is parallel to the grating lines.

To improve the single-mode tuning range and stable output power of the extended-cavity lasers it is important to have good Anti-Reflectance (AR) coatings on the laser's output facets [24-26]. Unfortunately, almost all of the commercial laser manufacturers use optical coatings on the facets in order to insure long lifetimes, and to tailor the laser's facet reflectance for optimum output power. These coatings obviously constrain the performance of any additional AR coatings that we might add. But even without removing the manufacturer's coatings we can achieve good AR coatings with a number of different coating designs. For example, we commonly use a two-layer coating that consists of $\mathrm{Al}_{2} \mathrm{O}_{3}$ plus $\mathrm{HfO}_{2}$ to achieve modal reflectance as low as $1 \times 10^{-3}$. With these coatings we obtain good operating conditions of extended-cavity red lasers with coarse tuning within $\pm 10 \mathrm{~nm}$ of the free-running laser wavelength. Starting with a $3 \mathrm{~mW}$, single spatial-mode, commercial laser, the extended-cavity systems typically provides $\approx 1.5 \mathrm{~mW}$ of usable power in a single spectral mode. These extended-cavity lasers typically have a free-running fast linewidth of about $30 \mathrm{kHz}$ for a $10 \mathrm{~cm}$ extended-cavity length. Linewidths are determined by direct beat note measurements.

To further narrow the laser's linewidth we use the Pound-Drever-Hall [27] method to lock one of our red lasers to a stable reference cavity. For fast electronic control of this laser's frequency an ADP electro-optic modulator is used inside the extended cavity. The use of the electro-optic modulator helps to suppress the coupling of frequency modulation to amplitude modulation in the diode laser system. When locked this laser has a fast-linewidth of about $500 \mathrm{~Hz}$ and a residual frequency jitter (due to vibrational perturbations of the reference cavity) of about $5 \mathrm{kHz}$ [18]. The laser linewidth with 
respect to the reference cavity may be decreased by extending the servo bandwidth beyond the present $1 \mathrm{MHz}$. Presently, the laser's linewidths do not contribute significantly to our observed calcium transition width $(\approx 65 \mathrm{kHz})$. Both the electronically stabilized laser and unlocked, but long $(\approx 30 \mathrm{~cm})$, extended cavity lasers give calcium resonance widths that are similar. The observed transition linewidth is presently dominated by transit and wavefront curvature effects. We also note that Simonsen [29] has reported an alternative laser narrowing scheme that combines optical-locking with a grating-tuned extended-cavity laser.

As is usual for diode lasers the spatial mode is both asymmetric and astigmatic. The mode also usually contains additional small aberrations that are caused by imperfections in the beam path (the holographic selector in particular). Some effort is then required to process this optical beam to obtain good quality optical wavefronts for saturation spectroscopy. At present we use beamforming optics that includes: a cylindrical lens ( $\mathrm{f} \approx 4 \mathrm{~m}$ ) to correct astigmatism, two sets of anamorphic prisms to shape the beam, and two spherical lenses for collimation. The resulting beam then has a nearly rectangular shape $4 \mathrm{~mm} \times 6 \mathrm{~mm}$ with a wavefront flatness of about 1 fringe.

\section{Results}

For typical operating conditions with the beam-cell system the measured calcium saturation-dip is about $1 \%$ of the laser power, while the Doppler background absorption is about $3 \%$. Using optical heterodyne techniques [30] produces saturated-absorption signals with high signal-to-noise ratios as shown in Fig. 2. So far the nar-

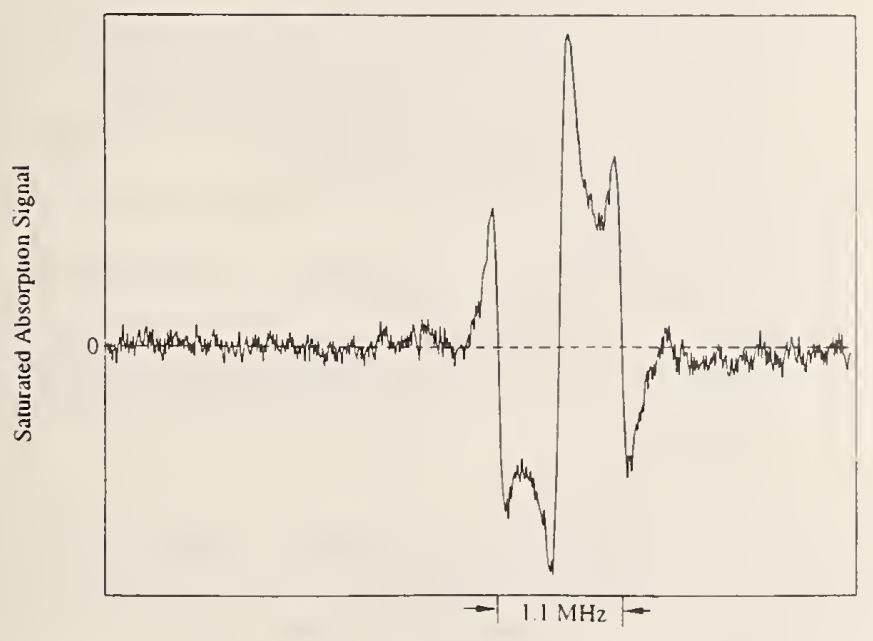

Laser Frequency

Fig. 2. Calcium saturated-absorption signal observed using the system diagrammed in Fig. 1. The lineshape results from the optical-heterodyne detection method using frequency modulation of the laser at $1.1 \mathrm{MHz}$. This example shows a calcium resonance with a $100 \mathrm{kHz}$ width with a nearly flat background. A good signal-tonoise ratio is achieved even using a $60 \mathrm{kHz}$ detection bandwidth rowest lines we have measured with our beam-cell system are $65 \mathrm{kHz}$ (FWHM) which corresponds to a resonance $\mathrm{Q} \approx 10^{10}$. These signals are observed with about $1 \mathrm{~mW}$ of optical power and provide a signal-to-noise ratio of about 30 in a $40 \mathrm{kHz}$ detection bandwidth.

If optimally used, the noise-limited-stability of a diode laser locked to this resonance would be $\sigma_{y}(\tau) \approx 3 \times 10^{-14} \tau^{-1 / 2}$. This estimate may be reasonable for short and medium averaging-times $(100 \mu \mathrm{s} \leq \tau \leq$ $100 \mathrm{~s})$ but will not persist for long averaging-times which will be limited by systematic effects. The time scale at which long-term systematic perturbations become important has not yet been determined for this system. Based on experience with similar systems, we might expect the stability to degrade for averaging times larger than a few hundred seconds. This projected stability of the calcium beam-cell saturated-absorption resonance is excellent and comes from a system that is still far from optimized.

With better quality optical beams we can anticipate narrowing the single-zone saturated-absorption signal by a factor of 2 or 3 . This improvement of the spatial mode will require higher quality windows and optics along the beam path as well as interferometric quality alignment in the interaction region. In the beam forming process a number of factors are traded against each other in order to achieve spectrally narrow saturation signals while maintaining a high signal-to-noise ratio. These tradeoffs include: limited optical power, poor initial spatial-mode quality, and the desire to have a large spot size (because of significant transit and wavefront-curvature broadening) $[31,32]$. A preferred method may be to use the optical Ramsey-fringe technique proposed by Baklanov et al. [33] and pioneered by Bergquist et al. [3,34]. These methods have already demonstrated linewidths as narrow as a few kilohertz $[3,7,8]$.

The most significant factors that limit the resolution and accuracy of non-cooled neutral-atom optical frequency wavelength references are velocity dependent shifts such as residual Doppler, second-order Doppler, transit and wavefront curvature effects. Other factors that will contribute smaller uncertainties include photon recoil (23 kHz splitting), gas lens [35], coupling of laser $\mathrm{AM}$ and FM, quadratic Zeeman $\left(10^{8} \mathrm{~Hz} / \mathrm{T}^{2}\right)$ and Stark $\left[\approx 1 \mathrm{~Hz}(\mathrm{~V} / \mathrm{cm})^{2}\right]$ shifts. Many of these effects are complicated by their dependence on the system geometry and atomic trajectories and will have to be evaluated in detail for any specific system realization. In particular the most troublesome shifts are caused by the wavefront curvature and second-order Doppler effects that are determined by averaging the atomic velocity distribution over the spatial distribution of the laser field. These effects will typically limit non-cooled optical frequency standards to an accuracy of about $1 \mathrm{kHz}$ [6,31].

The second-order Doppler shift for hot $(T \approx 900 \mathrm{~K})$ calcium atoms is significant $\left(\approx 2 \times 10^{-12}\right.$ for the most probable velocity of $\approx 740 \mathrm{~m} / \mathrm{s}$ ) and will have to be evaluated for any proposed standard. Fortunately, by using the optical transitions the atomic velocity distribution can be measured with some accuracy. The second-order Doppler shift can then be calculated and subtracted as 
is done for cesium atomic frequency standards. For these microwave primary standards uncertainties of a few percent of the second-order shift are achieved, but the uncertainties will likely be larger for the laser saturated-absorption case.

In evaluating the potential accuracy and precision of calcium standards there are still other unresolved questions, one of which is the possibility of pressure shifts of the $657 \mathrm{~nm}$ transition. While pushing towards the highest accuracy frequency standards it has been discovered that significant pressure shifts affect even trapped-ion systems with pressures as low as $10^{-8} \mathrm{~Pa}\left(\Delta \mathrm{v} / \mathrm{v} \approx 2 \times 10^{-13}\right.$ on an rf transition of $303 \mathrm{MHz}$ in Be) [36]. Similarly, Cs-Cs shifts have been measured in $C$ f fountain system $\left(\Delta v / v \approx 10^{-12}\right.$ for a cold Cs density of $\approx 3 \times 10^{9} \mathrm{~cm}^{-3}$ ) [37]. In unpublished data, Barger measured pressure shifts of the $657 \mathrm{~nm}$ saturatedabsorption transition due to argon and krypton collisions. The measured shifts were $\approx 68 \mathrm{kHz} / \mathrm{Pa}$ ( $9 \mathrm{MHz} /$ Torr) at low pressures. Unfortunately, the corresponding pressure shifts are not known for calcium perturbed by calcium, or other atoms that might be found in a typical vacuum system. The density of calcium atoms in the interaction region of our high flux beam is about $10^{12} / \mathrm{cm}^{3}\left(\approx 10^{-2} \mathrm{~Pa}\right)$ with a background gas pressure of about $7 \times 10^{-5} \mathrm{~Pa}$. If, for purposes of discussion, we assume a $\mathrm{Ca}-\mathrm{Ca}$ pressure shift of the same magnitude as the measured argon shift, our present system would have a pressure shift of $\approx 1 \mathrm{kHz}$. Linewidths measured as a function of calcium beam flux and system background pressure from $10^{-3} \mathrm{~Pa}$ to $10^{-4} \mathrm{~Pa}$ show no indication of pressure broadening. This limited diagnostic gives some indication that pressure shifts are not playing a signifcant role at our present resolution $(65 \mathrm{kHz})$, but these will have to be evaluated in future higher accuracy systems.

\section{Summary and future prospects}

Using diode lasers for high resolution saturation spectroscopy of calcium demonstrates the potential for a frequency/wavelength reference in the visible that could achieve very high precision with transportability. The calcium system should compete favorably with iodine stabilized lasers (scatter in frequency accuracy of good iodine cells is about $10 \mathrm{kHz}$ ) [38]. These initial results on calcium saturated-absorption in a beam-cell provides narrow linewidth signals with high signal-to-noise ratios. We can expect very high short-term stability from this system. Careful evaluation of all possible systematic effects, and intercomparisons of various system designs, will be required to determine the ultimate accuracy of the calcium saturated-absorption resonance.

It is clear that cold (low velocity) atoms will be required to achieve the ultimate resolution and accuracy. We are presently a long way from the $400 \mathrm{~Hz}$ naturallifetime-limited resolution that is possible on the $657 \mathrm{~nm}$ transition, but we expect that this will be achieved even with an all-diode-laser system. The $423 \mathrm{~nm}$ cooling transition in calcium is a very fortuitous match for frequencydoubling high-power diode lasers. Using $\mathrm{KNbO}_{3}$ in a ring build-up cavity and a $150 \mathrm{~mW}$ diode laser at $846 \mathrm{~nm}$, we have produced a usable beam of $35 \mathrm{~mW}$ at $423 \mathrm{~nm}$
[19]. To improve the long term stability and tuning of the blue light, we have injection-locked the $150 \mathrm{~mW}$ diode laser using an extended-cavity diode laser (output power $\approx 23 \mathrm{~mW}$ ) as the master oscillator. The injection locking narrows the high-power laser linewidth and allows continuous scans of about $7 \mathrm{GHz}$. The power and stability of the blue light which we have achieved should be adequate to test the fundamental resolution limits for calcium.

Acknowledgements. For their significant contributions to this effort on calcium and precision diode lasers we would like to thank J.C. Bergquist, J.L. Hall, R. Barger, F. Riehle, and J. Helmcke. We also thank N. Mackie and T. Zibrova for the excellent coatings on the diode lasers. This work was supported in part by the Air Force Office of Scientific Research.

\section{References}

I. U. Klingbeil, J. Kowalski, F. Träger, H.B. Wiegemann, G. zu Putlitz: Appl. Phys. 17, 199 (1978)

2. R.L. Barger, J.C. Bergquist, D.J. Glaze: J. Opt. Soc. Am. 68, 1634 (1978)

3. J.C. Bergquist, R.L. Barger, D.J. Glaze: In Laser Spectroscopy $I V$, ed. by H. Walther, K.W. Rothe, Springer Ser. Opt. Sci., Vol. 21 (Springer, Berlin, Heidelberg 1979) p. 120

4. J. Helmcke, D. Zevgolis, B.Ü. Yen: Appl. Phys. B 28. 83 (1991)

5. N. Ito, J. Ishikawa, A. Morinaga: J. Opt. Soc. Am. B 8, 1388 (1991)

6. A. Morinaga, F. Riehle, J. Ishikawa, J. Helmcke: Appl. Phys. B 48, 165 (1989)

7. R.L. Barger, J.C. Bergquist, T.C. English, D.J. Glaze: Appl. Phys. Lett. 34, 850 (1979)

8. R. Barger: Opt. Lett. 6, 145 (1981)

9. F. Riehle, J. Ishikawa, J. Helmcke: Phys. Rev. Lett. 61, 2092 (1988)

10. N. Beverini, F. Giammanco, E. Maccioni, F. Strumia, G. Vissani: J. Opt. Soc. Am. B 6, 2188 (1989)

11. T. Kurosu, F. Shimizu: Jpn. J. Appl. Phys. 29, 2127 (1990)

12. J. Helmcke, F. Riehle, J. Ishikawa, A. Witte, Th. Kisters, L.-L. Liu, X. Yuan: In Light-Induced Kinetic Effects on Atoms, Ions and Molecules, ed. by L. Moi (ETS Editrice, Pisa 1991)

13. K. Sengstock. U. Sterr, G. Hennig, D. Bettermann, J.H. Müller, W. Ertmer: Opt. Commun. 103, 73 (1993)

14. A.M. Akulshin, A.A. Celikov, V.L. Velichansky: Opt. Commun. 93, 54 (1992)

15. G.M. Tino, M. Barsanti, M. de Angelis, L. Gianfrani, M. Inguscio: Appl. Phys. B 55, 397 (1992)

16. CCDM (Comité Consultatif pour la Définition du Mètre) now recognizes the $657 \mathrm{~nm}$ transition as a working standard; Comité International des Poids et Mesures, Report of the 81 st Meeting, tome 60 (1992)

17. J. Helmcke, A. Morinaga, J. Ishikawa, F. Riehle: IEEE Trans. IM-38, 524 (1989)

18. R.W. Fox, H.G. Robinson, A.S. Zibrov, N. Mackie, J. Marquardt, J. Magyar, L.W. Hollberg: SPIE Proc. 1837, 360 (1992)

19. P. Gunter: SPIE Proc. 236, 8 (1980)

G.J. Dixon, C.E. Tanner, C.E. Wieman: Opt. Lett. 14, 732 (1989)

A. Hemmerich, D.H. McIntyre, C. Zimmerman. T.W. Hänsch: Opt. Lett. 15, 372 (1990)

G.P. Barwood, C.S. Edwards, P. Gill, H.A. Klein, W.R.C. Rowley: SPIE Proc. 1837, 27I (1992)

C.S. Weimer, J.H. Marquardt, R.W. Fox, H.G. Robinson, L. Hollberg: Proc. CSOISSA Summer School, Kiev (1993), in Special Issue of Ukr. Phys. J. (in press) 
20. W.M. Itano, J.C. Bergquist, D.J. Wineland: Science 237, 612 (1987)

21. C. Wieman, L. Hollberg: Rev. Sci. Instrum. 62, 1 (1991) and references therein

22. M. Ohtsu: Highly Coherent Semiconductor Lasers (Artech House, Norwood, MA 1992)

23. M.S. Soskin, V.B. Taranenko: Sov. J. Quant. Electron. 7. 298 (1977)

24. P. Zorabedian, W.R. Trutna Jr., L.S. Cutler: IEEE J. QE-23, 1855 (1987)

25. M.G. Boshier, D. Berkeland, E.A. Hinds, V. Sandoghdar: Opt. Commun. 85, 355 (1991)

26. R. Fox, G. Turk, N. Mackie, T. Zibrova, S. Waltman, J. Marquardt, A. Zibrov, C. Weimer, L. Hollberg, M.P. Sassi: In Solid State Lasers: New Development and Applications, NATO ASI Ser. (Plenum, New York) (in press)

27. R.W.P. Drever, J.L. Hall, F.V. Kowalski, J. Hough, G.M. Ford, A.J. Munley, H. Ward: Appl. Phys. B 31, 97 (1983)

28. L. Hollberg, R. Fox, N. Mackie, A.S. Zibrov, V.L. Velichansky, R. Ellingsen, H.G. Robinson: In Proc. Tenth Int'l. Conf. Laser Spectroscopy (World Scientific, Singapore 1992) p. 347

29. H.R. Simonsen: SPIE Proc. 1837, 250 (1992)
30. J.L. Hall, L. Hollberg, T. Baer, H.G. Robinson: Appl. Phys. Lett. 39, 680 (1981)

31. J.L. Hall: In Methodes de Spectroscopie sans Largeur Doppler de Niveaux Excites de Systemes Moleculaires Simples, ed. by J.C. Lehmann (CNRS, Paris 1974) p. 105

32. C.J. Bordé, J.L. Hall, C.V. Kunasz, D.G. Hummer: Phys. Rev. A 14, $236(1976)$

33. Ye. V. Baklanov, B.Ya. Dubetsky V.P. Chebotayev: Appl. Phys. 9, 171 (1976)

34. J.C. Berquist, S.A. Lee, J.L. Hall: Phys. Rev. Lett. 38, 159 (1977)

35. R. Felder: Metrologia 23, 101 (1986)

36. D.J. Wineland, J.C. Bergquist, J.J. Bollinger, W.M. Itano, F.L. Moore, J.M. Gilligan, M.G. Raizen, D.J. Heinzen, C.S. Weimer, C.H. Manney: In Laser Manipulation of Atoms and Ions, ed. by E. Arimondo, W.D. Phillips, F. Strumia (NorthHolland, Amsterdam 1992)

37. K. Gibble, S. Chu: Phys. Rev. Lett. 70, 1771 (1993)

38. J-M. Chartier, S. Picard-Fredin, A. Chartier: In Tenth Int'l. Conf. Laser Spectroscopy. (World Scientific, Singapore 1992) p. 106 


\title{
Sub-Doppler Frequency Measurements on OCS at $87 \mathrm{THz}(3.4 \mu \mathrm{m})$ with the CO Overtone Laser
}

\author{
A. DaX, ${ }^{*, 1}$ J. S. Wells, ${ }^{*}$ L. HollberG, ${ }^{*}$ A. G. MAKI, ${ }^{*, 2}$ AND W. Urban $\dagger$ \\ *Time and Frequency Division, National Institute of Standards and Technology, Boulder, Colorado \\ 80303; and †Institut für Angewandte Physik der Universität Bonn, Bonn, D-53115 Germany
}

\begin{abstract}
Sub-Doppler frequency measurements have been made on three transitions of OCS in the 87 $\mathrm{THz}$ region (near $2900 \mathrm{~cm}^{-1}$ ). The $\mathrm{CO}$ overtone laser was used as the saturating laser. Polarization spectroscopic techniques utilizing optical heterodyne detection were used to observe the features and subsequently provide the discriminant for locking the overtone laser to the OCS transitions. $\mathrm{A} \mathrm{CO}_{2}$ laser synthesizer was used to measure the frequency of the $\mathrm{CO}$ overtone laser and thereby measure the frequencies of the OCS lines. The resulting frequencies of the three new measurements are: $10^{\circ} 1-00^{\circ} 0 P(27), 87117278.496(50) \mathrm{MHz} ; 11^{1 / 1} 1-01^{1 /} 0 R(14), 87222001.143(70) \mathrm{MHz}$ for OCS; and for the $\mathrm{OC}^{34} \mathrm{~S} 10^{\circ} 1-00^{\circ} 0 P(9), 87010586.667(75) \mathrm{MHz}$, where the numbers in parentheses are the uncertainties in the last digits. These new numbers have been fitted along with more than 5700 other data points in our OCS data bank and improved constants have been obtained. These latest constants are used to calculate updated calibration tables containing values with much smaller uncertainties; three such tables are included. (c) 1994 Academic Press, Inc.
\end{abstract}

\section{INTRODUCTION}

The wide acceptance of the tunable diode laser (TDL) by the spectroscopic community in the late 1970s pointed to the need for frequency calibration standards to achieve the full potential of these devices. Since 1979, we have been involved in a major effort to provide frequency/wavenumber calibration standards based on heterodyne frequency measurements $(1,2)$.

Our heterodyne frequency measurement activity came to a temporary halt when we reached the $61.76-\mathrm{THz}\left(2060-\mathrm{cm}^{-1}\right)$ region, the upper limit of our $\Delta v=1 \mathrm{CO}$ laser (3). Some measurements at slightly higher frequencies were possible based on a doubling of the $\mathrm{CO}_{2}$ laser (4). It was the development of the DC discharge overtone $\mathrm{CO}$ laser at the Institut furr Angewandte Physik (IAP) in Bonn [W. Urban and coworkers $(5)]$ that made further progress possible. The most recent reports $(6,7)$ suggest the potential usefulness of this laser with over 330 transitions observed in the 2.6 to $4.1 \mu \mathrm{m}$ region. A long standing NIST-IAP collaboration was renewed in Bonn, where TDL-based heterodyne measurements were made on some OCS features in the $11^{1 e} 1$ $01^{1 e} 0$ and $11^{1 f} 1-01^{1 f} 0$ bands at $87 \mathrm{THz}(3.4 \mu \mathrm{m})(8)$. Between 1979 and 1990 , our measurements were all Doppler-limited with the exception of some measurements on $\mathrm{CO}(9)$ and $\mathrm{N}_{2} \mathrm{O}(10)$. In a separate effort, Fayt et al. (11) have made sub-Doppler measurements on those OCS transitions that are near the $9-\mu \mathrm{m} \mathrm{CO}{ }_{2}$ laser transitions.

These mostly Doppler-limited frequency measurements formed the foundation for the publication of a calibration atlas (2) which spans much of the region from 486 to $3120 \mathrm{~cm}^{-1}$ plus another small region $\left(4071-4352 \mathrm{~cm}^{-1}\right)$. The atlas has a spectral map

1 Guest researcher from Institut für Angewandte Physik der Universität Bonn, Bonn, D-53115 Germany.

${ }^{2}$ Address: 15012 24th Ave. SE, Mill Creek, WA 98012. 
and facing table format which we intend to update from time to time. Since a deliberate effort was made to keep the atlas at a manageable length, about three-quarters of the possible frequency entries were not included. The diskette in NIST Standard Reference Database 39 includes these omitted values (12). These calibration data will also be available next year in the over the world wide electronic network.

In order to further improve the atlas, more accurate frequency measurements are required. Consequently, we have begun sub-Doppler frequency measurements at 3.4 $\mu \mathrm{m}$. Our initial emphasis was on OCS, and we hope to include $\mathrm{N}_{2} \mathrm{O}$ later. Our colleagues in Bonn have also made sub-Doppler frequency measurements on OCS near $5 \mu \mathrm{m}$ (13). Both this paper and Ref. (14) describe our first OCS sub-Doppler experiments and results at NIST.

\section{SUB-DOPPLER EXPERIMENTS WITH THE CO OVERTONE LASER}

Two different experimental approaches were investigated in order to find the technique that is best suited to making measurements of OCS transitions near $3.4 \mu \mathrm{m}$. The first involved a normal saturated-absorption technique (15-23), which is much simpler to use and is potentially more accurate for frequency measurements (17) (provided that the transition moment of the molecule is sufficiently large). The second technique used polarization-selective optical saturation techniques (24-35) and produced a much better signal-to-noise ratio (SNR). However, it was considerably more cumbersome. For clarity concerning sub-Doppler spectroscopy and frequency measurements at $3 \mu \mathrm{m}$ with a $\mathrm{CO}$ overtone laser, we discuss elsewhere (14) both types of experiments showing the advantages, limitations, and additional details of each. Because of the weak transition moment involved and some technical problems in obtaining a clean modulation signal for third-derivative detection $(21,22)$, the saturatedabsorption technique was shelved for the time being.

In our NIST laboratory, we have developed a $\mathrm{CO}$ overtone laser similar to the one developed in Bonn (5). Our 1.87-m-long laser has a 450 line $/ \mathrm{mm}$ grating and a 10 $\mathrm{m}$ radius output coupler. The $\mathrm{LN}_{2}$-cooled gain section is about $1.09 \mathrm{~m}$ long, and the bore i.d. is $12 \mathrm{~mm}$. The operating partial pressures of the $\mathrm{He}, \mathrm{N}_{2}$, air, and $\mathrm{CO}$ are essentially the same as the Bonn papers indicate. (We can attest to the wisdom of using an aluminum storage cylinder for the $\mathrm{CO}$ to avoid problems $(6,7)$ arising from $\mathrm{Fe}(\mathrm{CO})_{5}$ in older iron cylinders.) The Bonn versions employ coupling from the laser utilizing the zero-order output of the grating. We chose to take the output from a dielectric coated $\mathrm{ZnSe}$ coupler. This compensated coupler (the second surface of the coupler is curved) minimizes the spreading of the output beam. In an effort to minimize the laser linewidth, we have inserted convection barriers to enclose the air path between the resonator optical elements and the Brewster windows of the gain tube. We have also inserted a surge reservoir between the mixing valves and the gas inlet to the laser tube to reduce pressure fluctuations caused by the flow meters.

\section{CONSIDERATIONS OF OPTICAL HETERODYNE POLARIZATION SPECTROSCOPY}

The polarization spectroscopy method was invented by Wieman and Hänsch (24) in 1976 for making sub-Doppler measurements. Since then, this versatile method has been used not only for several laser spectroscopic measurements, but also for laser stabilization $(25-33,35)$. The principle of operation is that a strong pump beam introduces an anisotropy in a sample; that is, the sample becomes dichroic and birefringent if the pump frequency is near a molecular resonance. A weak probe beam at 
the same frequency will suffer a change in its polarization if it interacts with the same molecules as the pump beam. By use of an appropriate selection method, we can observe this change in polarization at the low power levels necessary to avoid saturating the detector. The possible variations concerning the polarization of the pump and probe and the offset angle of the analyzer from a predetermined orientation produce different signal shapes and intensities for molecular $Q, R$, and $P$ transitions (24-30).

Because of the problems with the saturated absorption technique, we selected the optical heterodyne polarization spectroscopy technique $(20,24,30)$. Its physical principle is extensively explained in $(31)$. The main advantage of this technique is that a weak signal can be considerably enhanced by heterodyning the signal wave (amplitude $E_{\mathrm{s}}$ ) with a much stronger local oscillator wave (amplitude $E_{0}$ ), as long as $E_{0}$ (which can also contribute noise) is selected appropriately. To use this technique, we apply an intensity modulation to the pump beam and detect the modulation transfer to the probe beam. The result is a low-noise dispersion-shaped signal which can be symmetric about the molecular transition. The $J$-dependence of similar dispersion signals is calculated in Refs. $(20,24,27,30)$.

Since both birefringence and dichroism can contribute to the signal here, the most difficult problem is to obtain a line shape that is symmetric about the true line center $(24,32,33)$. The local oscillator wave $E_{0}$ can be obtained most conveniently from the probe beam itself by slightly uncrossing the analyzer with respect to the polarizer by an angle $\phi$. An improper choice of $\phi$ may be accompanied by asymmetries in the dispersion signal.

Excluding noise sources originating from the environment between the laser and detector for a moment, the SNR for this type of experiment increases as $1 / \sqrt{\xi}$, where $\xi$ is the extinction ratio of the analyzer $(20,24)$. This is limited by the laser power noise $\gamma P_{0}$ (where $P_{0}$ is the laser output power) and the detector noise $D_{\mathrm{r}}$. One can show $(14,34)$ that for $\gamma P_{0} \xi \ll D_{\mathrm{r}}$,

$$
\mathrm{SNR}_{\max }=\text { const. } \sqrt{\frac{P_{0}}{\gamma D_{\mathrm{r}}}} .
$$

A small extinction ratio of the analyzer is essential for a good signal-to-noise ratio. In the visible spectral region, extinction ratios of $10^{-7}$ are often cited $(24,35)$. Our Rochon-type analyzer had a theoretical extinction ratio of $\xi=10^{-5}(36)$. In practice it is not quite that good, depending on the optical beam path through the crystal. By using a two-stage analyzer, we have improved the extinction ratio considerably.

By using the Jones formalism (37) we have calculated the contributions to the detector response $I_{\text {det }}$ and find, by using linear approximations,

$$
\begin{aligned}
I_{\mathrm{det}}= & C e^{-\kappa k_{0} L} E_{0}^{2}\left\{\xi_{1} \xi_{2}-\xi_{1} \epsilon^{2}-\xi_{2} \epsilon^{2}+\epsilon^{2}-\epsilon k_{0} \Delta b_{\mathrm{i}}+\phi_{1}^{2}+\phi_{1} k_{0} \Delta b_{\mathrm{r}}\right. \\
& -\epsilon \Delta \kappa k_{0} L+\phi_{1} \Delta n k_{0} L+\frac{1}{2}\left[k_{0}^{2} \Delta n L \Delta b_{\mathrm{r}}+k_{0}^{2} \Delta \kappa L \Delta b_{\mathrm{i}}\right] \\
& \left.+\frac{1}{4}\left[\left(k_{0} \Delta n L\right)^{2}+\left(k_{0} \Delta b_{\mathrm{r}}\right)^{2}+\left(\Delta \kappa k_{0} L\right)^{2}+\left(k_{0} \Delta b_{\mathrm{i}}\right)^{2}\right]\right\},
\end{aligned}
$$

where $C$ is a proportionality constant, $\kappa$ is the susceptibility $\left(\kappa_{=} \kappa_{+}+\kappa_{-}\right), k_{0}=2 \pi$ / $\lambda, \lambda$ is the wavelength of the probe beam, and $L$ is the estimated overlap length of the crossed beams within the cell. The symbols $\xi_{1}$ and $\xi_{2}$ represent the extinction ratios for the first and second analyzer, respectively, $\epsilon$ is the ellipticity of the polarization after the polarizer, and $\Delta \kappa=\kappa_{+}-\kappa_{-}$and $\Delta n=n_{+}-n_{-}$are the susceptibility change and refractive index change of the anisotropic medium in the circular basis, respectively. 
$\Delta \kappa$ describes the dichroism and has a Lorentzian lineshape; $\Delta n$ describes the birefringence and has a dispersion lineshape; $\phi_{1}$ is the deviation from the crossing angle of the first analyzer; and $\Delta b_{\mathrm{r}}=b_{\mathrm{r}}^{+}-b_{\mathrm{r}}^{-}$(the real part) and $\Delta b_{\mathrm{i}}=b_{\mathrm{i}}^{+}-b_{\mathrm{i}}^{-}$(the imaginary part) describe the anisotropy of the cell windows under vacuum stress $(20,21,30)$.

Since both extinction ratios have similar values between $10^{-4}$ and $10^{-5}$, the term $\xi_{1} \xi_{2}$ is negligible. The same is usually true for all terms containing $\epsilon^{2}$ since we have found experimentally that $\epsilon<10^{-3}$. The signal term can be expressed as $\phi_{1} E_{0}\left(E_{0} \Delta n k_{0} L\right)$, showing the heterodyning of the signal amplitude $E_{0} \Delta n k_{0} L$ and the local oscillator amplitude $\phi_{1} E_{0}(31)$. The terms $\Delta \kappa,(\Delta \kappa)^{2}$, and $(\Delta n)^{2}$ may lead to asymmetry of the signal, and the remaining terms are responsible for the background.

\section{EXPERIMENTAL DETAILS}

The overtone laser is the starting point of our description of the block diagram in Fig. 1. The output of the overtone laser was directed by several mirrors to a wedged uncoated $\mathrm{ZnSe}$ beam splitter where the transmitted portion was used for the saturating beam. The beam reflected from the front surface was used for the linear probe beam, and the beam reflected from the back surface was directed through an acousto-optic modulator to the IR frequency synthesizer. Even if the frequencies of the $\mathrm{CO}$ laser and the OCS line did not overlap, it was sometimes possible to shift the laser to the required frequency by placing the acousto-optic modulator (AOM) in the laser beam before it reached the beam splitter.

As indicated in Fig. 1, the probe beam's polarization was rotated by $\pi / 2$ by the five-mirror variable rotator and was then focused by mirror 1 onto a reflecting wedged germanium plate polarizer $(23,38)$. The beam was recollimated by mirror 2 . This increased the polarization purity of the beam from 2000:1 at the laser output to greater than $650000: 1$. A four-mirror polarization conserving half-wave plate $(23,39)$ returned the polarization back to horizontal. The probe beam traversed the OCS cell at a small angle with respect to the cell axis and was focused by mirror 3 onto a second reflecting wedged germanium plate polarizer which acted as the first stage of the analyzer. Only the component polarized perpendicular to the plane of incidence was reflected by this $L$ unit along the detection path. The local oscillator wave was generated by tilting the base of this device to the offset angle $\phi_{1}$. A similar one-stage analyzer can also be found in Refs. $(25,34)$. These collinear signal and local oscillator beams were focused on the Rochon analyzer by mirror 4 and mirror 5 focused them on the detector.

Before considering the path of the pump beam, we point out that instead of the usual $\lambda / 4$ plate, we have used a two-component scheme to achieve circular polarization. Our two-component scheme has three advantages. First, and most important, it is possible to have some control over the degree of polarization (similar to using a Babinet Soleil compensator); second, it is nearly wavelength-independent; and, third, the device is purely reflective.

The path (shown in Fig. 1) of the saturating beam through our two-component device starts where it emerges from the $\mathrm{ZnSe}$ beam splitter. The beam then had its polarization rotated by a three-mirror variable polarization rotator $(40)$ to some arbitrary value, say $\Phi$ (the difference between a three-mirror rotator and a five-mirror rotator is that the latter conserves the polarization quality much better (23)). The beam was focused by mirror 6 onto a titanium plate, which affords an adjustable angle $\Theta$ of incidence relative to the incoming beam. The physics involved in the phase shift on reflection from the titanium plate is described in (23) and is more or less simply the physics of Fresnel reflection on a metal surface (4I). A measured polarization 


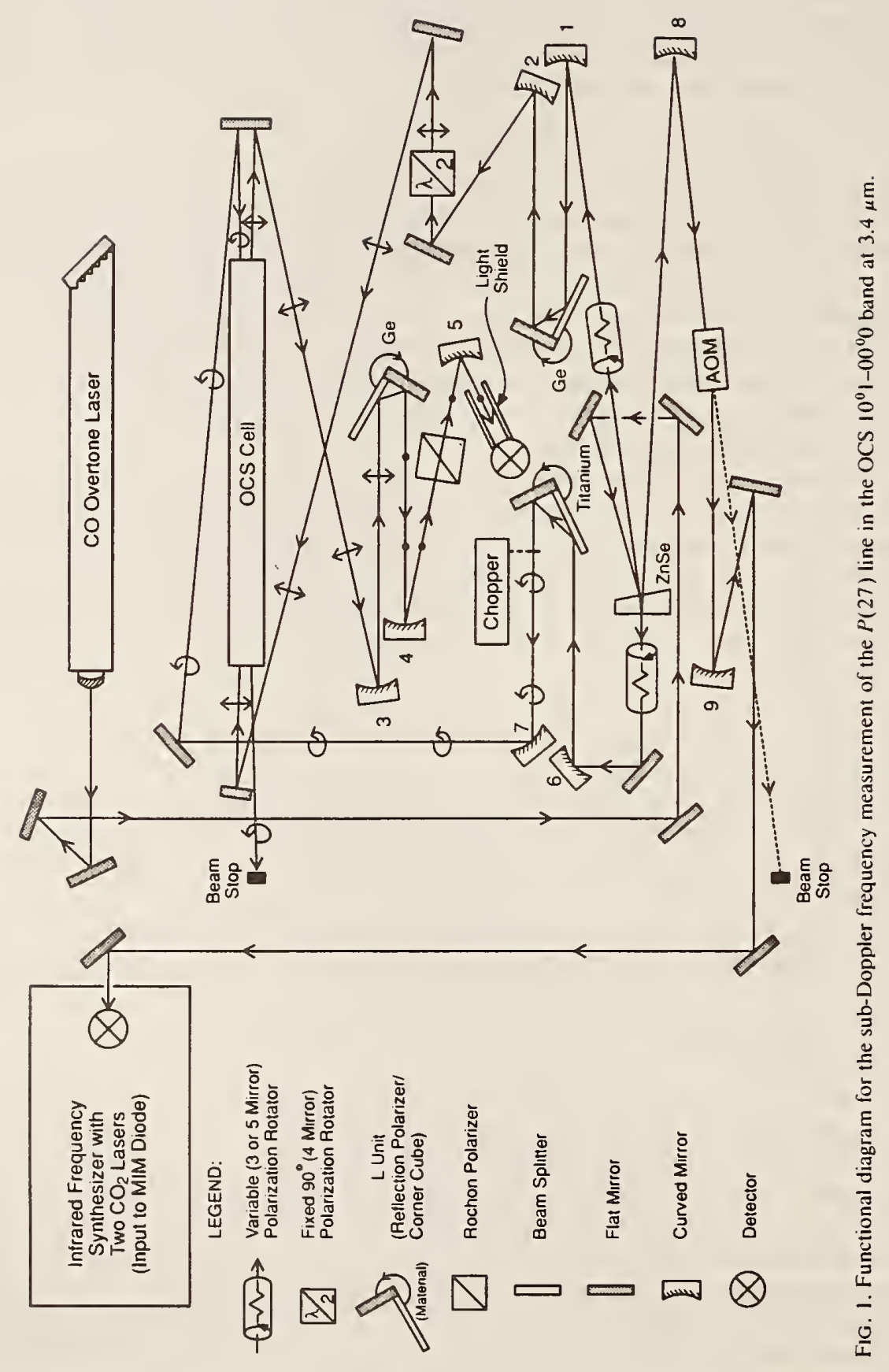




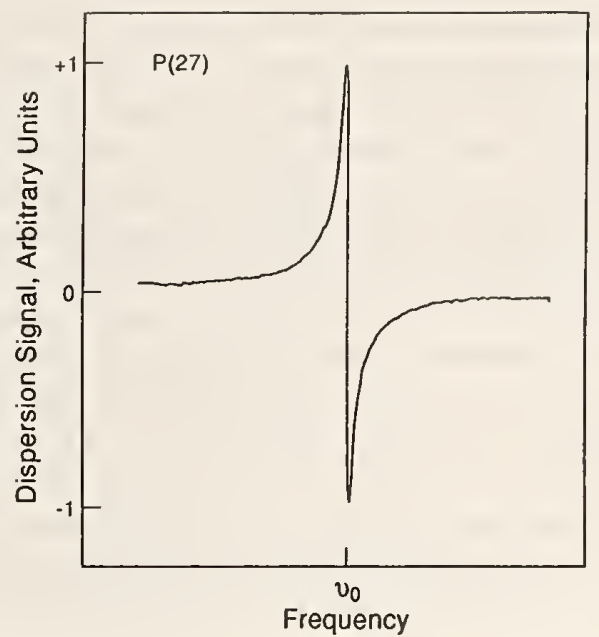

FIG. 2. Dispersion signal of the saturated $P(27)$ transition. The center frequency is $87117278.496(50)$ $\mathrm{MHz}$ and the peak to peak linewidth is about $700 \mathrm{kHz}$. The width of the scan shown here is about $45 \mathrm{MHz}$.

quality of $99.5 \%$ was achieved for the saturation beam by iterative adjustment of the angles, $\Phi$ and $\theta$. It is essential to have a good circular polarization quality here; otherwise, the pump wave can be considered to be composed of a circular and a linear part where dichroism can be accompanied with this linear pump wave. According to Ref. (25), such a dichroism changes the azimuth angle of the probe beam's linear polarization. This, in turn, increases the Lorentzian shaped contribution to the signal thus giving greater asymmetry to the detected dispersion signal. The saturation beam traversed the cell at a small angle with the cell axis. The crossing angle between the two beams was estimated to be about $1 \mathrm{mrad}$.

The elimination of stray light was tedious and is detailed in Ref. (14). When this was accomplished, we were able to measure three OCS transitions which had accidental overlaps with the $\mathrm{CO}$ overtone laser or its AOM shifted sidebands. Some of the data from the experiment on the $P(27) A$ line of OCS at $3.4 \mu \mathrm{m}$ are shown in Figs. 2 and

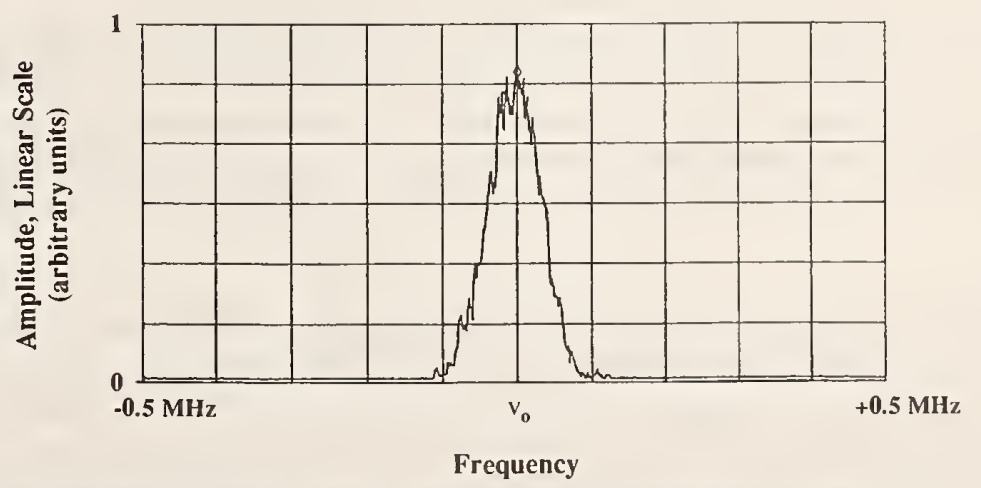

FIG. 3. Spectrum analyzer display of the time-averaged (100 scans) beatnote between the CO overtone laser which was locked to the $P(27)$ line and a reference from the infrared frequency synthesizer. The center frequency is $1171.900 \mathrm{MHz}$, the video bandwidth is $30 \mathrm{kHz}$, and the sweep time is $62.56 \mathrm{msec}$. 
3. Figure 2 shows the dispersion signal which was used to lock the $\mathrm{CO}$ overtone laser. The signal appears fairly symmetric.

In order to obtain a symmetric lineshape, we rotated the second analyzer (Rochon crystal) of our two-stage system relative to the first analyzer. Offset angles of $\phi_{2}<5^{\circ}$ were enough to achieve symmetric signals. Since the polarization of the probe beam after the first analyzer is elliptical, this technique bears some resemblance to the method employed by Delsard and Keller (26). They used a linear pump beam and a circular probe beam, and found that it was possible to change the signal continuously from an absorption shape to a dispersion shape simply by rotating their analyzer $45^{\circ}$.

Our quantitative description does not demonstrate that we can symmetrize the signal without changing the real zero-crossing of the Lamb dip. To do this would require the inclusion of a valid model which describes the cell windows under stress as well as our offset angle $\phi_{2}$. However, not only do the dispersion signals appear symmetric, but also another measurement using a different technique produced a result which agreed to within $20 \mathrm{kHz}$ with our result for the same $5.3-\mu \mathrm{m}$ OCS transition $(13,42)$.

Referring again to Fig. 1, the third beam from our wedged $\mathrm{ZnSe}$ beamsplitter was focused by mirror 8 on the AOM, and the shifted beam was recollimated and sent to the input of the infrared frequency synthesizer. The frequency synthesizer consists of two $\mathrm{CO}_{2}$ laser frequency standards, a microwave oscillator, a MIM diode, and a spectrum analyzer along with a marker oscillator. (See Ref. (2), pp. 17-21, or Refs. (3, $4,8)$.) Reference (14) contains details of the latest improvements of the $\mathrm{CO}_{2}$ laser systems.

Figure 3 shows the time averaged beatnote between the locked $\mathrm{CO}$ overtone laser and a reference synthesized from a combination of two $\mathrm{CO}_{2}$-stabilized laser standards. Aside from the frequency, which is the primary goal, two features are significant. The first is that the linewidth of the laser is about $100 \mathrm{kHz}$, which is considerably less than the $700-\mathrm{kHz}$ saturation feature. The relative phase and amplitudes of modulation of the reference lasers were adjusted to minimize the beatnote linewidth; hence, the linewidth here is an upper limit on that of the $\mathrm{CO}$ laser. The second feature (not discernible here) is the SNR of the beatnote which was generally observed to be between 25 and $30 \mathrm{~dB}$, and hence suitable for stabilizing the overtone laser to the synthesized reference. This makes it an ideal reference for use in a heterodyne measurement to measure the linewidth of TDLs and to use in frequency offset control in an alternate scheme with a line-narrowed TDL (43).

Dispersion signals similar to that in Fig. 2 were obtained for two other transitions. These measurements were made at pressures of about $1.3 \mathrm{~Pa}(10 \mathrm{mTorr})$ with an estimated overlap pathlength of about $1 \mathrm{~m}$ in a 1.5 -m-long absorption cell. The measurements were all made with the same sample of gas at room temperature. No isotopically enriched samples $\left({ }^{34} \mathrm{~S}\right.$ for example) were used in the measurements.

\section{RESULTS AND ANALYSIS}

Table I gives the three saturated-absorption frequency measurements made through the use of this polarization spectroscopic technique. Of course, this technique is limited to the measurement of transitions that are within the small tuning range of the $\mathrm{CO}$ laser lines, or within the range of the sideband produced by the acousto-optic modulator.

The $\mathrm{CO}_{2}$ laser frequencies used to calculate the observed frequencies given in Table I were taken from a recent reanalysis of the $\mathrm{CO}_{2}$ laser frequencies (44) and, for the lines used for this work, only differ from the frequencies given by Bradley et al. (45) 
TABLE I

The Measured Transition Frequencies for Carbonyl Sulfide

\begin{tabular}{|c|c|c|c|c|c|}
\hline $\begin{array}{l}\mathrm{CO} \\
\text { Trans. }^{\mathrm{a}}\end{array}$ & OCS & Transition & $\begin{array}{l}\text { Obs. Freq. }{ }^{\mathbf{b}} \\
(\mathrm{MHz})\end{array}$ & $\mathrm{CO}_{2}$ Freq. Stds. ${ }^{\mathrm{c}}$ & $\begin{array}{l}\text { Beat Freq. } \\
\text { (MHz) }\end{array}$ \\
\hline$P_{26}(9)$ & $P(27)$ & $10^{\circ} 1-00^{\circ} 0$ & $87117278.496 \pm 0.050$ & $2 \times R(8) \mathbf{I}+R(12) \mathbf{I}$ & -1261.908 \\
\hline$P_{26}(8)$ & $R(14)$ & $11^{1 f} 1-01^{15} 0$ & $87222001.143 \pm 0.070$ & $2 \times P(28) 1+P(32) \mathrm{II}$ & $-1056.507^{d}$ \\
\hline$P_{2 \sigma}(10)$ & $P(9)^{\mathrm{e}}$ & $10^{0} 1-00^{\circ} 0$ & $87010586.667 \pm 0.075$ & $2 \times R(28) 1+P(24) \mathrm{I}$ & -412.438 \\
\hline
\end{tabular}

${ }^{a}$ For the $\mathrm{CO}$ laser designation the subscript indicates the lower state vibrational quantum number and the number in parentheses is $J "$.

${ }^{b}$ The OCS observed frequency is given by the sum of the $\mathrm{CO}_{2}$ laser frequencies and the beat frequency.

${ }^{\mathrm{c}}$ For the $\mathrm{CO}_{2}$ laser designation the I indicates the $10 \mu \mathrm{m}$ band and the II indicates the $9 \mu \mathrm{m}$ band.

d For the measurement of the $R(14)$ line of the $11^{1 \mathrm{t}} 1-01^{1 \mathrm{t}} 0$ band a microwave frequency of $10999.988 \mathrm{MHz}$ was added to the $\mathrm{CO}_{2}$ laser frequencies.

${ }^{e}$ This transition is for ${ }^{16} \mathrm{O}^{12} \mathrm{C}^{34} \mathrm{~S}$; the other two are for ${ }^{16} \mathrm{O}^{12} \mathrm{C}^{32} \mathrm{~S}$.

by a few kilohertz. The uncertainties in the measurements were about $30 \mathrm{kHz}$ for the absolute frequencies of the synthesized references, or about $10 \mathrm{kHz}$ for each of the three $\mathrm{CO}_{2}$ laser harmonics. The remaining contribution to the uncertainty was that due to locating the line center. This depended on the ratio of asymmetry of the dispersion signal to the signal amplitude with an additional contribution due to the noise.

These measurements have been combined in a least-squares fit with the many other measurements on OCS that were used in Ref. (2). In addition, some more recent FTS

\section{TABLE II}

The Rovibrational Constants (in $\mathrm{cm}^{-1}$ ) Used to Fit the Data

\begin{tabular}{|c|c|c|c|c|}
\hline State & $B_{\mathrm{v}}$ & $D_{\mathrm{v}} \times 10^{8}$ & $H_{\mathrm{v}} \times 10^{14}$ & $L_{\mathrm{v}} \times 10^{17}$ \\
\hline \multicolumn{5}{|c|}{${ }^{16} \mathrm{O}^{12} \mathrm{C}^{32} \mathrm{~S}$} \\
\hline $00^{\circ} 0$ & $0.202856740854(398)^{\mathrm{a}}$ & $4.3406771(1187)$ & $-0.32619(1452)$ & \\
\hline $01^{1} 0$ & $0.203209834843(1006)$ & $4.4115112(1497)$ & $-0.25697(1803)$ & \\
\hline $10^{\circ} 1$ & $0.20110323850(5499)$ & $5.137499(5107)$ & $86.768(2420)$ & $-7.9212(5145)^{b}$ \\
\hline $11^{11}$ & $0.20151618528(6599)$ & $4.774825(3871)$ & $13.7979(8046)$ & $-0.42990(5429)$ \\
\hline \multicolumn{5}{|c|}{${ }^{16} \mathrm{O}^{12} \mathrm{C}^{34} \mathrm{~S}$} \\
\hline $00^{\circ} 0$ & $0.197898035007(3272)$ & $4.1411874(6101)$ & $-0.35171(8951)$ & \\
\hline $10^{\circ} 1$ & $0.19619768512(10560)$ & $5.000256(7002)$ & $68.962(1599)$ & $-2.6803(1161)$ \\
\hline State & $q_{v} \times 10^{4}$ & $q_{\mathrm{vj}} \times 10^{10}$ & $q_{\mathrm{v} J 1} \times 10^{14}$ & \\
\hline \multicolumn{5}{|c|}{${ }^{16} \mathrm{O}^{12} \mathrm{C}^{32} \mathrm{~S}$} \\
\hline $01^{\prime} 0$ & $2.121938676(253)$ & $1.424123(492)$ & $0.057395(2118$ & \\
\hline \multirow[t]{6}{*}{$11^{1} 1$} & $2.377549(462)$ & $10.9163(2087)$ & $3.0904(2204)$ & \\
\hline & \multicolumn{2}{|l|}{ Vib. Transition } & $\nu_{0}$ & \\
\hline & \multicolumn{4}{|l|}{${ }^{16} \mathrm{O}^{12} \mathrm{C}^{32} \mathrm{~S}$} \\
\hline & \multirow{2}{*}{\multicolumn{2}{|c|}{$\begin{array}{l}10^{\circ} 1-00^{\circ} 0 \\
11^{\prime} 1-01^{1} 0\end{array}$}} & \multicolumn{2}{|c|}{$2918.1050666(248)$} \\
\hline & & & \multicolumn{2}{|c|}{$2903.7175465(144)$} \\
\hline & ${ }^{16} \mathrm{O}^{12} \mathrm{C}^{34} \mathrm{~S}$ & $10^{\circ} 1-00^{\circ} 0$ & \multicolumn{2}{|c|}{$2906.0452748(75)$} \\
\hline
\end{tabular}

a The estimated uncertainty (one standard deviation) in the last digits is given in parentheses.

b The fit of the $10^{\circ} 1$ level of ${ }^{16} \mathrm{O}^{12} \mathrm{C}^{32} \mathrm{~S}$ also included $M_{v}=4.467( \pm 0.484) \times 10^{-21} \mathrm{~cm}^{-1}$ and $N_{\mathrm{v}}=-1.110( \pm 0.166) \times 10^{-25} \mathrm{~cm}^{-1}$. 
measurements were included in the fit in such a way as to allow the heterodyne measurements to determine the vibrational energy levels. The FTS measurements were necessary to determine the values of the higher order vibrational terms, i.e., the $L_{v}$ terms. Table II shows the vibrational constants that resulted from this fit. Enough digits are given for the constants to allow for correlation among the constants and to ensure that the observed frequencies (actually their deviations) are correctly calculated.

The least-squares analysis made use of the term equations

$$
\begin{aligned}
E_{v}=G_{v}+ & B_{v} J(J+1)-D_{v}\left[J(J+1)-l^{2}\right]^{2}+H_{v}\left[J(J+1)-l^{2}\right]^{3} \\
& +L_{v}\left[J(J+1)-l^{2}\right]^{4}+M_{v}\left[J(J+1)-l^{2}\right]^{5}+N_{v}\left[J(J+1)-l^{2}\right]^{6}
\end{aligned}
$$

and

$$
\nu_{0}=G_{v}^{\prime}-G_{v}^{\prime \prime}
$$

\begin{tabular}{|c|c|c|c|c|c|}
\hline$P$-Branch & $J^{n}$ & $R$-Branch & $P$-Branch & $J^{n}$ & $R$-Branch \\
\hline & 0 & $2918.507273(49)^{a}$ & $2898.038623(32)$ & 42 & $2932.195776(34)$ \\
\hline $2917.699353(50)$ & 1 & 2918.905971 (49) & $2897.484749(33)$ & 43 & $2932.444 \quad 165 \quad(35)$ \\
\hline $2917.290 \quad 134(49)$ & 2 & $2919.301 \quad 159$ (48) & $2896.927304(34)$ & 44 & $2932.688887(36)$ \\
\hline $2916.877410(49)$ & 3 & $2919.692836(48)$ & $2896.366284(35)$ & 45 & $2932.929936(37)$ \\
\hline $2916.461 \quad 181(48)$ & 4 & $2920.081000(47)$ & 2895.801689 (36) & 46 & $2933.167308(38)$ \\
\hline $2916.041448(48)$ & 5 & $2920.465649(46)$ & 2895.233517 (37) & 47 & $2933.401000(38)$ \\
\hline $2915.618211(47)$ & 6 & $2920.846780(44)$ & 2894.661765 (37) & 48 & 2933.631009 (39) \\
\hline $2915.191471(46)$ & 7 & $2921.224392(43)$ & $2894.086432(38)$ & 49 & $2933.857330(40)$ \\
\hline $2914.761227(44)$ & 8 & $2921.598482(41)$ & $2893.507516(39)$ & 50 & $2934.079960(42)$ \\
\hline $2914.327479(43)$ & 9 & 2921.969047 (39) & $2892.925016(40)$ & 51 & $2934.298895(44)$ \\
\hline $2913.890226(41)$ & 10 & 2922.336085 (37) & $2892.338931(42)$ & 52 & $2934.514131(46)$ \\
\hline 2913.449469 (39) & 11 & 2922.699591 (35) & $2891.749257(44)$ & 53 & $2934.725666(48)$ \\
\hline $2913.005206(37)$ & 12 & 2923.059564 (33) & $2891.155995(46)$ & 54 & $2934.933496(51)$ \\
\hline $2912.557436(35)$ & 13 & $2923.416000(31)$ & $2890.559 \quad 143(48)$ & 55 & $2935.137617(55)$ \\
\hline $2912.106 \quad 158$ (33) & 14 & 2923.768895 (29) & 2889.958699 (51) & 56 & $2935.338027(58)$ \\
\hline 2911.651371 (31) & 15 & $2924.118246(26)$ & 2889.354663 (55) & 57 & $2935.534721(62)$ \\
\hline 2911.193074 (29) & 16 & $2924.464049(24)$ & $2888.747032(58)$ & 58 & $2935.727697(66)$ \\
\hline $2910.731265(26)$ & 17 & 2924.806301 (21) & $2888.135807(62)$ & 59 & $2935.916952(71)$ \\
\hline $2910.265943(24)$ & 18 & $2925.144996(19)$ & $2887.520985(66)$ & 60 & $2936.102482(75)$ \\
\hline $2909.797105(21)$ & 19 & $2925.480133(16)$ & 2886.902566 (71) & 61 & $2936.284285(79)$ \\
\hline 2909.324751 (19) & 20 & $2925.811705(14)$ & $2886.280549(75)$ & 62 & 2936.462357 (83) \\
\hline $2908.848877(16)$ & 21 & $2926.139710(11)$ & 2885.654932 (79) & 63 & $2936.636695(88)$ \\
\hline $2908.369482(14)$ & 22 & $2926.464142(09)$ & 2885.025716 (83) & 64 & $2936.807297(91)$ \\
\hline $2907.886565(11)$ & 23 & $2926.784998(06)$ & $2884.392898(88)$ & 65 & 2936.974159 (95) \\
\hline $2907.400122(09)$ & 24 & $2927.102273(04)$ & 2883.756478 (91) & 66 & $2937.137279(98)$ \\
\hline $2906.910151(06)$ & 25 & $2927.415963(03)$ & 2883.116455 (95) & 67 & $2937.29665(10)$ \\
\hline $2906.416650(04)$ & 26 & $2927.726062(04)$ & $2882.472829(98)$ & 68 & $2937.45228(10)$ \\
\hline 2905.919618 (03) & 27 & $2928.032568(06)$ & $2881.82560(10)$ & 69 & $2937.60416\{11\}$ \\
\hline $2905.419051 \quad(04)$ & 28 & $2928.335475(08)$ & $2881.17476\{101$ & 70 & $2937.75228(11)$ \\
\hline $2904.914946(06)$ & 29 & $2928.634778(11)$ & $2880.52032(11)$ & 71 & $2937.89664(11)$ \\
\hline $2904.407303(08)$ & 30 & $2928.930474(13)$ & $2879.86227(11)$ & 72 & $2938.03725(11)$ \\
\hline $2903.896117(11)$ & 31 & $2929.222557(16)$ & $2879.20062(11)$ & 73 & $2938.17410(11)$ \\
\hline $2903.381387(13)$ & 32 & $2929.511023(18)$ & 2878.535351111 & 74 & $2938.30717(11)$ \\
\hline $2902.863111(16)$ & 33 & $2929.795867(20)$ & $2877.86648(11)$ & 75 & $2938.43649(11)$ \\
\hline $2902.341285(18)$ & 34 & $2930.077086(22)$ & $2877.19400(11)$ & 76 & $2938.56203(11)$ \\
\hline $2901.815908(20)$ & 35 & $2930.354674(24)$ & $2876.51791(11)$ & 77 & $2938.68381(11)$ \\
\hline $2901.286976(22)$ & 36 & $2930.628628(26)$ & $2875.83822(11)$ & 78 & $2938.80181(11)$ \\
\hline $2900.754 \quad 488(24)$ & 37 & $2930.898942(28)$ & $2875.15491(11)$ & 79 & $2938.91603(11)$ \\
\hline $2900.218442(26)$ & 38 & $2931.165613(30)$ & $2874.46799(11)$ & 80 & $2939.02647(11)$ \\
\hline $2899.678 \quad 834(28)$ & 39 & $2931.428636(31)$ & $2873.77746(11)$ & 81 & $2939.13314(11)$ \\
\hline 2899.135663 (30) & 40 & $2931.688007(32)$ & $2873.08332(11)$ & 82 & $2939.23602\{11\}$ \\
\hline $2898.588927(31)$ & 41 & $2931.943721(33)$ & $2872.38557(11)$ & 83 & $2939.33511(11)$ \\
\hline
\end{tabular}

TABLE III

Wavenumbers $\left(\mathrm{cm}^{-1}\right)$ Calculated for the $10^{0} 1-00^{0} 0$ Band of ${ }^{16} \mathrm{O}^{12} \mathrm{C}^{32} \mathrm{~S}$

a The estimated uncertainty in the last digits (twice the standard deviation) is given in parentheses. 
TABLE IV

Wavenumbers $\left(\mathrm{cm}^{-1}\right)$ Calculated for the $11^{1 / 5} 1-01^{1 / 0} 0$ Band of ${ }^{16} \mathrm{O}^{12} \mathrm{C}^{32} \mathrm{~S}$

\begin{tabular}{|c|c|c|c|c|c|}
\hline$P$-Branch & $J^{n}$ & $R$-Branch & $P$-Branch & $J^{\prime \prime}$ & $R$-Branch \\
\hline & 1 & $2904.520724(28)$ & $2883.746229(61)$ & 42 & $2917.994773(61)$ \\
\hline $2902.900922(28)$ & 2 & $2904.917267(27)$ & $2883.198259(61)$ & 43 & $2918.251229(61)$ \\
\hline $2902.487570(28)$ & 3 & $2905.310444(26)$ & $2882.646898(61)$ & 44 & $2918.504 \quad 195(62)$ \\
\hline $2902.070859(27)$ & 4 & $2905.700255(25)$ & $2882.092144(61)$ & 45 & $2918.753668(62)$ \\
\hline $2901.650790(26)$ & 5 & 2906.086697 (23) & $2881.533995(62)$ & 46 & $2918.999643(62)$ \\
\hline $2901.227363(25)$ & 6 & $2906.469769(21)$ & $2880.972448(62)$ & 47 & $2919.242116(62)$ \\
\hline $2900.800579(23)$ & 7 & 2906.849469 (19) & $2880.407503(62)$ & 48 & $2919.481083(62)$ \\
\hline $2900.370440(21)$ & 8 & $2907.225795(17)$ & $2879.839156(62)$ & 49 & $2919.716541(62)$ \\
\hline $2899.936944(19)$ & 9 & $2907.598746(15)$ & $2879.267407(62)$ & 50 & $2919.948484(62)$ \\
\hline $2899.500092(17)$ & 10 & $2907.968319(12)$ & $2878.692252(62)$ & 51 & $2920.176909(62)$ \\
\hline $2899.059885(15)$ & 11 & $2908.334512(10)$ & $2878.113691(62)$ & 52 & $2920.401811(63)$ \\
\hline $2898.616322(12)$ & 12 & $2908.697323(07)$ & $2877.531721(62)$ & 53 & $2920.623 \quad 188(63)$ \\
\hline $2898.169404(10)$ & 13 & $2909.056749(05)$ & $2876.946339(63)$ & 54 & $2920.841033(64)$ \\
\hline $2897.719131(07)$ & 14 & $2909.412789(04)$ & $2876.357545(63)$ & 55 & $2921.055344(64)$ \\
\hline 2897.265502 (05) & 15 & $2909.765440(05)$ & $2875.765336(64)$ & 56 & $2921.266 \quad 116(65)$ \\
\hline $2896.808517(04)$ & 16 & $2910.114699(08)$ & $2875.169711(64)$ & 57 & $2921.473346(66)$ \\
\hline $2896.348 \quad 175$ (05) & 17 & $2910.460563(11)$ & $2874.570666(65)$ & 58 & $2921.677028(67)$ \\
\hline $2895.884477(08)$ & 18 & 2910.803031 (14) & $2873.968201(66)$ & 59 & $2921.877 \quad 160(68)$ \\
\hline $2895.417421(11)$ & 19 & $2911.142098(17)$ & $2873.362313(67)$ & 60 & $2922.073736(69)$ \\
\hline $2894.947007(14)$ & 20 & $2911.477763(20)$ & $2872.753000(68)$ & 61 & $2922.266753(70)$ \\
\hline 2894.473235 (17) & 21 & $2911.810021(23)$ & $2872.140260(69)$ & 62 & 2922.456207 (71) \\
\hline $2893.996 \quad 104(20)$ & 22 & $2912.138871(27)$ & $2871.524092(70)$ & 63 & $2922.642094(72)$ \\
\hline $2893.515612(23)$ & 23 & $2912.464309(30)$ & 2870.904494 (71) & 64 & $2922.824410(73)$ \\
\hline $2893.031759(27)$ & 24 & 2912.786332 (33) & $2870.281463(72)$ & 65 & $2923.003150(75)$ \\
\hline $2892.544544(30)$ & 25 & $2913.104936(36)$ & $2869.654998(73)$ & 66 & $2923.178312(76)$ \\
\hline 2892.053966 (33) & 26 & $2913.420119(39)$ & $2869.025097(75)$ & 67 & 2923.349889 (77) \\
\hline $2891.560024(36)$ & 27 & 2913.731877 (41) & $2868.391757(76)$ & 68 & $2923.517880(78)$ \\
\hline $2891.062716(39)$ & 28 & $2914.040206(44)$ & $2867.754978(77)$ & 69 & $2923.682279(80)$ \\
\hline $2890.562041(41)$ & 29 & $2914.345103(46)$ & $2867.114757(78)$ & 70 & 2923.843084 (81) \\
\hline $2890.057999(44)$ & 30 & $2914.646564(48)$ & $2866.471092(80)$ & 71 & $2924.000289(81)$ \\
\hline $2889.550587(46)$ & 31 & $2914.944587(50)$ & $2865.823982(81)$ & 72 & $2924.153890(82)$ \\
\hline $2889.039804(48)$ & 32 & $2915.239166(52)$ & $2865.173424(81)$ & 73 & 2924.303885 (83) \\
\hline $2888.525648(50)$ & 33 & $2915.530299(54)$ & $2864.519418(82)$ & 74 & 2924.450269 (83) \\
\hline $2888.008119(52)$ & 34 & $2915.817982(55)$ & $2863.861960(83)$ & 75 & $2924.593037(84)$ \\
\hline $2887.487215(54)$ & 35 & $2916.102211(57)$ & $2863.201049(83)$ & 76 & $2924.732186(84)$ \\
\hline $2886.962933(55)$ & 36 & $2916.382982(58)$ & $2862.536684(84)$ & 77 & $2924.867712(86)$ \\
\hline $2886.435272(57)$ & 37 & $2916.660291(59)$ & 2861.868861 (84) & 78 & $2924.999610(88)$ \\
\hline $2885.904232(58)$ & 38 & $2916.934134(60)$ & $2861.197580(86)$ & 79 & $2925.127878(92)$ \\
\hline 2885.369809 (59) & 39 & $2917.204508(60)$ & $2860.522839(88)$ & 80 & 2925.252509 (99) \\
\hline $2884.832002(60)$ & 40 & 2917.471409 (61) & $2859.844 \quad 635(92)$ & 81 & $2925.37350 \quad(11)$ \\
\hline $2884.290809(60)$ & 41 & $2917.734832(61)$ & 2859.162967 (99) & 82 & $2925.49085 \quad\{13\}$ \\
\hline
\end{tabular}

a The estimated uncertainty in the last digits (twice the standard deviation) is given in parentheses.

In addition, for the $l=1$ states, the $l$-doubling was taken into account by adding the terms

$$
\pm\left[q_{v} J(J+1)-q_{v J} J^{2}(J+1)^{2}+q_{v J J} J^{3}(J+1)^{3}\right],
$$

where the upper sign (+) was used for the $f$ levels and the lower sign $(-)$ was used for the $e$ levels.

As discussed by Maki et al. (46), there is a weak resonance between the $10^{\circ} 1$ and $04^{0} 1$ states and a similar resonance between the $11^{1} 1$ and $05^{1} 1$ states. This resonance has been investigated in more detail by Fayt $(47,48)$. It causes the effective values of the $H_{v}, L_{v}, M_{v}$, and $N_{v}$ terms to be much larger than in the ground state; consequently, it was necessary to include those constants in the least-squares fits. Although it is surprising that an $N_{v}$ term should be so large that it is needed to fit the data, the value that we find for the $10^{0} 1$ state is not as large as that predicted by Fayt et al. (49) from their analysis. 
TABLE V

Wavenumbers $\left(\mathrm{cm}^{-1}\right)$ Calculated for the $10^{0} 1-00^{\circ} 0$ Band of ${ }^{16} \mathrm{O}^{12} \mathrm{C}^{34} \mathrm{~S}$

\begin{tabular}{|c|c|c|c|c|c|}
\hline P-Branch & $J^{\prime \prime}$ & $R$-Branch & P-Branch & $J^{n}$ & $R$-Branch \\
\hline & 0 & $2906.437670(15)^{a}$ & $2886.48394(10)$ & 42 & $2919.80787(10)$ \\
\hline $2905.649479(15)$ & 1 & $2906.826663(14)$ & $2885.94416(10)$ & 43 & $2920.05078(10)$ \\
\hline $2905.250283(15)$ & 2 & $2907.212253(13)$ & $2885.40090(10)$ & 44 & $2920.29012(10)$ \\
\hline $2904.847689(14)$ & 3 & $2907.594438(11)$ & $2884.85415(10)$ & 45 & $2920.525874(99)$ \\
\hline 2904.441696 (13) & 4 & $2907.973216(9)$ & $2884.30390(10)$ & 46 & 2920.758040 (99) \\
\hline $2904.032305(11)$ & 5 & $2908.348586(7)$ & $2883.750 \quad 170(99)$ & 47 & $2920.986614(98)$ \\
\hline $2903.619516 \mid 9\}$ & 6 & $2908.720544(5)$ & $2883.192940(99)$ & 48 & $2921.211593(98)$ \\
\hline $2903.203329(7)$ & 7 & $2909.089089(4)$ & $2882.632214(98)$ & 49 & $2921.432972(97)$ \\
\hline $2902.783745\{5\}$ & 8 & $2909.454218(5)$ & $2882.067989(98)$ & 50 & $292: .650749$ (97) \\
\hline $2902.360761 \mid 4\}$ & 9 & $2909.815928(8)$ & $2881.500263(97)$ & 51 & $2921.864919(96)$ \\
\hline $2901.934379(5)$ & 10 & $2910.174217(12)$ & $2880.929034(97)$ & 52 & $2922.075479(96)$ \\
\hline $2901.504597\{8\}$ & 11 & $2910.529080(16)$ & $2880.354301(96)$ & 53 & $2922.282426(96)$ \\
\hline $2901.071414(12)$ & 12 & $2910.880516(20)$ & $2879.776063(96)$ & 54 & $2922.485756(95)$ \\
\hline $2900.634829(16)$ & 13 & $2911.228519(25)$ & $2879.194318(96)$ & 55 & $2922.685467(95)$ \\
\hline $2900.194840(20)$ & 14 & $2911.573087(29)$ & $2878.609065(95)$ & 56 & 2922.881555 (94) \\
\hline $2899.751447(25)$ & 15 & $2911.914216(34)$ & $2878.020302(95)$ & 57 & $2923.074018(93)$ \\
\hline $2899.304647(29)$ & 16 & 2912.251902 (39) & $2877.428030(94)$ & 58 & 2923.262853 (93) \\
\hline $2898.854 \quad 438(34)$ & 17 & $2912.586141(44)$ & 2876.832247 (93) & 59 & $2923.448058(92)$ \\
\hline $2898.400820(39)$ & 18 & $2912.916928(48)$ & $2876.232953(93)$ & 60 & $2923.629630(91)$ \\
\hline $2897.943789(44)$ & 19 & $2913.244259\langle 53\rangle$ & $2875.630 \quad 147(92)$ & 61 & $2923.807567(91)$ \\
\hline $2897.483343(48)$ & 20 & $2913.568130(58)$ & $2875.023828\{91\}$ & 62 & $2923.981867(90)$ \\
\hline $2897.019480(53)$ & 21 & $2913.888536(62)$ & $2874.413997(91)$ & 63 & $2924.152528(90)$ \\
\hline $2896.552 \quad 197(58)$ & 22 & $2914.205472(67)$ & $2873.800653(90)$ & 64 & $2924.319546(91)$ \\
\hline $2896.081492(62)$ & 23 & $2914.518934(71)$ & $2873.183796(90)$ & 65 & $2924.482922(92)$ \\
\hline $2895.607363(67)$ & 24 & $2914.828917(75)$ & 2872.563426 (91) & 66 & 2924.642652 (94) \\
\hline $2895.129805(71)$ & 25 & $2915.135415(79)$ & $2871.939543(92)$ & 67 & $2924.798734(96)$ \\
\hline $2894.648817(75)$ & 26 & $2915.438424(83)$ & $2871.312147(94)$ & 68 & $2924.95117(10)$ \\
\hline $2894.164396(79)$ & 27 & $2915.737939(86)$ & $2870.681238(96)$ & 69 & $2925.09995(10)$ \\
\hline 2893.676538 (83) & 28 & $2916.033955(89)$ & $2870.046816(99)$ & 70 & $2925.24508(11)$ \\
\hline $2893.185240(86)$ & 29 & $2916.326466(91)$ & $2869.40888(10)$ & 71 & $2925.38655(11)$ \\
\hline $2892.690500(89)$ & 30 & $2916.615467(94)$ & $2868.76743(11)$ & 72 & $2925.52437(12)$ \\
\hline $2892.192314(91)$ & 31 & $2916.900954(96)$ & $2868.12247(11)$ & 73 & $2925.65852(12)$ \\
\hline $2891.690679(94)$ & 32 & $2917.182921(98)$ & $2867.47400(12)$ & 74 & 2925.78901 (13) \\
\hline $2891.185592(96)$ & 33 & $2917.461362(99)$ & $2866.82201(12)$ & 75 & $2925.91584(13)$ \\
\hline $2890.677049(98)$ & 34 & $2917.73627(10)$ & 2866.16651 (13) & 76 & $2926.03900(13)$ \\
\hline $2890.165048(99)$ & 35 & $2918.00765(10)$ & $2865.50749(13)$ & 77 & $2926.15849(13)$ \\
\hline $2889.64959\{10\}$ & 36 & $2918.27548(10)$ & $2864.84496(13)$ & 78 & $2926.27430(13)$ \\
\hline $2889.13066(10)$ & 37 & $2918.53977(10)$ & $2864.17891(13)$ & 79 & $2926.38643(13)$ \\
\hline $2888.60826(10)$ & 38 & $2918.80051(10)$ & 2863.50934 (13) & 80 & $2926.49488\{14\}$ \\
\hline $2888.08240(10)$ & 39 & $2919.05770(10)$ & $2862.83625(13)$ & 81 & $2926.59964\{1.4\}$ \\
\hline $2887.55306(10)$ & 40 & $2919.31132(10)$ & $2862.15963(14)$ & 82 & $2926.70070\{16\}$ \\
\hline $2887.02024(10)$ & 41 & $2919.56138(10)$ & $2861.47950(14)$ & 83 & $2926.79807(18)$ \\
\hline
\end{tabular}

a The estimated uncertainty in the last digits (twice the standard deviation) is given in parentheses.

The new constants are not very different from the previously determined constants $(2,46,49)$ because the one accurate heterodyne measurement for each vibrational transition does not change any one constant by very much, but rather changes all the constants by a small amount. However, those new constants, with the new variancecovariance matrix elements, can now be used to calculate more reliable transition frequencies and their uncertainties.

Tables III, IV, and V give new tables of OCS absorption line wavenumbers that can be used for calibration. Even though the table lists uncertainties that are twice the standard deviation, experience has shown us that they should be treated as if they were a single standard deviation. It would not be unusual if subsequent measurements of the high- $J$ transitions changed the numbers by two or three times the uncertainty given in the table. In order to further improve the reliability of the calibration numbers for these bands, it would be useful to make at least one more sub-Doppler frequency measurement for these bands at $J$ values that differ from the present measurements 
by at least 15 units. Until more sub-Doppler measurements are made, the calculated wavenumbers for transitions with $J$ values more than 10 units from the measured $J$ values should be treated with skepticism.

In the least-squares fit for the most abundant isotopic species over 5700 transitions were included although many of those transitions did not involve either the upper or lower state of the transitions measured in this work. For the $\mathrm{OC}^{34} \mathrm{~S}$ species, far fewer frequency measurements have been made, but again, all the data used in Refs. $(2,46)$ were included in the present fit along with some more recent FTS measurements.

\section{ACKNOWLEDGMENTS}

This work was supported in part by the NASA Office of Upper Atmospheric Research. The authors thank M. Winnewisser and co-workers for providing the FTS spectrum of OCS in this region. A. Dax was funded by the Deutsche Forschungsgemeinschaft and the Graduiertenkolleg "Wechselwirkung in Molekülen" in Bonn, Germany. The Institut für Angewandte Physik provided a large quantity of optics for this experiment. We thank B. Nelles and his co-workers from Carl Zeiss for polishing the Ti-mirror, and Mr. Koch from the Deutsche Titan GmbH Düsseldorf for making the substrate available to us. We also thank S. Gilbert of NIST for valuable suggestions regarding the paper.

RECEIVED: June 23, 1994

\section{REFERENCES}

1. J. S. Wells, F. R. Petersen, ANd A. G. Maki, Appl. Opt. 18, 3567-3573 (1979).

2. A. G. MAKI AND J. S. Wells, "Wavenumber Calibration Tables From Heterodyne Frequency Measurements," NIST Special Publication 821, U.S. Government Printing Office, Washington, DC, 1991.

3. J. S. Wells, M. SCHNEIDER, AND A. G. MAKI, J. Mol. Spectrosc. 140, 170-176 (1990).

4. A. G. Maki, J. S. Wells, AND D. A. Jennings, J. Mol. Spectrosc. 144, 224-229 (1990).

5. M. Gromoll-Bohle, W. Bohle, and W. Urban, Opt. Commun. 69, 409-413 (1989).

6. E. Bachem, A. Dax, A. Weidenfeller, M. Schneider, and W. Urban, Appl. Phys. B 57, 185-191 (1993).

7. T. GEORGE, Internal Report, Institut für Angewandte Physik, Universität Bonn, Germany, 1991. [Unpublished]

8. A. Dax, M. Murtz, J. S. Wells, M. Schneider, E. Bachem, W. Urban, and A. G. Maki, J. Mol. Spectrosc. 156, 98-103 (1992).

9. C. R. Pollock, F. R. Petersen, D. A. Jennings, J. S. Wells, and A. G. Maki, J. Mol. Spectrosc. 99, 357-368 (1983).

10. C. R. Pollock, F. R. Petersen, D. A. Jennings, J. S. Wells, and A. G. Maki, J. Mol. Spectrosc. 107, 62-71 (1984).

11. A. Fayt, J. G. LaHaye, J. Lemaire, F. Herlemont, and J. G. Bantegnie, J. Mol. Spectrosc. 140, 252-258 (1990).

12. A. G. MAKI AND J.S. WellS, NIST Standard Reference Data 39. [This can be purchased from Standard Reference Data, NIST Bldg 221 / Room A32, Gaithersburg, Maryland 20899, or telephoning (301) 975-2208]

13. T. George, M. H. Wappelhorst, S. Saupe, M. MUrtz, W. Urban, and A. G. Maki, J. Mol. Spectrosc., in press.

14. A. DaX, J. S. Wells, L. Hollberg, A. G. Maki, and W. Urban, NiST Tech. Note No. 1365, 1994.

15. V. P. Chebotayev, Phys. Rep. 119, 75-116 (1985).

I6. A. Clairon, O. ACEF, C. Chardonnet, And C. J. BordÉ, in "Frequency Standards and Metrology" (A. DeMarchi, Ed.), Springer, Berlin, 1989.

17. M. D. Levenson, "Introduction to Nonlinear Spectroscopy", Academic Press, New York, 1982.

18. B. Couillaud, in "Progress in Atomic Spectroscopy" (W. Hanle and H. Kleinpoppen, Eds.), Part C, Plenum Press, New York, 1984.

19. Murray Sargent Ill, Phys. Rev. A 14, 524-527 (1976).

20. R. E. TeEts, F. V. Kowalski, W. T. Hill, N. Carlson, AND T. W. HänSCH, SPIE 113, 80-87 (1977).

21. W. DEMTRÓDER, in "Laser Spectroscopy: Basic Concepts and Instrumentation," Springer, Berlin, 1981. [See for example, pp. 494-499.]

22. F. Bayer-Helms and J. HelmCKe, PTB-Bericht Me-17, Braunschweig, Germany, 1977. 
23. A. Dax, Ph.D. thesis, Institut für Angewandte Physik. Universität of Bonn. Germany, 1992.

24. C. Wieman and T. W. Hänsch, Phy:s. Rev. Lell. 36, II70-1173 (1976).

25. V. STERT AND R. FisCher, Appl. Phys. 17, ISI-154 (1978).

26. C. Delsart and J. C. Keller, in "Laser Spectroscopy III" (J. L. Hall and J. L. Carlsten, Eds.), Springer, Berlin. 1977.

27. S. SaIK an, J. Opt. Soc. Am. 68, 1184-1187 (1978).

28. D. Goddon, A. Groh, H. J. Hanses, M. Schneider, and W. Urban, J. Mol. Spectrosc. 147, 392397 (1991).

29. A. Groh, D. Goddon, M. Schneider, W. Zimmermann, and W. Urban, J. Mol. Specirosc. 146, $161-168(1991)$.

30. M. RaAb, G. Honing, W. Demtroder, and C. R. Vidal, J. Chem. Phys. 76, 4370-4386 ( 1982 ).

31. M. D. Levenson and G. L. Eesley, Appl. Phys. 19, 1-17 (1979).

32. C. Wieman and T. W. Hänsch, Phy's. Rev. A 22, 192-205 (1980).

33. Ch. Belfrage, P. Grafstrom, S. Kroll, and S. Svanberg, Phys. Scr. 27, 367-370 (1983).

34. W. BoHLE, internal report, Institut für Angewandte Physik, Universität Bonn, Germany, 1989. [Unpublished ]

35. M. RaAb and A. Weber, J. Opt. Soc. Am. B. 2, 1476-1479 (1985).

36. Bernhard Halle NaCHF. GmbH \& Co., Berlin Germany, data sheet, 1991; a copy can be obtained on request to one of the authors (JSW). It is also on deposit in the Editorial Office of this journal.

37. R. M. A. AZZAM, "Ellipsometry and Polarized Light," North-Holland, Amsterdam, 1977.

38. M. V. R. K. MurTy aNd R. P. SHukLa, Appl. Opt. 22, 1094-1098 (1983).

39. A. R. Chraplyvy, Appl. Opt. 15, 2022-2023 (i976).

40. C. E. GreniGer, Appl. Opt. 27, 774-776 (1988).

41. M. BOR, "Principles of Optics," 6th ed.. Pergamon Press. Oxford. 1987.

42. J. S. Wells, A. Dax, L. HollberG, and A. G. Maki, J. Mol. Spectrosc., in press.

43. M. MÚrtz, M. SChaefer, M. Schneider. J. S. Wells, W. Urban, U. Schiesse, and M. Tacke, Opt. Commun. 94, 551-556 (1992).

44. A. G. Maki, C-C. ChOU, K. M. Evenson, L. R. Zink, ANd J-T. Shy, J. Mol. Spectrosc. 167, 21 I214 (1994)

45. L. C. Bradley, K. L. SOOHOO, ANd C. FREED, IEEE J. Quantum Electron. QE-22, 234-267 ( 1986 ).

46. A. G. MaKi, J. S. Wells, and J. B. Burkholder, J. . Hol. Spectrosc. 147, 173-181 (1991).

47. A. FAYT, Ann. Soc. Sci. Brussels 82, 101-112 (1968).

48. A. FAYT, Ann. Soc. Sci. Brussels 84, 69-106 (1970).

49. A. Fayt, R. Vandenhaute, and J. G. Lahaye, J. Mol. Spectrosc. 119, 233-266 (1986). 


\title{
Sub-Doppler Frequency Measurements on OCS near 1689 and $1885 \mathrm{~cm}^{-1}$
}

\author{
J. S. Wells, A. Dax, ${ }^{1}$ L. Hollberg, and A. G. MaKI ${ }^{2}$ \\ Time and Frequency Division. National Institute of Standards and Technology, Boulder, Colorado 80303
}

\begin{abstract}
We have used an optical polarization heterodyne technique to measure the frequencies of two OCS lines, one near $5.3 \mu \mathrm{m}$ and the other near $5.9 \mu \mathrm{m}$. These sub-Doppler measurements gave $56533766.125 \pm 0.035 \mathrm{MHz}$ for the $2 \nu_{2}+\nu_{3} P(16)$ transition and $50627632.013 \pm 0.100 \mathrm{MHz}$ for the $2 \nu_{3} P(48)$ line. The $P(16)$ measurement is in excellent agreement ( $20 \mathrm{kHz}$ ) with a new result obtained with an alternate technique by a group at the University of Bonn. The $2 \nu_{3}$ measurement was combined with other frequency measurements to produce an improved calibration table for the $2 v_{3}$ OCS band near $1700 \mathrm{~cm}^{-1}$. These and other sub-Doppler frequency measurements in different spectral regions give improved frequencies for a number of OCS bands. These new frequencies will soon be incorporated in to calibration data that will be available over the Internet. c) 1995 Academic Press. Inc.
\end{abstract}

\section{INTRODUCTION}

The first phase of a joint NBS/NASA program (which began in $1979(1)$ ) to generate infrared frequency standards for the users of tunable lasers culminated in 1991 with the publication of a calibration atlas, NIST SP 821 (2). Although other new atlases (with some complementarity in frequency coverage) have recently been published ( 3 , 4 ), the NIST atlas is the only one in the mid-infrared based primarily on infrared frequency measurements. The NIST atlas has a spectral map/facing table format, and the facing table information is also available in the form of computer diskettes in NIST Reference Data Base 39 (5). About four times as many other frequencies (of transitions too weak and numerous to be included in the atlas proper) are included in the diskettes. A text (6), intended as a companion to Data Base 39 , contains references and other relevant information that also appears in the atlas. The frequency measurements used for the generation of tables in this atlas were based primarily on Doppler-limited spectra. The frequency measurements were based on $\mathrm{CO}_{2}$ lasers used as frequency standards.

Phase two of this on-going effort features three new developments, an ever increasing number of sub-Doppler frequency measurements, a revision of $\mathrm{CO}_{2}$ laser frequencies, and the dissemination of the atlas material on the new information superhighway.

Sub-Doppler frequency measurements on OCS include contributions in the $9-\mu \mathrm{m}$ region by Fayt et al. (7). More recently Urban's group at the Institut für Angewandte Physik in Bonn have extended their CO frequency measurement techniques at $5 \mu \mathrm{m}$ (8) to include OCS (9). The first NIST sub-Doppler measurements on OCS were made at $3.3 \mu \mathrm{m} \mathrm{(10).} \mathrm{The} \mathrm{initial} \mathrm{measurements} \mathrm{at} 5$ and $3.3 \mu \mathrm{m}$ relied on accidental coincidences between OCS and CO laser lines (both $\Delta v=1$ and $\Delta v=2$ transitions)

\footnotetext{
${ }^{1}$ Guest Researcher from Institute für Angewandte Physik der Universität Bonn, Bonn, D-53115 Germany.
}

${ }^{2}$ Present address: 1501224 Ave. S.E., Mill Creek, WA 98012. 
or on coincidences that were possible after shifting the $\mathrm{CO}$ frequency with an acoustooptic modulator (AOM).

The number of such accidental coincidences is rather small and the revolutionary development of a new frequency measurement system featuring tunable sidebands on a $\mathrm{CO}$ laser at the Institut für Angewandte Physik (Bonn) eliminates the need for coincidences. Many high quality new measurements are pending publication $(11,12)$ and others are being planned.

The precision of these sub-Doppler frequency measurements is quite high (uncertainties down to tens of kilohertz), and a new set of more accurate $\mathrm{CO}_{2}$ transition frequencies makes possible absolute frequency measurements with accuracies commensurate with this precision. This new set of $\mathrm{CO}_{2}$ laser frequencies was determined by a refit of all the frequency measurements for $\mathrm{CO}_{2}$ and its isotopomers by Maki et al. (13). It is based on the most accurate frequency measurements currently available. These new $\mathrm{CO}_{2}$ frequency values have been used in the sub-Doppler OCS frequency measurements on OCS. While the new OCS results are within the uncertainties of the frequencies listed in SP 821, they represent improved frequency values with much smaller uncertainties.

The results of new measurements are continually being added to the data bases used to calculate frequencies of transitions in the atlas, and the development of the information superhighway will permit the dissemination of the newly calculated frequencies to everyone in a more timely fashion. This first update of the calibration atlas will be available in 1995 on the Internet.

In the following, we describe two new sub-Doppler frequency measurements of OCS absorption lines, one at $5.3 \mu \mathrm{m}$ the other at $5.9 \mu \mathrm{m}$.

\section{EXPERIMENTAL DETAILS}

These 5-6 $\mu \mathrm{m}$ experiments were an extension of experiments at $3.3 \mu \mathrm{m}(10)$. Again, we have used the optical heterodyne polarization technique invented by Wieman and Hänsch (14). The essence of this technique is that a strong pump/saturation beam introduces anisotropy in a sample; that is, the sample becomes dichroic and birefringent when the pump beam frequency is near a molecular resonance. A counterpropagating probe beam will undergo a change in polarization orientation as the laser frequency approaches the vicinity of a molecular resonance. Under appropriate conditions one can detect the orientation changes in polarization and use the resulting dispersion signal as a discriminant for locking a laser frequency to the absorption frequency of a molecule of interest. One can divide a laser beam into several portions and use them for saturation and probing of a sample, and have a third portion for frequency measurement.

The basic scheme for the $5.3-\mu \mathrm{m}$ experiment is shown in Fig. 1 . The starting point is a liquid-nitrogen-cooled, flowing-gas $\mathrm{CO}$ laser which has been converted from 3.3 $\mu \mathrm{m}(\Delta v=2)$ operation to $\Delta v=1$ operation by substituting a 240 line $/ \mathrm{mm}$ grating and appropriate coupler for those used earlier. About $400 \mathrm{~mW}$ were available from the $P_{9}(6)$ transition, and polarization purity of the output beam was about $2000: 1$ as it left the laser. The beam from the $\mathrm{CO}$ laser was directed to an uncoated $\mathrm{ZnSe}$ beam splitter, and the transmitted beam as well as two reflected beams were used in the experiment.

The transmitted beam was passed through a novel two-stage device (equivalent to a quarterwave plate) which produced the circularly polarized saturating beam. This reflective device has a throughput of about $60 \%$ and produced a circularly polarized 


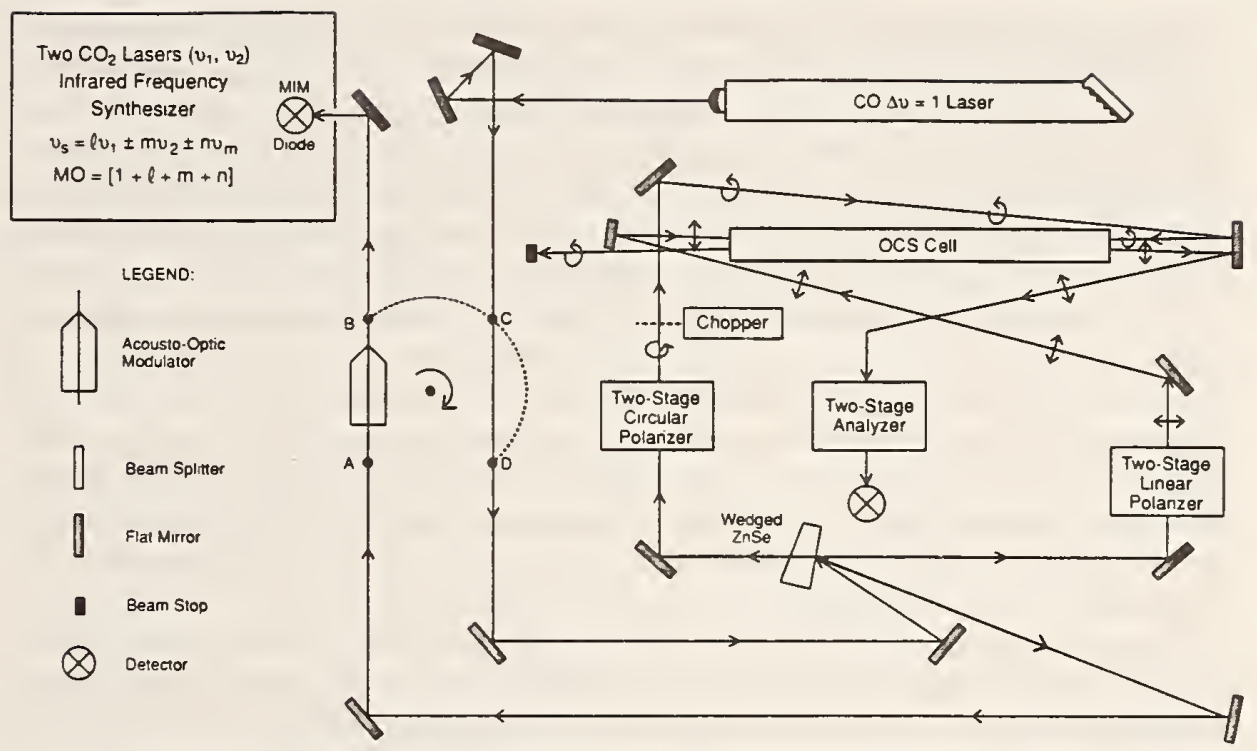

FIG. 1. Functional diagram for the optical polarization heterodyne spectroscopic experiment in the 5-6 $\mu \mathrm{m}$ region. The diagram has been stylized to a certain extent in the interest of simplicity.

beam with about $99.5: 1$ polarization purity as measured with a Rochon polarizer. The power transmitted through the beam splitter and polarizer was about $165 \mathrm{~mW}$ at the cell entrance. The saturating beam was chopped and the modulation transferred to the probe beam provided the signal at the detector.

The beam reflected from the first surface was used for the probe beam after passing through a two-stage device (with about $78 \%$ throughput) which increased its polarization purity to about $200000: 1$, again with the use of reflective elements. The details of both of these two-stage polarization devices appear elsewhere $(10,15)$. The power of the probe beam was about $50 \mathrm{~mW}$ at the absorption cell.

The reflected beam from the second surface was directed to the $\mathrm{CO}_{2}$ laser frequency synthesizer through an AOM in order to prevent feedback from the MIM diode to the $\mathrm{CO}$ laser. The $\mathrm{AOM}$ was coated for $3.3 \mu \mathrm{m}$, and this reduced power in the vicinity of the MIM to about $25 \mathrm{~mW}$.

Since details of the synthesizer appear in several places $(2,6,16,17)$; we briefly state that the synthesized frequency $\nu_{\mathrm{s}}$ is given by

$$
\nu_{\mathrm{s}}=l \nu_{1} \pm m \nu_{2} \pm n \nu_{\mathrm{m}},
$$

where we define $l, m$, and $n$ to be positive integers. A microwave frequency is represented by $\nu_{\mathrm{m}}$, and $\nu_{1}$ and $\nu_{2}$ are the new frequencies of the $\mathrm{CO}_{2}$ laser frequency standards. The $\mathrm{CO}_{2}$ lasers are now stabilized ( $1-f$ detection ) by means of $4.3-\mu \mathrm{m}$ fluorescence in an external absorption cell using the latest recommendations of Freed and co-workers (18). In general, it becomes more difficult (more stringent requirements on the impedence of the MIM and the sharpness of the antenna point) to synthesize a useful reference signal in the MIM diode as the mixing order increases. With the above definition of $l, m$, and $n$, the mixing order, MO, is defined as

$$
\mathrm{MO}=[1+l+m+n] \text {. }
$$


After traversing the cell, the probe beam is processed with the aid of a two-element analyzer, which is also discussed in Ref. (10). We have shown that the relevant part of the signal through the two-stage analyzer which reaches the detector is proportional to $\phi_{1} E_{0}\left(E_{0} \Delta n k_{0} L\right)$, where $\phi_{1}$ is the deviation of the crossing angle of the first analyzer, $E_{0}$ represents the field of the probe beam, $\Delta n$ describes the birefringence and has a dispersion characteristic, $k_{0}=2 \pi / \lambda$, and $L$ is the estimated overlap length of the slightly crossed beams within the cell. The signal amplitude is the term in parentheses, and the term $\phi_{1} E_{0}$ is the local oscillator amplitude. The main advantage of this heterodyne technique is that a weak signal can be enhanced a great deal by heterodyning it with a much stronger local oscillator. For the $P(16)$ measurement a comparatively large local oscillator power helped produce the dispersion signal shown in Fig. 2. The signal-to-noise ratio shown here is about $10000: 1$. The OCS pressure in the cell was $1.3 \mathrm{~Pa}$ ( $10 \mathrm{mTorr}$ ), and the effective beam overlap length in the OCS absorption cell was estimated to be $1 \mathrm{~m}$. This discriminant was used for locking the $\mathrm{CO}$ laser for the $P(16)$ frequency measurement.

The measurement of the $P(48)$ OCS line at $5.9 \mu \mathrm{m}$ was considerably more difficult, and the measurement uncertainty was increased. For this experiment, a direct overlap between $\mathrm{CO}$ and OCS did not exist and it was necessary to shift the frequency of the working beam $90.000 \mathrm{MHz}$ below the frequency of the $P_{15}(18)$ transition by first passing it through the AOM (path changed from A-B to C-D in Fig. 1). The 3.3- $\mu \mathrm{m}$ $A R$ coating on the AOM permitted an even smaller portion of the beam power to be transmitted than was the case at $5.3 \mu \mathrm{m}$. About $30 \mathrm{~mW}$ total was available for the three beams departing from our uncoated, wedged beam splitter. The power levels at the cell were about 13 and $4 \mathrm{~mW}$ for the saturation and probe beams, respectively, and $4 \mathrm{~mW}$ was available for generating the beatnote with the $\mathrm{CO}_{2}$ synthesizer. As a result of the lower power and a more than an order of magnitude reduction in detector sensitivity in going from 5.3 to $5.9 \mu \mathrm{m}$, the dispersion signal was not nearly as good as that for the $P(16)$ transition. A second difficulty was associated with the higher mixing order ( 8 in this case). The harmonic numbers were 4,2 , and 1 ; the need for

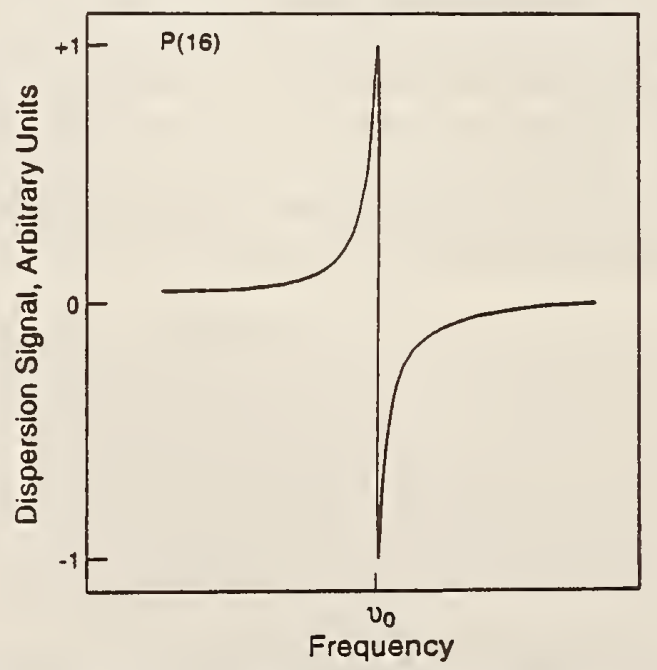

FIG. 2. Dispersion signal for the OCS $P(16)$ transition of the $02^{\circ} 1-00^{\circ} 0$ band. The peak-to-peak linewidth was about $600 \mathrm{kHz}$, and the width of the scan shown in the figure was estimated to be about $60 \mathrm{MHz}$ 
higher harmonics also contributed to a larger uncertainty than we had for the $P(16)$ measurement. For the $P(48)$ measurement, the pressure and effective pathlength were also $1.3 \mathrm{~Pa}$ and $1 \mathrm{~m}$.

In both instances, the frequency of the radiation in the cell was locked to a subDoppler feature of the molecular transition of interest, and a signal was generated in the $\mathrm{CO}_{2}$ synthesizer that was close to that transition frequency. A difference frequency beatnote, $\nu_{\text {if }}$ (between the synthesized frequency and the transition frequency) was measured on a spectrum analyzer. The OCS transition frequency was then

$$
\nu_{\mathrm{OCS}}=\nu_{\mathrm{s}} \pm \nu_{\mathrm{if}} .
$$

Both the Bonn measurement and our measurement of $P(16)$ used $\mathrm{CO}_{2}$ reference lasers of similar design which are not as accurate as the $\mathrm{CO}_{2}$ laser system used by Chardonnet $e t$ al. (19) for their very accurate measurements. Even though these two measurements agree to within $20 \mathrm{kHz}$, that does not mean that the absolute accuracy is that good. We believe that the uncertainties given here and in Ref. (9) are good estimates of the true uncertainties, regardless of how close the agreement may be. The uncertainty in the $P(48)$ measurement has a larger uncertainty for the reasons already discussed. The results of $P(16)$ and $P(48)$ measurements are shown in Table I.

\section{ANALYSIS AND RESULTS}

The present results were combined with earlier measurements in a least-squares fit. Earlier heterodyne measurements that involved the $2 \nu_{3}$ state are given in Refs. (2022 ), and those involving the $2 \nu_{2}+\nu_{3}$ state are given in Ref. (23). In addition, measurements that involve rotational transitions for these vibrational states were given by Vanek et al. (24).

The fit used the same Hamiltonian as that described by Maki et al. (23). For the $02^{\circ} 1-00^{\circ} 0$ transition the effect of $l$-type resonance was taken into account, but that resonance was not present for the $00^{\circ} 2-00^{\circ} 0$ transition.

The present new measurements are useful for determining much improved absolute frequencies for the levels of OCS that are near the same $J$ values as the measurements.

TABLE I

Measured Transition Frequencies for Carbonyl Sulfide

\begin{tabular}{|c|c|c|c|c|}
\hline $\begin{array}{l}\text { CO } \\
\text { Trans. }^{a}\end{array}$ & $\begin{array}{l}\text { OCS } \\
\text { Trans. }^{b}\end{array}$ & $\begin{array}{l}\text { Obs. Freq." } \\
(\mathrm{MHz})\end{array}$ & $\begin{array}{c}\mathrm{CO}_{2} \text { Laser }^{\mathrm{d}} \\
\text { Synthesis Scheme }\end{array}$ & $\begin{array}{c}\nu_{\mathrm{tF}} \\
(\mathrm{MHz})\end{array}$ \\
\hline $\begin{array}{l}P_{9}(6) \\
P_{15}(18)\end{array}$ & $\begin{array}{l}P(16) \mathrm{A} \\
P(48) \mathrm{A}\end{array}$ & $\begin{array}{l}56533766.125 \pm 0.035 \\
50627632.013 \pm 0.100\end{array}$ & $\begin{array}{c}P_{1}(30)+P_{1}(12)-\nu_{\mathrm{m}} \\
4 \times R_{\mathrm{t}}(6)-2 \times R_{11}(36)-\nu_{\mathrm{n}}\end{array}$ & $\begin{array}{r}307.596 \\
-449.840\end{array}$ \\
\hline
\end{tabular}

"For the CO laser designation the subscript indicates the lower state vibrational quantum number and $J "$ appears in parentheses.

${ }^{b}$ We are using the notations in Ref. (2) for these transitions. Thus for the $57 \mathrm{THz}$ transition, A represents the $02^{\prime \prime} 1-00^{\prime \prime} 0$ band. For the $51 \mathrm{THz}$ line, $\mathrm{A}$ is the designation for the $00^{\circ} 2-00^{\prime \prime} 0$ band.

' The observed OCS frequency is the sun of the synthesized frequency and the beatnote frequency.

${ }^{d}$ For the $\mathrm{CO}_{2}$ laser, the designation I indicates the $10 \mu \mathrm{m}$ band and II indicates the $9 \mu \mathrm{m}$ band.

e For the $P(16)$ measurement, $\nu_{\mathrm{m}}$ was $10000.000 \mathrm{MHz}$, for $P(48) \nu_{\mathrm{m}}$ was $10007.022 \mathrm{MHz}$. 
This can be seen in the uncertainties shown in Table II. The uncertainties become quite small for the transitions between $J=20$ and $J=65$ of $00^{\circ} 2-00^{\circ} 0$. For other values of $J$ the uncertainties approach the values given in Ref. (2) but are always smaller. Table II gives the calculated wavenumbers determined by the constants given by a least-squares fit of all the measurements available for the upper and lower states of the $00^{\circ} 2-00^{\circ} 0$ transitions. The constants from that fit are almost identical to those given by Maki and Wells (2), but there are significant changes in the correlation of the constants and in the variance-covariance matrix elements.

The earlier ( 9 ) sub-Doppler measurement of the $P(16)$ transition of $2 \nu_{2}+\nu_{3}$ has been confirmed by the present measurement, and the agreement can also be considered

TABLE II

Wavenumbers $\left(\mathrm{cm}^{-1}\right)$ Calculated for the $00^{0} 2-00^{0} 0$ Band of ${ }^{16} \mathrm{O}^{12} \mathrm{C}^{32} \mathrm{~S}$

\begin{tabular}{|c|c|c|c|c|c|}
\hline P-Branch & $J^{n}$ & $R$-Branch & P-Branch & $J^{\prime \prime}$ & $R \cdot$ Branch \\
\hline & 0 & $1711.379530(31)^{a}$ & $1690.309118(05)$ & 45 & $1726.972585(05)$ \\
\hline $1710.570547(31)$ & 1 & $1711.780357(31)$ & $1689.793863(05)$ & 46 & $1727.261570(05)$ \\
\hline $1710.162392(31)$ & 2 & $1712.178739(31)$. & $1689.276166(05)$ & 47 & $1727.548011(05)$ \\
\hline $1709.751796(31)$ & 3 & $1712.574674(31)$ & $1688.756026(05)$ & 48 & $1727.831907(05)$ \\
\hline $1709.338760(31)$ & 4 & $1712.968162(30)$ & $1688.233443(05)$ & 49 & $1728.113253(06)$ \\
\hline $1708.923286(31)$ & 5 & $1713.359202(30)$ & $1687.708414(05)$ & 50 & $1728.392047(06)$ \\
\hline $1708.505373(30)$ & 6 & $1713.747791(30)$ & $1687.180940(05)$ & 51 & $1728.668285(07)$ \\
\hline $1708.085023(30)$ & 7 & $1714.133929(29)$ & $1686.651018(06)$ & 52 & $1728.941964(08)$ \\
\hline $1707.662237(30)$ & 8 & $1714.517614(29)$ & $1686.118647(07)$ & 53 & $1729.213080(08)$ \\
\hline 1707.237015 (29) & 9 & $1714.898845(28)$ & $1685.583827(07)$ & 54 & $1729.481630(09)$ \\
\hline $1706.809358(29)$ & 10 & $1715.277620(28)$ & $1685.046556(08)$ & 55 & $1729.747611(10)$ \\
\hline $1706.379267(28)$ & 11 & $1715.653938(27)$ & $1684.506833(09)$ & 56 & $1730.011019(11)$ \\
\hline $1705.946741(28)$ & 12 & $1716.027797(27)$ & $1683.964656(10)$ & 57 & $1730.271850(13)$ \\
\hline $1705.511782(27)$ & 13 & $1716.399195(26)$ & $1683.420024(11)$ & 58 & $1730.530102(14)$ \\
\hline $1705.074391(27)$ & 14 & $1716.768132(25)$ & $1682.872936(13)$ & 59 & $1730.785770(15)$ \\
\hline $1704.634567(26)$ & 15 & $1717.134604(24)$ & $1682.323389(14)$ & 60 & $1731.038850(16)$ \\
\hline $1704.192311(25)$ & 16 & $1717.498610(24)$ & $1681.771383(15)$ & 61 & $1731.289340(18)$ \\
\hline $1703.747623(24)$ & 17 & $1717.860149(23)$ & $1681216917(16)$ & 62 & $1731.537235(19)$ \\
\hline $1703.300504(24)$ & 18 & $1718.219219(22)$ & $1680.659987(18)$ & 63 & $1731.782532(20)$ \\
\hline $1702.850954(23)$ & 19 & $1718.575817(21)$ & $1680.100594(19)$ & 64 & $1732.025227(22)$ \\
\hline $1702.398973(22)$ & 20 & $1718.929942(20)$ & $1679.538734(20)$ & 65 & $1732.265316(23)$ \\
\hline $1701944561(21)$ & 21 & $1719.281591(20)$ & $1678.974407(22)$ & 66 & $1732.502795(24)$ \\
\hline $1701.487719(20)$ & 22 & $1719.630764(19)$ & $1678.407611(23)$ & 67 & $1732.737660(25)$ \\
\hline $1701.028446(20)$ & 23 & $1719.977456(18)$ & $1677.838344(24)$ & 68 & $1732.969908(27)$ \\
\hline $1700.566743(19)$ & 24 & $1720.321667(17)$ & $1677266604(25)$ & 69 & $1733.199534(28)$ \\
\hline $1700.102609(18)$ & 25 & $1720.663395(16)$ & $1676.692389(27)$ & 70 & $1733.426534(29)$ \\
\hline $1699.636045(17)$ & 26 & $1721.002636(15)$ & $1676.115698(28)$ & 71 & $1733.650905(30)$ \\
\hline $1699.167050(16)$ & 27 & $1721.339389(15)$ & $1675.536528(29)$ & 72 & $1733.872643(31)$ \\
\hline $1698.695624(15)$ & 28 & $1721.673651(14)$ & $1674.954878(30)$ & 73 & $1734.091742(31)$ \\
\hline $1698.221767(15)$ & 29 & $1722.005420(13)$ & $1674.370746(31)$ & 74 & $1734 . j 08200(32)$ \\
\hline $1697.745479(14)$ & 30 & $1722.334694(12)$ & $1673784129(31)$ & 75 & $1734.522011(33)$ \\
\hline $1697.266760(13)$ & 31 & $1722.661471(12)$ & $1673.195026(32)$ & 76 & $1734.733173(33)$ \\
\hline $1696.785608(12)$ & 32 & $1722.985746(11)$ & $1672.603435(33)$ & 77 & $1734.941680(34)$ \\
\hline $1696.302025(12)$ & 33 & $1723.307519(10)$ & $1672.009353(33)$ & 78 & $1735.147528(34)$ \\
\hline $1695.816009(11)$ & 34 & $1723.626787(10)$ & $1671.412778(34)$ & 79 & $1735.350714(35)$ \\
\hline $1695.327559(10)$ & 35 & $1723.943546(09)$ & $1670.813709(34)$ & 80 & $1735.551232(35)$ \\
\hline $1694.836677(10)$ & 36 & $1724.257794(09)$ & $1670.212143(35)$ & 81 & $1735.749078(36)$ \\
\hline $1694.343360(09)$ & 37 & $1724.569529(08)$ & $1669.608078(36)$ & 82 & $1735.944249(37)$ \\
\hline $1693.847608(08)$ & 38 & $1724.878748(07)$ & $1669.001512(37)$ & 83 & $1736.136739(39)$ \\
\hline $1693.349421(08)$ & 39 & $1725.185448(07)$ & $1668.392441(38)$ & 84 & $1736.326544(41)$ \\
\hline $1692.848798(07)$ & 40 & $1725.489626(07)$ & $1667.780865(40)$ & 85 & $1736.513661(44)$ \\
\hline $1692.345739(07)$ & 41 & $1725.791279(06)$ & $1667,166781(42)$ & 86 & $1736.698083(48)$ \\
\hline $1691.840242(06)$ & 42 & $1726.090404(06)$ & $1666.550186(45)$ & 87 & $1736.879807(53)$ \\
\hline $1691.332307(06)$ & 43 & $1726.386999(05)$ & $1665.931078(49)$ & 88 & $1737.058829(59)$ \\
\hline $1690.821932(06)$ & 44 & $1726.681060(05)$ & $1665.309454(54)$ & 89 & $1737.235143(66)$ \\
\hline
\end{tabular}

a The estimated uncertainty in the last digits (twice the standard deviation) is given in parentheses. 
as a confirmation of the accuracy of the other sub-Doppler measurement of this paper and of Ref. (10). The present measurements do not make a significant difference to the transition wavenumbers calculated for the $2 \nu_{2}+\nu_{3}$ band given as Table IV in the paper by George et al. (9).

\section{ACKNOWLEDGMENTS}

This work was supported in part by the NASA Office of Upper Atmospheric Research. A. Dax was funded by the Deutsche Forschungsgemeinschaft and the Graduiertenkolleg "Wechselwirkung in Molekülen" in Bonn, Germany. We are grateful to the Institut für Angewandte Physik of the University of Bonn for temporary loan of a large quantity of optics for this experiment.

RECEIVED: September 6, 1994

\section{REFERENCES}

1. J. S. Wells, F. R. Petersen, AND A. G. MaKi, Appl. Opt. 18, 3567-3573 (1979).

2. A. G. MAKI AND J. S. WellS, "Wavenumber Calibration Tables from Heterodyne Frequency Measurements," Natl. Inst. Stand. Technol. Special Publication 821, U.S. Government Printing Office, Washington, DC, 1991.

3. G. Guelach ViLI AND K. Narahari RaO, "Handbook of Infrared Standards," Academic Press, Otlando, FL, 1986.

4. G. Guelachvili and K. Narahari Rao, "Handbook of Infrared Standards II," Academic Press, Boston, 1993.

5. A. G. MAKI AND J. S. WELLS, NIST Standard Reference Data 39. [ This may be purchased by contacting Standard Reference Data, NIST, Bldg. 221/Room A32, Gaithersburg, MD 20899, or telephoning (301) 975-2208

6. A. G. Maki and J. S. WellS, J. Res. Natl. Inst. Stand. Technol. 97, 409-470 (1992).

7. A. Fayt, J. G. LaHaye, J. Lemaire, F. Herlemont, and J. G. Bantegnie, J. Mol. Spectrosc. 140, 252-258 (1990).

8. T. George, S. Saupe, M. H. Wappelhorst, and W. Urban, Appl. Phys. B. 59, 159-166 (1994).

9. T. George, M. H. Wappelhorst, S. Saupe, M. MUrtz, W. Urban, and A. G. Maki, J. Mol. Spectrosc. 167, 419-428 (1994).

10. A. Dax, J. S. Wells, L. Hollberg, A. G. MaKi, and W. URban, J. Mol. Spectrosc. 168, 416-428 (1994).

11. B. Meyer, S. Saupe, M. H. Wappelhorst, T. George, F. Kunnemann, M. Schneider, M. Havenith, W. Urban, and J. Legrand, Appl. Phys. B., submitted for publication.

12. S. SAUPE, B. MeYer, M. H. WAPPelhorst, W. URBAN, AND A. G. MAKI, in preparation.

13. A. G. MAKI, C-C. Chou, K. M. Evenson, L. R. ZinK, AND J-T. Shy, J. Mol. Spectrosc. 167, $211-$ 224 (1994).

14. C. Wieman and T. W. Hänsch, Phys. Rev. Lett., 1170-1173 (1976).

15. A. Dax, J. S. Wells, L. HollberG, A. G. Mak, and W. Urban, NiST Tech. Note 1365 (1994).

16. A. Hinz, J. S. Wells, AND A. G. MAKI, Z. Phys. D 5, 351-358 (1987).

17. J. S. Wells, M. SChNeIder, AND A. G. MaKI, J. Mol. Spectrosc. 132, 422-428 (1988).

18. K. L. SOOHOO, C. FreEd, J. E. ThOMAS, AND H. A. HAUS, IEEE J. Quantum Electron. QE-21, 1159$1171(1985)$.

19. Ch. Chardonnet, A. VAn Lerberghe, And Ch. J. Borde, Opt. Commun. 58, 333-337 (1986).

20. J. S. Wells, F. R. Petersen, A. G. Maki, And D. J. SuKle, Appl. Opt. 20, 1676-1684 (1981); 20, $2874(1981)$.

21. J. S. Wells, F. R. Petersen, And A. G. Maki, J. Mol. Spectrosc. 98, 404-412 (1983).

22. J. S. Wells, M. D. VANEK, AND A. G. MAKI, J. Mol. Spectrosc. 135, 84-88 (1989).

23. A. G. MaKi, WM. B. Olson, J. S. Wells, AND M. D. VANeK, J. Mol. Spectrosc. 130, 69-80 (1988).

24. M. D. Vanek, D. A. Jennings, J. S. Wells, and A. G. MaKı, J. Mol. Spectrosc. 138, 79-83 (1989). 


\title{
Detection of methane in air using diode-laser pumped difference-frequency generation near $3.2 \mu \mathrm{m}$
}

\author{
K. P. Petrov ${ }^{1}$, S. Waltman', U. Simon ${ }^{1}$, R. F. Curl ${ }^{1}$, F. K. Tittel ${ }^{1}$, E. J. Dlugokencky ${ }^{3}$, L. Hollberg ${ }^{2}$ \\ 'Departments of Electrical and Computer Engineering, and Chemistry, Rice University, Houston, TX 77251-1892, USA \\ (Fax: + 1-713/524-5237, E-mail: FKT@rice.edu) \\ Time and Frequency Division, National Institute of Standards and Technology, 325 Broadway, Boulder, CO 80303, USA \\ ${ }^{3}$ National Oceanic and Atmospheric Administration, 325 Broadway, Boulder, CO 80303, USA
}

Received: 23 June 1995/Accepted: 1 July 1995

Abstract. Spectroscopic detection of the methane in natural air using an $800 \mathrm{~nm}$ diode laser and a diode-pumped $1064 \mathrm{~nm}$ Nd:YAG laser to produce tunable light near $3.2 \mu \mathrm{m}$ is reported. The lasers were pump sources for ring-cavity-enhanced tunable difference-frequency mixing in $\mathrm{AgGaS}_{2}$. IR frequency tuning between 3076 and $3183 \mathrm{~cm}^{-1}$ was performed by crystal rotation and tuning of the extended-cavity diode laser. Feedback stabilization of the $I R$ power reduced intensity noise below the detector noise level. Direct absorption and wavelength-modulation (2f) spectroscopy of the methane in natural air at $10.7 \mathrm{kPa}$ (80 torr) were performed in a $1 \mathrm{~m}$ single-pass cell with $1 \mu \mathrm{W}$ probe power. Methane has also been detected using a $3.2 \mu \mathrm{m}$ confocal build-up cavity in conjunction with an intracavity absorption cell. The best methane detection limit observed was $12 \mathrm{ppb} \mathrm{m}(\mathrm{Hz})^{-1 / 2}$.

PACS: $07.65 ; 33.00 ; 42.60 ; 42.65 ; 42.80$

This work was done to develop a diode-laser-based technique for sensitive detection of environmentally important atmospheric trace gases such as methane, carbon monoxide, nitrous oxide. and nitric oxide. Several measurements of optical absorption in methane using tunable near-infrared lasers have been reported recently. Lucchesini et al. have used diode lasers to access $3 v_{1}+v_{3}+\left(v_{2}\right.$ or $\left.v_{4}\right)$ combination-overtone bands of methane near $790 \mathrm{~nm}$, and the $2 v_{1}+2 v_{3}$ band near $860 \mathrm{~nm}$ [1]. Scott et al. have investigated the possibility of methane detection with the use of a $1.34 \mu \mathrm{m} \mathrm{Nd}$ : YAG laser which can access the $v_{1}+2 v_{3}$ combination-overtone band [2]. Uehara and Tai [3] have been used a diode laser to detect methane in air by monitoring absorption in the $2 v_{3}$-stretch vibrationovertone band near $1.66 \mu \mathrm{m}$, achieving a detection limit of $0.3 \mathrm{pfmm}$ with a signal averaging time of $1.3 \mathrm{~s}$. This corresponds to $680 \mathrm{ppbm}(\mathrm{Hz})^{-1 / 2}$. Pavone and Inguscio [4] observed a component of the methane combination band corresponding to a third overtone at $866 \mathrm{~nm}$.
The fundamental $v_{3}$ band of methane near $3.2 \mu \mathrm{m}$, however, has transitions that are as much as a factor of 160 stronger than those of the first overtone band and may be better suited for sensitive detection. The maximum line intensity and the typical pressure broadening coefficient of methane in this band is $2.13 \times 10^{-19} \mathrm{~cm}$, and $0.027 \mathrm{MHz} \mathrm{Pa}^{-1}$, respectively, which corresponds to a peak absorption of $0.005 \mathrm{~m}^{-1} \mathrm{ppm}^{-1}$ in air at $1 \mathrm{~atm}$ near $3067 \mathrm{~cm}^{-1}$ [5]. Given the typical $1.8 \mathrm{ppm}$ natural abundance of methane in air the absorption coefficient to be measured is $0.009 \mathrm{~m}^{-1}$. The fundamental $v_{3}$ band of methane is accessible by either conventional spectroscopy using the carbon monoxide overtone laser [6], the heliumneon laser near $3.39 \mu \mathrm{m}[7]$, lead-salt diode lasers, colorcenter lasers, or with $\mathrm{Ar}^{+}$-dye laser difference-frequency generation [8]. These infrared laser sources are also suitable for sensitive atmospheric trace gas detection. McManus et al. [9] demonstrated an atmospheric methane measurement instrument using a Zeeman-split helium-neon laser with a sensitivity of $20 \mathrm{ppb}$ with a signal averaging time of $1 \mathrm{~s}\left(40 \mathrm{ppb}(\mathrm{Hz})^{-1 / 2}\right)$. Simultaneous detection of methane and other gas species in air has been accomplished with the use of a compact lead-salt diode laser spectrometer that included a multipass absorption cell [10]. A detection limit for methane of $4 \mathrm{ppb}$ m near $8 \mu \mathrm{m}$ with a signal averaging time of $3 \mathrm{~s}$ was reported $\left(14 \mathrm{ppb} \mathrm{m}(\mathrm{Hz})^{-1 / 2}\right)$.

However, each of the mid-infrared laser sources mentioned above suffers from its own specific practical drawbacks súch as large physical size. lack of portability, high cost, high power consumption. poor tunability. or need for cryogenic cooling. On the other hand. the use of commercial single-frequency short wavelength diode lasers as pump sources offers benefits of small size, reliability, low cost, and low power consumption. These diode lasers also offer good amplitude and frequency stability which are important in the design of a compact and robust gas sensor. Several new interesting diode lasers operating in the 2.7 to $3.9 \mu \mathrm{m}$ wavelength region have been developed $[11+13]$ but are not yet commercially available. These lasers may still require cooling with liquid nitrogen for normal operation which is sometimes a 
practical drawback. Therefore, CW Difference-Frequency Generation (DFG) pumped by visible and near-infrared diode lasers at room temperature remains an attractive technique for generation of tunable mid-infrared light [14].

In this paper, the applicability of $\mathrm{CW}$ diode-pumped DFG to the detection of methane in air is investigated. The detection limit is based upon the measured performance characteristics of the IR probe source and detector. A significant technical difficulty in applying DFG to spectroscopic detection is its low conversion efficiency when pumped directly by most single-frequency $\mathrm{CW}$ semiconductor lasers. Traveling-wave semiconductor amplifiers have been successfully applied to boost effective optical pump power [15]. In earlier work [16] we reported tunable CW mid-infrared DFG with output power in excess of $3 \mu \mathrm{W}$ pumped by relatively low-power near-infrared diode lasers. A compact ring enhancement cavity was used in order to increase effective signal power available for difference-frequency mixing.

In this work, an improved and more robust design of the build-up cavity was implemented. Direct absorption and wavelength-modulation ( $2 f$ ) spectroscopy of methane in air at $10.7 \mathrm{kPa}$ (80 torr) were performed in a $1 \mathrm{~m}$ singlepass cell with $1 \mu \mathrm{W}$ probe power. The detection sensitivity of $12 \mathrm{ppbm}(\mathrm{Hz})^{-1 / 2}$ was limited by detector noise. In an effort to improve detection sensitivity, we tested the effectiveness of a confocal $3.2 \mu \mathrm{m}$ enhancement cavity in combination with an intracavity absorption cell.

\section{Diode-pumped cavity-enhanced $3.2 \mu \mathrm{m} \mathrm{DFG}$ source}

A schematic diagram of the experimental setup is shown in Fig. 1. Two different compact lasers were used for pumping the difference-frequency IR source: a $500 \mathrm{~mW}$ diode-pumped monolithic Nd:YAG ring laser at $1064 \mathrm{~nm}$ (signal), and a $20 \mathrm{~mW}$ extended-ca vity diode laser (ECDL) at $800 \mathrm{~nm}$ (pump). In later experiments, the ECDL was replaced with a $100 \mathrm{~mW}$ solitary laser diode in order to increase the IR power and allow fast wavelength modulation. The pump and signal laser beams were combined in a polarizing beamsplitter cube after spatial mode matching. They were then focused into a $5.5 \mathrm{~mm}$ antireflectioncoated $\mathrm{AgGaS}_{2}$ crystal through the input coupler of a bow-tie enhancement cavity. The cavity was designed to build up the $1064 \mathrm{~nm}$ light because bulk absorption in the crystal at that wavelength is lower than at $800 \mathrm{~nm}$ and scanning is simpler. The input coupler was a plano-concave fused silica substrate coated for high transmittance near $800 \mathrm{~nm}$ and $3.4 \%$ input coupling at $1064 \mathrm{~nm}$. The radius of curvature of the concave side was $100 \mathrm{~mm}$. The $\mathrm{CaF}_{2}$ output coupler had the same dimensions and was coated for high reflection at $1064 \mathrm{~nm}$. The transmittance of the output coupler was measured to be $41 \%$ near $3.2 \mu \mathrm{m}$ using light from a carbon monoxide overtone laser. The long arm of the cavity was not a conventional bow-tie in that a Littrow prism reflector was used instead of a flat mirror. The function of this element was to eliminate multiple passes of the $800 \mathrm{~nm}$ light in the cavity. Use of a flat mirror resulted in a small portion of the diode laser

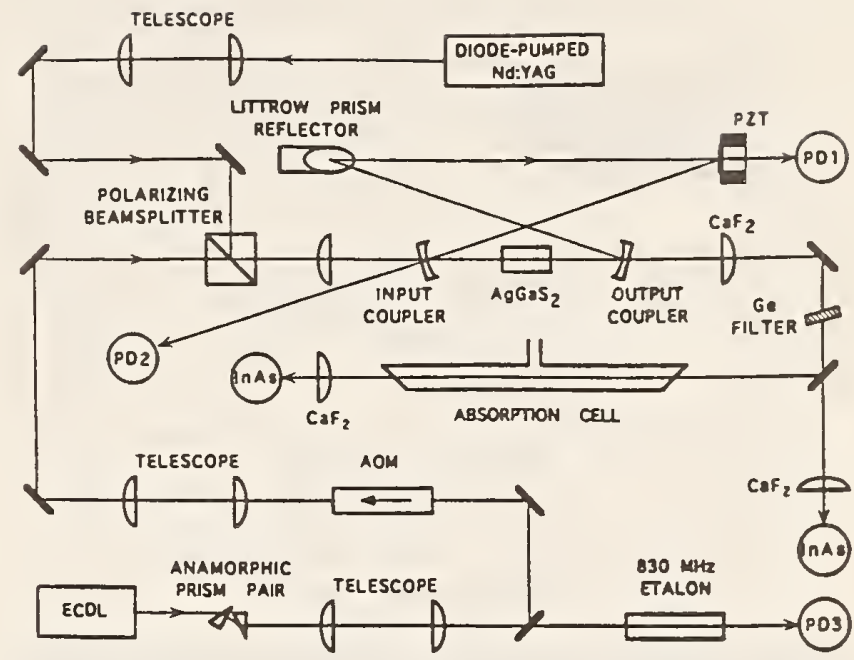

Fig. 1. Experimental setup for spectroscopic detection of methane near $3.2 \mu \mathrm{m}$ in air. The $\mathrm{AgGaS}_{2}$ crystal was placed into a bow-tie build-up cavity which was locked to resonance at $1064 \mathrm{~nm}$. The $1064 \mathrm{~nm}$ light was supplied by a diode-pumped Nd:YAG laser. The tunable $800 \mathrm{~nm}$ light was supplied by either a grating tuned extended-cavity diode laser or a solitary laser diode. The IR power was stabilized by adjusting the diode laser power with an AOM

light circulating in the cavity which produced a systematic ripple in the IR power when the diode was tuned. The bow-tie cavity design with the addition of a Littrow prism reflector provides several advantages: it employs a minimum number of intraca vity elements, is easy to align, has flexible mode diameter control, which is important for reaching optimum DFG conversion efficiency [17], and eliminates cavity resonance at the pump wavelength.

The Nd:YAG beam was spatially mode-matched to the cavity and the reflected power was monitored by a silicon photodetector PD2 (Fig. 1). Optimization of the cavity mode diameter allowed us to achieve a $90 \%$ input coupling efficiency limited mainly by the impedance mismatch of the input coupler transmittance. However, in normal operation a typical input coupling efficiency of between $75 \%$ and $85 \%$ was observed. The intracavity Nd: YAG power was monitored by a silicon photodetector PD1 which detected a $0.011 \%$ transmission through a PZT-driven flat cavity mirror (Fig. 1). The cavity buildup was 16 with the mixing crystal and 144 without. This corresponds to $6.1 \%$ and $0.7 \%$ excess cavity loss. Transmission losses in the crystal for the ordinary beam at $1064 \mathrm{~nm}$ have increased from $1.5 \%$ to $5.4 \%$ due to additional surface and bulk losses in the one-year time interval since the previous work [16]. Present measurements revealed $2.5 \%$ total reflection loss at $1064 \mathrm{~nm}$ compared to an immeasurably small reflection previously. This suggests that the index of refraction at the surface has changed. The manufacturer suggests that these changes are induced by exposure to near-UV light.

The $3.2 \mu \mathrm{m}$ output beam (idler) was collimated by a $\mathrm{CaF}_{2}$ lens. filtered by a broadband antireflection-coated germanium filter to remove pump and signal light, and focused by another $\mathrm{CaF}_{2}$ lens onto a room-temperature InAs detector. A maximum of $6 \mu \mathrm{W} I \mathrm{R}$ power was measured with $40 \mathrm{~mW}$ pump power incident on the crystal, and 


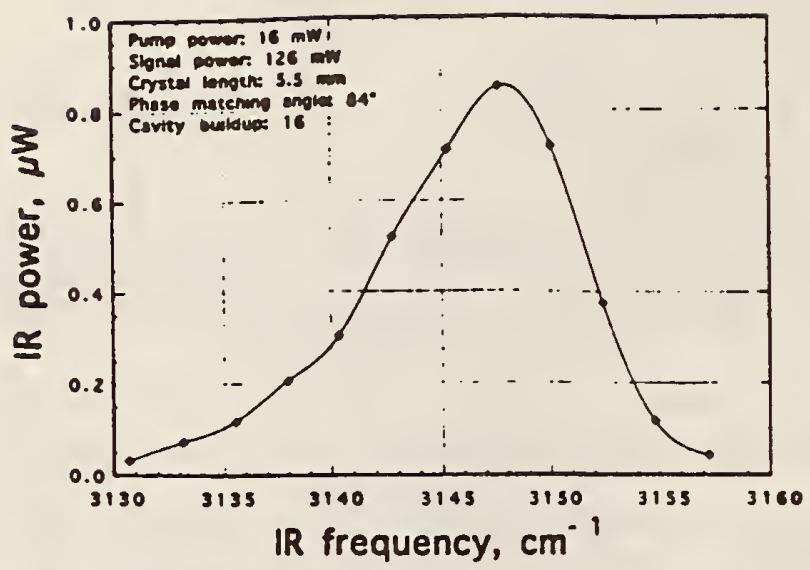

Fig. 2. Measured DFG power with pump wavelength near $800 \mathrm{~nm}$ for fixed phase matching angle. The full width at half maximum (FWHM) of the peak is $11 \mathrm{~cm}^{-1}$. The asymmetry is a result of pumping with focused Gaussian beams

$230 \mathrm{~mW}$ signal power in front of the cavity, which corresponds to $3.75 \mathrm{~W}$ intracavity signal power. Operation with more than $4.2 \mathrm{~W}$ intracavity $\mathrm{Nd}: \mathrm{YAG}$ power was achieved, accompanied by noticeable thermal lensing in the mixing crystal. Frequency tuning of the idler wave was performed by tuning of the pump laser and crystal angle. At a fixed phasematching angle, frequency tuning over a range of approximately $10 \mathrm{~cm}^{-1}$ was possible as is apparent from Fig. 2. Therefore, short frequency scans of $10 \mathrm{GHz}$ for spectroscopic measurements did not require crystal rotation. The observed asymmetry of the phasematching peak is a result of pumping with focused Gaussian beams [18]. Tuning of the pump laser from $801.3 \mathrm{~nm}$ to $795.2 \mathrm{~nm}$ and changing the internal phasematching angle of the crystal from $80^{\circ}$ to $90^{\circ}$ shifted the peak output IR power from 3076 to $3183 \mathrm{~cm}^{-1}$. Operation at infrared frequencies below $3076 \mathrm{~cm}^{-1}$ was possible with phasematching angles below $80^{\circ}$ but at the cost of more than $25 \%$ reduction in the output power due to larger beam walkoff. decreasing effective non-linear coefficient. and increasing Fresnel reflection losses at the AR-coated crystal surfaces which decreased the cavity build-up.

In the experiments described below the source was scanned by sweeping the frequency of the ECDL or solitary laser diode. In the ECDL, continuous mode-hop-free frequency tuning of $20 \mathrm{GHz}$ was performed by electronically adjusted synchronous rotation and translation of the PZT-driven tuning mirror. The Littman configuration of the ECDL allowed tuning without steering of the output beam. The solitary laser diode was tuned by current and temperature control. Frequency scans of $10 \mathrm{GHz}$ and wavelength modulation were performed by modulating the injection current. Fig. 3 shows a single-sweep $2 f$ spectrum near $3086 \mathrm{~cm}^{-1}$ of $75.3 \mathrm{ppm}$ methane in air at $13.3 \mathrm{kPa}(100 \mathrm{torr})$ in a $59 \mathrm{~cm}$ single-pass cell. The spectrum was acquired by scanning the solitary diode pump laser. A low-finesse $830 \mathrm{MHz}$ etalon was used for monitoring the scans.

The primary objective of the spectroscopic measurements was to determine a detection limit for methane in

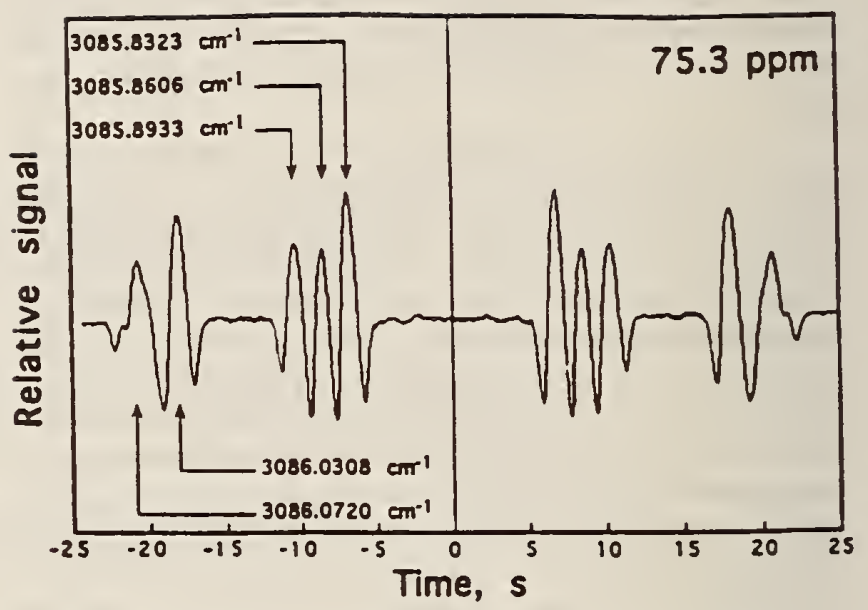

Fig. 3. Wavelength-modulation (2f) single-sweep spectrum of $75.3 \mathrm{ppm}$ methane in $13.3 \mathrm{kPa}$ (100 torr) air in a $59 \mathrm{~cm}$ cell. The spectrum was acquired by tuning of the solitary diode pump laser. Modulation frequency was $2 \mathrm{kHz}$ lock-in time constant was $0.1 \mathrm{~s}$. The vertical solid line at the center indicates the point of sweep reversal

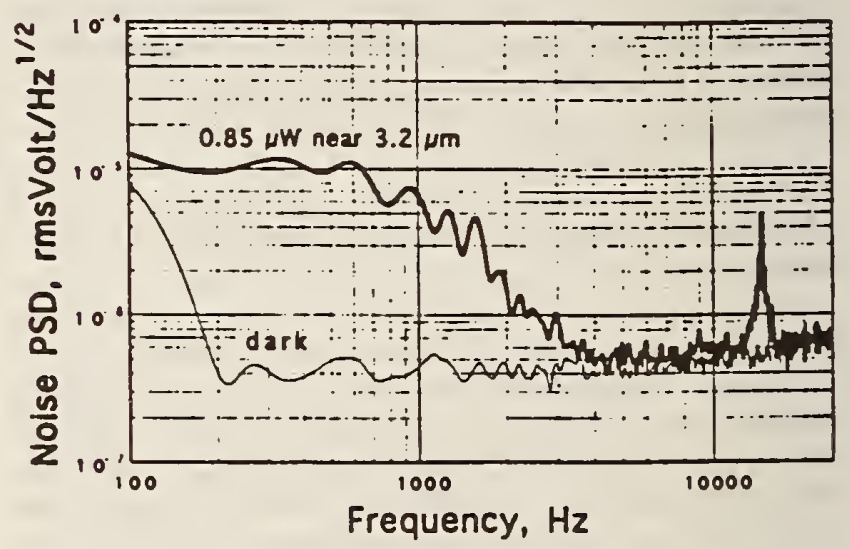

Fig. 4. The power spectral density of the notse'from the InSb photodetector/preamplifier measured with a fast Fourier transform analyzer. The upper curve was taken when the detector was exposed to $0.85 \mu \mathrm{W}$ of IR DFG radiation near $3.2 \mu \mathrm{m}$. Note that the buildup castly locking notse dominates at frequencies below $3 \mathrm{kHz}$ The detector/preamplifier responsivity at $3.2 \mu \mathrm{m}$ was $1.2 \times 10^{\mathrm{s}} \mathrm{VW}^{-1}$

air using light produced by difference-frequency generation pumped by tunable diode lasers and to identify the sources of noise. Figure 4 shows plots of power spectral density of the amplitude noise in the IR detector signal acquired with a fast Fourier transform analyzer. In our $2 f$ spectroscopic measurements, the maximum modulation frequency of the ECDL was limited to $\sim 100 \mathrm{~Hz}$ resulting in demodulation frequencies below $200 \mathrm{~Hz}$ where the cavity locking noise still dominated.

In later experıments, a servo loop was used to reduce intensity noise in the IR beam. A portion of the IR beam was focused onto a room-temperature InAs reference detector, and the feedback adjustment of the diode laser power was performed by an acousto-optic modulator. This allowed the effects of the Nd:YAG build-up cavity noise, the amplitude modulation of the diode laser result- 
ing from frequency tuning, and background fringes to be cancelled out.

In addition to single-pass absorption measurements we tested the effectiveness of a confocal enhancement cavity to improve the detection sensitivity. The cavity mirrors were plano-concave quartz substrates with $250 \mathrm{~mm}$ radius of curvature coated for high reflection at $3.4 \mu \mathrm{m}$. A transmittance of $1 \%$ was measured for each mirror using a probe beam from the DFG source. The finesse of the empty cavity was measured to be 213 which suggested a $0.5 \%$ additional loss in each mirror. For spectroscopic measurements an $18 \mathrm{~cm}$ long absorption cell with Brewster windows was placed into the cavity, reducing the cavity finesse to 182 , which corresponds to an additional loss of about $0.25 \%$ per round trip. The cavity was dither-locked to resonance at the idler wavelength by controlling a PZT-driven mirror. In order to maintain the locking when the idler frequency was tuned, the diode laser sweep and modulation signals were supplied to the PZT controller that locked the $3.2 \mu \mathrm{m}$ build-up cavity. Only $10 \%$ of the IR power from the DFG source could be coupled into the cavity because of poor spatial mode matching. Typical idler power delivered to the detector was only about $80 \mathrm{nW}$. With improved spatial mode matching of the IR beam, it should be possible to achieve $80 \%$ coupling into the cavity and deliver about $0.8 \mu \mathrm{W}$ probe power to the detector.

\section{Spectroscopic detection of methane in air}

Direct absorption and wavelength-modulation $(2 f)$ spectroscopy of both pure methane and methane in air was performed with and without IR cavity enhancement. We used three calibrated mixtures of methane with natural air; these had mixing ratios of $75.3,10.8$ and $1.8 \mathrm{ppm}$. The last was natural air sampled on a mountain ridge.

Figure 5 shows experimental wavelength-modulation (2f) spectra of methane in natural air sample at $13.3 \mathrm{kPa}$ (100 torr) in a $59 \mathrm{~cm}$ single-pass cell near $3086 \mathrm{~cm}^{-1}$. This center frequency was chosen because of the presence of six strong distinct absorption lines of methane. For example, the transition at $3085.8323 \mathrm{~cm}^{-1}$ has a line intensity of $1.70 \times 10^{-19} \mathrm{~cm}$. It is only $20 \%$ weaker than the transition at $3067.3000 \mathrm{~cm}^{-1}$ which is the strongest in the band [5]. Tuning of the source to the frequency of this strongest line was possible at the cost of reduction in IR power output. For each of the spectra, the amplitude of the frequency modulation was optimized to produce the maximum $2 f$ signal size. The spectra were taken without IR power stabilization.

Figure 6 shows direct absorption spectrum of the methane in natural air at 80 torr in a $1 \mathrm{~m}$ single-pass cell. It was acquired using a signal-averaging bandwidth of $1 \mathrm{~Hz}$, and IR power stabilization. Atmospheric pressurebroadened methane in the laboratory air between the power stabilizer beamsplitter and the sample cell is visible in the baseline trace. The baseline slope is due to interference from a secondary reflection from the power stabilizer beamsplitter. Based upon the observed signal-to-noise ratio, a detection limit (signal-to-noise ratio of 1) of $12 \mathrm{ppb} \mathrm{m}(\mathrm{Hz})^{-1 / 2}$ can be determined; it is in good agree-

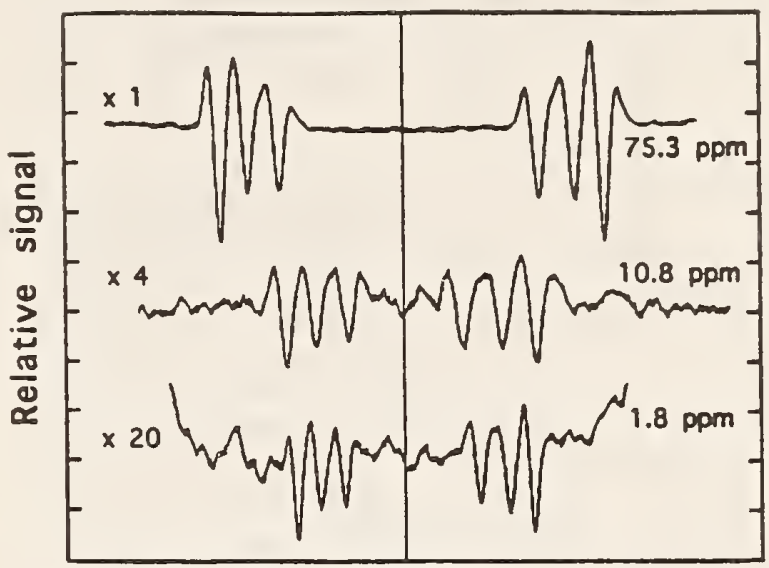

Time

Fig. 5. Wavelength-modulation (2f) spectra of methane in air at $13.3 \mathrm{kPa}$ (100 torr) in a $59 \mathrm{~cm}$ cell, obtained with $0.85 \mu \mathrm{W}$ of IR probe power near $3086 \mathrm{~cm}^{-1}$. Modulation frequency was $100 \mathrm{~Hz}$. Sweep times were $50 \mathrm{~s}, 50$ and $500 \mathrm{~s}$, and lock-in amplifier time constants were $1 \mathrm{~s}, 1 \mathrm{~s}$, and $3 \mathrm{~s}$, respectively. The build-up cavity locking noise can be seen in the spectrum of $1.8 \mathrm{ppm}$ methane. The scan widths were not the same for the 3 traces. The traces have been offset vertically for clarity

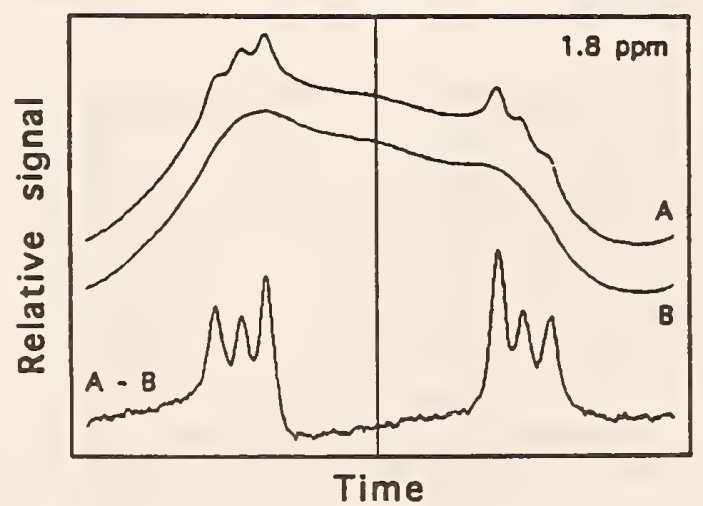

Fig. 6. Direct absorption spectrum of the methane in natural air near $3086 \mathrm{~cm}^{-1}$ at $10.7 \mathrm{kPa}$ (80 torr) in a $1 \mathrm{~m}$ single-pass cell $(A)$, and the evacuated cell $(B)$. Atmospheric pressure-broadened methane in the laboratory air between the power stabilizer beamsplitter and the sample cell is visible in the baseline trace $(B)$. The traces have been offset vertically for clarity. Both are 2000 sweep averages resulting in $1 \mathrm{~Hz}$ noise bandwidth for $(A-B)$. The detection limit of $12 \mathrm{ppb} \mathrm{m}(\mathrm{Hz})^{-1 / 2}$ can be inferred

ment with the measured InAs detector noise. This corresponds to an absorbance root-mean-square noise of $5.1 \times 10^{-5}(\mathrm{~Hz})^{-1 / 2}$. The detection limit can be improved. First, more IR probe power can be generated. For example, a commercial $1 \mathrm{~W}$ single-frequency master oscillator power amplifier (MOPA) can be used as a pump source at $800 \mathrm{~nm}$. Also, a somewhat longer mixing crystal can be used. Second, better output coupler transmittance can be obtained. We have measured $60 \%$ transmittance at $3.2 \mu \mathrm{m}$ for the replacement output coupler and $41 \%$ for that used in the experiment. Third, an IR detector with lower noise equivalent power (NEP) can be used. The room-temperature InAs detectors used in the experiment had NEPs of $17 \mathrm{pW}(\mathrm{Hz})^{-1 / 2}$ at $3.2 \mu \mathrm{m}$, which is a factor 


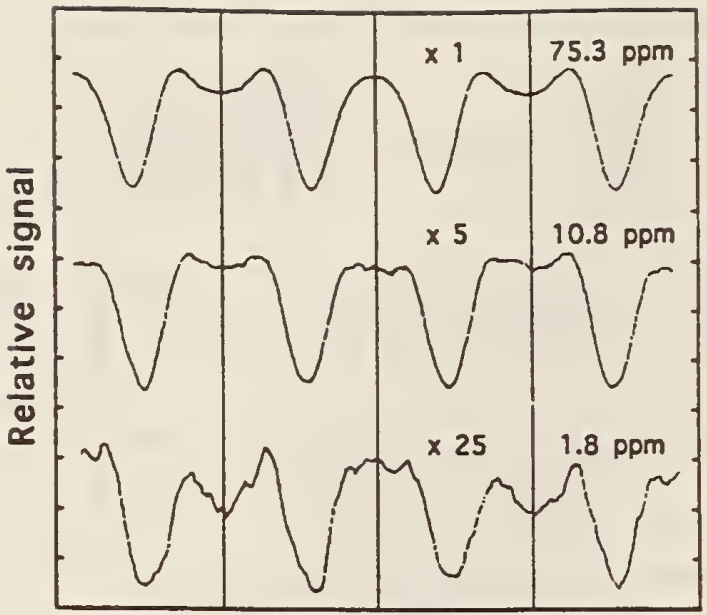

Time

Fig. 7. Wavelength-modulation (2f) spectra of methane in air at $13.3 \mathrm{kPa}$ (100 torr) in an $18 \mathrm{~cm}$ Brewster window cell inside a confocal enhancement cavity near $3.2 \mu \mathrm{m}$. The frequency of the transition is $3086.0308 \mathrm{~cm}^{-1}$. The detected IR power was $80 \mathrm{nW}$, sweep time was $10 \mathrm{~s}$, lock-in amplifier time constant was $0.3 \mathrm{~s}$. A modulation frequency of $10 \mathrm{~Hz}$ was used to maintain locking of the IR cavity. The IR frequency sweep range was approximately $3 \mathrm{GHz}$ in order to operate with reliable locking. The traces have been offset vertically for clarity. The vertical solid lines indicate points of sweep reversal

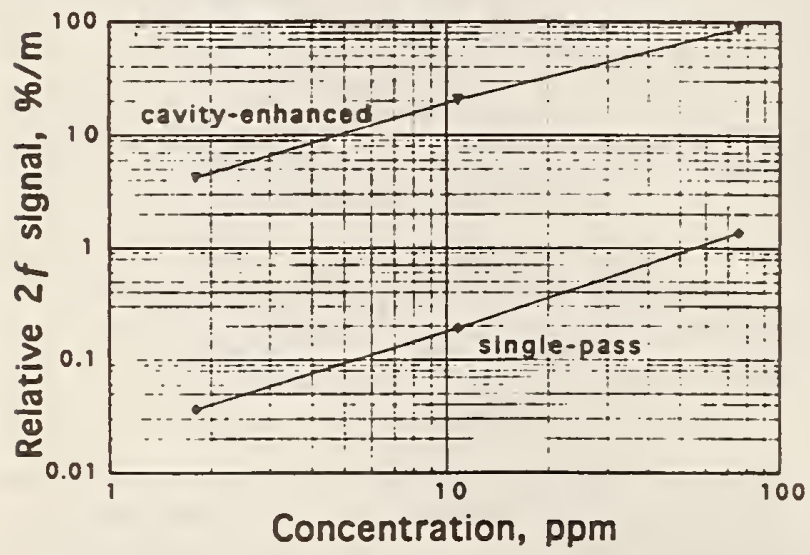

Fig. 8. Measured relative $2 f$ signal amplitude per unit cell length $P_{2 s} /\left(P_{0}, L\right)$ in the case of single-pass and cavity-enhanced detection, versus concentration of methane in air at $13.3 \mathrm{kPa}$ (100 torr). The enhancement factor (max. 116) is in good agreement with the measured cavity finesse

of 5 larger than typical NEP of cooled InSb detectors. Fourth, either a multipass absorption cell or a build-up cavity can be used to increase effective path length.

The confocal enhancement cavity near $3.2 \mu \mathrm{m}$ described above was used to increase the relative magnitude of the absorption signal. The effective number of passes in such cavity is

$N=2 \frac{F}{\pi}$

where $F$ is the cavity finesse, and the factor 2 accounts for two passes through an intracavity absorption cell per round trip. Given the previously measured value of cavity finesse $F=182$, the number of passes should be $N=116$, which is in good agreement with the number obtained by comparing the fractional $2 f$ signal per centimeter of cell length detected with cavity enhancement (Fig. 7) to that detected in the single-pass configuration (Fig. 5). The experimental enhancement factor for lower intracavity methane concentrations is in agreement with the experimental value of cavity finesse (Fig. 8). Higher concentrations of methane introduce more absorption per round trip thus decreasing the cavity finesse and the related effective number of passes which is the case with the 75.3 ppm sample.

\section{Conclusion}

In summary, spectroscopic detection of the methane in natural air (1.8 ppmv) using diode-laser pumped cavityenhanced $\mathrm{CW}$ tunable difference-frequency generation near $3.2 \mu \mathrm{m}$ has been performed by four methods: direct absorption spectroscopy, second-harmonic detection wavelength modulation spectroscopy, cavity-enhanced second-harmonic detection wavelength modulation spectroscopy, and direct absorption spectroscopy with power stabilization. The spectroscopic DFG source was pumped with an $800 \mathrm{~nm}$ diode laser and a diode-pumped $1064 \mathrm{~nm}$ Nd:YAG laser. It delivered a maximum of $6 \mu \mathrm{W}$ of narrowband infrared light with $40 \mathrm{~mW}$ pump power and $230 \mathrm{~mW}$ signal power and was tunable from 3076 to $3183 \mathrm{~cm}^{-1}$.

With no cryogenic components, we observed a noise equivalent concentration for the detection of methane in air at 80 torr of $12 \mathrm{ppb}(\mathrm{Hz})^{-1 / 2}$ using a $1 \mathrm{~m}$ cell and direct absorption spectroscopy with power stabilization. This corresponds to an absorbance root-mean-square noise of $5.1 \times 10^{-5}(\mathrm{~Hz})^{-1 / 2}$. We have observed the methane in natural air at atmospheric pressure, as can be seen from the baseline trace in Fig. 6 . The competing effects of increased methane density and pressure broadening compared to 80 torr cancel out so the expected detection limit would be the same.

By using an output coupler with higher transmittance, and using thermoelectrically cooled $\mathrm{HgCdTe}$ detectors with 5 times better noise equivalent power, we expect to be able to observe a noise equivalent column density of $1.6 \mathrm{ppbm}(\mathrm{Hz})^{-1 / 2}$. Proper spatial mode matching into the $3.2 \mu \mathrm{m}$ build-up cavity would yield an effective path length of $15 \mathrm{~m}$ and thus a predicted detection limit of $0.1 \mathrm{ppb}(\mathrm{Hz})^{-1 / 2}$. Alternatively, a White cell could also be used. The choice of build-up cavity or multipass cell would be dictated by application-dependent constraints such as physical size and sample volume. With either the build-up cavity or a multipass cell stray interference fringes or other baseline effects may limit the actual performance before the predicted $0.1 \mathrm{ppb}(\mathrm{Hz})^{-1 / 2}$ detection limit is reached.

Acknowledgements. The authors are grateful to Joe Wells for helping with measurements requiring the use of the carbon monoxide laser and Tamara Zibrova for the special coatings. The work was supported in part by the National Science Foundation, and the Robert A. Welch Foundation. 


\section{References}

1. A. Lucchesini, I. Longo, C. Gabbanini, S. Gozzini, L. Moi: Appl. Opt. 32, 5211 (1993)

2. J.C. Scott, R.A. Maddever. A.T. Paton: Appl. Opt. 31, 815 (1992)

3. K. Uehara, H. Tai: Appl. Opt. 31, 809 (1992)

4. F.S. Pavone. M. Inguscio: Appl. Phys. B 56, 118 (1993)

5. GEISA database, Laboratoire de Meteorologie Dynamique DU C.N.R.S., Ecole Polytechnique, F-91128 Palaiseau Cedex, France

6. E. Bachem, A. Dax, T. Fink, A. Weidenfeller, M. Schneider, W. Urban: Appl. Phys. B 57, 185 (1993)

7. C.B. Moore: Appl. Opt. 4, 252 (1965)

8. A.S. Pine: J. Opt. Soc. Am. 66, 97 (1976)

9. J.B. McManus, C.E. Kolb, P.L. Kebabian: Appl. Opt. 28, 5016 (1989)
10. C.R. Webster, R.D. May, C. A. Trimble, R. G. Chave, J. Kendall: Appl. Opt. 33, 454 (1994)

11. H.K. Choi. G.W. Turner, Z.L. Liau: Appl. Phys. Lett. 65, 2251 (1994)

12. A.N. Baranov, A.N. Imenkov, V.V. Sherstnev, Yu. P. Yakovlev: Appl. Phys. Lett. 64, 2480 (1994)

13. J. Faist, F. Capasso, D.L. Sivco, C. Sirtori, A.L. Hutchinson, A. Y. Cho: Science 246. 553 (April 1994)

14. U. Simon, C.E. Miller, C.C. Bradley, R.G. Hulet, R.F. Curl, F.K. Tittel: Opt. Lett. 18, 1062 (1993)

15. U. Simon, F.K. Tittel, L. Goldberg: Opt. Lett. 18, 1931 (1993)

16. U. Simon, S. Waltman, 1. Loa, L. Hollberg, F. Tittel: J. Opt. Soc. Am. B 12, 323 (1995)

17. Tran-Ba-Chu, M. Broyer: J. Phys. (Paris) 46, 523 (1985)

18. G.D. Boyd, D.A. Kleinman: J. Appl. Phys. 39, 3597 (1968) 


\title{
Precise measurement of methane in air using diode-pumped 3.4- $\mu \mathrm{m}$ difference-frequency generation in PPLN
}

\author{
K.P. Petrov ${ }^{1}$, S. Waltman ${ }^{2}$, E.J. Dlugokencky ${ }^{3}$, M. Arbore ${ }^{4}$, M.M. Fejer ${ }^{4}$, F.K. Tittel ${ }^{1}$, L.W. Hollberg ${ }^{2}$ \\ 'Rice Quantum Institute, Rice University, Houston, TX 77251-1892, USA \\ (Fax:+1-713/524-5237,E-mail: fkt@rice.edu) \\ ${ }^{2}$ National Institute of Standards and Technology, Boulder, CO 80303, USA \\ (Fax: + I-303/497-7845) \\ ${ }^{3}$ National Oceanic and Atmospheric Administration, Climate Monitoring and Diagnostics Laboratory, Boulder, CO 80303 , USA \\ (Fax: + 1-303/497-6975) \\ ${ }^{4}$ Edward L. Ginzton Laboratory, Stanford University, Stanford. CA 94305-4085. USA \\ (Fax: + I-415/723-2666)
}

Received: 2 October 1996

\begin{abstract}
Fast, accurate measurement of the methane mixing ratio in natural air samples using a compact solid-state $3.4-\mu \mathrm{m}$ difference-frequency spectrometer is reported. The spectrometer employed bulk periodically poled lithium niobate (PPLN) pumped by a solitary diode laser at $808 \mathrm{~nm}$ and a diode-pumped monolithic ring Nd:YAG laser at $1064 \mathrm{~nm}$, and a $300 \mathrm{~cm}^{3}$ volume multi-pass absorption cell with an 18 $\mathrm{m}$ path length. The methane mixing ratio was determined by comparing the direct optical absorption measured in the sample with that measured in a reference gas at 100 torr and room temperature. Relative accuracy of better than $1 \mathrm{ppb}$ (parts in $10^{9}$, by mole fraction) was achieved in measurements of natural air that contained $1700-1900 \mathrm{ppb}$ methane. The typical measurement time for each sample was 60 seconds. The accuracy was limited by residual interference fringes in the multi-pass cell that resulted from scattering. Without the use of reference samples, the relative accuracy was $20 \mathrm{ppb}$; it was limited by the long-term reproducibility of the spectroscopic baseline, which was affected by drift in the optical alignment coupled to changes in the ambient temperature. This work demonstrates the use of diode-pumped difference-frequency generation (DFG) in PPLN in a high-precision infrared spectrometer. Compact, room-temperature solid-state gas sensors can be built based on this technology, for accurate real-time measurements of trace gases in the $3-5 \mu \mathrm{m}$ spectroscopic region.
\end{abstract}

PACS: $07.65 ; 33.00 ; 42.60 ; 42.65 ; 42.80$

Precise measurements of the global distribution of trace greenhouse gases such as $\mathrm{CH}_{4}, \mathrm{CO}_{2}$, and $\mathrm{N}_{2} \mathrm{O}$ provide some of the best-known constraints on their global budgets. i.e. sources to, and removal from, the atmosphere. For example, the NOAA Climate Monitoring and Diagnostics Laboratory (CMDL) operates a globally distributed network of surface air sampling sites from which more than 7000 air samples are analyzed for $\mathrm{CH}_{4}$ each year [1]. The current measurement technique, gas chromatography (GC), is robust and precise (with a relative precision of $\sim 0.1 \%$ ), but it is slow, requiring approximately 15 minutes for each measurement. Expansion of the sampling network at the surface into the vertical, a necessary step to better constrain the global $\mathrm{CH}_{4}$ budget [2], would overwhelm the current analysis capacity.

Infrared laser spectroscopy is a uniquely effective method for the measurement of trace gas concentrations because it combines high precision, remote sensing capabilities, and fast response. These features can benefit applications in which many gas samples are analyzed or time-dependent changes in gas concentration are monitored. Several instruments have been developed based on lead-salt diode lasers that offer detection sensitivities down to $0.05 \mathrm{ppb}$ (parts in $10^{9}$, by mole fraction) for several trace species in air at atmospheric or reduced pressure [3-5]. However, lead-salt diode lasers require cooling to liquid nitrogen temperatures, have problems with mode jumps and multi-mode operation, and often require a large monochromator for mode selection, which in many applications are considerable disadvantages. The need for cryogenic cooling can potentially be eliminated in infrared spectrometers based on InAsSb [6] and InGaAsSb [7] semiconductor lasers, which have recently seen considerable development. They hold the promise of potential single-frequency operation with output powers in excess of $1 \mathrm{~mW}$ at temperatures that can be reached with Peltier coolers. However, single-frequency, single-spatial-mode lasers are not currently available. An attractive alternative to mid-infrared diode lasers is difference-frequency generation (DFG). Difference-frequency mixing of $\mathrm{Ar}^{+}$and dye [8], also dye and $\mathrm{Ti}_{2} \mathrm{Al}_{2} \mathrm{O}_{3}$ [9] lasers was effectively used for high-resolution infrared spectroscopy of stable molecules and short-lived free radicals. Several feasibility tests have recently indicated that DFG-based spectrometers pumped by commercial near-infrared diode lasers can cover most of the spectroscopic fingerprint region from 3 to $18 \mu \mathrm{m}$. In particular, typical linewidths of less than $50 \mathrm{MHz}$ and output powers from 0.5 to $30 \mu \mathrm{W}$ have been reported for diode-pumped DFG sources operating near $3 \mu \mathrm{m} \mathrm{[10],} 4 \mu \mathrm{m}$ [11] $.5 \mu \mathrm{m}$ [12], and $9 \mu \mathrm{m}[13]$. In these experiments, spectroscopic measurements of methane and carbon monoxide in natural air have indicated that detector-limited sensitivity can be achieved, corresponding to minimum detectable column densities of less than $10 \mathrm{ppbm}(\mathrm{Hz})^{-1 / 2}$. Waveguide DFG sources hold promise for 
higher output powers, but no practical spectroscopic system has yet been reported.

The purpose of this work was to design and test an all-solidstate room-temperature IR spectrometer for fast measurement of atmospheric methane with a precision of $<1 \mathrm{ppb}$, and to investigate the long-term stability of its calibration. We report the operation of such a compact spectrometer, based on quasi-phase-matched DFG at $3.4 \mu \mathrm{m}$ in bulk periodically poled lithium niobate (PPLN) pumped by a solitary diode laser and a diode-pumped Nd:YAG laser. The spectrometer was used for measurements of the methane mixing ratio in natural air samples with $<1 \mathrm{ppb}$ relative accuracy with signal averaging time of 60 seconds. This is the first reported application of PPLN in a high-precision infrared spectrometer.

\section{Experimental setup}

Figure 1 shows a schematic diagram of the DFG spectrometer used for $\mathrm{CH}_{4}$ measurement from natural air samples. It employed two compact laser sources: a $100 \mathrm{~mW}$ solitary Fabry-Perot diode laser at $808 \mathrm{~nm}$ (pump) and a $500 \mathrm{~mW}$ diode-pumped monolithic ring Nd:YAG laser at $1064 \mathrm{~nm}$ (signal). The single-frequency outputs of these two lasers were mode-matched, combined by a dichroic beamsplitter, and focused into an uncoated PPLN crystal. The $10 \mathrm{~mm}$ by- $10 \mathrm{~mm}$ crystal was cut from a 0.5 -mm-thick wafer of lithium niobate with a $21 \mu \mathrm{m}$ periodic domain grating fabricated by electric field poling [14]. The input facets were cut $13^{\circ}$ relative to the grating lines, making it possible to use effective grating periods between $21 \mu \mathrm{m}$ and $24 \mu \mathrm{m}$ with external incidence angles below $35^{\circ}$. This configuration allows room-temperature generation of $3-5 \mu \mathrm{m}$ radiation by mixing near-infrared diode lasers with a $\mathrm{Nd}$ :YAG laser at
$1064 \mathrm{~nm}$ while using the largest nonlinear coefficient in lithium niobate, $d_{33}=27 \mathrm{pm} \mathrm{V}^{-1}$, for mixing three extraordinarily polarized waves. Quasi-phase-matched generation of $3.35 \mu \mathrm{m}$ radiation (idler) was achieved by using an effective grating period of $22.2 \mu \mathrm{m}$, with an external incidence angle of $13.2^{\circ}$.

The idler was collimated by an uncoated $\mathrm{CaF}_{2}$ lens. With $70 \mathrm{~mW}$ of diode power at $808 \mathrm{~nm}$ and $500 \mathrm{~mW} \mathrm{Nd:YAG}$ power at $1064 \mathrm{~nm}$ incident on the uncoated mixing crystal, a maximum of $3.4 \mu \mathrm{W}$ idler power was measured. Because the PPLN crystal was oriented such that the incident beams propagated at an angle with respect to the grating $k$-vector, a walkoff between the idler beam and the incident beams limited the interaction length to approximately $7 \mathrm{~mm}$. This phase-velocity walkoff [15] effect, analogous to Poynting vector walkoff in birefringent materials, could be avoided by using a $22.2-\mu \mathrm{m}$ grating period at incidence close to normal.

Approximately $40 \%$ of the IR output was deflected by an uncoated $\mathrm{ZnSe}$ wedge and measured at a liquid-nitrogencooled InSb detector. The beam transmitted through the wedge was focused into a multi-pass absorption cell which provided an $18 \mathrm{~m}$ optical path length with 90 passes through the sample volume of $300 \mathrm{~cm}^{3}$. The cell had a tilted $\mathrm{BaF}_{2}$ window and a measured optical throughput of $41 \%$ which corresponds to a mirror reflectance of $99.1 \%$. The cell was connected to a rotary pump and a manifold that allowed switching between two high-pressure aluminum cylinders containing natural air that had been previously calibrated for methane at CMDL. The pressure in the cell was maintained near 100 torr, as measured by a capacitive manometer with 0.01 torr resolution and 0.2 torr accuracy. The cell temperature was measured using a mercury thermometer with $0.1{ }^{\circ} \mathrm{C}$ resolution. After the cell, the DFG beam was collected by an off-axis parabol-

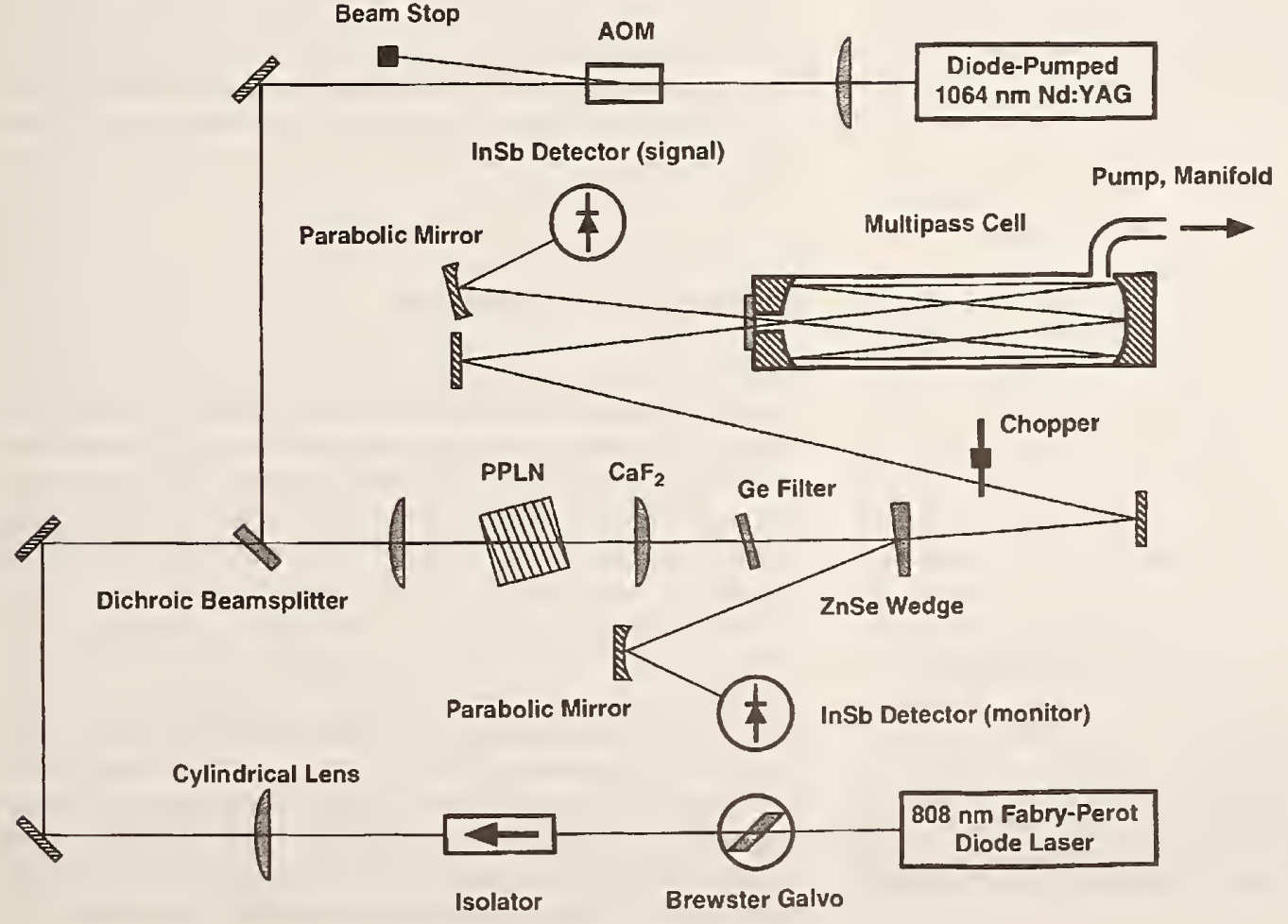

Fig. 1. Schematic diagram of a compact $3.4 \mu \mathrm{m}$ spectrometer based on bulk PPLN pumped by two solid-state lasers. The spectrometer was used to measure the methane mixing ratio in natural air at 100 torr and room temperature relative to a reference gas to better than $1 \mathrm{ppb}$ in 60 seconds 
ic mirror and focused onto a second liquid-nitrogen-cooled InSb detector with a $1 \times 1 \mathrm{~mm}^{2}$ active area. The detector had a noise-equivalent power (NEP) of $1.7 \mathrm{pW}(\mathrm{Hz})^{-1 / 2}$ at $3.4 \mu \mathrm{m}$.

\section{Reduction of interference and noise}

In addition to the basic elements described above, several other components were required to improve the output stability and tuning characteristics of the DFG spectrometer. Initial measurements showed that the linearity of the frequency sweep of the pump laser was degraded by weak optical feedback from scattering in the optical isolator. This resulted in distortion of the observed spectral lineshapes and an unstable baseline profile. To reduce the unwanted distortion, a galvodriven Brewster plate [16] was placed in the diode laser beam

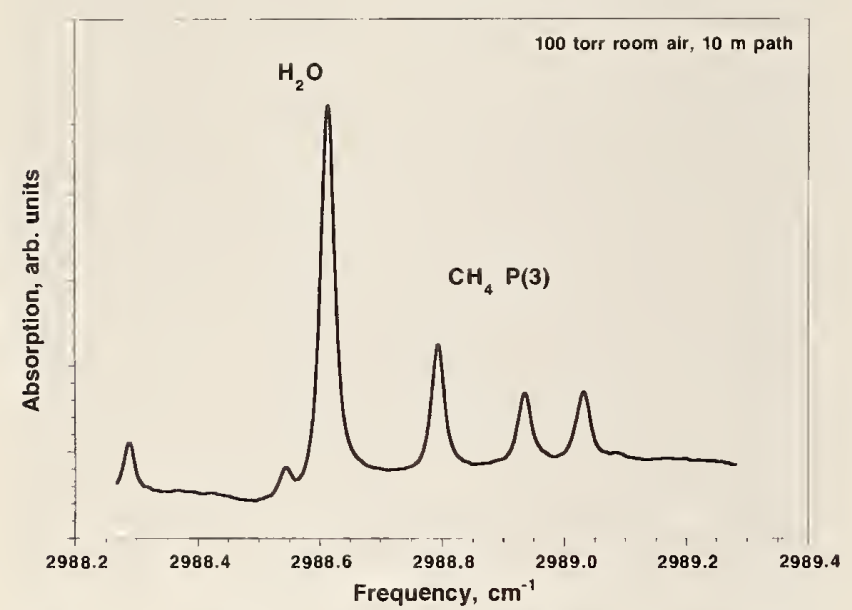

Fig. 2. Survey absorption spectrum of room air near $3.35 \mu \mathrm{m}$ at 100 torr in a 10-m multi-pass cell. The observed transitions belong to water $\left(2988.6 \mathrm{~cm}^{-1}\right.$ and lower) and the $\mathrm{P}(3)$ group of methane $\left(2988.8 \mathrm{~cm}^{-1}\right.$ and higher). The trace shown is a 1024-sweep average with baseline subtracted. The sweep rate was $100 \mathrm{~Hz}$

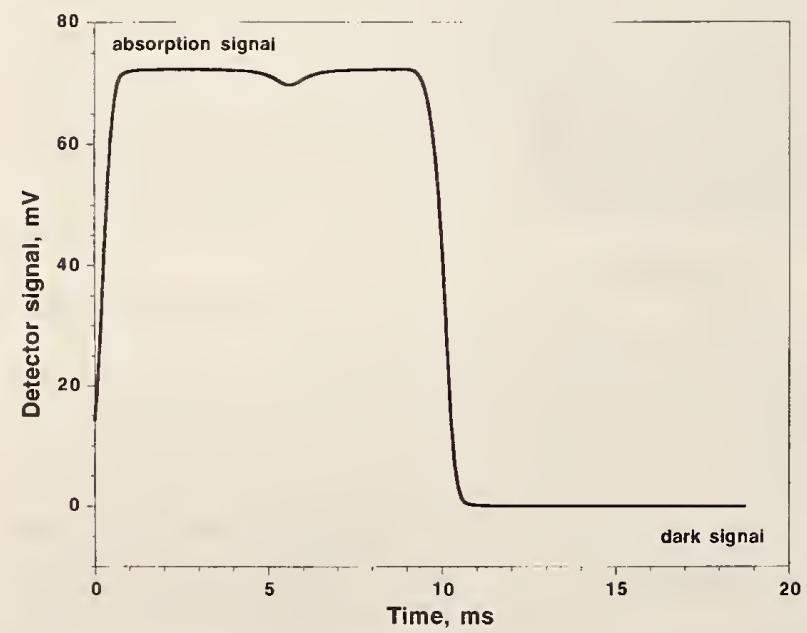

Fig. 3. A single period of a typical waveform seen by the signal InSb detector. A direct-absorption spectrum of an isolated methane line was recorded during the first $10 \mathrm{~ms}$. Over the next $10 \mathrm{~ms}$, the beam was blocked by the chopper and the dark voltage was recorded. The average of the dark voltage was subtracted from the entire trace before the isolator. The Brewster plate was rocked at $5 \mathrm{~Hz}$ to scramble the phase of the optical feedback. This did not remove the feedback, but it did ensure a reproducible baseline for absorption spectra which were averaged over many scans.

We observed alignment-dependent optical interference fringes in the multi-pass absorption cell. They were caused by scattering from beam spots in close proximity to the output window at the center of the front mirror. The smallest magnitude of these fringes was approximately $5 \times 10^{-4}$ absorption units. To cancel the fringes, a voice coil was attached to the cell. The coil was driven by a sine-wave generator tuned to an acoustic resonance in the cell near $800 \mathrm{~Hz}$. Vibration of the cell mirrors resulted in an excursion of fringes that was larger than their period. This reduced the magnitude of the interference in the averaged absorption signal by more than a factor of 10 . No evidence of drift in the alignment of the cell mirrors was found during 5 weeks of experiment.

The commercial InSb detector that sampled the DFG power before the multi-pass absorption cell was modified to have a $77 \mathrm{~K}$ quartz filter and an aperture. The dc-coupled detector output was subtracted from a precision voltage reference, and the filtered error signal was used to control an acousto-optic modulator placed in the Nd:YAG beam. This servo removed amplitude modulation associated with frequency scans, beam offset caused by motion of the Brewster plate, acoustic vibration, and alignment drift. However, since the servo-stabilized output of the monitor detector includes dark voltage, the output DFG power still changed as the dark voltage changed with temperature. Temperature stabilization of the entire system, including the background seen by the monitor detector, could improve the power stability. After the wedge beamsplitter, the IR beam path to the first detector was nearly equal to the beam path outside the multi-pass cell to the second detector, making it possible to cancel out absorption by methane in ambient air. Considering that temporal fluctuations of the methane concentration in room air can easily exceed $10 \%$, improper cancellation of stray absorption would result in signal errors that are unacceptable in this precision application. Nitrogen-purging of the spectrometer would further reduce stray absorption caused by spatial fluctuations of methane concentration.

\section{Control and measurement}

The temperature of the Fabry-Perot diode laser was stabilized for operation with a center wavelength of $808 \mathrm{~nm}$. Fine wavelength tuning and scans were performed by modulation of the laser drive current. The maximum available fine-tuning range of $35 \mathrm{GHz}$ without mode-hops was sufficient for simultaneous observation of several strong molecular transitions of methane and water (Fig. 2). For absorption measurements, a tuning range of $7 \mathrm{GHz}$ was adequate to capture a single methane peak. The DFG signal detected after the multi-pass absorption cell was low-pass filtered below $10 \mathrm{kHz}$ and digitized by a 16-bit analog-to-digital converter. The frequency sweep was triggered by a $50 \mathrm{~Hz}$ chopper, such that the IR beam was blocked during retrace of the frequency sweep. This arrangement permitted sampling of the signal detector's dark voltage and the absorption trace during each sweep cycle, as seen in Fig. 3. The $50 \mathrm{~Hz}$ sweep rate was chosen to combine 


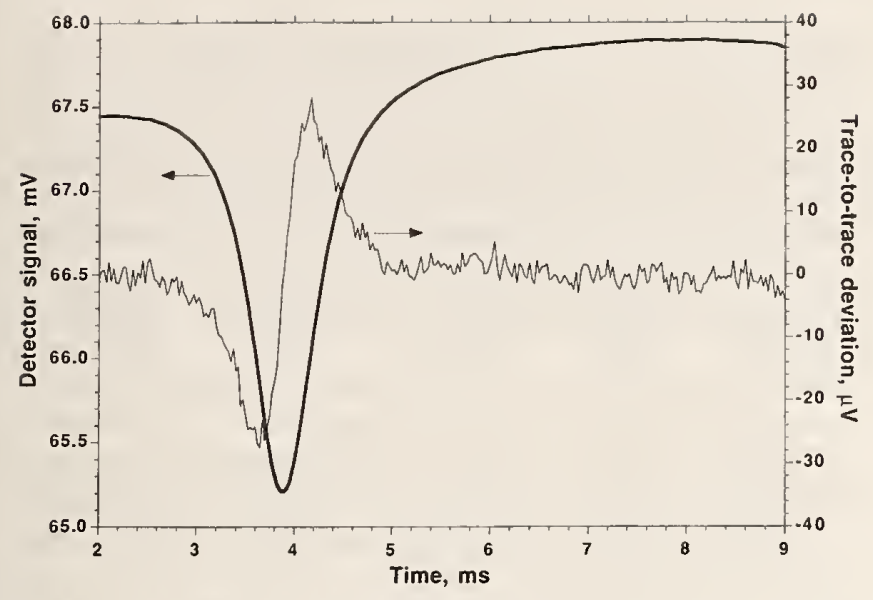

Fig. 4. A close-up of an absorption peak (thick solid line) similar to that shown in Fig. 3. The thin solid trace in the middle is the difference between absorption traces acquired 5 minutes apart. Detector noise can be seen in the flat part of the trace. The anti-symmetric feature is a result of frequency drift

minimal distortion of the signal by the $10 \mathrm{kHz}$ low-pass filter and reasonably short measurement time.

Each measurement consisted of a 3000 sweep average acquired over 60 seconds. An example of such measurement - a trace of detector voltage versus time-is shown in Fig. 4. The thick solid trace was acquired with the use of cancellation of interference fringes in the multi-pass cell. The thin solid line is the difference between two such traces acquired 5 minutes apart. The preamplifier had a single-pole low-pass filter with a $3 \mathrm{~dB}$ corner frequency of $10 \mathrm{kHz}$. The equivelant noise bandwidth of the average of 3000 sweeps was $5.2 \mathrm{~Hz}$. Infrared detector noise measured in this bandwidth corresponds to $8 \times 10^{-6} \mathrm{rms}$ absorption which can be easily observed in the thin trace because of the large dynamic range $(16+11$ bits $)$ available in the averaged signal. The peak amplitude was extracted from the absorption trace by using nonlinear least-squares fitting. Prior to fitting, the retrace average of the dark voltage was subtracted from the entire trace. The parameters of the fitting included the peak position, pressure- and Doppler-broadened linewidths $\Delta v_{\mathrm{p}}$ and $\Delta v_{\mathrm{T}}$, and a linear fit to the baseline. Voigt line shape was assumed, and a rational approximation [17] was used to compute the normalized absorption profile. Absorption $\alpha$ was determined by normalizing the peak amplitude to the value of the fitted baseline taken at the center of the peak. Values of the absorption for the various calibrated air samples used in the experiment ranged between $3 \%$ and $4 \%$. Corrected for total pressure and temperature, $T$, of each sample, the absorption was assumed to be proportional to the methane mole fraction in that sample [here $V\left(0 ; \Delta v_{\mathrm{p}} / \Delta v_{\mathrm{T}}\right)$ is the peak value of the normalized Voigt function]:

$C \propto-T \ln (1-\alpha) V\left(0 ; \frac{\Delta v_{\mathrm{p}}}{\Delta v_{\mathrm{T}}}\right)$.

Such correction was necessary because the cell temperature was observed to change by more than $1{ }^{\circ} \mathrm{C}$ during the experiment, and the fill-to-fill variations in sample pressure were as large as 0.5 torr. The constant of proportionality is omitted in the above formula, since it depends on quantities such as the line intensity and optical path length - each known to $\pm 2 \%$, at best. The sample pressure and pressure-broadening coefficient, both known to better than $\pm 0.2 \%$, are included in the above formula in the form of $\Delta v_{p}$, and their combined effect on the value of the normalized Voigt function is less than $0.04 \%$. Initially, an attempt was made to determine the constant of proportionality in the above formula. With the use of a single air sample with known methane mixing ratio this should be possible, and the result could be used to measure $\mathrm{CH}_{4}$ from other samples. We have found, however, that the day-to-day reproducibility of the spectroscopic baseline, which depended on alignment and was strongly coupled to changes in ambient temperature, limited the accuracy of such measurement to about $1 \%$. This demonstrated that measurements with $1 \%$ absolute accuracy would be possible, and in many cases such accuracy is more than adequate. However, our specific application required precision of better than $0.1 \%$. We have therefore resorted to using a reference sample before (or after) each unknown sample. This is a somewhat less attractive technique because it requires an additional gas transfer and roughly doubles the measurement time. In some applications, however, this is acceptable and could be used to verify whether the instrument is working properly. It is important to point out that this technique does not solve the problem of baseline drift; it only makes the drift insignificant on the time scale of an individual measurement.

\section{Performance testing and results}

The performance of the DFG spectrometer was tested by using four high-pressure aluminum cylinders filled with dry air, as supplied by NOAA CMDL. All four cylinders had been calibrated for methane at CMDL via gas chromatography. The precision of the measurements was typically better than $1 \mathrm{ppb}$, and methane mole fractions were assigned relative to the CMDL methane scale [1]. The testing was performed in two parts. In the first part, two cylinders (identification numbers 30516 and 37057 ) were supplied with their methane mole fractions. The second part was blind; the two authors performing the spectroscopic measurements (KPP and SW) were not told the methane mole fractions in the two other cylinders (64040 and 30482).

The methane mole fractions assigned to the cylinders used in the first test were $1775.3 \pm 0.1 \mathrm{ppb}$ for cylinder 30516 and $1896.7 \pm 1.3 \mathrm{ppb}$ for cylinder 37057 . Air samples from each cylinder were analyzed continuously for 5 minutes, with a 15 minute pause between the samples. The pause was made to completely evacuate the multi-pass cell and to let the newly injected sample reach thermal equilibrium with the cell. These measurements were performed without cancellation of fringes in the cell. Figure 5 shows the measured absorption by methane as a function of time. The sample temperature of $25.0 \pm 0.1^{\circ} \mathrm{C}$ though not actively stabilized, was not observed to change in this test. When corrected for differences in the sample pressure as explained above, the ratio of methane mole fractions is found to be $1.0681 \pm 0.0015$, versus $1.0684 \pm 0.0007$ as calculated from the gas chromatograph data. As suspected, the primary source of large deviations among individual measurements is interference fringes in the multi-pass absorption cell. Although weak, they still produced baseline features clearly distinguishable from detector noise, and they affected the precision 
with which the fitting parameters were determined. Since no changes in the ambient temperature were observed during this test, the measurements were not affected by drift in optical alignment.

In the subsequent blind tests, cylinders 30482 and 64040 were analyzed. Cancellation of interference fringes in the multi-pass cell was used this time, as the need for it had been clearly realized. Measurements of optical absorption in cylinder 30482 relative to cylinder 30516 (reference) gave a methane mixing ratio of $1781.1 \pm 0.8 \mathrm{ppb}$ after correction for pressure and temperature (Fig. 6). The $\mathrm{CH}_{4}$ mixing ratio assigned to that cylinder by $\mathrm{CMDL}$ was $1781.9 \pm 0.9 \mathrm{ppb}$. A summary of data obtained for all samples analyzed in the experiment is given in Table 1. Since

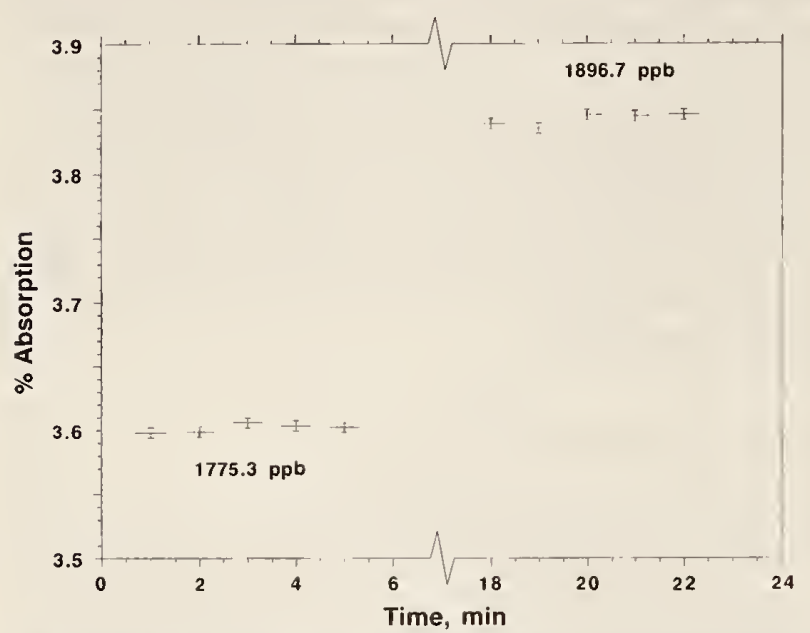

Fig. 5. Peak absorption by $\mathrm{CH}_{4}$ versus time, measured for two air samples previously calibrated for methane (indicated on the plot). The measured ratio of mole fractions is $1.0681 \pm 0.0015$, after correction for differences in pressure. This can be compared with the ratio of $1.0684 \pm 0.0007$, determined from the $\mathrm{CH}_{4}$ mole fractions assigned to the samples. Cancellation of interference was not used in this data, hence the large error bar

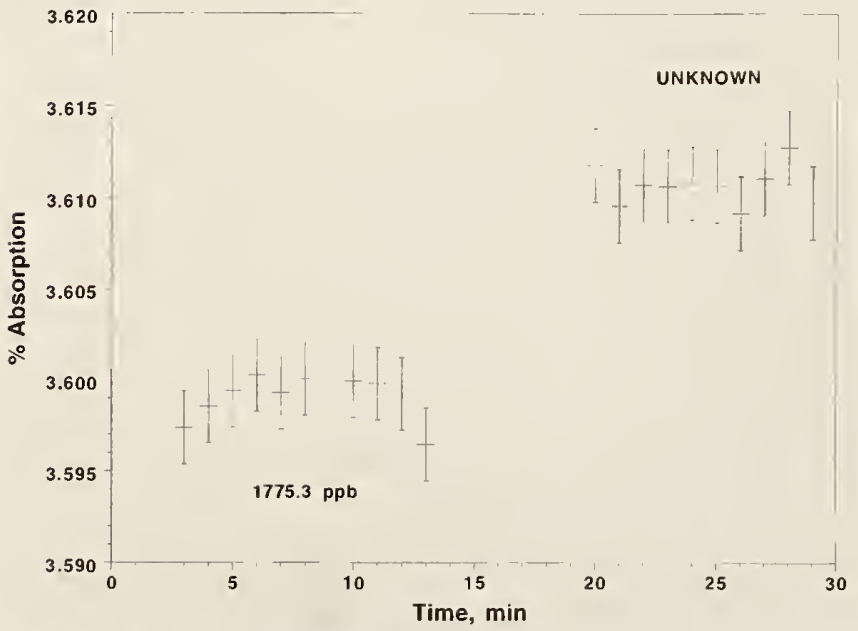

Fig. 6. Peak absorption by $\mathrm{CH}_{4}$ versus time for air from two high-pressure cylinders. The 10 measurements plotted on the lower left from cylinder 30516 (with a $\mathrm{CH}_{4}$ mole fraction of $1775.3 \mathrm{ppb}$ assigned by CMDL) were used as a reference. The 10 measurements in the upper right were for cylinder 30482 which was analyzed as an unknown. The spectroscopic result for the unknown was $1781.1 \pm 0.8 \mathrm{ppb}$; this compares well with the value of $1781.9 \pm 0.9 \mathrm{ppb}$ assigned by CMDL
Table 1. Summary of methane mixing ratios measured using the $3.4 \mu \mathrm{m}$ DFG spectrometer. Four samples were available in the experiment, and one of them (30516) was used as a reference in all measurements. The methane mixing ratio in this sample was assumed to be known exactly. Standard deviation shown for the DFG data therefore only includes errors of measurement of absorption, temperature, and pressure. Sample 37057 was measured without cancellation of interference in the multi-pass cell, hence the large error bar

\begin{tabular}{ccccc}
\hline $\begin{array}{c}\text { cylinder } \\
\text { identification } \\
\text { number }\end{array}$ & $\begin{array}{c}\mathrm{CDMLCH}_{4} \\
\text { assignment } \\
(\mathrm{ppb})\end{array}$ & $\begin{array}{c}\text { standard } \\
\text { deviation } \\
(\mathrm{ppb})\end{array}$ & $\begin{array}{c}3.4 \mu \mathrm{m} \text { DFG } \\
\text { measurement } \\
(\mathrm{ppb})\end{array}$ & $\begin{array}{c}\text { standard } \\
\text { deviation } \\
(\mathrm{ppb})\end{array}$ \\
\hline 64040 & 1753.8 & 0.2 & 1754.3 & 0.8 \\
30516 & 1775.3 & 0.1 & used as areference & $\mathrm{n} / \mathrm{a}$ \\
30482 & 1781.9 & 0.9 & 1781.1 & 0.8 \\
37057 & 1896.7 & 1.3 & 1896.2 & 3.0 \\
\hline
\end{tabular}

cylinder 30516 was used as a reference in all measurements, its methane mixing ratio of $1775.3 \mathrm{ppb}$ was assumed to be known exactly. Therefore the uncertainty in the mixing ratio determined by CMDL is not included in the errors given for spectroscopic measurements. The errors quoted in Table 1 represent instead the combined uncertainties in the measured optical absorption, temperature, and pressure.

The data suggest that the combined uncertainty of the spectroscopic measurements over 1 minute is less than $1 \mathrm{ppb}$ for a typical ambient methane mixing ratio of $1700-1900 \mathrm{ppb}$. This is equivalent to about $0.06 \% \mathrm{rms}$ relative accuracy. Taking into account the conditions of our experiment ( $18 \mathrm{~m}$ path length, $5.2 \mathrm{~Hz}$ bandwidth) we estimate the noise-equivalent methane column density of $6.3 \mathrm{ppbm}(\mathrm{Hz})^{-1 / 2}$.

\section{Summary}

We have demonstrated a precise spectroscopic application of diode-pumped difference-frequency generation. A compact 3.4- $\mu \mathrm{m}$ spectrometer employed a 1-cm-long PPLN crystal with a $21-\mu \mathrm{m}$ domain grating period, pumped by two compact solid-state lasers: a $100-\mathrm{mW}$ solitary laser diode at $808 \mathrm{~nm}$ (pump), and a diode-pumped monolithic ring Nd:YAG laser at $1064.5 \mathrm{~nm}$ (signal). With a maximum total output power of $3.4 \mu \mathrm{W}$ at $3.4 \mu \mathrm{m}$, the DFG source of the spectrometer performed continuous frequency scans of more than $35 \mathrm{GHz}$, and could be tuned over more than $60 \mathrm{~cm}^{-1}$ by scanning the temperature the pump laser. The use of a galvo-driven Brewster plate allowed compensation of feedback distortion of the frequency sweep. Sampling and servo control of the output power allowed cancellation of the amplitude modulation and noise due to frequency sweep, acoustic noise, and alignment drift. The spectrometer employed a compact $18 \mathrm{~m}$ multi-pass cell which had an optical throughput of $41 \%$.

The measurements were made relative to the CMDL standard methane scale. With a measurement time of 60 seconds, direct-absorption spectroscopy of methane in natural air samples was performed, and the methane mixing ratios were determined with a precision of better than $1 \mathrm{ppb}$. The precision was limited by residual interference fringes in the multi-pass cell. The peak-to-peak magnitude of the fringes corresponds to a signal-to-noise ratio of 510 for the meas- 
ured optical absorption of $\sim 3.5 \%$. Without a reference gas, the relative accuracy of $20 \mathrm{ppb}$ was limited by the long-term stability and reproducibility of the spectroscopic baseline. The baseline profile depended on the optical alignment, which drifted following changes in the ambient temperature.

This precision spectrometer can benefit applications where many similar gas samples are analyzed. In tracegas-monitoring applications that require fast measurements with an accuracy of down to $1 \%$, such a spectrometer can employ room-temperature InAs infrared detectors and operate without reference samples. Diode-pumped DFG in PPLN can effectively replace lead-salt diode lasers and gas lasers in spectroscopic applications requiring low-noise, single-frequency sources that are continuously tunable in the $3-5 \mu \mathrm{m}$ region, and eliminate the need for cryogenic cooling.

Acknowledgements. The authors are grateful to Charlie Hultgren of New Focus, Inc. and Richard Fox at NIST Boulder for helpful discussions. The work was supported in part by the NOAA Climate Monitoring and Diagnostics Laboratory, the National Aeronautics and Space Administration, the Advanced Research Projects Agency through the Center for Nonlinear Optical Materials at Stanford University, the Joint Services Electronics Program, the National Science Foundation, the Texas Advanced Technology Program, and the Robert A. Welch Foundation.

\section{References}

1. E.J. Dlugokencky, L.P. Steele, P.M. Lang, K.A. Masarie: J. Geophys. Res. 99, 17021 (1994)

2. 1. Fung, J. John, J. Lerner, E. Matthews, M. Prather, L.P. Steele, P.J. Fraser: J. Geophys. Res. 96, 13033 (1991)

3. J. Reid, M. El-Sherbiny, B.K. Garside, E.A. Balik: Appl. Opt. 19, 3349 (1980)

4. C.R. Webster, R.D. May, C.A. Trimble, R.G. Chave, J. Kendall: App1. Opt. 33, 454 (1994)

5. M.S. Zahniser, D.D. Nelson, J.B. McManus, P.L. Kebabian: Phil. Trans. R. Soc. Lond. A 351, 371 (1995)

6. A. Popov, B. Scheumann, R. Mücke, A. Baranov, V. Sherstnev Y. Yakovlev, P. Werle: Infrared Physics and Technology 37, 117 (1996)

7. D.Z. Garbuzov, R.U. Martinelli, R.J. Menna, P.K. York, H. Lee S.Y. Narayan, J.C. Connolly: Appl. Phys. Lett. 67, 1346 (1995)

8. A.S. Pine: J. Opt. Soc. Am. 66, 97 (1976)

9. C.E. Miller, W.C. Eckhoff, R.F. Curl: J. Mol. Struct. 352, 435 (1995)

10. K.P. Petrov, S. Waltman, U. Simon, R.F. Curl, F.K. Tittel, E.J. Dlugokencky, L. Hollberg: Appl. Phys. B 61, 553 (1995)

11. A. Balakrishnan, S.Sanders, S. DeMars, J. Webjörn, D.W. Nam, R.J. Lang, D.G. Mehuys, R.G. Waarts, D.F. Welch: Opt. Lett. 21, 952 (1996)

12. K.P. Petrov, L. Goldberg, W.K. Burns, R.F. Curl, F.K. Tittel: Opt. Lett. 21, 86 (1996)

13. K.P. Petrov, L. Goldberg, R.F. Curl, F.K. Tittel: Opt. Lett. 21, 1451 (1996)

14. L.E. Myers, R.C. Eckardt, M.M. Fejer, R.L. Byer, W.S. Bosenberg, J.W. Pierce: J. Opt. Soc. Am. B 12, 2102 (1995)

15. M.M. Fejer, G.A. Magel, D.H. Jundt, R.L. Byer: IEEE J. Quan. Elec. 28, 2631 (1992)

16. C.R. Webster: J. Opt. Soc. Am. B 2, 1464 (1985)

17. J. Humlicek: J. Quant. Spectrosc. Radiat. Transfer 21, 309 (1979) 


\title{
Reactive uptake of $\mathrm{NO}_{3}$ on pure water and ionic solutions
}

\author{
Yinon Rudich, Ranajit K. Talukdarl and A. R. Ravishankara ${ }^{1,2}$ \\ Aeronomy Laboratory, National Oceanic and Atmospheric Administration, Boulder, Colorado
}

\author{
R. W. Fox \\ Time and Frequency Division, National Institute of Standards and Technology, Boulder, Colorado
}

\begin{abstract}
The reactive uptake coefficients $(\gamma)$ of $\mathrm{NO}_{3}$ onto pure water and dilute solutions of $\mathrm{NaCl}, \mathrm{NaBr}$, and $\mathrm{NaNO}_{2}$ were measured using a wetted-wall flow-tube setup combined with a long-path absorption cell for the detection of $\mathrm{NO}_{3}$. The measured $\gamma$ values were in the range $1.5 \times 10^{-4}-6 \times 10^{-3}$, depending on the salt concentration in the water. By measuring $\gamma$ as a function of salt concentration, $\mathrm{HD}_{\ell}^{0.5}$ for $\mathrm{NO}_{3}$ in water was determined to be $(1.9 \pm 0.4) \times 10^{-3} \mathrm{M} \mathrm{atm}{ }^{-1} \mathrm{~cm}$ $\mathrm{s}^{-0.5}$ at $273 \mathrm{~K}$, assuming that the rate coefficient for the reaction of $\mathrm{NO}_{3}$ with $\mathrm{Cl}^{-}$is $2.76 \times 10^{6} \mathrm{M}^{-1}$ $\mathrm{s}^{-1}$ at $273 \mathrm{~K}$. The Henry's law coefficient for $\mathrm{NO}_{3}$ in water is estimated to be $0.6 \pm 0.3 \mathrm{M} \mathrm{atm}^{-1}$, assuming that the diffusion coefficient of $\mathrm{NO}_{3}$ in water is $D_{\ell}=(1.0 \pm 0.5) \times 10^{-5} \mathrm{~cm}^{2} \mathrm{~s}^{-1}$. Uptake of $\mathrm{NO}_{3}$ on pure water is interpreted as due to reaction of $\mathrm{NO}_{3(\mathrm{aq})}$ with $\mathrm{H}_{2} \mathrm{O}_{(\ell)}$ to produce $\mathrm{HNO}_{3}$ and $\mathrm{OH}$ in the liquid phase. Implications of these findings to the chemistry of $\mathrm{NO}_{3}$ in the troposphere are also discussed.
\end{abstract}

\section{Introduction}

The $\mathrm{NO}_{3}$ radical is a major tropospheric nighttime oxidant and its gas phase reactions are important loss pathways for many compounds during the night [Atkinson, 1991; Wayne et al., 1991]. $\mathrm{NO}_{3}$ can also be important in initiating radical reactions within cloud droplets [Chameides, 1986a,b; Lelieveld and Crutzen, 1991; Mozurkewich, 1986] and in aerosols [Pederson, 1995]. $\mathrm{NO}_{3}$ scavenging by wet aerosols may explain the short lifetime of $\mathrm{NO}_{3}$ that was observed in the troposphere [Heikes and Thompson, 1983; Mihelcic et al., 1993; Noxon, 1983], especially during episodes of high humidity and fog [Platt et al., 1981, 1984].

Odd nitrogen species such as $\mathrm{NO}$ and $\mathrm{NO}_{2}$ have limited solubility in liquid water [DeMore et al., 1994]. Thus the main removal pathways of $\mathrm{NO}_{\mathrm{x}}$ species from the atmosphere are believed to be via the formation of $\mathrm{HNO}_{3}$, which is removed by both dry and wet deposition. $\mathrm{HNO}_{3}$ is formed in the atmosphere either by the gas phase reaction of $\mathrm{OH}$ with $\mathrm{NO}_{2}$ or heterogeneous hydrolysis of $\mathrm{N}_{2} \mathrm{O}_{5}$. Since the $\mathrm{NO}_{x}$ family plays very important roles in various reaction cycles in the atmosphere, the removal pathways for the members of this family from the atmosphere needs to be well known. The $\mathrm{NO}_{3}$ radical, which is formed by reaction of $\mathrm{NO}_{2}$ and ozone, may also provide a removal pathway of $\mathrm{NO}_{x}$ from the atmosphere through its gas phase and heterogeneous loss processes.

The uptake of a compound into a liquid is determined by diffusion, its solubility and its liquid phase reactions [Danckwerts, 1951; Hanson et al., 1994; Schwartz, 1986]. To model the heterogeneous chemistry of $\mathrm{NO}_{3}$ accurately, properties

\footnotetext{
${ }^{1}$ Also at the Cooperative Institute for Research in Environmental Sciences, University of Colorado, Boulder.

${ }^{2}$ Also at the Department of Chemistry and Biochemistry, University of Colorado, Boulder.
}

Copyright 1996 by the American Geophysical Union.

Paper number 96JD01844.

0148-0227/96/96JD-01844\$09.00 such as Henry's law coefficient $(H)$ and the reactive uptake coefficients $(\gamma)$ must be known accurately. In previous studies, $\mathrm{H}$ for $\mathrm{NO}_{3}$ was estimated to be between $3 \times 10^{-2}$ and $2 \times 10^{5} \mathrm{M} \mathrm{atm}^{-1}$ [Chameides, 1986a,b; Jacob, 1986; Lelieveld and Crutzen, 1991; Mozurkewich, 1986]. To our knowledge, the only experimentally determined value of $\mathrm{H}$, the Henry's law coefficient for $\mathrm{NO}_{3}, \mathrm{H}=$ 1.8 $\pm 1.5 \mathrm{M} \mathrm{atm}^{-1}$ is that reported in the thesis of Thomas [1992].

To understand the nighttime processing of $\mathrm{NO}_{3}$ in the troposphere and its impact on cloud and fog chemistry, we have undertaken the studies of its uptake on pure water and on some representative ionic solutions relevant to the marine troposphere and to cloud droplets. In this paper we report measurements of the uptake coefficients of $\mathrm{NO}_{3}$ by water and by aqueous solutions of $\mathrm{NaCl}, \mathrm{NaBr}$, and $\mathrm{NaNO}_{2}$. From these measurements we also determine $\mathrm{HD}_{\ell} 0.5$ for $\mathrm{NO}_{3}$ in water and discuss the atmospheric implications of these results.

\section{Experimental and Data Analysis}

\section{Setup}

Uptake of $\mathrm{NO}_{3}$ by distilled water $(p \mathrm{H} \sim 7)$ and by $\mathrm{NaCl}(0.01$ $0.5 M), \mathrm{NaNO}_{2}(0.0005-0.0038 M)$, and $\mathrm{NaBr}(0.001-0.01 M)$ solutions was measured. The experimental setup was an adaptation of the one described by Utter et al. [1992], and only details specific to the current experiment are described here. It consisted of a vertical wetted-wall flow tube, a movable injector through which $\mathrm{NO}_{3}$ is introduced, a long path absorption cell, and a stabilized diode laser system. A schematic diagram of the apparatus is shown in Figure 1. The reactor was $85 \mathrm{~cm}$ long with an internal diameter of $1.9 \mathrm{~cm}$. The first $60-\mathrm{cm}$ section enabled thermal and water vapor equilibration between the gas flow and the liquid film. The uptake coefficients were measured in the lowest $25-\mathrm{cm}$ section of the flow tube, where the injector could move up to $18 \mathrm{~cm}$. About $5 \mathrm{~L}$ of liquid were stored in a $12-\mathrm{L}$ bulb above the system and the rate of flow through the reactor was controlled using a Teflon needle valve. The liquid was continuously pumped back to the reservoir by a Teflon gear pump. 


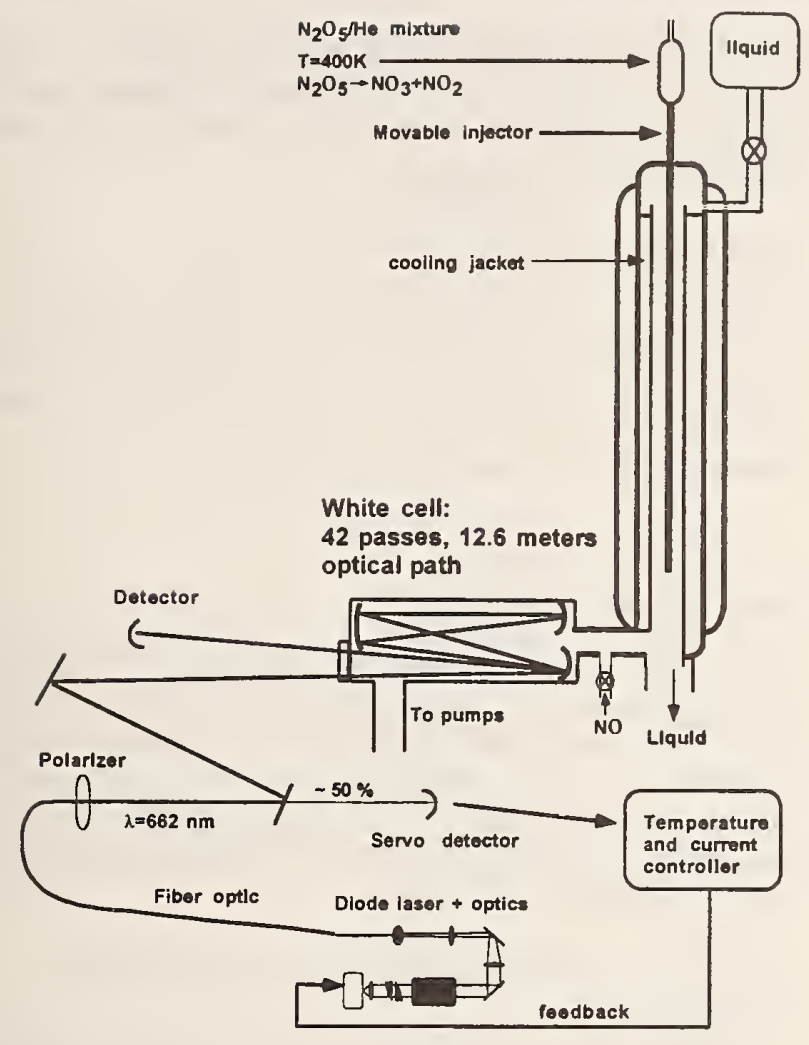

Figure 1. A schematic diagram of the experimental apparatus.

The $\mathrm{NO}_{3}$ radical was generated by thermal dissociation of $\mathrm{N}_{2} \mathrm{O}_{5}$ in an oven mounted on top of the injector and maintained at a constant temperature of $400 \mathrm{~K}$. High purity He was passed through an $\mathrm{N}_{2} \mathrm{O}_{5}$ trap, which was kept at $\sim 220 \mathrm{~K}$, to generate a mixture of $\mathrm{N}_{2} \mathrm{O}_{5}$ in He. This mixture was flowed to the oven via Teflon tubing. $\mathrm{NO}_{3}$ concentrations of $2 \times 10^{-11}-1 \times 10^{12} \mathrm{~cm}^{-3}$ were used.

The concentration of $\mathrm{NO}_{3}$ coming out of the wetted-wall reactor was determined as a function of exposure time to the liquid surface by measuring the attenuation of light at $662 \mathrm{~nm}$, from a diode laser, in a long-path absorption cell [White, 1942; 1976]. The cell was covered with a glass jacket with an inner diameter of $2.3 \mathrm{~cm}$ and a total volume of about $130 \mathrm{~cm}^{3}$. The dielectric coated mirrors had a reflectance of $>99 \%$ between 450 and $700 \mathrm{~nm}$. The distance between the mirrors of the cell was 30 $\mathrm{cm}$. The beam was multipassed, 42 times, to get a total path length of $12.6 \mathrm{~m}$.

The temperature and current of the diode laser were adjusted to give single-mode operation near $662 \mathrm{~nm}$, the peak of the $\mathrm{NO}_{3}$ absorption [Wayne et al., 1991]. An optical fiber was used to spatially filter and relay the beam to the White cell. To minimize optical feedback-induced amplitude noise, the fiber ends were polished at an $8^{\circ}$ angle, and an optical isolator was used. Approximately $60 \%$ of the laser power ( $4 \mathrm{~mW}$ total power) was coupled into the fiber by using a spherical lens, an anamorphic prism pair, and a telescope $(\times 3)$, in addition to the collimating and focusing objectives.

At the fiber output, approximately $50 \%$ of the power was directed to a photodiode for an amplitude stabilization servo. A stable offset current was subtracted from the photocurrent to provide an error signal, and then fed back to the laser's injection current with a bandwidth of $100 \mathrm{kHz}$. The offset current was derived from a stable, well-filtered voltage reference and a precision low temperature-coefficient resistor. The remaining laser power was mode-matched to the waist of the White cell, and the cell output directed to a photodiode.

The fiber caused the beam polarization to vary slightly with temperature and acoustic noise, which resulted in amplitude noise on the signal photocurrent due to the polarization sensitivity of the optics. This noise was minimized by using a polarizer immediately after the collimated fiber output but before sampling the beam for the servo.

The laser's wavelength was tuned to the $\mathrm{NO}_{3}$ absorption line using a diode array spectrometer and a Neon lamp as a wavelength reference. The correction current for the amplitude stabilization $(<0.1 \mathrm{~mA})$ did not significantly change the wavelength. The laser's mode structure and wavelength were periodically checked with an optical multichannel analyzer.

The output of the detector was averaged for $3 \mathrm{~s}$ and read by a digital voltmeter; the voltmeter signal was monitored by a computer for further analysis. The stability of the detection system was about $8 \times 10^{-5}$, limited by vibrations of the multipass cell or optical feedback to the laser. This corresponds to a sensitivity of $\sim 5 \times 10^{9}$ molecules $\mathrm{cm}^{-3}$, using $\sigma(662 \mathrm{~nm})=2 \times 10^{-17}$ $\mathrm{cm}^{2}$ [Wayne et al., 1991].

Water vapor ( 4.5 torr in these experiments) absorbs $662 \mathrm{~nm}$ light. Therefore to obtain $I_{0}$, the light level in the absence of $\mathrm{NO}_{3}$, NO was added to the gas flow at the entrance to the cell to completely convert $\mathrm{NO}_{3}$ to $\mathrm{NO}_{2}$. Such $I_{0}$ values were measured for each injector position before and after taking a reading with $\mathrm{NO}_{3}$.

All experiments were performed at $273 \pm 1 \mathrm{~K}$ and at a total pressure of $9-14$ torr. Typical liquid flow rates were $2-3 \mathrm{~cm}^{3} \mathrm{~s}^{-1}$, implying a Reynold's number of $~ 80$ and liquid flow velocities of $\sim 10-15 \mathrm{~cm} \mathrm{~s}^{-1}$. Typical liquid film thickness was calculated to be about $0.025 \mathrm{~cm}$. Care was taken to minimize ripples on the water film by controlling the liquid flow rate, and maintaining constant wetting of the wall. Flow velocities of the gas were typically $\sim 1000 \mathrm{~cm} \mathrm{~s}^{-1}$ but were varied from 650 to $1600 \mathrm{~cm} \mathrm{~s}^{-1}$. A flow velocity of $\sim 1000 \mathrm{~cm} \mathrm{~s}^{-1}$ corresponds to a Reynold's number of $\sim 100$. The liquid solutions were bubbled with He for at least 15 hours to remove dissolved $\mathrm{N}_{2}$ and $\mathrm{O}_{2}$. Removal of these dissolved gases helped maintain constant unperturbed liquid flow and minimize possible reactive uptake of $\mathrm{NO}_{3}$ by $\mathrm{O}_{2}^{-}$. To maintain a well-defined temperature in the flowing liquid and to avoid rapid evaporation of the film, we added water vapor from a saturator to the main He flow. The temperature of the gas, as well of as the liquid film, were measured in several of the runs and found to be $273 \pm 1 \mathrm{~K}$.

Ozone was prepared by a commercial ozone generator using high purity (>99.995\%) oxygen, which was passed through a molecular sieve trap at $195 \mathrm{~K}$ to remove water. $\mathrm{N}_{2} \mathrm{O}_{5}$ was synthesized by the reaction of ozone with $\mathrm{NO}_{2}$. The ozone/O mixture from the ozone generator was mixed with $\mathrm{NO}_{2}$ which was formed in situ by the reaction of $\mathrm{O}_{2}$ with pure NO. The gases were mixed in a $50 \mathrm{~cm}$ long, $3.8 \mathrm{~cm}$ inner-diameter reactor at a pressure of 800 torr and the resulting gas mixture was passed through a trap at dry ice temperature to collect $\mathrm{N}_{2} \mathrm{O}_{5}$. High purity $\mathrm{He}(>99.9995 \%)$ and chemically pure (ASC reagent grade, $>99 \%$ ) salts were used throughout the experiments.

\section{Data Analysis}

The uptake coefficients of $\mathrm{NO}_{3}$ were calculated from the measured first-order rate coefficients for the loss of $\mathrm{NO}_{3}$ from the gas phase, $k_{w}$. The loss of $\mathrm{NO}_{3}$ from the gas phase is described by 


$$
\left[N O_{3}\right]_{z_{1}}=\left[N O_{3}\right]_{z_{2}} \exp \left(-k_{w} z / c\right)
$$

where $\left[\mathrm{NO}_{3}\right]_{\mathrm{zl}}$ and $\left[\mathrm{NO}_{3}\right]_{\mathrm{z2}}$ are the $\mathrm{NO}_{3}$ concentrations at injector positions $z_{1}$ and $z_{2}$, with $z=z_{2}-z_{1}$ and $c$ is the gas flow velocity. A plot of $\ln \left[\mathrm{NO}_{3}\right]$ versus relative injector position $(\mathrm{z})$ yielded a straight line with a slope of $-k_{w} / \mathrm{c}$. Then $k_{w}$ was corrected for the radial concentration gradient generated by the uptake of $\mathrm{NO}_{3}$ into the liquid, using the method developed by Brown [Brown, 1978; Hanson et al., 1992].

To determine the true wall loss rate coefficient, $k_{w}^{c o r r}$, the gas phase diffusion coefficients of $\mathrm{NO}_{3}$ in $\mathrm{He}$ and $\mathrm{H}_{2} \mathrm{O}$ are needed. Since these diffusion coefficients were not measured before, they were calculated [Mason and Monchick, 1962; Monchick and Mason, 1961] using estimated Lennard-Jones parameters for $\mathrm{NO}_{3}$ ( $\sigma=3.77 \AA, \varepsilon / K=395 \mathrm{~K}$ [Patrick and Golden, 1983]) to be 100 and 370 torr $\mathrm{cm}^{2} \mathrm{~s}^{-1}$, respectively. Variation of $\varepsilon / K$, the well depth, by a factor of 2 changed the diffusion coefficients by only $10 \%$. The calculated diffusion coefficient of $\mathrm{NO}_{3}$ was between 13 and $17 \mathrm{~cm}^{2} \mathrm{~s}^{-1}$ under our experimental conditions (4.5 torr $\mathrm{H}_{2} \mathrm{O}, 5-10$ torr $\mathrm{He}$ ). In addition to calculating them, we measured the pressure-independent diffusion coefficients of $\mathrm{NO}_{3}$ in $\mathrm{He}$ and water vapor under our experimental conditions ( $Y$. Rudich et al., Uptake of $\mathrm{NO}_{3}$ on KI Solutions: Rate Coefficient for the $\mathrm{NO}_{3}+$ I- Reaction and Gas-Phase Diffusion Coefficients for $\mathrm{NO}_{3}$, Submitted to Chemical Physics Letters, 1996). The calculated values agreed very well with the measurements.

The reactive uptake coefficient $\gamma$ was calculated from the corrected first-order rate coefficient, $k_{w}^{c o r r}$, using the relation for a cylindrical reactor [Howard, 1979]

$$
\gamma=2 r k_{w}^{c o r r} / \omega
$$

where $\omega$ is the average molecular speed of $\mathrm{NO}_{3}$ and $r$ is the flow tube radius. Here, $k_{w}$ was measured 10-25 times for each salt concentration, while varying pressure and liquid flow velocities. Such measurements were repeated at various salt concentrations. The relationship between $\gamma$ and reactive loss in the aqueous phase is given by (e.g., Hanson et al. 1992)

$$
\frac{1}{\gamma}=\frac{1}{\alpha}+\frac{\omega}{4 R T \mathrm{H} \sqrt{D_{\ell}} \sqrt{k_{\ell}}}
$$

where $\alpha$ is the mass accommodation coefficient, $H$ is Henry's law coefficient $\left(M \mathrm{~atm}^{-1}\right), \quad T$ is the temperature $(\mathrm{K}), R$ is the gas constant ( $\mathrm{L}$ atm $\mathrm{mol}^{-1} \mathrm{~K}^{-1}$ ), and $k_{\ell}$ is the first-order loss rate coefficient of $\mathrm{NO}_{3}$ due to reaction in the aqueous phase $(\ell)$, that is, $k_{\ell}=k^{\mathrm{II}} \times$ [salt] $\times f$ ( $k^{\mathrm{I} 1}$ is the second-order rate coefficient for reaction of $\mathrm{NO}_{3}$ with the salt in the aqueous phase, and $f$ is the activity coefficient of the reactive ion in the salt solution). $D_{\ell}$ is the diffusion coefficient of $\mathrm{NO}_{3}$ in liquid water $\left(\mathrm{cm}^{2} \mathrm{~s}^{-1}\right)$.

In cases where multiple reactions can simultaneously take place in the aqueous phase, equation (3) should be modified to include all reactive channels:

$$
\frac{1}{\gamma}=\frac{1}{\alpha}+\frac{\omega}{4 R T \mathrm{H} \sqrt{D_{\ell}} \sqrt{\sum_{i} k_{\ell i}}}
$$

In equation (4) $k_{\ell i}$ is the first-order loss rate coefficient for reaction of $\mathrm{NO}_{3}$ with species $R_{i}$ in the aqueous phase $(\ell)$, i.e., $k_{\ell i}=k_{i}{ }^{\mathrm{II}}\left[R_{i}\right] \times f_{i}$ ( $k_{i}{ }^{\mathrm{Il}}$ is the second-order rate coefficient for reaction of $\mathrm{NO}_{3}$ with species $i$ in the aqueous phase). The summation is over all the reactants. In cases where the uptake is not limited by mass accommodation, i.e., $\gamma<<\alpha$, equation (4) reduces to

$$
\gamma=\frac{4 R T}{\omega} \mathrm{H} \sqrt{D_{\ell}} \sqrt{\sum_{i} k_{\ell i}}
$$

When $\mathrm{NO}_{3}$ was lost by more than one reaction, $\gamma$ was measured as a function of the concentration of one reactant $i$, while the concentrations of the other $j$ reactants were held constant. Under these conditions,

$$
\gamma^{2}=\mathrm{H}^{2} D_{\ell}\left[\frac{4 R T}{\omega}\right]^{2} \sum_{j} k_{\ell}+\mathrm{H}^{2} D_{\ell}\left[\frac{4 R T}{\omega}\right]^{2} k_{\ell i}
$$

a plot of measured $\gamma 2$ versus $[(4 R T) / \omega]^{2} k_{\ell i}=[(4 R T) / \omega]^{2} k_{i}^{I I}\left[X_{i}\right]$ yields $H^{2} D_{\ell}$ as the slope and $H^{2} D_{\ell}[(4 R T) / \omega]^{2} \sum_{j} k_{\ell j}$ as the intercept. For this analysis, $k_{i}^{\prime \prime}$ must be known.

\section{Results and Discussion}

First-order decay plots of $\mathrm{NO}_{3}$ for a few $\mathrm{NaCl}$ concentrations and pure water are shown in Figure 2. From such plots, $\gamma$ for that set of conditions was calculated. The measured uptake coefficients of $\mathrm{NO}_{3}$ on water and $\mathrm{NaCl}, \mathrm{NaBr}$, and $\mathrm{NaNO}_{2}$ solutions are given in Table 1. The error bars in Figure 2 are the standard deviation of the mean of $10-25$ measurements of $\gamma$ for each salt concentration.

\section{Determination of $\mathrm{HD}{ }_{\ell}^{0.5}$ and Measurements of Liquid Phase Rate Coefficients}

To extract $\mathrm{HD}_{\ell}^{0.5}$ for $\mathrm{NO}_{3}$ in water from reactive uptake coefficients measurements, at least one aqueous phase rate constant $\left(k^{\mathrm{II}}\right)$ has to be known. The reaction of $\mathrm{NO}_{3}$ with $\mathrm{Cl}^{-}$

$$
\mathrm{NO}_{3}(\mathrm{aq})+\mathrm{Cl}^{-}(\mathrm{aq}) \stackrel{k_{1}}{\longrightarrow} \mathrm{NO}_{3}^{-}(\mathrm{aq})+\mathrm{Cl}_{(\mathrm{aq})}
$$

has been previously studied by Exner et al. [1992] as a function of temperature and by Neta and Huie [1986] and Kim and Hamill [1976] at $298 \mathrm{~K}$. The values of $k_{1}$ at $298 \mathrm{~K}$ reported by Neta and Huie and by Kim and Hamill are 7-10 times higher than the value reported by Exner et al. This difference was attributed to the effect of high ionic strength of the $\mathrm{NaNO}_{3}$ solutions used in the pulsed radiolysis studies [Exner et al., 1992]. Therefore, we used the rate coefficient, $k_{1}(T)=(1.9 \pm 0.2) \times 10^{13} \exp [-$ $(4300 \pm 500) / T] M^{-1} \mathrm{~s}^{-1}$, measured by Exner et al. at low ionic strength for the analysis of our results.

A loss of $\mathrm{NO}_{3}$ on water was observed even in the absence of $\mathrm{NaCl}$. This observation points to a possibility that $\mathrm{NO}_{3}$ is lost due to reaction with the water. To account for this additional loss, we used equation (6) to extract $\mathrm{H} D_{\ell}^{0.5}$

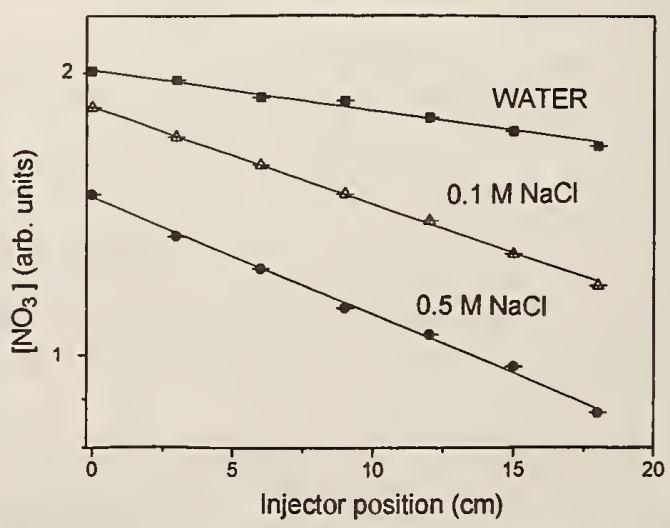

Figure 2. Plots of $\mathrm{NO}_{3}$ concentrations as a function of exposure to pure water and solutions of $\mathrm{NaCl}$ : squares, pure water; triangles, $0.1 \mathrm{M} \mathrm{NaCl}$; circles, $0.5 \mathrm{M} \mathrm{NaCl}$. 
Table 1. Measured Values of $\gamma$ Over Various lonic Solutions Along With Experimental Parameters

\begin{tabular}{cccccc}
\hline & $\begin{array}{c}\text { [salt }] \\
M\end{array}$ & $\begin{array}{c}\text { Activity } \\
(f \times[\text { salt }]\end{array}$ & $\begin{array}{c}\text { Total pressure, } \\
\text { torr }\end{array}$ & $\begin{array}{c}\text { Flow Velocities, } \\
\mathrm{cm} \mathrm{s}^{-1}\end{array}$ & $\gamma \times 10^{3} \mathrm{~b}$ \\
\hline $\mathrm{H}_{2} \mathrm{O}$ & 0.000 & 0.0000 & $10.0-16.2$ & $580-1000$ & $0.20 \pm 0.10$ \\
$\mathrm{NaCl}$ & 0.0087 & 0.0079 & $11.8-14.0$ & $640-1020$ & $0.78 \pm 0.12$ \\
& 0.0096 & 0.0087 & $12.0-14.75$ & $700-960$ & $0.85 \pm 0.12$ \\
& 0.0101 & 0.0091 & $11.5-14.5$ & $620-860$ & $1.14 \pm 0.12$ \\
& 0.0123 & 0.0111 & $10.2-14.7$ & $730-1500$ & $0.67 \pm 0.20$ \\
& 0.0150 & 0.0136 & $8.1-10.3$ & $860-1500$ & $1.39 \pm 0.25$ \\
& 0.0506 & 0.0458 & $10.4-14.6$ & $650-1000$ & $1.98 \pm 0.28$ \\
& 0.0790 & 0.0717 & $12.2-13.0$ & $920-1100$ & $0.21 \pm 0.06$ \\
& 0.100 & 0.0904 & $7.2-12.8$ & $500-1380$ & $2.73 \pm 0.40$ \\
& 0.2090 & 0.1890 & $11.6-13.3$ & $920-1180$ & $3.01 \pm 0.53$ \\
& 0.2620 & 0.2370 & $9.2-13.8$ & $730-1300$ & $4.21 \pm 0.53$ \\
& 0.5000 & 0.4520 & $6.6-9.1$ & $830-1870$ & $4.70 \pm 0.65$ \\
$\mathrm{NaNO}_{2}$ & 0.5000 & 0.4520 & $11.5-13.0$ & $910-1050$ & $6.00 \pm 1.50$ \\
& 0.0005 & 0.0005 & $10.5-14.5$ & $620-1000$ & $1.74 \pm 0.20$ \\
& 0.0010 & 0.0010 & $11.3-13.5$ & $660-950$ & $2.75 \pm 0.53$ \\
& 0.0011 & 0.0010 & $11.0-13.5$ & $690-960$ & $2.61 \pm 0.52$ \\
& 0.0038 & 0.0037 & $10.1-12.5$ & $730-1050$ & $4.6 \pm 1.2$ \\
$\mathrm{NaBr}$ & 0.0010 & 0.0010 & $10.6-13.8$ & $520-800$ & $1.56 \pm 0.26$ \\
& 0.0040 & 0.0037 & $11.1-13.6$ & $700-800$ & $3.22 \pm 0.22$ \\
& 0.0083 & 0.0076 & $10.7-12.6$ & $790-1330$ & $5.32 \pm 0.58$ \\
& 0.0100 & 0.0090 & $11.7-13.1$ & $660-780$ & $5.5 \pm 1.3$ \\
\hline
\end{tabular}

${ }^{a} \mathrm{Here} f$ is the activity coefficient of ions in the solution.

${ }^{b}$ The quoted errors are $2 \sigma$ in the standard deviation of the mean of many measurements and do not include any systematic error. Precision of each measurement is much smaller.

$$
\gamma^{2}=\left[\frac{4 R T}{\omega}\right]^{2} H^{2} D_{l}\left[k_{f 4}\left[H_{2} \mathrm{O}\right]+k_{1}[N a C l] \times f\right]
$$

where $k_{f 4}\left[\mathrm{H}_{2} \mathrm{O}\right]$ is the first-order loss rate coefficient of $\mathrm{NO}_{3}$ in the liquid phase due to its reaction with water. Figure 3 shows a plot of $\gamma^{2}$ versus $[(4 R T) / \omega]^{2} \mathrm{H}^{2} D_{l}\left[k_{44}\left[\mathrm{H}_{2} \mathrm{O}\right]+k_{1}[\mathrm{NaCl}] \times f\right]$, where $k_{1}=2.76 \times 10^{6} \mathrm{M}^{-1} \mathrm{~s}^{-1}$ at $273 \mathrm{~K}$ [Exner et al., 1992]. By fitting these data to a straight line, we obtain $\mathrm{H} D_{\ell}^{0.5}=(1.9 \pm 0.4) \times 10^{-3} \mathrm{M} \mathrm{atm}^{-1}$ $\mathrm{cm} \mathrm{s}^{-0.5}$ for $\mathrm{NO}_{3}$ in water. Taking the value of $D_{2}$ for $\mathrm{NO}_{3}$ in water to be $\mathrm{D} \ell=(1.0 \pm 0.5) \times 10^{-5} \mathrm{~cm}^{2} \mathrm{~s}^{-1}$, we obtain $\mathrm{H}=0.6 \pm 0.3 \mathrm{M}$ $\mathrm{atm}^{-1}$.

Using our measured value of $H D_{\ell}^{0.5}$, we can extract the rate coefficients of other aqueous phase reactions, such as the reactions of $\mathrm{NO}_{3}$ with $\mathrm{NO}_{2}^{-}$and $\mathrm{Br}^{-}$by measuring $\gamma$ as a function of their concentration in water. The rate coefficients for the reactions

(R2)

$\mathrm{NO}_{3(2 a)}+\mathrm{Br}_{(2 \mathrm{aq})} \stackrel{k_{2}}{\longrightarrow} \mathrm{NO}_{3_{(2 \mathrm{a})}^{-}}+\mathrm{Br}_{(\mathrm{aq})}$

and

(R3) $\quad \mathrm{NO}_{3(\mathrm{aq})}+\mathrm{NO}_{2}^{\left.-{ }_{(2 \mathrm{q}}\right)} \stackrel{\mathrm{k}_{3}}{\longrightarrow} \mathrm{NO}_{3_{(\mathrm{aq})}^{-}}+\mathrm{NO}_{2(\mathrm{aq})}$

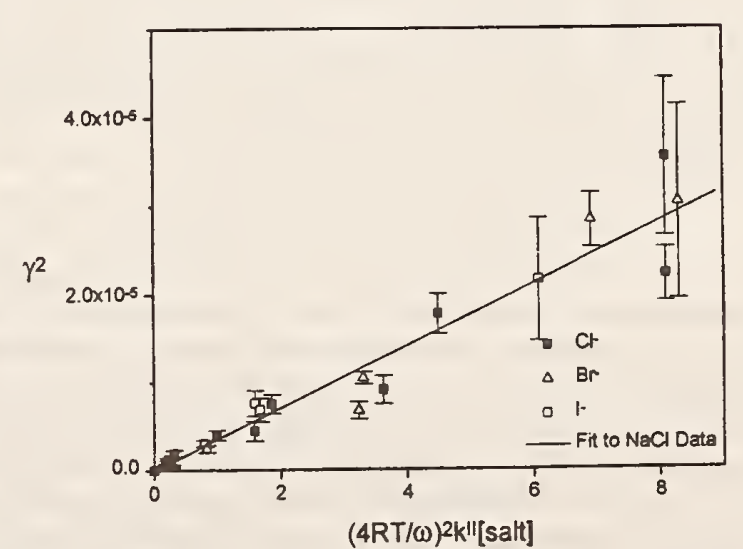

Figure 3. A plot of $\gamma^{2}$ versus $(4 R T / \omega)^{2}\left(k_{\operatorname{sen}}[\right.$ salt $\mid \times f)$ for water, $\mathrm{NaCl}, \mathrm{NaBr}$, and $\mathrm{NaNO}_{2}$. The solid line is a weighted fit of the $\mathrm{NaCl}$ data, and from the slope we calculate $H D_{\ell}^{0.5}=$ $(1.9 \pm 0.4) \times 10^{-3} \mathrm{M} \mathrm{atm}^{-1} \mathrm{~cm} \mathrm{~s}^{-0.5}$. 
have not been measured under experimental conditions that are applicable to ours [Daniels, 1969; Neta and Huie, 1986]. The measured $\gamma$ of $\mathrm{NO}_{3}$ on $\mathrm{Br}^{-}$and $\mathrm{NO}_{2}^{-}$solutions were fitted to equation (6) using the $\mathrm{H} D_{\ell}^{0.5}$ value obtained above to extract $k_{2}$ and $k_{3}$. The derived values for $k_{2}$ and $k_{3}$ are used in Figure 3, where $\gamma^{2}$ values for $\mathrm{NaBr}$ and $\mathrm{NaNO}_{2}$ solutions are plotted against $[4 R T / \omega]^{2} \mathrm{k}_{\ell \mathrm{i}}$ (where $\mathrm{i}$ can be either 2 or 3 ). Since we have measured $k_{2}$ and $k_{3}$ relative to $k_{1}$, the ratios $k_{2} / k_{1}$ and $k_{3} / k_{1}$ are more accurate than the absolute values. We report $k_{2} / k_{1}=37 \pm 4$ and $k_{3} / k_{1}=67 \pm 7$, for dilute $\mathrm{NaBr}$ and $\mathrm{NaNO}_{2}$ solutions, at $p \mathrm{H} \sim 6$ and $273 \pm 1 \mathrm{~K}$.

The majority of the experiments discussed here were obtained by using solutions which were bubbled with $\mathrm{He}$ to remove dissolved oxygen. To simulate conditions relevant to the atmosphere, some experiments were carried out with water saturated with $\mathrm{O}_{2}$. No differences in the measured uptake coefficients were observed in these experiments. The effect of ionic strength and dissolved oxygen on these measurements will be discussed in a separate publication ( $T$. Imamura et al., manuscript in preparation, 1996).

\section{Uptake of $\mathrm{NO}_{3}$ on Pure Water}

A value of $\gamma=\left(2.0_{-0.5}^{+1.0} 0\right) \times 10^{-4}$ was measured for the uptake of $\mathrm{NO}_{3}$ by pure water. The first-order decays for $\mathrm{NO}_{3}$ on pure water were exponential (see Figure 2) and did not show effects of saturation. Using $\mathrm{H} D_{\ell}^{0.5}$ that was obtained from the measured uptake coefficients, saturation is expected to occur within [Hanson et al., 1992] less than 100 ps, given by

$$
\tau_{\text {sat }}=\left[\frac{4 \mathrm{H} R T}{\omega}\right]^{2} D_{\ell}<100 p s
$$

(assuming $\alpha=1$ ). This time is much smaller than the contact time between $\mathrm{NO}_{3}$ and the water-film in our experiments (18-26 ms). Hence the observed uptake of $\mathrm{NO}_{3}$ on pure water implies that a reactive loss is taking place. The reaction of $\mathrm{NO}_{3}$ with $\mathrm{OH}^{-}$ $\left(k(273 \mathrm{~K})=4 \times 10^{7} M^{-1} \mathrm{~s}^{-1}\right.$ [Exner et al., 1992]) cannot account for the observed uptake of $\mathrm{NO}_{3}$ in pure water at $p \mathrm{H}$ of $\sim 7$. Further, the measured uptake coefficient onto water did not change when the $p H$ was $\sim 6$ or even $\sim 5$. In addition, we have used ionchromatography to analyze the water sample for possible presence of other reactive ions prior to the uptake measurements. The analysis revealed that none of the ions present in the water were in concentrations that could lead to the measured loss rate of $\mathrm{NO}_{3}$. After exposure to $\mathrm{NO}_{3}, \mathrm{NO}_{3}{ }^{-}$ions were detected in the water. However, since $\mathrm{N}_{2} \mathrm{O}_{5}$ is also present in our gas flow and is efficiently taken up by water, we cannot attribute the presence of the nitrate ion as solely due to uptake of $\mathrm{NO}_{3}$.

The gas phase loss of $\mathrm{NO}_{3}$ due to the reaction

$$
\mathrm{NO}_{3}+\mathrm{NO}_{2}+\mathrm{M} \stackrel{k_{\text {rec }}}{\longrightarrow} \mathrm{N}_{2} \mathrm{O}_{5}+\mathrm{M} \text {, }
$$

followed by rapid uptake of $\mathrm{N}_{2} \mathrm{O}_{5}$ into the liquid can potentially contribute to the measured loss rate coefficient of $\mathrm{NO}_{3}$ in the absence of reactants in the water. We measured $k_{\text {rec }}$ under our experimental conditions by adding known amounts of $\mathrm{NO}_{2}$ $\left(\left[\mathrm{NO}_{2}\right]=2.5-27 \times 10^{12} \mathrm{~cm}^{-3}\right.$ ) to the gas flow and by measuring the enhancement in the rate constant for the loss of $\mathrm{NO}_{3}$ (see Figure 4). The slope of a plot of the measured loss rate constant as a function of $\left[\mathrm{NO}_{2}\right]$ resulted in $k_{\text {rec }}=(3.0 \pm 0.2) \times 10^{-13} \mathrm{~cm}^{3}$ molecule ${ }^{-1} \mathrm{~s}^{-1}$ (where the error is $1 \sigma$ due to precision of the measurements), corresponding to a loss rate constant of less than $0.5 \mathrm{~s}^{-1}$ under most of our experimental conditions. The loss of $\mathrm{NO}_{3}$ due to recombination with $\mathrm{NO}_{2}$ is negligible compared to the measured loss of $\mathrm{NO}_{3}$. The intercept of the plot did not

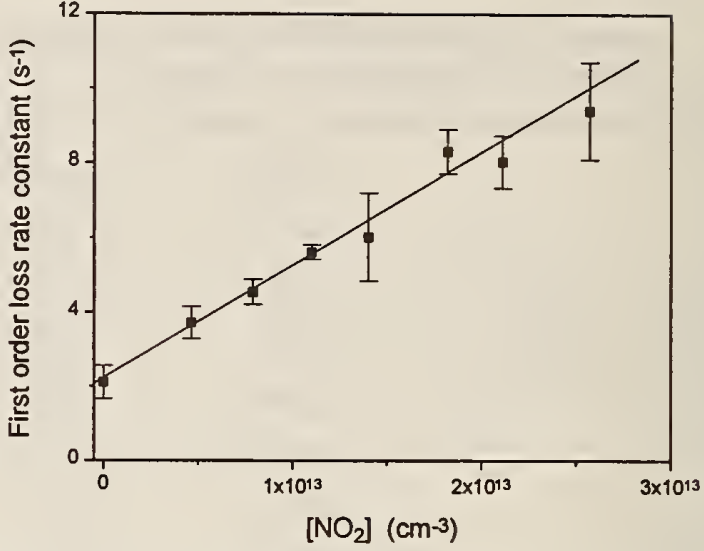

Figure 4. First-order rate coefficients of $\mathrm{NO}_{3}$ in the presence of excess $\mathrm{NO}_{2}$. The initial concentration of $\mathrm{NO}_{3}$ in this experiment were $1.3 \times 10^{12} \mathrm{~cm}^{-3}$, while $\left[\mathrm{NO}_{2}\right]$ ranged between $4.6 \times 10^{12}$ and $2.6 \times 10^{13} \mathrm{~cm}^{-3}$. The slope of this line is the second-order rate coefficient for the recombination of $\mathrm{NO}_{3}$ with $\mathrm{NO}_{2}$, $k_{\text {rec }}=(3.0 \pm 0.2) \times 10^{-13} \mathrm{~cm}^{-3}$ molecule ${ }^{-1} \mathrm{~s}^{-1}$, where the error bars denote only precision. The intercept corresponds to uptake of $\mathrm{NO}_{3}$ on pure water, $\gamma=1.2 \times 10^{-4}$. This intercept did not change upon changing $\left[\mathrm{NO}_{3}\right]$ by a factor of 2 .

change when the gas phase concentration of $\mathrm{NO}_{3}$ was 2 times higher. This further indicates that there were no major loss processes other than the reactive loss on the water film and that $\mathrm{NO}_{2}$ concentrations in the gas flow were comparable to that of $\mathrm{NO}_{3}$. Finally, a plot of the measured loss rate constants versus $\left[\mathrm{NO}_{3}\right]$ resulted in a similar values of $k_{\text {rec}}$.

Therefore we suggest that the observed value of $\gamma$ measured with pure water is caused by reactive uptake of $\mathrm{NO}_{3}$ due to the reaction of $\mathrm{NO}_{3(\mathrm{aq})}$ with water:

$$
\mathrm{NO}_{3(\mathrm{aq})}+\mathrm{H}_{2} \mathrm{O}_{(\iota)} \underset{k_{r_{4}}}{\stackrel{k_{f 4}}{\rightleftarrows}} \mathrm{HNO}_{3(\mathrm{aq})}+\mathrm{OH}_{(\mathrm{aq})}
$$

Based on kinetic and thermodynamic properties of the forward and reverse reactions (see the appendix), we estimate the rate coefficient $k_{f 4}$ to be about $6 M^{-1} \mathrm{~s}^{-1}$ at $298 \mathrm{~K}$ and $1.5 M^{-1} \mathrm{~s}^{-1}$ at 273 $\mathrm{K}$. In principle, it should be possible to extract $k_{/ 4}$ from the intercept of Figure 3. However, since $k_{f 4}\left[\mathrm{H}_{2} \mathrm{O}\right]$ is very small compared to the uncertainties in some of the measured $\gamma$ values, there is a very large error associated with the value of the intercept. For that reason, we use the values actually measured for $\gamma$ to extract $k_{f 4}$. The measured value of $\gamma$ in our experiments corresponds to $k_{f 4} \approx 23_{-13}^{+30} M^{-1} \mathrm{~s}^{-1}$ and is different from the value calculated from thermodynamic properties. This discrepancy may result either from errors associated with the thermodynamic parameters that we used or by another, unknown, loss process that occurs either in the gas phase or in the liquid. It should be noted that a change of $\approx 750 \mathrm{cal} / \mathrm{mol}$ in $\Delta G^{\circ}$ for reaction (R4) can account for the observed difference.

Our interpretation that the $\gamma$ measured on pure water is due to reaction (R4), critically depends on the rate coefficient for the reaction of $\mathrm{NO}_{3}$ with liquid water. Jiang et al. [1992] reported the rate coefficients of $k_{f 4}$ and $k_{r 4}$ to be $5.3 \times 10^{5}$ and $5.3 \times 10^{7} M^{-1}$ $\mathrm{s}^{-1}$, respectively, while our measured $\gamma$ values on liquid $\mathrm{H}_{2} \mathrm{O}$ suggest $k_{f 4}$ to be $k_{f 4} \approx 23_{-13}^{+30} M^{-1} \mathrm{~s}^{-1}$ at $273 \mathrm{~K}$. It appears that the value of $k_{f 4}$ quoted by Jiang et al. is too high. Katsamura et al. [1991] measured $k_{r 4}=(1.4 \pm 0.1) \times 10^{8} M^{-1} \mathrm{~s}^{-1}$. Using this value of $k_{r 4}$ and the $\Delta G^{0}$ for reaction (R4) calculated in the appendix, we 
obtain a value of $k_{f 4}$ of $6 M^{-1} \mathrm{~s}^{-1}$ at $298 \mathrm{~K}$. The redox potential for $\mathrm{NO}_{3}$ calculated using our estimated $\Delta G_{f}^{0}$ for $\mathrm{NO}_{3(\mathrm{aq})}$ and $\Delta G_{f}^{0}$ for $\mathrm{NO}_{3}^{-}(\mathrm{aq})$ is $2.38 \mathrm{~V}$, which is in excellent agreement with the value calculated by Berdnikov and Bazhin [1970] of $2.3 \pm 0.1 \mathrm{~V}$. This value is lower than the value of $2.67 \mathrm{~V}$, which was calculated by Jiang et al. [1992], based on what we believe is an erroneous value for $k_{/ 4}$. Finally, the value of $k_{r 4}=(1.4 \pm 0.1) \times 10^{8}$ $M^{-1} \mathrm{~s}^{-1}$ reported by Katsamura et al. [1991] and used by us is consistent with the rate coefficient for the high pressure limit of the $\mathrm{OH}+\mathrm{HNO}_{3}$ reaction in the gas phase [DeMore et al., 1994].

\section{Possible Sources of Error}

The major sources of uncertainty in these experiments are the fluctuations in the water vapor pressure in the flow tube and ripples on the liquid film. The water vapor pressure in the flow tube influences the $\mathrm{NO}_{3}$ gas phase diffusion coefficient and the gas flow velocity [Hanson et al., 1992; Utter et al., 1992]. Ripples on the water film may also introduce an error in the measured $\gamma$ due to enhanced transport of $\mathrm{NO}_{3}$ caused by. eddies near the wall. The effect of ripples is discussed by Hanson et al. [1992]. To minimize these uncertainties, slow liquid flows (Reynold's number of $\sim 80$ ) and almost laminar gas flows (Reynold's number $\sim 100$ ) were employed. We estimate that these phenomena can contribute $10 \%$ error to our measurements. To minimize fluctuations in the water vapor pressure, we added He saturated with water vapor to the flow tube. This procedure suppressed evaporation and cooling of the liquid film. In addition, we allowed a long time for the gas flow to equilibrate with the water film. Also, the experiments were carried out with slower liquid flows and at the highest possible pressures, so that fluctuations in water pressure had minimal effect. High uptake coefficients measured at high pressures are uncertain because $k_{w}$ is close to the diffusion controlled loss rate coefficient, $k_{\text {diff }}$ [Hanson et al., 1992]. Under those conditions, the uncertainties in $k_{\text {diff }}$ contribute to the error in the diffusion correction due to radial concentration gradient [Brown, 1978].

Finally, $\mathrm{NO}_{2}$, which is also present in the $\mathrm{NO}_{3}$ source, can enter the liquid water and form $\mathrm{NO}_{2}^{-}(\mathrm{aq})$. Then $\mathrm{NO}_{2}^{-}$can react with $\mathrm{NO}_{3}$ and slightly enhance its uptake. Using the known low solubility constant of $\mathrm{NO}_{2}$ [Lee and Schwartz, 1981; Schwartz and White, 1983], we calculate a $\gamma<1 \times 10^{-5}$ for this process.

\section{Atmospheric Implications}

Three results and conclusions from this study that are relevant to the atmosphere are (1) $\mathrm{NO}_{3}$ is taken up by liquid water via a reactive process that produces $\mathrm{OH}$ radicals in solution, (2) the physicochemical parameters, such as $H D_{\ell}^{0.5}$ and $k_{j}^{11}$, which are necessary for deriving the reactive uptake of $\mathrm{NO}_{3}$ into water containing various reactants are determined and (3) the solubility of $\mathrm{NO}_{3}$ in water is small and the previous controversy about the magnitude of this quantity is settled. Of these, the production of $\mathrm{OH}$ oxidant from $\mathrm{NO}_{3}$ uptake is probably the most important conclusion. Here, we discuss the implications of these findings to the chemistry of the lower atmosphere, including those of the marine boundary layer and polar troposphere.

The solubility of $\mathrm{NO}_{3}$ in water has been a disputed issue, with the assumed values ranging from $3 \times 10^{-2}$ to $2 \times 10^{5} \mathrm{M} \mathrm{atm}^{-1}$ [Chameides, 1986a,b; Jacob, 1986; Lelieveld and Crutzen, 1991; Mozurkewich, 1986]. Our value of $\mathrm{H}=0.6 \pm 0.3 \mathrm{M} \mathrm{atm}^{-1}$ is much lower than what has been assumed in many atmospheric calculations. This number is uncertain by $\sim 50 \%$, mostly because of the uncertainties in the rate coefficient for the reaction of $\mathrm{NO}_{3}$ with $\mathrm{Cl}^{-}$and the value of the diffusion coefficient of $\mathrm{NO}_{3}$ in liquid water. Once these quantities are determined by some independent method, our measurement can be used to derive a more accurate value of $\mathrm{H}$. To our knowledge, only Thomas [1992] has previously reported a value of $H$. Thomas could not derive a precise value of $H$ because of experimental difficulties. However, her results greatly constrained the value of $\mathrm{H}$ and is in agreement with ours. Our measured value of $\mathrm{H}$ places a limit on the heterogeneous uptake coefficients that are possible.

We have measured $\mathrm{H} D_{\ell}^{0.5}$, the quantity that is most important for calculating the uptake of $\mathrm{NO}_{3}$ by water. If $\mathrm{HD}{ }_{l}^{0.5}$ is known, the uptake coefficient can be calculated using equation (4) for a known composition of solution. We can calculate the rate of removal of $\mathrm{NO}_{3}$ from the gas phase and the rate of product formation in the liquid phase using the $\mathrm{HD}{ }_{\ell}^{0.5}$ value determined here, if the concentrations of dissolved species in the liquid droplets and the rate coefficients for their reactions with $\mathrm{NO}_{3}$ are known. Even in the absence of additional reactive species in the liquid water droplets, there will be loss of $\mathrm{NO}_{3}$ due to its reaction with water. This loss alone leads to an uptake coefficient, $\gamma$, of $\sim 2 \times 10^{-4}$. The presence of other reactive species in the water droplet will increase $\gamma$.

If the surface area of the water droplets in air is known, the loss rate of $\mathrm{NO}_{3}$ can be calculated. For example, we calculate the $\mathrm{NO}_{3}$ lifetime due to heterogeneous loss to be of the order of 5 min in clouds by assuming a surface area of $-3 \times 10^{-3} \mathrm{~cm}^{2}$ per $\mathrm{cm}^{3}$ of air (e.g., 40 particles $\mathrm{cm}^{-3}$ with $50 \mu \mathrm{m}$ diameter [Graedel and Crutzen, 1993]). In fog, where the drop size is smaller than in a cloud, the diffuso-reactive length is close to the drop radius [Hanson et al., 1994; Ravishankara and Hanson, 1994] and leads to effective uptake coefficients that are a factor of 2 - 3 smaller than in large droplets. The lifetime calculated here is in reasonable agreement with the short lifetime observed for $\mathrm{NO}_{3}$ in the troposphere [Mihelcic et al., 1993; Platt et al., 1981]. Under certain conditions, $\mathrm{NO}_{3}$ will be in equilibrium with $\mathrm{N}_{2} \mathrm{O}_{5}$. Then, removal of $\mathrm{N}_{2} \mathrm{O}_{5}$ by heterogeneous hydrolysis will affect gas phase $\mathrm{NO}_{3}$ concentrations. As temperature increases to make $\left[\mathrm{NO}_{3}\right] /\left[\mathrm{N}_{2} \mathrm{O}_{5}\right]>1$, the effect of heterogeneous $\mathrm{N}_{2} \mathrm{O}_{5}$ uptake on the $\mathrm{NO}_{3}$ concentration becomes smaller. Such conditions are reached when $T \geq 285 \mathrm{~K}$ and for low $\mathrm{NO}_{2}$ mixing ratios ( $<20 \mathrm{pptv}$ ).

In the marine boundary layer (MBL), sea-salt aerosols will be the medium for heterogeneous reactions. For sea-salt aerosols with $\left[\mathrm{Cl}^{-}\right] \cong 1 \mathrm{M}$ and $[\mathrm{Br}-] \cong 5 \times 10^{-3} \mathrm{M}$, the uptake coefficient will be $\gamma \sim 1 \times 10^{-2}$. Under these conditions, the uptake will be restricted by interfacial mass transport for particles of size up to about $15 \mu \mathrm{m}$ [Schwartz, 1986]. For particles above this size, gas phase diffusion will be the rate limiting step. In the presence of sea-salt aerosols, the major loss process for $\mathrm{NO}_{3}$ will be removal by the these particles. Its rapid heterogeneous removal in MBL may be responsible for the low concentrations of $\mathrm{NO}_{3}$ in this region. Low concentrations of $\mathrm{NO}_{3}$ in the MBL may also help explain the observed diumal variation in the abundance of dimethyl sulfide (DMS) in spite of the large rate coefficient for the reaction of $\mathrm{NO}_{3}$ with DMS [Saltzman and Cooper, 1988]. If the sea-salt aerosols are indeed suppressing $\mathrm{NO}_{3}$ abundance in the $\mathrm{MBL}, \mathrm{NO}_{3}$ may not be a major oxidizer of DMS.

Outside of the MBL, based on the measured values of the rate constants and estimated concentrations of reactive species in tropospheric liquid droplets, loss of $\mathrm{NO}_{3}$ via its direct reaction with water may be an important loss mechanism for $\mathrm{NO}_{3}$. Liquid phase reactions of $\mathrm{NO}_{3}$ with reactants other than water can also lead to the formation of very reactive oxidants within the 
droplets. These oxidants can be $\mathrm{Cl}$ (reaction (R1)), $\mathrm{Br}$ (reaction (R2)), or $\mathrm{OH}$ (due to reaction (R4)). The radical products can initiate reactions of various organic and inorganic species dissolved in rain and fog drops or in sea-salt aerosols. Here we very briefly and qualitatively explore the consequences of such oxidant production.

Platt et al. [1984] hypothesized that there should be a mechanism for converting $\mathrm{NO}_{3}$ to $\mathrm{HNO}_{3}$ in the liquid phase. Our work very strongly suggests that the direct reaction of $\mathrm{NO}_{3}$ with water is responsible for what was observed by Platt et al. The importance of this finding is that not only there is a reaction between liquid $\mathrm{H}_{2} \mathrm{O}$ and $\mathrm{NO}_{3}$, but also the product of this reaction is an oxidant in the liquid phase. This is in contrast to $\mathrm{NO}_{3}$ removal via formation of $\mathrm{N}_{2} \mathrm{O}_{5}$ and its subsequent hydrolysis, which results in only formation of $\mathrm{HNO}_{3}$.

Chameides [1986a,b] has shown that $\mathrm{NO}_{3}$ can initiate an oxidation chain within cloud droplets that can convert S(IV) to $\mathrm{S}(\mathrm{VI})$. However, Henry's law coefficient, $\mathrm{H}=12 \mathrm{M} \mathrm{atm}^{-1}$, used in these calculations was larger than the one measured here and reaction (4) was not considered. Therefore the role of $\mathrm{NO}_{3}$ in rain chemistry, sulfur chemistry, and chemical processes within seasalt aerosols must be reevaluated using the lower solubility measured here and the reaction of $\mathrm{NO}_{3}$ with liquid water to produce $\mathrm{OH}$. Reevaporation of $\mathrm{NO}_{3}$ from the droplets that was suggested by Mozurkewich [1986] will not be significant because the uptake of $\mathrm{NO}_{3}$ even in pure water is reactive, i.e., not determined by physical solubility.

Uptake of $\mathrm{NO}_{3}$ into sea-salt aerosols and cloud droplets can directly oxidize $\mathrm{SO}_{2(\mathrm{aq})}, \mathrm{HSO}_{3}{ }^{-}$, and $\mathrm{SO}_{3}{ }^{2-}$ dissolved in them and also indirectly via reactions of $\mathrm{Cl}_{2}^{-}, \mathrm{Br}_{2}{ }^{-}$, and $\mathrm{OH}$ that are produced by the $\mathrm{NO}_{3}$ reactions in the solution. These reactions produce $\mathrm{SO}_{3}{ }^{-}$, which is capable of further initiating catalytic oxidation reactions of S(IV). Production of $\mathrm{SO}_{3}{ }^{-}, \mathrm{Cl}_{2}{ }^{-}, \mathrm{OH}$, and $\mathrm{Br}_{2}{ }^{-}$in the sea-salt aerosols can initiate a mechanism similar to that proposed by Mozurkewich [1995] for release of halogens (chlorine, bromine, and possibly iodine) by oxidation of halide ions via involvement of Caro's acid in the liquid phase. It will be interesting to investigate the role of such a mechanism in the Arctic springtime troposphere. Mozurkewich's mechanism is based on daytime radicals such as $\mathrm{OH}$ and $\mathrm{HO}_{2}$ as initiators. However, $\mathrm{NO}_{3}$ can be important during the dark.

The above discussion is very qualitative and highlights the many possible consequences of our laboratory findings. A quantitative assessment of the impact of the reaction of $\mathrm{NO}_{3}$ with liquid water (oxidant production), the refined values of $\mathrm{H}$, and the derived values of $H D_{\ell}^{0.5}$ require more sophisticated atmospheric modeling calculations.

Appendix: Estimation of Henry's Law Coefficient for $\mathrm{NO}_{3}$ in Water, $k f_{4}$ and $\gamma$ Due to Reaction of $\mathrm{NO}_{3}$ with $\mathrm{H}_{2} \mathrm{O}$

In our experiments, we measured $\mathrm{HD}_{\ell}^{0.5}$ for $\mathrm{NO}_{3}$ in aqueous solutions. To estimate Henry's law coefficient for $\mathrm{NO}_{3}$ in water from this value, the diffusion coefficient of $\mathrm{NO}_{3}$ in water must be known or estimated. Crudely, the diffusion coefficient in the aqueous phase can be estimated by the equation [Atkins, 1990]

$$
D_{\ell}=\frac{k T}{6 \pi \eta \mathrm{R}^{*}}
$$

Where $\eta$ is the viscosity of the liquid $(\eta(273 K)=1.787 \mathrm{cP}), T$ is the temperature, $k$ is Boltzmann constant and $\mathrm{R}^{*}$ is the effective radius of the solvated molecule. Since the exact value of $R^{*}$ is unknown, we assume it to be $1.2 \pm 0.6 \AA$ and obtain $\mathrm{D}_{\ell}\left(\mathrm{NO}_{3(\mathrm{aq})}\right)=$ $(1.0 \pm 0.5) \times 10^{-5} \mathrm{~cm}^{2} \mathrm{~s}^{-1}$. Such a diffusion coefficient yields $\mathrm{H}=$
$0.6 \pm 0.3 \mathrm{M} \mathrm{atm}^{-1}$. A better knowledge of the diffusion coefficient of $\mathrm{NO}_{3}$ in water will allow a better calculation of $\mathrm{H}$ and to reduce the uncertainties in the value determined here.

The reaction of $\mathrm{NO}_{3(\mathrm{aq})}$ with $\mathrm{H}_{2} \mathrm{O}$ can be considered to be a two-step process:

1. Solvation of $\mathrm{NO}_{3}$ in water

$$
\mathrm{NO}_{3(\mathrm{~g})} \stackrel{\mathrm{H}}{\rightleftarrows} \mathrm{NO}_{3 \text { (aq) }}
$$

2. Reaction of aqueous $\mathrm{NO}_{3 \text { (aq) }}$ with $\mathrm{H}_{2} \mathrm{O}$

$$
\mathrm{NO}_{3(\mathrm{aq})}+\mathrm{H}_{2} \mathrm{O}_{(\mathrm{aq})} \underset{k_{r 4}}{\stackrel{k_{f 4}}{\rightleftarrows}} \mathrm{HNO}_{3(\mathrm{aq})}+\mathrm{OH}_{(\mathrm{aq})}
$$

Calculating the standard free energy change, $\Delta G^{0}$, of step (R4) would allow us to evaluate $k_{f 4}$. The $\Delta \mathrm{G}^{0}$ value for reaction (R4) can be estimated from the free energy change in the formation of individual species. The standard free energy change, $\Delta \mathrm{G}_{f}^{*}$ of compounds in the liquid phase are calculated by $\Delta \mathrm{G}_{j}^{*}=\Delta \mathrm{G}_{f}^{0}+$ $\Delta \mathrm{G}^{\#}{ }_{\text {sol }}$, where $\Delta \mathrm{G}_{\text {sol }}^{\#}$ is the $\Delta \mathrm{G}$ due to solvation, which is calculated using Henry's law coefficient and $\Delta \mathrm{G}^{0}$ is the standard energy of formation in the gas phase. Although the standard state for solutions is molality, we will use molarity throughout this calculation, since for dilute aqueous solutions, these quantities are essentially the same.

For $\mathrm{NO}_{3}, \Delta G_{\text {sol }}^{\#}=-R T \ln (\mathrm{H})=0.27_{+0.40}^{-0.24} \mathrm{kcal} \mathrm{mol}^{-1}$ using $\mathrm{H}=$ $0.6 \pm 0.3 \mathrm{M} \mathrm{atm}^{-1} . \Delta G_{f}^{0}$ of $\mathrm{NO}_{3(\mathrm{~g})}$ is $27.75 \mathrm{kcal} \mathrm{mol}^{-1}$ [Chase et al., 1985]. Hence $\Delta G_{f}{ }_{f}$ of $\mathrm{NO}_{3(\mathrm{aq})}=27.75+0.27=28.0_{+0.40}^{-0.24} \mathrm{kcal} \mathrm{mol}^{-1}$. For $\mathrm{HNO}_{3(\mathrm{aq})}$ the physical Henry's law coefficient is needed. There are three equilibria processes that must be taken into account, i.e., Henry's law partition of the nonionized acid $\mathrm{H}_{\mathrm{HNO}_{3}}$

$$
\mathrm{HNO}_{3(\mathrm{~g})} \rightleftarrows \mathrm{HNO}_{3}(\mathrm{aq})
$$

followed by the ionization equilibrium, $K_{\text {eq }}$

$$
\mathrm{HNO}_{3(\mathrm{aq})} \rightleftarrows \mathrm{H}^{+}{ }_{(\mathrm{qq})}+\mathrm{NO}_{3}^{-}{ }_{(\mathrm{qq})}
$$

These can be combined to give the overall equilibrium for nitric acid $K_{H}$

$$
\mathrm{HNO}_{3(\mathrm{~g})} \rightleftarrows \mathrm{H}^{+}{ }_{(\mathrm{qq})}+\mathrm{NO}_{3}^{-}(\mathrm{aq})
$$

Several calculated values for $K_{\mathrm{H}}\left(M^{2} \mathrm{~atm}^{-1}\right)$ at $298 \mathrm{~K}$ exist: $3.26 \times 10^{7}$ [Schwartz and White, 1981], 2.45 $\times 10^{6}$ [Brimblecombe and Clegg, 1988], and $2.66 \times 10^{6}$ [Tang et al., 1988]. For this calculation, we will use the most recent value of $K_{\mathrm{H}}=2.45 \times 10^{6}$. $K_{\text {eq }}$ has been measured to be $20 \mathrm{M}$ [Redlich et al., 1968]. Henry's law constant for $\mathrm{HNO}_{3}$ can be calculated by [Schwartz and White, 1981] to be

$$
\mathrm{H}_{\mathrm{HNO}_{3}}=K_{\mathrm{H}} / K_{\text {eq }}=1.22 \times 10^{5} \mathrm{Matm}^{-1}
$$

Knowing $\mathrm{H}_{\mathrm{HNO}_{3}}$ and $\Delta G_{f}^{3}$ of $\mathrm{HNO}_{3(\mathrm{~g})}=-17.72 \mathrm{kcal} \mathrm{mol}{ }^{-1}$, we calculate $\Delta G_{f}^{*}$ of $\mathrm{HNO}_{3(\mathrm{aq})}=-17.72-R \ln \left(\mathrm{H}_{\mathrm{HNO}_{3}}\right)=-24.66 \mathrm{kcal}$ $\mathrm{mol}^{-1}$. (Note that the $\Delta G_{f}^{\circ}$ for $\mathrm{HNO}_{3(\mathrm{aq})}$ in the CRC Handbook is actually that for $\mathrm{NO}_{3}^{-}{ }_{(\mathrm{aq})}$ ).

Using known $\Delta G_{f}^{*}$ for $\mathrm{OH}_{(\mathrm{aq})}$, and $\mathrm{H}_{2} \mathrm{O}_{\text {(aq) }}(6.2$ and $-56.5 \mathrm{kcal}$ $\mathrm{mol}^{-1}$, respectively [Golden et al., 1990]), $\Delta G^{0}$ for reaction (R4) is calculated to be $\Delta G^{\circ}=10.0 \pm 0.3 \mathrm{kcal} \mathrm{mol}^{-1}$. This standard free energy change yields an equilibrium constant $K_{2}=k_{f 4} / k_{r 4}=$ $\exp (-\Delta G / R T)=4.3 \times 10^{-8}$ at $298 \mathrm{~K}$. Assuming $k_{r 4}=(1.4 \pm 0.1) \times 10^{8}$ $M^{-1} \mathrm{~s}^{-1}$ [Katsamura et al., 1991], $k_{f 4} \cong 6_{-2}^{+4} M^{-1} \mathrm{~s}^{-1}$ is obtained at $298 \mathrm{~K}$. With $\Delta G^{\circ}=10.0 \pm 0.3 \mathrm{kcal} \mathrm{mol}^{-1}, k_{f 4}$ at $273 \mathrm{~K}$ is calculated to be $k_{f 4}=1.3 \mathrm{M}^{-1} \mathrm{~s}^{-1}$, corresponding to $\gamma=5 \times 10^{-5}$.

Acknowledgments. We thank David R. Hanson and Edward R. Lovejoy for their valuable help throughout this work and Paul Wine for valuable discussions. The authors also acknowledge 
the help of Richard B. Norton for the ion chromatographic analysis of the liquid samples. This work was performed while Yinon Rudich held a National Research Council-NOAA Postdoctoral Research Associateship. This work is funded in part by NOAA's Climate and Global Change program

\section{References}

Atkins, P.W., Physical Chemistry, Oxford University Press, New York, 1990.

Atkinson, R., Kinetics and mechanisms of the gas-phase reactions of the $\mathrm{NO}_{3}$ radical with organic compounds, $J$. Phys. Chem. Ref. Data, 20, 459-507, 1991.

Berdnikov, V.M., and N.M. Bazhin, Oxidation-reduction potentials of certain inorganic radicals in aqueous solutions, Russ. J. Phys. Chem. Engl. Transl., 44, 395-398, 1970.

Brimblecombe, P., and S. Clegg, The solubility and behavior of acid gases in the marine aerosol, J. Atmos. Chem., 7, 1-18, 1988.

Brown, R.L., Tubular flow reactors with first-order kinetics, $J$. Res. Natl. Bur. Stand. U.S., 83, 1-8, 1978.

Chameides, W.L., Possible role of $\mathrm{NO}_{3}$ in the nighttime chemistry of a cloud, J. Geophys. Res., 91, 5331-5337, 1986a.

Chameides, W.L., Reply to comment on "Possible role of $\mathrm{NO}_{3}$ in the nighttlme chemistry of a cloud", J. Geophys. Res., 91, 14,571-14,572, 1986b.

Chase, M.W., Jr. , C.A. Davis, J.R. Downey, Jr., D.J. Frurip, R.A. McDonald, and A.N. Syverud, JANAF thermochemical tables, J. Phys. Chem. Ref. Data, 14, Suppl. I, 1985.

Danckwerts, P.V., Absorption by simultaneous diffusion and chemical reaction into particles of various shapes and into falling drops, Trans. Faraday Soc., 47, 1014, 1951.

Daniels, M., Radiation chemistry of the aqueous nitrate system, III, Pulse electron radiolysis of concentrated sodium nitrate solutions, J. Phys. Chem., 73, 3710-3717, 1969.

DeMore, W.B., S.P. Sander, D.M. Golden, R.F. Hampson, M.J. Kurylo, C.J. Howard, A.R. Ravishankara, C.E. Kolb, and M.J. Molina, Chemical kinetics and photochemical data for use in stratospheric modeling, Evaluation 11, JPL publ. 9426, 1994.

Exner, M., H. Herrmann, and R. Zellner, Laser-based studies of reactions of the nitrate radical in aqueous solution, $B e r$. Bunsenges. Phys. Chem., 96, 470-477, 1992.

Golden, D.M., V.M. Bierbaum, and C.H. Howard, Comments on "Reevaluation of the bond-dissociation energies for $\mathrm{H}-\mathrm{OH}$, $\mathrm{H}-\mathrm{OOH}, \mathrm{H}-\mathrm{OO}^{-}, \mathrm{H}-\mathrm{O}, \mathrm{H}-\mathrm{OO}^{-}$and $\mathrm{H}-\mathrm{OO} "$, J. Phys. Chem., 94, 5413-5415, 1990.

Graedel, T.E., and P.J. Crutzen, Atmospheric Change - An Earth System Perspective, W.H. Freeman, New York, 1993.

Hanson, D.R., J.B. Burkholder, C.J. Howard, and A.R. Ravishankara, Measurement of $\mathrm{OH}$ and $\mathrm{HO}_{2}$ radical uptake coefficients on water and sulfuric acid Surfaces, J. Phys. Chem., 96, 4979-4985, 1992.

Hanson, D.R., A.R. Ravishankara, and S. Solomon, Heterogeneous reactions in sulfuric acid aerosols: A framework for model calculations, J. Geophys. Res., 99, 3615-3629, 1994.

Heikes, B.G., and A.M. Thompson, Effects of heterogeneous processes on $\mathrm{NO}_{3}, \mathrm{HONO}$ and $\mathrm{HNO}_{3}$ chemistry in the troposphere, J. Geophys. Res., 88, 10,883-10,895, 1983.
Howard, C.J., Kinetic measurements using flow Tubes, J. Phys. Chem., 83, 3-9, 1979.

Jacob, D.J., Chemistry of $\mathrm{OH}$ in remote clouds and its role in the production of formic acid and peroxymonosulfate, $J$. Geophys. Res., 91, 9807-9826, 1986.

Jiang, P.Y., Y. Katsamura, K. Ishigure, and Y. Yoshida, Reduction potential of the nitrate radical in aqueous solution, Inorg. Chem., 31, 5135-5136, 1992.

Katsamura, K., P.Y. Jiang, R. Nagaishi, T. Oishi, K. Ishigure, and $\mathrm{Y}$. Yoshida, Pulse radiolysis study of aqueous nitric acid solutions: Formation mechanism, yield, and reactivity of $\mathrm{NO}_{3}$, J. Phys. Chem., 95, 4435-4439, 1991.

Kim, K.J., and W.H. Hamill, Direct and indirect effects in pulse irradiated concentrated aqueous solutions of chloride and sulfate ions, J. Phys. Chem., 80, 2320-2325, 1976.

Lee, Y.N., and S.E. Schwartz, Reaction kinetics of nitrogen dioxide with liquid water at low partial pressure, J. Phys. Chem., 85, 840-848, 1981.

Lelieveld, J., and P.J. Crutzen, The role of clou's in tropospheric chemistry, J. Atmos. Chem., 12, 229-267, 1;i.

Mason, E.A., and L. Monchick, Transport properties of polar-gas mixtures, J. Chem. Phys., 36, 2746-2757, 1962.

Mihelcic, D., D. Klemp, P. Musgen, A.W. Patz, and A. VoltzThomas, Simultaneous measurements of peroxy and nitrate radicals at Schauinsland, J. Atmos. Chem., 16, 313-335, 1993.

Monchick, L., and E.A. Mason, Transport properties of polar gases, J. Chem. Phys., 35, 1676-1697, 1961.

Mozurkewich, M., Comment on "Possible role of $\mathrm{NO}_{3}$ in the nighttime chemistry of a cloud", J. Geophys. Res., 91, 14,569-14,570, 1986.

Mozurkewich, M., Mechanisms for the release of halogens from sea-salt particles by free radical reactions, J. Geophys. Res., 100, 14,199-14,207, 1995.

Neta, P., and R.E. Huie, Rate constants for reactions of $\mathrm{NO}_{3}$ radicals in aqueous solutions, $J$. Phys. Chem., 90, 4644-4648, 1986.

Noxon, J.F., $\mathrm{NO}_{3}$ and $\mathrm{NO}_{2}$ in the mid-pacific troposphere, $J$. Geophys. Res., 88, 11,017-11,021, 1983.

Patrick, R., and D.M. Golden, Third-order rate constants of atmospheric importance, Int. J. Chem. Kinet., 15, 1189-1227, 1983.

Pederson, T., Nighttime hydrogen peroxide production on sulfuric-acid aerosols involving nitrate and sulfate radicals, Geophys. Res. Lett., 22, 1497-1499, 1995.

Platt, U., D. Perner, J. Schroder, C. Kessler, and A. Toenissen, The diurnal variation of $\mathrm{NO}_{3}, J$. Geophys. Res., 86, 11,96511,970, 1981.

Platt, U.F., A.W. Winer, H.W. Bierman, R. Atkinson, and J.N. Pitts Jr., Measurement of nitrate radical concentrations in continental air, Environ. Sci. Technol., 18, 365-369, 1984.

Ravishankara, A.R., and D.R. Hanson, Chemistry in sulfate aerosols, in Low Temperature Chemistry of the Atmosphere, NATO ASI Ser., edited by G.K. Moortgart, et al., SpringerVerlag, Berlin, 1994.

Redlich, O., R.W. Duerst, and A. Merbach, lonization of strong electrolytes, XI, The molecular states of nitric acid and perchloric acid, J. Chem. Phys., 49, 2986-2994, 1968.

Saltzman, E.S., and D.J. Cooper, Shipboard measuremnts of atmospheric dimethylsulfide and hydrogen sulfide in the caribbean and gulf of mexico, J. Atmos. Chem., 7, 191-209, 1988. 
Schwartz, S.E., Mass-transport considerations pertinent to aqueous phase reactions of gases in liquid-water clouds, in NATO ASI Ser., edited by W. Jaeschke, pp. 415-471, Springer-Verlag, New York, 1986.

Schwartz, S.E., and W.H. White, Solubility equilibria of the nitrogen oxides and oxyacids in dilute aqueous solution, in Advances is Environmental Science and Technology, edited by J.R. Pfafflin and E.N. Ziegler, pp. 1-45, Gordon and Breach, New York, 1981.

Schwartz, S.E., and W.H. White, Kinetics of reactive dissolution of nitrogen oxides into aqueous solution, Adv. Environ. Sci. Technol., 12, 1-116, 1983.

Tang, I.N., H.R. Munkelwitz, and J.H. Lee, Vapor-liquid equilibrium measurements for dilute nitric acid solutions, Atmos. Environ., 22, 2579-2585, 1988.

Thomas, K., Ph.D. Thesis, Univ. of Wuppertal, Germany, 1992.

Utter, R.G., J.B. Burkholder, C.J. Howard, and A.R.
Ravishankara, Measurement of the mass accommodation coefficient of ozone on aqueous surfaces, J. Phys. Chem., 96, 4973-4979, 1992.

Wayne, R.P., et al., The nitrate radical: physics, chemistry and the atmosphere, Atmos. Environ., 25A, 1-203, 1991.

White, J.U., Long optical paths of large aperture, J. Opt. Soc. Am., 32, 285-288, 1942.

White, J.U., Very long optical paths in air, J. Opt. Soc. Am., 66, 411-416, 1976.

A.R. Ravishankara, Y. Rudich and R.K Talukdar, Aeronomy Laboratory, NOAA, R/E/AL2, 325 Broadway, Boulder CO 80303. (e-mail: ravi@al.noaa.gov, yrudich@al.noaa.gov, talukdar@al.noaa.gov)

R.W. Fox, Time and Frequency Division, NIST, 325 Broadway, Boulder CO80303. (e-mail: rich@nist.gov)

(Received November 15, 1995; revised May 18, 1996; accepted May 28, 1996.) 


\title{
An All-Diode-Laser Optical Frequency Reference Using Laser-Trapped Calcium
}

\author{
C. W. Oates, M. Stephens, and L. Hollberg \\ Time and Frequency Division \\ National Institute of Standards and Technology \\ 325 Broadway \\ Boulder, CO 80303
}

\begin{abstract}
$\underline{\text { Abstract }}$
The $657 \mathrm{~nm}$ calcium intercombination line is an attractive optical frequency/ength reference due to its high line $Q\left(10^{12}\right)$ and insensitivity to external perturbations. In this paper we describe progress towards an all-diode-laserbased reference using laser-trapped $\mathrm{Ca}$. To generate $35 \mathrm{~mW}$ of $423 \mathrm{~nm}$ trap light, we double the frequency of a $150 \mathrm{~mW}$, $846 \mathrm{~nm}$ diode laser with a nonlinear crystal $\left(\mathrm{KNbO}_{3}\right)$ inside a resonant build-up cavity. We use this light to trap $2 \times 10^{7}$ atoms in a compach, magneto-optic trap. Spectroscopy of the narrow $657 \mathrm{~nm}$ transition is performed with a frequencystabilized diode laser system. With the trapped atoms we have measured optical Ramsey fringes with a linewidth of 6 $\mathrm{kHz}$. We discuss future improvements as well as potential performance which could achieve a fractional frequency instability of $<3 \times 10^{-15}$.
\end{abstract}

\section{Introduction}

Due to their narrow linewidth and insensitivity to external perturbations, the intercombination lines in alkaline earth atoms are attractive optical frequency/length references.[1,2] From preliminary work on the lowest lying ${ }^{1} \mathrm{~S}_{0}-{ }^{3} \mathrm{P}_{1}$ lines in $\mathrm{Ba}, \mathrm{Ca}$, and $\mathrm{Mg}$, potential uncertainties in the determination of the line centers were estimated to be $<1 \mathrm{~Hz}$ in the best cases.[3,4,5] This predicted performance is particularly attractive when compared with that of two well-known systems, the iodine-stabilized HeNe laser at 633 $\mathrm{nm}$, and the two-photon system in $\mathrm{Rb}$ at $778 \mathrm{~nm}$, which have uncertainties $>1 \mathrm{kHz} .[6,7]$ Thus, there is a strong impetus for further investigations and development of these alkaline earth-based references.

In particular, the $657 \mathrm{~nm}$ intercombination line ${ }^{1} \mathrm{~S}_{0}$ $(m=0) \rightarrow{ }^{3} P_{1}(m=0)$ in atomic calcium is an excellent candidate due to its high line $\mathrm{Q}\left(10^{12}\right)$ and convenient wavelength. $\mathrm{Ca}$ also has a strong ${ }^{1} \mathrm{~S}_{0} \rightarrow{ }^{1} \mathrm{P}_{1}$ transition at 423 $\mathrm{nm}$ which is well suited for laser cooling and trapping.

Contribution of U.S. Government; not subject to copyright.
The use of laser-cooled samples offers two benefits for a $\mathrm{Ca}$ frequency reference: (i) the extended interaction time needed to resolve the $400 \mathrm{~Hz}$ (FWHM) natural linewidth of the 657 nm transition, and (ii) greatly reduced systematic errors. Another attractive feature of $\mathrm{Ca}$ is that the $657 \mathrm{~nm}$ and 423 $\mathrm{nm}$ transitions are accessible with semiconductor laser systems, enabling a compact, reliable, and low power frequency/length reference. These factors led the BIPM in 1994 to recommend that this be one of those transitions used for the realization of the meter.[6]

The first high resolution Ramsey spectroscopy on the narrow $657 \mathrm{~nm}$ transition was performed in 1979 where a frequency-stabilized dye laser was used to achieve line widths as narrow as $6 \mathrm{kHz}$.[1] Since then there have been numerous measurements using thermal atoms, lasercooled atoms, and even laser-trapped atoms, with resolutions just now approaching the natural linewidth.[3,8] Recently PTB has used an optical frequency synthesis system to measure the absolute frequency of this transition with an uncertainty of less than $450 \mathrm{~Hz}$,[9] maling it the most precisely known optical reference. There are presently several groups around the world developing optical frequency/length references based on this transition.

At NIST our work has focused on the development of a such a reference based on semiconductor laser systems (most previous work used dye lasers at $423 \mathrm{~nm}$ and $657 \mathrm{~nm}$ ). This required construction of a diode laser system at $657 \mathrm{~nm}$ with high frequency stability and high power, and another at $423 \mathrm{~nm}$ which can generate sufficient blue light for use in a Ca magneto-optic trap (MOT)[10]. Additionally, we have designed a simple, compact MOT which stores samples of $-2 \times 10^{7}$ atoms suitable for high-resolution spectroscopy at $657 \mathrm{~nm}$.

This paper will first describe our present apparatus, beginning with the diode laser systems. We will then present some preliminary spectroscopic results which indicate that, when optimized, our frequency reference could attain a fractional frequency instability of $\left\langle 3 \times 10^{-14}\right.$ in $1 \mathrm{sec}$. Finally, we will outline some modifications to this initial experimental setup which promise much improved performance in the near future. 


\section{Trapping Ca with Diode Lasers}

There were two important technical challenges in the construction of a compact, diode-laser-based Ca trap. The first was to generate blue light with sufficient power, tunability, and frequency stability to trap $\mathrm{Ca}$. Since there are no suitable semiconductor laser sources at $423 \mathrm{~nm}$, we instead double the frequency of a high-power diode at 846 $\mathrm{nm}$. Figure 1 shows the apparatus used to generate the blue light.[11] A $150 \mathrm{~mW}$ beam from a solitary diode is sent through beam-shaping optics and an optical isolator to a resonant cavity containing a $\mathrm{KNbO}_{3}$ nonlinear crystal cooled to $-10^{\circ} \mathrm{C}$. With $105 \mathrm{~mW}$ incident on the build-up cavity ( $x 20)$ the crystal produces $30 \mathrm{~mW}$ of light at $423 \mathrm{~nm}$. We control the frequency of the high-power diode by injectionlocking it with a lower power $(20 \mathrm{~mW})$ extended-cavity diode laser ( $E C D L)$. Long-term drifts of the laser frequency are suppressed by locking the master ECDL to an absorption signal derived from our $\mathrm{Ca}$ beam apparatus.

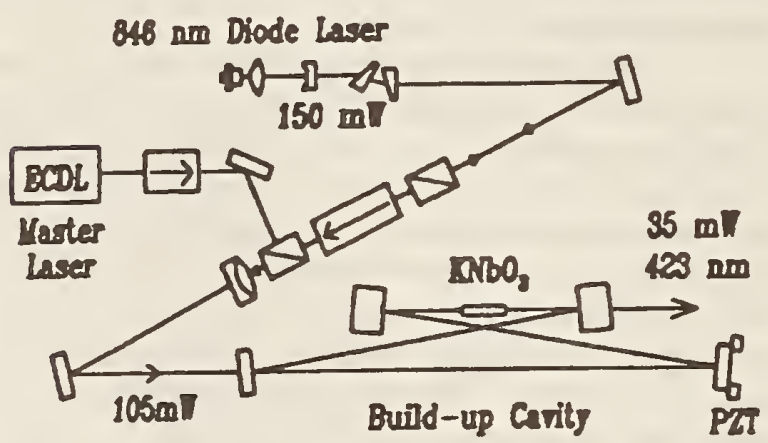

Figure 1. Schematic of the frequency-doubling apparatus which generates $>30 \mathrm{~mW}$ of $423 \mathrm{~nm}$ light.

The second important challenge was to find a compact design for our Ca MOT. Simple vapor cell MOT designs which are commonly used for $\mathrm{Cs}$ and $\mathrm{Rb}$ are difficult for $\mathrm{Ca}$, since $\mathrm{Ca}$ must be heated to over $400^{\circ} \mathrm{C}$ to generate adequate vapor pressure for trap loading. Previous Ca MOT designs $[3,12]$ relied instead on loading from laserslowed atomic beams. They achieved efficient loading via the technique of Zeeman slowing, which keeps the atoms resonant with the slowing laser beams as their velocities are reduced. The Zeeman slowing technique, however, requires large magnetic field coils. We chose instead to modify a design first demonstrated at Stanford[13] using $\mathrm{Li}$ atoms and more recently at PTB using $\mathrm{Ca}[7]$, which traps slow atoms directly from an atomic beam without using a Zeeman slower. Such a trap compensates the reduced range of trapped atomic velocities by placing the trap much closer to the atomic source, thus increasing the flux of atoms through the trap region.

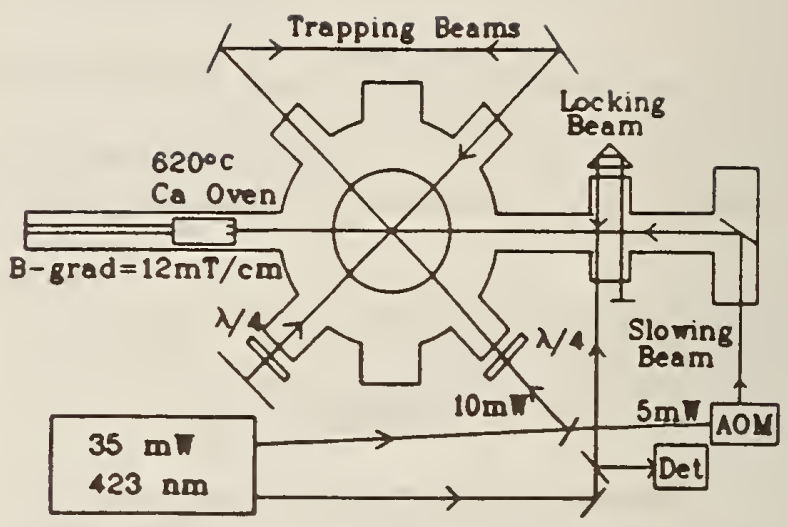

Figure 2. Design of a compact Ca MOT. A retroreflected vertical beam is omitted for clarity.

In our design (shown in Figure 2) the trap is located $13 \mathrm{~cm}$ from the oven nozzle, which operates at $620^{\circ} \mathrm{C}$. We use $8 \mathrm{~mW}$ of $423 \mathrm{~nm}$ light in a single horizontal beam ( $1 \mathrm{~cm}$ diameter) which is recirculated and retroreflected to generate the two perpendicular horizontal trapping beams, set at $45^{\circ}$ relative to the atomic beam axis. The retroreflected vertical trapping beam contains $2 \mathrm{~mW}$ of blue power. We generate the large magnetic field gradient $(12 \mathrm{mT} / \mathrm{cm})$ required by our trap by sending 150 amperes of current through a water-cooled pair of anti-Helmholtz coils. Under these conditions our MOT fills with more than $10^{6} \mathrm{Ca}$ atoms with a loading time constant of $20 \mathrm{~ms}$, apparently limited by optical pumping to the ' $D_{2}$ state which lies below the ${ }^{\prime} P_{1}$ state. We observe the highest level of blue fluorescence from the trapped atoms with the blue light detuned $-30 \mathrm{MHz}$ below the $423 \mathrm{~nm}$ resonance.

In previous versions of this type of MOT[7,12] one or more frequency sidebands were added to the trapping light in order to extend the capture velocity, thus increasing the loading rate. We instead implemented a slowing beam counter-propagating to the atomic beam, which slows atoms (without benefit of Zeeman slowing coils) before they reach the trapping region. We could not however, simply send this light through a window downstream from the oven. A window in this position quickly becomes coated with $\mathrm{Ca}$ from the atomic beam and no longer transmits the blue slowing beam. To avoid this problem we send the slowing light through a window on the side (see Figure 2) and reflect it off a mirror located inside the vacuum system downstream from the oven. It turns out that layers of deposited Ca make a fairly decent mirror ( $\sim 70 \%$ reflectivity) at $423 \mathrm{~nm}$, so the mirror continues to reflect the slowing beam even after becoming coated with $\mathrm{Ca}$. Tuning the frequency of this beam $110 \mathrm{MHz}$ to the red of the cooling transition gives a 
tenfold increase in the loading rate, producing laser-cooled samples of $-2 \times 10^{7}$ atoms in $-20 \mathrm{~ms}$.

We used the narrow $657 \mathrm{~nm}$ transition to measure the temperature of the cold atom cloud via the first-order Doppler effect. Typical temperatures of the trapped atoms range from 2-3 mK, depending on trap laser intensity and detuning. These temperatures are higher than those routinely achieved in alkali traps due to the absence of Zeeman structure (and hence polarization gradient cooling) in the $\mathrm{Ca}$ ground-state. Without such structure, the minimum achievable temperature is set by the Doppler cooling limit.[14] For $\mathrm{Ca}$ this limit is $0.8 \mathrm{mK}$, which corresponds to a root-mean-square velocity of $40 \mathrm{~cm} / \mathrm{s}$ (see ref. [3] for further discussion).

\section{High-Power, Frequency-Stabilized $657 \mathrm{~nm}$ Diode Laser System}

The requirements for the red diode system are (i) its frequency be sufficiently stable to resolve the $400 \mathrm{~Hz}$ natural linewidth of the intercombination line and (ii) its power be greater than $10 \mathrm{~mW}$ to excite this weak transition by the Ramsey method.

To achieve sufficient frequency stability we started with an extended-cavity diode laser at $657 \mathrm{~nm}$, which yielded $3 \mathrm{~mW}$ output power (see Figure 3). To reduce the

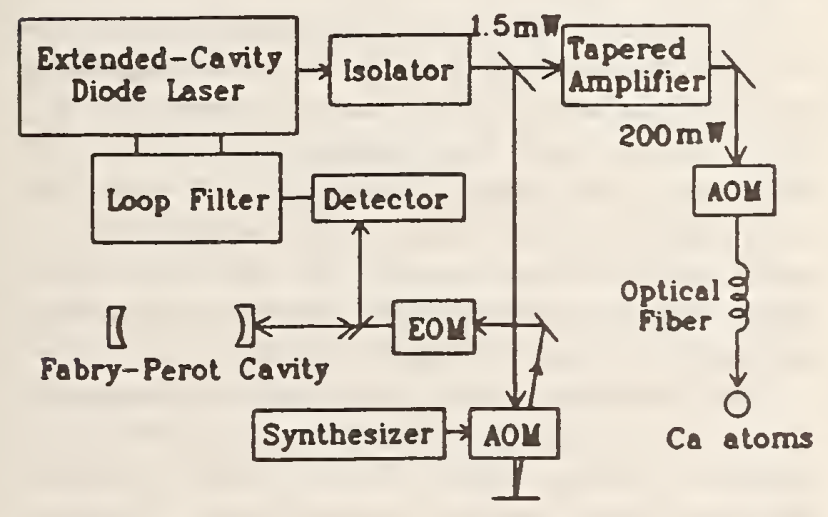

Figure 3. Block diagram of the frequency-stabilized laser system at $657 \mathrm{~nm}$ used for high resolution spectroscopy.

$100 \mathrm{kHz}$ linewidth of this laser, we lock its frequency to a resonance of a high-finesse, Fabry-Perot cavity ( $3 \mathrm{GHz}$ FSR and $50 \mathrm{kHz}$ fringe FWHM). We send $100 \mu \mathrm{W}$ of the laser output to this cavity and use the Pound-Drever-Hall technique[14] to derive a high signal-to-noise error signal using a modulation frequency of $13.3 \mathrm{MHz}$. We then filter this signal to correct laser frequency fluctuations via the laser current (fast corrections) and a PZT-driven mirror on the laser cavity (slow corrections). A servo bandwidth of over $3 \mathrm{MHz}$ yields fast laser linewidths of $<100 \mathrm{~Hz}$, as inferred from the error signal relative to the cavity. To reduce long-term frequency fluctuations due to drifts of the reference cavity, we constructed the cavity spacer out of ultra-low expansion (ULE) material and placed it inside a vacuum chamber. This chamber was then placed inside a thermally-stabilized, vibrationally-isolated environment. To enable precise tuning of the laser frequency relative to the cavity resonance, we use a double-passed, acousto-optic modulator (AOM) before the cavity.

We increased the $657 \mathrm{~nm}$ laser power available for Ca spectroscopy by using $2 \mathrm{~mW}$ of light from the stabilized ECDL to injection-lock a semiconductor tapered amplifier(TA). The stabilized ECDL serves as the master laser and the tapered amplifier as the power amplifier in a master-oscillator power-amplifier (MOPA) configuration. With 1.9 amperes of current in the TA, the MOPA produces a remarkable $240 \mathrm{~mW}$ of light without adding significant phase noise to the frequency-stabilized light. After spatially filtering the output with an optical fiber, however, we have typically only 10-20 mW of usable light, a level adequate though somewhat low for the Ramsey spectroscopy. The MOPA system also allows us to stabilize the intensity level of the light after the fiber by feeding back to the TA current.

As a first test of this system we performed spectroscopy on a thermal beam of calcium. To achieve high resolution while maintaining good signal-to-noise ratio, we used the optical Ramsey fringe technique.[16] This technique is analagous to the microwave Ramsey technique but uses four (rather than two) spatially-separated interaction regions in order to cancel the first-order Doppler effect. The distance between the four traveling waves (along with the most probable velocity of the atomic beam) then sets the spectral resolution for a given measurement. We generated Ramsey lineshapes by measuring the induced atomic beam fluorescence downstream from the interaction region.

Figure 4 shows an example of a Ramsey fringe taken at a probe power level of $8 \mathrm{~mW}$ ( 40 scans averaged total data accumulation time of 1 minute). Here the spacing between each pair of beams was $15 \mathrm{~mm}$, which yields a Ramsey resolution of $11.5 \mathrm{kHz}$ for our most probable velocity of $700 \mathrm{~m} / \mathrm{s}$. Actually seen here are the two recoil components for this transition, which are separated by 23.1 $\mathrm{kHz}$. Our Ramsey resolution of $11.5 \mathrm{kHz}$ was chosen so the fringe patterns of the two components would interfere constructively in order to give the best contrast, which was about $25 \%$.

Note that such a signal can be used as an optical frequency reference simply by locking a laser to one of the two recoil components, one of which can be suppressed, if so desired.[17] If limited only by the signal-to-noise ratio demonstrated in Figure 4, one should attain a fractional frequency uncertainty of $5 \times 10^{-13}$ in $1 \mathrm{sec}$. Workers at PTB 


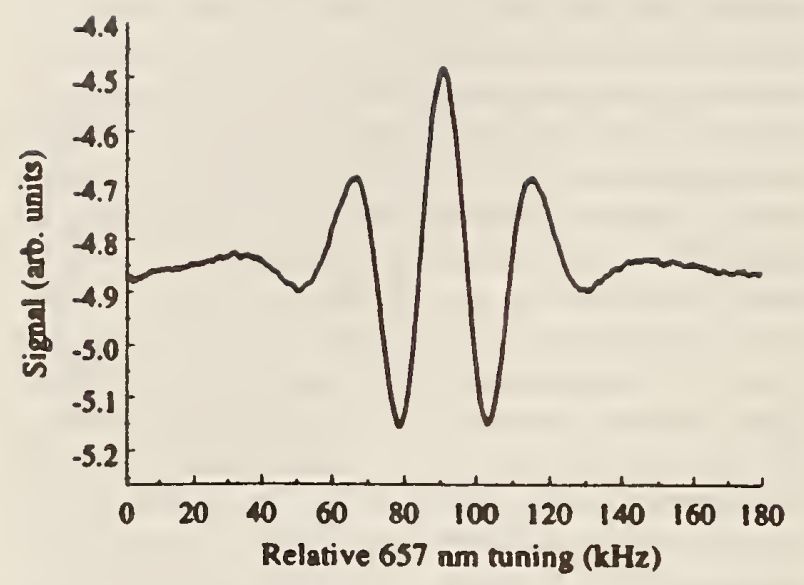

Figure 4. Optical Ramsey fringes in a $\mathrm{Ca}$ atomic beam.

are presently constructing a compact version of this type of device to use as a transportable frequency reference.[8] They have determined, however, that a laser locked to a Ramsey fringe derived from an atomic beam is susceptible to drifts as large as $1 \mathrm{kHz}$, which seriously compromises its long-term performance.[8]

\section{High-Resolution Spectroscopy with Laser-Cooled Ca Atoms}

Presently we perform spectroscopy on the narrow red transition using laser-cooled and trapped atoms. Optical Ramsey spectroscopy with trapped samples differs from that in a beam in that one must separate the four interaction zones temporally rather than spatially. $[3,4]$ Thus this second spectroscopic investigation uses four pulsed traveling waves (two in one direction followed by two in the opposite) to excite the narrow transition. In this case, the time, $T$, between the pulses of each pulse pair determines the Ramsey fringe resolution, $\Delta v=1 /(4 T)$. An added complication is that we must turn off the blue trap light during this excitation time so that the relevant atomic levels are unperturbed.

A typical measurement cycle commences with 5-10 ms for trapping, after which we shut off the trap light and magnetic field gradient. Next we illuminate the atoms with four pulses (controlled by two AOM's) from our frequencystabilized $657 \mathrm{~nm}$ laser system. The cycle ends with a 300 $\mu$ s detection period, during which we collect red fluorescence with a photomultiplier tube. We typically repeat this cycle 200 times for each frequency data point.

Figure 5 shows a preliminary scan taken (without chopping the magnetic field gradient) with a total pulse separation of $80 \mu$ s, which yields a Ramsey resolution of 6
$\mathrm{kHz}$. One immediately notices that many more finges are present than we saw in the case of atomic beam Ramsey spectroscopy. In a thermal beam the effective pulse separation time as seen by an atom depends on its velocity. Due to the wide range of velocities in an atomic beam, the resultant lineshape was in fact the superposition of fringe patterns with different resolutions, which washed out all but the central fringe. For samples of trapped atoms, however, all atoms see the same time separation between pulses, so the individual contributions add coherently, leading to a lineshape such as that seen in Figure 5.

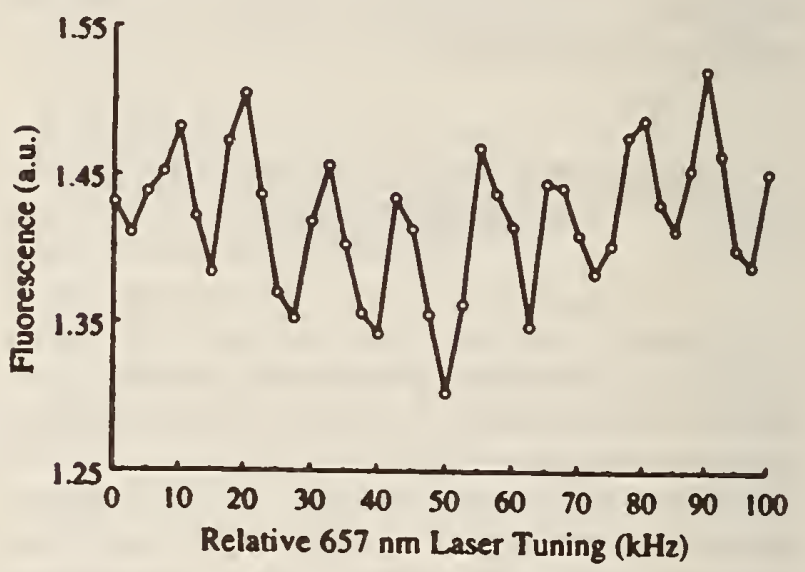

Figure 5. Optical Ramsey fringes at $657 \mathrm{~nm}$ using lasertrapped $\mathrm{Ca}$ atoms.

The actual situation is even more complicated due to the fact that we again have the two recoil components, thus producing two full fringe patterns (separated by 23.1 $\mathbf{k H z}$ ). To have good fringe contrast, the Ramsey fringe period needs to be chosen to be an integral fraction of the recoil splitting (or one of the recoil components must be suppressed). In either case, one can then determine the central fringe (i.e. the line center) for each component by taking spectra at several different resolutions.

The signal-to-noise ratio in this preliminary scan was far from shot-noise limited and should be greatly improved in the near future. For our current apparatus, a shot-noise limited lineshape should yield a fractional uncertainty of $3 \times 10^{-14}$ in $1 \mathrm{sec}$, a level comparable to one demonstrated in a similar setup at PTB using dye lasers.[8] A laser locked to such a fringe could then provide an excellent length reference. For use as a frequency reference, however, the frequency stability of the locked laser would first have to be transferred to the microwave domain using presently unavailable optical frequency-synthesis techniques. 


\section{Future Work and Prospects}

While our present apparatus provides good performance, there are several aspects which can be readily improved. We presently collect less than $2 \%$ of the total fluorescence, so improved collection optics could increase our signal by at least an order of magnitude. To increase the signal further we are presently implementing a different excitation strategy which first uses a resonant blue pulse to measure the number of atoms in the sample before the four red pulses.[4] Immediately following the last red pulse, a second blue pulse can read out the fraction of atoms still in the ground state after the Ramsey interaction. Since we can scatter hundreds of blue photons per atom per blue pulse, we can have a much larger signal. Moreover, this signal is normalized to the number of atoms in the sample, reducing noise induced by shot-to-shot, trap-number fluctuations.

These improvements can, in principle, reduce the uncertainty of our optical frequency reference by one or more orders of magnitude. What will be the ultimate performance of such a system? This remains an open question as there have yet to be detailed studies of systematic effects associated with an optical reference based on trapped atoms. Independent estimates $[4,8]$ predict that known systematic effects should contribute $<1 \mathrm{~Hz}$ (or a fractional frequency uncertainty of $2.5 \times 10^{-15}$ ), but several effects such as those due to cold-atom collisions[18] have not yet been investigated. Such studies will require comparisons between independent systems. The first $\mathrm{Ca}$ intercomparison, between a portable thermal beam apparatus constructed by PTB and our apparatus here, is scheduled for later this year.

\section{Acknowledgements}

We thank our colleagues in the group of $\mathrm{J}$. Helmcke and F. Riehle at PTB, R.W. Fox and J.C. Bergquist at NIST, and J.L. Hall at JILA for useful discussions and suggestions. We also gratefully acknowledge the financial support of AFOSR.

\section{References}

[1] R.L. Barger, J.C. Bergquist, T.C. English, and D.J. Glaze, "Resolution of photon-recoil structure of the 6573-A calcium line in an atomic beam with optical Ramsey fringes," Appl. Phys. Lett., vol. 34, pp. 12-14, June 1979.

[2] J.L. Hall, M. Zhu, and P. Buch, "Prospects for using laser-prepared atomic fountains for optical frequency standards applications," J. Opt. Soc. Amer., vol. 6, pp. 2194-2205, Nov. 1994.
[3] Th. Kisters, K. Zeiske, F. Riehle, and J. Helmcke, "High-resolution spectroscopy with laser-cooled and trapped calcium atoms," Appl. Phys. B, vol. 59, pp. 89-98, Aug. 1994.

[4] K. Sengstock, U. Sterr, J. H. Muller, V. Rieger, D. Bettermann. and W. Ertmer, "Optical Ramsey spectroscopy on laser-trapped and thermal Mg atoms," Appl. Phys. B, vol. 59, pp. 99-115, Aug. 1994.

[5] A.M. Akulshin, A.A. Celikov, and V.L. Velichansky, "Nonlinear Doppler-free spectroscopy of the $6^{1} S_{1}-6^{3} \mathrm{P}_{1}$ intercombination line in barium." Opt. Comm., vol. 93, pp. 54-58, Sept. 1992.

[6] T. Quinn, "Mise en Pratique of the Definition of the Metre (1992)," Metrologia, vol. 30, pp. 523-541, Jan. 1994.

[7] D. Touahri, O. Acef, A. Clairon, J.-J. Zondy, R. Felder, L. Hilico, B. de Beauvoir, F. Biraben, and F. Nez, "Frequency measurement of the $5 S_{1 / 2}(F=3)-5 D_{5 / 2}(F=5)$ two-photon transition in rubidium," Opt. Comm., vol. 133, pp. 471-478, Jan. 1997.

[8] F. Riehle, H. Schnatz, B. Lipphardt, P. Kersten, T. Trebst, G. Zinner, and J. Helmcke, "Optical Calcium Frequency-Standard: Status and Prospects," in PTB-Bericht Opt-51, Proceedings of "Frequency Standards Based on Laser-Manipulated Atoms and Ions," Schierke, Germany, Feb. 12-14, 1996, pp. 11-20.

[9] H. Schnatz, B. Lipphardt, J. Helmcke, F. Riehle, G. Zinner, "First phase-coherent frequency measurement of coherent visible radiation," Phys. Rev. Lett., vol. 76, pp. 1821, Jan. 1996.

[10] E.L. Raab, M. Prentiss, A. Cable, S. Chu, and D.E. Pritchard, "Trapping of neutral sodium atoms with radiation pressure," Phys. Rev. Lett. vol. 59, pp. 2631-2634, Dec. 1987.

[11] R.W. Fox, L. Hollberg, and A.S. Zibrov, "Semiconductor Diode Lasers," in Experimental Methods in the Physical Sciences, vol. 29C, (F.B. Dunning, and R.G. Hulet, ed.) New York:Academic Press, 1997, ch. 4, pp. 77. 100. Leo Hollberg, V. L. Velichansky, C.S. Weimer, and R. W. Fox, "High-Accuracy Spectroscopy with Semiconductor Lasers: Application to Laser-frequency Stabilization," in Frequency Control of Semiconductor Lasers, (Motoichi Ohtsu, ed.). New York:John Wiley \& Sons, Inc, 1996, ch 3, pp. 73-93.

[12] Takayuki Kurosu and Fujio Shimizu, "Laser cooling and trapping of calcium and strontium," Japanese J. Of Applied Phys., vol. 29, pp. L2127-2129, Nov. 1990. 
[13] Brian P. Anderson and Mark A. Kasevich, "Enhanced loading of a magneto-optic trap from an atomic beam," Phys. Rev. A, vol. 50, pp R3581-R3584, Nov. 1994.

[14]. T. W. Hansch and A.L. Schawlow, "Cooling of gases by laser radiation," Opt. Comm., vol. 13, pp. 68-69, Jan. 1975. D.J. Wineland and Wayne M. ltano, "Laser cooling of atoms," Phys. Rev. A, vol. 20, pp. 1521-1540, Oct. 1979.

[15] R.W.P. Drever, J. L. Hall, F.V. Kowalski, J.Hough, G.M. Ford, A.J. Munley, and H. Ward, "Laser Phase and Frequency Stabilization Using an Optical Resonator," Appl. Phys. B, vol. 31, pp.97-105, June 1983.

[16] Ye. V. Baklanov, B. Ya. Dubetsky, V. P. Chebotayev, "Non-linear Ramsey resonance in the optical region," Appl. Phys., vol. 9, pp. 171-173, Feb. 1976.

J.C. Bergquist, S.A. Lee, and J.L. Hall, "Saturated absorption with spatially separated laser fields: observation of optical Ramsey fringes," Phys. Rev. Lett., vol. 38, pp. 159-162, Jan. 1977. Ch. J. Borde, Ch. Salomon, S. Avrillier, A. Van Lerberghe, Ch. Breant, D. Bassi, and G. Scoles, "Optical Ramsey fringes with traveling waves," Phys. Rev. A, vol. 30, pp. 1836-1848, Oct. 1984.

[17] F. Riehle, J. Ishikawa, and J. Helmcke, "Suppression of a recoil component in nonlinear Doppler-free spectroscopy," Phys. Rev. Letl., vol. 61, pp. 2092-2095, Oct. 1988. U. Ster, K. Sengstock, J.H. Muller, D. Betterman, W. Ertmer, "The magnesium Ramsey interferometer: applications and prospects," Appl. Phys. B, vol. 54, pp. 341-346, May 1992.

[18] K. Gibble and S. Chu, "Laser-cooled Cs frequency standard and a measurement fo the frequency shift due to ultracold collisions," Phys. Rev. Lett., vol. 70, pp. 17711774, March 1993. 


\section{Optical Synthesis and Extended Wave Length Coverage}




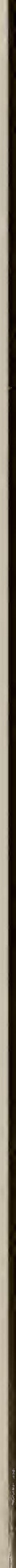


Precise optical frequency references and difference frequency
measurements with diode lasers

S. Waltman, A. Romanovsky, J. Wells, R.W. Fox, and L. Hollberg

National Institute of Standards and Technology Boulder, CO 80303

M.P. Sassi

Instituto di Metrologia

Torino, Italy

H.G. Robinson

Duke University, Department of Physics Durham, NC

\section{ABSTRACT}

Heterodyne methods have been used in conjunction with molecular calculations to accurately determine the wavelengths of more than 35,000 infrared transitions. We have used high speed whisker contact Schottky diodes to extend this technology to the $0.8 \mu \mathrm{m}$ spectral region. Using microwave harmonic mixing we demonstrate that it is possible to detect beat notes between diode lasers to frequencies as high as $400 \mathrm{GHz}$.

\section{INTRODOCTION}

Optical heterodyne techniques can play a fundamental role in precision spectroscopy and laser frequency control. Increasing demand for precise frequency measurements in the visible and near-infrared regions of the spectrum have resulted in a number of ideas for optical frequency synthesis. ${ }^{1-4}$ These proposals often require several tunable narrow-linewidth laser sources. For purely practical reasons these sources might best be supplied by diode lasers. Achieving optical frequency synthesis and measurement will require high-accuracy optical frequency references, very narrow linewidth, well-controlled lasers, and the ability to make frequency difference measurements over extremely large frequency intervals. We have explored and continue to explore some of the promising optical frequency references that are compatible with diode lasers. Of particular interest in this area are the narrow intercombination transitions in the group-II atoms such as calcium, ${ }^{5,6}$ barium, ${ }^{7}$ and strontium. ${ }^{8}$ Our effort at NIST on diode-laser compatible frequency/wavelength references has focused primarily on the calcium transition at $657 \mathrm{~nm}$ and on laser-cooled cesium. ${ }^{9,10}$

'Work of United States Government. Not subject to copyright. 
An effort in measuring the frequencies of molecular transitions in the infrared has just been completed at NIST by J. Wells and A. Maki.11-13 They determined the absolute frequencies of more than 35,000 molecular transitions in the wavelength range between 486 and $4352 \mathrm{~cm}^{-1}$. In their measurement system tuneable lead-salt diode lasers were used to probe the molecular transitions, and heterodyne techniques were used to measure the frequency of the diode laser relative to known stabilized gas laser frequencies. These newly tabulated frequencies now provide a precise calibration atlas for wavelengths in the infrared. This work demonstrates the utility of using heterodyne methods for precision spectroscopy.

We have been exploring the potential for extending these heterodyne techniques using diode lasers to larger frequency intervals and up into the visible and near-visible spectral regions. Some time ago $H$. U. Daniel, J. Bergquist, and collaborators ${ }^{14.15}$ demonstrated that very small Schottky diodes could be used to measure difference frequencies of visible lasers to hundreds of gigahertz. Their devices were similar to ours which were actually designed as millimeter-wave detectors for applications such as radio astronomy. Following their lead we have been using these high-speed, whisker-contact schottky diodes simultaneously as photodetectors and microwave harmonic mixers. These detectors consist of small, recessed Pt-Au islands on a GaAs epilayer grown on a GaAs substrate. The area of the gold islands is about $1.5 \mu \mathrm{m}^{2}$ which gives a capacitance of about $2.6 \mathrm{fF}$ and a $3 \mathrm{~dB}$ corner frequency for millimeterwaves of about $3.7 \mathrm{THz}$. The useful optical detection bandwidth has not yet been fully determined.

\section{EXPERIMENTAL DETAILS}

The experimental apparatus is diagrammed in Fig. 1 . It consists of two $830 \mathrm{~nm}$, AlGaAs lasers that are optically locked ${ }^{16}$ to different modes of a single confocal Fabry-Perot cavity. The lasers' outputs are combined and focused on the schottky diode along with the output from the microwave source. The schottky diode generates the laser beat frequency as well as the harmonics of the microwave source. The laser beat note is detected directly or is mixed down by heterodyning with the microwave frequency or one of its harmonics. The microwaves are supplied by a klystron (at about $47 \mathrm{GHz}$ ) or by a synthesizer.

A picture of the beatnote between two diode lasers and the eighth harmonic of a $47 \mathrm{GHz}$ klystron is shown in Fig. 2. With an optical frequency difference of $382 \mathrm{GHz}$, this beatnote demonstrates the good signal-to-noise ratio that can be achieved with this detection system even though the schottky diode is not at all designed for optical detection. The typical experimental parameters were $\approx 5 \mathrm{~mW}$ in each of the two lasers beams, $\approx 200 \mathrm{mw}$ of klystron power, and a spectrum analyzer resolution bandwidth of $10 \mathrm{kHz}$. The detector can be biased to enhance the mixing and/or harmonic generation efficiency. However, we have found that when the microwave power on the detector is high the bias has very little effect on the detected signal-to-noise ratio. A large experimental uncertainty in the actual microwave power that is coupled onto the detector remains, but we typically operate the detector so that 


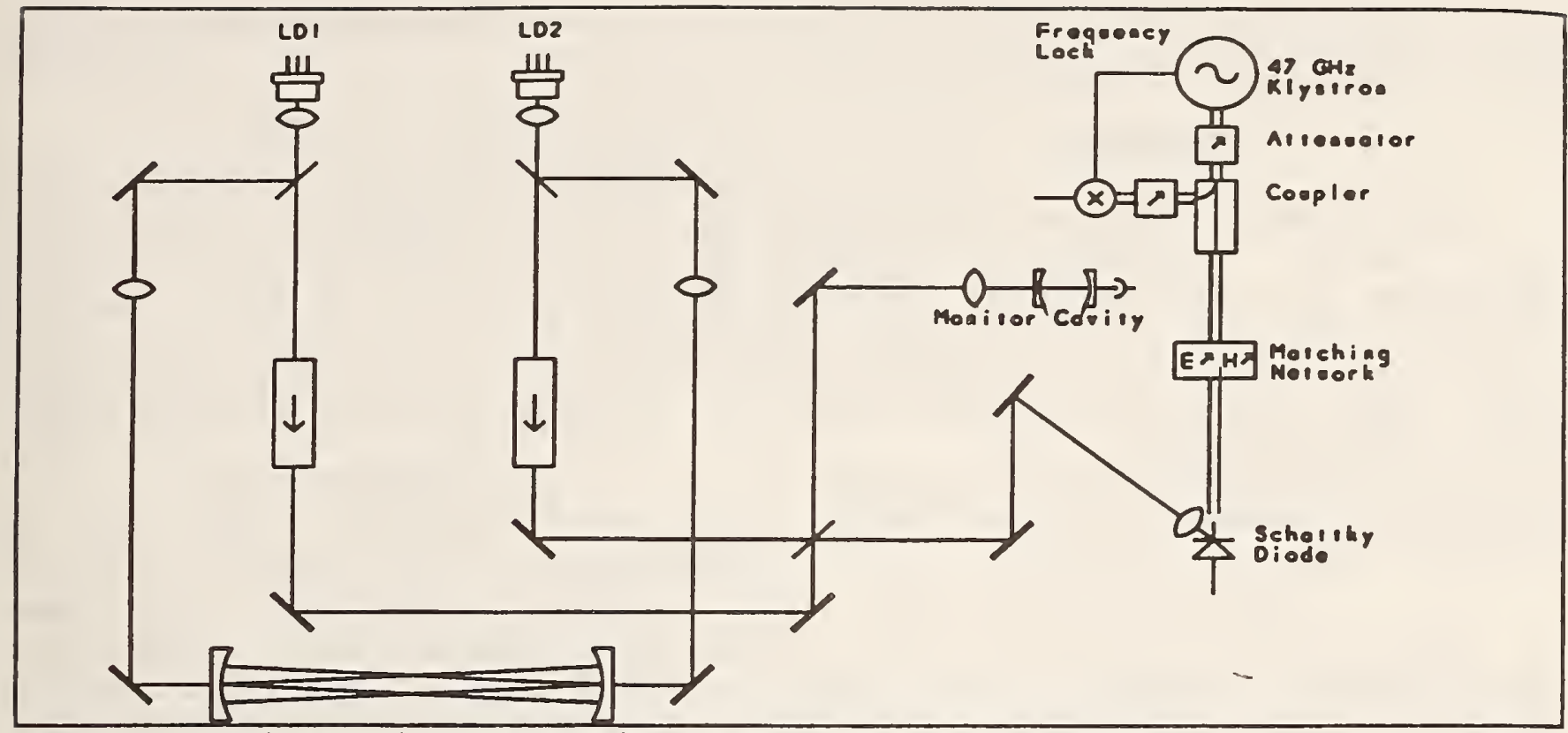

Fig. 1. Optical-microwave mixing experimental diagram.

the rectified current from the microwaves is about $1.5 \mathrm{~mA}$. The direct optical detection efficiency of the laser light, as measured from the $\mathrm{DC}$ photocurrent, is about $20 \mu \mathrm{A} / \mathrm{mW}$. Even though this value is quite low compared to silicon photodiodes $(\approx 450 \mu \mathrm{A} / \mathrm{mW}$ at these wavelengths) we measure good signal-to-noise ratios on the beatnotes. With the lasers off, the dominant noise from the detector is shot noise in the rectified microwave current.

The good signal-to-noise ratio is partly the result of the narrow spectral width of the opticallylocked diode lasers, which allows us to use a $10 \mathrm{kHz}$ bandwidth. We measured short-term laser linewidths between 1 and $10 \mathrm{kHz} .^{10}$ The laser beatnote was about $70 \mathrm{~dB}$ above the background noise in a $10 \mathrm{kHz}$ bandwidth when both lasers are optically locked. This background noise is from residual high frequency noise on the lasers. By locking both lasers to the same optical cavity we remove most of the problems of cavity vibration and drift. Even so, the lasers still have substantial low frequency jitter (approximately 100 $\mathrm{kHz}$ ) and drift, which are mainly due to the low finesse of the optical locking cavity and mechanical instabilities in the feedback phases. The present optical locking cavity

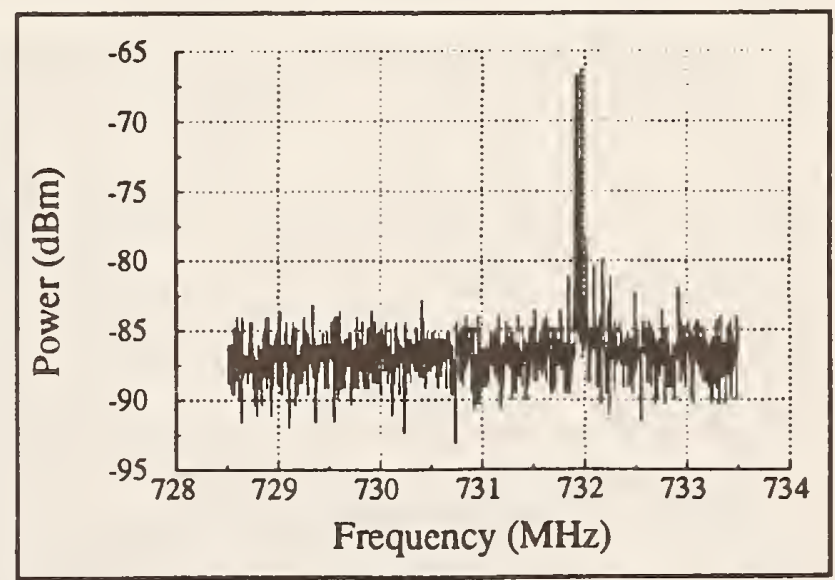

Fig. 2. Beatnote at $382 \mathrm{GHz}$ between two diode lasers detected relative to the eighth harmonic of a $47 \mathrm{GHz}$ klystron. Spectrum analyzer resolution bandwidth was $10 \mathrm{kHz}$. 
has a Eree spectral range of $300 \mathrm{MHz}$ and a finesse of about 30 .

\section{RESULTS}

By mixing the laser-laser beat note with increasingly higher harmonics of the klystron, we have observed the beat note between the two diode lasers out to almost 400 GHz. A plot of the signal-to-noise ratio (SNR) as a

function of the difference frequency between the lasers is shown in Fig. 3.

The data points in Fig. 3 correspond to the harmonics of the 47 $\mathrm{GHz}$ klystron. The signal-to-noise ratio in the baseband response of the detector in direct measurements of the laser-laser beatnote is essentially flat out to about $27 \mathrm{GHz}$. The actual electrical bandwidth of the present schottky diode mount is flat to about $8 \mathrm{GHz}$. This is presently limited by the imperfect matching of the whisker and diode mount to the $50 \Omega$ transmission line. Better matching would be helpful, but is not necessary because the signal-to-noise ratio is limited by noise, not by signal, out to $27 \mathrm{GHz}$. This $27 \mathrm{GHz}$ spans more than half of the $47 \mathrm{GHz}$ local oscillator frequency. Thus we have, at least in principle, complete coverage with this detector for any laser beat note between $\mathrm{dc}$ and $400 \mathrm{Ghz}$. In practice, for IF frequencies above about $12 \mathrm{GHz}$ an additional mixing stage with a standard microwave mixer can be helpful.

We do not know whether the rolloff we see at higher frequencies in Fig. 3 is due to some optical detection bandwidth, or whether it is due to the harmonic generation efficiency. Most probably it is the result of both. Evidence for this is seen in Fig. 4 which shows the signal-to-noise ratio as a function of harmonic number for two different LO frequencies ( $10 \mathrm{GHz}$ and $47 \mathrm{GHz}$ ). Plotted in this manner, the $47 \mathrm{GHz}$ data fall off faster than the $10 \mathrm{GHz}$ data. In contrast, if these data are plotted as a function of the laser beat frequency, the signal-to-noise ratio of the $10 \mathrm{GHz}$ data falls off very fast as the frequency approaches $100 \mathrm{GHz}$, while the $47 \mathrm{GHz}$ data fall

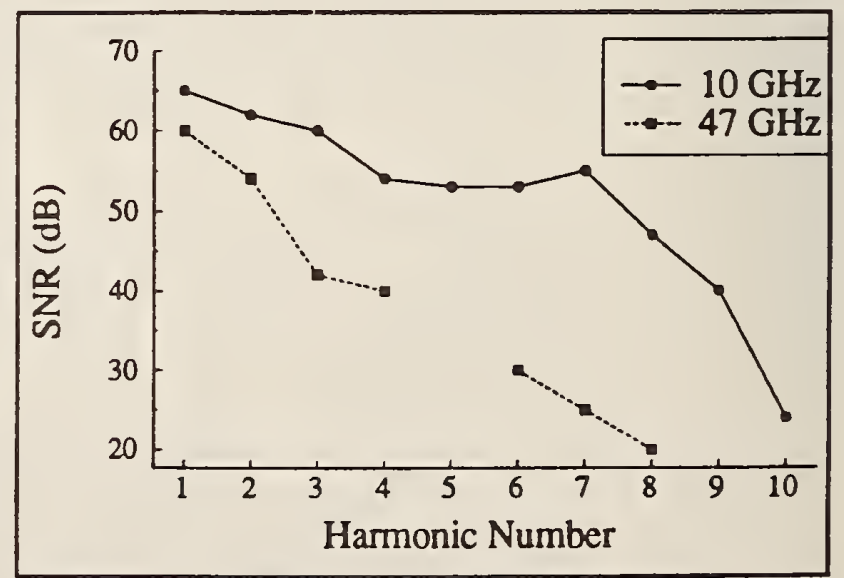

Fig. 4. Beatnote signal-to-noise ratio as a function of microwave harmonic for two different microwave frequencies, 10 and 47 $\mathrm{GHz}$. 
more slowly and extend out to about $400 \mathrm{GHz}$.

In order to better understand the general applicability of this technique we have looked at the wavelength dependence of the dc optical detection efficiency for our diodes. These results are shown in Fig. 5 and indicate that the optical detection efficiency is relatively flat between 600 and $850 \mathrm{~nm}$.

\section{CONCLUSION}

Our results demonstrate that we can now directly measure the frequency difference between tunable diode lasers out to at least $400 \mathrm{GHz}$ with a signal-to-noise ratio greater than $20 \mathrm{~dB}$ in a $10 \mathrm{kHz}$ bandwidth. Although we have a long way to go in direct measurement of optical frequencies, $382 \mathrm{GHz}$ corresponds to $0.1 \%$ of the optical laser frequency and is significantly higher than has been measured with other kinds of optical detectors. We are hopeful that by using higher LO frequencies we can push the useful beatnote detection bandwidth well beyond our present $400 \mathrm{GHz}^{14}$

\section{ACKNOWLEDGMENTS}

We gratefully acknowledge the many helpful discussions with $J$. Bergquist, K. Evenson, and J.L. Hall. This work was supported in part by NASA and AFOSR.

\section{REFERENCES}

1. D.A. Jennings, C.R. Pollock, F.R. Petersen, R.E. Drullinger, K.M. Evenson, J.S. Wells, J.L. Hall, H.P. Layer, "Direct frequency measurement of the I2-stabilized He-Ne 473-THz (633-nm) laser," Optics Lett. , Vol. 8, pp. 136-138, 1983.

2. D.J. Wineland, "Laser-to-microwave frequency division using synchrotron radiation," J. Appl. Phys, Vol. 50, pp. 2528-2532, 1979.

3. H. Telle, D. Meschede, T. Hansch, "Realization of a new concept for visible frequency division: phase locking of harmonic and sum frequencies," Optics Lett., Vol. 15, pp. 532-534, 1990.

4. N.C. Wong, "Optical frequency division using an optical parametric oscillator," Optics Lett., Vol. 15, pp. 1129-1131, 1990.

5. J.C.Bergquist, R.L.Barger D.J.Glaze, Laser Spectroscopy IV, H. Walther and K.W. Rothe eds., Springer-Verlag, 120, 1979.

6. J. Helmcke, A. Morinaga, J. Ishikawa and F. Riehle, "Optical frequency standards," IEEE Trans. Inst. Meas., Vol. 38, pp. 524-532, 1989 .

7. A.M. Akulshin, A.A. Celikov and V.L. Velichansky, "Nonlinear Dopplerfree spectroscopy of the $6{ }^{1} \mathrm{~S}_{0}-6^{3} \mathrm{P}_{1}$ intercombination transition in barium," Optics Comm., vol. 93, pp. 54-58, 1992. 
8. G.M. Tino, M. Barsanti, M. de Angelis, L. Gianfrani and M. Ingusicio, "Spectroscopy of the $689 \mathrm{~nm}$ intercombination line of strontium using and extended-cavity InGaP/InGaAlp diode laser," Appl Phys.B. pp. 397-400, 1992 .

9. L. Hollberg, R. Fox, N. Mackie, A.S. Zibrov, V.L. Velichansky, R. Ellingsen, and H.G. Robinson, "Diode Lasers and Spectroscopic Applications," Tenth International conference on Laser Spectroscopy, M. Ducloy, E. Giacobino and G. Camy, pp. 347-352, World Scientific, 1992. 10. R.W. Fox et. al. in these proceedings.

11. A. Dax, M. Mürtz, J. S. Wells, M. Schneider, E. Bachem, W. Urban and A. G. Maki, "Extension of Heterodyne Frequency Measurements on OCS to $87 \mathrm{THz}\left(2900 \mathrm{~cm}^{-1}\right)$," J. Mol. Spectroc. (accepted).

12. Arthur G. Maki and Joseph S. Wells, Frequency Calibration Tables From Heterodyne Frequency Measurements, NIST Special Publication 821, 654 pages, 1991. (printed March 1992).

13. Arthur G. Maki and Joseph S. Wells, "New Frequency Calibration Tables from Heterodyne Frequency Measurements, " J. Res. NIST, Vol. 97, pp. 409-470, 1992; \& NIST Standard Reference Database \#39, Wavelength Calibration Tables (1992). (The NIST SDR 39 four floppy disk companion set of tables of reference data is available upon request from NIST). 14. H.-U. Daniel, B. Maurer and M. Steiner, "A broadband Schottky Point contact mixer for visible laser light and microwave harmonics," Appl. Phys. B, Vol. 30, pp. 189-193, 1983.

15. J.C. Bergquist, H.-U. Daniel, "A wideband frequency-offset-locked dye laser spectrometer using a schottky barrier mixer," Opts. Comm., Vol. 48, pp. 327-333, 1984 .

16. B.Dahmani, L.Hollberg, R.Drullinger, "Frequency stabilization of semiconductor lasers by resonant optical feedback, " Opt. Lett., Vol. 12, p. 876,1987 . 


\title{
Proposed sum-and-difference method for optical-frequency measurement in the near infrared
}

\author{
D. A. Van Baak* and L. Hollberg \\ National Institute of Standards and Technology, 325 Broadway, Boulder, Colorado 80303
}

Received March 4, 1994

\begin{abstract}
We propose a method for the determination of optical frequencies in the near infrared that is based on the nonlinear generation of the optical sum and difference frequencies of two near-infrared lasers followed by the comparison of the sum and difference frequencies with standards in the visible and in the far infrared, respectively. We also address questions of practicability and discuss some examples open to the method.
\end{abstract}

The impressive stability exhibited by lasers locked to atomic and molecular standards has created a set of frequency fixed points, and some of these frequencies have been tied to the Cs-based definition of the second through the use of intricate frequency-transfer chains. Because of the complexity of such measurements, there is ample motivation both to exploit the standard frequencies already established by them and to find simpler frequency-measurement techniques applicable to general frequency targets. Here we highlight a method especially suitable for measuring optical frequencies in the near infrared. The basic idea is that, given two lasers of stable but unknown frequency, knowledge of the sum and the difference of their frequencies suffices to determine both unknown frequencies. For laser frequencies in the near infrared it is often possible to arrange for both the sum frequency to lie sufficiently near a standard in the visible and the difference frequency to lie sufficiently near a standard in the far infrared for such a measurement to be performed. Furthermore, the required nonlinear processes of sum- and difference-frequency generation can be accomplished with adequate efficiency. We conclude with examples of possible frequency measurements near laser wavelengths of $1.338,1.153$, and $1.126 \mu \mathrm{m}$.

The method proposed here is an extension of the bisection method proposed by Telle et al. ${ }^{1}$ and demonstrated by Wynands et $a l^{2}{ }^{2}$ and obeys the same algebra as the optical parametric oscillator technique proposed and demonstrated by Wong ${ }^{3}$ and by Lee and Wong, ${ }^{4}$ respectively. A special case of the method has already been demonstrated by Kourogi et al. ${ }^{5}$ We start by assuming that $\nu_{1}$ and $\nu_{2}$ are the frequencies of two stable lasers in the near infrared; of these, we treat one as the target frequency to be measured and the other as a free variable. If milliwatt beams from these two lasers are available, then the use of two separate nonlinear processes will permit the simultaneous creation of nanowatt beams of the optical sum $\left(\nu_{s}=\nu_{1}+\nu_{2}\right)$ and difference $\left(\nu_{d}=\nu_{1}-\nu_{2}\right)$ frequencies. The idea is to choose the free-variable near-infrared frequency such that both the sum and the difference frequencies fall at (or sufficiently near) standard optical frequencies previously measured with respect to the Cs-based standard. In general, the variation of only one free parameter would not allow two such matching conditions to be met, but here we take advantage of three levels of frequency flexibility. On the first level there is a growing list of well-known frequencies in the visible, ${ }^{6}$ with frequency spacings of the order of $20 \mathrm{THz}$, which can be compared with the sum frequency. On the second level there is a large list of lines of $\mathrm{CO}_{2}$ lasers ${ }^{7}$ in the $25-33-\mathrm{THz}$ range, with typical frequency spacing of the order of $20 \mathrm{GHz}$, which can be compared with the difference frequency. On the third level there is the ability to measure a heterodyne beat note of more than $400 \mathrm{GHz}$ in the visible, ${ }^{8}$ and more than $30 \mathrm{GHz}$ near $\mathrm{CO}_{2}$ wavelengths, ${ }^{9}$ between a nonlinearly derived sum or difference frequency and a visible or $\mathrm{CO}_{2}$-based standard. The result is that significant fractions of the near infrared are accessible to the proposed technique.

The easiest way to visualize the possibilities open to the method is to draw the two-dimensional $\nu_{1} \nu_{2}$ plane, in which (through the convention $\nu_{2}<\nu_{1}$ ) the arena of interest is the lower $45^{\circ}$ wedge of the first quadrant. In the plane, lines of slope -1 represent the loci of constant sum frequency; lines of slope +1 , the loci of constant difference frequency. Figure 1 shows such a plane, with some of the useful standards in the visible shown as candidate sum frequencies and with the $\mathrm{CO}_{2}$-laser lines shown as a band of candidate difference frequencies. Each intersection of a visible line with the $\mathrm{CO}_{2}$ band defines projections onto the $\nu_{1}$ and the $\nu_{2}$ axes, giving two regions (each approximately $4 \mathrm{THz}$ wide) accessible to the method; each new visible standard developed will add another two such regions. The present state of visible standards, with spacings greater than $20 \mathrm{THz}$, combined with a range of only approximately $8 \mathrm{THz}$ of available $\mathrm{CO}_{2}$ lines, implies that certain frequencies $\nu_{1}$ or $\nu_{2}$ are not yet measurable by the method.

Apart from the need for visible and far-infrared standards for comparison, this method requires ways to generate adequate power levels at the sum and the difference frequencies to be heterodyned against those standards. The nonlinear processes used will 


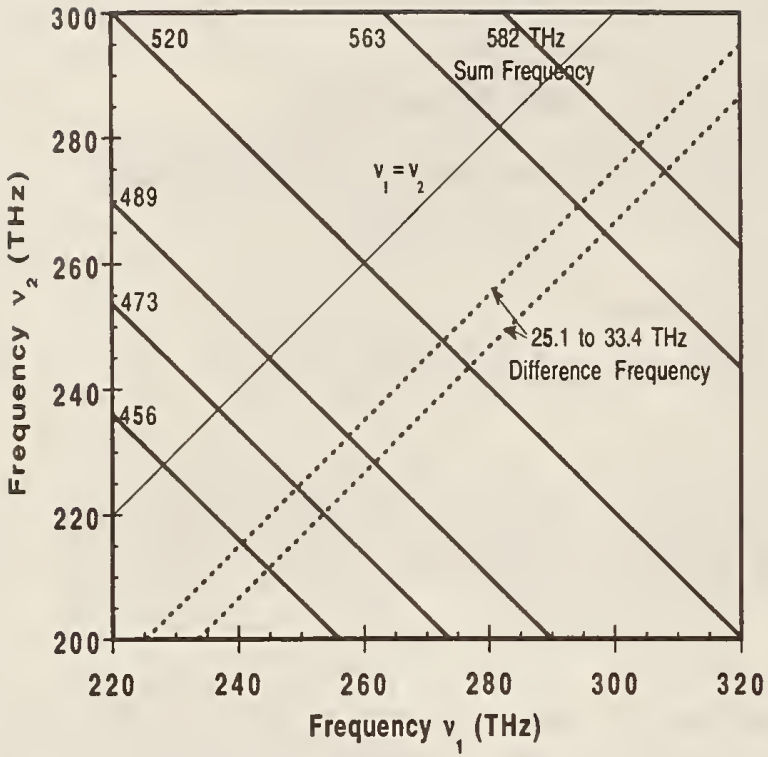

Fig. 1. A portion of the $\nu_{1} \nu_{2}$-frequency sum-anddifference plane, which shows loci of constant sum frequency for some interesting visible-laser frequencies, and the band of constant difference frequencies available from $\mathrm{CO}_{2}$ lasers.

thus need to be phase matched, either by angle or by temperature tuning. For generating the sum frequency one solution is temperature-tuned noncritically phase-matched sum-frequency generation in lithium niobate $\left(\mathrm{LiNbO}_{3}\right)$. The dispersion curves for the pure material ${ }^{10}$ give phase-matching loci (for the $o+o \rightarrow e$ process, where $o$ and $e$ denote ordinary and extraordinary axes) shown in the $\nu_{1} \nu_{2}$ plane in Fig. 2; the temperatures required are feasible (and will differ somewhat if congruently melting, or $\mathrm{MgO}$-doped, material is used). For generating the difference frequency the nonlinear material must be transparent beyond $10 \mu \mathrm{m}$; one attractive possibility is silver selenogallate $^{11}\left(\mathrm{AgGaSe}_{2}\right)$. Its dispersion curves ${ }^{12}$ give the room-temperature phase-matching locus for noncritically phase-matched difference-frequency generation (for the $e-o \rightarrow o$ process) shown in Fig. 2. The extent to which $\mathrm{AgGaSe}_{2}$ can be temperature tuned is not yet known; angle tuning of the material $\left(\theta<90^{\circ}\right)$ makes accessible the region above and to the left of the locus shown. For regions inaccessible to $\mathrm{AgGaSe}_{2}$ a useful material (although of smaller nonlinear coefficient) is proustite ${ }^{13}\left(\mathrm{Ag}_{3} \mathrm{AsS}_{3}\right)$, which at room temperature will give angle-tuned $e-o \rightarrow o$ difference-frequency generation in the required region for angles $\theta=16-20^{\circ} .^{14}$

One more choice can be exercised in the measurement of the difference frequency $\nu_{d}=\nu_{1}-\nu_{2}$. The most obvious method is to illuminate the nonlinear material with input beams at frequencies $\nu_{1}$ and $\nu_{2}$ and to extract the output beam at $\nu_{d}$. This beam, and one from a $\mathrm{CO}_{2}$ laser, can then illuminate a far-infrared photodetector to generate the beat signal. An alternative method is to use the nonlinear material as an upconverter, illuminating it with frequency $\nu_{2}$ and a beam from the $\mathrm{CO}_{2}$ laser, to produce an output $\nu_{\mathrm{up}}=\nu_{2}+\nu_{\mathrm{CO}_{2}}$; this output can be combined with the beam of frequency $\nu_{1}$ to form a beat note on a photodetector sensitive in the near infrared (where detectors of greater sensitivity and bandwidth are available). The phase-matching conditions and the polarization requirements on the nonlinear material are the same for the two alternatives.

One of the attractions of the proposed method is that the only use made of the sum and the difference frequencies is to compare them by direct heterodyning with reference lasers in the visible and the far infrared. Given the power available from visible and $\mathrm{CO}_{2}$ standards serving as local oscillators, the generation of very modest powers at the sum and the difference frequencies will suffice to give adequate beat signals at the detectors. For example, sumfrequency generation ${ }^{15}$ in a 5 -cm crystal of $\mathrm{LiNbO}_{3}$ with two $10-\mathrm{mW}$ input beams will give an output power of the order of $10 \mathrm{nW}$; when combined on a photodetector with a visible-standard beam of power $100 \mu \mathrm{W}$, this output will generate a beat note with a signal-to-noise ratio ${ }^{16}$ of approximately $48 \mathrm{~dB}$ in a bandwidth of $100 \mathrm{kHz}$. Similarly, even angle-tuned upconversion in a 0.5 -cm-long sample of proustite $^{17}$ is efficient enough that a $10-\mathrm{mW}$ beam at $\nu_{2}$, combined with a $\mathrm{CO}_{2}$ beam of $100 \mathrm{~mW} / \mathrm{mm}^{2}$, will give an upconverted output of the order of $10 \mathrm{nW}$; when combined on a photodetector with a $10-\mathrm{mW}$ beam at frequency $\nu_{1}$, this output suffices to give a signal-to-noise ratio of $52 \mathrm{~dB}$ in the same bandwidth. Such beat-note signal strengths are sufficient to permit tight phase locking of the lasers at frequencies $\nu_{1}$ and $\nu_{2}$ to the visible and $\mathrm{CO}_{2}$ standards, if desired. Thus there is no need to create new transfer-standard oscillators at the sum and the difference frequencies.

The first application of this sum-and-difference method was demonstrated by Kourogi et al. ${ }^{5}$ who locked a solid-state laser at $1.06 \mu \mathrm{m}$ and a diode laser

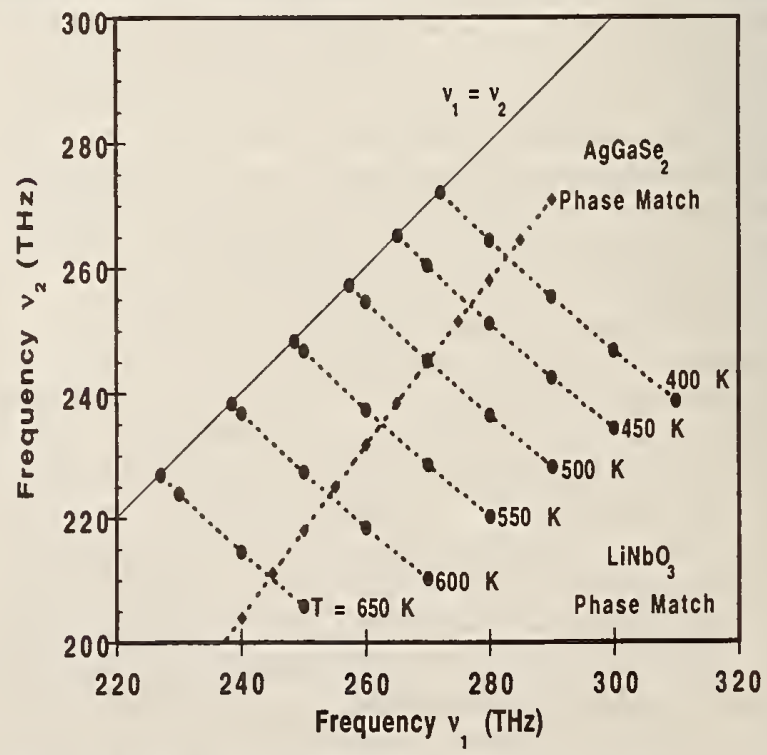

Fig. 2. Phase-matching loci in the $\nu_{1} \nu_{2}$-frequency plane. The family of curves gives the phase-matching loci for temperature-tuned type-I sum-frequency generation in pure $\mathrm{LiNbO}_{3}$, calculated with the data of Ref. 10 . The dashed curve gives the phase-matching locus for type-I difference-frequency generation in $\mathrm{AgGaSe}_{2}$ at room temperature, calculated with the data of Ref. 12 . 
at $1.55 \mu \mathrm{m}$ by beating their sum frequency against an $\mathrm{I}_{2}$-stabilized 633 -nm $\mathrm{He}-\mathrm{Ne}$ laser and their difference frequency against a $\mathrm{CH}_{4}$-stabilized 3.39- $\mu \mathrm{m}$ $\mathrm{He}-\mathrm{Ne}$ laser. With $\nu_{s}$ and $\nu_{d}$ thus fixed there is no slack in the system, and it is indeed fortunate that the required $\nu_{1}$ falls within the range of $\mathrm{Nd}$-based solid-state lasers and that the resulting $\nu_{2}$ is of such interest to the optical-fiber communication band.

As another possible application of the method, we mention the availability of diode-pumped monolithic Nd:YAG lasers ${ }^{18}$ at wavelengths of $1.064,1.319$, and $1.338 \mu \mathrm{m}$; the first two of these have had their doubled frequencies stabilized to absorption lines in iodine cells, ${ }^{19}$ and because of their compact size and their excellent passive stability all three are attractive candidates for absolute-frequency measurements. The three frequencies available are near $281.63,227.26$, and $224.06 \mathrm{THz}$, respectively. With the available $\mathrm{CO}_{2}$ lines the last of these is directly accessible to the sum-and-difference technique: if we choose the $1.338-\mu \mathrm{m}$ laser to give $\nu_{2}=224.06 \mathrm{THz}$ and build a $1.201-\mu \mathrm{m}$ laser (using available diode lasers or the $\mathrm{Cr}$-doped forsterite ${ }^{20}$ laser system) to provide $\nu_{1}=249.55 \mathrm{THz}$, then the sum frequency lies at the 473.61-THz 633-nm He-Ne standard, and the difference lies at $25.49-\mathrm{THz}$, near enough to the 25.475- $\mathrm{THz} P(38)$ line of the ${ }^{13} \mathrm{CO}_{2}$ hot band.

Another example is a remeasurement of the $1.153-\mu \mathrm{m} \mathrm{He}-\mathrm{Ne}$ laser frequency (whose second harmonic, stabilized by absorption in iodine, has already provided a $520-\mathrm{THz}$ standard in the visible). The direct remeasurement of the frequency $\nu_{1}=$ $260.103 \mathrm{THz}$ at $1.153 \mu \mathrm{m}$ is possible, given a commercially available diode-laser source at $1.305 \mu \mathrm{m}$ to provide $\nu_{2}=229.77 \mathrm{THz}$, because the sum frequency of $489.880 \mathrm{THz}$ matches the $\mathrm{I}_{2}$-stabilized 612-nm $\mathrm{He}-\mathrm{Ne}$ visible standard, and the difference frequency of $30.326 \mathrm{THz}$ can be compared with the ${ }^{13} \mathrm{CO}_{2} P(8)$ line at $30.315 \mathrm{THz}$. This remeasurement could provide a very tight consistency check on frequencies already measured by means of more complicated frequency chains.

A final example is a measurement of the $266.188-\mathrm{THz}$ frequency of the $1.126-\mu \mathrm{m} \mathrm{Nd}: \mathrm{FAP}$ laser, whose fourth harmonic matches the clock transition of the mercury ion-trap standard. ${ }^{21}$ The use of a commercially available $1.446-\mu \mathrm{m}, 207.394-$ $\mathrm{THz}$ diode-laser system would yield a sum frequency within $30 \mathrm{GHz}$ of the $633-\mathrm{nm} \mathrm{I}_{2}$-stabilized $\mathrm{He}-\mathrm{Ne}$ standard and a difference frequency of $58.794 \mathrm{THz}$ at the $P_{5}(13)$ line of the ${ }^{12} \mathrm{C}^{16} \mathrm{O}$ laser. ${ }^{22}$ This carbon monoxide frequency could in turn be stabilized with a metal-insulator-metal diode to double and to mix with the $29.407-\mathrm{THz} R(28)$ line of the ${ }^{12} \mathrm{C}^{16} \mathrm{O}_{2}$ laser. The necessary sum- and difference-frequency generation can be phase matched by temperature tuning in $\mathrm{LiNbO}_{3}$ and by angle tuning in $\mathrm{AgGaS}_{2}$, respectively.

We thank U. Simon and J. L. Hall for stimulating discussions and NASA and the U.S. Air Force Office of Scientific Research for supporting this research.

*Permanent address, Department of Physics, Calvin College, Grand Rapids, Michigan 49546.

\section{References}

1. H. R. Telle, D. Meschede, and T. W. Hänsch, Opt. Lett. 15, 532 (1990).

2. R. Wynands, T. Mukai, and T. W. Hänsch, Opt. Lett. 17, 1749 (1992).

3. N. C. Wong, Opt. Lett. 15, 1129 (1990).

4. D. Lee and N. C. Wong, Opt. Lett. 17, 13 (1992).

5. M. Kourogi, K. Nakagawa, and M. Ohtsu, IEEE J. Quantum Electron. 29, 2693 (1993).

6. Examples include the $\mathrm{I}_{2}$-stabilized $473-\mathrm{THz}(633-\mathrm{nm})$, $490-\mathrm{THz}(612-\mathrm{nm})$, and $520-\mathrm{THz}$ (doubled $1.15-\mu \mathrm{m}$ ) $\mathrm{He}-\mathrm{Ne}$ lasers ["Documents concerning the new definition of the metre," Metrologia 19, 163 (1984)] and the Rb-stabilized 385-THz (778-nm) standard [F. Nez, F. Biraben, R. Felder, and Y. Millerioux, Opt. Commun. 102, 432 (1993)]. Standards of accurately measured wavelengths include the $456-\mathrm{THz}(657-\mathrm{nm})$ Ca-stabilized and the 582-THz (514-nm) I I $_{2}$-stabilized $\mathrm{Ar}^{+}$systems. A standard likely to be measured soon is the $563-\mathrm{THz}$ (532-nm) $\mathrm{I}_{2}$-stabilized doubled Nd:YAG system.

7. More than $400 \mathrm{CO}_{2}$ lines are listed in A. G. Maki and J. S. Wells, Natl. Inst. Stand. Technol. Spec. Publ. 821, 9 (1991).

8. S. Waltman, A. Romanovsky, J. Wells, R. W. Fox, L. Hollberg, M. P. Sassi, and H. G. Robinson, Proc. Soc. Photo-Opt. Instrum. Eng. 1837, 386 (1992); Waltman has now extended heterodyne techniques beyond $600 \mathrm{GHz}$.

9. H.-W. Hübers, G. W. Schwaab, and H. P. Röser, J. Appl. Phys. 75, 4243 (1994).

10. M. V. Hobden and J. Warner, Phys. Lett. 22, 243 (1966).

11. R. S. Feigelson and R. K. Route, Opt. Eng. 26, 113 (1987); R. C. Eckhart, Y. X. Fan, R. L. Byer, R. K. Route, R. S. Feigelson, and J. van der Laan, Appl. Phys. Lett. 47, 786 (1985).

12. H. Kildal and J. C. Mikkelsen, Opt. Commun. 9, 315 (1973).

13. K. F. Hulme, O. Jones, P. H. Davies, and M. V. Hobden, Appl. Phys. Lett. 10, 133 (1967).

14. M. V. Hobden, Opto-electronics 1, 159 (1969).

15. T. H. Jeys, A. A. Brailove, and A. Mooradian, Appl. Opt. 28, 2588 (1989).

16. K. Nakagawa, M. Kourogi, and M. Ohtsu, Appl. Phys. B 57, 425 (1993).

17. J. Warner, Appl. Phys. Lett. 12, 222 (1968).

18. T. J. Kane and R. L. Byer, Opt. Lett. 10, 65 (1985); W. R. Trutna, Jr., D. K. Donald, and M. Nazarathy, Opt. Lett. 12, 248 (1987); A. B. Neilson, W. A. Clarkson, and D. C. Hanna, Opt. Lett. 18, 1426 (1993).

19. A. Arie, S. Schiller, E. K. Gustafson, and R. L. Byer, Opt. Lett. 17, 1204 (1992); A. Arie, M. L. Bortz, M. M. Fejer, and R. L. Byer, Opt. Lett. 18, 1757 (1993); J. L. Hall, in Proceedings of the Eleventh International Conference on Laser Spectroscopy, L. A. Bloomfield, T. F. Gallagher, and D. J. Larson, eds. (American Institute of Physics, New York, 1994).

20. V. Petricevic, S. K. Gayen and R. R. Alfano, Appl. Opt. 28, 1609 (1989); Opt. Lett. 14, 612 (1989).

21. J. C. Bergquist, F. Diedrich, W. M. Itano, and D. J. Wineland, in Frequency Standards and Metrology, A. De Marchi, ed. (Springer-Verlag, Berlin, 1989).

22. M. Schneider and J. S. Wells, J. Mol. Spectrosc. 139, 432 (1990). 


\title{
External-cavity difference-frequency source near $3.2 \mu \mathrm{m}$, based on combining a tunable diode laser with a diode-pumped $\mathrm{Nd}$ :YAG laser in $\mathrm{AgGaS}_{2}$
}

\author{
U. Simon \\ Department of Electrical and Computer Engineering, Rice University, Houston, Texas 77251-1892
}

S. Waltman

Time and Frequency Division, National Institute of Standards and Technology, 235 Broadway, Boulder, Colorado 80303

I. Loa and F. K. Tittel

Department of Electrical and Computer Engineering, Rice University, Houston, Texas 77251-1892

\author{
L. Hollberg
}

Time and Frequency Division, National Institute of Standards and Technology, 325 Broadway, Boulder, Colorado 80303

Received April 29, 1994; revised manuscript received August 26, 1994

\begin{abstract}
$\mathrm{AgGaS}_{2}$ has been used to generate more than $2 \mu \mathrm{W}$ of $\mathrm{cw}$ mid-infrared radiation near $3.2 \mu \mathrm{m}$ by differencefrequency mixing of the outputs of an extended-cavity diode laser near $795 \mathrm{~nm}$ (pump wave) an a compact diode-pumped Nd:YAG laser at $1064 \mathrm{~nm}$ (signal wave). An external ring enhancement cavity was used to build up the signal power inside the nonlinear crystal by as much as 14.5 times. The novel mid-infrared source incorporating a single diode laser could be angle-tuned from 3.155 to $3.423 \mu \mathrm{m}$ (from 3170 to $2921 \mathrm{~cm}^{-1}$ ). This system was used to detect the Doppler-broadened fundamental $\nu_{3}$-asymmetric stretch vibration of methane $\left(\mathrm{CH}_{4}\right)$ by both direct and wavelength-modulation absorption spectroscopy.
\end{abstract}

\section{INTRODUCTION}

Difference-frequency generation (DFG) of continuouswave (cw) tunable infrared single-frequency radiation has proved to be a useful technique for high-resolution infrared spectroscopy but was limited until recently to wavelengths shorter than $5 \mu \mathrm{m}$ by the infrared transmission characteristics of the available nonlinear optical materials $\left(\mathrm{LiNbO}_{3}\right.$ and $\left.\mathrm{LiIO}_{3}\right){ }^{1}$ Recent advances in the growth and the fabrication of nonlinear optical materials, such as $\mathrm{AgGaS}$ and $\mathrm{AgGaSe}$, now offer a convenient means for extending the wavelength coverage of DFG sources to a wider wavelength range $(3-18 \mu \mathrm{m})^{2,3}$

Semiconductor diode lasers are particularly attractive as pump sources in the nonlinear DFG process, as their compact size and ease of operation will permit the construction of portable and robust mid-infrared laser sources that are especially suitable for environmental remote sensing, pollution monitoring, chemical analysis, and medical research. Until now the nonlinear frequency conversion efficiency of all-diode-laser DFG sources has been limited by the relatively low output power of commercially available single-mode III-V diode lasers. We reported several nanowatts of $\mathrm{cw}$ midinfrared radiation ${ }^{4}$ near $5 \mu \mathrm{m}$, obtained by differencefrequency mixing of two visible-near-infrared diode lasers (2 and $10 \mathrm{~mW}$ ) in $\mathrm{AgGaS}_{2}$. Using KTP as the nonlinear optical material, Wang and Ohtsu ${ }^{5}$ generated as much as $300 \mathrm{nW}$ of near-infrared radiation $(\sim 1.6 \mu \mathrm{m})$ from two diode lasers $(50 \mathrm{~mW}$ each). One way to increase the infrared DFG output power is to use optical semiconductor amplifiers to boost the power of the single-mode diode lasers to the watt level. Recently we demonstrated difference-frequency mixing of a highpower, GaAlAs, tapered, traveling-wave, semiconductor amplifier ${ }^{6}$ with a $\mathrm{cw}$ Ti: $\mathrm{Al}_{2} \mathrm{O}_{3}$ laser in a $45-\mathrm{mm}$-long $\mathrm{AgGaS}_{2}$ crystal cut for type-I noncritical phase matching at room temperature. The diode amplifier was injection seeded by a low-power, single-mode, index-guided diode laser and provided $1.5 \mathrm{~W}$ of $860-\mathrm{nm}$ radiation for the DFG mixing experiment. As much as $47 \mu \mathrm{W}$ of cw infrared radiation and $89 \mu \mathrm{W}$ of pulsed infrared radiation, tunable near $4.3 \mu \mathrm{m}$, was generated. Alternatively, the infrared DFG output power can be increased by use of the high circulating fields inside optical resonators with the nonlinear crystal placed either in an external (passive) enhancement cavity or in one of the pump-laser cavities. The latter approach was investigated by Dixon and co-workers ${ }^{7}$ with a nonlinear KTP or $\mathrm{AgGaS}_{2}$ crystal introduced into a Nd:YAG laser cavity.

In this paper we describe a DFG source that uses a tunable extended-cavity diode laser (ECDL) near $795 \mathrm{~nm}$ (500-kHz linewidth), a diode-pumped monolithic Nd:YAG laser at $1064 \mathrm{~nm}(5-\mathrm{kHz}$ linewidth), and an external ring enhancement cavity around a $\mathrm{AgGaS}_{2}$ crystal. The enhancement cavity resonates the signal wavelength 


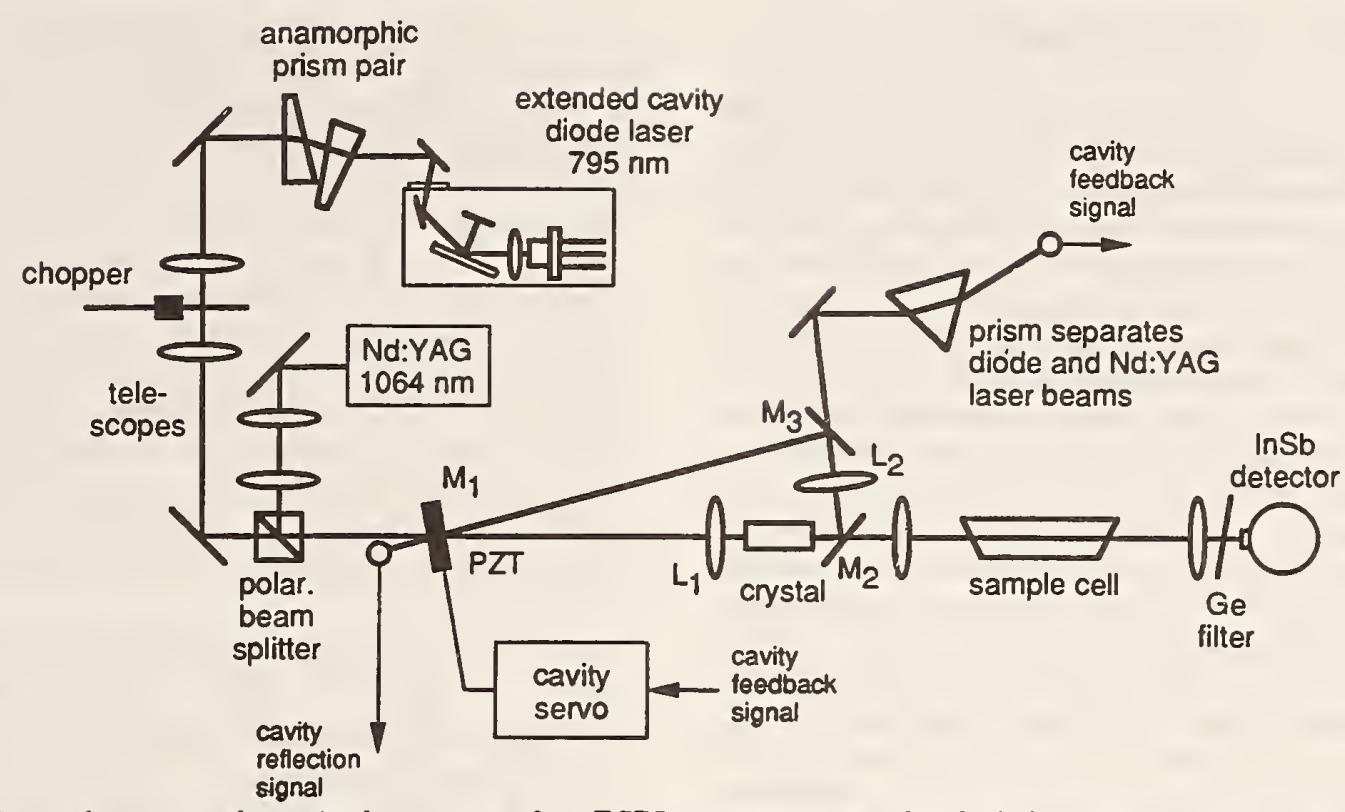

Fig. 1. Experimental setup used to mix the outputs of an ECDL near $795 \mathrm{~nm}$ and a diode-laser-pumped Nd:YAG laser at $1064 \mathrm{~nm}$ in $\mathrm{AgGaS}_{2}$ cut for $90^{\circ}$ type-I phase matching. The Nd:YAG laser radiation inside the mixing crystal is resonantly enhanced by a three-mirror ring buildup cavity.

(Nd:YAG laser), thereby increasing the signal power present inside the mixing crystal. The infrared power scales as the product of the signal and the pump powers, and hence its enhancement depends linearly on the buildup factor.

\section{EXPERIMENTAL SETUP}

The experimental diagram in Fig. 1 shows the ring buildup cavity, the ECDL, and the diode-pumped Nd:YAG laser. The $\mathrm{ECDL}^{8}$ uses an index-guided, quantum-well laser, ${ }^{9}$ a 0.60 N.A. collimating objective, and a reflection grating (2400 lines $/ \mathrm{mm}$ ) used in the Littman configuration. To improve the tuning characteristics of the ECDL, the output facet of the laser chip was antireflection (AR) coated with $\mathrm{Al}_{2} \mathrm{O}_{3}$ and $\mathrm{HfO}_{2}$. Most high-power diode lasers are supplied from the manufacturer with reduced-reflectance coatings on the front facet and highreflectance coatings on the back facet. These standard high-power lasers will work well in an ECDL configuration, but further reduction of the front facet reflectance permits a broader tuning range. An anamorphic prism pair $(6 \times)$ and a telescope were used to match the spatial mode of the ECDL approximately to the Nd:YAG laser mode in the ring buildup resonator.

The signal wave was provided by a cw diode-pumped monolithic Nd:YAG ring laser ${ }^{10}$ at $1064 \mathrm{~nm}$. Pump- and signal-wave polarizations were perpendicular for type-I phase matching in $\mathrm{AgGaS}_{2}$. Both beams were spatially overlapped with a polarizing beam splitter. Telescopes were used in both beam paths to match the beam waists at the center of the crystal. The beam waists (half-width at $1 / e^{2}$ of maximum) were set to $\sim 20 \mu \mathrm{m}$ in both vertical and horizontal planes, which is close to optimum focusing. ${ }^{2}$

To minimize thermal effects on the nonlinear conversion efficiency, the signal wave at $1064 \mathrm{~nm}$ rather than the pump wave at $795 \mathrm{~nm}$ was resonated in the buildup cavity. Owing to poor thermal conductivity $\left(\sim 0.115 \mathrm{~W} \mathrm{~cm}^{-1} \mathrm{~K}^{-1}\right), \mathrm{AgGaS}_{2}$ suffers from thermal lensing problems, especially if high-power pump sources at wavelengths close to the absorption-band edge of the material $(\sim 500 \mathrm{~nm})$ are used. ${ }^{11}$ Both to avoid degradation of the wave fronts by thermal lensing effects and to have a broad angle tuning range, we chose a short $\mathrm{AgGaS}_{2}$ mixing crystal ( $5 \mathrm{~mm}$ long) in this experiment. After AR coatings were applied to the crystal facets, the total linear absorption loss of the $\mathrm{AgGaS}_{2}$ crystal at $1064 \mathrm{~nm}$ was measured to be $\sim 1.5 \%$.

The ring buildup cavity consisted of three flat mirrors $\left(\mathrm{M}_{1}-\mathrm{M}_{3}\right)$ and two intracavity lenses $\left(\mathrm{L}_{1}, f_{L_{1}}=50 \mathrm{~mm}\right.$; $\mathrm{L}_{2}, f_{L_{2}}=25 \mathrm{~mm}$ ) together with a 5-mm-long $\mathrm{AgGaS}_{2}$ crystal cut for type-I $90^{\circ}$ phase matching. With a crystal aperture of $5 \mathrm{~mm}$ this configuration gave us access to phase-matching angles between $90^{\circ}$ and $67^{\circ}$. The total length of the ring cavity was $\sim 28 \mathrm{~cm}$, and the full angle $\Theta$ on mirror $M_{1}$ was $\sim 3^{\circ}$. The facets of the nonlinear mixing crystal were coated with a single-layer $\mathrm{Al}_{2} \mathrm{O}_{3}$ $\mathrm{AR}$ coating for low loss at $1064 \mathrm{~nm}\left(R \approx 4 \times 10^{-5}\right)$. In spite of the quality of the coatings, passive losses from the lenses ( $R<0.25 \%$ per surface, nominally) reduced the cavity buildup. However, the cavity setup shown in Fig. 1 could be realized almost exclusively with standard Nd:YAG laser optics. Mirrors $\mathrm{M}_{2}$ and $\mathrm{M}_{3}$ were highreflectance coated (nominally $R \sim 98.5 \%$ and $R \sim 99.7 \%$, respectively) for $s$-polarized light at $1064 \mathrm{~nm}$. The infrared radiation generated inside the crystal was coupled out through mirror $\mathrm{M}_{2}$, which was an $\mathrm{Al}_{2} \mathrm{O}_{3}$ substrate high-reflectance coated for $1064 \mathrm{~nm}$ with transmission $T \approx 78 \%$ for the generated difference-frequency radiation near $3 \mu \mathrm{m}$. Mirror $\mathrm{M}_{1}$ was used as the input coupler, with $T \approx 4 \%$ (nominally) at $1064 \mathrm{~nm}$ chosen to impedance match $^{12}$ the buildup cavity to the signal-wave losses. Approximately $85 \%$ of the diode laser power at $795 \mathrm{~nm}$ incident upon the cavity could be coupled through $\mathrm{M}_{1}$. 


\section{RESULTS AND DISCUSSION}

With no mixing crystal inside the buildup cavity, approximately $75 \%$ of the incident Nd:YAG laser power was coupled into the cavity and enhanced by a factor of $\sim 24.5$, corresponding to a total cavity round-trip loss of $\sim 4.1 \%$. After the crystal was introduced into the cavity, the coupling increased to $\sim 83 \%$, indicating a better match of the input coupler transmission to the total cavity round-trip loss. However, owing to the additional absorption of the mixing crystal, the buildup factor decreased to $\sim 14.5$, corresponding to a total cavity round-trip loss of $\sim 6.9 \%$.

The coupling efficiency of the Nd:YAG laser power into the buildup cavity was determined from the power reflected off the input coupler $\mathrm{M}_{1}$ while the buildup cavity was tuned over the resonance with the piezoelectrictransducer- (PZT-) driven mirror $M_{1}$. The circulating power inside the cavity could be inferred from the $\mathrm{Nd}$ :YAG laser power that leaked through mirror $\mathrm{M}_{3}$. After traversing a $60^{\circ}$ prism that separated the Nd:YAG laser beam from the diode laser beam, the transmitted signal was also used to lock the cavity resonance to the $\mathrm{Nd}$ :YAG laser frequency with a standard modulationcontrol scheme.

The difference-frequency radiation generated in the $\mathrm{AgGaS}_{2}$ crystal was collimated outside the buildup cavity with a $\mathrm{CaF}_{2}$ lens (5-cm focal length) and then detected by a liquid- $\mathrm{N}_{2}$-cooled photovoltaic detector (InSb with a 1-mm-diameter area and a factory calibrated responsivity of $\sim 1.248 \mathrm{~A} / \mathrm{W}$ at $3 \mu \mathrm{m}$ ). A broadband AR-coated germanium filter blocked pump and signal waves while transmitting the $3 \cdot \mu \mathrm{m}$ difference-frequency radiation.

Using $1064.504 \mathrm{~nm}$ as the signal wave, we found type-I $90^{\circ}$ phase matching at a diode laser wavelength of $795.5 \mathrm{~nm}$, corresponding to a difference-frequency wavelength of $\sim 3.148 \mu \mathrm{m}\left(3176.7 \mathrm{~cm}^{-1}\right)$. The laser wavelengths were determined with a spectral resolution of $\sim 30 \mathrm{MHz}$ and an absolute accuracy of $\sim 500 \mathrm{MHz}$ with the radiation coupled via a single-mode probe fiber into a lambdameter. ${ }^{13}$ The DFG phase-matching bandwidth at a phase-matching angle of $90^{\circ}$ is shown in Fig. 2. This was recorded by tuning the wavelength of the ECDL. The large phase-matching bandwidth of $\sim 8.8 \mathrm{~cm}^{-1}$ (FWHM) is in good agreement with the theoretically calculated value of $8.1 \mathrm{~cm}^{-1}$, which results mainly from the short length $(5 \mathrm{~mm})$ of the nonlinear optical mixing crystal.

Figure 3 depicts the mid-infrared DFG power at $90^{\circ}$ phase matching for a fixed diode-laser power of $12.1 \mathrm{~mW}$ (measured after the input coupler of the buildup cavity) as a function of the Nd:YAG laser power incident upon the enhancement cavity. The Nd:YAG laser power was varied by the relative angle between a polarization rotator and an analyzer. Values shown are corrected for the 3- $\mu \mathrm{m}$ transmission loss of the optical components external to the cavity ( $T \approx 89 \%$ ), but not for the transmission loss of the infrared light in the output coupler of the buildup cavity ( $T \approx 78 \%$ ). The plot shows the expected linear dependence of the generated infrared power on the signal power. However, the experimentally determined slope efficiency of $\sim 4.1 \mathrm{nW} / \mathrm{mW}$ at $12.1 \mathrm{~mW}$ of diode laser power is approximately four times less than the value that was predicted based on the theoretical model (given in Refs. 2 and 3) assuming ideal Gaussian beams and an effective nonlinear coefficient of $12 \times 10^{-12} \mathrm{~m} / \mathrm{V}$ for type-I $90^{\circ}$ phase matching in $\mathrm{AgGaS}_{2}$. This deviation between experiment and theory was also found in previous $\mathrm{AgGaS}_{2}$ DFG experiments. ${ }^{6}$ A maximum cw DFG power of $\sim 1.7 \mu \mathrm{W}$ was obtained after the external cavity with $\sim 13.6 \mathrm{~mW}$ of diode laser power and $360 \mathrm{~mW}$ of Nd:YAG laser power. Taking into account the buildup cavity output coupler transmission at $3 \mu \mathrm{m}$, this corresponds to $\sim 2.1 \mu \mathrm{W}$ of $\mathrm{DFG}$ power after the mixing crystal. Even at the high-signal-power levels circulating inside the buildup cavity, the infrared DFG power was the same for both cw and pulsed modes of operation. For cw operation the buildup cavity resonance was locked to

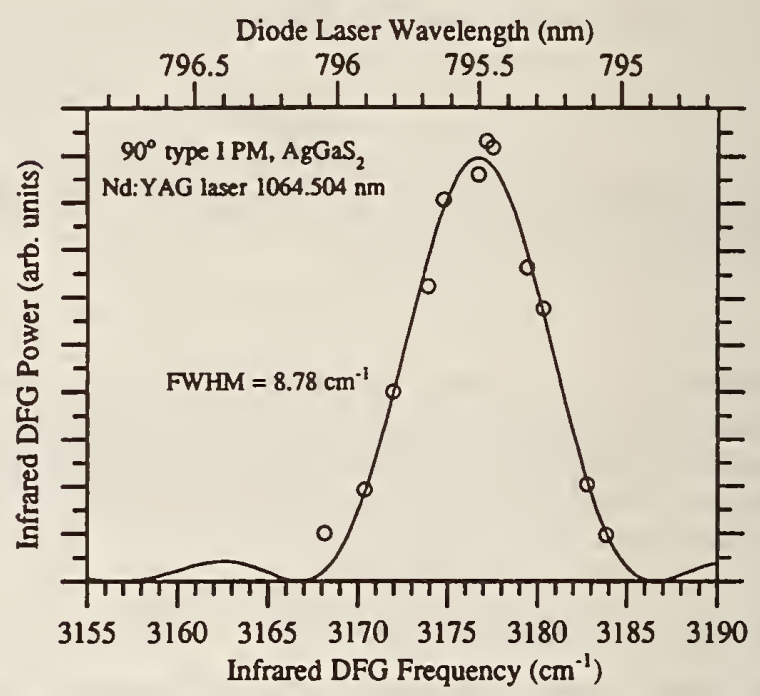

Fig. 2. Phase-matching bandwidth of the DFG mixing process at a phase-matching angle of $90^{\circ}$, recorded with the diode laser wavelength tuned. The large bandwidth of $>8 \mathrm{~cm}^{-1}$ (FWHM) is due to the 5 - $\mathrm{mm}$ length of the crystal used in the nonlinear optical mixing.

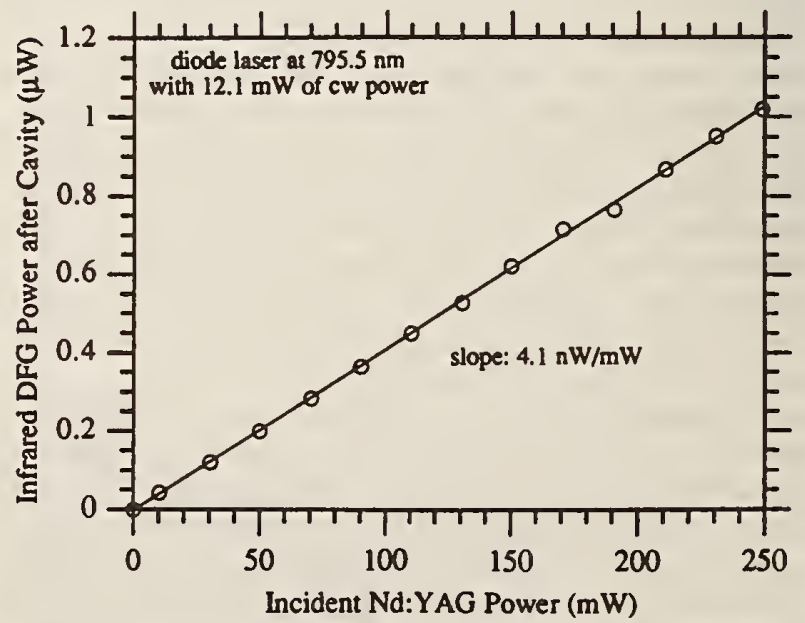

Fig. 3. Generated infrared DFG power as a function of the Nd:YAG laser power incident upon the enhancement cavity. For this measurement the diode laser power was fixed at $12.1 \mathrm{~mW}$ (measured after the input coupler of the buildup cavity). Values shown are corrected for transmission losses of the optical components external to the buildup cavity at a wavelength of $3 \mu \mathrm{m}$. 


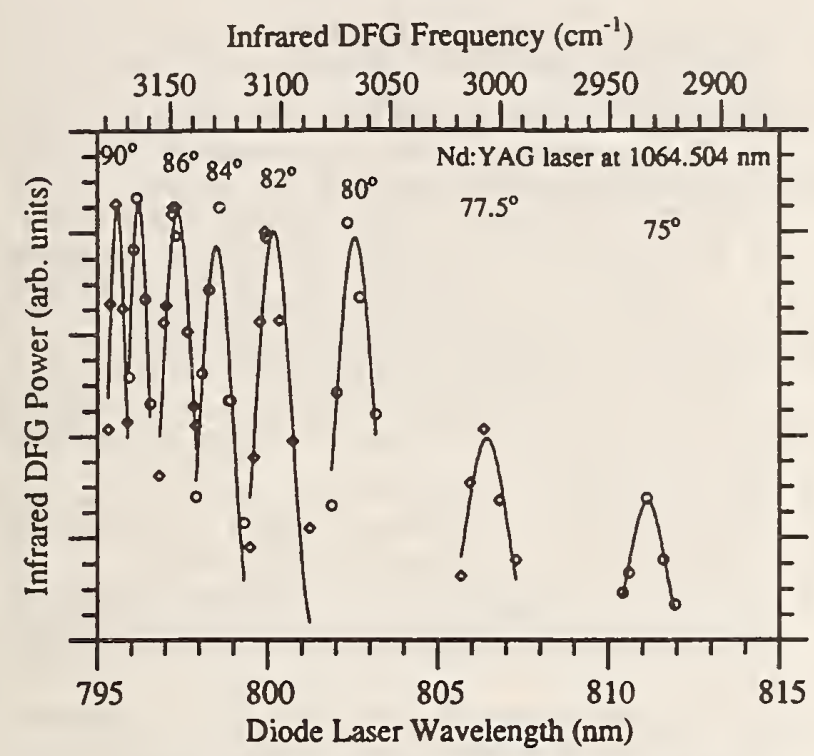

Fig. 4. Tuning range and power output of the infrared DFG radiation. Infrared tuning was accomplished by variation of the wavelength of the diode laser for a given orientation of the mixing crystal.

the Nd:YAG laser while pulsed operation was obtained by sweeping the cavity over its resonance in $\sim 0.4 \mathrm{~ms}$ and detecting the transmitted infrared signal. This indicates that, even at circulating power levels of $>3.5 \mathrm{~W}$ inside the cavity, no degradation of the nonlinear conversion efficiency by thermal lensing effects was observed.

With the wavelength of the ECDL tuned from 795 to $812 \mathrm{~nm}$ (over $\sim 17 \mathrm{~nm}$ ) and the phase-matching angle of the mixing crystal adjusted (over $\sim 15^{\circ}$ ), the infrared wavelength could be tuned from 3.155 to $3.423 \mu \mathrm{m}$ $\left(3170-2921 \mathrm{~cm}^{-1}\right)$. Figure 4 shows the relative infrared DFG power as a function of the diode laser and infrared wavelengths over the entire tuning range of the diode laser. We recorded the tuning curve by setting the phase-matching angle to a given value and tuning the diode laser over the DFG phase-matching bandwidth. The DFG power levels shown here are normalized to the incident diode-laser power, which varied from 10 to $15 \mathrm{~mW}$ over this tuning range. We achieved continuous infrared tuning ranges of $>40 \mathrm{GHz}$ without mode hopping by scanning the piezoelectric transducer on the ECDL. Figure 4 indicates that for phase-matching angles above $80^{\circ}$ the effective phase-matching length ${ }^{14}$ is actually larger than the physical length of the mixing crystal, which results in a nearly constant infrared DFG power between $90^{\circ}$ and $80^{\circ}$. For phase-matching angles smaller than $80^{\circ}$ the effective phase-matching length drops below the physical length of the crystal because of walk-off effects, resulting in an equivalent drop of the generated infrared power. Figure 5 shows the experimentally measured phase-matching points compared with the values theoretically calculated from the Sellmeier coefficients given in Ref. 15. Rotating the nonlinear crystal in the buildup cavity introduced a shift of the laser beams. Therefore at each crystal angle a realignment of the cavity was required. This can be avoided, however, by use of two crystals in a walk-off compensated cavity configuration as demonstrated by Bosenberg et al. ${ }^{16}$
To demonstrate the applicability of this compact allsolid-state DFG source, we recorded a portion of the absorption spectrum of the fundamental $\nu_{3}$-asymmetric stretch vibration of methane $\left(\mathrm{CH}_{4}\right)$ around $3.2 \mu \mathrm{m}$ (see Fig. 6). The phase-matching angle was set to $90^{\circ}$ and the ECDL wavelength was tuned over an $\sim 30-\mathrm{GHz}$ portion of the DFG phase-matching bandwidth. Only $100 \mathrm{~mW}$ of incident Nd:YAG laser power was used to record this spectrum, which corresponds to an infrared power level of $\sim 0.45 \mu \mathrm{W}$. The methane pressure in the $50 \mathrm{-cm}$-long absorption cell was set to the vapor pressure of methane at $77 \mathrm{~K}(1333 \mathrm{~Pa}, 10$ Torr $)$ with a liquid- $\mathrm{N}_{2}$-cooled reservoir. Figure 6 depicts a portion of the recorded methane absorption spectrum and demonstrates the high signalto-noise ratios that could readily be obtained. Trace 1 of Fig. 6 shows a (single-sweep) background-free absorption spectrum recorded by wavelength modulation of the diode laser (cavity length), with the $2 f$-absorption signal detected by lock-in techniques. Trace 2 of Fig. 6 shows

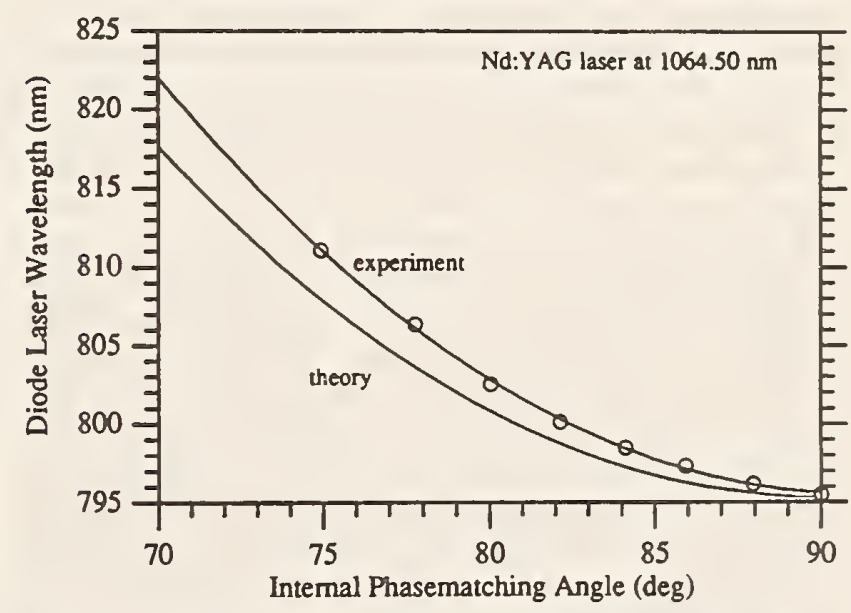

Fig. 5. Comparison of the experimentally determined (data points, circles; fit, top solid curve) and theoretically calculated (bottom curve) phase-matching angles. The calculated values are based on the Sellmeier equations given in Ref. 15.

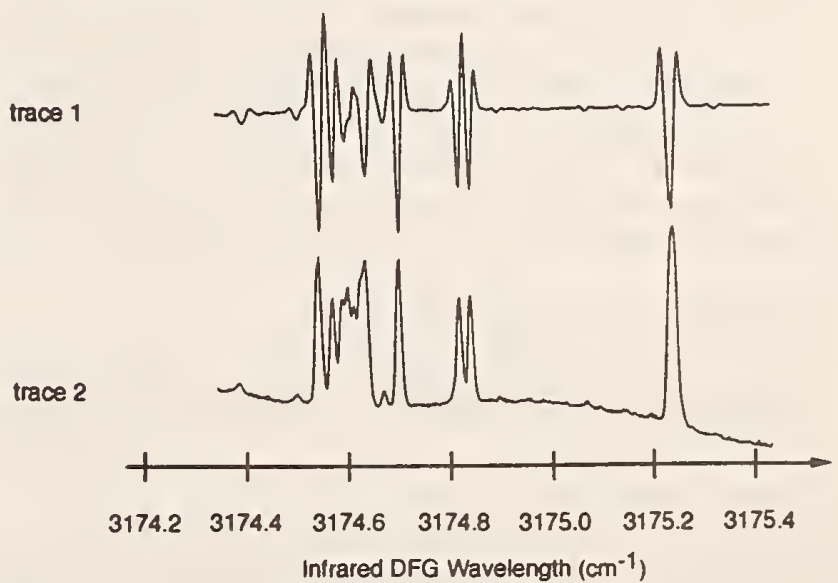

Fig. 6. Methane absorption spectrum near $3.2 \mu \mathrm{m}$ (fundamental $\nu_{3}$-asymmetric stretching motion), obtained with a $50-\mathrm{cm}-\mathrm{long}$ absorption cell and a methane pressure of $\sim 10$ Torr. Trace 1, 2f-absorption signal (single sweep) recorded with a lock-in amplifier. The spectrum was recorded at a phase-matching angle of $90^{\circ}$ with the diode laser wavelength tuned over a $30-\mathrm{GHz}$ portion of the phase-matching bandwidth; trace 2 , a direct absorption signal obtained by the averaging of 10 sweeps. 
the direct absorption spectrum of the same lines recorded with a digital oscilloscope averaging 10 sweeps. The buildup cavity was resonant only at $1064 \mathrm{~nm}$, with no measurable buildup at the diode laser wavelength. Thus no channeling on the baseline of the methane spectrum was observed when the diode laser wavelength was tuned.

\section{CONCLUSION}

In conclusion, more than $2 \mu \mathrm{W}$ of $\mathrm{cw}$ tunable infrared radiation near $3.2 \mu \mathrm{m}$ have been generated by differencefrequency mixing an ECDL (pump source) near $795 \mathrm{~nm}$ and a diode-pumped Nd:YAG laser (signal source) in $\mathrm{AgGaS}_{2}$. The signal source was resonated in a ring enhancement cavity, resulting in buildup factors for the signal power of as much as 14.5. The compact infrared DFG source (footprint of $0.5 \mathrm{~m}^{2}$ ) could be tuned from 3.155 to $3.423 \mu \mathrm{m}$ ( 3170 to $2921 \mathrm{~cm}^{-1}$ ), which coincides with the fundamental $\mathrm{CH}$ stretch vibrations of the vast majority of molecular organic compounds. The DFG source was used to detect the fundamental $\nu_{3}$-asymmetric stretching motion of methane by direct and modulation spectroscopy. Methane was chosen in this feasibility study because of its convenience and its environmental significance (it is an important greenhouse gas that supports ozone formation in polluted air). With the Nd:YAG laser line at $1.32 \mu \mathrm{m}$ as the signal wave and a tunable high-power ECDL near $855 \mathrm{~nm}$ as the pump wave, angle-tunable midinfrared radiation from 2.42 to $2.57 \mu \mathrm{m}$ (phase-matching angles $90-75^{\circ}$ ) could be generated. The cavity-enhanced DFG source demonstrated here is a useful system whenever low power consumption, relatively low cost, and no-water cooling are important and for wavelength regions in which high-power semiconductor GaAlAs optical amplifiers ${ }^{6}$ are not available. This makes it an attractive tool for wide range of applications in the mid-infrared wavelength range.

\section{ACKNOWLEDGMENTS}

This work has been supported in part by the Robert $\mathrm{A}$. Welch Foundation, the National Science Foundation, the U.S. Air Force Office of Scientific Research, and NASA. The authors thank J. L. Hall for significant contributions and Lightwave Electronics, Inc., for providing the diode-pumped Nd:YAG laser ${ }^{10}$ used in this work. U. Simon gratefully acknowledges the support of the A. v. Humboldt-Foundation by a F. Lynen Fellowship.

\section{REFERENCES AND NOTES}

1. A. S. Pine, "Doppler-limited molecular spectroscopy by difference-frequency mixing," J. Opt. Soc. Am. 64, 1683
(1974); M. G. Bawendi, B. D. Rehfuss, and T. Oka, "Laboratory observation of hot bands of $\mathrm{H}_{3}{ }^{+}$, J. Chem. Phys. 93, 6200 (1990).

2. P. Canarelli, Z. Benko, R. F. Curl, and F. K. Tittel, "A continuous-wave infrared laser spectrometer based on difference-frequency generation in $\mathrm{AgGaS}_{2}$ for highresolution spectroscopy," J. Opt. Soc. Am. B 9, 197 (1992).

3. A. H. Hielscher, C. E. Miller, D. C. Bayard, U. Simon, K. P. Smolka, R. F. Curl, and F. K. Tittel, "Optimization of a midinfrared high-resolution difference-frequency laser spectrometer," J. Opt. Soc. Am. B 9, 1962 (1992).

4. U. Simon, C. E. Miller, C. C. Bradley, R. G. Hulet, R. F. Curl, and F. K. Tittel, "Difference-frequency generation in AgGaS, by use of single-mode diode-laser pump sources," Opt. Lett. 18, 1062 (1993).

5. W. Wang and M. Ohtsu, "Frequency-tunable sum- and difference-frequency generation by using two diode lasers in a KTP crystal," Opt. Commun. 102, 304 (1993).

6. U. Simon, F. K. Tittel, and L. Goldberg, "Differencefrequency mixing in $\mathrm{AgGaS}_{2}$ by use of a high-power $\mathrm{GaAlAs}$ tapered semiconductor amplifier at $860 \mathrm{~nm},{ }^{,}$Opt. Lett. 18, (1993).

7. F. J. Effenberger and G. J. Dixon, " $2.95 \mu \mathrm{m}$ intracavity difference-frequency laser," in Digest of Topical Meeting on Advanced Solid State Lasers (Optical Society of America, Washington, D.C., 1992), p. 59; "Intracavity difference frequency generation," in Lasers and Electro-Optics, Vol. 8 of 1994 OSA Technical Digest Series (Optical Society of America, Washington, D.C., 1994), p. 380.

8. C. E. Wieman and L. Hollberg, "Using diode lasers in atomic physics," Rev. Sci. Instrum. 62, 1 (1991).

9. SDL, Inc., Model SDL5410C (mentioned to help to specify experimental parameters; other sources may be suitable).

10. Lightwave Electronics, Inc., Model 122-1064-500-F, $500 \mathrm{~mW}$ (mentioned to help to specify experimental parameters; other sources may be suitable).

11. C. E. Miller, W. C. Eckhoff, U. Simon, F. K. Tittel, and R. F. Curl, "Difference-frequency laser spectrometer using $\mathrm{AgGaS}_{2}$ : problems encountered in power scaling," in Nonlinear Optics for High-Speed Electronics and Optical Frequency Conversion, N. Peyghambarian, H. Everitt, and R. C. Eckardt, eds., Proc. Soc. Photo-Opt. Instrum. Eng. 2145, 282 (1994).

12. W. J. Kozlovsky, C. D. Nabors, and R. L. Byer, "Efficient second harmonic generation of a diode-pumped cw Nd:YAG laser using monolithic $\mathrm{MgO}: \mathrm{LiNbO}_{3}$ external resonant cavities," J. Quantum Electron. 24, 913 (1988).

13. J. L. Hall and S. A. Lee, "Interferometric real-time display of cw dye laser wavelength with sub-Doppler accuracy," Appl. Phys. Lett. 29, 367 (1976).

14. R. L. Byer and R. L. Herbst, "Parametric oscillation and mixing," in Nonlinear Infrared Generation, V. R. Shen, ed. (Springer-Verlag, New York, 1977), pp. 81-137.

15. Y. X. Fan, R. C. Eckardt, R. L. Byer, R. K. Route, and R. S. Feigelson, "AgGaS 2 infrared parametric oscillator," Appl. Phys. Lett. 45, 313 (1984).

16. W. R. Bosenberg, W. S. Pelouch, and C. L. Tang, "Highefficiency and narrow-linewidth operation of a two-crystal $\beta-\mathrm{BaB}_{2} \mathrm{O}_{4}$ optical parametric oscillator," Appl. Phys. Lett. 55, 1952 (1989). 


\title{
Division by 3 of optical frequencies by use of difference-frequency generation in noncritically phase-matched $\mathrm{RbTiOAsO}_{4}$
}

\author{
Olivier Pfister \\ JILA, Nationol Institute of Stondords ond Technology ond University of Colorodo, Boulder, Colorado 80309-0440 \\ Manfred Mürtz \\ Institute für Angewondte Physik der Universitöt Bonn, D-53115 Bonn, Germany
}

Joseph S. Wells and Leo Hollberg

Notionol Institute of Stondords ond Technology, Boulder, Colorodo 80303

James T. Murray

Opticol Sciences Center, University of Arizono, Tucson, Arizono 85721-0001

Received March 7, 1996

\begin{abstract}
A new scheme for coherently connecting optical frequencies in a 3:1 ratio has been demonstrated. To phase lock a Nd:YAG laser at $1064 \mathrm{~nm}$ with a $\mathrm{CO}$ overtone laser at $3192 \mathrm{~nm}$, we generated their difference frequency in $\mathrm{RbTiOAsO}_{4}$ (RTA) and beat it against the second harmonic of $3192 \mathrm{~nm}$ that was generated in $\mathrm{AgGaSe}_{2}$.
\end{abstract}

The ability to measure optical frequencies is a critical technology for tests of QED, as demonstrated in the beautiful experiments of Hänsch and co-workers ${ }^{1}$ and Biraben and co-workers. ${ }^{2}$ A number of promising new optical frequency references have been proposed in recent years, including trapped ions $\mathrm{s}^{3,4}$ and cooled $\mathrm{Ca}$ atoms. ${ }^{5}$ One expects higher precision and accuracy with standards at optical frequencies rather than the Cs primary frequency standard at $9.2 \mathrm{GHz}$. The ultimate linewidth of the $\mathrm{Hg}^{+}$-based system, for example, is $1 \mathrm{~Hz}$ at $10^{15} \mathrm{~Hz}$, with a projected stability of $10^{-15} \tau^{-1 / 2}$ ( $\tau$ is the averaging time). A serious limitation to the feasibility of optical references is that it is difficult to measure optical frequencies and to transfer the stability to lower frequencies, where it is compatible with electronics. This is also what makes the connection of these potential new references to each other and to the microwave standards an interesting if formidable task.

A few modern ideas for optical frequency synthesis are now being developed, including the optical cyclotron, ${ }^{6}$ the bisection method of Telle et al. ${ }^{7}$ four-wave mixing techniques ${ }^{8}$ and optical comb generators. ${ }^{9}$ These ideas often feature an attractive way to address the problem, which is the use of nonlinear mixing crystals together with solid-state and diode lasers. Divide-by-3 schemes are of particular interest for many reasons. First, as Wong discussed, ${ }^{10}$ building a frequency chain that could go from the Cs clock up to visible frequencies might require only a few lasers if $2: 1$ and $3: 1$ optical parametric oscillation (OPO) frequency dividers were combined. Also, a 3:1 frequency connection is a convenient way to jump from tunable visible lasers (diodes, Ti:sapphire) to the gas lasers that are established IR frequency standards ( $\mathrm{He}-\mathrm{Ne}$ laser locked on $\mathrm{CH}_{4}$ at $3.39 \mu \mathrm{m}, \mathrm{CO}_{2}$ laser locked on
$\mathrm{OsO}_{4}$ at $10 \mu \mathrm{m}$ ). Finally, we could combine a $3: 1$ divider with the twice-frequency-doubled Nd:FAP laser $(1126 \mathrm{~nm})$, which will be used at the National Institute of Standards and Technology to excite the $282-\mathrm{nm} \mathrm{Hg}^{+}$transition. ${ }^{11}$ Such a combination would produce a wavelength of $3378 \mathrm{~nm}$, which is close to the methane reference. If this divide-by-3 system were implemented with the 1126-nm wavelength (one fourth of the $\mathrm{Hg}^{+}$"clock" transition), we could readily generate an array of reference lines across the visible and the near IR. Adjacent elements of this array would be spaced by one twelfth of the $\mathrm{Hg}^{+}$transition frequency. In this case the wavelengths would be approximately $3378,1689,1126,844,676,563,483,422$, $375,338,307$, and $282 \mathrm{~nm}$. For the present study, with a Nd:YAG laser instead of a Nd:FAP laser, the wavelengths would be $3192,1596,1064,798,638,532$, $456,399,355,319,290$, and $266 \mathrm{~nm}$. In part to test the feasibility of these schemes, we have already used difference frequency generation ( $\mathrm{DFG}$ ) to generate wavelengths close to some of those listed above: For example, a diode laser at 657 or $800 \mathrm{~nm}$ and a Nd:YAG laser at $1064 \mathrm{~nm}$ have been mixed to produce 1700 or $3200 \mathrm{~nm},{ }^{12}$ respectively. Recent high-precision measurements ${ }^{13}$ of an $\mathrm{I}_{2}$ electronic transition near $532 \mathrm{~nm}$ do make the Nd:YAG laser an interesting starting point.

A 3:1 connection (through second-order frequency mixing) is achievable with two input waves, such as sum-frequency generation $(f+2 f \mapsto 3 f)$ or DFG $(3 f-f \mapsto 2 f, 3 f-2 f \mapsto f)$, or with a single input wave, that is, OPO $(3 f \mapsto f+2 f)$. An exact connection requires another mixing stage to lock the 3:1 ratio (the phase-matching requirements of the crystal are not precise enough). We need two different mixings of $(f, 2 f, 3 f)$ to guarantee a division by 3 . 
For example, we can use OPO $(3 f \mapsto f+2 f)$ and second-harmonic generation (SHG, $f \mapsto 2 f$ ), or we can use DFG $(3 f-f \mapsto 2 f)$ and SHG $(f \mapsto 2 f)$, to obtain a beat note at $2 f$, which can then be used to phase lock the OPO resonator to the $3 f$ pump or to lock the two DFG in put lasers together, respectively. The OPO beat note is actually much less sensitive to pump frequency noise than is the DFG beat note, for which the fluctuations of two independent lasers do not cancel each other. However, because of the additional problems related to OPO threshold, and because two good laser sources are available, we chose to build a DFG-SHG setup first. The frequencies involved are

$$
f(1064 \mathrm{~nm})-f(3192 \mathrm{~nm}) \mapsto f(1596 \mathrm{~nm}) .
$$

The input wavelengths were provided by a diodepumped Nd:YAG monolithic ring laser (output power $530 \mathrm{~mW}$ ) and by a $\mathrm{CO}$ overtone laser. ${ }^{14} \mathrm{CO}$ overtone lasers are not common but are nonetheless remarkable mid-IR sources. They were first developed in W. Urban's group in Bonn, Germany. They provide laser oscillation on 400 rotational lines, spread over 28 vibrational $\lrcorner v=2$ transitions between 2.6 and $4.1 \mu \mathrm{m}$, with an output power as high as $200 \mathrm{~mW}$ in our case. Fortuitously, three times the frequency of the $3193.5-\mathrm{nm} P_{21}(14)$ line is only $4 \mathrm{GHz}$ away from the center of the Nd:YAG gain curve. This falls well within the $30-\mathrm{GHz}$ temperature-tuning range of the Nd:YAG monolithic oscillator.

Of the many crystals that permit phase matching for interaction (1) with reasonable nonlinear coefficients and low absorption $\left(\mathrm{LiNbO}_{3}, \mathrm{KNbO}_{3}, \mathrm{AgGaS}\right.$, proustite), none gives noncritical phase matching at a convenient temperature, which is a prerequisite if one hopes eventually to build a cw OPO system. Alkali metal-titanyl arsenate crystals (KTA, RTA, CTA) are of prime interest here: While they retain all the properties of their better-known parent KTP (reasonable nonlinearity, reduced walk-off in the $X-Y$ plane, high optical damage threshold, very high ferroelectric Curie temperature), they lack the phosphate-ion vibrational resonances that lead to significant absorption at $3.5 \mu \mathrm{m} .^{15}$ Also, $\mathrm{RTA}^{16}$ gives noncritical phase matching at room temperature for ${ }^{17}$

$$
f(1064 \mathrm{~nm})-f(3137 \mathrm{~nm}) \mapsto f(1610 \mathrm{~nm}) .
$$

We observed that interaction (1) is phase matched in RTA at $335^{\circ} \mathrm{C}$. We predicted this temperature by measuring the phase-matching bandwidth at $3 \mu \mathrm{m}$ by tuning the $\mathrm{CO}$ overtone laser through its $v=23 \rightarrow 21$ emission band. A least-squares fit with a sinc ${ }^{2}$ function gave us a $552 \pm 25 \mathrm{GHz}$ FWHM bandwidth, which, when divided by a temperature bandwidth of $100^{\circ} \mathrm{C}$ (obtained from KTP's temperature dependence ${ }^{18}$ ), yields an approximate temperature-tuning coefficient of $-5.52 \mathrm{GHz} /{ }^{\circ} \mathrm{C}$. Thus, to displace the phase-matching central wavelength from $3137 \mathrm{~nm}$ [interaction (2)] at room temperature to $3193.5 \mathrm{~nm}$ $(-1691 \mathrm{GHz})$, one should increase the temperature by $306{ }^{\circ} \mathrm{C}$. This is in fair agreement with the experimental determination and indicates that KTP's temperature dependence is a good first guess for arsenates as well. This large temperature acceptance gives sluggish temperature tuning, but, on the other hand, the sensitivity of the DFG interaction to temperature fluctuations is very low. We calculated the second-derivative FWHM angular bandwidth to be $8.9^{\circ}$. Our attempt to measure it was not successful because of the inhomogeneity of our crystal.

The propagation in the biaxial crystal was along the $X$ axis. Since the interaction is of type II (oee) in the $X-Y$ plane, the polarizations were along the $Z$ axis for the $3192-\mathrm{nm}$ ordinary beam and along the $Y$ axis for the 1064- and 1596-nm extraordinary beams. The $7.25 \mathrm{~mm} \times 5 \mathrm{~mm} \times 5 \mathrm{~mm}$ RTA sample that we used was cut at $30^{\circ}$ from the $Z$ axis, so we had to use it sideways (normal axis at $30^{\circ}$ from the $X$ axis) with an $\sim 65^{\circ}$ incidence angle (Fig. 1). This alignment is close to Brewster incidence for the $p$-polarized light at $3192 \mathrm{~nm}$, but it gives Fresnel losses of $\sim 33 \%$ on the uncoated faces for the two other, $s$-polarized, beams. It also results in significant astigmatism, since the light was focused in the crystal. (Calculated waist sizes were $23 \mu \mathrm{m}$ for the Nd:YAG beam and $40 \mu \mathrm{m}$ for the $\mathrm{CO}$ beam.) The experimental setup is diagrammed in Fig. 2: The two pump beams were combined by means of a dichroic mirror (DM) and a $35-\mu \mathrm{m}$-diameter pinhole placed at the common waist position. A removable mirror mount of kinematic design allowed us to send the two beams into the RTA crystal, which was enclosed in a Au-plated $\mathrm{Cu}$ oven and heated to $330-340^{\circ} \mathrm{C}$. Two fusedsilica mirrors, with transmittances of $\sim 10^{-4}$ at $1064 \mathrm{~nm}$ and $90 \%$ at $1.6 \mu \mathrm{m}$, were used between the crystal and the detector to block the powerful Nd:YAG radiation and also to absorb the lessintense $3.2 \mu \mathrm{m}$ light. Part of the $\mathrm{CO}$ beam was simultaneously frequency doubled in a $\mathrm{AgGaSe}_{2}$ crystal. This produced a second $1.6-\mu \mathrm{m}$ beam, which was overlapped on a fast photodetector with the DFG signal. The $(R \sim 1 / 3)$ beam splitter on the $\mathrm{CO}$ laser beam path was chosen to maximize the power on the beat detector:

$$
P_{\text {beat }} \propto\left[\left(T P_{1}\right)^{2} R P_{1} P_{3}\right]^{1 / 2} \propto R^{1 / 2}(1-\mathrm{R}) .
$$

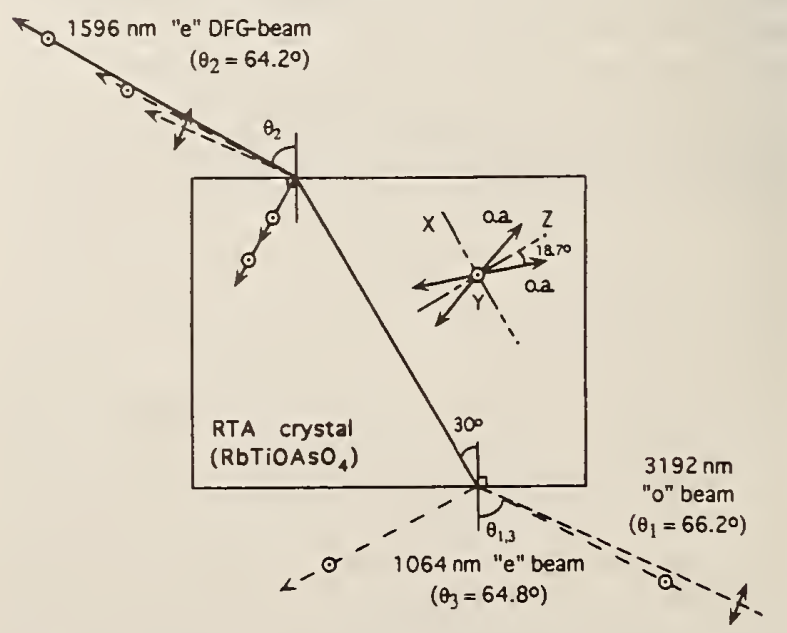

Fig. 1. RTA crystal: beam alignments and polarizations, optical axes (o.a.), and principal axes $(X, Y, Z)$. 


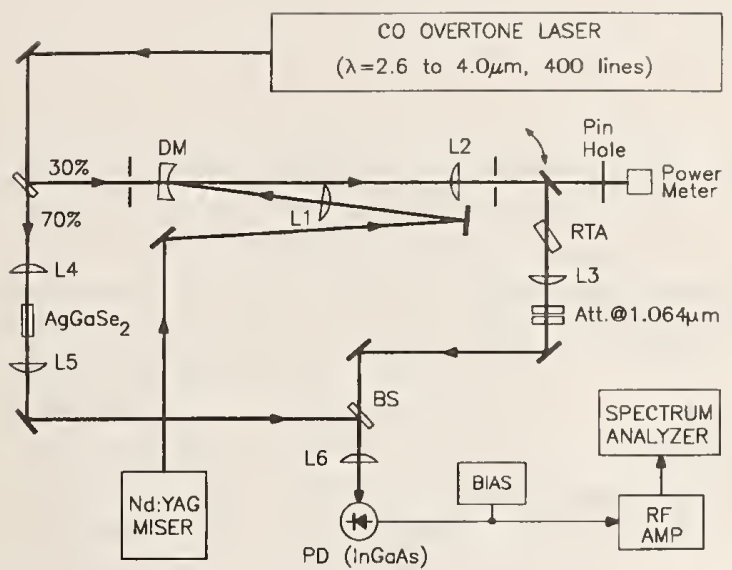

Fig. 2. Experimental setup: L1-L6, lenses; BS, beam splitter; PD, photodiode.

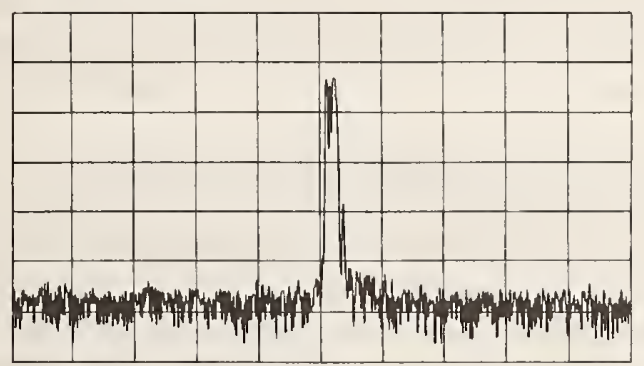

Fig. 3. Spectrum of the beat note between DFG and SHG 1596-nm waves. Vertical scale, $10 \mathrm{~dB} /$ division; horizontal scale, $500 \mathrm{kHz} /$ division; resolution bandwidth $30 \mathrm{kHz}$; center frequency $6 \mathrm{MHz}$.

The powers at $1596 \mathrm{~nm}$ at the photodetector were $25 \mathrm{nW}$ for the DFG beam and $1 \mu \mathrm{W}$ for the SHG beam. A $60-\mu$ m-diameter InGaAs photodiode (bandwidth $5 \mathrm{GHz}$ ) was used to detect the beat note, which was amplified ( $40 \mathrm{~dB}$ gain, $60 \mathrm{MHz}$ bandwidth) and sent to a spectrum analyzer (Fig. 3 ). The $40-\mathrm{dB}$ signalto-noise ratio should be adequate to phase lock the two lasers together. Fast frequency control of the $\mathrm{CO}$ laser is now being developed for this purpose. A frequency lock between the lasers has been established and gives a beat note with a $150-\mathrm{kHz}$ linewidth (owing to the jittering of a narrower peak under acoustical and discharge noise in the CO laser) and a $1-\mathrm{kHz}$ center frequency stability.

We have demonstrated a coherent frequency connection in an exact 3:1 ratio between 1064 and $3192 \mathrm{~nm}$. The fact that RTA noncritically phase matches this process is encouraging for the extension of this research to an OPO system. We also look forward to realizing the $1126-3378-\mathrm{nm}$ link on the way to connecting the $\mathrm{Hg}^{+}$282-nm transition to the primary Cs standard.

We thank G. M. Loiacono (Crystal Associates, Inc.) for growing the RTA crystal, R. A. Stolzenberger (Crystal Associates, Inc.) for information about RTA, and S. Waltman and J. L. Hall for significant contributions to this research. This research is supported in part by the U.S. Air Force Office of Scientific Research. M. Mürtz acknowledges support from the German
Science Foundation; O. Pfister acknowledges a Lavoisier grant from the Ministère des Affaires Etrangères of France.

\section{References}

1. T. Andreae, W. König, R. Wynands, F. Schmidt-Kaler, C. Zimmermann, D. Meschede, and T. W. Hänsch, Phys. Rev. Lett. 69, 1923 (1992).

2. F. Nez, M. D. Plimmer, S. Bourzeix, L. Julien, F. Biraben, R. Felder, O. Acef, J. J. Zondy, P. Laurent, A. Clairon, M. Abed, Y. Millerioux, and P. Juncar, Phys. Rev. Lett. 69, 2326 (1992).

3. D. J. Wineland, J. C. Bergquist, W. M. Itano, F. Diedrich, and C. S. Weimer, in The Hydrogen Atom, G. F. Bassani, M. Inguscio, and T. W. Hänsch, eds. (Springer-Verlag, Berlin, 1989), p. 123.

4. E. Peik, G. Hollemann, and H. Walther, Phys. Rev. A 49, 402 (1994); H. Lehmitz, J. Hattendorf-Ledwoch, R. Blatt, and H. Harde, Phys. Rev. Lett. 62, 2108 (1989); G. P. Barwood, C. S. Edwards, P. Gill, G. Huang, H. A. Klein, and W. R. C. Rowley, IEEE Trans. Instrum. Meas. 44, 117 (1995); B. G. Whitford, K. J. Siemsen, A. A. Madej, and J. D. Sankey, Opt. Lett. 19, 356 (1994).

5. H. Schnatz, B. Lipphardt, J. Helmcke, F. Riehle, and G. Zinner, Phys. Rev. Lett. 76, 18 (1996).

6. J. C. Bergquist and D. J. Wineland, in Proceedings of the Thirty-Third Annual Symposium on Frequency Control (Electronic Industries Association, Washington, D.C., 1979), p. 494.

7. H. R. Telle, D. Meschede, and T. W. Hänsch, Opt. Lett. 15, 532 (1990).

8. H. R. Telle, in Proceedings of the Twelfth International Conference on Laser Spectroscopy, M. Inguscio, M. Allegrini, and A. Sasso, eds. (World Scientific, Singapore, 1996), p. 230.

9. M. Kourogi, K. Nakagawa, and M. Ohtsu, IEEE J. Quantum Electron. 29, 2693 (1993).

10. N. C. Wong, Appl. Phys. B 61, 143 (1995); Opt. Lett. 17, 1155 (1992).

11. F. C. Cruz, M. Rauner, J. H. Marquardt, L. Hollberg, and J. C. Bergquist, in Proceedings of the Fifth Symposium on Frequency Standards Metrology, J. C. Bergquist, ed. (World Scientific, Singapore, 1996), p. 511 .

12. U. Simon, S. Waltman, I. Loa, F. K. Tittel, and L. Hollberg, J. Opt. Soc. Am. B 12, 323 (1995).

13. P. A. Jungner, S. Swartz, M. Eickoff, J. Ye, J. L. Hall, and S. Waltman, IEEE Trans. Instrum. Meas. 44, 151 (1995).

14. A. Dax, J. S. Wells, L. Hollberg, A. G. Maki, and W. Urban, J. Mol. Spectrosc. 168, 416 (1994); E. Bachem, A. Dax, T. Fink, A. Weidenfeller, M. Schneider, and W. Urban, Appl. Phys. B 57, 185 (1993).

15. J. C. Jacco and G. M. Loiacono, Appl. Phys. Lett. 58, 560 (1991); K. Kato, IEEE J. Quantum Electron. 27, 1137 (1991).

16. L. T. Cheng, L. K. Cheng, and J. D. Bierlein, in Growth, Characterization, and Applications of Laser Host and Nonlinear Crystals II, B. H. Chai, ed., Proc. SPIE 1863, 43 (1993).

17. RTA Sellmeier coefficients published in Ref. 16 are not accurate at $3 \mu \mathrm{m}$. Interaction (2) was measured by R. A. Stolzenberger, Crystal Associates, Inc.

18. K. Kato, IEEE J. Quantum. Electron. 28, 1974 (1992). 
Demonstration of a phase-lockable microwave to submillimeter-wave sweeper

\author{
S. B. Waltman, L. W. Hollberg \\ National Institute of Standards and Technology \\ Boulder, Colorado 80303-3328 \\ K. A. McIntosh \\ Lincoln Laboratory \\ Massachusetts Institute of Technology \\ Lexington, Massachusetts 02173-9108 \\ E. R. Brown \\ Defense Advanced Research Projects Agency \\ Arlington, Virginia 22203-1714
}

\begin{abstract}
The development of low-temperature-grown GaAs photomixers enables the construction of a microwave to submillimeter-wave source capable of large frequency sweeps. By utilizing semiconductor diode lasers to drive the photomixer, this source is all solid-state and compact, and has small power consumption. Frequency stabilization of the semiconductor diode lasers allows this source to be phase-locked to an external microwave reference. Two $805 \mathrm{~nm}$ extended-cavity-diode lasers are mixed in a low-temperature-grown GaAs photoconductive photomixer. The differencefrequency mixing product is radiated by a planar spiral antenna and collimated by a Si lens. This output is phase-locked to a microwave reference by downconverting it in a whisker-contacted Schottky-barrier diode harmonic mixer and using the output to offset-phase-lock one laser to the other. The photomixer output power is $300 \mathrm{nW}$ at $200 \mathrm{GHz}$ and $10 \mathrm{nW}$ at 1.6 $\mathrm{THz}$, as measured by a $4 \mathrm{~K}$ InSb bolometer calibrated with a methanol laser and a power meter at 526 and $812 \mathrm{GHz}$.
\end{abstract}

\title{
1. INTRODUCTION
}

The earliest and possibly the most important application of submillimeter-wave radiation was spectroscopy.' As submillimeter-wave sources have become more compact and robush, spectroscopic applications outside the laboratory, such as remote sensing for atmospheric science and astrophysics, have become practical. The most serviceable present submillimeter-wave source is a $50-100 \mathrm{GHz}$ Gunn diode oscillator harmonically multiplied with a whisker-contacted Schattky-diode varactor. However, this source is not yet electrically tunable over even a $10 \%$ bandwidth with high power. ${ }^{2}$

Optical heterodyne conversion, or photomixing, was proposed as a method for producing coherent microwave and millimeter-wave radiation over three decades ago. ${ }^{3}$ But application of this method was delayed until the development of suitable photomixers. Such photomixers have recently become possible with the advent of low-temperature-grown (LTG) GaAs with photoconductive response, sub-picosecond electron-hole recombination time, and $>10^{5} \mathrm{~V} \cdot \mathrm{cm}^{-1} \mathrm{dc}$ breakdown field properties." Photomixers made from this material have produced as much as about $3 \mu \mathrm{W}$ of output power with a $3 \mathrm{~dB}$ bandwidth of $650 \mathrm{GHz}^{5.6}$

One advantage of photomixing over other ways of generating millimeter and submillimeter-wave radiation is the ease of tuning the output frequency by tuning one of the pump lasers. Diode lasers with a single-mode frequency sweep range of $1.4 \mathrm{THz}$ at $100 \mathrm{~Hz}$ sweep rate have been demonstrated. Since the photomixer conversion efficiency is approximately constant below its comer frequency, multi-octave frequency sweeps with minimal amplitude variation are possible.

A nice demonstration of the spectroscopic capabilities of these photomixers has been made by A. Pine. ${ }^{7}$ A spectrometer based on LTG GaAs photomixers was used to study $\mathrm{SO}_{2}$ self broadening coefficients in the $0.1-1.0 \mathrm{THz}$ 
frequency range. In this instrument, the photomixer was driven by two single-mode dye lasers which were pumped by an argon ion laser. Subsequent work demonstrated operation of LTG GaAs photomixers pumped by semiconductor diode lasers. ${ }^{6}$ Semiconductor diode lasers are ideal for driving photomixers because they are compact, efficient, tunable, and relatively inexpensive. They also have sufficient output power and can be spectrally narrow. The extended-cavity tunable diode lasers used in this work occupy $780 \mathrm{~cm}^{3}$, consume less than $1 \mathrm{~W}$ of electrical power, and can be mechanically tuned over at least $14 \mathrm{THz}$.

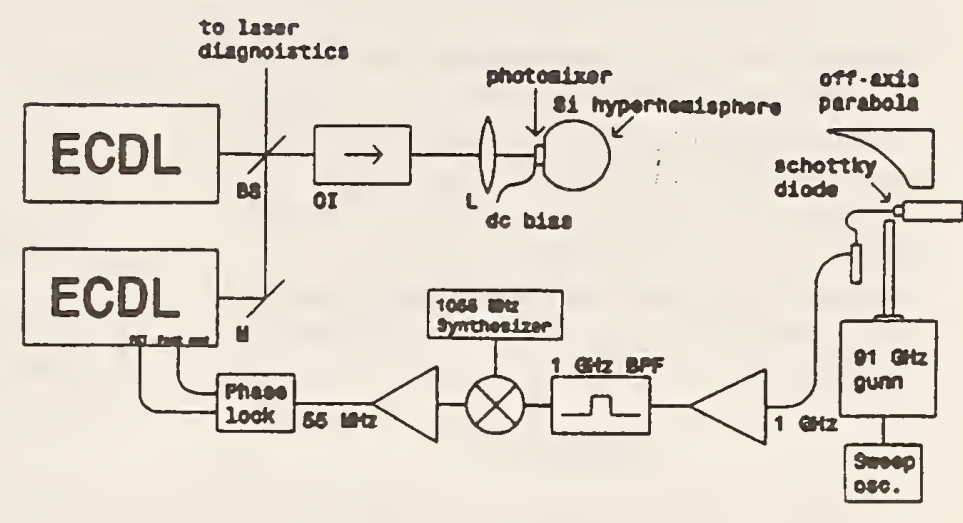

Figure 1. Experimental setup. The extended-cavity diode lasers use commercial $100 \mathrm{~mW}, 805 \mathrm{~nm}$ devices.

The line width and stability of the radiation produced by the photomixer is determined by the lasers used to drive it. The lasers in this work have linewidths of approximately $100 \mathrm{kHz}$ and low-frequency jitter of a few megahertz. By coherently detecting the radiation, we can phase-lock the offset between the two lasers to a harmonic of a microwave source. The linewidth, stability, and accuracy of the millimeter and submillimeter radiation are then determined by the phase noise, stability, and accuracy of the microwave source.

\section{EXPERIMENT DESCRIPTION}

The photomixers used in this work consist of interdigitated electrodes on an epitaxial layer of LTG GaAs. The electrodes are $0.2 \mu \mathrm{m}$ wide, separated by $1.8 \mu \mathrm{m}$ wide gaps, and cover a total area of $8 \times 8 \mu \mathrm{m}$. The electrodes drive a threeturn self-complementary spiral antenna. The output radiation propagates through the GaAs substrate and is coupled out of the substrate and partially collimated by a hyperhemispherical Si lens mounted in contact with the back of the GaAs chip.

The experimental setup is illustrated in Figure 1. The photomixer is driven by two extended-cavity diode lasers (ECDLs). Each is a commercial $100 \mathrm{~mW} 805 \mathrm{~nm}$ diode laser with an anti-reflection coating on the output facet. The extended cavities are in a Littman configuration, ${ }^{8}$ and the output frequency of the laser is adjurted by tilt and translation of the mirror. Adjustment screws on the mirror mount provide coarse tuning with a range of $14 \mathrm{THz}$, and piezoelectric actuators provide fine tuning. Single mode scans of $19 \mathrm{GHz}$ are possible in the present system by electronically matching the ratio of tilt and translation to synchronize the bandpass frequency determined by the grating with the frequency of an extended-cavity mode. This tuning range is determined by the $9 \mu \mathrm{m}$ translation range of the piezoelectric actuators used and the cavity dimensions. Special lasers designed for long-scanning range have demonstrated multi-terahertz continuous scans. For phase-locking, electronic feedback to the injection current provides fast frequency control with a $3 \mathrm{~dB}$ bandwidth of approximately $2 \mathrm{MHz}$.

The two laser beams are combined by a beam splitter and then circularized with an anamorphic prism pair. After passing through two Faraday isolators, the combined beam is resized by a telescope and focused on the photomixer by a lens. The beam size and lens focal length were chosen to produce a $7 \mu \mathrm{m}$ spot size on the photomixer. Typical powers from each of the lasers are $9 \mathrm{~mW}$ and $22 \mathrm{~mW}$. The performances of the two lasers differ due to differences in the quality of their anti-reflection coatings.

The dc photocurrent and the millimeter-wave output power both increase with increasing de bias across the photomixer electrodes. At $30 \mathrm{~V}$ bias the responsivity was $14 \mathrm{~mA}$ w. Electrical or thermal burnout of the photomixer becomes likely at higher bias voltages or at total optical power levels above $100 \mathrm{~mW}$. 
The output radiation of the photomixer is focused by an off-axis paraboloid onto the long-wire whisker antenna of a point-contact Schottky diode. A $91 \mathrm{GHz}$ local oscillator signal from a Gunn diode is also coupled onto the whisker. The Schortky diode down-converts the millimeter or submillimeter radiation from the photomixer by mixing it with a harmonic of the local oscillator. The downconverted signal near $1 \mathrm{GHz}$ is amplified, filtered, and then sent to a spectrum analyzer and a phaselocking circuit.

\section{RESULTS}

The power produced by the photomixer was measured by focusing it onto an InSb bolometer with a polyethylene lens; the result of these measurements is shown in Figure 2. The bolometer's response was calibrated at $526 \mathrm{GHz}$ and $812 \mathrm{GHz}$ by using an attenuated methanol laser whose full output power was measured separately with a broadband power meter.

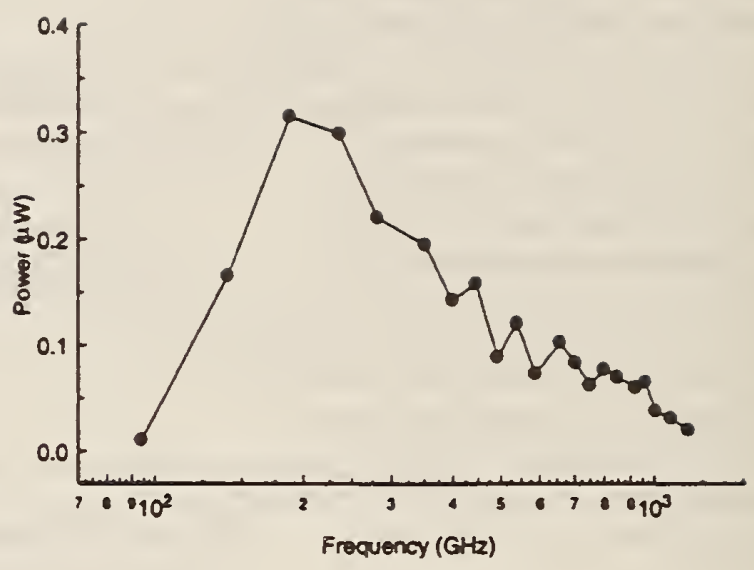

Figure 2. Photomixer output vs. frequency. Power measurements were made with $24 \mathrm{~K}$ InSb bolometer which was calibrated at 526 and $812 \mathrm{GHz}$ with a methanol laser.

Figure 3 shows the signal-to-noise ratio of the down-converted signal as a function of local oscillator harmonic number. The signal-to-noise ratio in the present system is sufficient to maintain an offset phase lock of one of the lasers to the other for harmonic numbers up to 5 . Figure 4 shows the down-converted signal with the lasers locked using the fourth harmonic, an offset of $366 \mathrm{GHz}$. The linewidth of the submillimeter wave signal is limited by the phase noise of the 91 $\mathrm{GHz}$ Gunn diode local oscillator. If the Gunn diode had been locked to a low phase-noise synthesis chain, the linewidth could have been as narrow as a few hertz. ${ }^{10}$

One method for sweeping the photomixer's output frequency while maintaining a phase-lock is to mix the downconverted output from the harmonic mixer with a variable frequency before sending it to the phase-locking circuit. This topology was tested for offset-frequency laser phase-locks with frequency differences up to $18 \mathrm{GHz}$ by using a fast photodetector in place of the photomixer. Phase-locked sweeps encompassing the full synthesizer band of $7 \mathrm{GHz}$ worked reliably. When the synthesizer switched bands, the if output was briefly absent. Unless the phase-locking integrator was carefully nulled, the system failed to automatically relock after the band switch. If it relocked, then it was possible to sweep over the full $19 \mathrm{GHz}$ electronic tuning range of the lasers. However, without a tracking filter or a single-sideband mixer, the signal-to-noise ratio of the signal at the phase detector was degraded by $3 \mathrm{~dB}$ by the noise from the unwanted mixing sideband. These minor difficulties could be overcome in a system specifically designed for long-range scans.

A second method for sweeping the photomixer's output frequency while maintaining a phase lock is to sweep the $91 \mathrm{GHz}$ local oscillator's frequency. This allows a fixedfrequency filter to be used to reject the unwanted mixing sideband. This approach was tested with the photomixer's

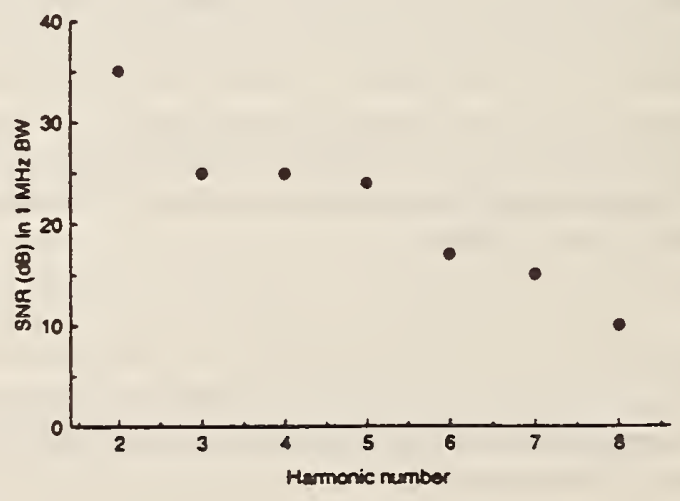

Figure 3. Detected signal-to-noise ratio (SNR) vs. $91 \mathrm{GHz}$ local oscillator harmonic number. Phase-locks are possible for harmonic numbers less than six. 
output phase-locked to the fourth harmonic of the $91 \mathrm{GHz}$ local oscillator. The Gunn diode oscillaror used in this experiment had only $1.10 \mathrm{GHz}$ of bias tuning range. Applying a voltage ramp to the Gunn diode bias produced a phaselocked sweep of $4 \mathrm{GHz}$ span centered at $364 \mathrm{GHz}$.

\section{FUTURE PROSPECTS}

The present system would allow free-running sweeps with a width over $1 \mathrm{THz}$ with an appropriate laser. For phase-locked sweeps, sweeps broader than $4 \mathrm{GHz}$ should be possible with a local oscillator which has more tuning range. The loss of local oscillator power that comes with a broader tuning range should not be a problem, since the Gunn diode had significantly more power than was necessary for these experiments.

Figure 4 shows that power measurements made by observing the down-converted signal amplitude could have a noise floor of $3 \mathrm{fW} / \mathrm{Hz}$. This is much lower than a

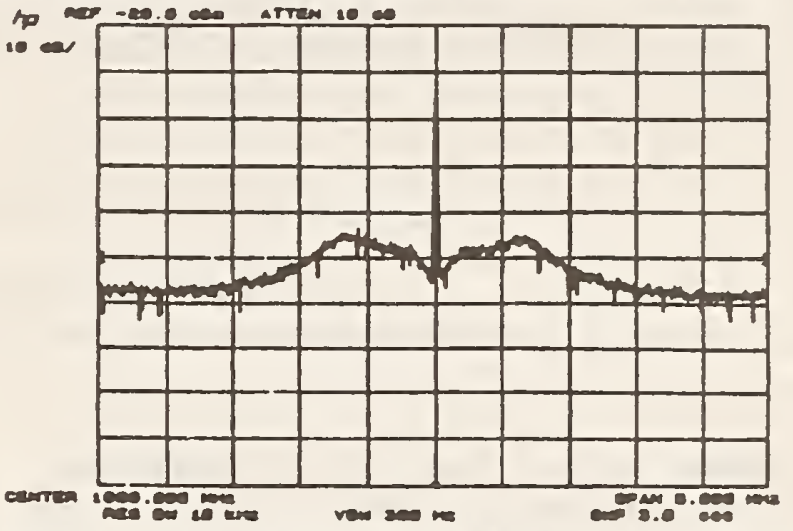

Figure 4. Power spectrum of phase-lock using the fourth harmonic of the $91 \mathrm{GHz}$ local oscillator. cryogenic Si bolometer with a NEP of $0.13 \mathrm{pW} / \mathrm{Hz} .^{7}$ Compared to cryogenic bolometers, heterodyne detection also has the practical advantage of room-temperature operation.

As the speed of LTG GaAs material improves, photomixers with greater bandwidth will become possible. Some recent LTG GaAs samples have shown photoconductive lifetimes on the order of $100 \mathrm{fs}$, raising hopes for photomixers with bandwidths of several terahertz. Improvements in the photomixer design may also increase their burnout threshold, so stronger pumps can be used to increase the output power. When combined with semiconductor diode lasers, these photomixers will make possible a broadly tunable, narrow-linewidth millimeter-wave source which will also be compact and transportable. This in turn would allow new spectroscopic and coherent-detection applications.

\section{ACKNOWLEDGMENTS}

At NIST this work was supported by the Air Force Office of Scientific Research. At Lincoln Laboratories this work was supponed by NASA through the Jet Propulsion Laboratory. The authors thank Lyndon Zink and Ken Evenson for supplying assistance, equipment, and laboratory space.

\section{REFERENCES}

1. F. C. DeLucia, T. M. Goyette, "Terahertz source requirements for molecular spectroscopy," Nonlinear Optics for High-Speed Electronics and Optical Frequency Conversion, pp. 239-247, SPIE, Los Angeles, CA, USA, 1994.

2. M. A. Frerking, "Submillimeter Source Needs for NASA Missions," Nonlinear Optics for High-Speed Electronics and Optical Frequency Conversion, pp. 222-229, SPIE, Los Angeles, CA, USA, 1994.

3. R. H. Pantell, J. M. DiDomenico, O. Svelto, J. N. Weaver, "The Theory of Optical Mixing in Semi-conductors," Proceedings of the 3rd Intemational Conference on Quantum Electronics, P. Grivet, N. Bloembergen, Eds., pp. 18111818, Columbia Univ. Press, 1963.

4. E. R. Brown, F. W. Smith, K. A. McIntosh, J. Appl. Phys. 73, 1480-1484 (1993).

5. E. R. Brown, K. A. McIntosh, K. B. Nichols, C. L. Dennis, Appl. Phys. Lett. 66, 285-287 (1995).

6. K. A. Mclntosh, et al., Appl. Phys. Lett. 67, 3844-3846 (1995).

7. A. S. Pine, R. D. Suenram, E. R. Brown, K. A. McIntosh, Journal of Molecular Spectroscopy 175, 37-47 (1996).

8. M. G. Littman, H. J. Metcalf, Applied Optics 17, 2224-2227 (1978).

9. K. M. Evenson, et al., IEEE Joumal of Quantum Electronics QE-13, $442-444$ (1977).

10. F. L. Walls, C. M. Felton, "High Spectral Purity X-Band Source," Fourty-Fourth Annual Symposium on Frequency Control, pp. 542-547, Baltimore, Maryland, 1990. 


\title{
A $1 \mathrm{GHz}$ OPTICAL-DELAY-LINE OSCILLATOR DRIVEN BY A DIODE LASER
}

\author{
J. Kitching, L. Hollberg and F. L. Walls \\ Time and Frequency Division, 847.10, \\ National Institute of Standards and Technology, 325 Broadway, Boulder, CO 80303
}

Abstract: Experimental results are presented on a bybrid optical/electronic oscillator which uses an optical delay line to generate high spectral purity microwave signals. At Fourier frequencies above $10 \mathrm{kHz}$, the single sideband (SSB) phase noise spectrum decreases as roughly $1 / f^{2}$ attaining a value of $-138 \mathrm{~dB}$ below the carrier in a 1 Hz bandwidtb ( $\mathrm{dBc} / \mathrm{Bz}$ ) at 20 $\mathrm{kBz}$ offet. The origin of this noise is in part the fundamental shot noise on the light itself, although other optical noise sources such as double Rayleigh scattering and stimulated Brillouin scattering also appear to be important under certain operating conditions. The frequency stability over several hours is dominated by changes in the fiber ambient temperature witb a coeffi. cient of about $10^{-5} / \mathrm{K}$ originating mostly from changes in the fiber refractive index with temperature.

\section{Introduction}

Stable S-band and X-band oscillators play a role in numerous applications ranging from precision metrology to optical communication systems. There are several traditional methods of generating low phase noise oscillation at these frequencies. Bulk acoustic wave (BAW) oscillators, oscillating at 5 or $10 \mathrm{MBz}$ can be multiplied up to $\mathrm{GHz}$ frequencies. Although the initial BAW oscillator may be extremely stable, the multiplication process usually adds substantial additional noise, particularly at frequencies far from the carrier, which seriously degrades the original performance of the oscillator. Sufface acoustic wave (SAW) oscillators bave been used to directly generate oscillation in the frequency range of $100 \mathrm{MHz}$ to a few $\mathrm{GHz}$ with somewhat inferior close-in phase noise performance than low frequeocy BAW oscillators. For even bigher frequencies, up to tens of $\mathrm{GHz}$, dielectric resonator

\footnotetext{
${ }^{1}$ Contribution of U.S. Government; not subject to copyright.
}

oscillator (DRO's) are most often used; sapphire is the dielectric material of choice for obtaining the low. est phase noise. While these metbods bave been, on the whole, successful, particularly in producing signals with extremely low phase noise, their lack of tunability and difficulty of fabrication limit their usefulness in many critical applications.

Recently, a novel bybrid optical/electronic approacb to this problem was proposed $[1,2]$. This uses an optical fiber delay line to achieve pbase noise performance competitive with the best commercially available synthesizers. These optical-delay-line oscillators (ODLO's) or light-induced microwave oscillators also have a number of highly desirable features not available in otber oscillators: they are bigbly tunable; bave oscillation frequencies which can potentially range from bundreds of $\mathrm{kHz}$ to tens of $\mathrm{GHz}$; can have moderately low phase noise; have both optical and electronic inputs and outputs facilitating their integration with other optical systems; and can be constructed with no precision components other than commercially available optical fiber. Another important aspect of these oscillators is that their phase noise performance is independent of oscillation frequency making them excellent candidates for generating microwave signals above $10 \mathrm{GHz}$. Recent experimental results for an $800 \mathrm{MHz}$ oscillator $[3,4]$ have demonstrated a SSB phase noise of less than $-140 \mathrm{dBc} / \mathrm{Bz}$ at $30 \mathrm{kBz}$ frequency offset and many future improvements seem likely.

\section{The Optical Delay Line Oscillator}

The ODLO takes advantage of the extremely low loss and long delay times which can be achieved in optical fibers in order to provide the frequency discrimination necessary for low phase noise oscillation. A diagram of one of the oscillators built at NIST is shown in Figure 1. An electronic signal is first translated into amplitude modulation on an optical carrier generated by 


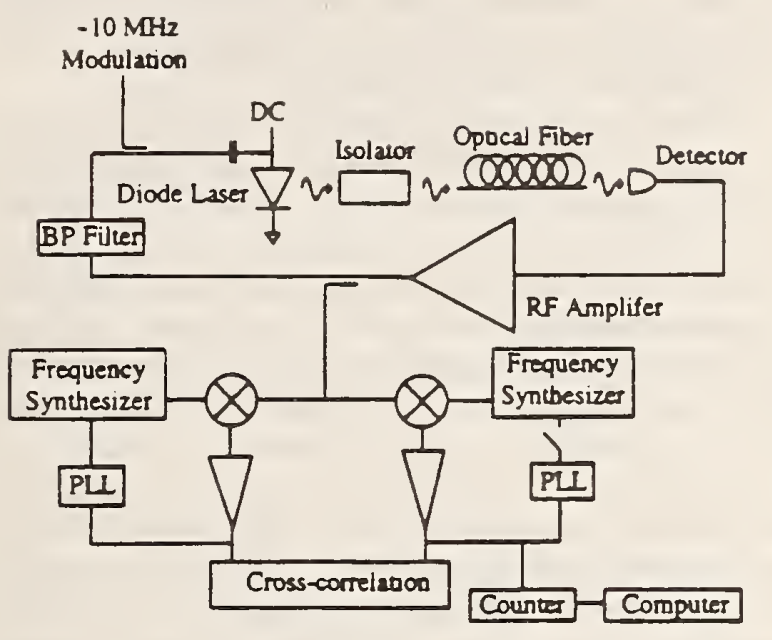

Figure 1: Experimental setup of one of the NIST op tical delay line oscillators

a laser. This can be done either by directly modulating the injection current of a semiconductor laser or by using an electro-optic modulator (EOM) to amplitude modulate an externally generated $\mathrm{Cr}$ beam. The light is passed through a long optical fiber and then sent into a high speed photodetector which converts the optical signal back into an electronic signal. The electronic signal is then amplified, filtered, and sent back into the amplitude modulator. This feedback loop defines RF "modes" which satisfy the condition that the roundtrip phase shift through the loop is a multiple of $2 \pi$. For a fiber length of $1 \mathrm{~km}$, these modes are spaced by about $200 \mathrm{kHs}$.

The transfer of RF modulation into amplitude modulated light and then back into a RF signal usually involves some loss of RF power making the loop gain substantially less than unity. However, as the amplifier gain is increased one of the modes will eventually reach threshold when the loop gain is equal to unity and will start to oscillate. Which mode oscillates depends on the tuning of the RF filter. The filter gain profile acts very much like the wavelength-dependent gain provided by an atomic transition in a laser. Assuming saturation properties analogous to bomogeneous broadening in a laser, when the mode at the peak of the gain profile sees unity gain, it will start to oscillate, saturating the gain, and bringing the system into equilibrium. Adjacent modes are (usually) suppressed by some large factor below the oscillating mode since the mode amplitude is enormously sensitive to the net gain in the region around threshold. How and where the gain saturation occurs depends on the exact configuration of the oscillator, as will be discussed below.

An important property of this type of oscillator is its ability to produce oscillation with low phase noise. This is true because very long delays are possible in an optical fiber with very little loss. A typical single-mode optical fiber can transmit $1.3 \mu \mathrm{m}$ light with as little as $0.4 \mathrm{~dB} / \mathrm{km}$ of optical power loss. Since the optical power modulation is proportional to the current modulation, this corresponds to $0.8 \mathrm{~dB} / \mathrm{km}$ of RF power loss intrinsic to the fiber (as opposed to $\approx 1000 \mathrm{~dB} / \mathrm{km}$ for a delay line made of semi-rigid coaxial waveguide, for example). As a result of this low loss and long delay, the effective fractional RF energy loss per unit time in the fiber can be very small. The bandwidth of optical fiber is also extremely large, so in principle very high frequency modulation can be delayed without significant increase in the RF loss. If we assume perfectly efficient coupling of RF power into optical modulation (that is, one optical photon per electron) and then back into RF power at the detector end, then the limiting loss mechanism in the loop is the fiber loss and the $Q$ of the cold (no gain) RF "cavity" can be as high as $10^{6}$ at a frequency of $10 \mathrm{GHs}$. In fact, if the light could be modulated at the optical fiber bandwidth of $\approx 10 \mathrm{THs}$ (by using two lasers at different wavelengths, for example), the intrinsic fiber $Q$ for the ODLO could be as bigh as $10^{9}$. For practical fiber lengths, the loop losses are usually dominated by coupling the RF power into and out of the optical domain, making the actual RF cavity $Q$ in a real device much smaller (a few tens of thousands).

An analysis of the ODLO's RF power spectrum bas been made by Yao and Maleki [3], who include amplifier thermal noise, photon shot noise, and laser noise in their model. They find that the RF power spectral density close to the oscillating mode is equal to

$$
S_{\varphi f}\left(f^{\prime}\right)=\frac{\delta}{(\delta / 2 \tau)^{2}+\left(2 \pi \tau f^{\prime}\right)^{2}},
$$

for $2 \pi \tau f^{\prime} \ll 1$, where $f^{\prime}$ is the frequency deviation from the nominal oscillation frequency, $T$ is the loop delay time and $1 / \delta$ is the open-loop RF signal-to-noise ratio at the amplifier input. If the dominant noise source is assumed to be shot noise (this is often the case) then $\delta=2 e I_{p h} R / P_{p f}$, where $e$ is the charge on an electron, $I_{p h}$ is the DC photocurrent (a few $m A$ ), $R$ is the amplifier input resitance, and $P_{\rho f}$ is the RF power of the detector photocurrent modulation. For typical values of $I_{p h}(2 \mathrm{~mA})$, modulation index $(0.3)$, and fiber 
length $(1.5 \mathrm{~km})$, this results in a single sideband phase noise of about $-144 \mathrm{dBc} / \mathrm{Hz}$ at a frequency offset of 20 kHz. This is competitive with current state-of-the-art commercial oscillators. An interesting aspect of this analysis is that the phase noise does not depend on the oscillation frequency. If the mode-selection filter is moved from $1 \mathrm{GHz}$ to $10 \mathrm{GHz}$ or $50 \mathrm{GHz}$, the phase noise, in principle, stays the same.

\section{Oscillator Description}

We now turn to a description of the specific oscillator which bas been built at NIST (Figure 1). This oscillator incorporates a number of features which make it different from the oscillator described in previous publications [3]. The main difference is that a semiconductor distributed Bragg reflector (DBR) laser, lasing at $850 \mathrm{~nm}$, is used instead of a YA laser and EOM. The laser injection current is directly modulated in order to transfer the RF signal to optical modulation. This implementation offers a number of advantages as well as disadvantages over electro-optic modulation. The most obvious advantages are compactness and lower cost. In addition, if pumped far enough above threshold, semiconductor lasers can have amplitude noise close to, or even in some cases below, the sbot noise limit, which makes them ideal for use in the ODLO. One disadvantage with using diode lasers is the lower optical output power which limits the signal-to-noise ratio at the photodetector. In addition, the maximum frequency at which diode lasers can be modulated is typically somewhat lower than can be obtained in state-of-the-art EOM's.

The output from the laser was sent into $1.5 \mathrm{~km}$ of single-mode fiber and then into a high speed PIN photodiode. One technological issue encountered was the diffculty in obtaining high speed photodiodes which can operate with incident optical powers over a few $\mathrm{mW}$. If the laser noise is close to the shot noise limit, then the signal-to-noise ratio at the photodetector improves as the optical power detected increases. As a result, in order to obtain the best phase noise performance, it is desirable to operate at the highest optical power possible. Although the laser used in the experiment was capable of generating $100 \mathrm{~mW}$ of optical power, it had to be used at a lower power and the light sometimes attenuated in order to avoid overloading the detector (and also, as described below, to reduce optical scattering in the fiber). Our best results were acheived with an output power of $95 \mathrm{~mW}$ and resulting detector $D C$ photocurrent of $1.6 \mathrm{~mA}$. The currentto-current DC efficiency from laser to photodetector was $1.2 \%$. The low efficiency was mostly the result of an optical attenuator placed intentionally in the beam path in order to reduce the DC photocurrent to a level acceptable to the photodiode.

The output from the photodetector was sent into a series of amplification stages, the final stage having a $1 \mathrm{~dB}$ compression output power of $79 \mathrm{~mW}$. This final stage provided the saturating element in the loop, lim. iting the RF power so that the power modulation on the laser was about $50 \%$ of the total power. Filtering at $1 \mathrm{GHz}$ was accomplisbed by stub matching the $50 \Omega$ line impedance to the laser impedance of a few ohms which provided the additional benefit of increasing the RF coupling into the laser. The tuning stub filter bad a FWHM of $10 \mathrm{MHz}$ about a $1.002 \mathrm{GHz}$ center frequency. It is interesting to note that this impedance matching (which can also be implemented on the detector end) can actually result in RF gain [6] in the loop despite the presence of optical loss. Thus it seems possible to avoid the use of RF amplifiers altogether in this type of oscillator, drawing energy instead from the DC bias circuits of the laser and detector.

\section{Sources of Noise}

In this section, the sources of noise which affect the performance of an OLDO are reviewed. In addition to the three sources mentioned above, we found two other factors related to light scattering in the fiber which limit the performance of the oscillator under certain operating conditions. These scattering mechanisms are likely to be more important in our oscillator, which uses light at $850 \mathrm{~nm}$, than in an oscillator using light at $1.3 \mu \mathrm{m}$ or $1.5 \mu \mathrm{m}$.

The critical issue is the signal-tonoise ratio at the input to the RF amplifier under open-loop conditions. This signal-to-noise ratio determines not only the oscillator phase noise but also, for a given filter bandwidth, the RF power in the side modes. The three noise sources mentioned previously are thermal noise in the RF amplifier, shot noise resulting from the graininess of the laser light, and excess noise generated by the laser. When refered to the amplifier input, the noiseto-signal due to these sources is given by [4]

$$
\delta=\left[4 k_{B} T(N F)+2 e I_{p h} R+N_{R N N} I_{p h}^{3} R\right] / P_{00 c}
$$

where $k_{B}$ is Boltzmann's constant, $T$ is the temperature, $N F$ is the amplifer noise figure, and $N_{R I N}$ is the relative intensity noise of the laser. For many semiconductor lasers operating at a few times the threshold current, and with the DC detector current into $50 \Omega$ above a few $\mathrm{mA}$, and the dominant noise source 


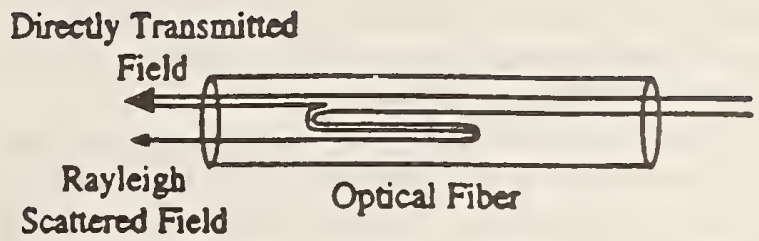

Figure 2: Double Rayleigh scattering in the optical fiber generates excess photocurrent noise when the scattered field beats with the directly transmitted field.

is shot noise. Assuming a constant modulation index on the laser light, the signal-to-noise ratio is then maximized by increasing the laser output power to its highest level and reducing the optical losses between laser and detector to a minimum. This strategy runs into two difficulties. Firstly, as mentioned above, high speed photodiodes can usually handle only limited optical power. Secondly, and more importantly, however, when high power is sent through the fiber, optical scattering mechanisms contribute additional noise and degrade the oscillator performance.

The first scattering mechanism which was found to be important in our $850 \mathrm{~nm}$ oscillator was stimulated Brillouin scattering (SBS). In this process, the optical field scatters off acoustic phonons in the fiber generating a backward propagating optical field red-sbifted by $21 \mathrm{GHz}$ from the input. The threshold input power for this process in our fiber was about $9 \mathrm{~mW}$ if no modulation was applied to the laser (if all of the optical power was concentrated in a single spectral region). When the optical power was increased above this level, the SBS gain in the reverse direction was larger than the fiber loss, and the counter-propagating field was amplified. When there was a significant SBS, the amplitude noise of the transmitted light increased dramatically by several orders of magnitude. It is clear, therefore, that this process imposes a limitation on the optical power which can be used in the oscillator and therefore on the maximum signal-to-noise ratio which can be achieved. Under oscillating conditions, signifcantly more than $9 \mathrm{~mW}$ of optical power could be sent through the fiber without observing the effects of SBS. This is because the laser was being modulated with a large modulation index (both $A M$ and PM) which distributed the optical power among several sidebands and therefore reduced the power at any one frequency.

A second, and more serious problem, was that of

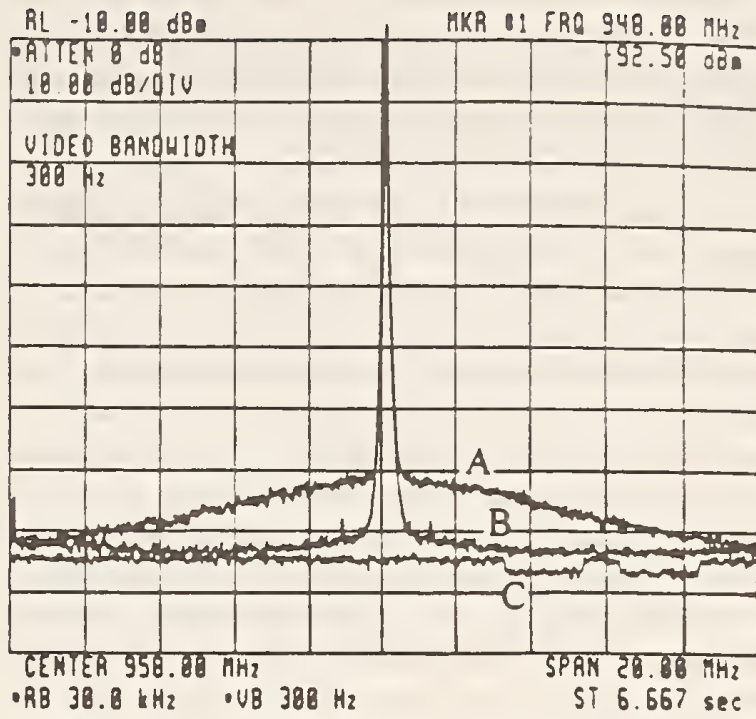

Figure 3: Excess noise about the RF carrier generated by double Rayleigh scattering. Trace $A$ show the increased photocurrent noise when modulation at 958 MHs is applied to the laser. Trace B is under identical conditions to $\mathrm{A}$ except with additional injection current modulation at $10 \mathrm{MHs}$. Trace $\mathrm{C}$ indicates the noise level with no RF modulation and the background amplifier noise level.

double Rayleigh scattering (DRS) in the fiber [7]. DRS occurs because a small fraction of the optical power propagating in the fiber is Rayleigh scattered twice resulting in a weak, delayed replica copropagating with the original field in the fiber (see Figure 2). In fibers longer than the coherence length of the laser, this causes additional noise resulting from phase fluctuations of the laser. Because of the long delay, the phase noise of the scattered field is not coherent with the noise of the directly transmitted field and when the two fields are detected simultaneously on a photodetector, excess photocurrent noise is generated. When a single optical frequency is present in the fiber, this noise can be seen at low frequencies in a bandwidth roughly equal to the laser linewidth (a few $\mathrm{MEs}$ ). If the laser is being modulated (that is, the oscillator is oscillating), AM noise is also generated around the detected RF modulation as a result of the scattered optical sideband field beating with the directly transmitted optical carrier (and vice-versa). Figure 3 shows this effect. For this measurement, the loop was open and a RF signal from a synthesizer at $958 \mathrm{MHs}$ was sent into the laser injection current. The photodiode signal was then detected with a spectrum analyser. Trace 
$C$ indicates the noise power with no modulation (the two small steps on the right indicate the background amplifier noise power). With modulation at $958 \mathrm{MBz}$ applied (trace $A$ ), the noise around the carrier clearly increases as a result of the DRS.

There are several ways of dealing with this problem. Since the noise is a replica of the laser linewidtb (which is true if the laser's coberence length is much shorter than the fiber length), changing the linewidth will obviously affect the noise. Reducing the linewidth will concentrate more of the noise close to the modulation ignal thereby increasing the phase noise of the oscillator close to the carrier. On the other hand, increasing the laser phase noise (by adding low-frequency noise to the injection current, for example) will brosden the noise bandwidth seen in Figure 3, Trace $A$, thereby reducing the noise close to the modulation frequency and improving the oscillator performance. This was indeed the case.

A more successful solution, however, was to add ad. ditional modulation to the laser injection current at a frequency different from the oscillation frequency [8]. This spreads the optical power throughout a number of FM ridebands (separated by this new modulation frequency) while it propagates through the fiber. When the light is detected, part of the noise originally neas the oscillation frequency is transferred to near the modulation sidebands. As a result, the $A M$ noise around the original $1 \mathrm{GH} s$ signal is reduced by a factor roughly equal to the number of sidebands generated by the second modulation signal. This reduction is clearly seen in Figure 3, trace B, which is taken under identical conditions as trace $A$, except that current modulation of $10 \mathrm{MHz}$ is added to the laser injection current in addition to the $958 \mathrm{MHz}$ original modulation. A reduction in the DRS amplitude noise can be clearly seen in the figure. The added phase noise on the transmitted $958 \mathrm{MHz}$ signal was reduced by over an order of magnitude at a frequency $10 \mathrm{kHz}$ from the carrier. Thus it seems possible to reduce, if not eliminate altogether, the effects of DRS noise using this modulation technique.

Acoustic vibrations were also found to generate oscillator noise, mostly at offset frequencies below about $1 \mathrm{kH}$. Although we expect that compression-induced changes in the fiber refractive index are in part responsible, it was also found that the oscillator frequency was very sensitive to the position of the optical beam on the photodetector. It is thought that this sensitivity may be a result of the detector capacitance or carrier transit time changing slightly as the location of the charge-carrier generation was changed. As a result, acoustic noise changing both the detector position and also the beam pointing direction would be expected to generate phase noise in the oscillator. The use of fiber-coupled detectors might reduce the sensitivity to acoustic vibrations.

A final source of additional noise originating with the fiber results from temperature luctuations causing the fiber length and refractive index, and therefore the oscillation frequency, to change. The temperature dependence of the refractive index for glass is about $10^{-5} / \mathrm{K}[9]$ resulting in a similar coefficient for the frequency deviation of the oscillator. Thus it is expected that temperature stabilization of the fiber of the order of $\approx 100 \mu \mathrm{K}$ will be necessary to reduce the frequency fluctuations on long time scales to below one part in $10^{\circ}$.

\section{Experimental Results}

When the RF gain was increased enough to overcome the optical loss and output coupling, the system oscillated stably in a single frequency mode. A typical mode spectrum, taken with the oscillation frequency at $1 \mathrm{GHz}$, is shown in Figure 4. The power in the side

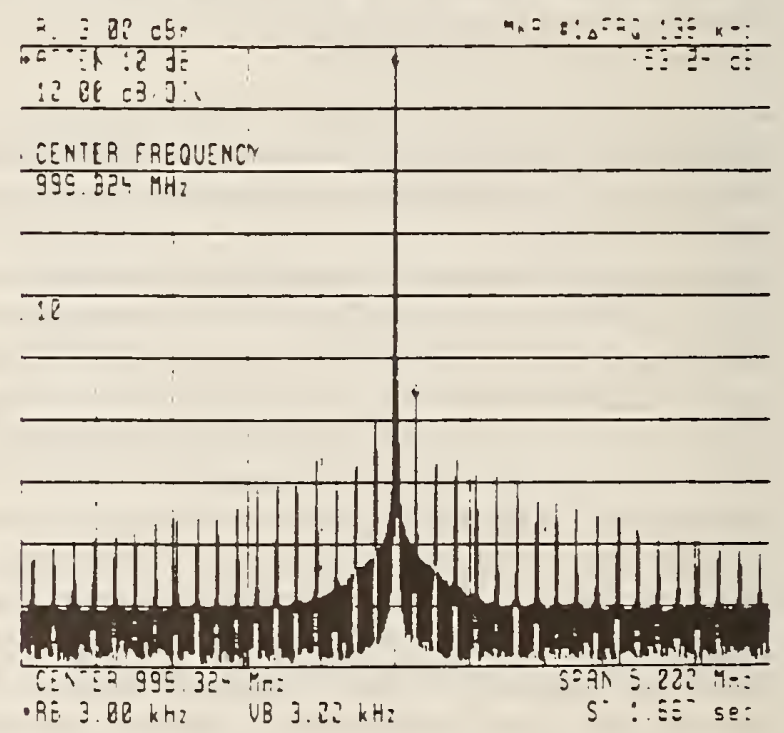

Figure 4: Mode spectrum of $1 \mathrm{GHs}$ oscillator.

modes closest to the main mode was typically between 50 and $60 \mathrm{~dB}$ below the main mode power (although under the optimum conditions described above, a side mode suppression of $62 \mathrm{~dB}$ was measured). The side mode suppression agreed reasonably well with the fundamental theoretical prediction which depends on the filter bandwidth, open loop signal-to-noise ratio and 


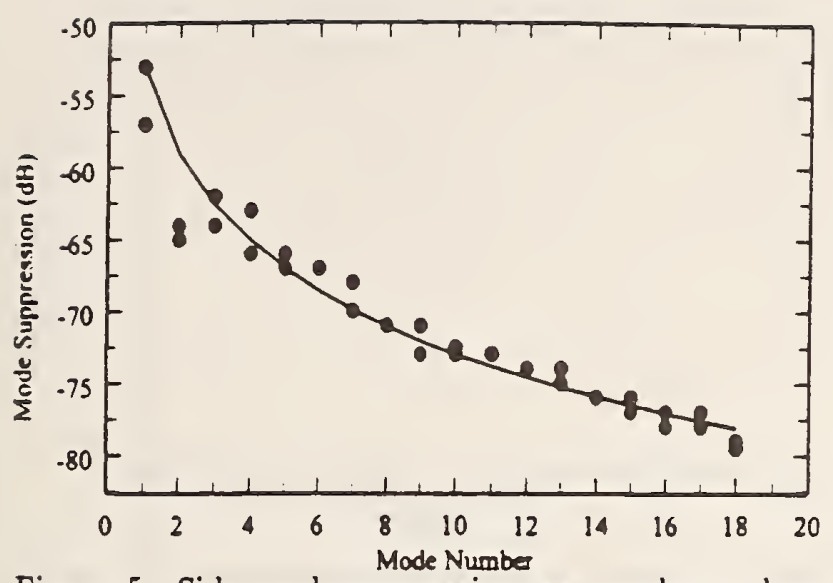

Figure 5: Side mode euppression vs. mode number for $1 \mathrm{GH}$ oscillator. The solid line indicates the theoretically predicted $1 / n^{2}$ dependence of the side mode power on the mode number $n$.

mode spacing. A plot of the side-mode suppression against mode number, $n$, is shown in Figure 5 , with the solid line indicating the theoretical $1 / n^{2}$ dependence.

In order to accurately characterire the phase noise of the ODLO, a three-cornered-hat cross-correlation measurement was made [10]. The measurement system is shown in Figure 1 and used two frequency synthesisers as reference oscillators which were phaselocked to the OLDO at frequencies below about 100 $\mathrm{Hz}$. The resulting measured ODLO-SSB phase noise spectrum, taken with $14 \mathrm{MHz}$ modulation applied to the laser in order to reduce the noise from double Rayleigh scattering, is shown in Figure 6. For this measurement, 1000 averages of the cross-correlation signal were performed and the noise level calibrated with a $1 \mathrm{GHz}$ noise standard [11]. The calibration was checked by frequency-modulating the ODLO with a known amplitude at $5 \mathrm{kHz}$ and comparing the crosscorrelation with the resulting calculated phase modulation power. Phase noise of $-138 \mathrm{dBc} / \mathrm{Bz}$ at $20 \mathrm{kBz}$ was measured for the $1 \mathrm{GHz}$ oscillator. The plot also shows the calculated phase noise which should result from $1.6 \mathrm{~mA}$ of DC detector photocurrent, $50 \%$ optical intensity modulation under oscillating conditions, and open-loop detection noise at the shot noise limit. Clearly the measured noise is somewhat higher. The origin of this excess noise is likely a combination of double Rayleigh scattering and thermal noise in the RF amplifiers. The theoretical phase noise increase estimated from measurements of the DRS fiber noise (with the $14 \mathrm{MHz}$ modulation applied to the laser) is also shown in the figure.

The oscillator frequency drift on longer time scales was also measured by mixing the ODLO output with the output from a frequency synthesiser and sending the resulting signal into a frequency counter, with the assumption that the crystal oscillatora driving the synthesiser and counter were more stable on long time scales than the ODLO. The ODLO frequency over a period of several hours is shown in Figure 7. One data point was taken every second with a dead time of about 200 ms between measurements. A drift in the abso lute frequency by $10 \mathrm{kHz}$ can be seen in the figure. This drift was correlated with changes in the ambient temperature of the fiber with a measured temperature coefficient of about $10^{-5} / \mathrm{K}$ which agrees well with the temperature dependence of the effective refractive index for the glass used in optical fibers [9].

\section{Conclusions}

The preliminary investigations described above and previously published results $[2,3,4]$, suggest that optical delay line oscillators may be competitive alter. natives to crystal and dielectric resonator oscillators if oscillation frequencies can be increased beyond 10 GHs. Their tunability and ease of construction are additional advantages. The development of these oscillators is still in its infancy and substantial room for improvement remains. Specifically, the phase noise performance can almost certainly be improved by using higher optical powers from the laser and better optical coupling from laser to detector. Diode lasers appear compatible with ODLO's and do not themselves seem to cause any fundamental problems. High performance lasers [12] having large modulation bandwid ths in addition to useful output powers would be a great asset in the development of these ODLO's. To some extent, this improvement hinges on being able to find high speed photodiodes which can handle tens of $\mathrm{mW}$ of optical input power.

Additional reductions in the fiber-generated noise could be obtained by using optics at $1.3 \mu \mathrm{m}$ rather than $850 \mathrm{~nm}$. Although lasers at this wavelength tend to be slightly lower in optical output power, optical fibers have substantially less loss and scattering mechanisms such as stimulated Brillouin scattering and double Rayleigh scattering should be much weaker. A system with these design improvements is under construction at NIST. This system should produce even higher frequency oscillation with phase noise close to the fundamental limit set by the shot noise on the laser light. 


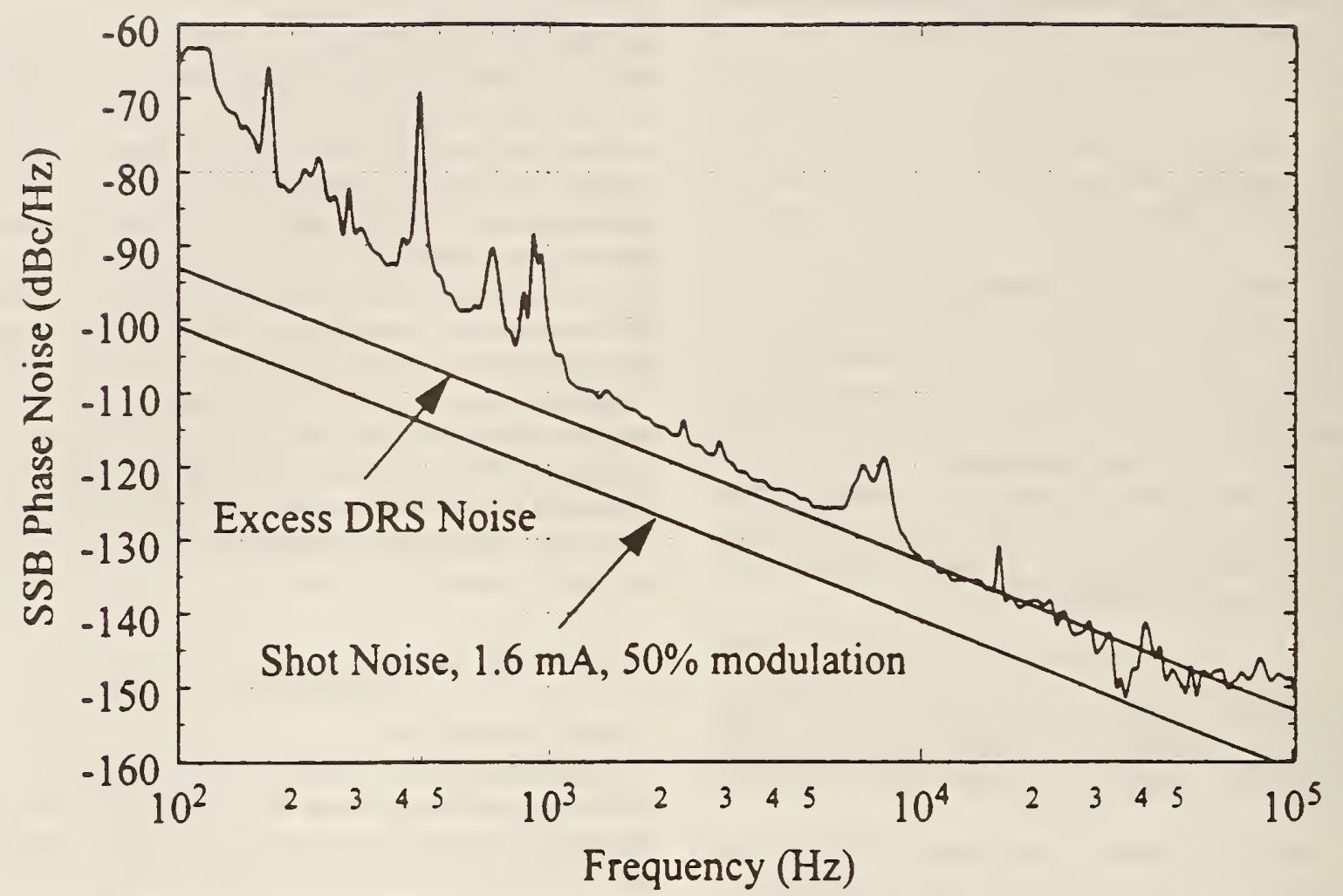

Figure 6: Single-sideband phase noise spectrum for a $1 \mathrm{GH}$ optical-delay-line oscillator. The measurement was made using the three-cornered-hat crose-correlation technique with 1000 averages. Current modulation at 14 $\mathrm{MHz}$ was applied to the laser to reduce the noise from double Rayleigh scattering.

\section{Acknowledgements}

This work was funded in part by the AFOSR. We are grateful to $D$. Welch at Spectra-Diode Labs for the donation of the laser used in the experiment. We also acknowledge useful discussions with S. Waltman and R. Shuker.

\section{References}

[1] A. Neyer and E. Voges, "High-Frequency ElectroOptic Oscillator Using an Integrated Interferometer," Appl. Phys. Lett., vol. 40, pp. 6-8, Jan., 1982.

[2] X. S. Yao and L. Maleki, "High Frequency Optical Subcarrier Generator," Electron. Lett., vol. 30, pp. 1525-6, Sept. 1994.

[3] S. Yao and L. Maleki, "Characteristics and Performance of a Novel Photonic Oscillator," Proc. of 49th Annual IEEE Frequency Control Symposium, San Francisco, CA, May 31 - June 2, 1995.
[4] X. S. Yao and L. Maleki, "Converting Light into Spectrally Pure Microwave Oscillation," Opt. Lett., vol. 21, pp. 483-5, April, 1996.

[5] The product information given is for technical reference only and does not constitute an endorsement of the laser or detector used in the experiment.

[6] M. F. Lewis, "Novel RF Oscillator Using Optical Components," Electron. Lett., vol. 28, pp. 31-2, Jan., 1992.

[7] S. Wu, A. Yariv, B. Blauvelt and N. Kwong, "Theoretical and Experimental Investigation of Conversion of Phase Noise to Intensity Noise by Rayleigh Scattering in Optical Fibers," Appl. Phys. Lett., vol. 59, pp. 1156-1158, Sept., 1991.

[8] A. Yariv, H. Blauvelt and S. Wu, "A Reduction of Interferometric Phase-to-Intensity Conversion Noise in Fiber Links by Large Index Phase Modulation of the Optical Beam, " J. Lightwave Tech., vol. 10, pp. 978-81, July, 1992. 


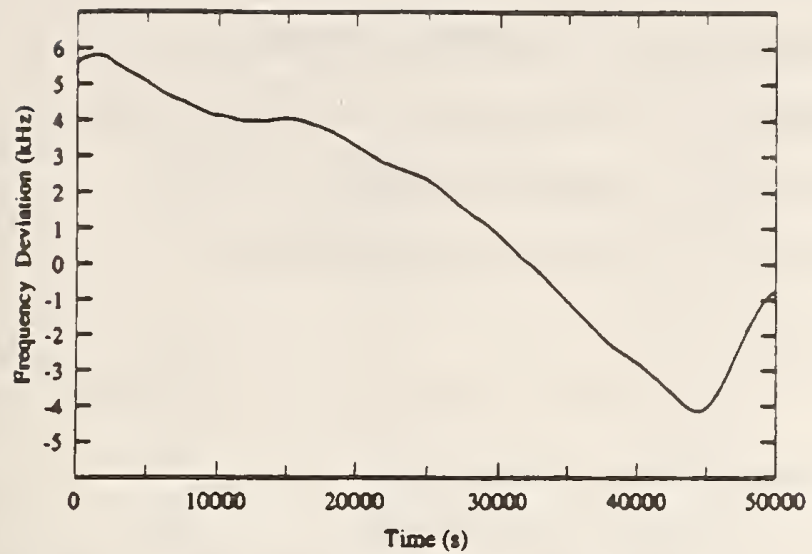

Figure 7: Optical-delay-line oscillator frequency variation over several hours. The frequency drift wa correlated with the fiber ambient temperature with a coefficient of about $10^{-8} / \mathrm{K}$.

[9] J. J. Carr, S. L. Saikkonen and D. H. Williams, "Refractive Index Measurements on Single-Mode Fiber as Functions of Product Parameters, Tenvile Stress, and Temperature," Fiber and Integrated Optics, vol. 9, pp. 393-396, 1990.

[10] W. F. Walls, "Cross-Correlation Phase Noise Measurements," Proc. of 46th Annual IEEE Frequency Control Symposium, Bershey, PA, May 27-29, 1992, pp. 257-261.

[11] F. L. Walls, "Secondary Standard for PM and AM Noise at 5, 10, and $100 \mathrm{MBz}$, Proc. of 46th Annual IEEE Frequency Control Symposium, Hershey, PA, May 27-29, 1992, pp. 290-299.

[12] T. R. Chen, P. C. Chen, J. Ungar and N. Bar-Chaim, "High Power Operation of MultiQuantum Well DFB Lasers at $1.3 \mu \mathrm{m},{ }^{n}$ Elerton. Lett., vol. 31, Pp. 1344-5, 1995; J-M Verdiell, D. F. Welch, B. Plano and K. Duncan, "BighPerformance $1.3 \mu \mathrm{m}$ Lasers for Analog and Digital A pplications," Proc. LEOS 1995 Annual Meeting, pp. $169-70,1995$. 


\title{
Continuous-wave frequency tripling and quadrupling by simultaneous three-wave mixings in periodically poled crystals: application to a two-step 1.19-10.71- $\mu \mathrm{m}$ frequency bridge
}

\author{
O. Pfister \\ IILA, Natianal Institute af Standards and Technalagy and University af Calarada, Baulder, Calarada 80309-0440 \\ J. S. Wells, L. Hollberg, L. Zink, and D. A. Van Baak* \\ Natianal Institute of Standards and Technalagy, Baulder. Calarada 80303 \\ M. D. Levenson \\ Department af Electrical and Camputer Engineering, Rice University, Houstan, Texas 77251 \\ W. R. Bosenberg \\ Lightwave Electronics Corporatian, Mauntain View. Califarnia 94043 \\ Received April 3, 1997

\begin{abstract}
We observed $\mathrm{cw}$ third-harmonic generation in a periodically poled $\mathrm{LiNbO}_{3}$ crystal by cascading optimally phase-matched second-harmonic and sum-frequency generation. Other processes, such as fourth-harmonic generation, are allowed by the flexibility of quasi-phase matching. We demonstrate a divide-by-nine (1.19$10.71-\mu \mathrm{m})$ frequency chain that uses only two lasers.
\end{abstract}

Precise measurement of optical frequencies requires connecting laser frequencies to well-known standards by means of a frequency chain. Because of the large frequency intervals involved, these systems can be extremely complex, but they are required for future optical clocks and tests of fundamental physics. ${ }^{1}$ Therefore there is a need for new and efficient optical mixing schemes compatible with $\mathrm{cw}$, low-power frequency-stabilized lasers. Our interest is also in frequency connections in a 3:1 ratio, which would bridge gaps between excellent existing standards such as the $\mathrm{CO}_{2}$ laser locked on $\mathrm{OsO}_{4}$ at $10 \mu \mathrm{m}$, the $\mathrm{He}-\mathrm{Ne}$ laser locked on $\mathrm{CH}_{4}$ at $3.39 \mu \mathrm{m}$, and the $\mathrm{Nd}: Y A G$ laser locked on $\mathrm{I}_{2}$ at $1 \mu \mathrm{m}$.

Nonlinear mixing in crystals is a good way to connect optical frequencies. ${ }^{2}$ One promising technique is quasi-phase matching ${ }^{3,4}$ (QPM) in periodically poled (PP) crystals, ${ }^{5}$ such as $\mathrm{LiNbO}_{3}$ (LN), $\mathrm{KTiOPO}_{4}$ (KTP), and $\mathrm{RbTiOAsO}_{4}$ (RTA). QPM has well-known advantages such as access to the largest nonlinear coefficients, suppressed walk-off, and great flexibility in the choice of the wavelengths involved in the mixing.

Because of these properties, simultaneous QPM of different interactions is more probable than one might at first suppose. We envisaged third-harmonic generation (THG) by cascading ${ }^{6}$ second-harmonic generation (SHG; $\omega \mapsto 2 \omega$ ) and sum-frequency generation (SFG; $\omega+2 \omega \mapsto 3 \omega$ ), which of course realizes a 3:1 frequency connection. Another possibility (using a single input beam as well) is fourth-harmonic generation (FHG) by cascading SHG twice. Considering simple poling (50\% duty-cycle period) and first-order collinear $\mathrm{QPM}^{4}$ as well as birefringent phase matching (BPM), ${ }^{7}$ and allowing different polarizations, we find 12 THG and 10 FHG coincidences in PP LN (Fig. 1 and Table 1), 8 and 2 coincidences in PP KTP, and 14 and 5 in PP RTA (Table 2).

Given our low initial power $P(\omega)$, the THG power is $P(3 \omega)=\eta_{\mathrm{SHG}} \eta_{\mathrm{SFG}} P(\omega)^{3}$, where $\eta_{\mathrm{SHG}}$, SFG are the SHG and SFG efficiencies, each of the form $\left[\chi^{(2)} \operatorname{sinc}(\Delta \mathbf{k}\right.$. $\mathbf{l} / 2)]^{2} . \quad \Delta \mathbf{k}$ is the phase-mismatch vector and $\mathbf{l}$ is a vector of modulus the effective crystal's length, perpendicular to the walls of the poled domains. Although this model takes into account noncollinear QPM (angle tuning $)^{4}$ and the temperature dependence of the

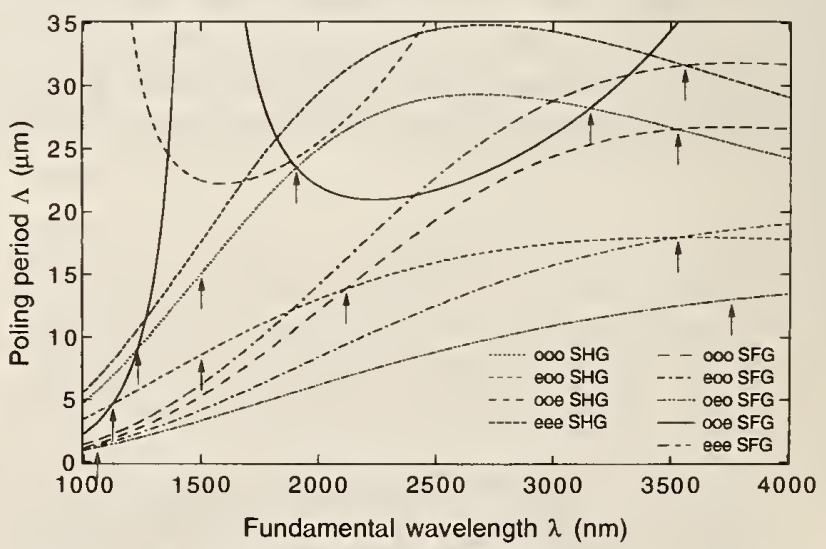

Fig. 1. QPM curves for THG in PP LN: The optimal poling period $\Lambda$ is calculated versus the input wavelength $\lambda$ for SHG and SFG and several polarizations. These curves are based on the Sellmeier coefficients ${ }^{8}$ that gave predictions closest to our experimental results at room temperature. Arrows point to the THG coincidences. As in the tables, polarizations $(o, e)$ are given for $\omega \omega 2 \omega$ and $\omega 2 \omega 3 \omega$. 
Table 1. THG and FHG Coincidences in PP $\mathbf{L N}^{a}$

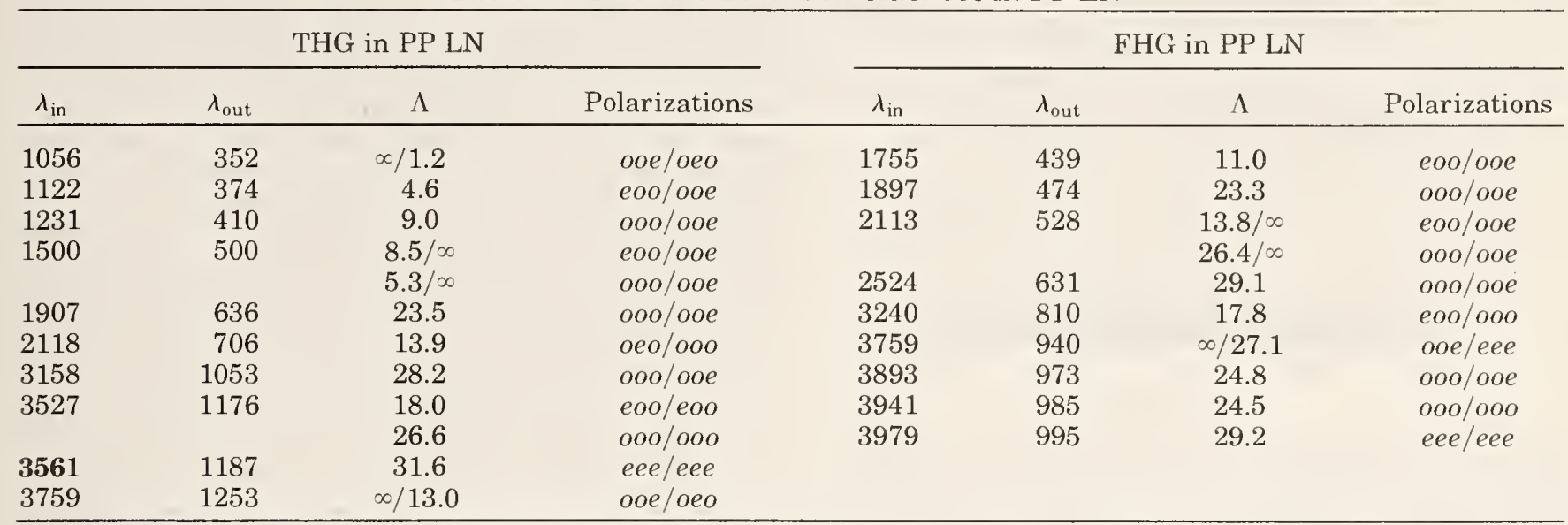

${ }^{a} \lambda_{\text {in, out }}$ in nanometers; poling period $\Lambda$ (or $\Lambda_{\mathrm{SHG}} / \Lambda_{\mathrm{SFG}}$ ) in micrometers. $\Lambda \rightarrow \infty$ indicates BPM. Polarizations $(o, e)$ are given for $\omega \omega 2 \omega / \omega 2 \omega 3 \omega$ for THG and for $\omega \omega 2 \omega / 2 \omega 2 \omega 4 \omega$ for FHG. Nonlinear efficiencies $\propto\left(d_{22}\right)^{2}(o o o)<\left(d_{31}\right)^{2}(o o e, o e o, e o o) \ll\left(d_{33}\right)^{2}(e e e)$. The boldface number is the wavelength at which the experiment was performed (see text).

Table 2. THG and FHG Coincidences in PP KTP and PP RTA ${ }^{a}$

\begin{tabular}{|c|c|c|c|}
\hline$\lambda_{\text {in }}$ & $\lambda_{\text {out }}$ & $\Lambda$ & Polarizations \\
\hline \multicolumn{4}{|c|}{ THG in PP RTA } \\
\hline \multirow[t]{2}{*}{1143} & \multirow[t]{2}{*}{381} & $\infty / 1.7$ & $z x x / x x z$ \\
\hline & & $\infty / 5.9$ & $z x x / z x x$ \\
\hline 1244 & 415 & $\infty / 2.2$ & $z y y / y y z$ \\
\hline 1368 & 456 & $6.0 / \infty$ & $x x z / x z x$ \\
\hline 1492 & 497 & $7.4 / \infty$ & $y y z / y z y$ \\
\hline 1720 & 573 & 54.7 & $z x x / z x x$ \\
\hline 1916 & 639 & 75.1 & $z y y / z y y$ \\
\hline 2196 & 732 & $67.2 / \infty$ & $z x x / z x x$ \\
\hline 3312 & 1104 & 1290 & $z y y / z y y$ \\
\hline 3394 & 1131 & $\infty / 12.4$ & $z y y / y y z$ \\
\hline 3603 & 1201 & $\infty / \infty$ & $z x x / z x x$ \\
\hline 3604 & 1201 & $\infty / 12.3$ & $z x x / x x z$ \\
\hline 3630 & 1210 & 455 & $z y y / z y y$ \\
\hline 3658 & 1219 & 43.4 & $z z z / z z z$ \\
\hline \multicolumn{4}{|c|}{ FHG in PP RTA } \\
\hline 2424 & 606 & 12.6 & $x x z / z z z$ \\
\hline 2501 & 625 & 13.9 & $y y z / z z z$ \\
\hline 3394 & 849 & $\infty / 8.9$ & $z y y / y y z$ \\
\hline 3604 & 901 & $\infty / 17.3$ & $z x x / x x z$ \\
\hline 4091 & 1023 & 40.1 & $z z z / z z z$ \\
\hline \multicolumn{4}{|c|}{ THG in PP KTP } \\
\hline 1082 & 361 & $\infty / 4.6$ & $z y y / z y y$ \\
\hline 1213 & 404 & $4.6 / \infty$ & $x x z / x z x$ \\
\hline 1325 & 442 & $5.6 / \infty$ & $y y z / y z y$ \\
\hline 1473 & 491 & 36.5 & $z x x / z x x$ \\
\hline 1623 & 541 & 47.8 & $z y y / z y y$ \\
\hline 1824 & 608 & $40.9 / \infty$ & $z x x / z x x$ \\
\hline \multirow[t]{2}{*}{2052} & 684 & $\infty / 6.0$ & $z y y / y y z$ \\
\hline & & $59.0 / \infty$ & $z y y / z y y$ \\
\hline \multicolumn{4}{|c|}{ FHG in PP KTP } \\
\hline 2161 & 540 & 9.8 & $x x z / z z z$ \\
\hline 2227 & 557 & 10.8 & $y y z / z z z$ \\
\hline
\end{tabular}

${ }^{a}$ Same as in Table 1. Nonlinear efficiencies $\propto\left(d_{31}\right)^{2}(x x z, x z x$, $z x x)<\left(d_{32}\right)^{2}(y y z, y z y, z y y) \ll\left(d_{33}\right)^{2}(z z z)$. Note, in RTA, the THG and FHG of the $\mathrm{He}-\mathrm{Ne} / \mathrm{CH}_{4}$ standard (3392 $\mathrm{nm}$ ) and the rare BPM-BPM THG $(3603 \mathrm{~nm})$.

indices ${ }^{8,9}$ and of the length ${ }^{10}$ of the crystal, it did not fit our experimental results well, probably because the Sellmeier coefficients ${ }^{8,9}$ are not accurate beyond $3 \mu \mathrm{m}$. Still, one set of coefficients ${ }^{9}$ enabled us to predict qualitatively that higher temperatures should give THG at longer wavelengths and incidences closer to normal, a prediction confirmed by the experiment.

We demonstrated experimentally THG coincidence eee/eee in PP LN (Fig. 1, Table 1). A CO overtone laser, ${ }^{11,12}$ emitting on its $v^{\prime}=30 \rightarrow v=28$ band (3.54$3.61 \mu \mathrm{m}$ ) with an output power of $\leq 250 \mathrm{~mW}$, was focused to a $50-\mu \mathrm{m}$ waist in our $20 \mathrm{~mm} \times 15 \mathrm{~mm} \times$ $0.5-\mathrm{mm}$ PP LN sample. The crystal was poled with a period $\Lambda=31.5 \mu \mathrm{m}$ and antireflection coated at 1.8 and $1.2 \mu \mathrm{m}$. The output SHG at $1.8 \mu \mathrm{m}$ was measured with an InGaAs photodiode with a $2.2-\mu \mathrm{m}$ cutoff. The efficiency inside the crystal was $P(2 \omega) / P(\omega)^{2} \geq$ $5.410^{-4} \mathrm{~W}^{-1}$. The third-harmonic signal was then detected and measured with an optical spectrum analyzer. The light was brought to it by a $400-\mu \mathrm{m}$ diameter multimode fiber. This large fiber core was most convenient for optimizing the mixing (crystal angles, focusing) for maximum THG signal.

By measuring the THG efficiency for different $\mathrm{CO}$ laser lines, we determined that the optimum THG coincidence point occurs at a shorter wavelength than expected from the theory (Table 1). At room temperature the THG coincidence point for our crystal is at $<3.54 \mu \mathrm{m}$, which is in the gap between the $29 \rightarrow 27$ and the $30 \rightarrow 28$ bands of our CO overtone laser. Even so, $\sim 200 \mathrm{~mW}$ out of the CO laser still produces a maximum THG power of $\sim 0.5 \mathrm{nW}$ at $22{ }^{\circ} \mathrm{C}$ and $\sim 15$-deg incidence. We were then able to temperature tune the optimum coincidence point to $3.56 \mu \mathrm{m}\left[P_{28}(9)\right]$, for a temperature of $133{ }^{\circ} \mathrm{C}$ and nearly normal incidence. We obtained a THG power of $\sim 7.3 \mathrm{nW}$ (sufficient for phase-locking purposes) for a $\mathrm{CO}$ power of $195 \mathrm{~mW}$ (Fig. 2), which corresponds to a THG efficiency inside the crystal of $P(3 \omega) / P(\omega)^{3} \leq 10^{-6} \mathrm{~W}^{-2}$. This optimized efficiency corresponds to normal incidence, i.e., maximum interaction length (collinear QPM) in our crystal.

As the first stage in using THG for optical frequency synthesis we made a two-step, divide-by-nine frequency conversion between 10.71 and $1.19 \mu \mathrm{m}$. The CO overtone laser, emitting at $84 \mathrm{THz}$ on $P_{28}(11)(3.57 \mu \mathrm{m})$, 


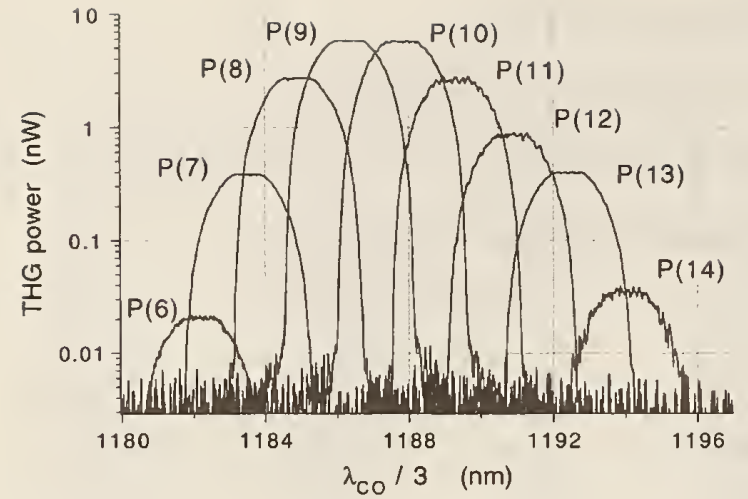

Fig. 2. THG power, at $133^{\circ} \mathrm{C}$, for several $\mathrm{CO}$ overtone frequencies. The width of the signals is the resolution of the optical analyzer, fixed by the fiber's diameter (input slit).

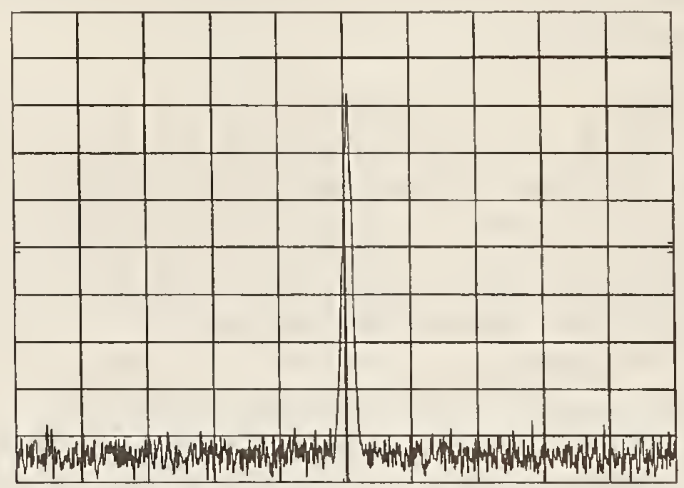

Fig. 3. MIM-diode voltage of beat note $f_{\mathrm{CO}}-3 f_{\mathrm{CO}_{2}}$. Vertical linear scale. Center frequency, $796 \mathrm{MHz}$; scan, $10 \mathrm{MHz}$; resolution and video bandwidths, 100 and $30 \mathrm{kHz}$, respectively.

was frequency tripled in PP LN to $252 \mathrm{THz}(1.19 \mu \mathrm{m})$. It was also connected to a ${ }^{12} \mathrm{C}^{18} \mathrm{O}_{2}$ laser emitting on $P_{\mathrm{I}}(40)(28 \mathrm{THz}, 10.71 \mu \mathrm{m})$ by use of a metalinsulator-metal (MIM) diode; the electrical IF signal from the diode corresponded to the beat note between the tripled $\mathrm{CO}_{2}$ laser and the $\mathrm{CO}$ laser. ${ }^{12}$ The two laser beams were focused on the MIM diode at orthogonal angles, as has proved to be optimum. ${ }^{13}$ The laser powers and the resultant rectified voltages in the MIM were $70 \mathrm{~mW}$ and $0.5 \mathrm{mV}$ for the CO laser and $200 \mathrm{~mW}$ and $5 \mathrm{mV}$ for the $\mathrm{CO}_{2}$ laser. We obtained a $796-\mathrm{MHz}$ beat note with a signal-to-noise ratio of $\sim 25 \mathrm{~dB}$ in a $100-\mathrm{kHz}$ detection bandwidth (Fig. 3), which should be adequate for phase locking the CO laser. $\mathrm{CO}_{2}$ lasers have now been stabilized to the hertz level on the short term, with subhertz Flicker plateaus and reproducibilities of $<10 \mathrm{~Hz} \cdot{ }^{14}$ Phase locking a $\mathrm{CO}$ laser to such a reference would yield a short-term stability of $\sim 10 \mathrm{~Hz}$ at $1.19 \mu \mathrm{m}$.

We have demonstrated a new use of QPM in PP crystals for optical frequency mixing. One can remove the constraint of finding coincidences by juxtaposing different gratings in the same crystal, each grating corresponding to each desired mixing. This procedure can also lead to an optical equivalent of $\mathrm{rf}$ diode mixers that generate the sum, difference, and second harmonics of two input frequencies. We have observed all four of these mixings (occurring at different angles and QPM orders) out of a single-grating PP LN crystal with inputs from an 800-nm diode and a 1064-nm Nd:YAG laser. More generally, Fejer et al. proposed an elegant design method of phase matching curves by Fourier analysis of spatial poling frequencies. ${ }^{4}$ Hence QPM opens many new possibilities in optical frequency synthesis and high-resolution spectroscopy, especially because these applications do not usually require high power. Optical beat notes can be detected with $\geq 1 \mathrm{nW}$ of power, and ultrahigh-sensitivity saturation spectroscopy can now be achieved with low initial power by use of buildup in high-finesse optical resonators. ${ }^{15}$

We thank L. E. Myers, R. W. Fox, S. Waltman, A. Bredsford, K. Petrov, and J. L. Hall for significant contributions to this research, which is supported in part by the U.S. Air Force Office of Scientific Research.

*Permanent address, Calvin College, Grand Rapids, Michigan 49546.

\section{References}

1. A detailed list of references is given in Ref. 2 .

2. O. Pfister, M. Mürtz, J. S. Wells, L. Hollberg, and J. T. Murray, Opt. Lett. 21, 1387 (1996), and references therein.

3. J. A. Armstrong, N. Bloembergen, J. Ducuing, and P. S. Pershan, Phys. Rev. 127, 1918 (1962).

4. M. M. Fejer, G. A. Magel, D. H. Jundt, and R. L. Byer, IEEE J. Quantum Electron. 28, 2631 (1992).

5. L. E. Myers, R. C. Eckardt, M. M. Fejer, R. L. Byer, W. R. Bosenberg, and J. W. Pierce, J. Opt. Soc. Am. B 12, 2102 (1995), and references therein.

6. For cascading three-wave mixings, see E. Yablonovitch, C. Flytzanis, and N. Bloembergen, Phys. Rev. Lett. 29, 865 (1972); R. DeSalvo, D. J. Hagan, M. Sheik-Bahae, G. Stegeman, and E. Van Stryland, Opt. Lett. 17, 28 (1992). Optimally phase-matched cascading three-wave mixings in nonresonant crystals (as here) is new: $M$. Asobe, I. Yokohama, H. Itoh, and T. Kaino, Opt. Lett. 22, 274 (1997)

7. We are grateful to one of the referees for pointing out the existence of hybrid QPM-BPM coincidences in a crystal whose length is partially poled.

8. M. V. Hobden and J. Warner, Phys. Lett. 22, 243 (1966).

9. G. J. Edwards and M. Lawrence, Opt. Quantum Electron. 16, 373 (1984).

10. P. K. Gallagher, H. M. O'Bryan, E. M. Gyorgy, and J. T. Krause, Ferroelectrics 75, 71 (1987).

11. E. Bachem, A. Dax, T. Fink, A. Weidenfeller, M. Schneider, and W. Urban, Appl. Phys. B 57, 185 (1993).

12. A. Dax, J. S. Wells, L. Hollberg, A. G. Maki, and W. Urban, J. Mol. Spectrosc. 168, 416 (1994).

13. K. M. Evenson, M. Inguscio, and D. A. Jennings, J. Appl. Phys. 57, 956 (1985).

14. V. Bernard, P. E. Durand, T. George, H. W. Nicolaisen, A. Amy-Klein, and Ch. Chardonnet, IEEE J. Quantum Electron. 31, 1913 (1995); O. Acef, Opt. Commun. 134, 479 (1997).

15. J. Ye, L. S. Ma, and J. L. Hall, IEEE Trans. Instrum. Meas. 46, 178 (1997). 
Part V

\section{Multiphoton Interactions and Optical Coherences}




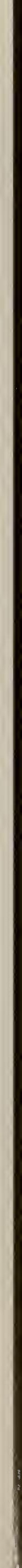




\title{
Optical probing of cold trapped atoms
}

\author{
R. W. Fox, S. L. Gilbert, L. Hollberg, and J. H. Marquardt \\ National Institute of Standards and Technology, Boulder, Colorado 80303
}

H. G. Robinson

Department of Physics, Duke University, Durham, North Carolina 27706

Received April 6, 1993

\begin{abstract}
Transitions between excited states of laser-cooled and laser-trapped rubidium and cesium atoms are probed by use of fiber and diode lasers. High-resolution Doppler-free spectra are detected by observation of the absorption and fluorescence of light from the intermediate level of two-step cascade systems. The optical double-resonance spectra show Autler-Townes splitting in the weak probe limit and more complicated spectra for a strongly coupled three-level system.
\end{abstract}

The potential, and even the requirement, for the use of laser-cooled atoms in the highest-resolution optical spectroscopy is well recognized, ${ }^{1}$ but little high-resolution optical spectroscopy has been done on laser-cooled neutral atoms. This is in marked contrast to impressive demonstrations of extremely high-resolution optical spectroscopy on laser-cooled trapped ions. ${ }^{2}$ With room-temperature atoms and Doppler-free techniques such as saturated absorption and two-photon transitions, the ultimate resolution and spectroscopic accuracy is usually limited by velocity-dependent effects, such as transit time broadening, second-order Doppler shift, and wavefront curvature. ${ }^{3}$ These limitations can be avoided by the use of low-velocity atoms. Trapped-atom systems also operate at low pressures, which should significantly reduce the pressure shifts that often plague gas-cell wavelength measurements and stability.

Two important technological advances have greatly simplified experiments with low-velocity atoms. These are magneto-optic traps ${ }^{4}$ (also known as Zeeman-shift optical traps) and the implementation of these traps in a relatively simple vapor cell. ${ }^{5}$ Recent research with neutral atom traps has demonstrated very-high-resolution microwave spectra, ${ }^{6}$ narrow Raman spectra, ${ }^{7}$ and optical spectroscopy out of the ground state. ${ }^{8}$ To our knowledge, our experiments demonstrate the first high-resolution optical spectroscopy of excited states of laser-trapped and laser-cooled alkali atoms. By probing the excited state of the trapping transition, we have an independent diagnostic of the conditions of the trapped atoms.

In our experiments, cesium or rubidium atoms are trapped in separate vapor cells by use of diode lasers tuned to resonance lines of the atoms. Additional lasers are then used to probe the trapped atoms in the excited state of the trapping-cooling transition. The transitions of interest are shown on simplified energy-level diagrams in Fig. 1. Most of the experimental conditions are common to both systems and are similar to those described in the literature. ${ }^{5,9}$ Small ion-pumped cell traps operate at background alkali pressures that range from $10^{-5}$ to $10^{-7} \mathrm{~Pa}\left(\approx 10^{-7}-10^{-9}\right.$ Torr $)$. The trapping laser beams are approximately $1 \mathrm{~cm}$ in diameter, with $\sim 2-5 \mathrm{~mW}$ in each beam. In each experiment, an elliptically polarized probe beam copropagates with one of the trapping beams. These systems typically confine between $10^{7}$ and $10^{8}$ atoms in a $1-2-\mathrm{mm}$ diameter cloud at temperatures of a few hundred microkelvins.

The study of rubidium is part of a program to develop a wavelength standard for optical communications in the $1.5-\mu \mathrm{m}$ region. ${ }^{10}$ Atoms are trapped with the use of two 780-nm diode lasers. The trapping laser is tuned to the low-frequency side of the $5 S_{1 / 2}, F=2 \rightarrow 5 P_{3 / 2}, F=3$ rubidium $\left({ }^{87} \mathrm{Rb}\right)$ cycling transition and cools and traps the atoms. The repumping laser, tuned to the $5 S_{1 / 2}, F=1 \rightarrow 5 P_{3 / 2}, F=$ 2 transition, prevents the atoms from accumulating in the $F=1$ ground state. The trapping laser was locked to the low-frequency side of the cooling transition by use of a signal from a saturated-absorption spectrometer. The $1.529-\mu \mathrm{m} 5 P_{3 / 2} \rightarrow 4 D_{5 / 2}$ transitions were probed with the use of a tunable erbiumdoped fiber laser that has a free-running linewidth of $1 \mathrm{MHz} .{ }^{11}$ We observed these transitions by measuring their effect on the 780-nm trap fluorescence. When the fiber laser is tuned into resonance, it removes population from the $5 P, F=3$ level and

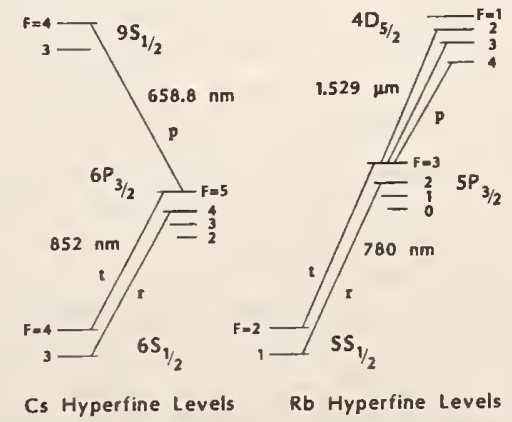

Fig. 1. Diagram of relevant energy levels of cesium $\left({ }^{133} \mathrm{Cs}\right)$ and rubidium $\left({ }^{87} \mathrm{Rb}\right)$ with the trapping-cooling $(\mathrm{t})$, repumping (r), and probe (p) transitions indicated. 
causes a reduction in the $780-\mathrm{nm}$ fluorescent light emitted by the trapped atoms. The fiber laser's effect on this fluorescence can be quite large for the $5 P, F=3 \rightarrow 4 D, F=3$ and 2 transitions because it optically pumps the atoms into the $5 S, F=1$ state, removing them from the strongly fluorescing $5 S, F=2 \rightarrow 5 P, F=3 \rightarrow 5 S, F=2$ cycle. The atoms must then be pumped back into the cycling transition by the repumping laser. When the repumping rate is relatively small, this depopulation technique greatly enhances the sensitivity for detection of weak signals. ${ }^{12}$ The $5 P, F=3 \rightarrow 4 D$, $F=4$ signal, however, is not enhanced because this transition does not optically pump the atoms into the $F=1$ ground state. The $5 S, F=2,5 P, F=3$ and $4 D, F=4$ levels compose a closed system of three levels that are strongly coupled in the presence of the two laser fields. A small modulation was applied to the fiber-laser frequency, and the first derivative of the 780-nm trap fluorescence was obtained by use of phase-sensitive detection. Figure 2(a) shows this derivative signal as the $1.529-\mu \mathrm{m}$ fiber laser is scanned through the resonances.

The trapped atoms in the cesium experiment are probed by observation of absorption out of the upper state of the $6 S_{1 / 2}, F=4 \rightarrow 6 P_{3 / 2}, F=5$ trapping-cooling transition. We used both direct absorption and derivative techniques to interrogate the red $6 P_{3 / 2}, F=5 \rightarrow 9 S_{1 / 2}, F=4$ transition at $658.8 \mathrm{~nm}$. Approximately $1.5 \mathrm{~mW}$ of power at $658.8 \mathrm{~nm}$ was provided by an extended-cavity diode laser that was frequency stabilized to a reference cavity, resulting in a short-term linewidth of $\sim 500 \mathrm{~Hz}$. The laser's asymmetric spatial mode was filtered by a single-mode fiber used to deliver the light to the trap. The probe beam diameter was set to be approximately the same size as the trappedatom cloud. After frequency stabilization, spatial filtering, and amplitude noise subtraction, the probe laser power at the trap was $\sim 40 \mu \mathrm{W}$. Absorption by the trapped cesium atoms was typically $1 \%$ and provided a signal-to-noise ratio of $\sim 10$ in a $200-\mathrm{kHz}$ detection bandwidth. The power in the probe laser is low enough so that it does not appear to perturb the trap dynamics or the absorption line shape. We accomplished precise detuning of the 852-nm cesium trapping laser by offset locking the laser (using an acousto-optic modulator) to a saturatedabsorption crossover resonance. Figure 2(b) shows cesium absorption of the 658.8-nm probe light.

These cesium and rubidium cascade two-photon transitions have high signal-to-noise ratios and, because of the laser cooling, are free of Doppler broadening and associated effects. The obvious double-peaked nature of these signals is due to Autler-Townes splitting of the $P$ states that is caused by the relatively strong trapping laser light. ${ }^{13-15}$ The cesium $6 P, F=5 \rightarrow 9 S, F=4$ transition and the rubidium $5 P, F=3 \rightarrow 4 D, F=$ 3 and $F=2$ transitions are representative of the moderately weak probe regime, in which the Rabi frequency owing to the probe light is much less than the Rabi frequency owing to the trapping laser. The $5 P, F=3 \rightarrow 4 D, F=4$ rubidium transition is in the moderately strong probe regime because of the larger matrix element for this transition. This strong coupling results in an asymmetic line shape. The observed linewidths are influenced by power broadening owing to the lasers, the magneticfield gradient that causes splitting of the Zeeman levels, and nonuniform laser-field strength across the volume of the trap. From our measurements of linewidths with the magnetic field off, we conclude that broadening that results from the splitting of Zeeman levels is less than $1 \mathrm{MHz}$. We estimate that the residual Doppler broadening is less than $300 \mathrm{kHz}$. It is actually remarkable that the experimental line shapes are so narrow; linewidths as narrow as $3.1 \mathrm{MHz}$ FWHM were observed on the $658.8-\mathrm{nm}$ transition in cesium. This is to be compared with a natural width for the $P$ state of $5.3 \mathrm{MHz}$. The ultimate probe transition linewidth is not affected by the intermediate-state lifetime; instead, it is limited by the natural linewidth of the upper level, ${ }^{15}$ which is $\sim 0.97 \mathrm{MHz}$ for the $9 S_{1 / 2}$ state of cesium and $\sim 1.8 \mathrm{MHz}$ for the $4 D_{5 / 2}$ state of rubidium.

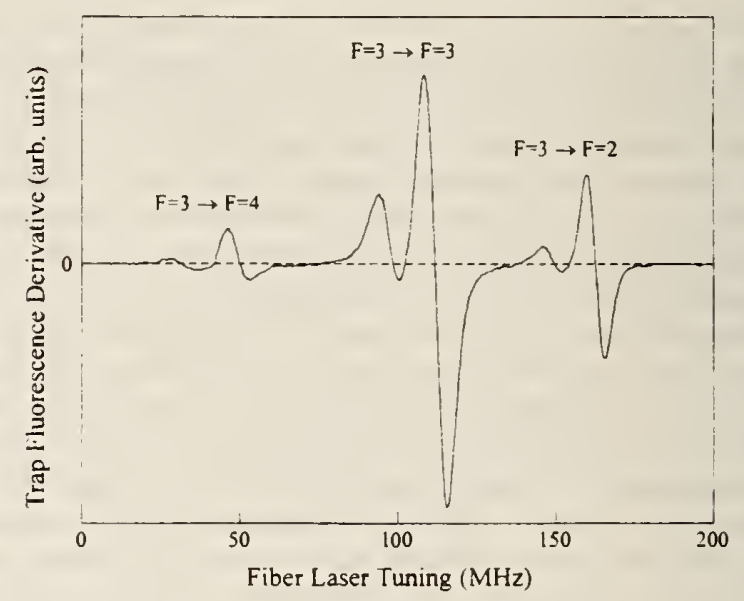

(a)

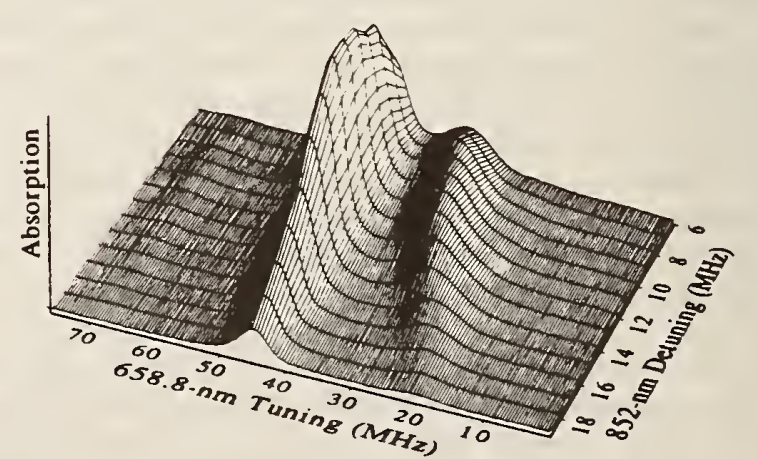

(b)

Fig. 2. (a) Rubidium line shape showing the derivative of the $780-\mathrm{nm}$ fluorescence signal as the $1.529-\mu \mathrm{m}$ fiber laser was scanned through the $5 P_{3 / 2}, F=3 \rightarrow 4 D_{5 / 2}, F=4$, 3 , and 2 transitions. The trapping laser detuning was $-11 \pm 1 \mathrm{MHz}$, and the Rabi frequency was $8.5 \pm 0.5 \mathrm{MHz}$. The fiber-laser intensity was $0.7 \pm 0.2 \mathrm{~mW} / \mathrm{cm}^{2}$. (b) Cesium absorption at $658.8 \mathrm{~nm}$ as a function of the probe laser frequency for various trap laser detunings. We improved the signal-to-noise ratio by averaging 200 scans at each detuning. The large peaks on successive lines were aligned to display clearly the change in splitting. 


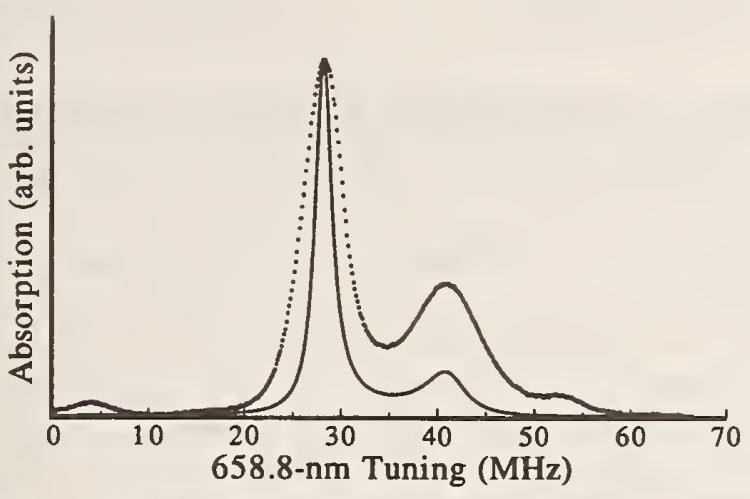

Fig. 3. Theoretical cesium-absorption line shape and experimental data points for $9-\mathrm{MHz}$ detuning of the trapping laser and a Rabi frequency of $11 \mathrm{MHz}$. The experimental data show sidebands at 4 and $54 \mathrm{MHz}$ that result from $25-\mathrm{MHz}$ modulation of the probe laser. These sidebands are used for determining the frequency scale.

A density matrix model with five energy levels was used to calculate theoretical line shapes for the twophoton resonances. Three of these levels, $|1\rangle,|2\rangle$, and $|3\rangle$ with energies $E 1<E 2<E 3$, form the cascadelevel system that is coherently excited by two lasers. The remaining two levels, $|4\rangle$ and $|5\rangle$, are artificial levels that represent decay channels from $|2\rangle$ and $|3\rangle$ and can themselves decay only to the ground state $|1\rangle$. These artificial levels approximate the real effects of decay through other hyperfine levels and the repumping by a third laser. The parameters entering the theory include the trap laser detuning from resonance, the probe laser frequency (which was swept), the Rabi frequencies for the two applied laser fields, and the six relaxation coefficients between states. The relaxation coefficients and radiative decay constants come from known Einstein $A$ values. Our analysis follows that of previous research ${ }^{13}$ with some extensions.

To make the analysis tractable, we assume motionless atoms, uniform laser intensity, no collisions, and no trap dynamics. A simplified model of Zeeman broadening and magnetic-field gradients has been included. The general features of the theoretical line shape agree with the experimental results (see Fig. 3), but quantitative agreement is not found within experimental uncertainties. Our model contains no free parameters, other than signal size, and generally gives line shapes that are too narrow. Quantitative agreement of the line shapes should yield illuminating details of the dynamics of the trapped atoms, but will require more sophisticated models of the conditions within the trap. The most significant simplification in our model is the lack of averaging over the three-dimensional atom density and laser field and hence over the variation in Rabi frequency. These spatial variations may account for the difference between observed and calculated linewidths.

The potential accuracy in determining the line center in these narrow two-photon transitions is yet to be determined. Though essentially free from systematic effects owing to pressure, Doppler, transit, and wave-front perturbations, the transitions are affected by ac Stark shifts resulting from the trapping laser and, to a lesser extent, by the trap's magnetic field. These problems could be reduced by temporarily turning off the trapping fields while probing the atoms with weak laser fields.

These experiments have demonstrated the potential for high-resolution optical detection of narrow transitions in trapped neutral atoms. Experimental line shapes give good qualitative and fair quantitative agreement with calculations based on a simple model. Trapped neutral-atom spectroscopy yields Doppler-free signals and diagnostic information about the conditions of the trapped atoms; the Autler-Townes-split line shape provides information on the average square of the laser electric field that the atoms experience. As with trapped ions, we can anticipate that spectroscopy in these systems will soon measure resonances for which the accuracy and precision are not limited by the atom's environment.

We gratefully acknowledge the helpful comments of C. Wieman, C. Weimer, J. L. Hall, and J. Bollinger and the support of the U.S. Air Force Office of Scientific Research, NASA, and the Naval Command, Control and Ocean Surveillance Center.

\section{References}

1. J. L. Hall, M. Zhu, and P. Buch, J. Opt. Soc. Am. B 6, 2194 (1989); W. Ertmer and S. Penselin, Metrologia 22, 195 (1986).

2. J. C. Bergquist, W. M. Itano, F. Elsner, M. G. Raizen, and D. J. Wineland, in Light Induced Kinetic Effects in Atoms, Ions and Molecules, L. Moi, ed. (ETS Editrice, Pisa, Italy, 1991), p. 291; W. Nagourney, N. Ya, and H. Demelt, Opt. Commun. 79, 176 (1990).

3. C. J. Bordé, J. L. Hall, C. V. Kunasz, and D. G. Hummer, Phys. Rev. A 14, 236 (1976).

4. E. L. Raab, M. Prentiss, A. Cable, S. Chu, and D. Pritchard, Phys. Rev. Lett. 59, 2631 (1987).

5. C. Monroe, W. Swann, H. Robinson, and C. Wieman, Phys. Rev. Lett. 65, 1571 (1990).

6. C. Monroe, H. Robinson, and C. Wieman, Opt. Lett. 16, 50 (1991); A. Clairon, C. Salomon, S. Guellati, and W. D. Phillips, Europhys. Lett. 16, 165 (1991); K. Gibble and S. Chu, Phys. Rev. Lett. 70, 1771 (1993).

7. D. Grison, B. Lounis, C. Salomon, J. Courtois, and G. Grynberg, Europhys. Lett. 15, 149 (1991); J. Tabosa, G. Chan, and H. Kimble, Phys. Rev. Lett. 66, 3245 (1991); L. Hilco, C. Fabre, and E. Giacobino, Europhys. Lett. 18, 685 (1992).

8. M. Zhu, C. W. Oates, and J. L. Hall, Opt. Lett. 18, 1186 (1993).

9. K. Lindquist, M. Stephens, and C. Wieman, Phys. Rev. A 46, 4082 (1992).

10. S. L. Gilbert, Proc. Soc. Photo-Opt. Instrum. Eng. 1837, 146 (1993).

11. S. L. Gilbert, Opt. Lett. 16, 150 (1991).

12. D. J. Wineland and W. M. Itano, Phys. Lett. 82A, 75 (1981).

13. S. H. Autler and C. H. Townes, Phys. Rev. 100, 703 (1955); R. M. Whitley and C. R. Stroud, Jr., Phys. Rev. A 14, 1498 (1976).

14. J. L. Picqué and J. Pinard, J. Phys. B 9, L77 (1976).

15. P. R. Hemmer, B. W. Peuse, F. Y. Wu, J. E. Thomas, and S. Ezekiel, Opt. Lett. 6. 531 (1981). 


\title{
Experimental Demonstration of Laser Oscillation without Population Inversion via Quantum Interference in $\mathbf{R b}$
}

\author{
A. S. Zibrov, ${ }^{1,2,4,5}$ M. D. Lukin, ${ }^{1,2,3}$ D. E. Nikonov, ${ }^{1,2,3}$ L. Hollberg, ${ }^{4}$ M. O. Scully, ${ }^{1,2,3}$ V. L. Velichansky, ${ }^{5}$ \\ and H. G. Robinson ${ }^{4}$ \\ ${ }^{1}$ Department of Physics, Texas A\&M University. College Station, Texas 77843 \\ ${ }^{2}$ Texas Laser Lab, HARC, The Woodlands. Texas 77381 \\ ${ }^{3}$ Max-Planck-Institut für Quantenoptik, D-85748 Garching. Germany \\ ${ }^{4}$ National Institute of Standards and Technology, Boulder, Colorado 80303 \\ ${ }^{5}$ Lebedev Institute of Physics, Moscow, 117924 Russia
}

(Received 23 February 1995)

\begin{abstract}
Laser oscillation without population inversion is demonstrated experimentally in a V-type atomic configuration within the $D_{1}$ and $D_{2}$ lines of Rb vapor. It is shown that the effect is due to the atomic interference. The experimental results, as first predicted by careful theoretical analysis, are in a good agreement with detailed calculations.
\end{abstract}

PACS numbers: 42.50.-p, 42.55.-f

In the present work, laser oscillation without population inversion (LWI) [1] is reported for the first time. Our demonstration makes use of noninversion gain in a Vtype [2] $\mathrm{cw}$ amplifier. In order to obtain a clear proofof-principle demonstration of LWI oscillation, we carried out a set of experiments which include the following: the observation of inversionless amplification of a weak probe light by a coherently prepared atomic medium, experimental verification of the absence of inversion, and, finally, observation of noninversion laser oscillation in a cavity.

Several recent experiments $[4,5]$ have already demonstrated the possibility of noninversion amplification. These experiments were based on observation of amplification in $\Lambda$-type systems [6] which involved coherent population trapping and can therefore be viewed as amplification with inversion in a dressed state basis.

$\mathrm{V}$-type inversionless amplification is itself conceptually interesting in that the physics of resonant V-type LWI is the cancellation of absorption via quantum interference. And there is, in general, no dressed state basis in which there is a "hidden" inversion. Furthermore, the present work is a technical advance, in that it is the first observation of gain without inversion using low power $\mathrm{cw}$ diode lasers.

The present experiment was suggested and modeled by a detailed theoretical analysis, the results of which may be understood by considering the four-level model of Fig. 1(a), although the actual calculations were carried out using a realistic model of $\mathrm{Rb}^{87}$ with all hyperfine and Zeeman sublevels included. Two sublevels of the ground state are coupled to a pair of excited states via three fields. A strong driving field with Rabi frequency $\Omega_{c}$ and a weak probe field with Rabi frequency $\Omega_{a}$ are assumed to be quasimonochromatic. These fields have linewidths $\Delta \nu_{c}$ and $\Delta \nu_{a}$, both of which are much less than atomic radiative decay rates. The third (pump) field is taken to be incoherent, i.e., it has a very broad linewidth $\left(\Delta \nu_{\text {pump }} \gg \gamma_{a}, \gamma_{c}\right)$ and is represented by an incoherent pumping rate $r$ [see Fig. 1(a)].

In the absence of the incoherent pump field, almost all the population is optically pumped into the state $b^{\prime}$ by the strong driving field. The incoherent pump destroys this optical pumping by populating the upper state $a$, and thus state $b$, via spontaneous decay. Hence the population difference $\rho_{a a}-\rho_{b b}$ is determined by the rates with

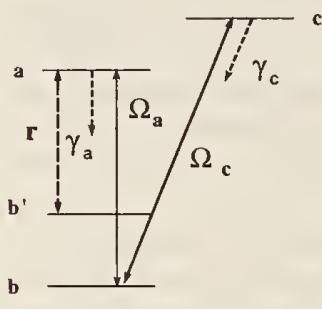

(a)

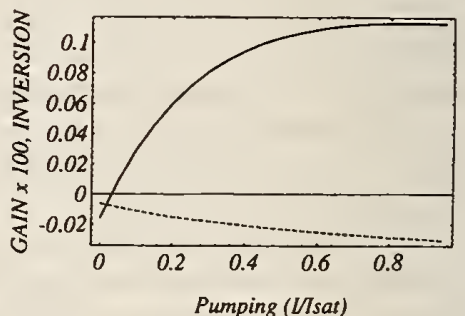

(b)
FIG. 1. (a) Simplified four-level model for lasing without inversion. In our realization of the model in $\mathrm{Rb}^{87}|a\rangle$ corresponds to sublevels $\left|P_{1 / 2}, F=2\right\rangle,|c\rangle=\left|P_{3 / 2}, F=2\right\rangle$ and the two sublevels of ground state $|b\rangle=\left|S_{1 / 2}, F=1\right\rangle$ and $\left|b^{\prime}\right\rangle=\left|S_{1 / 2}, F=2\right\rangle$. Driving field $\Omega_{c}$ is tuned to the $D_{2}$ resonance from the $F=1$ ground state to $F=2$ of excited state, probe field $\Omega_{a}$ couples the same sublevels of the ground state with levels having $F=2$ of the $P_{1 / 2}$ excited state. Polarizations of the fields are described in the text. (b) Results of numerical modeling for the realistic 32-level scheme of $\mathrm{Rb}$. Gain $\times 8 \pi / N \lambda^{2} L$ (solid line) and inversion per atom (dashed line) as a function of the incoherent $\Delta \nu=50 \mathrm{MHz}$ pump laser intensity; parameters are $\left|\Omega_{c}\right|=30 \mathrm{MHz}, H_{z}=10^{-4} \mathrm{~T}$. All fields are in the exact resonance with corresponding transitions in the center of the Doppler profile. Inversion is defined as the sum of populations of levels $P_{1 / 2}, F=$ 2. $M_{F}=0, \pm 1$ minus populations of ground state sublevels having $F=1$ with corresponding $M_{F}$. Incoherent pumping rate is expressed in terms of Rabi frequency and a linewidth of a pumping laser as $r=2\left|\Omega_{\text {pump }}\right|^{2} /\left(\Delta \nu+\gamma_{a} / 2\right) \gamma_{a}$. 
which atoms leave and decay into the states $a$ and $b$. Note that in the limit of a weak probe field, atoms can leave state $b$ only via state $c$. Thus the population difference above is determined by the ratio of decay rates from level $a$ to level $b$ and from level $c$ to level $b^{\prime}$. If level $c$ decays more slowly than level $a$, an inversion on the transition coupled by the weak probe field cannot be created.

Amplification of a weak probe field on the $a$ to $b$ transition is. however, possible, due to the presence of atomic coherence between upper levels $a$ and $c$. The physical origin of this mechanism is to be interpreted as a quantum interference cancellation of absorption. Alternatively, reduction of absorption and enhanced gain can be viewed as a result of a Fano-type interference between the dressed states [2].

The gain (absorption) coefficient for a weak probe field on the $a$ to $b$ transition is proportional to the imaginary part of the off-diagonal element of the atomic density matrix $\rho_{a b}$. In the weak probe field limit, and under conditions of exact resonance, we obtain the gain

$$
\begin{aligned}
G= & \frac{3 \lambda^{2} N L \gamma_{a}}{4 \pi} \frac{\operatorname{Im} \rho_{a b}}{\Omega_{a}}=\frac{3 \lambda^{2} N L \gamma_{a}}{4 \pi} \\
& \times \frac{\left(\rho_{a a}^{0}-\rho_{b b}^{0}\right)+\left|\Omega_{c}\right|^{2} / \gamma_{a c} \gamma_{b c}\left(\rho_{b b}^{0}-\rho_{c c}^{0}\right)}{\gamma_{a b}+\left|\Omega_{c}\right|^{2} / \gamma_{a c}},
\end{aligned}
$$

where $\rho_{i i}^{0}$ is the population of level $i$ calculated to the zeroth order in the probe field, $\gamma_{i j}$ is the relaxation rate of the density matrix element $\rho_{i j}, N$ is the density of atoms. $L$ is cell length, and $\lambda$ is the probe laser wavelength.

From the second equality in Eq. (1) we see that there are two contributions of the upper-level coherence which modify the usual gain or absorption. The first is the dynamical Stark effect [7], studied theoretically

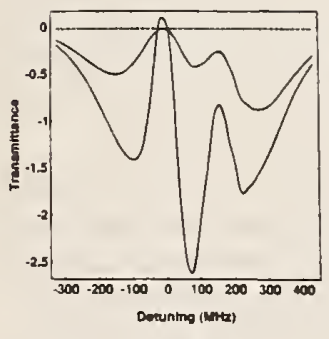

(a)

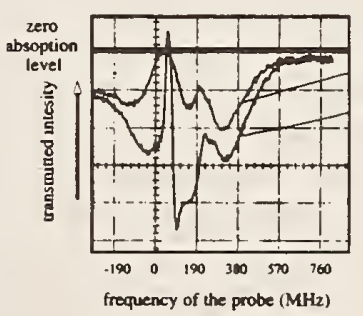

(b)

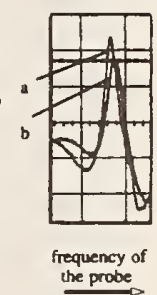

(c)
FIG. 2. (a) Calculated absorption (gain) coefficient for a weak probe field as a function of its frequency in the vicinity of $1 \rightarrow 2^{\prime}$ resonance of the $D_{1}$ line. Parameters and scaling correspond to those of Fig. 1(b). Detuning is from the center of the absorption line. Upper curve. without incoherent pump; lower curve, $r=0.4$. (b) Experimentally measured transmission of the probe laser as a function of its frequency. Curve $a$, without the incoherent laser; curve $b$, with incoherent laser. Estimated parameters for the experiment are $\Omega_{c} \sim$ $30 \mathrm{MHz}, \Omega_{a} \sim 0.1 \mathrm{MHz}, r \sim 0.4$. (c) Experimental results for transmission of the probe field showing the influence of a probe laser linewidth on its transmission spectrum. Curve $a$, without modulation of current; curve $b$, with modulation of current $\left(\Delta \nu_{p} \sim 10 \mathrm{MHz}\right)$. and experimentally in a $\mathrm{V}$-type configuration in [3]; it is responsible for the $\left|\Omega_{c}\right|^{2}$ term in the denominator.

There is, however, another important effect which modifies the properties of the $\mathrm{V}$ scheme. This contribution is represented by the term proportional to the $\left|\Omega_{c}\right|^{2}$ in the numerator of Eq. (1) and is precisely due to the quantum interference of absorption mentioned above. It follows from Eq. (1) that absorption interferes destructively if $\rho_{b b}-\rho_{c c}>0$. This leads to a reduction of absorption and enhances the gain. As a result the probe field can be amplified even if $\rho_{a a}-\rho_{b b}<0$. We would like to underscore the fact that quantum interference is the only reason for inversionless gain in the $\mathrm{V}$ scheme, i.e., there is no inversion in any dressed state picture (provided the drive and the probe fields are equally detuned from resonance).

Motivated by these simple considerations, we choose for the present experiment in $\mathrm{Rb}^{87}$ the following combination of the fields. The coherent drive is $\hat{x}$ polarized and couples sublevel $1[8]$ of the $S_{1 / 2}$ state with sublevels of the $P_{3 / 2}$ state (the power ranged from 1 to $20 \mathrm{~mW}$ in $0.9 \mathrm{~mm} \mathrm{spot}$ ). The probe laser is $\hat{z}$ polarized and couples sublevels $1 \rightarrow 2^{\prime}$ (the probe power ranged from 0.5 to $50 \mu \mathrm{W}$ in a $0.7 \mathrm{~mm}$ spot). The third beam (incoherent pump) comes from a solitary diode laser that had a broad variable linewidth of $50-200 \mathrm{MHz}$, an output power $10 \mathrm{~mW}$, and a diameter in the cell of $8 \mathrm{~mm}$, which encompassed both the probe and pump beams. All fields propagate in the same direction, hence, for sufficiently strong drive field the amplification peak is essentially Doppler free.

To obtain amplification without inversion in our scheme, a weak magnetic field in the $z$ direction is required. This field destroys Zeeman coherences within the $F=2$ ground state manifold created by an $\hat{x}$ polarized incoherent pumping field. Without the magnetic field population tends to collect in certain coherent superpositions of sublevels of this manifold. We found that the optimum range (for the given linewidth of the incoherent field) of the magnetic field $H_{z}$ is of order $10^{-4} \mathrm{~T}$.

To investigate the possibility of LWI in this scheme we have numerically solved density matrix equations for the 32-level scheme of $\mathrm{Rb}$, taking into account all relevant Clebsch-Gordan and Racah coefficients, field polarizations, Zeeman sublevels, and Doppler broadening. The results of the modeling are presented in Fig. I(b). The results, for this particular field configuration, turn out to be very similar to those obtained from the simple four-level model of Fig. 1(a). As shown in Fig. 1(b) the gain increases as a function of the incoherent pump intensity, while inversion is negative and decreases within incoherent pump intensity.

A remarkable feature of the amplification peak in Figs. 2(a)-2(c) is its narrow width. This is determined in our case by the Rabi frequency of the driving laser. In contrast. the optical pumping peaks are broader. since their width is determined by the optical pumping rate 
of the driving field and the slow ground state relaxation rate. The calculated spectrum for the probe transmission is presented in the Fig. 2(a). Agreement between theory and experiment is excellent.

In the experiment we use a $4 \mathrm{~cm}$ long Brewster cell containing natural $\mathrm{Rb}$ heated to $\sim 60^{\circ} \mathrm{C}$. The driving and probe lasers are tunable extended cavity diode lasers, having short-term linewidths on the order of $100 \mathrm{kHz}$. The drive and probe beams were separated after the cell with a diffraction grating.

In the first set of experiments the transmission of the probe field was measured as a function of its frequency. The frequency of the driving laser was tuned within the Doppler profile of the $D_{2}$ absorption line, while the frequency of the probe laser was swept through the $D_{1}$ line. The results of the transmission measurements are shown in the Fig. 2(b). This figure shows the portion of the transmission spectrum which corresponds to the coupling of the probe field to the $1 \rightarrow 2^{\prime}$ transition within the $D_{1}$ line.

The first experiment was done without the incoherent pump laser [curve $a$ in Fig. 2(b)]. On the broad Doppler profile apparent in the transmission spectrum of the probe beam we see two transmission peaks. They are due to the optical pumping of specific velocity groups by the drive laser. There are three such groups within the velocity distribution; for one of these groups the drive field is resonant with $1 \rightarrow 2^{\prime \prime}$ transition [left peak in Fig. 2(b)] and for others it couples levels 1 with $1^{\prime \prime}$ and 1 with $0^{\prime \prime}$. Since the frequency separation between the last two transitions is small, both of these groups contribute to the right optical pumping peak.

In the second experiment [curve $b$ in Fig. 2(b)] the incoherent pump was added and its frequency was tuned such that it couples $2 \rightarrow 2^{\prime}$ transition. We clearly observe an increase in the transmission of the probe corresponding to amplification of the weak probe field in the vicinity of $1 \rightarrow 2^{\prime \prime}$ optical pumping peak. The amplification occurs in spite of the fact that the results of optical pumping

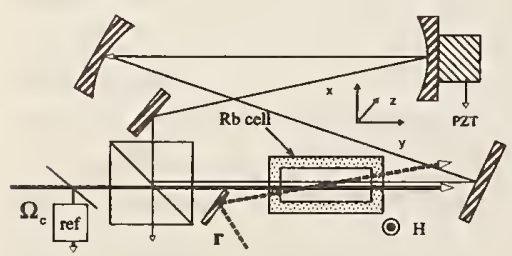

(a)

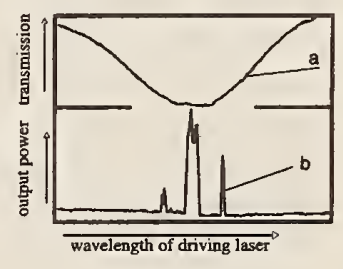

(b)
FIG. 3. (a) Schematic of the ring resonator closed by a polarization beam splitter cube for the LWI oscillator experiment; ref is a reference cell with $\mathrm{Rb}$; output from the cavity is analyzed using a monochromator. (b) Dependence of the driving field absorption (curve " $a$ ") in the reference cell and the corresponding dependence of the cavity output power at $794 \mathrm{~nm}$ (curve " $b$ ") as a function of the frequency of a driving field. Three peaks of the curve $a$ correspond to the three longitudinal modes of the cavity (with frequency separation $\sim 170 \mathrm{MHz}$ ). are destroyed by the incoherent laser. Because of the reverse optical pumping effect, the absorption of the probe increases in all other parts of the spectrum. We also notice that the amplification peak has a much narrower width [ $\sim 30 \mathrm{MHz}$ in Fig. 2(b)] than the purc optical pumping peaks of curve $a$. With present experimental conditions we observe approximately $8 \%$ to $16 \%$ gain per pass.

To verify the absence of a population inversion we carry out an experiment with different linewidths for the probe laser. Since the probe field is weak, it does not affect the population distribution in our system. However, its phase fluctuations do affect the coherent contribution to the gain coefficient [9]. This is due to fact that phases of atomic coherences cannot follow fast phase fluctuations of the fields and therefore, on average, coherent contributions are reduced. Indeed, the probe laser linewidth adds to the decay rate of the upper-level coherence. Consequently, if $\Delta \nu_{a} \geq \gamma_{a, b}$, the linewidth contribution dominates the coherence decay rate, and therefore reduces the contribution due to atomic interference in the gain coefficient. In the limit $\Delta \nu_{a} \gg \gamma_{a, b}$ the interference contribution vanishes, and therefore amplification without inversion should disappear.

In our original experiment the linewidths of probe and driving lasers are relatively narrow $(\Delta \nu \sim$ $100 \mathrm{kHz} \ll \gamma_{a}=5.4 \mathrm{MHz}, \gamma_{b}=5.6 \mathrm{MHz}$ ). However, we can change these linewidths from $100 \mathrm{kHz}$ to $50 \mathrm{MHz}$ by modulating the diode laser's injection current with a noise signal. Figure 2(c) shows the result of such a modulation of the current to the probe laser. In this experiment all three lasers are present in the cell, and therefore without the additional modulation we see the typical amplification peak [curve $a$ in Fig. 2(c)]. In the presence of the current modulation the linewidth of the probe laser was $\sim 10 \mathrm{MHz}$, and we observe that the amplification disappears (curve $b$ ). Since the populations of the levels are not changed, this proves that the observed amplification is not due to population inversion, i.e., it is LWI due to quantum interference effects.

Having observed the amplification of a weak probe field and having verified the absence of a population inversion, we are able to demonstrate the buildup of laser oscillations in a cavity at the frequency corresponding to the $1 \rightarrow 2^{\prime}$ transition of the $D_{1}$ line. To this end the Rb cell was placed inside the ring cavity [Fig. 3(a)]. One mirror of this cavity was formed by a polarization cube in order to allow for a substantial transmission of $\hat{x}$-polarized driving field, and, at the same time, high reflectance for a $\hat{z}$ polarized lasing field. The laser radiation at $794 \mathrm{~nm}$ was selected at the cavity output by using a monochromator. The frequencies of driving and incoherent pump lasers were controlled by using additional reference cells with $\mathrm{Rb}$. The typical dependence of the output power from the cavity at $794 \mathrm{~nm}$ and corresponding absorption of the driving field in the reference cell is presented in the Fig. 3(b) as a function of driving field frequency. When driving laser $(780 \mathrm{~nm})$ is tuned near the center 
of the $D_{2}$ absorption line $\left(1 \rightarrow 2^{\prime \prime}\right.$ transition), the laser oscillation at $794 \mathrm{~nm}$ appears in the ring cavity. This laser field oscillates at the frequency corresponding to the $1 \rightarrow 2^{\prime}$ transition of the $D_{1}$ line as was verified by making a beat note with an independent laser. The beat note measurement shows a direct one-to-one correspondence of the tuning of $795 \mathrm{~nm}$ laser with the frequency of the driving laser, which again confirms that the observed lasing is caused by coherent interaction of the drive laser with atoms. This tuning, along with the fact that the incoherent pump is spectrally broad, rules out the possibility that the lasing is due to Raman gain. Under conditions similar to that of the pump-probe experiment except with no probe present we observed approximately $30 \mu \mathrm{W}$ of lasing power at $794 \mathrm{~nm}$.

In conclusion, we have experimentally shown inversionless amplification and lasing in a Rb gas cell coherently prepared by a cw diode laser. To prove that the gain is "without inversion" we performed measurements with various linewidths of the probe laser, showing that when the linewidth of the probe laser exceeds the radiative decay rate, the amplification peak disappears. This establishes the LWI nature of the observed amplification and the absence of population inversion. And, finally, selfgenerated laser oscillation was observed when the inversionless gain medium was placed inside a laser cavity that was resonant with the appropriate transition of the $D_{1}$ line.

The authors gratefully acknowledge useful discussions with E. Arimondo, M. Fleischhauer, E. Fry, T. Hänsch, and A. Weis, valuable assistance of T. Zibrova, and the support from the Office of Naval Research, the Welch Foundation, the Texas Advanced Research Program, and the Air Force Office of Scientific Research.

[1] For recent theoretical work on LWI in multilevel schemes see, e.g., O. A. Kocharovskaya and Ya. I. Khanin, Pis'ma Zh. Exp. Teor. Fiz. 48, 581 (1988) [JETP Lett. 48, 630 (1988)]; S. E. Harris, Phys. Rev. Lett. 62, 1033 (1989); M. O. Scully, S.-Y. Zhu, and A. Gavrielides, ibid. 62, 2813 (1989); A. Imamoğlu, J.E. Field, and S. E. Harris, ibid. 66. 1154 (1991). For a review of the subject, see O. Kocharovskaya, Phys. Rep. 219, 175 (1992)

[2] Interaction of optical fields in a V-type atomic configuration was studied long ago [3] but recently attracted renewed attention and was investigated theoretically in the context of LWI, see, e.g., O. Kocharovskaya and P. Mandel, Opt. Commun. 84, 179 (1991); M. Fleischhauer et al., Phys. Rev. A 46. 1468 (1992): W. Tan, W. Lu, and R. G. Harrison, ibid. 46, 3613 (1992); G. A. Wilson, K. K. Meduri, P. B. Sellin, and T.W. Mossberg, ibid. 50, 3394 (1994); M. Fleischhauer, T. Mclllrath, and M. O. Scully, Appl. Phys. B (to be published).

[3] I. M. Beterov and V.P. Chebotaev, Sov. Phys. JETP Lett. 9. 127 (1969); T. Hänsch et al., Z. Phys. 226, 293 (1969); T. Hänsch and P. Toschek, ibid. 236, 213 (1970); M. S. Feld and A. Javan, Phys. Rev. 177, 540 (1969).

[4] Xingfu Li et al., in Proceedings of the International Conference on Lasers '92, Houston, 1992 (STS Press, McLean. VA 1992), p. 446; A. Nottelmann, C. Peters, and W. Lange, Phys. Rev. Lett. 70, 1783 (1993); E. S. Fry et al., ibid. 70, 3235 (1993); W. E. van der Veer et al., ibid. 70, 3243 (1993).

[5] Some interesting experimental studies of LWI continue to be a source of discussion. For example, in the interesting experiments of J. Gao et al. [Optics Commun. 93, 323 (1992)] and Gao et al. [ibid. 110, 590 (1994)] it is difficult to assess experimental evidence for the absence of inversion. Moreover, theoretical modeling which takes into account Zeeman sublevels shows that simple LWI of the type studied theoretically by L. Narducci et al. [Opt. Commun. 81, 379 (1991); 86, 324 (1991)] cannot, in fact, be observed under the conditions of these experiments, see, e.g., G.M. Meyer et al., Quantum Opt. 6, 231 (1994). Interpretation of some of the results in the recent experiment of J.A. Kleinfeld and D. A. Streater, Phys. Rev. A 49, 4301 (1994), are also complicated from the theoretical point of view for a similar reason. Nevertheless, coherence effects were observed in these experiments. Further theoretical considerations along these lines will be published elsewhere.

[6] G. Alzetta, A. Gozzini, L. Moi, and G. Orriols. Nuovo Cimento B 36, 5 (1976); E. Arimondo and G. Orriols, Nuovo Cimento Lett. 17, 333 (1976); H. R. Gray, R. M. Whitley, and C. R. Stroud, Jr., Opt. Lett. 3, 218 (1978).

[7] S H. Autler and C. H. Townes, Phys. Rev. 100, 707 (1955).

[8] In the present paper we use the following notations to distinguish different sublevels of $\mathrm{Rb}^{87} . \quad 1$ and 2 denote the $F=1$ and $F=2$ hyperfine components of the $S_{1 / 2}$ ground state, $1^{\prime}$ and $2^{\prime}$ are hyperfine components of the first excited state $P_{1 / 2}$, and $0^{\prime \prime}, \mathrm{I}^{\prime \prime}, 2^{\prime \prime}$, and $3^{\prime \prime}$ are sublevels of the $P_{3 / 2}$ state with corresponding $F$

[9] G.S. Agarwal, Phys. Rev. A 18, 1490 (1978); B.J. Dalton and P. L. Knight, Optics Commun. 42, 411 (1982); M. Fleischhauer et al., ibid. 110, 351 (1994). 


\title{
Line shapes of cascade two-photon transitions in a cesium magneto-optic trap
}

\author{
J. H. Marquardt, H. G. Robinson, and L. Hollberg \\ National Institute of Standards and Technology, Boulder, Colorado 80303
}

Received August 16, 1995; revised manuscript received November 30, 1995

We present a study of a two-photon double-resonance transition in cold cesium atoms in a magneto-optic trap. A closed five-level density matrix model of the system is developed to characterize the double-resonance Autler-Townes-split absorption line shape. Comparing this model with experimental spectra, we gain insight into the dominant process involved in trapping atoms, in particular, the importance of the interference of the six trapping laser beams. This system is used to probe transient effects in the trap as well as collective effects of the trapped atom cloud resulting from the intentional misalignment of the trapping beams.

\section{INTRODUCTION}

We investigate the detailed line shapes of cascade twophoton transitions in laser-cooled and trapped atoms. There has been much research on the use of cold, trapped atoms for microwave frequency standards, ${ }^{1}$ ultracold atom collisions, ${ }^{2}$ and spectroscopic dynamics of cold, trapped atoms. ${ }^{3}$ Some of this research has lead to surprising results in terms of narrow optical resonances, evidence of quantized motion, atomic localization in atom traps, ${ }^{4}$ and other effects. Various mechanisms that produce low temperatures in these traps are now understood. In addition, some theoretical models are now able to calculate detailed line shapes of the spectroscopic features observed on the cooling and trapping transitions. ${ }^{5}$ Other effects, including some aspects of the collective behavior of trapped atoms and unusually long relaxation times remain unexplained. Further detailed spectroscopic investigations of cold, trapped atoms promise new surprises as we learn more about their environment.

Our experiment consists of optically probing cold, trapped cesium atoms, which are excited to the $6 P_{3 / 2}$ state by the absorption of the cooling and trapping laser. A probe laser, tuned to the $6 P_{3 / 2} \rightarrow 9 S_{1 / 2}$ transition at $658.8 \mathrm{~nm}$, is sent through the trapped atoms, creating a doubly resonant two-photon transition (Fig. 1). The probe field is weak and nonperturbing and completely independent of the trapping laser fields. In our experimental work we observed relatively narrow Rabi-split two-photon line shapes that are reminiscent of atomic beam studies of Gray and Stroud and of Hemmer et al. ${ }^{6}$ except that in our case the atoms are cooled and trapped by the resonant radiation and a weak magnetic field. The system consists of magnetic-field gradients and strong laser fields at $852 \mathrm{~nm}$ necessary for the trapping of the atoms in the usual $\sigma^{+}-\sigma^{-}$magneto-optic trap (MOT) configuration. The signal that we observe is the absorption of the $658.8-\mathrm{nm}$ laser as it is tuned across the two-photon resonance. Because the splitting and the shape of the absorption signal depend on the properties of the trap, understanding the features of the line shape provides knowledge of the local environment of the atoms. Independently probing the perturbing effects of the trapping fields will be important in future realizations of optical frequency and length standards based on laser-cooled and trapped atoms.

Initially, we developed a simple theoretical model describing this double-resonance experiment, ${ }^{7}$ which showed the qualitative features of the experimental line shape but quantitatively was not in good agreement with the experimental results. We have improved the model by including important additional effects, such as the three-dimensional nature of laser field and intensity distributions and the effects of laser beam phase fluctuation. In this paper we present a detailed description of this improved model and its comparison with the experimental data.

\section{EXPERIMENT}

The cesium atoms are cooled and trapped in a standard vapor-cell MOT. Our MOT is similar to those described in the literature ${ }^{8}$ and is shown schematically in our experimental setup in Fig. 2. We use two extended-cavity diode lasers for the trap, one relatively powerful laser $(\sim 30 \mathrm{~mW})$ for cooling and trapping and a second laser $(\sim 1 \mathrm{~mW})$ to recirculate atoms that have fallen into the $F=3$ hyperfine ground state. The cooling and trapping light is tuned to the low-frequency side of the $6 S_{1 / 2}$, $F=4 \rightarrow 6 P_{3 / 2}, F=5$ cycling transition. To lock the trapping laser at a specific detuning, the laser beam is sent through an acousto-optic modulator (AOM), where a small percentage of the light is frequency shifted and sent through a separate cesium cell used for saturated absorption. The laser is locked to the saturated-absorption signal corresponding to the nearest crossover resonance to the $6 S_{1 / 2}, F=4 \rightarrow 6 P_{3 / 2}, F=5$ transition. The frequency of the trapping laser is controlled by use of a if synthesizer to drive the AOM. The repumping laser is tuned to the $6 S_{1 / 2}, F=3 \rightarrow 6 P_{3 / 2}, F=3$ or $F=4$ transition and locked to the side of the cesium Doppler profile in a second, separate cesium cell. The $6 P_{3 / 2}$ state of the trapped atoms is then interrogated by direct absorption of a diode laser tuned to the excited $6 P_{3 / 2}, F=5 \rightarrow 9 S_{1 / 2}, F=4$ transition at $658.8 \mathrm{~nm}$. Approximately $1.5 \mathrm{~mW}$ of 658.8 $\mathrm{nm}$ light was provided by an extended-cavity diode laser. 


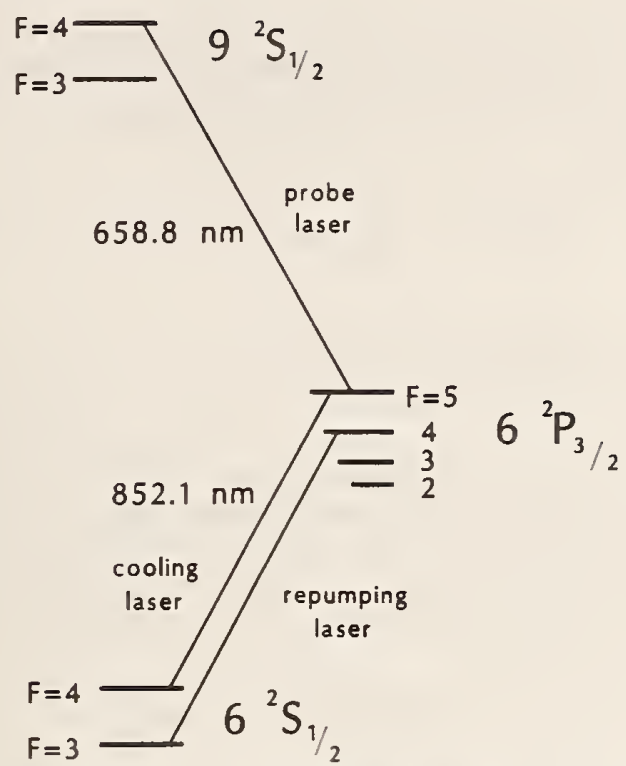

Fig. 1. Relevant cesium energy levels and the hyperfine structure, showing the lasers used for the magneto-optic trap and the cascade two-photon transition.

This laser was frequency stabilized to a reference cavity, which resulted in a short-term linewidth of $\sim 500 \mathrm{~Hz}$ with low-frequency jitter of $\sim 5 \mathrm{kHz}$. The laser's spatial mode was filtered by an optical fiber, which delivered the light to the experiment. The probe laser was elliptically polarized and copropagated with one of the six trapping beams. The diameter of the probe beam was set approximately equal to the diameter of the trapped atom cloud. After spatial filtering, frequency stabilization, and amplitude noise subtraction, the probe laser power available at the MOT was $\sim 40 \mu \mathrm{W}$. The absorption on the $658.8-\mathrm{nm}$ transition by the cesium-atom cloud $\left(\sim 10^{8}\right.$ cold atoms) was typically $\sim 1 \%$. (Fluorescence detection could have been chosen as well but would have required good imaging optics, filtering of the strong $852-\mathrm{nm}$ light, etc. Absorption detection was chosen because of its relative simplicity.) The power of the probe beam was low enough that it did not appear to perturb the trap dynam. ics or the profile of the absorption line shape.

A typical absorption spectrum of the 658.8-nm probe laser is shown in Fig. 3. The doubly peaked profile of this transition is attributed to the optical Autler-Townes effect (ac-Stark splitting). Because of the strong trapping laser fields, we expect a doublet structure of the coupled ground and the first excited states. These states exhibit a well-defined generalized Rabi splitting that depends on the detuning and the Rabi frequency of the trapping laser and the decay rates of the atomic states (the Rabi frequency is given by $\alpha_{21}=\mu_{21} E_{21} / \hbar$, where $\mu_{21}$ is the dipole matrix element and $E_{21}$ is the amplitude of the laser field driving the $6 S_{1 / 2} \rightarrow 6 P_{3 / 2}$ transition).

The characteristics of the line shape were investigated as functions of various trap parameters. For example, Fig. 4 shows the variation of individual $658.8-\mathrm{nm}$ absorption line shapes with detuning of the trapping laser frequency. In this figure the centers of the larger peak were aligned (in frequency space) to show clearly the dependence of the splitting on the trap detuning. We can compare these individual line shapes with theory to evaluate our model of the trapping environment. In particular, we studied the Autler-Townes splitting, the peak height ratios, and the widths of the absorption peaks as a function of the trap parameters. This gave valuable information about the local fields in the trap. The detuning of the trapping laser was limited by the range of stable trap operation. The overall height of the signal in the figure varied with the number of trapped atoms, obviously a function of the detuning. The maximum signal corresponded to a magnetic field gradient of $\sim 15 \times 10^{-4} \mathrm{~T} / \mathrm{cm}(\sim 15 \mathrm{G} / \mathrm{cm})$ and a detuning of $\sim 2 \Gamma$ to the low-frequency side of the transition $(\Gamma / 2 \pi=5.1 \mathrm{MHz}$ is the natural linewidth for this transition in cesium).

\section{THEORY}

We have developed a theoretical model of the two-photon transition that includes many of the physical processes that affect the atomic spectra of the trapped atoms. It

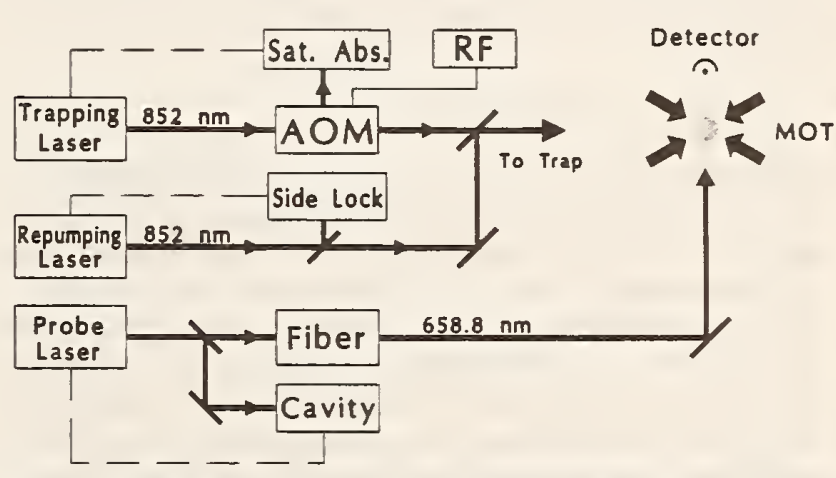

Fig. 2. Schematic diagram of the experimental laser setup. The trapping laser is locked to a saturated-absorption crossover feature in a separate cesium cell. The AOM is used to frequency offset the lock point from the saturated-absorption (Sat. Abs.) feature. The repumping laser is locked to the side of the Doppler profile in a different cesium cell. The dashed lines represent servo loops needed to lock the lasers. The vertical pair of trapping beams and the magnetic-field coils are not shown. The detectors are used to monitor direct absorption of the probe beam and trap fluorescence.

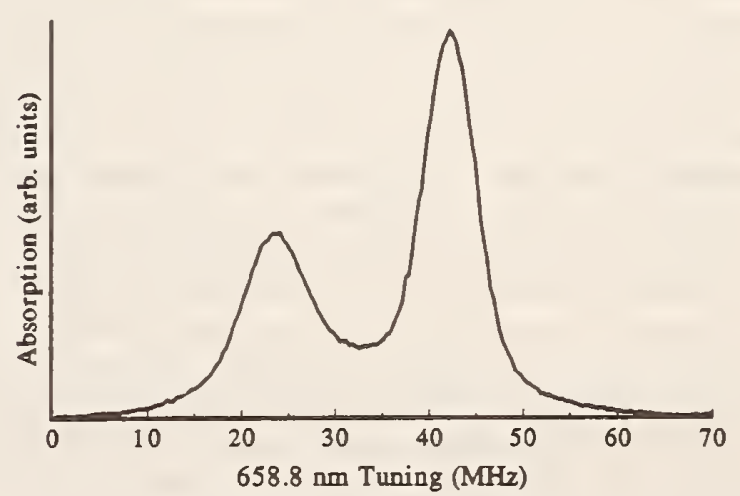

Fig. 3. Typical absorption spectrum taken in our MOT with standard $\sigma^{-} / \sigma^{-}$laser polarizations. The trap detuning $\left(\delta_{21} / 2 \pi\right)$ was $10 \mathrm{MHz} ; \partial \mathbf{B}_{\text {trap }} / \partial z=16 \times 10^{-4} \mathrm{~T} / \mathrm{cm}(16 \mathrm{G} / \mathrm{cm})$. Cloud diameter $\sim 3.5 \mathrm{~mm}, \sim 2.5 \mathrm{~mm}$ in height, $-10^{8}$ atoms, effective Rabi frequency $\alpha_{21}$ of $15.5 \mathrm{MHz}$. The 658.8-nm laser is swept through the resonance with the trapping lasers kept fixed. The $x$ axis shows the relative tuning of the 658.8-nm laser. The selection of the origin is arbitrary. To improve the signal-to-noise ratio this spectrum is averaged over 20 scans. 


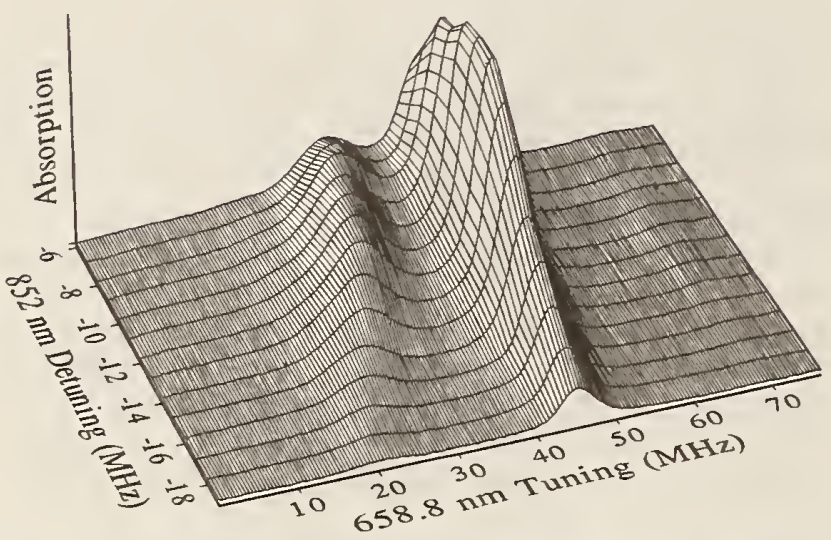

Fig. 4. Probe absorption as a function of trapping laser detuning, $\delta_{21} / 2 \pi$. The large peaks were aligned to show clearly the dependence of the cascade two-photon line shape on the trap laser detuning. The signal height depends on the number of atoms in the trap, a function of the detuning. The small features at $\sim 20$ and $\sim 70 \mathrm{MHz}$ are sidebands put on the 658.8-nm laser as a frequency reference. These spectra were averaged over 200 scans to improve the signal-to-noise ratio. The figure is adapted from Ref. 7.

would be extremely difficult to analyze the complete system theoretically, including all relevant atomic levels in cesium, six strong laser fields, laser polarizations, the three-dimensional variation of the magnetic field $\mathbf{B}_{\text {trap }}$, and the distribution and the residual motion of the cold atoms in the trap. To analyze a more tractable system we have made some assumptions and simplifications. We have assumed (to a good approximation) that the laser fields are plane waves, the atoms are motionless, and the collisional effects are negligible. We include spatial effects for the three-dimensional trapping laser fields and the variation of $\mathbf{B}_{\text {trap }}$ over the trapping volume. The Zeeman structure for each radiatively coupled level is approximated by use of only the $m_{F}= \pm F$ (stretched state) Zeeman coefficient for each of the three coupled levels. The validity of our assumptions and simplifications is discussed later in this paper. We have further simplified the complicated cesium energy-level spectra to a closed five-level system (Fig. 5). Three of these levels, $|1\rangle,|2\rangle$, and $|3\rangle$, correspond to the three coupled levels of the doubly resonant, cascade configurations $6 S_{1 / 2}, F=4 \rightarrow 6 P_{3 / 2}, F=5 \rightarrow 9 S_{1 / 2}, F=4$. The remaining two levels, $|4\rangle$ and $|5\rangle$, are artificial and represent all other decay channels out of the cascade system, which are due to the other atomic states and to the relevant hyperfine structure of the three coupled levels. The hyperfine structure permits population decay out of the $6 S_{1 / 2}, F=4 \rightarrow 6 P_{3 / 2}, F=5$ cycling transition by offresonant absorption and decay to the $6 S_{1 / 2}, F=3$ ground state. This loss mechanism and the presence of the repumping laser are represented in our model by the addition of level |5). The rate at which atoms enter the other hyperfine states can be calculated and is represented in the model by $\gamma_{25}$. Similarly, we have added level $|4\rangle$ to represent other decay channels out of the excited, $9 S_{1 / 2}, F=4$, state to the other noncoupled states. This rate is represented by $\gamma_{34}$ and shown in Fig. 5. Following the standard approach, we model our system by using the density matrix formalism. ${ }^{9}$ Some additions and modifications to this approach are necessary for the conditions relevant to this experiment.

The time evolution of the density operator can be represented by

$$
\begin{array}{ll}
\frac{\mathrm{d} \rho_{n n}}{\mathrm{~d} t}=-\frac{i}{\hbar}[\hat{H}, \hat{\rho}]_{n n}+\sum_{k}\left\{\gamma_{k n} \rho_{k k}-\gamma_{n k} \rho_{n n}\right\} & \text { for } n=m, \\
\frac{\mathrm{d} \rho_{n m}}{\mathrm{~d} t}=-\frac{i}{\hbar}[\hat{H}, \hat{\rho}]_{n m}-\Gamma_{n m} \rho_{n m} & \text { for } n \neq m .
\end{array}
$$

Here the $\hat{H}$ is the Hamiltonian operator of the system and $\gamma_{n m}$ is the decay rate from state $n$ to state $m$. (In this representation $\gamma_{n m} \neq \gamma_{m n}$. If state $n$ is a higher-energy state than state $m$, then $\gamma_{m n}=0$.) The damping rate $\Gamma_{n m}$ of the off-diagonal elements can be written as

$$
\Gamma_{n m}=\frac{1}{2}\left(\gamma_{n}+\gamma_{m}\right)+\gamma_{n m}{ }^{\mathrm{col}}
$$

where $\gamma_{n}$ and $\gamma_{m}$ represent the total decay rates out of levels $n$ and $m$ and $\gamma_{n m}{ }^{\text {col }}$ is the dipole dephasing term that is due to processes such as collisions (which we neglect).

We can write the Hamiltonian operator as

$$
\hat{H}=\hat{H}_{0}+\hat{V}(t),
$$

where $\hat{H}_{0}$ is the atomic Hamiltonian and $\hat{V}(t)$ represents the interaction energy of the atom with the electromagnetic field. $\hat{V}(t)$ can be represented by

$$
\begin{aligned}
V_{n m}(t) & =\mathcal{E}_{n m} \cos \Omega_{n m} t \\
& =\frac{-\boldsymbol{\mu}_{n m} \cdot \mathbf{E}_{n m}}{2}\left[\exp \left(-i \Omega_{n m} t\right)+\exp \left(i \Omega_{n m} t\right)\right],
\end{aligned}
$$

where $\mathbf{E}_{n m}$ is the incident laser field with frequency $\Omega_{n m}$ and $\mu_{n m}$ is the dipole matrix element corresponding to the transition from level $m$ to level $n$. The rotatingwave approximation allows us to neglect the last term in Eq. (4). After inserting the full Hamiltonian $\hat{H}$ into Eqs. (1), we can write

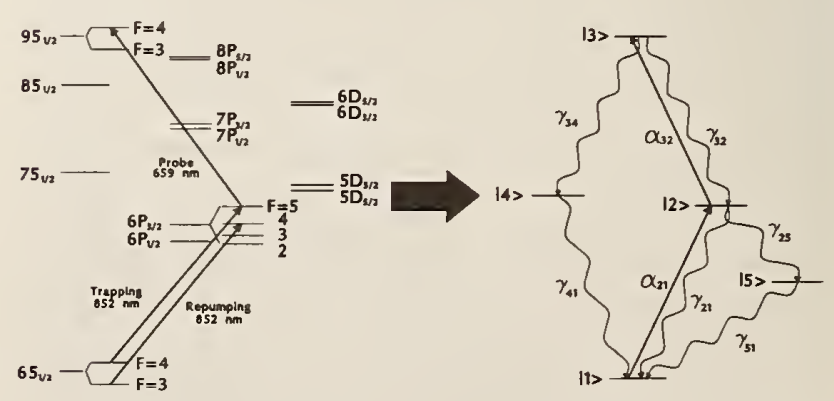

\section{Cesium Energy Levels}

Model Energy Levels

Fig. 5. Relevant cesium energy-level diagram showing the cascade two-photon transition, the lasers used in the experiment, and the corresponding energy levels of the model. The energy levels of the model consist of three levels that are coupled by laser radiation, $|1\rangle,|2\rangle$, and $|3\rangle$, and two artificial levels, $|4\rangle$ and $|5\rangle$, representing all the other decay channels out of the cascade system. The $\gamma$ are the decay rates between levels, and the $\alpha$ are the Rabi frequencies of the lasers used in the model. 


$$
\begin{aligned}
\left(\frac{\partial}{\partial t}+i \omega_{n m}\right) \rho_{n m} & +\frac{i}{\hbar} \sum_{k}\left(V_{n k} \rho_{k m}-\rho_{n k} V_{k m}\right) \\
& =\left\{\begin{array}{ll}
\sum_{k}\left(\gamma_{k n} \rho_{k k}-\gamma_{n k} \rho_{n n}\right) & \text { for } n=m \\
-\Gamma_{n m} \rho_{n m} & \text { for } n \neq m
\end{array},\right.
\end{aligned}
$$

where $\omega_{n m}=E_{n}-E_{m} / \hbar$ is the transition frequency between levels $n$ and $m$. Introducing the slowly varying quantity $\sigma_{n m}$, such that

$$
\rho_{n m}(t)=\sigma_{n m}(t) \exp \left(-i \Omega_{n m} t\right)
$$

we look for steady-state ( $\left.\mathrm{d} \sigma_{n m} / \mathrm{d} t=0\right)$ solutions to Eq. (5); these yield

$$
\begin{gathered}
\sum_{k}\left(\gamma_{k n} \sigma_{k k}-\gamma_{n k} \sigma_{n n}\right)+\frac{i}{2} \sum_{k}\left(\alpha_{n k} \sigma_{k n}-\sigma_{n k} \alpha_{k n}\right)=0 \\
\text { for } n=m, \\
{\left[i\left(\Omega_{n m}-\omega_{n m}\right)-\Gamma_{n m}\right] \sigma_{n m}+\frac{i}{2} \sum_{k}\left(\alpha_{n k} \sigma_{k m}-\sigma_{n k} \alpha_{k m}\right)=0} \\
\text { for } n \neq m, \quad
\end{gathered}
$$

where $\Omega_{n m}=-\Omega_{m n}$ and $\alpha_{n m}=-\mathcal{E}_{n m} / \hbar$ in this notation. Equations (7) describe a $25 \times 25$ matrix for our fivelevel system. Fortunately, many of the levels are not coupled by radiation or decay, so some simplifications can be made. In the steady state many of the density matrix states can be written in terms of other states. For example $\sigma_{44}$ can be eliminated by use of the relation for $\mathrm{d} \sigma_{44} / \mathrm{d} t$ :

$$
\frac{\mathrm{d} \sigma_{44}}{\mathrm{~d} t}=\gamma_{41} \sigma_{44}-\gamma_{34} \sigma_{33}=0 \rightarrow \sigma_{44}=\frac{\gamma_{34}}{\gamma_{41}} \sigma_{33} .
$$

Similarly, the other states that are not coupled by laser radiation can be represented in terms of coupled states. This simplifies the $25 \times 25$ matrix into a $9 \times 9$ matrix (shown in Table 1), which can be solved for each of the remaining density matrix components. In the table $\delta_{n m}=\Omega_{n m}-\omega_{n m}$ represents the detuning of the lasers from exact resonance for the given transition $\left(\delta_{21}<0\right.$ for trap operation). The signal is then calculated as a function of the probe laser detuning $\delta_{32}$. Solving this $9 \times 9$ density matrix gives the specific values of $\sigma_{n m}$. The signal that we observe experimentally is the absorption from level $|2\rangle$ to level $|3\rangle$, which is dependent on the imaginary part of $\sigma_{32}$.

\section{NUMERICAL IMPLEMENTATION OF THE THEORY}

This solution for the imaginary part of $\sigma_{32}$ gives only part of the total line shape because the three-dimensional nature of the trapping laser and the magnetic fields of the MOT have not yet been included. Because the diameter of the probe beam is set approximately equal to the size of the cloud of trapped atoms, every atom in the trap can contribute to the absorption line shape. The energy levels of the atoms in the trap are position dependent because of both the magnetic-field gradient and the wavelength scale variations of the electric field and polarization. The magnetic-field gradient $\partial \mathbf{B}_{\text {trap }} / \partial \mathbf{r}$ effectively alters the detuning of the trapping laser $\delta_{21}$ as a function of the distance from the center of the trap (where $\left|\mathbf{B}_{\text {trap }}\right|=0$ ). Thus, for a fixed trap detuning, the actual detuning of the trapping laser depends on atomic Zeeman structure as

$$
\delta_{21}{ }^{\prime}=\delta_{21}+\frac{\mu_{B}}{h} \mathbf{B}_{\mathrm{trap}}(\rho, z)\left(g_{F^{\prime}=5} m_{F^{\prime}}-g_{F=4} m_{F}\right) .
$$

Here $\mathbf{B}_{\text {trap }}(\rho, z)$ is the local magnetic field at point $(\rho, z)$, $g_{F^{\prime}=5}=0.4$ and $g_{F=4}=0.25$ are the Landé $g$ factors, $\mu_{B}$ is the Bohr magneton, and $m_{F}$ and $m_{F}$ are the Zeeman sublevels of the excited state and the ground state, respectively. For the anti-Helmholtz configuration the magnetic-field strength $\left|\mathbf{B}_{\text {trap }}\right|$ varies approximately linearly with distance from the center of the trap. The atoms at the center of the trap have a detuning that corresponds to the trapping laser detuning, $\delta_{21}{ }^{\prime}(\rho=0)=\delta_{21}$, whereas other atoms experience a different detuning, depending on the magnetic-field gradient and the position of the atom. We call the most probable value of $\hat{\delta}_{21}$ the effective detuning. Larger magnetic fields correspond to smaller effective detunings (for $\delta_{21}<0$ ), which naturally affect the cascade two-photon line shape. Of course, it is possible to switch off the magnetic field to eliminate the magnetic-field-dependent effects, but we dedicate most of this discussion to the steady state, which includes the magnetic field.

Unlike that of the magnetic field of the trap, which varies over macroscopic dimensions, the electric field's

\begin{tabular}{|c|c|c|c|c|c|c|c|c|c|}
\hline & $\sigma_{33}$ & $\sigma_{32}$ & $\sigma_{31}$ & $\sigma_{21}$ & $\sigma_{11}$ & $\sigma_{12}$ & $\sigma_{13}$ & $\sigma_{23}$ & $\sigma_{22}$ \\
\hline$\sigma_{33}$ & $-\gamma_{34}-\gamma_{32}$ & $-i \alpha_{23} / 2$ & 0 & 0 & 0 & 0 & 0 & $i \alpha_{32} / 2$ & 0 \\
\hline$\sigma_{32}$ & $-i \alpha_{32} / 2$ & $i \delta_{32}-\Gamma_{32}$ & $-i \alpha_{12} / 2$ & 0 & 0 & 0 & 0 & 0 & $i \alpha_{32 / 2}$ \\
\hline$\sigma_{31}$ & 0 & $-i \alpha_{21} / 2$ & $i\left(\delta_{32}+\delta_{21}\right)-\Gamma_{31}$ & $i \alpha_{32} / 2$ & 0 & 0 & 0 & 0 & 0 \\
\hline$\sigma_{21}$ & 0 & 0 & $i \alpha_{23} / 2$ & $i \delta_{21}-\Gamma_{21}$ & $i \alpha_{21} / 2$ & 0 & 0 & 0 & $-i \alpha_{21}{ }^{\prime} 2$ \\
\hline$\sigma_{11}$ & $\gamma_{34}$ & 0 & 0 & $i \alpha_{12} / 2$ & 0 & $-i \alpha_{21} / 2$ & 0 & 0 & $\gamma_{21}+\gamma_{25}$ \\
\hline$\sigma_{12}$ & 0 & 0 & 0 & 0 & $-i \alpha_{12} / 2$ & $-i \delta_{21}-\Gamma_{21}$ & $-i \alpha_{32} / 2$ & 0 & $i \alpha_{12} / 2$ \\
\hline$\sigma_{13}$ & 0 & 0 & 0 & 0 & 0 & $-i \alpha_{23} / 2$ & $-i\left(\delta_{32}+\delta_{21}\right)-\Gamma_{31}$ & $i \alpha_{12}$ & 0 \\
\hline$\sigma_{23}$ & $i \alpha_{23} / 2$ & 0 & 0 & 0 & 0 & 0 & $i \alpha_{21} / 2$ & $-i \hat{\delta}_{32}-\Gamma_{32}$ & $-i \alpha_{23} / 2$ \\
\hline$\sigma_{22}$ & $\gamma_{32}$ & $i \alpha_{32} / 2$ & 0 & $-i \alpha_{21} / 2$ & 0 & $i \alpha_{12} / 2$ & 0 & $-i \alpha_{32} / 2$ & $-\gamma_{21}-\gamma_{25}$ \\
\hline
\end{tabular}
magnitude changes on a scale smaller than the optical wavelength. The interference of the laser fields in the trap is generated by the intersection of the six circularly polarized laser beams. This interference pattern depends on the relative amplitudes and phases of the six beams and varies significantly, both in magnitude and in polarization, over an optical wavelength. In our model

Table 1. Density Matrix 
we neglect any polarization dependencies that would contribute to optical pumping of the atoms to other Zeeman states. A more exact model that includes all the magnetic sublevels and polarization dependencies of the transitions was not attempted because of its complexity. We can write the $\sigma^{+}$and $\sigma^{-}$beams as

$$
\begin{aligned}
& \mathcal{E}_{\sigma^{+}}(z)=E_{0}\left[\hat{i} \cos \left(k z-\omega t+\phi_{1}\right)-\hat{j} \sin \left(k z-\omega t+\phi_{1}\right)\right], \\
& \mathcal{E}_{\sigma^{-}}(z)=E_{0}\left[\hat{i} \cos \left(k z-\omega t+\phi_{2}\right)+\hat{j} \sin \left(k z-\omega t+\phi_{2}\right)\right],
\end{aligned}
$$

for waves traveling along the positive $z$ axis. The total field is given by the vector sum of the six intersecting beams with the proper polarization and direction. Initially, choosing all the phases to be $0\left(\phi_{\mathrm{n}}=0\right)$ allows us to write the total field for our MOT as

$$
\begin{aligned}
\mathcal{E}_{t o t}= & 2 E_{0} \cos \omega t[\hat{i}(\cos k z+\sin k y)+\hat{j}(\cos k x-\sin k z) \\
& +\hat{k}(\cos k y+\sin k x)] .
\end{aligned}
$$

We are interested in the time-averaged magnitude of this three-dimensional field, which can be written as

$$
\begin{aligned}
\left|\mathbf{E}_{\text {laser }}\right|= & \sqrt{\mathcal{E}_{\text {tot }} \cdot \mathcal{E}_{\text {tot }}{ }^{*}} \\
= & E_{0}[6+4(\cos k z \sin k y-\cos k x \sin k z \\
& +\cos k y \sin k x)]^{1 / 2} .
\end{aligned}
$$

Figure 6(a) shows Eq. (12) plotted as a function of $x$ and $y$ with a fixed value for $z(k z=3 \pi / 4)$. The actual interference pattern depends on the relative phase of each of the six trapping beams and can easily be included in Eq. (12). In the experiment the relative phase of each of the laser beams is unknown and is continually changing owing to mechanical vibrations. These vibrations are important because of the finite sweep speed of the probe laser (generally $<200 \mathrm{~Hz}$ ) and must be taken into account.

Using the laser field distribution above, we generated a Monte Carlo histogram of the probability of finding a specific laser field $\left|\mathbf{E}_{\text {laser }}\right|$ in the trap. An initial histogram was calculated with a specific phase distribution $\left(\phi_{n}=0\right)$. To approximate phase-changing mechanical vibrations, we calculated separate histograms for many different phase distributions. These were combined and normalized to form a composite distribution representative of the electric fields in the trap. This composite distribution is shown in Fig. 6(b). With this distribution we can integrate over the fields with the proper weighting of the Rabi frequencies to give the final line shape.

Because of the spatial dependence of the trap's magnetic and electric fields, we need to include all the fields in the spatial distribution to calculate the measured twophoton line shape. We accomplish this by numerically integrating over the magnetic-field contributions while simultaneously integrating over the microscopic interference pattern of the electric field. The total line shape can be represented as a sum of many individual line shapes, each of which corresponds to atoms in different electric and magnetic fields. These line shapes are additionally weighted to ensure that each individual line-shape contribution reflects the number of atoms present in similar fields. We assume that the distribution of atoms is proportional to the intensity of the local electric field. ${ }^{10} \mathrm{By}$ stepping through the possible values of the electric and the magnetic fields and calculating a separate line shape for each value, we combine these line shapes with the proper weighting to create a final line shape. This absorption line shape $S$ can be represented by

$$
S \propto \iiint \operatorname{Im}\left[\sigma_{32}\right] P(E) I \rho \mathrm{d} E \mathrm{~d} \rho \mathrm{d} z
$$

where $P(E)$ is the probability of finding a certain electric field $E$ in the trap [from Fig. 6(b)], $I$ is the intensity of the radiation, and $\rho$ and $z$ are the radial and the axial positions, respectively, in the trap. ${ }^{11}$ We numerically integrate relation (13) over all electric and magnetic fields (all positions in the trap). This procedure produces a line shape that we can compare with experiment. This position-dependent integration $\left(\mathbf{E}_{\text {laser }}, \phi, \mathbf{B}_{\text {trap }}\right.$ ) broadens the Rabi-split line shape but does not wash it out. Although each group of atoms in the trap corresponds to a different Rabi splitting, the composite line shape has a single, well-defined value for the splitting. This single Rabi splitting represents the weighted average of the in-

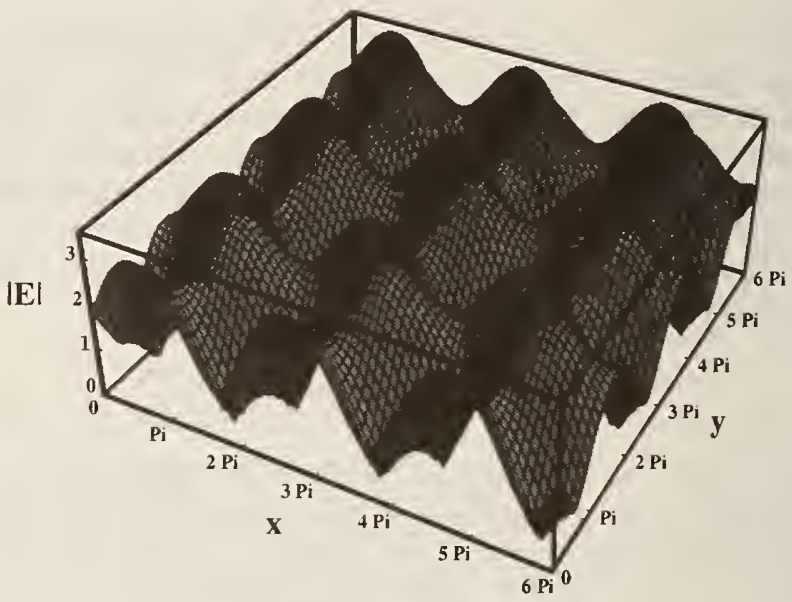

(a)

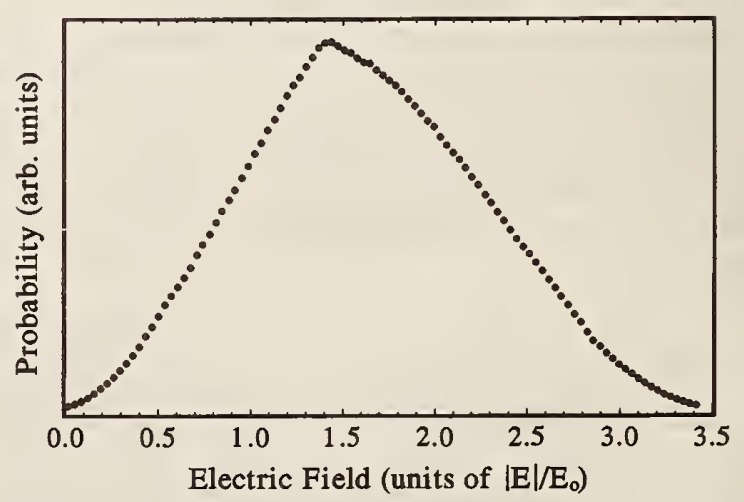

(b)

Fig. 6. (a) Local, small-scale structure of the magnitude of the net trapping laser field $\left|\mathbf{E}_{\text {laser }}\right|$ for the standard threedimensional $\sigma^{+}-\sigma^{-}$MOT configuration. The $x$ and the $y$ axes show the structure over several optical periods for $k z$ fixed at $3 \pi / 4$. (b) Composite Monte Carlo distribution of the probability of finding a certain $\left|\mathbf{E}_{\text {laser }}\right|$ field averaged over random phases in units of $\left|\mathbf{E}_{\text {laser }}\right| / E_{0}$. 
dividual line shapes and can be represented by a single effective Rabi frequency.

The important parameters in the numerical model are the decay rates of the cesium levels, the Rabi frequencies for the trapping and the probing laser fields, the detuning of the trapping laser, the magnetic field gradient, and the physical size and shape of the atom cloud. All these parameters are known or measured experimentally. When the atom density is chosen to be proportional to the local field intensity, the model contains no other free parameters except for the signal size, which is used for normalization. For convenient comparisons the height of the larger peak in the model is set equal to the height of the corresponding peak in the experimental data. The Rabi frequency for the probe and the trapping lasers can be calculated from the intensity of the laser beams. At low intensity the probe beam can be considered nonperturbing, although the model is able to calculate the line shape for any value of the probe's Rabi frequency. We control the detuning of the trapping laser experimentally by locking the laser to a saturated absorption signal with a variable-frequency offset. The magnetic-field gradient is calculated from the knowledge of the current and the geometry of the coils. The size of the trap is measured with a CCD camera, which detects the fluorescence of the trapped atoms (in the near infrared at $852 \mathrm{~nm}$ ). The decay rates of the coupled states are known experimentally, and the decay rates of the uncoupled, artificial states can be estimated by use of simple realistic assumptions. There are many decay paths out of the $9 S_{1 / 2}$ state, and the decay rate depends on which decay path the atom takes. An effective decay rate was estimated with the knowledge of the branching ratios of the possible decay paths. Table 2 shows the range of the values for the important trap parameters, an estimation of the uncertainties, and the qualitative effect of these uncertainties on the line shape.

We turn our attention to the splitting of the levels as a function of various trap parameters. If we first apply a perturbation approach for the probe laser $\left(\alpha_{32} \approx 0\right)$ and assume a single Rabi frequency across the trap, we can calculate the position of the peaks by solving for the maxima of the imaginary part of $\sigma_{32}$ \{that is, setting $\partial\left(\operatorname{Im}\left[\sigma_{32}\right]\right) / \partial \delta_{32}=0$ and solving for $\left.\delta_{32}\right\}$. We get an analytic solution for the special case of $\delta_{21}=0$ (the solution for $\delta_{21} \neq 0$ can be solved numerically). The solution for this case has three values, $\delta_{32}=0$ and

$$
\begin{aligned}
\delta_{32}= & \pm\left\{-\frac{\alpha_{21}^{2}}{4}\left(\frac{\gamma_{2}+\gamma_{3}}{\gamma_{3}}\right)-\frac{1}{16}\left(\gamma_{2}+\gamma_{3}\right)^{2}\right. \\
& \left.+\frac{\alpha_{21}\left(\gamma_{2}+2 \gamma_{3}\right)}{8 \gamma_{3}}\left[\alpha_{21}^{2}+\frac{1}{4} \gamma_{3}\left(\gamma_{2}+\gamma_{3}\right)\right]^{1 / 2}\right\}^{\mathrm{V} / 2} .
\end{aligned}
$$

Equation (14) has two values, which represent the Rabisplit peaks; the value $\delta_{32}=0$ is a local minimum. The splitting and the widths of the peaks depend only on the total lifetimes of the coupled levels, not on the artificial levels for this perturbation approach. This is true for all values of $\delta_{21}$. However, the amplitude of the absorption line shape depends on $\delta_{21}$. This amplitude dependence becomes important when one is considering the whole ensemble of atoms. Therefore the contribution to the total line shape of each Rabi frequency changes as a function of the detuning. At larger detunings the atoms in the larger fields contribute more to the line shape, causing an increase in the effective Rabi frequency compared with those of the line shapes at smaller detunings. This makes it impossible to choose a single value of the electric field to model the splitting dependence on the detuning.

\section{COMPARISONS OF THE MODEL AND EXPERIMENT}

Our theoretical model works fairly well in reproducing the experimental line shapes. Figure 7 shows a comparison of the theoretical model and the experimental line shape for three different trap detunings $\delta_{21}$. Including the spatial variations of $\mathbf{E}_{\text {laser }}$ and $\mathbf{B}_{\text {trap }}$ and averaging over the phase fluctuations broaden the line shapes as expected and significantly improve the agreement between theory and experiment. For these spectra the best agreement between the model and the experiment occurs with an effective Rabi frequency of $\sim 15.5 \mathrm{MHz}$. The model reproduces the experimental line shape very accurately at smaller detunings [Fig. 7(a)]. At larger detunings the discrepancies between theory and experiment become more apparent. For most conditions the model predicts a line shape that exhibits the proper linewidths and splittings, but the amplitude of the smaller peak tends to be too small relative to the experimental line shape. The dashed curve in Fig. 7(b) shows the result of the model with a single Rabi frequency under the same conditions as for the solid curve. One can easily see the

Table 2. Important Parameters, Range of Experimental Values, Their Estimated Experimental Uncertainties, and the Qualitative Effect on the Theory ${ }^{a}$

\begin{tabular}{lll}
\hline Parameter & Value & $\begin{array}{c}\text { Estimated } \\
\text { Uncertainty }\end{array}$ \\
\hline Trap diameter $(2 \rho)$ & $2.5-4.0 \mathrm{~mm}$ & $\pm 0.3 \mathrm{~mm}$ \\
Trap height $(2 z)$ & $1.3-3.0 \mathrm{~mm}$ & $\pm 0.3 \mathrm{~mm}$ \\
Magnetic field & $12-20 \times 10^{-4} \mathrm{~T} / \mathrm{cm}$ & $\pm 0.5 \times 10^{-4} \mathrm{~T} / \mathrm{cm}$ \\
Detuning $\left(\left|\delta_{21} / 2 \pi\right|\right)$ & $4-16 \mathrm{MHz}$ & $\pm 0.1 \mathrm{MHz}$ \\
Rabi frequency & & Small \\
$\alpha_{21}$ & $12-19 \mathrm{MHz}$ & $\pm 1 \mathrm{MHz}$ \\
$\alpha_{32}$ & $\sim 0.5 \mathrm{MHz}$ & $\pm 0.1 \mathrm{MHz}$ \\
$\gamma_{n m}$ & Various & $\pm 1-10 \%$ \\
\hline
\end{tabular}

\footnotetext{
${ }^{a}$ The decay rates $\gamma_{n m}$ are well known for the lower-lying levels, but the uncertainties increase for the higher-lying states.
} 


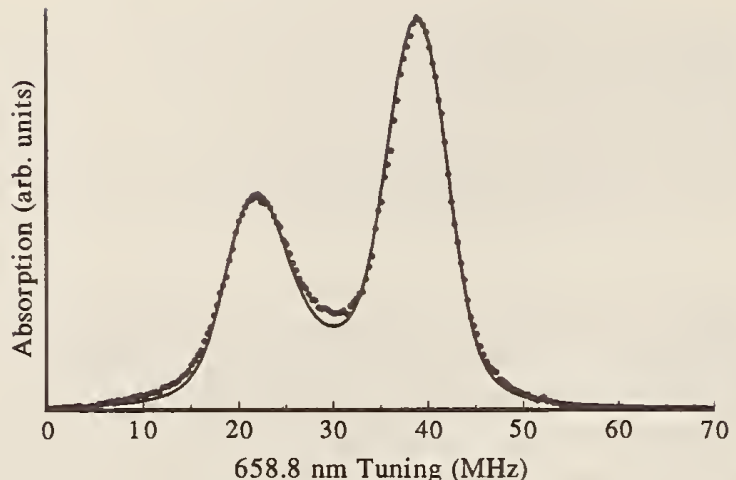

(a)

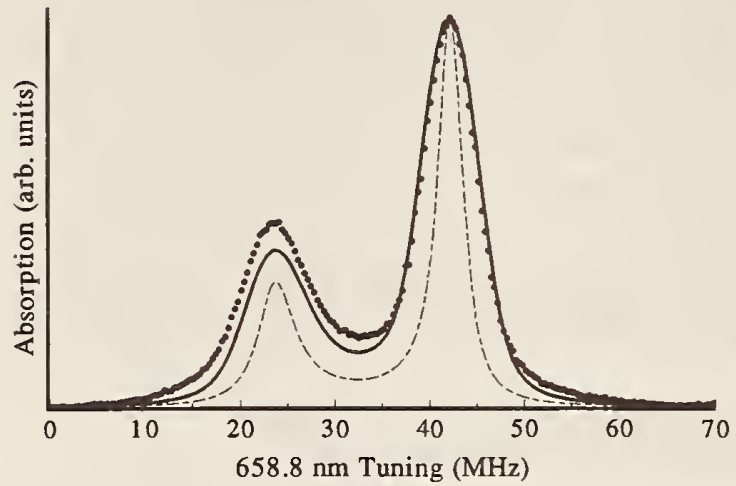

(b)

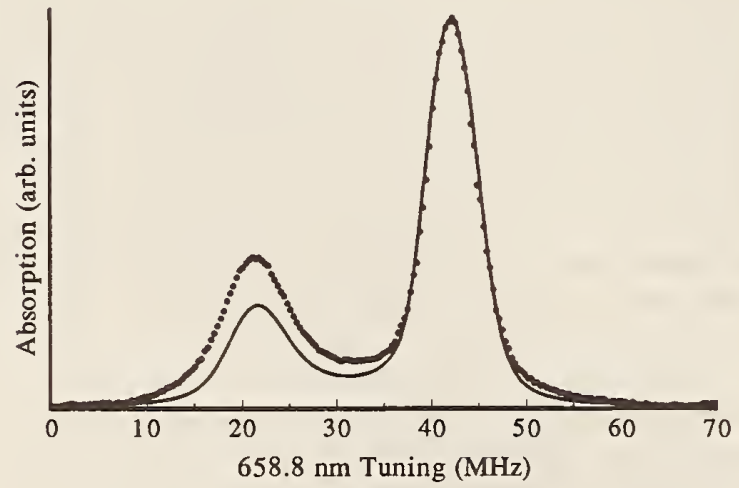

(c)

Fig. 7. Experimental and theoretical line shapes with a field gradient of $16 \times 10^{-4} \mathrm{~T} / \mathrm{cm}$, an atom cloud diameter of $\sim 3.0 \mathrm{~mm}$, and $\alpha_{\text {effective }}=15.5 \mathrm{MHz}$ for various trap laser detunings $\delta_{21}$. (a) $\delta_{21} / 2 \pi=-7 \mathrm{MHz}$, (b) $\delta_{21} / 2 \pi=-10 \mathrm{MHz}$, and (c) $\delta_{21} / 2 \pi=-13 \mathrm{MHz}$. The experimental data are represented by the dots, and the solid curve is the model. The dashed curve in (b) is the theoretical prediction with the same parameters as the solid curve but for a single Rabi irequency.

importance of the electric-field interference pattern and the phase fluctuations by comparing the two predicted line shapes with the experimental data. The electricfield effects broaden the line shape and improve the relative peak height agreement with the experiment. We have chosen model parameters that are realistic for our experiment. (It is possible to change the parameters to unrealistic values to improve the apparent agreement.)

We have investigated the Autler-Townes splitting as a function of the detuning. Inasmuch as the Zeeman shifts of the atomic energy levels increase the complexity of the model, we investigated the splitting both with and with- out the magnetic-field gradient. The line shapes with the magnetic-field gradient turned off are similar to those shown in Fig. 7, except that the splitting is slightly larger, as expected, because of the larger effective detuning. Figure 8 shows the line-shape splitting as a function of detuning with the magnetic field turned off. The solid curve represents the predicted line-shape splitting. Although the model does a good job of predicting the splitting, a systematic structure at large detunings is not accounted for by the model. It does, however, predict the dependence of the splitting more accurately than if we included only a single Rabi frequency. A study of the splitting with the magnetic field present exhibited similar results, albeit with slightly larger discrepancies between theory and experiment at large detunings. This seems to indicate a need for a more comprehensive treatment of the Zeeman structure.

The two peaks in the Autler-Townes line shape can be interpreted as representing different types of twophoton transition. ${ }^{12}$ In the limit where the pump and the probe lasers are very weak $\left(\alpha_{21} \approx 0, \alpha_{32} \approx 0\right)$ and the decay to the artificial levels is negligible (a closed three-level system), the absorption peaks can be interpreted in terms of coherent and incoherent transitions. The smaller peak can be represented by a term that is proportional to $\sigma_{22}$, which requires buildup of a population in the $6 P_{3 / 2}$ state. This represents an incoherent twophoton double-resonance peak. The larger, narrow peak can be represented as a term proportional to $\sigma_{21}$ and corresponds to a two-photon transition with no population in the $6 P_{3 / 2}$ state. To satisfy the conditions for this second type of transition, coherence must be preserved. If the coherence degrades, the transition probability decreases for the coherent peak but increases for the incoherent transition. Therefore, if there are excess relative phase fluctuations, the coherent peak suffers relative to the incoherent peak (the relative height of the smaller peak increases). Most perturbing effects in the laboratory would destroy coherence, thus increasing the apparent relative size of the smaller peak. This effect appears to be small for the laser linewidths ( $\leq 500 \mathrm{kHz}$ for the trapping laser and $\leq 1 \mathrm{kHz}$ for the probe, as opposed to linewidths of $5.1 \mathrm{MHz}$ for the $6 P_{3 / 2}$ state and $\sim 1 \mathrm{MHz}$ for the $9 S_{1 / 2}$ state) and the collisional effects present in our experi-

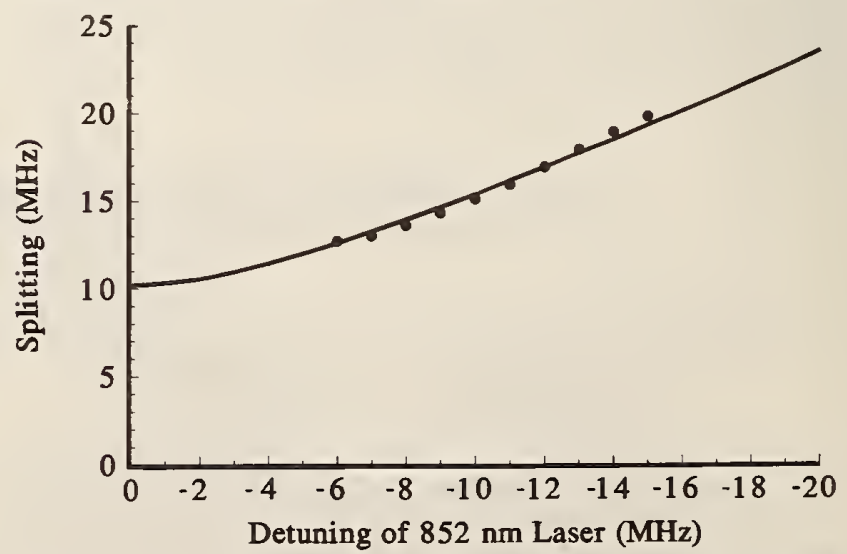

Fig. 8. Splitting of the line shape versus the detuning of the $852-\mathrm{nm}$ laser with the magnetic-field gradient turned off. The solid curve is the numerical result of the model. The error bars on the data are roughly equal to the size of the circles. 
ment, and it cannot explain the discrepancies between theory and experiment. Inasmuch as the model and the experiment agree well at small detunings but less so at large detunings, the discrepancy between theory and experiment is in some way dependent on trap conditions. At large detunings we might expect other effects to begin to play a larger role, which would increase the effective Rabi frequency, such as atom localization.

We made a number of simplifications that need to be justified. The first assumption is that the atoms are stationary. In the case of cold trapped cesium atoms the residual Doppler width is small compared with the natural linewidth of the transition (for a sample of cesium atoms cooled to the Doppler limit, the residual Doppler width is $\sim 250 \mathrm{kHz}$ ). For Doppler widths in standard cesium traps this effect is small and can be neglected. Similarly, the movement of atoms across the interference pattern of the electric field can be neglected because of the fast cycling frequency and slow atomic velocities. If the intensities of the six laser beams in the trap are not exactly equal or are not uniform across the trapping region (because of the spatial mode variation across the laser beam inherent for diode lasers, for example), the electric field distribution differs from that shown in Fig. 6(b). The peak in the distribution changes position slightly, but the effect on the line shape is surprisingly small. Inasmuch as the Zeeman contribution to the total detuning is small compared with the actual detuning, the effect of other Zeeman levels is expected to be small. Further, as we assume maximum $m_{F}$ values in the model, inclusion of lower Zeeman coefficients would increase the effective detuning, which tends to degrade the agreement between theory and experiment. To validate further the assumption of large $m_{F}$ values, a study was done to investigate the polarization of the three-dimensional electric-field distribution. Although for the standard $\sigma^{+}-\sigma^{-}$configuration the electric field is linear everywhere in space for $\phi_{n}=0$ [Eq. (11)], changing the relative phases of the laser beams changed the interference pattern. For most choices of the relative phase, the electric field was not linear across the trap but was elliptically polarized. This would tend to optically pump the trapped atoms into the maximum $m_{F}$ states. This is consistent with our assumption of using only the $m_{F}= \pm F$ state for each of the coupled states. Other simplifications in the model involved the assumption of plane waves. Because the diameters of the laser beams are large $(\sim 1.5 \mathrm{~cm})$ relative to the size of the trap and are well collimated, the approximation of plane waves seems accurate. The inclusion of laser beam geometry would be more important for predictions of other phenomena of trap dynamics that are not confined to the very center of the intersecting beams.

The cascaded two-photon probe also proved useful for studying some of the effects of MOT dynamics. We have used this probe to study some transient properties of the trap following a change in the magnetic field. The trapped atoms took a surprisingly long time to reach equilibrium. We could easily see these properties by monitoring the splitting of the lines as a function of delay after the magnetic field was changed or shut off. The magnetic field in our trap can be switched off in less than $1 \mathrm{~ms}$, yet the splitting of the lines took much longer to reach equilibrium ( $\gg 10 \mathrm{~ms}$ ). Long time constants in MOT dynamics have also been observed in other laboratories but remain largely unexplained. The slow (order of a few milliseconds) redistribution of atoms between $\sigma^{-}$ and $\sigma^{-}$potential wells has been reported in a four-beam optical lattice and a longitudinal magnetic field. ${ }^{13}$ In our six-beam, $\sigma^{+}-\sigma^{-}$MOT there are no true $\sigma^{+} / \sigma^{-}$potential wells, but the long time constants may be due to the redistribution of atoms in the trap as the result of other localization or collective effects.

We have also used this system to probe a trap configuration consisting of balls and orbiting rings of atoms that are due to the intentional misalignment of the trapping beams (as seen in other laboratories). ${ }^{14}$ This configuration consists of the standard $\sigma^{-}-\sigma^{-}$MOT with two of the pairs of retroreflected laser beams intentionally misaligned in one plane, creating a racetrack composed of

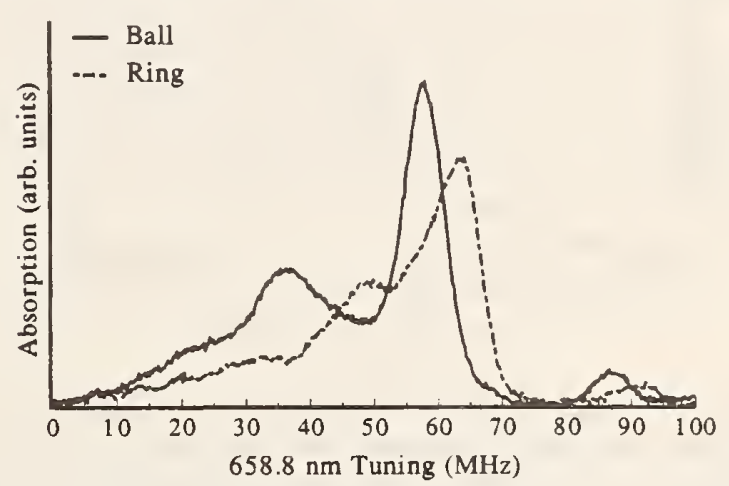

Fig. 9. 658.8-nm absorption spectra of the ring of atoms and the ball of atoms. The MOT parameters were $\delta_{21} / 2 \pi=-8.5 \mathrm{MHz}$ and $\partial \mathbf{B}_{\text {trap }} / \partial z=15 \times 10^{-4} \mathrm{~T} / \mathrm{cm}(15 \mathrm{G} / \mathrm{cm})$. The difference between the shapes of the two spectra is due to the large magnetic field at the location of the ring and the electric field amplitude drop-off of the laser beams. The shift in frequency is due primarily to the Doppler shift of the resonant frequency of the moving atoms. The signal size reflects the smaller number of atoms in the ring that intersect the probe laser. The additional features at $\sim 90$ and $\sim 25 \mathrm{MHz}$ are sidebands on the probe laser, which are used as a frequency scale. These signals were averaged over 20 scans.

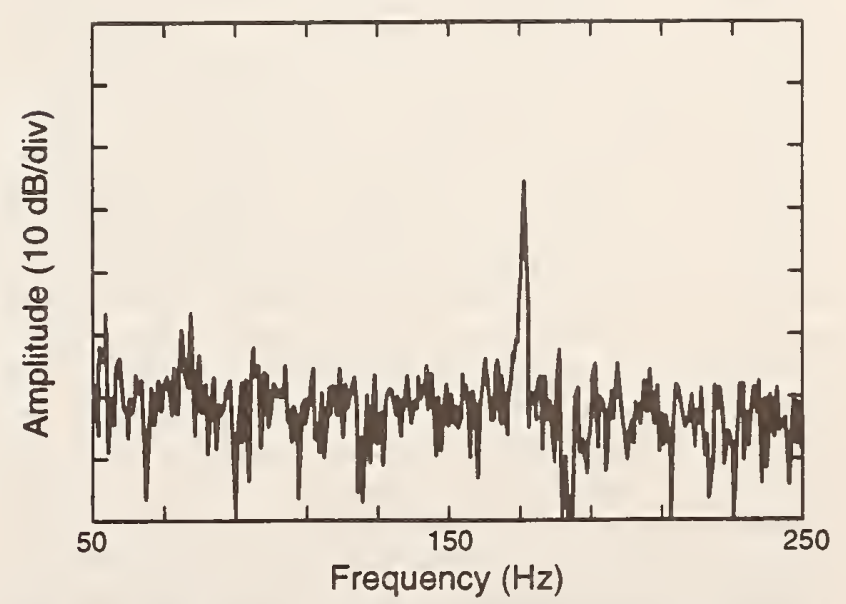

Fig. 10. FFT spectrum of the fluorescence of the MOT showing the narrow frequency component at $170 \mathrm{~Hz}$ corresponding to the rotational frequency of the orbiting atoms. The horizontal scale is $-19.5 \mathrm{~Hz}$ /division, and the resolution bandwidth is $0.5 \mathrm{~Hz}$. The figure was averaged over 20 scans. 


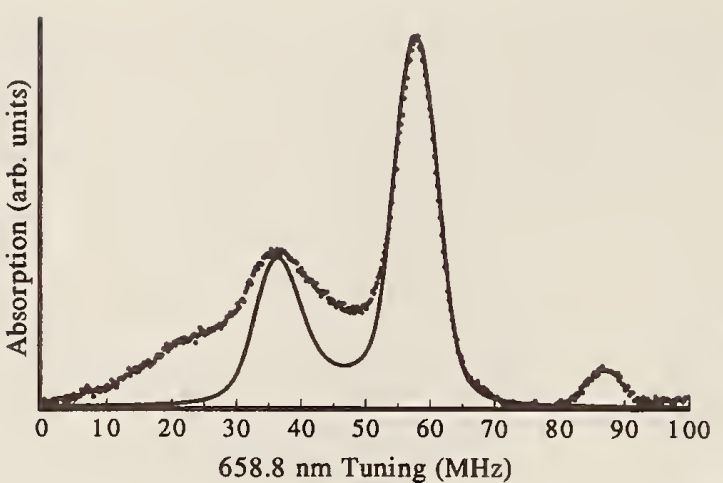

(a)

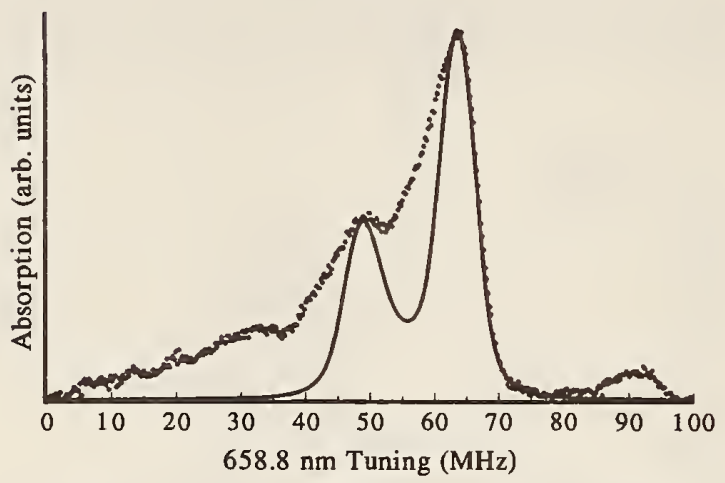

(b)

Fig. 11. Comparison of the model and the experiment for the 658.8-nm absorption for (a) the $z$ ball and (b) the $z$ ring. The data and the trap parameters are the same as in Fig. 9. This model contains no width in the velocity distribution of the orbiting atoms.

four of the laser beams. This configuration shows collective effects of the trapped atoms in which the atoms orbit in stable rings around the center of the trap (which may or may not contain a separate ball of atoms). Our system was aligned to create a ball and ring of atoms in the vertical plane of the experiment. We studied this configuration by observing the differences in the absorption spectra of the 658.8-nm light as it was sent through the ball of atoms versus the ring of atoms. We can compare these two absorption spectra to investigate the environment specific to this particular setup (Fig. 9). Three main properties of the spectra in this figure merit comment. First, owing to the smaller number of trapped atoms in the ball and the ring, the signal-to-noise ratio is much lower than that of previous spectra (the features at $\sim 90$ and $\sim 25 \mathrm{MHz}$ are sidebands on the probe laser, which are used as a frequency scale). Second, the Autler-Townes splitting of the line shape of the ball is different from that of the ring. This is expected because of large magnetic fields at the radial position of the rings, as well as the smaller Rabi frequency, which is due to the Gaussian nature of the laser beams (the diameter of the rings was usually $\sim 0.6-1 \mathrm{~cm}$, depending on parameters). Finally, the large frequency shift in the absorption spectra of the orbiting atoms is due mainly to the Doppler shift (there is a small frequency shift from the magnetic fields, as well). With the knowledge of the magnetic-field shift, the smaller Rabi frequency of the laser beams, and the diameter of the orbiting rings, the period of the orbit can be calculated from the Doppler shift. The rotational frequency of atoms in our trap was usually between $\sim 120$ and $200 \mathrm{~Hz}$. We used an independent method for determining the rotational frequency of the rings, as well. The bandwidth of the optical detection was fast enough that we could observe the time dependence of the absorption signal as the atoms orbited. This signal was studied in both the time and the frequency domains. A fast Fourier transform (FFT) was used to display the Fourier spectrum of the absorption signal and the signal from the photodetector monitoring trap fluorescence. The FFT spectrum showed a strong and narrow peak at the frequency of the orbiting atoms. Figure 10 shows a typical output of the FFT corresponding to a rotation frequency of $170 \mathrm{~Hz}$. Under most conditions this orbit had a remarkably high $Q$ of several hundred. Figure 11 shows the experimental and modeled line shapes for the two cases of the ring of atoms and the ball of atoms. Our model was able to predict accurately the relative splitting and the relative heights of the line shapes, but the widths of the signal showed poorer agreement.

\section{CONCLUSION}

We have observed and modeled an Autler-Townes-split optical double-resonance transition for cold atoms in a MOT. The model reproduces the experimental twophoton line shape fairly well under many conditions, particularly for small detunings of the trapping laser and large magnetic-field gradients. It also accurately predicts the splitting of the line shape as a function of the detuning of the trapping laser. The discrepancies between theory and experiment for larger detunings seem to suggest that, although many of the important aspects of the trap, such as the three-dimensional properties of the electric and the magnetic fields, are included in the model, more complexity is needed. We have also used this system as a useful diagnostic for the investigation of collective effects of atoms in a magneto-optic trap. In addition, using the probe laser, we observed transient effects with long time constants that indicate unexplained optical physics in samples of cold trapped atoms.

\section{ACKNOWLEDGMENTS}

We acknowledge the helpful comments and discussions of S. Gilbert, R. Fox, and P. Gould and the support of the U.S. Air Force Office of Scientific Research.

\section{REFERENCES AND NOTES}

1. K. Gibble and S. Chu, "Future slow-atom frequency standards," Metrologia 29, 201-212 (1992).

2. See, for example, P. S. Julienne, A. M. Smith, and K. Burnett, "Theory of collisions between laser cooled atoms," Adv. At. Mol. Opt. Phys. 30, 141-198 (1993).

3. See, for example, feature on laser cooling and trapping of atoms, J. Opt. Soc. Am. B 6(11) (1989).

4. J. W. R. Tabosa, G. Chen, Z. Hu, R. B. Lee, and H. J. Kimble, "Nonlinear spectroscopy of cold atoms in a spontaneousforce optical trap," Phys. Rev. Lett. 66, 3245-3248 (1991); G. Grynberg, B. Lounis, P. Verkerk, J.-Y. Courtis, and C. Salomon, "Quantized motion of cold cesium atoms in twoand three-dimensional optical potentials," Phys. Rev. Lett. 70, 2249-2252 (1993); P. S. Jessen, C. Gerz, P. D. Lett, W. D. Phillips, S. L. Rolston, R. J. C. Spreeuw, and C. I. Westbrook, 
"Observation of quantized motion of Rb atoms in an optical field," Phys. Rev. Lett. 69, 49-52 (1992); A. Hemmerich and T. W. Hänsch, "Two-dimensional atomic crystal bound by light," Phys. Rev. Lett. 70, 410-413 (1993).

5. P. Marte, R. Dum, R. Taieb, and P. Zoller, "Resonance fluorescence from one-dimensional molasses," Phys. Rev. A 47, 1378-1390 (1993); J. Guo, "Contribution of energy continuum states to probe absorption signal of atoms in onedimensional optical molasses," Phys. Rev. A 49, 3934-3942 (1994); J.-Y. Courtois and G. Grynberg, "Probe transmission in one-dimensional optical molasses: theory for linearly crossed polarized cooling beams," Phys. Rev. A 46, 7060-7078 (1992).

6. H. R. Gray and C. R. Stroud, Jr., "Autler-Townes effect in double optical resonance," Opt. Commun. 25, 359-362 (1978); P. R. Hemmer, B. W. Peuse, F. Y. Wu, J. E. Thomas, and S. Ezekiel, "Precision atomic-beam studies of atom-field interactions," Opt. Lett. 6, 531-533 (1981).

7. R. W. Fox, S. L. Gilbert, L. Hollberg, J. H. Marquardt, and H. G. Robinson, "Optical probing of cold trapped atoms," Opt. Lett. 18, 1456-1458 (1993).

8. C. Monroe, W. Swann, H. Robinson, and C. Wieman, "Very cold trapped atoms in a vapor cell," Phys. Rev. Lett. 65, 1571-1574 (1990).

9. V. M. Fain and Ya. I. Khanin, Quantum Electronics (MIT Press, Cambridge, Mass., 1969); R. M. Whitley and C. R.
Stroud, "Double optical resonance," Phys. Rev. A 14, $1498-1513$ (1976).

10. Initially we assumed that the atom distribution in the atom cloud was random, but we found that the agreement between theory and experiment improved by assuming that the atom density is proportional to the local field intensity. The enhancement of the atom density in the higher fields is expected because of the dipole force that is due to the red-detuned $\left(\delta_{21}<0\right)$ laser field.

11. We use cylindrical coordinates. The actual shape of the cloud in our trap is a somewhat irregular, oblate spheroid. The height of the atom cloud ( $z$ direction) is approximately two thirds of the diameter and is fairly well represented by a cylindrical volume.

12. S. Stegholm, Foundations of Laser Spectroscopy (Wiley, New York, 1984).

13. D. R. Meacher, S. Guibal, C. Mennerat, J.-Y. Courtis, K. I. Pestas, and G. Grynberg, "Paramagnetism in a cesium optical lattice," Phys. Rev. Lett. 74, 1958-1961 (1995).

14. D. W. Sesko, T. G. Walker, and C. E. Wieman, "Behavior of neutral atoms in a spontaneous force trap," J. Opt. Soc. Am. B 8, 946-958 (1991); V. S. Bagnato, L. G. Marcassa, M. Oria, G. I. Surdutovich, R. Vitlina, and S. C. Zilio, "Spatial distribution of atoms in a magneto-optical trap," Phys. Rev. A 48, 3771-3775 (1993). 


\title{
Population- and Coherence-Induced Gain and Self-Oscillations in Alkali Vapor
}

\author{
A.S. Zibrovab.c, H.G. Robinson, ${ }^{a}$, V.L. Velichanskyc, V.V. Vasiliev", \\ L. Hollberg, E. Arimondo , M.D. Lukin ${ }^{b}$, and M.O. Scully \\ a National Institute of Standards and Technology, Boulder, CO, USA \\ ${ }^{b}$ Texas A\&M University, College Station, TX 77843 \\ ' Lebedev Institute of Physics, Moscow, II7924 Russia \\ 'Dipartimento di Fisica. Universita di Pisa, Pisa, Italy
}

\section{Abstract}

Gain and oscillations are observed in a 3-level, $\Lambda$ configuration when $\mathrm{Rb}$ atoms are driven with a strong, coherent, laser field and a broadband repumping laser. The system automatically generates an output beam that has a beatnote at the frequency of the ground state hyperfine splitting $(6.8 \mathrm{GHz})$. Gain is due to both population and coherence effects in a Raman configuration. The experimental system is extremely simple and shows promise of providing a compact, Rb-stabilized microwave oscillator.

Large Raman gain signals are observed in the very simple experimental setup diagrammed in Fig. 1. The beam from a single-mode diode laser DL1 (drive laser) is passed through a $\mathrm{Rb}$ cell and is then detected on a fast photodiode. DL2 is a solitary diode-laser that can be spectrally broadened by adding noise to the injection current. These two input beams propagate through the cell (either in the same or opposite directions) at a small angle that allows separation of the beams and avoids feedback.

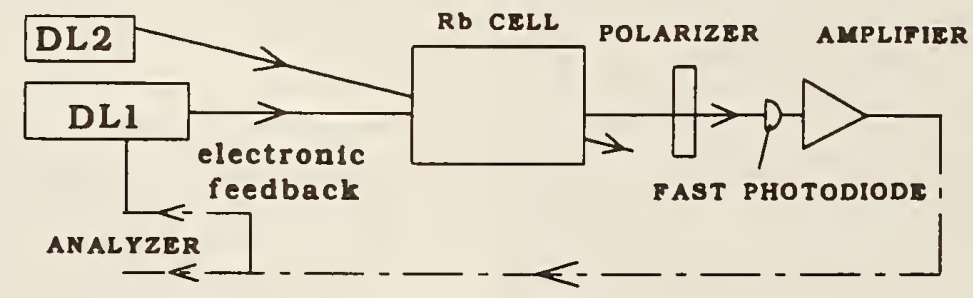

Fig. 1 Experimental setup consists of two diode laser beams, a $\mathrm{Rb}$ cell, a fast photodetector, and a microwave spectrum analyzer used to display the signal. The dotted line indicates the electronic feedback path that is used in some of the experiments.

Raman scattering of the drive laser in the $\mathrm{Rb}$ vapor generates a beam that is collinear with the drive but is frequency-shifted by the ground state hyperfine splitting ( 3.0 and 6.8 $\mathrm{GHz}$ for $\mathrm{Rb}^{85}$ and $\mathrm{Rb}^{87}$ ). The fast photodiode simply detects the beatnote between the drive laser and the Raman shifted beam. The function of the repumping laser (DL2) is to return the population to the initial ground-state hyperfine level, thus controlling the optical pumping effects of the drive laser. The tuning of the broadband repumping laser is not critical; any 
transition (for example, different $\mathrm{P}$ state) that will return population to the initial hyperfine ground state can be used. In this way the repumping laser serves the same purpose as the buffer gas in the earlier Raman laser experiments. $\{2\}$

Figure 2 shows a typical signal that is detected with the fast photodiode. In this example the power of the drive laser was $\sim 13 \mathrm{~mW}$, tuned to resonance on the $\mathrm{Dl}, \mathrm{F} \rightarrow \mathrm{F}^{\prime}\left(2 \rightarrow 2^{\prime}\right)$ transition; the repumping laser was about $8 \mathrm{~mW}$, broadened to $\sim 500 \mathrm{MHz}$ spectral width and tuned approximately to the Dl $(1 \rightarrow 2$ ') transition. The spot diameters in the center of the $6 \mathrm{~cm}$ long cell were $\sim 500 \mu \mathrm{m}$ for the drive and $\sim 3 \mathrm{~mm}$ for the repump. In this example the beatnote between the self-generated Raman beam and the drive laser has a signal-to-noise ratio of about $40 \mathrm{~dB}$ with a detection bandwidth of $300 \mathrm{kHz}$.
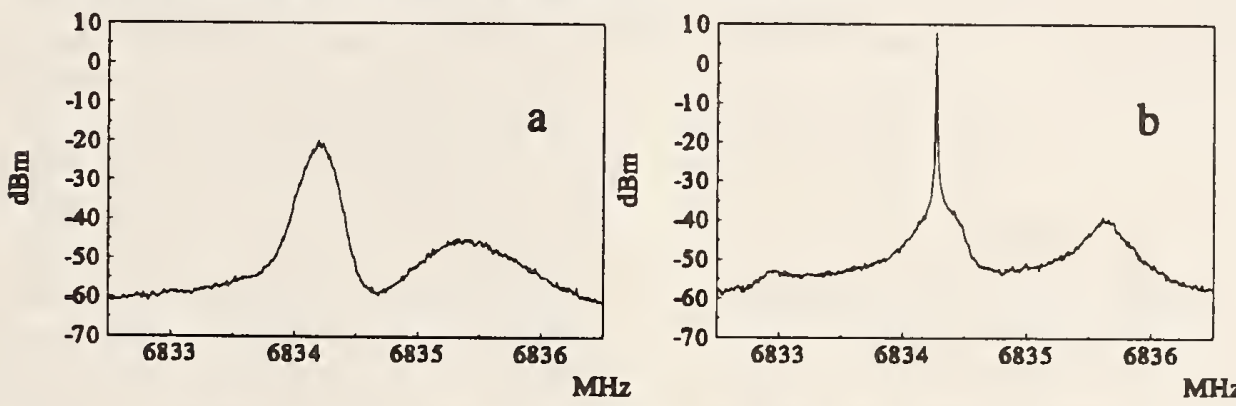

Fig. 2 Signal at $6.8 \mathrm{GHz}$ from the photodetector as displayed on a microwave spectrum analyzer. Part (a) shows the beatnote between the self-generated Raman beam and the drive laser with no electronic feedback, while in (b) the spectrum is narrowed when the electronic feedback at $6.8 \mathrm{GHz}$ causes the system to self-oscillate.

Our approach is reminiscent of the previous work of P. Kumar ${ }^{1,2}$ and P. Hemmer, ${ }^{3}$ who have demonstrated Raman gain and lasing in Na vapor heat-pipes using optical cavities. Our system is distinguished from previous work in that it does not require an optical cavity, nor a buffer gas. Also, an electronic beatnote signal is generated directly at the hyperfine difference frequency, which can be use to create a narrow self-oscillation at the hyperfine frequency. The system is very simple and in its minimum configuration requires only requires a diode laser, a photodiode and a $\mathrm{Rb}$ cell. It does not require a microwave oscillator and it provides a stable oscillation at the frequency defined by the atomic hyperfine splitting.

The gain in this system results from both population and coherence effects, and is remarkably high and robust. We have observed gain in both the Stokes and anti-Stokes configurations, on both the D1 and D2 lines, and within and outside the Doppler profiles. For some configurations the system also shows gain at twice the ground-state hyperfine splitting (13.6 GHz for $\mathrm{Rb}$ ) which is most likely due to gain in the double- $\Lambda$ configuration. ${ }^{3}$

Comparison of theoretical and experimental lineshapes is possible for both the microwave spectrum and the optical gain/loss spectrum. A few theoretical models have been used with an attempt to determine the most important physical effects: hyperfine structure, Zeeman structure, optical coherences, propagation, and Doppler broadening. At least for some range of the experimental parameters, the theory reproduces the experimental lineshapes reasonably well.

This simple system generates a signal with a very good signal-to-noise ratio and with a 
fairly narrow linewidth as a microwave oscillator. An obvious application of the Raman selfoscillator would be as a compact, $\mathrm{Rb}$ frequency standard; however a number of questions remain to be answered before this can be realized. In particular, can the problems associated with AC Stark shifts be minimized to the point that the atomically stabilized microwave oscillator has useful stability? Stark shifts resulting from the drive laser power are the main perturbation to the frequency of the Rb self-oscillator. These shifts are complicated by the multilevel nature of $\mathrm{Rb}$, the variation of intensity across and along the laser beam, and their dependence on the detunings of the lasers from resonance. As a baseline test we set up the $\mathrm{Rb}$ self-oscillator without any optimization and let it run without any control on the laser frequencies, cell temperature or laser power levels. Using a frequency counter to record the oscillation frequency allows a measurement of the Allan variance of the self-oscillator as shown below.

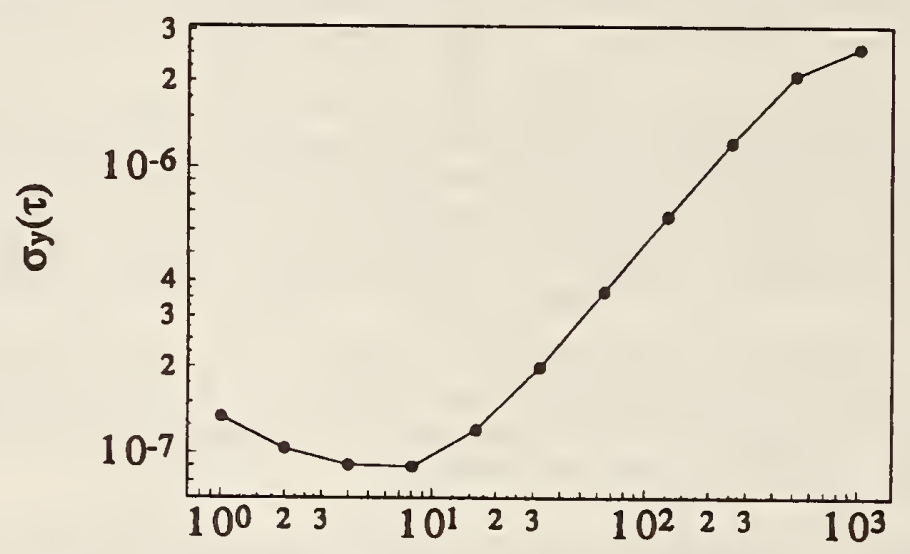

Figure 3. Data shows a typical square-root of the Allan variance of the frequency of the freerunning $\mathrm{Rb}$ self-oscillator as a function of averaging time $(\tau)$ in seconds.

The stability of the frequency is good for a free-running oscillator, but is obviously not of frequency-standard quality in this free-running state. Active control could easily be implemented but with some penalty in complexity. Perhaps there are laser tunings and powers that can be uses to minimize the effects of the AC Stark shift. A potentially important curiosity of the system is that the oscillation frequency does not appear to have first-order sensitivity to acceleration. This may be advantageous in some very high acceleration environments.

We gratefully acknowledge numerous helpfui comments from D. Gauthier and P. Hemmer, and the support of the Air Force Office of Scientific Research.

References

1. P. Kumar and J.H. Shapiro, Opt. Lett. 10,226 (1985).

2. M. Poelker, P. Kumar, and S.-T. Ho, Opt. Lett. 16, 1853 (1991).

3. J. Donoghue, M. Cronin-Golomb, J. Kane and P. Hemmer, Opt. Lett. 17, 1313 (1991). 


\title{
Experimental Demonstration of Enhanced Index of Refraction via Quantum Coherence in $\mathbf{R b}$
}

\author{
A. S. Zibrov, ${ }^{1,2,4,5}$ M. D. Lukin, ${ }^{1,2,3}$ L. Hollberg, ${ }^{4}$ D. E. Nikonov, ${ }^{1,2,3}$ M. O. Scully, ${ }^{1,2,3}$ H. G. Robinson, ${ }^{4}$ \\ and V.L. Velichansky ${ }^{5}$ \\ 'Department of Physics, Texas A\&M University, College Station, Texas 77843 \\ ${ }^{2}$ Texas Laser Laboratory, HARC, The Woodlands, Texas 77381 \\ ${ }^{3}$ Max-Planck-Institut für Quantenoptik, D-85748 Garching, Germany \\ ${ }^{4}$ National Institute of Standards and Technology, Boulder, Colorado 80303 \\ ${ }^{5}$ Lebedev Institute of Physics, 117924 Moscow, Russia
}

(Received 11 December 1995)

\begin{abstract}
We present a proof-of-principle experiment demonstrating a resonant enhancement of the index of refraction accompanied by vanishing absorption in a cell containing a coherently prepared $\mathrm{Rb}$ vapor. The results are in good agreement with detailed theoretical predictions. [S0031-9007(96)00142-1]
\end{abstract}

PACS numbers: 42.50.Ar, 42.55.-f

In the present Letter we report the first demonstration of a resonant enhancement of the index of refraction without absorption [1]. Surprising and counterintuitive effects involving quantum coherence and interference have recently become laboratory realities. For example, phenomena such as electromagnetically induced transparency (EIT) [2] and lasing without population inversion (LWI) [3], predicted theoretically at the end of the 1980s have now been demonstrated experimentally [4-7]. In both of the above effects quantum coherence dramatically modifies the absorptive properties of the medium. The unusual behavior of the dispersive part of the susceptibility of coherently prepared medium is no less intriguing. For example, it was shown theoretically that it is possible to have a completely transparent medium with large dispersion (i.e., rapid variation of index of refraction with frequency) or with large index of refraction [1]. Both of these effects can be achieved using atomic phase coherence in a resonant medium, which is normally optically thick. Coherence effects, however, allow us to prepare an optical medium such that the medium has vanishing absorption, while the dispersive part of the susceptibility is enhanced.

The interest in these phenomena is due, on one hand, to the fundamental nature of coherence effects and, on the other hand, to possible applications. For example, the dispersive properties of coherently prepared media have been studied with an eye toward generation of pulses with very slow group velocity [8], effective control of nonlinearities [9], high precision magnetometry [10], and laser acceleration of particles [1].

Several recent experiments [11] have demonstrated the large dispersion of the index of refraction accompanying EIT. Index enhancement, however, allows not only for large dispersion, but also for a large refractive index itself, while maintaining a transparent medium.

The conceptual foundation of the present experiment can be understood by considering the simple $\Lambda$ atomic configuration of Fig. 1. The coherent strong driving field with Rabi frequency $(\Omega)$ and weak coherent probe $\left(\Omega_{p}\right)$ allow us to prepare the atom in a coherent superposition of states $b$ and $c$ [12]. When the detunings of these two fields from their respective atomic resonances are equal, EIT is obtained. The incoherent pumping (represented by the rate $r$ ) alters this coherent superposition by pumping some of the population into other states. Depending upon the actual parameters of the system this may result in gain, loss, or complete transparency for the probe field.

The linear gain (absorption) coefficient $(G)$ is proportional to the imaginary part of complex susceptibility $\left(\chi^{\prime \prime}\right)$. In the case when the driving field is in exact resonance with transition $a \rightarrow c$ it is given for this particular three-level configuration by [13]

$$
G=\kappa \frac{\gamma_{b c} A\left(\gamma_{c b} \gamma_{a b}+|\Omega|^{2}-\Delta^{2}\right)+\Delta^{2} B\left(\gamma_{a b}+\gamma_{b c}\right)}{\left(\gamma_{b c} \gamma_{a b}+|\Omega|^{2}-\Delta^{2}\right)^{2}+\Delta^{2}\left(\gamma_{a b}+\gamma_{b c}\right)^{2}}
$$

where $\kappa=3 \lambda^{2} N \gamma_{a}^{\prime} L / 4 \pi$, and $A$ and $B$ are given by

$$
\begin{gathered}
A=\left[1+\left(\gamma_{a}^{\prime}+\gamma_{a}^{\prime \prime}\right) / 2 \gamma_{b c}\right] \rho_{a a}-\rho_{b b}, \\
B=\rho_{a a}-\rho_{b b} .
\end{gathered}
$$

Here $\rho_{i i}$ is the population of level $i$ calculated to the zeroth order in the probe field, $\gamma_{i j}$ is the relaxation rate of the density matrix element $\rho_{i j}, N$ is the density of atoms, $L$ is the length of the cell, $\lambda$ is the wavelength,
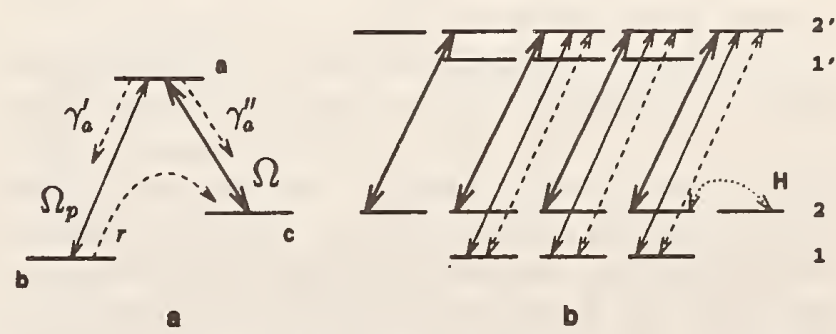

FIG. 1. (a) Simplified three-level model for index enhancement. (b) The actual level scheme of $D_{1}$ absorption line in $\mathrm{Rb}^{87}$ and the optical fields used in the present experiment. Energy separation between manifolds 1 and 2 is equivalent to $6.8 \mathrm{GHz}$. 
$\Delta$ is the detuning of the probe laser, and atomic decays $\left(\gamma_{a}^{\prime}, \gamma_{a}^{\prime \prime}\right)$ are indicated in Fig. 1(a). For the case $\Delta=0$ we see that, in the presence of the coherent driving field, the gain (which is in that case proportional to $A$ ) can be positive even if most population remains in the ground state $b$, i.e., even if $B<0$. That is, a weak probe field undergoes amplification without the need of population inversion $[7,14]$. If the probe field is detuned from atomic resonance, the gain coefficient decreases rapidly, and vanishes when

$$
\Delta=\Delta_{0}= \pm \sqrt{\frac{A \gamma_{b c}\left(\Omega^{2}+\gamma_{a b} \gamma_{b c}\right)}{A \gamma_{b c}-B\left(\gamma_{a b}+\gamma_{b c}\right)}} .
$$

Let us turn now to the real part of the complex susceptibility $\left(\chi^{\prime}\right)$, i.e., to the resonant index of refraction. It results, in particular, in the phase shift $(\phi)$ for the probe light transmitted through the resonant vapor, which is proportional to $\chi^{\prime}$ :

$$
\phi=-\frac{\kappa}{2} \Delta \frac{B\left(\gamma_{c b} \gamma_{a b}+|\Omega|^{2}-\Delta^{2}\right)-A\left(\gamma_{a b}+\gamma_{b c}\right) \gamma_{b c}}{\left(\gamma_{c b} \gamma_{a b}+|\Omega|^{2}-\Delta^{2}\right)^{2}+\Delta^{2}\left(\gamma_{a b}+\gamma_{b c}\right)^{2}} .
$$

The phase shift is equal to zero when the probe field is tuned precisely to the resonance with the $a \rightarrow b$ transition, but for nonzero probe field detuning it can become quite large. To be more specific, we consider the system with decay rates and pumping as indicated in Fig. 1(a) [13]. In this case and if we take $r \sim \gamma_{a}^{\prime} \sim$ $\gamma_{a}^{\prime \prime}, \Omega \gg \sqrt{\gamma_{a b} r}$, the gain coefficient vanishes at $\Delta_{0} \sim$ $\pm \sqrt{\left|\Omega_{d}\right|^{2}+\gamma_{a b} \gamma_{b c}}$. At this point the phase shift is on the order of

$$
\phi\left(\Delta_{0}\right) \sim(\kappa / 12) / \Delta_{0} .
$$

It follows, therefore, that a medium can become transparent at the point where the resonant index of refraction has a large value.

A $\Lambda$-type atomic configuration can be realized within the $D_{1}$ absorption line of $\mathrm{Rb}$ as shown in Fig. 1(b). Here right circularly polarized coherent driving and probe fields are tuned close to the $2 \rightarrow 2^{\prime}$ and $1 \rightarrow 2^{\prime}$ transitions, respectively [15]. The incoherent pumping out of the states 1 is accomplished by a broadband right circularly polarized field which couples the transition $1 \rightarrow 2^{\prime}$. All of the fields are copropagating, which allows for the reduction of Doppler broadening.

We note here that optical properties of the real atomic systems are, in general, different from those of simplified 3- or 4-level models due to the presence of many hyperfine and Zeeman sublevels. In particular, optical pumping can play an important role. Indeed, in the case when both driving and pumping fields are present, atoms can be optically pumped into the state $F=2, M_{F}=+2$ [Fig. 1(b)]. To avoid the trapping of the population in this state a weak magnetic field $\left(\sim 2 \times 10^{-4} \mathrm{~T}\right)$ is used, which mixes the populations via Larmor precession.

In order to account for realistic experimental conditions we developed a numerical model, wherein the field polar- izations, the presence of hyperfine and Zeeman sublevels and Doppler broadening are taken into account. This is done by numerically solving the density matrix equations for the 16-level system of Fig. 1(b) and averaging over a Maxwell velocity distribution. The numerical analysis showed that for parameters typical of our experiment the effect of index enhancement depends upon the tuning of the drive laser within the Doppler absorption profile. This is the case when the intensity of a driving field is relatively low and the ground state relaxation rate is sufficiently high (determined in our case by the incoherent pump rate). Under these conditions we found the index enhancement is larger if the driving field is detuned to either side of the center of the Doppler profile by roughly a third of the Doppler width.

The results of the calculations are presented in Fig. 2 . They clearly show that there is a region (indicated by $I$ ) where absorption is canceled and, at the same time, the real part of susceptibility (i.e., the phase shift) increases due to the presence of the drive and pump fields.

In the experiment we used a $4 \mathrm{~cm}$ long cell containing natural $\mathrm{Rb}$. Drive and probe lasers were extended cavity diode lasers at $794 \mathrm{~nm}$ with linewidths of about $100 \mathrm{kHz}$. The powers of these beams in the region of the cell were on the order of $10 \mathrm{~mW}$ and $5 \mu \mathrm{W}$, respectively, and the corresponding spot sizes in the same region were $2 \mathrm{~mm}$ and $100 \mu \mathrm{m}$. The angle between two beams did not exceed $5 \times 10^{-3} \mathrm{rad}$. The pump laser was a solitary laser diode $(794 \mathrm{~nm})$ with output power $\approx 5 \mathrm{~mW}$. Its linewidth was additionally broadened to about $150 \mathrm{MHz}$

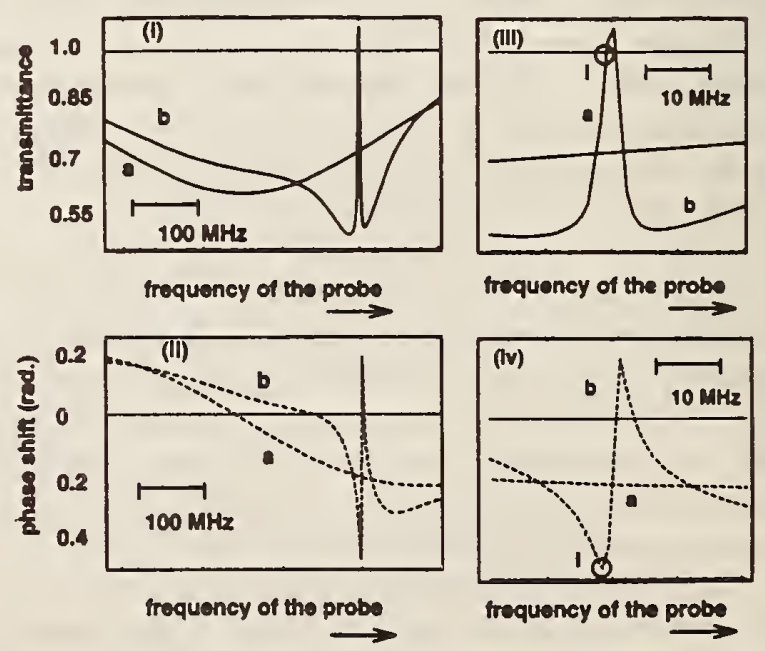

FIG. 2. Calculated absorption coefficient (i,iii) and phase shift (ii,iv) of the probe field in the $\mathrm{Rb}$ vapor. In all figures curve (a) corresponds to the case without driving and pumping fields, curve $(b)$ corresponds to the case when these two fields are present. Parameters for numerical modeling are $\Omega=16 \mathrm{MHz}$, Doppler width is $500 \mathrm{MHz}$, incoherent pump corresponds to $r=0.5 \mathrm{MHz}$, and magnetic field $H_{x}=2 \times 10^{-4} \mathrm{~T}$. Natural linewidth of the $\mathrm{Rb} D_{1}$ absorption line is $5.4 \mathrm{MHz}$. 
by modulating the diode's input current with noise. The size of the pump beam was on the order of $3 \times 5 \mathrm{~mm}$.

The resonant index of refraction was determined via phase-shift measurements using a Mach-Zehnder interferometer (see Fig. 3). When the interferometer arms are of nearly equal length the difference current of photodetectors PD1 and PD2 is proportional to

$$
S_{\text {signal }} \sim I_{0} \exp (G / 2) \sin \left(\phi+\phi_{0}\right),
$$

where $I_{0}$ is the intensity of the probe field and $\phi_{0}$ is a constant phase shift determined by a balance of the interferometer arms. A piezoelectric transducer is used to vary $\phi_{0}$ and thus obtain the scaling of the signal.

The first experiments were carried out with the low $\mathrm{Rb}$ density, such that maximum probe absorption was on the order of $40 \%$. Under these conditions the signal given by Eq. (7) is the phase shift of the probe field in the $\mathrm{Rb}$ cell, provided that the arms of the interferometer are appropriately balanced. The absorption of the probe field was detected simultaneously. Without the drive and incoherent pump fields, the absorption and the phase shift show the ordinary features of Doppler-broadened twolevel atomic resonance (curve $a$ in Fig. 4). When the drive field is present and tuned within the Doppler profile, the absorption spectrum of the probe laser exhibits a narrow transmission peak at the frequency corresponding to that of a two-photon resonance condition. When the incoherent pump is added the narrow gain peak appears (curve $b$ in Fig. 4). If the probe field is detuned slightly from the two-photon resonance (point $I$ in Fig. 4) the medium is transparent; i.e., it has neither loss nor gain.

The phase shift, which is proportional to the real part of susceptibility, is shown in Figs. 4(ii) and 4(iv). It can be quite large in the transparency region. In particular, we note that at point $I$ the change in the refractive index exceeds that found in the same system without the driving field. That is, the real part of the susceptibility is enhanced at the point where the absorption vanishes. We note the good agreement between the theoretical predictions of Fig. 2 and the experimental observations of Fig. 4.

Having observed and analyzed the absorption and dispersion in an optically thin medium we were also able to demonstrate the enhancement of refractive index in an optically dense medium. To accomplish this phase shift and absorption measurements were carried out for

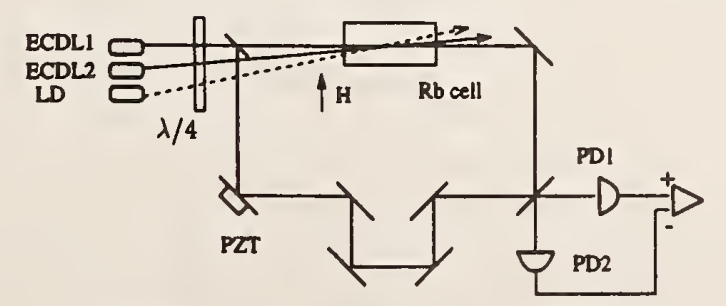

FIG. 3. Schematic of experimental setup. ECDLl and ECDL2 are probe and driving lasers, respectively; LD is an incoherent pump laser. a variety of temperatures. Figure 5 shows the value of the phase shift as a function of atomic density. For low temperatures the phase shift in the coherently prepared transparent $\mathrm{Rb}$ vapor is somewhat larger than the maximum phase shift in the usual $\mathrm{Rb}$ resonant vapor. For temperatures $T>80^{\circ} \mathrm{C}\left(N>3 \times 10^{11} \mathrm{~cm}^{-3}\right)$ the $\mathrm{Rb}$ absorbs $100 \%$ of the probe light near the resonance, i.e., in the region where the refractive index has its maximum. When the drive and incoherent pump are present the system is transparent and displays large phase shifts. The inset in Fig. 5 shows the transmission of the probe laser (curve $A$ ) and corresponding output $S_{\text {sig }}$ of the phase shift measurement (curve $B$ ) at a $\mathrm{Rb}$ cell temperature $\sim 87^{\circ} \mathrm{C}$. In the presence of coherent drive and incoherent pumping, the narrow transparency peak appears (curve $a$ ) on an otherwise flat, completely absorbing background. The corresponding phase shift (curve $b$ ) measurements display the oscillations which correspond to large values of phase shift. Under the present experimental conditions we observed the phase shifts up to $\approx 7 \pi$ at the point of complete transparency, which corresponds to the resonant change in the refractive index $\Delta n \sim 10^{-4}$. We note that such a phase shift is not observable in a usual absorber in the immediate vicinity of resonance because of the resonant absorption.

We remark here that quite large phase shifts accompanied by relatively good transparency can be obtained in a usual nondriven atomic system (e.g., within the $\mathrm{Rb}$ $D_{1}$ line without coherent preparation) at large detunings. This happens because the real part of the susceptibility $\chi^{\prime}$ decreases slower than $\chi^{\prime \prime}$ with detuning. However, for large detunings, $\chi^{\prime}$ of a usual absorber increases with density much slower than the resonant $\chi^{\prime}$. In particular, for $N \sim 10^{12} \mathrm{~cm}^{-3}$ we observed that the absorption of the
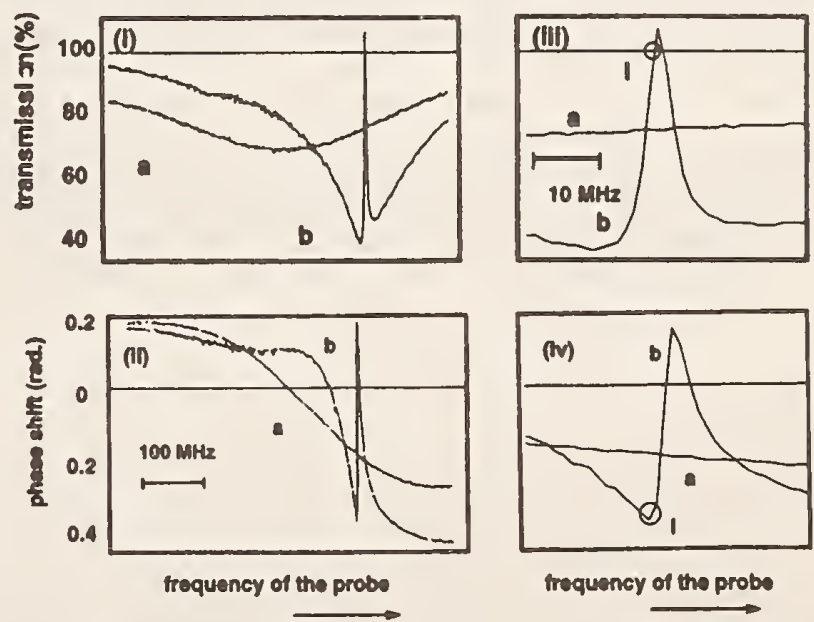

FIG. 4. Transmitted intensity (i,iii) of the probe light in the $\mathrm{Rb}$ cell and the difference current of photodetectors, converted into the phase shift (ii,iv). In all figures curve $(a)$ was observed without driving and pumping fields, curve $(b)$ was observed when those two fields were present. 


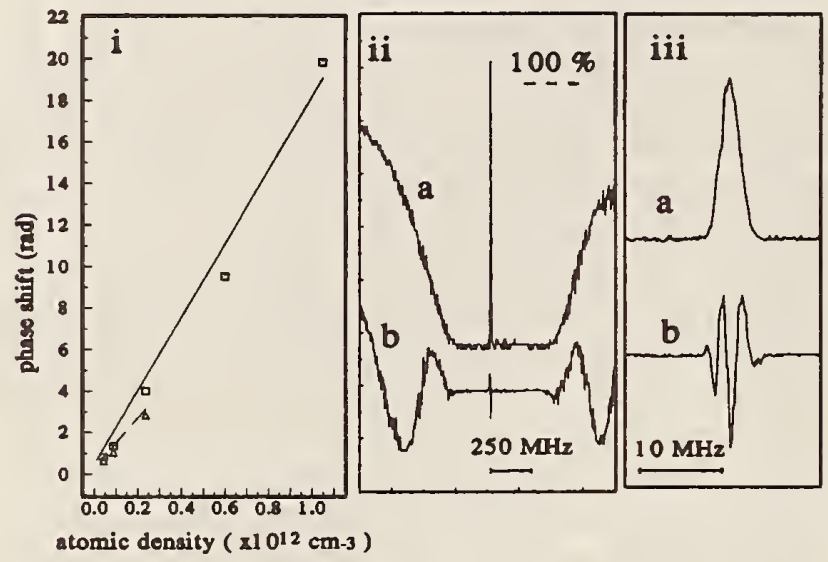

FIG. 5. (i) The phase shifts in Rb vapor as a function of a vapor density. $\square$ is the phase shift in coherently prepared $R b$ at the point of complete transparency; $\Delta$ is the maximum value of phase shift in the same system without coherent preparation. (ii) Transmission of the probe (curve $a$ ) and corresponding quadrature component of interferometer signal (curve $b$ ) in an optically dense $\mathrm{Rb}$ vapor with driving and incoherent pumping fields present. (iii) The enlarged central part of (ii).

probe field in the nondriven $\mathrm{Rb}$ vapor is essentially zero at detuning $\sim 15 \mathrm{GHz}$. In this region the measured phase shift was approximately $1.5 \mathrm{rad}$.

In conclusion, we have presented an experimental demonstration of an enhancement of the refractive index in a coherently prepared atomic medium accompanied by vanishing absorption. The experimental results were predicted by and are in good agreement with a detailed theoretical analysis.

The authors gratefully acknowledge discussions with E. Arimondo, H. Briegel, M. Fleischhauer, E. Fry, and T. Hänsch, valuable assistance of T. Zibrova, and the support from the Office of Naval Research, the Welch Foundation, the Texas Advanced Research and Technology Program, and the Air Force Office of Scientific Research.

[1] M. O. Scully, Phys. Rev. Lett. 67, 1855 (1991); M. O. Scully and S.-Y. Zhu, Opt. Commun. 87, 134 (1992); M. Fleischhauer et al., ibid. 87, 109 (1992); A.D. Wilson-Gordon and H. Friedman, ibid. 94, 238 (1992);
M. Fleischhauer et al., Phys. Rev. A 46, 1468 (1992); U. Rathe et al., ibid. 47, 4994 (1993); J. Dowling and C. Bowden, Phys. Rev. Lett. 70, 1421 (1993); A. Manka et al., ibid. 73, 1789 (1994); O. Kocharvoskaya, P. Mandel, and M. O. Scully, ibid. 74, 2451 (1995).

[2] S. E. Harris, J. E. Field, and A. Imamoglu, Phys. Rev. Lett. 64, 1107 (1990).

[3] O. Kocharovskaya and Y. Khanin, JETP Lett. 48, 630 (1988); S. E. Harris, Phys. Rev. Lett. 62, 1033 (1989); M. O. Scully, S.-Y. Zhu, and A. Gavrielides, ibid. 62, 2813 (1989).

[4] K.-J. Boller, A. Imamoglu, and S.E. Harris, Phys. Rev. Lett. 66, 2593 (1991).

[5] A. Nottelmann, C. Peters, and W. Lange, Phys. Rev. Lett. 70, 1783 (1933); E. S. Fry et al., ibid. 70, 3235 (1993); W. E. van der Veer et al., ibid. 70, 3243 (1993).

[6] A.S. Zibrov et al., Phys. Rev. Lett. 75, 1499 (1995).

[7] G. G. Padmabandu et al., Phys. Rev. Lett. 76, 2053 (1996).

[8] S. E. Harris, J.E. Field, and A. Kasapi, Phys. Rev. A 46, R29 (1992).

[9] S. E. Harris, Opt. Lett. 19, 2018 (1994).

[10] M.O. Scully and M. Fleischhauer, Phys. Rev. Lett. 69 , 1360 (1992).

[11] M. Xiao et al., Phys. Rev. Lett. 74, 666 (1995); R. Moseley et al., ibid. 74, 670 (1995); D. Meschede et al., in Proceedings of CLEO/QELS '95 Boston 1995.

[12] G. Alzetta, A. Gozzini, L. Moi, and G. Orriols, Nuovo Cimento Soc Ital. Fis. 36B, 5 (1976); E. Arimondo and G. Orriols, Nuovo Cimento Lett. 17, 333 (1976); H.R. Gray, R. M. Whitley, and C. R. Stroud, Jr., Opt. Lett. 3, 218 (1978).

[13] We note here that the results for $\chi^{\prime}$ and $\chi^{\prime \prime}$ depend upon the particular choice of the model, e.g., upon the particular incoherent pump mechanism. In the present three-level model, which we use to illustrate the physics, we assume an indirect pump mechanism which populates level $c$ via a "one way" pumping from level $b$.

[14] A. Imamoğlu, J. E. Field, and S. E. Harris, Phys. Rev. Lett. 66, 1154 (1991).

[15] In such a configuration probe and driving fields form three effective $\Lambda$ systems with the Rb $D_{1}$ manifold [Fig. 1(b)]. Note that the situation would be different if we used fields with linear polarizations: In that case fields form only two effective $\Lambda$ systems (since the transition 2, $m_{F}=0 \rightarrow 2^{\prime} m_{F}=0$ is forbidden) which implies a large absorption of the probe field on the transition $1, m_{F}=$ $0 \rightarrow 2^{\prime} m_{F}=0$. 


\title{
Spectroscopy in Dense Coherent Media: Line Narrowing and Interference Effects
}

\author{
M. D. Lukin, ${ }^{1,4}$ M. Fleischhauer, ${ }^{1,3}$ A. S. Zibrov, ${ }^{1,2,5}$ H. G. Robinson, ${ }^{2}$ V. L. Velichansky, ${ }^{1,5}$ \\ L. Hollberg, ${ }^{2}$ and M. O. Scully ${ }^{1,4}$ \\ ${ }^{1}$ Department of Physics, Texas A \& M University, College Station, Texas 77843 \\ ${ }^{2}$ National Institute for Standards and Technology, Boulder, Colorado 80303 \\ ${ }^{3}$ Sektion Physik, Universität München, D-80333 München, Germany \\ ${ }^{4}$ Max-Planck Institut für Quantenoptik, D-85748 Garching, Germany' \\ ${ }^{5}$ Lebedev Institute of Physics, Moscow, Russia
}

(Received 18 April 1997)

\begin{abstract}
Spectroscopic properties of coherently prepared, optically dense atomic media are studied experimentally and analyzed theoretically. It is shown that in such media the power broadening of the resonances can be substantially reduced. A density-dependent spectral narrowing of the electromagnetically induced transparency (EIT) window and novel, even narrower, resonances superimposed on the EIT line are observed in dense Rb vapor. A nonlinear two-photon spectroscopic technique based on coherent atomic media and combining high resolution with a large signal-to-noise ratio seems feasible. [S0031-9007(97)04278-6]
\end{abstract}

PACS numbers: $42.50 .-\mathrm{p}, 42.62 . \mathrm{Fi}$

In conventional methods of resonant spectroscopy, optically thin ensembles of atoms or molecules are probed with lasers of limited intensity [1]. Low intensities are used in order to avoid power broadening and frequency shifts. In general, as the Rabi frequency of the resonant electromagnetic field exceeds the natural width of the transition under consideration, the width of the resonance increases, resulting in a rapid loss of resolution. This effect is especially profound when transitions between long-lived states are considered, in which case the necessity of low-intensity light fields results in an unfavorably low signal-to-noise ratio [2]. An example of such a resonance is a two-photon transition in a $\Lambda$-type atomic configuration [3].

In the present Letter we study experimentally and describe theoretically the spectroscopic properties of dense ensembles of atoms coherently prepared and probed by strong optical fields. We observe and discuss the narrowing of power-broadened dark resonances [4,5] associated with electromagnetically induced transparency (EIT). Moreover, when the atomic density becomes sufficiently large, we observe novel, even narrower structures superimposed on the EIT line. We find that these resonances are due to interference induced by a new field component arising from resonantly enhanced coherent Raman scattering [6]. We show that, in contrast to simple transmission measurements, the characteristics of these novel spectral features are determined by the dispersive properties of the dense phase-coherent medium [7]. In particular, their width can be orders of magnitude smaller than the usual power-broadened limit; that is, power broadening can be compensated to a large extent. This makes feasible a new regime of two-photon spectroscopy, in which high resolution can be achieved with higher intensities and therefore with lower noise than in conventional methods.

Before proceeding we note that it has already been recognized that sometimes it is advantageous to do spec- troscopy in optically thick samples [8]. As will be seen, the essential new features of the present system are twofold: the absorption cancellation due to quantum interference [5] and the importance of the dispersive and nonlinear optical properties of a coherently prepared medium. The present technique is also related to a coherent anti-Stokes Raman spectroscopy (CARS) [6]. Our method, however, differs qualitatively from the latter since in our case it is essential to operate near the single photon resonance. As a result the spectral resolution is determined mostly by the linear susceptibility rather than the $\chi^{(3)}$ nonlinearity. We further note that the advantage of using the dispersion of EIT resonances for spectroscopic measurements has been pointed out in a recent group delay experiment [9]. Our approach is conceptually different in that it utilizes a combination of linear and nonlinear response on cw fields.

The conceptual foundations of the present work can be understood by considering a simple 3-level $\Lambda$ system as shown in Fig. 1(a). A resonant driving field on the $a \rightarrow c$ transition with complex amplitude $E=E_{0} e^{-i \omega_{d} t}$ and Rabi frequency $\Omega=\wp_{a c} E_{0} / 2 \hbar$ produces symmetric Autler-Townes dressed states with a frequency separation
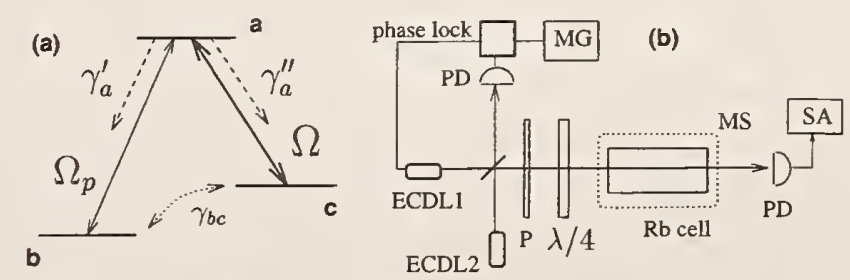

FIG. 1. (a) Simplified 3-level model for EIT. (b) Experimental setup (schematic). ECDLl and ECDL2 are extended-cavity diode lasers (probe and driving lasers, respectively). PD: photodetector, $\mathrm{P}$ : polarizer, $\mathrm{MG}$ : microwave synthesizer, and SA: spectrum analyzer. The Rb cell is inside magnetic shields. 
of $2 \Omega . \wp_{a c}$ is the dipole moment of the $a \rightarrow c$ transition and $\omega_{d}$ the drive-field frequency. Interference of transitions from the ground state to the two dressed states causes a reduction of absorption of a probe field $E_{p}$ tuned to the center of the $b \rightarrow a$ transition. This is the essence of dark resonances [4] and EIT [5]. The EIT window in the single-atom response is power broadened and its spectral width approaches $2 \Omega$. As we will now demonstrate, this conclusion is not valid in the case of transmission through a thick atomic ensemble [10].

The spectroscopic properties of a dense vapor of coherent $\Lambda$-type $\mathrm{Rb}$ atoms were studied in the experiment indicated in Fig. I(b). Two extended-cavity diode lasers, a drive laser and a probe laser, were phase locked with a frequency offset $\left(\omega_{0} \approx \omega_{\mathrm{HFS}}=6.83 \mathrm{GHz}\right)$ determined by a tunable microwave-frequency synthesizer. Two collinear laser beams were passed through a $5 \mathrm{~cm}$ long cell containing natural $\mathrm{Rb}$, and the transmitted power was detected by a fast photodetector (PD). Both beams were of identical circular polarization. The powers of the drive and probe beams in the cell ranged from 5 to $10 \mathrm{~mW}$ and 0.05 to $0.1 \mathrm{~mW}$ with spot sizes of 5 and $3 \mathrm{~mm}$, respectively. The frequency of the drive laser was tuned to the center of the $S_{1 / 2}, F=2 \rightarrow P_{1 / 2}, F=2$ transition of the $\mathrm{Rb} D_{1}$ line, while the frequency of the probe laser was scanned across the $S_{1 / 2}, F=1 \rightarrow P_{1 / 2}, F=2$ transition by tuning the synthesizer frequency. In such a configuration probe and drive fields create a $\Lambda$-type system [Fig. 1(a)] within the Rb $D_{1}$ manifold.

In the present experiment the signal was recorded by monitoring the amplitude of the time-dependent component of the detector current at frequency $\omega_{0}=\omega_{p}-\omega_{d}$ corresponding to the difference frequency between probe and drive fields. In the case of a dilute medium the heterodyne signal at the photo detector is proportional to the transmitted probe-field amplitude.

At relatively low values of the atomic density the observed signal shows the usual absorptive features corresponding to EIT [Fig. 2(a)]. When the beat-note frequency of the lasers matches the resonant frequency of the $b \rightarrow c$ transition, the medium becomes transparent displaying an EIT-type resonance [curve $i$ in Fig. 2(a)] with a nearly symmetric line shape. The peculiar feature of this resonance is that its width decreases with the atomic density. At higher densities the transparency window becomes substantially asymmetric. Within this window, additional few kilohertz-wide resonances [Fig. 2(b)] were clearly resolved. Their characteristics such as position, width, and amplitude depend on the strength of the drive field and on the atomic density. Furthermore their position was found to be very sensitive to atomic level shifts, induced, for example, by weak magnetic fields. Under the conditions of dense media, resonances as narrow as $3 \mathrm{kHz}$ were observed with a high signal-to-noise ratio. For the data presented here, the estimated Rabi frequencies of drive and probe fields were correspondingly on the order of 10 and $2 \mathrm{MHz}$.
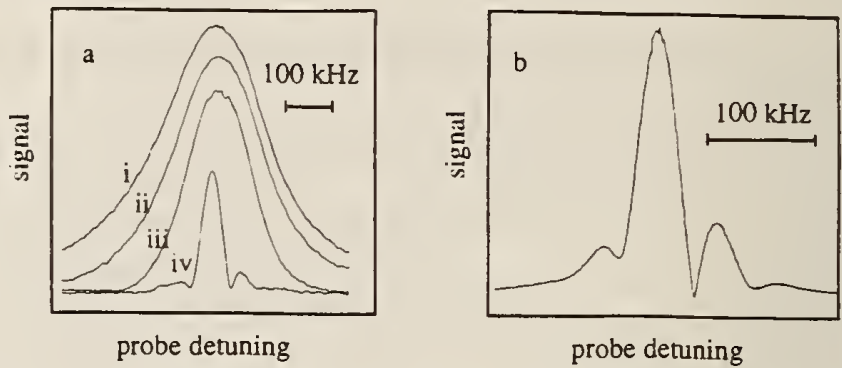

FIG. 2. Measured signal as a function of a relative laser detuning for various atomic densities. (a) Curves $i-i v$ correspond to atomic densities $N \sim 6 \times 10^{10}, 1 \times 10^{11}, 2 \times 10^{13}$, and $6 \times 10^{11} \mathrm{~cm}^{-3}$, respectively. Estimated Rabi frequencies are 10 and $2 \mathrm{MHz}$ for drive and probe. (b) Extra resonances in optically dense $\mathrm{Rb}$ vapor. Drive-field Rabi frequency is $13 \mathrm{MHz}$ and atomic density is $\sim 10^{12} \mathrm{~cm}^{-3}$. Scan time was a few seconds. The estimated time-of-flight broadening in the probe and drive beams is $\sim 15$ and $\sim 8 \mathrm{kHz}$, respectively. There is no external magnetic field. Natural linewidth of the $\mathrm{Rb} D_{1}$ line is 5.4 MHz. Doppler broadening is $\sim 500 \mathrm{MHz}$.

The narrowing of the dark resonances with increasing density has a nature similar to that of Ref. [8] and can easily be understood from an idealized 3-level model in which probe and drive interact solely with the respective transitions $a \rightarrow b$ and $a \rightarrow c$. For simplicity let us assume, in addition, a spatially homogeneous Rabi frequency of the drive field. The intensity transmission of a monochromatic probe of wavelength $\lambda$ through a vapor cell of length $L$ is described by $T=\exp \left\{-\chi^{\prime \prime} k L\right\}$. Here $k=2 \pi / \lambda$ and $\chi^{\prime \prime}$ is the imaginary part of the susceptibility $\chi$, which for the idealized. homogeneously broadened 3-level $\Lambda$ system and a weak $\left(\Omega_{p}<\Omega\right)$ field is given by [5]

$$
\chi=\eta \gamma_{a}^{\prime} \frac{i \gamma_{b c}-\left(\Delta-\Delta_{d}\right)}{\left.\left.\left[\gamma_{a b}+i\right\lrcorner\right]\left[\gamma_{b c}+i(\lrcorner-\Delta_{d}\right)\right]+\Omega^{2}},
$$

where $\eta=3 N \lambda^{3} / 4 \pi^{2}, N$ is the atomic density, $\Delta$ is the detuning of the probe laser, and $\Delta_{d}$ is the detuning of the drive laser. $\gamma_{a}^{\prime}, \gamma_{a}^{\prime \prime}$ are the radiative decay rates as per Fig. I(a), and $\gamma_{\mu \nu}$ are the decay rates of the respective coherences.

In the low density limit $(\eta k L \ll 1)$ the spectral features of the transmission coincide exactly with those of $\chi^{\prime \prime}$. In the high density limit, however, only spectral components which are very close to the center of the transparency are transmitted. In order to estimate the effective width of the EIT window, we expand $\chi^{\prime \prime}$ around the point of maximum transmission $[\Delta=(1-$ $\left.\gamma_{b c} / \gamma_{a b}\right) \Delta_{d}$ ] assuming the usual EIT conditions: $\Omega^{2} \gg$ $\gamma_{a b} \gamma_{b c}, \Delta_{d} \gamma_{b c}, \Delta_{d}^{2} \gamma_{b c} / \gamma_{a b}$, and $\gamma_{a b} \gg \gamma_{b c}$. The resulting transmission spectrum is a Gaussian function of the detuning. Its characteristic half width

$$
\lrcorner \omega_{\text {trans }}=\frac{\Omega^{2}}{\sqrt{\gamma_{a b} \gamma_{a}^{\prime}}} \frac{1}{\sqrt{\eta k L}}
$$

scales inversely with the square root of the density-length product and can become substantially smaller than the single-atom power-broadened width. 
Let us now turn to the additional resonances. We show that these resonances are due to a new field generated by resonantly enhanced coherent Raman scattering [see Fig. 3(a) and Ref. [11]] and that they reflect the dispersive properties of the medium. The mechanism of this process can be understood if we note that the strong driving field couples the excited state to both ground state hyperfine levels. The interaction of the drive and probe fields with the resonant transitions generates coherence between the ground states. In the presence of this coherence, the interaction of the drive with the offresonant transition $S_{1 / 2}, F=1 \rightarrow P_{1 / 2}, F=2$ leads to a nonlinear generation of a new field $E_{n}$ with frequency $\omega_{n}=2 \omega_{d}-\omega_{p}$. This process is very similar to the recently observed frequency conversion in coherent atomic media in Ref. [12]. For a large density-length product, the output amplitude of the new field $E_{n}$ can be of the same order as that of the probe field, $E_{p}$. At the photodetector, the new field gives rise to an additional component to the beat note at frequency $\omega_{0}$. Taking this contribution into account we find that the signal power at $\omega_{0}$ in the present heterodyne detection scheme is given by

$$
S_{\text {sig }}^{2} \propto\left|E_{d} E_{n}^{*}+E_{d}^{*} E_{p}\right|^{2} .
$$

To understand the nature of the additional resonances, in particular, their relatively narrow width, let us consider again the simplified 3-level model discussed before. We now, however, take the nonresonant couplings and the associated parametric process into account. Let us further assume that the probe and "new" field are much weaker than the drive field, and that the drive field $\left(\Delta_{d}=0\right)$ is undepleted. These assumptions (which will not be used in the later numerical simulations) allow for a simplified discussion of the relevant physics. In this case we arrive at the system of equations

$$
\frac{2}{k} \frac{\partial}{\partial z}\left[\begin{array}{l}
E_{p} \\
E_{n}^{*}
\end{array}\right]=\left[\begin{array}{ll}
a_{11} & a_{12} \\
a_{21} & a_{22}
\end{array}\right]\left[\begin{array}{c}
E_{p} \\
E_{n}^{*}
\end{array}\right] .
$$

The coefficient $a_{11}$ is proportional to the linear susceptibility of the resonant field $E_{p}$ and $a_{22}$ to that of the off-resonant field $E_{n}$. The cross-coupling coefficients $a_{12}$ and $a_{21}$ describe the resonantly enhanced $\chi^{(3)}$-type nonlinearity. Under near-resonance conditions and in the absence of Doppler broadening they are given by $a_{11}=$
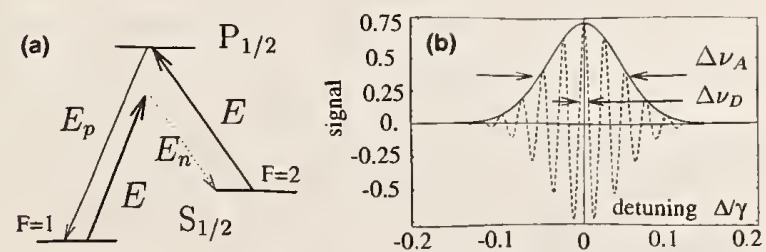

FIG. 3. (a) 3-wave mixing leading to parametric generation of field $E_{n}$ within the $\mathrm{Rb} D_{1}$ line. (b) Illustration of the difference between absorption and dispersion spectroscopy based on EIT in optically dense medium. Solid curve (characteristic width $\Delta \nu_{A}$ ) illustrates typical absorption signal, while dashed curve (width $\Delta \nu_{D}$ ) shows the dispersion signal. $i \chi \approx-i \eta \gamma_{a}^{\prime}\left(\Delta-\Omega^{2} / \omega_{0}\right) / \Omega^{2}-\eta \gamma_{a}^{\prime} \gamma_{b c} / \Omega^{2}, a_{22} \approx$ $-i \eta \gamma_{a}^{\prime \prime} / 2 \omega_{0}$, and $a_{12}=a_{21}^{*} \approx-i \eta \gamma_{a}^{\prime} / \omega_{0} \times E_{d}^{2} /\left|E_{d}\right|^{2}$. Solving this linear propagation problem and substituting the solution into Eq. (3) we find that the beat note at the photodetector contains an interference term with an amplitude depending on the probe laser detuning via the phase shift $\Delta \phi=\chi^{\prime} k L / 2$, which modulates the usual transmission profile and gives rise to extra resonances. Hence the characteristics of the new resonances are determined by the dispersive properties of the EIT medium [7].

Since the index of refraction close to the point of maximum transmission is a linear function of the probe detuning [7], the phase shift of the transmitted field is also linear in $\Delta$. For a large density-length product the phase shift accumulated over the cell length can easily surpass $2 \pi$ already for a very small detuning from the two-photon resonance. Hence the interference term $\left(\sim \exp \left\{-\chi^{\prime \prime} k L / 2\right\} \sin \Delta \phi\right)$ in the beat note at the photodetector rapidly oscillates with $\Delta$ as indicated in Fig. 3(b). We define a characteristic width $\Delta \omega_{\text {dis }}$

$$
\Delta \omega_{\mathrm{dis}}=\pi \frac{\Omega^{2}}{\gamma_{a}^{\prime}} \frac{1}{\eta k L},
$$

as the detuning from the line center at which the phase of a probe laser shifts by $\pi / 2$. This expression scales inversely with the density-length product itself instead of its square root as in Eq. (2). Thus we conclude that in dense media the width of interferometric fringes ("dispersive" width $\Delta \omega_{\text {dis }}$ ) can be considerably smaller than the characteristic width of the EIT transmission Eq. (2). Because of the parametric generation and the homodyne detection technique both "absorptive" and "dispersive" widths are observed in our experiment.

To make a detailed comparison with the experiment we consider a theoretical model in which the 3-wave mixing process is included together with Doppler broadening and the full (hyperfine) level structure of $\mathrm{Rb}^{87}$. The propagation equations for the three fields were solved numerically. The atomic polarization was calculated from the density matrix equations with a Floquet ansatz. To truncate the resulting hierarchy, the beat note frequency $\omega_{0}$ was assumed to be large compared to the relevant decay rates and Rabi frequencies. As shown in Fig. 4 the result of this calculation is in good agreement with the experimental data. In particular, the additional narrow resonances indeed have a width close to the "dispersive" width in a dense medium, Eq. (5). Finally, the observation of the extra resonances coincided with the appearance of a new field component $E_{n}$ as was verified by making a beat note between the output field and an independent laser.

Before concluding we remark on the possible implications of the compensation of power broadening in dense media. To this end we estimate the theoretical limit of this compensation by considering an ideal 3-level $\Lambda$ type medium with a large density-length product, such that the probe-field intensity is attenuated at line center by $1 / e$. In this case, which corresponds to a maximum 


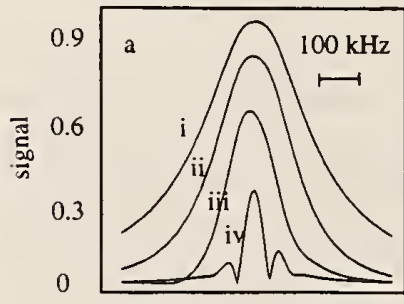

probe detuning

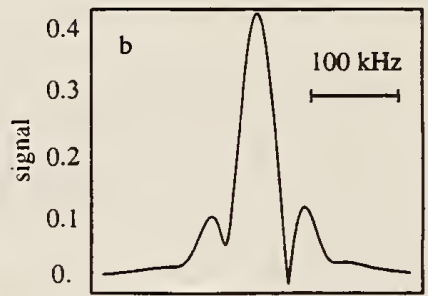

probe detuning
FIG. 4. Calculated signal [Eq. (3)] as a function of probe detuning. (a) Curves $i-i v$ correspond to atomic densities of the Fig. 3(a). (b) Extra resonances under the conditions corresponding to those of Fig. 3(b). All parameters correspond to Fig. 3. Relaxation rate of ground state coherences is taken to be $15 \mathrm{kHz}$, and relaxation rate of ground state populations is $8 \mathrm{kHz}$. Signal is normalized such that unity corresponds to undepleted probe and drive fields.

signal-to-noise ratio at the output, the "absorptive" and "dispersive" widths approach

$$
\Delta \omega_{\text {trans }} \rightarrow \Omega \sqrt{\frac{\gamma_{b c}}{\gamma_{a b}}}, \quad \Delta \omega_{\text {dis }} \rightarrow \pi \gamma_{b c} .
$$

which implies that under idealized conditions the effect of power broadening can be completely compensated in a dispersive measurement [13]. For a realistic experimental situation this conclusion is certainly too optimistic due to light shifts from off-resonant levels and/or due to effects of collisional broadening of the ground state transition (e.g., for the maximal laser power used in our experiments residual power broadening of the resonances became observable at about $\sim 20 \mathrm{kHz}$ ). In general these effects limit the "useful" laser intensity [14]. We further note that the particular heterodyne detection scheme used here yields a rather complicated, asymmetric line shape and is therefore not ideal for the determination of the atomic line center. It is, however, likely that modification of the detection scheme [15] can lead to more symmetric line shape and therefore to improved precision for the line center determination.

In conclusion, we have shown that a spectroscopic technique based on dense ensembles of coherent atoms can be much less sensitive to power broadening, and thus may combine high resolution with large intensities resulting in an enhanced signal-to-noise ratio.

The authors gratefully acknowledge useful discussions with E. Arimondo, Yu. Rostovtsev, and G. Welch, the valuable assistance of $\mathrm{T}$. Zibrova, and the support from the Office of Naval Research, the Welch Foundation, the Texas Advanced Research and Technology Program, and the Air Force Office of Scientific Research. M.F. thanks the Alexander-von-Humboldt foundation for financial support.

[1] W. Demtröder, Laser Spectroscopy: Basic Concepts and Instrumentation (Springer, Berlin, 1996).
[2] J. Vanier and C. Audoin, The Quantum Physics of Atomic Frequency Standards (A. Hilger, Bristol, 1989).

[3] The narrow features of two-photon transitions were exploited in many experiments for optically thin samples and weak fields: M.S. Feld and A. Javan, Phys. Rev. 177, 177 (1964); W. Gawlik et al., Phys. Lett. 48A, 283 (1974); R. P. Hackel and S. Ezekiel, Phys. Rev. Lett. 42, 1736 (1979); J.E. Thomas et al., Phys. Rev. Lett. 48, 867 (1982); M. Kailova et al., Phys. Rev. A 32, 207 (1985); A. Akulshin, A. Celikov, and V. Velichansky, Opt. Commun. 84, 139 (1991).

[4] G. Alzetta, A. Gozzini, L. Moi, and G. Orriols, Nuovo Cimento 36B, 5 (1976); E. Arimondo and G. Orriols, Nuovo Cimento Lett. 17, 333 (1976); H.R. Gray, R.M. Whitley, and C.R. Stroud, Jr., Opt. Lett. 3, 218 (1978).

[5] K.-J. Boller, A. Imamoğlu, and S.E. Harris, Phys. Rev. Lett. 66, 2593 (1991); for review see S.E. Harris, Phys. Today 50, No. 7, 36 (1997).

[6] Y.R. Shen, The Principles of Nonlinear Optics (John Wiley \& Sons, New York, 1984).

[7] Dispersive properties of coherent media have been studied in M. O. Scully, Phys. Rev. Lett. 67, 1855 (1991); S.E. Harris, J.E. Field, and A. Kasapi, Phys. Rev. A 46, R29 (1992); M. Xiao et al., Phys. Rev. Lett. 74, 666 (1995); R. Moseley et al., Phys. Rev. Lett. 74, 670 (1995); O. Schmidt et al., Phys. Rev. A 53, R27 (1996); A. S. Zibrov et al., Phys. Rev. Lett. 76, 3935 (1996).

[8] S. Svanberg et al., Opt. Lett. 11, 138 (1986); S. Svanberg et al., J. Opt. Soc. Am. B 4, 462 (1987); O. Di LorenzoFilho et al., Opt. Lett. 16, 1768 (1991).

[9] A. Kasapi et al., Phys. Rev. A 53, 4547 (1996).

[10] Various aspects of propagation in dense EIT media were studied in S.E. Harris, Phys. Rev. Lett. 72, 52 (1994); M. Fleischhauer, Phys. Rev. Lett. 72, 989 (1994); M. Jain et al., Phys. Rev. Lett. 75, 4385 (1995); A. Kasapi et al., Phys. Rev. Lett. 74, 2447 (1995); S. E. Harris and ZhenFei Luo, Phys. Rev. A 52, R928 (1995); M. B. Gomy, B.G. Matisov, and Yu. V. Rozhdestvenskii, Sov. Phys. JETP 68, 728 (1989).

[11] Y. Li and M. Xiao, Opt. Lett. 21, 1064 (1996).

[12] M. Jain et al., Phys. Rev. Lett. 77, 4326 (1996); we also point out the relevant studies of P. Hemmer et al., Opt. Lett. 20, 982 (1995).

[13] M. O. Scully and M. Fleischhauer, Phys. Rev. Lett. 69, 1360 (1992).

[14] We note here that our results indicate that the ac-stark shift and accompanying power broadening due the offresonant $\left(\Delta_{\mathrm{HFS}} \sim 800 \mathrm{MHz}\right)$ states of the $F=1, P_{1 / 2}$ hyperfine manifold is substantially reduced for the present choice of polarizations, since the dark states associated with two different upper levels $P_{1 / 2}, F=1$ and $F=2$ are identical. For example, for parameters of Fig. 2 a naive estimate yields for shifts and broadening values $\Omega^{2} / \Delta_{\mathrm{HFS}} \sim 200 \mathrm{kHz}$, which is more than an order of magnitude larger than the experimentally observed value.

[15] For example, detecting the generated field itself can combine a symmetric line shape with a resolution given by the dispersive width. 


\section{MIST Technical Publications}

\section{Periodical}

Journal of Research of the National Institute of Standards and Technology-Reports NIST research and development in those disciplines of the physical and engineering sciences in which the Institute is active. These include physics, chemistry, engineering, mathematics, and computer sciences. Papers cover a broad range of subjects, with major emphasis on measurement methodology and the basic technology underlying standardization. Also included from time to time are survey articles on topics closely related to the Institute's technical and scientific programs. Issued six times a year.

\section{Nonperiodicals}

Monographs-Major contributions to the technical literature on various subjects related to the Institute's scientific and technical activities.

Handbooks-Recommended codes of engineering and industrial practice (including safety codes) developed in cooperation with interested industries, professional organizations, and regulatory bodies.

Special Publications-Include proceedings of conferences sponsored by NIST, NIST annual reports, and other special publications appropriate to this grouping such as wall charts, pocket cards, and bibliographies.

Applied Mathematics Series-Mathematical tables, manuals, and studies of special interest to physicists, engineers, chemists, biologists, mathematicians, computer programmers, and others engaged in scientific and technical work.

National Standard Reference Data Series-Provides quantitative data on the physical and chemical properties of materials, compiled from the world's literature and critically evaluated. Developed under a worldwide program coordinated by NIST under the authority of the National Standard Data Act (Public Law 90-396). NOTE: The Journal of Physical and Chemical Reference Data (JPCRD) is published bimonthly for NIST by the American Chemical Society (ACS) and the American Institute of Physics (AIP). Subscriptions, reprints, and supplements are available from ACS, 1155 Sixteenth St., NW, Washington, DC 20056.

Building Science Series-Disseminates technical information developed at the Institute on building materials, components, systems, and whole structures. The series presents research results, test methods, and performance criteria related to the structural and environmental functions and the durability and safety characteristics of building elements and systems.

Technical Notes-Studies or reports which are complete in themselves but restrictive in their treatment of a subject. Analogous to monographs but not so comprehensive in scope or definitive in treatment of the subject area. Often serve as a vehicle for final reports of work performed at NIST under the sponsorship of other government agencies.

Voluntary Product Standards-Developed under procedures published by the Department of Commerce in Part 10, Title 15, of the Code of Federal Regulations. The standards establish nationally recognized requirements for products, and provide all concerned interests with a basis for common understanding of the characteristics of the products. NIST administers this program in support of the efforts of privatesector standardizing organizations.

Consumer Information Series-Practical information, based on NIST research and experience, covering areas of interest to the consumer. Easily understandable language and illustrations provide useful background knowledge for shopping in today's technological marketplace.

Order the above NIST publications from: Superintendent of Documents, Government Printing Office, Washington, DC 20402.

Order the following NIST publications-FIPS and NISTIRs-from the National Technical Information Service, Springfield, VA 22161.

Federal Information Processing Standards Publications (FIPS PUB)-Publications in this series collectively constitute the Federal Information Processing Standards Register. The Register serves as the official source of information in the Federal Government regarding standards issued by NIST pursuant to the Federal Property and Administrative Services Act of 1949 as amended, Public Law 89-306 (79 Stat. 1127), and as implemented by Executive Order 11717 (38 FR 12315, dated May 11, 1973) and Part 6 of Title 15 CFR (Code of Federal Regulations).

NIST Interagency Reports (NISTIR)-A special series of interim or final reports on work performed by NIST for outside sponsors (both government and non-government). In general, initial distribution is handled by the sponsor; public distribution is by the National Technical Information Service, Springfield, VA 22161, in paper copy or microfiche form. 
U.S. Department of Commerce

National Institute of Standards and Technology

325 Broadway

Boulder, Colorado 80303-3328

Official Business

Penalty for Private Use, $\$ 300$ 